ANLESD/TM-30, Vol. 2

\title{
Experimental Evaluation of Oxygen-Enriched Air and Emulsified Fuels in a Single-Cylinder Diesel Engine
}

\section{Volume 2: Data Sets}

by R.R. Sekar, W.W. Marr, R.L. Cole, and T.J. Marciniak

Energy Systems Division,

Argonne National Laboratory, 9700 South Cass Avenue, Argonne, Illinois 60439

November 1991

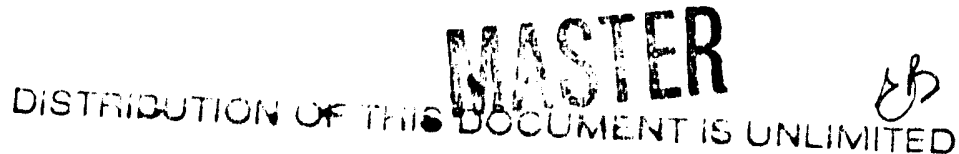

Work sponsored by United States Department of Energy,

Assistant Secretary for Conservation and Renewable Energy, Office of Industrial Technologies 


\section{Contents}

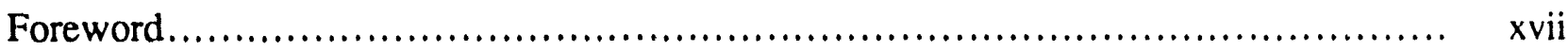

Appendix A: Data Package of Diesel Engine Tests with Oxygen-Enriched Air and

Water-Emulsified Fuels: Series I Tests ...............................

A.1 Tabulation of Test Data in the Order of Test Sequence ....................... A-3

A.2 Grouping of Test Data................................................. A-11

A.3 Graphic Display of Test Data for No. 2 Fuel ................................ A-17

A.4 Graphic Display of Test Data for No. 4 Fuel .................................. A-29

A.5 Comparison of Test Data for No. 2 and No. 4 Fuels......................... A-39

A.6 Graphic Display of Cylinder Pressures and Results of Heat-Release Analyses for No. 2 Fuel ........................................................ A -55

A.7 Graphic Display of Cylinder Pressures and Results of Heat-Release Analyses for No. 4 Fuel ........................................................ A 69

A.8 Comparison of Calculated Ignition Delays for No. 2 and No. 4 Fuels........... A-81

A.9 Graphic Display of Thermal Efficiency for No. 2 Fuel............................ A-89

A.10 Graphic Display of Thermal Efficiency for No. 4 Fuel........................... A-95

A.11 Comparison of Thermal Efficiency for No. 2 and No. 4 Fuels ................. A-101

A.12 Graphic Display of Smoke Emissions................................... A-109

Appendix B: Data Package of Diesel Engine Tests with Oxygen-Enriched Air and

Water-Emulsified Fuels: Series II Tests .............................. B-1

B.1 Tabulation of Test Data as a Function of Injection Time ...................... B-3

B.2 Results of Analyses of Data from Emissions Repeatability Tests ............... B-21

B.3 Graphic Display of Test Data for No. 2 Fuel .............................. B-29

B.4 Graphic Display of Cylinder Pressure Data and Results of Heat-Release Analysis for No. 2 Fuel ............................................. B-57

B.5 Graphic Display of Data from Emissions Repeatability Tests .................. B-79

B.6 Graphic Display of Test Data for No. 4 Fuel .............................. B 85

B.7 Graphic Display of Cylinder Pressure Data and Results of Heat-Release Analysis for No. 4 Fuel .............................................. B-105

\section{Figures}

A.1 Maximum Attainable Power vs. Oxygen Concentration, No. 2 Fuel ................. A-19

A.2 Maximum Attainable Power vs. Oxygen Concentration, No. 4 Fuel ................. A-19

A.3 Effect of Oxygen Level on Maximum Power Output and Peak Cylinder Pressure, Half Load .................. .................................. A-20

A.4 Maximum Attainable Power, No. 2 Fuel-Water Emulsion........................ A-20) 


\section{Figures (Cont'd)}

A.5 Nitrogen Oxides vs. Brake Horsepower, No. 2 Fuel, 50\% Load, No Water........ A-21

A.6 Nitrogen Oxides vs. Brake Horsepower, No. 2 Fuel, $100 \%$ Load, No Water...... A-21

A.7 Nitrogen Oxides vs. Water Content, No. 2 Fuel, $21 \%$ Oxygen.................... A-22

A.8 Nitrogen Oxides vs. Water Content, No. 2 Fuel, 50\% Load, 25\% Oxygen ......... A-22

A.9 Nitrogen Oxides vs. Water Content, No. 2 Fuel, 50\% Load, 35\% Oxygen ........ A-23

A.10 Nitrogen Oxides vs. Water Content, No. 2 Fuel, 50\% Load, 30\% Oxygen .......... A-23

A.11 Nitrogen Oxides vs. Water Content, No. 2 Fuel, $100 \%$ Load, 30\% Oxygen......... A-24

A.12 Nitrogen Oxides vs. Water Content, No. 2 Fuel, 100\% Load, 35\% Oxygen........ A-24

A.13 Particulate Matter vs. Water Content, No. 2 Fuel, 21\% Oxygen...................... A-25

A.14 Particulate Matter vs. Water Content, No. 2 Fuel, 100\% Load, 35\% Oxygen...... A-25

A.15 Particulate Matter vs. Brake Horsepower, No. 2 Fuel, 50\% Load, No Water...... A-26

A.16 Particulate Matter vs. Brake Horsepower, No. 2 Fuel, 100\% Load, No Water .... A-26

A.17 Ignition Delay vs. Water Content, No. 2 Fuel, 100\% Load, 25\% Oxygen ........... A-27

A.18 Maximum Attainable Power, No. 4 Diesel Fuel ................................... A-31

A.19 Nitrogen Oxides vs. Oxygen Concentration, No. 4 Fuel, No Water............... A-31

A.20 Nitrogen Oxides vs. Brake Horsepower, No. 4 Fuel, $100 \%$ Load, No Water ...... A-32

A.21 Nitrogen Oxides vs. Water Content, No. 4 Fuel, $21 \%$ Oxygen..................... A-32

A.22 Nitrogen Oxides vs. Water Content, No. 4 Fuel, 50\% Load, 25\% Oxygen ......... A-33

A.23 Nitrogen Oxides vs. Water Content, No. 4 Fuel, 50\% Load, 35\% Oxygen......... A-33

A.24 Nitrogen Oxides vs. Water Content, No. 4 Fuel, 100\% Load, 35\% Oxygen........ A-34

A.25 Particulate Matter vs. Brake Horsepower, No. 4 Fuel, 50\% Load, No Water...... A-34

A.26 Particulate Matter vs. Brake Horsepower, No. 4 Fuel, 100\% Load, No Water ..... A-35

A.27 Particulate Matter vs. Water Content, No. 4 Fuel, 21\% Oxygen..................... A-35

A.28 Particulate Matter vs. Water Content, No. 4 Fuel, 50\% Lcad, 25\% Oxygen ......... A-36

A.29 Particulate Matter vs. Water Content, No. 4 Fuel, 50\% Load, 30\% Oxygen........ A-36

A.30 Particulate Matter vs. Water Content, No. 4 Fuel, 100\% Load, 25\% Oxygen....... A-37

A.31 Particulate Matter vs. Water Content, No. 4 Fuel, $100 \%$ Load, 30\% Oxygen....... A-37

A.32 Particulate Matter vs. Water Content, No. 4 Fuel, $100 \%$ Load, 35\% Oxygen....... A-38

A.33 Particulate Matter vs. Water Content, No. 4 Fuel, 50\% Load, 35\% Oxygen ........ A-38

A.34 Nitrogen Oxides vs. Brake Horsepower, 50\% Load, No Water, 25\% Oxygen ..... A-41

A.35 Nitrogen Oxides vs. Brake Horsepower, 50\% Load, No Water, 30\% Oxygen ..... A-41

A.36 Nitrogen Oxides vs. Brake Horsepower, 50\% Load, No Water, 35\% Oxygen .... A-42

A.37 Nitrogen Oxides vs. Brake Horsepower, $100 \%$ Load, No Water,

$25 \%$ Oxygen ......................................................... A 42

A.38 Nitrogen Oxides vs. Brake Horsepower, $100 \%$ Load, No Water, $35 \%$ Oxygen ..................................................... A 43

A.39 Nitrogen Oxides vs. Water Content, 50\% Load, 25 Horsepower, $30 \%$ Oxygen

A -43

A.40 Nitrogen Oxides vs. Water Content, 50\% Load, 25 Horsepower,

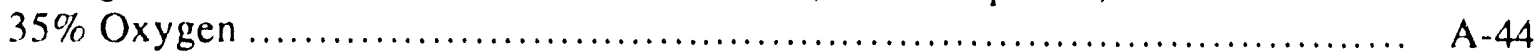

A.41 Nitrogen Oxides vs. Water Content, 50\% Load, 45 Horsepower, $35 \%$ Oxygen 


\section{Figures (Cont'd)}

A.42 Nitrogen Oxides vs. "Nater Content, 50\% Load, 60 Horsepower, $30 \%$ Oxygen

A.43 Nitrogen Oxides vs. Water Content, $100 \%$ Load, 50 Horsepower, $21 \%$ Oxygen .

A.44 Nitrogen Oxides vs. Water Content, 100\% Load, 50 Horsepower, $35 \%$ Oxygen

A.45 Particulate Matter vs. Brake Horsepower, 50\% Load, No Water, $25 \%$ Oxygen

A.46 Particulate Matter vs. Brake Horsepower, 100\% Load, No Water, $30 \%$ Oxygen

A.47 Particulate Matter vs. Brake Horsepower, 50\% Load, No Water, $35 \%$ Oxygen

A.48 Particulate Matter vs. Brake Horsepower, $100 \%$ Load, No Water, $25 \%$ Oxygen

A.49 Particulate Matter vs. Brake Horsepower, 50\% Load, No Water, $30 \%$ Oxygen

A.50 Particulate Matter vs. Brake Horsepower, $100 \%$ Load, No Water, $35 \%$ Oxygen

A.51 Particulate-Matter Emissions, 50\% Load, 25 Brake Horsepower, No Water.

A.52 Particulate Matter vs. Water Content, 50\% Load, 25 Horsepower, $21 \%$ Oxygen

A.53 Particulate Matter vs. Water Content, 50\% Load, 25 Horsepower, $25 \%$ Oxygen

A.54 Particulate Matter vs. Water Content, 50\% Load, 25 Horsepower, $30 \%$ Oxygen

A.55 Particulate Matter vs. Water Content, 50\% Load, 25 Horsepower, $35 \%$ Oxygen

A.56 Particulate Matter vs. Water Content, 100\% Load, 50 Horsepower, $21 \%$ Oxygen

A.57 Particulate Matter vs. Water Content, 100\% Load, 50 Horsepower, $30 \%$ Oxygen

A.58 Particulate-Matter Emissions, 1U0\% Load, 50 Horsepower, 35\% Oxygen

A.59 Particulate Matter vs. Water Content, 50\% Load, 45 Horsepower, 35\% Oxygen

A.60 Particulate Matter vs. Water Content, 50\% Load, 60 Horsepower, $30 \%$ Oxygen

A.61 Peak Pressure, 100\% Load, 50 Horsepower, $21 \%$ Oxygen

A.62 Effect of Water Content on Slope of Cylinder Pressure Change, No. 2 Fuel, $100 \%$ Load, $21 \%$ Oxygen, \#37 vs. \#45

A.63 Effect of Oxygen Level and Water Content on Slope of Cylinder Pressure Change, No. 2 Fuel, $100 \%$ Load, \#37 vs. \#94 


\section{Figures (Cont'd)}

A.64 Effect of Water Content on Cylinder Pressure, No. 2 Fuel, 100\% Load, $21 \%$ Oxygen, \#37 vs. \#45

A.65 Effect of Oxygen Level and Power on Cylinder Pressure, No. 2 Fuel, $100 \%$ Load, No Water, \#37 vs. \#92.

A.66 Effect of Water Content and Oxygen Level on Cylinder Pressure, No. 4 Fuel, $100 \%$ Load, $21 \%$ Oxygen, \#28 vs. \#24

A.67 Effect of Water Content on Cumulative Heat Release, No. 2 Fuel, $100 \%$ Load, 50 Horsepower, $21 \%$ Oxygen

A.68 Effect of Oxygen Level and Power on Cumulative Heat Release, No. 2 Fuel, $100 \%$ Load, No Water.

A.69 Effect of Water Content and Oxygen Level on Cumulative Heat Release, No. 2 Fuel, 100\% Load, 50 Horsepower.

A.70 Effect of Oxygen Level, Water Content, and Power on Cumulative Heat Release, No. 2 Fuel, $100 \%$ Load .

A.71 Heat Release, No. 2 Fuel, 100\% Load, 50 Horsepower.............................. A-61

A.72 Heat Release, No. 2 Fuel, 100\% Load, 21\% Oxygen............................. A-62

A.73 Heat Release, No. 2 Fuel, 100\% Load, No Water.............................. A-62

A.74 Heat Release, No. 2 Fuel, 100\% Load, 50 Horsepower, No Water................... A-63

A.75 Heat Release, No. 2 Fuel, 100\% Load, 50 Horsepower......................... A-63

A.76 Heat Release, No. 2 Fuel, 100\% Load .......................................... A-64

A.77 Ignition Delay vs. Oxygen Concentration, No. 2 Fuel, No Water ................... A-64

A.78 Ignition Delay vs. Brake Horsepower, No. 2 Fuel, 50\% Load, No Water .......... A-65

A.79 Ignition Delay vs. Water Content, No. 2 Fuel, $100 \%$ Load, 30\% Oxygen .......... A-65

A.80 Ignition Delay vs. Water Content, No. 2 Fuel, 50\% Load, 25 Horsepower......... A-66

A.81 Ignition Delay vs. Water Content, No. 2 Fuel, 21\% Oxygen........................... A-66

A.82 Ignition Delay vs. Water Content, No. 2 Fuel, 50\% Load, 30\% Oxygen........... A-67

A.83 Ignition Delay vs. Water Content, No. 2 Fuel, 50\% Load, 35\% Oxygen........... A-67

A.84 Peak Cylinder Pressure, No. 4 Fuel, 100\% Load, No Water ....................... A-71

A.85 Pressure, No. 4 Fuel, 100\% Load, No Water, \#28 vs. \#102 ...................... A-71

A.86 Pressure, No. 4 Fuel, 100\% Load, No Water, \#28 vs. \#104 ...................... A-72

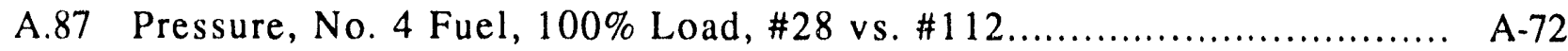

A.88 Slope of Cylinder Pressure Change, No. 4 Fuel, 100\% Load, 21\% Oxygen, \#28 vs. \#24

A.89 Heat Release, No. 4 Fuel, 100\% Load, 21\% Oxygen......................... A-73

A.90 Heat Release, No. 4 Fuel, 100\% Load, No Water, 50 Horsepower..................... A-74

A.91 Heat Release, No. 4 Fuel, 100\% Load, No Water............................... A-74

A.92 Heat Release, No. 4 Fuel, 100\% Load, 50 Horsepower.......................... A-75

A.93 Heat Release, No. 4 Fuel, 100\% Load ........................................ A-75

A.94 Heat Release, No. 4 Fuel, 100\% Load, 50 Horsepower............................ A-76

A.95 Cumulative Heat Release, No. 4 Fuel, 100\% Load, 50 Hor,epower,

$21 \%$ Oxygen 


\section{Figures (Cont'd)}

A.96 Cumulative Heat Release, No. 4 Fuel, 100\% Load, 50 Horsepower,

No Water

A-77

A.97 Cumulative Heat Release, No. 4 Fuel, 100\% Load, No Water.

A-77

A.98 Cumulative Heat Release, No. 4 Fuel, 100\% Load, 50 Horsepower

A-78

A.99 Cumulative Heat Release, No. 4 Fuel, 100\% Load.

A-78

A.100 Ignition Delay vs. Oxygen Concentration, No. 4 Fuel, No Water.

A-79

A.101 Ignition Delay vs. Brake Horsepower, No. 4 Fuel, 100\% Load, No Water.........

A-79

A.102 Ignition Delay vs. Water Content, No. 4 Fuel, $21 \%$ Oxygen.

A-80

A.103 Ignition Delay vs. Brake Horsepower, 50\% Load, No Water, 25\% Oxygen ........ A-83

A.104 Ignition Delay vs. Brake Horsepower, 50\% Load, No Water, 30\% Oxygen .........

A.105 Ignition Delay vs. Brake Horsepower, 50\% Load, No Water, 35\% Oxygen .........

A.106 Ignition Delay vs. Brake Horsepower, $100 \%$ Load, No Water, 30\% Oxygen........

A.107 Ignition Delay vs. Brake Horsepower, 100\% Load, No Water, 35\% Oxygen........

A.108 Ignition Delay vs. Water Content, 50\% Load, 25 Horsepower, $21 \%$ Oxygen........

A.109 Ignition Delay vs. Water Content, 50\% Load, 25 Horsepower, 30\% Oxygen........

A.110 Ignition Delay vs. Water Content, 50\% Load, 25 Horsepower, 35\% Oxygen.......

A.111 Ignition Delay vs. Water Content, 50\% Load, 45 Horsepower, 35\% Oxygen........

A.112 Ignition Delay vs. Water Content, $50 \%$ Load, 60 Horsepower, $30 \%$ Oxygen........

A.113 Ignition Delay vs. Water Content, $100 \%$ Load, 50 Horsepower, $30 \%$ Oxygen.....

A.114 Ignition Delay vs. Water Content, $100 \%$ Load, 50 Horsepower, 35\% Oxygen......

A.115 Thermal Efficiency vs. Brake Horsepower, No. 2 Fuel, 50\% Load, No Water.....

A.116 Thermal Efficiency vs. Water Content, No. 2 Fuel, 50\% Load, 25\% Oxygen .......

A.117 Thermal Efficiency vs. Water Content, No. 2 Fuel, 50\% Load, 35\% Oxygen .......

A.118 Thermal Efficiency vs. Brake Horsepower, No. 2 Fuel, 100\% Load,

No Water

A-83

A- 84

A- 84

A-85

A-85

A-86

A-86

A-87

A- 87

A- 88

A-88

A-91

A-91

A-92

A-92

A.119 Thermal Efficiency vs. Water Content, No. 2 Fuel, 100\% Load, 25\% Oxygen...... A-93

A.120 Thermal Efficiency vs. Water Content, No. 2 Fuel, 100\% Load, 35\% Oxygen..... A-93

A.121 Thermal Efficiency vs. Water Content, No. 2 Fuel, $100 \%$ Load,

50 Horsepower ..............................................................

A.122 Thermal Efficiency vs. Brake Horsepower, No. 4 Fuel, 50\% Load, No Water......

A.123 Thermal Efficiency vs. Water Content, No. 4 Fuel, 21\% Oxygen....................

A.124 Thermal Efficiency vs. Water Content, No. 4 Fuel, 50\% Load, 25\% Oxygen .......

A.125 Thermal Efficiency vs. Brake Horsepower, No. 4 Fuel, $100 \%$ Load,

No Water

A-94

A-97

A-97

A-98

A-98

4.126 Thermal Efficiency vs. Water Content, No. 4 Fuel, 100\% Load,

50 Horsepower

A- 99

A .127 Thermal Efficiency vs. Water Content, No. 4 Fuel, 100\% Load, 25\% Oxygen..... A-99

‘.128 Thermal Efficiency vs. Water Content, No. 4 Fuel, 100\% Load, 30\% Oxygen..... A-100

A.129 Thermal Efficiency vs. Water Content, No. 4 Fuel, $100 \%$ Load, 35\% Oxygen..... A-1(0)

A 130 Thermal Efficiency vs. Brake Horsepower, $50 \%$ Load, No Water, $25 \%$ Oxygen

A-103

A. 131 Thermal Efficiency vs. Brake Horsepower, 50\% Load, No Water, $30 \%$ Oxygen 


\section{Figures (Cont'd)}

A.132 Thermal Efficiency vs. Water Content, 50\% Load, 25 Horsepower, $21 \%$ Oxygen ....

A.133 Thermal Efficiency vs. Water Content, $50 \%$ Load, 25 Horsepower, $25 \%$ Oxygen .

A.134 Thermal Efficiency vs. Water Content, $50 \%$ Load, 45 Horsepower, $35 \%$ Oxygen ....

A.135 Thermal Efficiency vs. Oxygen Concentration, 50\% Load, 25 Horsepower, No Water

A.136 Thermal Efficiency vs. Brake Horsepower, $100 \%$ Load, No Water, $25 \%$ Oxygen

A.137 Thermal Efficiency vs. Brake Horsepower, $100 \%$ Load, No Water, $30 \%$ Oxygen

A.138 Thermal Efficiency vs. Brake Horsepower, 100\% Load, No Water, $35 \%$ Oxygen .

A.139 Thermal Efficiency vs. Oxygen Concentration, 100\% Load, 50 Horsepower, No Water.....

A.140 Thermal Efficiency vs. Water Content, 100\% Load, 50 Horsepower, $35 \%$ Oxygen

A.141 Smoke Emission vs. Water Content, No. 2 Fuel, 50\% Load, 25 Horsepower A-111

A.142 Smoke Emission vs. Water Content, No. 2 Fuel, 21\% Oxygen. A-111

A.143 Smoke Emission vs. Water Content, No. 2 Fuel, 100\% Load, 50 Horsepower.

A.144 Smoke Emission vs. Brake Horsepower, No. 2 Fuel, $100 \%$ Load, No Water. A-112

A.145 Smoke Emission vs. Water Content, No. 4 Fuel, 50\% Load, 25 Horsepower. A-113

A.146 Smoke Emission vs. Water Content, No. 4 Fuel, $21 \%$ Oxygen A-113

A.147 Smoke Emission vs. Water Content, No. 4 Fuel, $100 \%$ Load, 30\% Oxygen A-114

A.148 Smoke Emission vs. Water Content, No. 4 Fuel, 100\% Load, 35\% Oxygen A-114

A.149 Smoke Emission vs. Oxygen Concentration, 50\% Load, 25 Horsepower, No Water

A.150 Smoke Emission vs. Water Content, 50\% Load, 25 Horsepower, $30 \%$ Oxygen .

A.151 Smoke Emission vs. Brake Horsepower, 100\% Load, No Water, $35 \%$ Oxygen

B.1 Effect of Injection Timing on Exhaust Temperature, 25 Brake Horsepower, No. 2 Diesel, No Water

B.2 Effect of Injection Timing on Peak Cyïnder Pressure, 25 Brake Horsepower, No. 2 Diesel, No Water.

B.3 Effect of Injection Timing on Ignition Delay, 25 Brake Horsepower, No. 2 Diesel, No Water

B.4 Effect of Injection Timing on Thermal Efficiency, 25 Brake Horsepower, No. 2 Diesel, No Water 


\section{Figures (Cont'd)}

B.5 Effect of Injection Timing on Smoke Emission, 25 Brake Horsepower, No. 2

Diesel, No Water ........................................................

B-33

B.6 Effect of Injection Timing on Particulate Matter, 25 Brake Horsepower, No. 2

Diesel, No Water, 21\% Oxygen............................................. B-33

B.7 Effect of Injection Timing on Particulate Matter, 25 Brake Horsepower, No. 2 Diesel, No Water, 25\% Oxygen.................................. B-34

B.8 Effect of Injection Timing on $\mathrm{NO}_{\mathrm{x}}, 25$ Brake Horsepower, No. 2 Diesel, No Water, $21 \%$ Oxygen ....

B.9 Effect of Injection Timing on $\mathrm{NO}_{\mathrm{x}}, 25$ Brake Horsepower, No. 2 Diesel, No Water, $25 \%$ Oxygen ....

B-35

B.10 Effect of Injection Timing on Exhaust Air Flow, 25 Brake Horsepower, No. 2 Diesel, No Water, $21 \%$ Oxygen............................................

B.11 Effect of Injection Timing on Exhaust Air Flow, 25 Brake Horsepower, No. 2 Diesel, No Water, 25\% Oxygen.

B.12 Effect of Injection Timing on Brake-Specific Fuel Consumption, 25 Brake Horsepower, No. 2 Diesel, No Water, 21\% Oxygen.............................

B.13 Effect of Injection Timing on Brake-Specific Fuel Consumption, 25 Brake Horsepower, No. 2 Diesel, No Water, 25\% Oxygen...............................

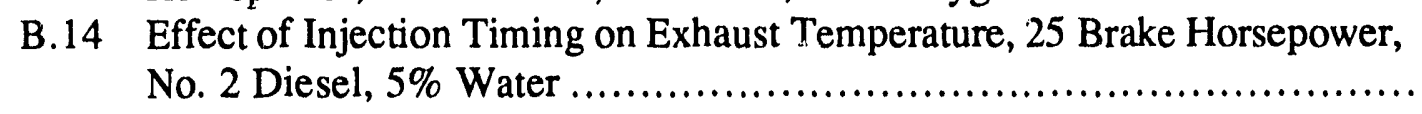

B.14 Effect of Injection Timing on Exhaust Temperature, 25 Brake Horsepower,
No. 2 Diesel, $5 \%$ Water $\ldots \ldots \ldots \ldots \ldots \ldots \ldots \ldots \ldots \ldots \ldots \ldots \ldots \ldots \ldots \ldots \ldots \ldots \ldots \ldots \ldots \ldots \ldots \ldots \ldots \ldots \ldots \ldots \ldots \ldots \ldots \ldots \ldots \ldots \ldots$

B.15 Effect of Injection Timing on Peak Cylinder Pressure, 25 Brake Horsepower, No. 2 Diesel, $5 \%$ Water.

B.16 Effect of Injection Timing on Ignition Delay, 25 Brake Horsepower, No. 2 Diesel, 5\% Water.....

B.17 Effect of Injection Timing on Thermal Efficiency, 25 Brake Horsepower, No. 2 Diesel, $5 \%$ Water.

B.18 Effect of Injection Timing on Smoke Emission, 25 Brake Horsepower, No. 2 Diesel, $5 \%$ Water.

B.19 Effect of Injection Timing on Particulate Matter, 25 Brake Horsepower, No. 2 Diesel, 5\% Water, $21 \%$ Oxygen.

B.20 Effect of Injection Timing on Particulate Matter, 25 Brake Horsepower, No. 2 Diesel, $5 \%$ Water, $26 \%$ Oxygen.

B.21 Effect of Injection Timing on $\mathrm{NO}_{\mathrm{x}}, 25 \mathrm{Brake}$ Horsepower, No. 2 Diesel, $5 \%$ Water, $21 \%$ Oxygen....................................................

B.22 Effect of Injection Timing on $\mathrm{NO}_{\mathrm{x}}, 25$ Brake Horsepower, No. 2 Diesel, $5 \%$ Water, $26 \%$ Oxygen.

B.23 Effect of Injection Timing on Exhaust Air Flow, 25 Brake Horsepower, No. 2 Diesel, $5 \%$ Water, $21 \%$ Oxygen

B.24 Effect of Injection Timing on Exhaust Air Flow, 25 Brake Horsepower, No. 2 Diesel, $5 \%$ Water, $26 \%$ Oxygen 


\section{Figures (Cont'd)}

B.25 Effect of Injection Timing on Brake-Specific Fuel Consumption, 25 Brake Horsepower, No. 2 Diesel, 5\% Water, 21\% Oxygen.

B.26 Effect of Injection Timing on Brake-Specific Fuel Consumption, 25 Brake Horsepower, No. 2 Diesel, 5\% Water, 26\% Oxygen.

B.27 Effect of Injection Timing on Exhaust Temperature, 50 Brake Horsepower, No. 2 Diesel, No Water

B.28 Effect of Injection Timing on Peak Cylinder Pressure, 50 Brake Horsepower, No. 2 Diesel, No Water

B.29 Effect of Injection Timing on Ignition Delay, 50 Brake Horsepower, No. 2 Diesel, No Water

B.30 Effect of Injection Timing on Thermal Efficiency, 50 Brake Horsepower, No. 2 Diesel, No Water

B.31 Effect of Injection Timing on Smoke Emission, 50 Brake Horsepower, No. 2 Diesel, No Water.

B.32 Effect of Injection Timing on Particulate Matter, 50 Brake Horsepower, No. 2 Diesel, No Water, $21 \%$ Oxygen.

B.33 Effect of Injection Timing on Particulate Matter, 50 Brake Horsepower, No. 2 Diesel, No Water, 25\% Oxygen.

B.34 Effect of Injection Timing on $\mathrm{NO}_{\mathrm{x}}, 50$ Brake Horsepower, No. 2 Diesel, No Water, $21 \%$ Oxygen....

B.35 Effect of Injection Timing on $\mathrm{NO}_{\mathrm{x}}, 50$ Brake Horsepower, No. 2 Diesel, No Water, $25 \%$ Oxygen...

B.36 Effect of Injection Timing on Exhaust Air Flow, 50 Brake Horsepower, No. 2 Diesel, No Water, $21 \%$ Oxygen.

B.37 Effect of Injection Timing on Exhaust Air Flow, 50 Brake Horsepower, No. 2 Diesel, No Water, $25 \%$ Oxygen.

B.38 Effect of Injection Timing on Brake-Specific Fuel Consumption, 50 Brake Horsepower, No. 2 Diesel, No Water, 21\% Oxygen

B.39 Effect of Injection Timing on Brake-Specific Fuel Consumption, 50 Brake Horsepower, No. 2 Diesel, No Water, 25\% Oxygen

B.40 Effect of Injection Timing on Exhaust Temperature, 50 Brake Horsepower, No. 2 Diesel, 5\% Water

B.41 Effect of Injection Timing on Peak Cylinder Pressure, 50 Brake Horsepower, No. 2 Diesel, $5 \%$ Water.

B.42 Effect of Injection Timing on Ignition Delay, 50 Brake Horsepower, No. 2 Diesel, 5\% Water.

B.43 Effect of Injection Timing on Thermal Efficiency, 50 Brake Horsepower, No. 2 Diesel, 5\% Water.

B.44 Effect of Injection Timing on Smoke, 50 Brake Horsepower, No. 2 Diesel, $5 \%$ Water 


\section{Figures (Cont'd)}

B.45 Effect of Injection Timing on Particulate Matter, 50 Brake Horsepower, No. 2 Diesel, 5\% Water, $21 \%$ Oxygen.............................................

B.46 Effect of Injection Timing on Particulate Matter, 50 Brake Horsepower, No. 2 Diesel, $5 \%$ Water, $25 \%$ Oxygen...........................................

B.47 Effect of Injection Timing on $\mathrm{NO}_{\mathrm{x}}, 50$ Brake Horsepower, No. 2 Diesel, $5 \%$ Water, $21 \%$ Oxygen.

B.48 Effect of Injection Timing on $\mathrm{NO}_{\mathrm{x}}, 50$ Brake Horsepower, No. 2 Diesel, $5 \%$ Water, $25 \%$ Oxygen.

B.49 Effect of Injection Timing on Exhaust Air Flow, 50 Brake Horsepower, No. 2 Diesel, 5\% Water, $21 \%$ Oxygen.

B.50 Effect of Injection Timing on Exhaust Air Flow, 50 Brake Horsepower, No. 2 Diesel, 5\% Water, 25\% Oxygen....

B.51 Effect of Injection Timing on Brake-Specific Fuel Consumption, 50 Brake Horsepower, No. 2 Diesel, $5 \%$ Water, $21 \%$ Oxygen ....

B.52 Effect of Injection Timing on Brake-Specific Fuel Consumption, 50 Brake Horsepower, No. 2 Diesel, 5\% Water, 25\% Oxygen

B 53 Effect of Fuel Injection Timing on Cylinder Pressure, No. 2 Fuel, Full Load, No Water, $21 \%$ Oxygen ...................................................

B.54 Effect of Fuel Injection Timing on Cylinder Pressure, No. 2 Fuel, Full Load,

No Water, $25 \%$ Oxygen .....................................................

B.55 Effect of Fuel Injection Timing on Cumulative Heat Release, No. 2 Fuel, Full Load, No Water, $21 \%$ Oxygen ............................................

B.56 Effect of Fuel Injection Timing on Cumulative Heat Release, No. 2 Fuel, Full

Load, No Water, 25\% Oxygen ....

B.57 Effect of Fuel Injection Timing on Slope of Cylinder Pressure Change, No. 2

Fuel, Full Load, No Water, 21\% Oxygen....

B.58 Effect of Fuel Injection Timing on Slope of Cylinder Pressure Change, No. 2 Fuel, Full Load, No Water, 25\% Oxygen....

B.59 Effect of Fuel Injection Timing on Heat Release, No. 2 Fuel, Full Load, No Water, $21 \%$ Oxygen

B.60 Effect of Fuel Injection Timing on Heat Release, No. 2 Fuel, Full Load, No Water, $25 \%$ Oxygen

B.61 Effect of Fuel Injection Timing on Pressure, No. 2 Fuel, Half Load, No Water, $21 \%$ Oxygen

B.62 Effect of Fuel Injection Timing on Slope of Cylinder Pressure Change, No. 2 Fuel, Half Load, No Water, 21\% Oxygen

B.63 Effect of Fuel Injection Timing on Heat Release, No. 2 Fuel, Half Load, No Water, 2:\% Oxygen ....

B.64 Effect of Fuel Injection Timing on Cumulative Heat Release, No. 2 Fuel, Half I oad, No Water, $21 \%$ Oxygen 


\section{Figures (Cont'd)}

B.65 Effect of Fuel Injection Timing on Pressure, No. 2 Fuel, Half Load,

No Water, $25 \%$ Oxygen ....

B-65

B.66 Effect of Fuel Injection Timing on Slope of Cylinder Pressure Change, No. 2

Fuel, Half Load, No Water, $25 \%$ Oxygen

B-65

B.67 Effect of Fuel Injection Timing on Heat Release, No. 2 Fuel, Half Load,

No Water, $25 \%$ Oxygen .

B-66

B.68 Effect of Fuel Injection Timing on Cumulative Heat Release, No. 2 Fuel, Half

Load, No Water, 25\% Oxygen

B-66

B.69 Effect of Fuel Injection Timing on Pressure, No. 2 Fuel, Full Load, 5\% Water, $21 \%$ Oxygen

B.70 Effect of Fuel Injection Timing on Slope of Cylinder Pressure Change, No. 2

Fuel, Full Load, 5\% Water, $21 \%$ Oxygen.

B-67

B.71 Effect of Fuel Injection Timing on Heat Release, No. 2 Fuel, Full Load,

$5 \%$ Water, $21 \%$ Oxygen.

B-67

B.72 Effect of Fuel Injection Timing on Cumulative Heat Release, No. 2 Fuel, Full Load, $5 \%$ Water, $21 \%$ Oxygen.

B-68

B-68

B.73 Effect of Fuel Injection Timing on Pressure, No. 2 Fuel, Full Load, 5\% Water, $25 \%$ Oxygen

B.74 Effect of Fuel Injection Timing on Slope of Cylinder Pressure Change, No. 2

Fuel, Full Load, 5\% Water, 25\% Oxygen.

B-69

B.75 Effect of Fuel Injection Timing on Heat Release, No. 2 Fuel, Full Load, $5 \%$ Water, $25 \%$ Oxygen.

B.76 Effect of Fuel Injection Timing on Cumulative Heat Release, No. 2 Fuel, Full

Load, 5\% Water, 25\% Oxygen

B-70

B.77 Effect of Fuel Injection Timing on Pressure, No. 2 Fuel, Half Load,

$5 \%$ Water, $21 \%$ Oxygen...

B-70

B.78 Effect of Fuel Injection Timing on Slope of Cylinder Pressure Change, No. 2

Fuel, Half Load, 5\% Water, 21\% Oxygen

B-71

B.79 Effect of Fuel Injection Timing on Heat Release, No. 2 Fuel, Half Load, $5 \%$ Water, $21 \%$ Oxygen.

B-71

B-72

B.80 Effect of Fuel Injection Timing on Cumulative Heat Release, No. 2 Fuel, Half Load, $5 \%$ Water, $21 \%$ Oxygen

B-72

B.81 Effect of Fuel Injection Timing on Pressure, No. 2 Fuel, Half Load, $5 \%$ Water, $26 \%$ Oxygen.

B.82 Effect of Fuel Injection Timing on Slope of Cylinder Pressure Change, No. 2 Fuel, Half Load, 5\% Water, 26\% Oxygen .

B.83 Effect of Fuel Injection Timing on Heat Release, No. 2 Fuel, Half Load, $5 \%$ Water, $26 \%$ Oxygen.

B.84 Effect of Fuel Injection Timing on Cumulative Heat Releasc, No. 2 Fuel, Half

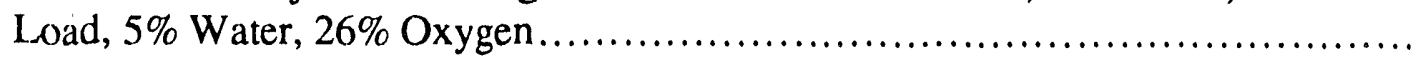

B.85 Heat-Release Rate, Run 213

B-75

B.86 Heat-Release Rate, Run 209

B-75 


\section{Figures (Cont'd)}

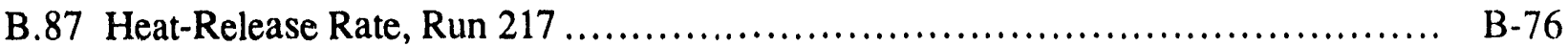

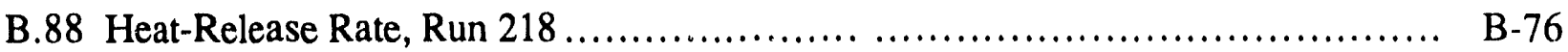

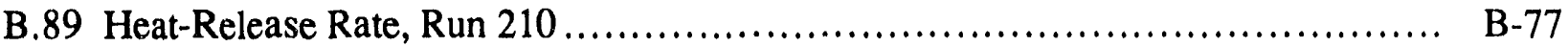

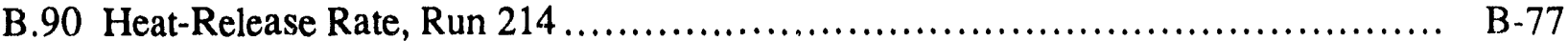

B.91 Emission Repeatability Tests, 50 Brake Horsepower, No. 2 Fuel, No Water, 22 deg. btdc Injection Timing, $\mathrm{NO}_{\mathbf{x}}$ Measurements......................... B-81

B.92 Emission Repeatability Tests, 50 Brake Horsepower, No. 2 Fuel, No Water, 22 deg. btdc Injection Timing, Particulate-Matter Measurements.

B.93 Emission Repeatability Tests, 50 Brake Horsepower, No. 2 Fuel, No Water, 22 deg. btdc Injection Timing, Smoke Measurements .......................... B. 82

B.94 Emission Repeatability Tests, 25 Brake Horsepower, No. 2 Fuel, No Water, $27 \mathrm{deg}$. btdc Injection Timing, $\mathrm{NO}_{\mathrm{x}}$ Measurements

B.95 Emission Repeatability Tests, 25 Brake Horsepower, No. 2 Fuel, No Water, 27 deg. btdc Injection Timing, Particulate-Matter Measurements.

B.96 Emission Repeatability Tests, 25 Brake Horsepower, No. 2 Fuel, No Water, $27 \mathrm{deg}$. btdc Injection Timing, Smoke Measurements

B.97 Effect of Injection Timing on Exhaust Temperature, 25 Brake Horsepower, No. 4 Fuel, No Water

B.98 Effect of Injection Timing on Peak Pressure, 25 Brake Horsepower, No. 4 Fuel, No Water.

B.99 Effect of Injection Timing on Ignition Delay, 25 Brake Horsepuwer, No. 4 Fuel, No Water

B.100 Effect of Injection Timing on Thermal Efficiency, 25 Brake Horsepower, No. 4 Fuel, No Water.

B.101 Effect of Injection Timing on Particulate-Matter Emission, 25 Brake Horsepower, No. 4 Fuel, No Water.

B.102 Effect of Injection Timing on $\mathrm{NO}_{\mathrm{x}}$ Emission, 25 Brake Horsepower, No. 4 Fuel, No Water....

B.103 Effect of Injection Timing on Smoke Emission, 25 Brake Horsepower, No. 4 Fuel, No Water.

B.104 Effects of Injection Timing on Brake-Specific Fuel Consumption, 25 Brake Horsepower, No. 4 Fuel, No Water.

B.105 Effects of Injection Timing on Exhaust Air Flow, 25 Brake Horsepower, No. 4 Fuel, No Water

B.106 Effect of Injection Timing on Exhaust Temperature, 25 Brake Horsepower, No. 4 Fuel, 5\% Water

B.107 Effect of Injection Timing on Cylinder Pressure, 25 Brake Horsepower, No. 4 Fuel, 5\% Water.

B. 108 Effect of Injection Timing on Ignition Delay, 25 Brake Horsepower, No. 4 Fuel, $5 \%$ Water. 


\section{Figures (Cont'd)}

B.109 Effect of Injection Timing on Thermal Efficiency, 25 Brake Horsepower, No. 4 Fuel, 5\% Water.

B.110 Effect of Injection Timing on Particulate-Matter Emission, 25 Brake Horsepower, No. 4 Fuel, 5\% Water.

B.111 Effect of Injection Timing on $\mathrm{NO}_{\mathrm{x}}$ Emission, 25 Brake Horsepower, No. 4 Fuel, 5\% Water.

B.112 Effect of Injection Timing on Smoke Emission, 25 Brake Horsepower, No. 4 Fuel, 5\% Water.

B.113 Effect of Injection Timing on Brake-Specific Fuel Consumption, 25 Brake Horsepower, No. 4 Fuei, $5 \%$ Water

B.114 Effect of Injection Timing on Exhaust Air Flow, 25 Brake Horsepower, No. 4 Fuel, 5\% Water.

B.115 Effect of Injtetion Timing on Exhaust Temperature, 50 Brake Horsepower, No. 4 Fuel, No Water

B.116 Effect of Injection Timing on Cylinder Pressure, 50 Brake Horsepower, No. 4 Fuel, No Water

B.117 Effect of Injection Timing on Ignition Delay, 50 Brake Horsepower, No. 4 Fuel, No Water.

B.118 Effect of Injection Timing on Thermal Efficiency, 50 Brake Horsepower, No. 4 Fuel, No Water

B.119 Effect of Injection Timing on Particulate-Matter Emission, 50 Brake Horsepower, No. 4 Fuel, No Water.

B. 120 Effect of Injection Timing on $\mathrm{NO}_{\mathrm{x}}$ Emission, $5 \cap$ Brake Horsepower, No. 4 Fuel, No Water

B.121 Effect of Injection Timing on Smoke Emission, 50 Brake Horsepower, No. 4 Fuel, No Water

B.122 Effect of Injection Timing on Brake-Specific Fuel Consumption, 50 Brake Horsepower, No. 4 Fuel, No Water.

B.123 Effect of Injection Timing on Exhaust Air Flow, 50 Brake Horsepower, No. 4 Fuel, No Water.

B.124 Effect of Injection Timing on Exhaust Temperature, 50 Brake Horsepower, No. 4 Fuel, $5 \%$ Water

B.125 Effeci of Injection Timing on Cylinder Pressure, 50 Brake Horsepower, No. 4 Fuel, 5\% Water

B.126 Effect of Injection Timing on Ignition Delay, 50 Brake Horsepower, No. 4 Fuel, 5\% Water

B.127 Effect of Injection Timing on Thermal Efficiency, 50 Brake Horsepower, No. 4 Fuel, $5 \%$ Water

B.128 Effect of Injection Timing on Particulate-Matter Emission, 50 Brake Horsepower, No. 4 Fuel, $5 \%$ Water. 


\section{Figures (Cont'd)}

B.129 Effect of Injection Timing on $\mathrm{NO}_{\mathrm{x}}$ Emission, 50 Brake Horsepower, No. 4

Fuel, $5 \%$ Water........................................................ B-103

B.130 Effect of Injection Timing on Smoke Emission, 50 Brake Horsepower, No. 4 Fuel, 5\% Water........................................................ B-103

B.131 Effect of Injection Timing on Brake-Specific Fuel Consumption, 50 Brake Horsepower, No. 4 Fuel, 5\% Water..................................... B-104

B.132 Effect of Injection Timing on Exhaust Air Flow, 50 Brake Horsepower, No. 4 Fuel, 5\% Water ................................................ B-104

B.133 Pressure, No. 4 Fuel, Full Load, No Water, 21\% Oxygen........................ B-107

B.134 Slope of Cylinder Pressure Change, No. 4 Fuel, Full Load, No Water,

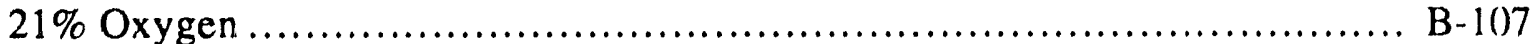

B.135 Heat Release, No. 4 Fuel, Full Load, No Water, 21\% Oxygen.................... B-108

B.136 Cumulative Heat Release, No. 4 Fuel, Full Load, No Water, 21\% Oxygen.......... B-108

B.137 Pressure, No. 4 Fuel, Full Load, No Water, 25\% Oxygen....................... B-109

B.138 Slope of Cylinder Pressure Change, No. 4 Fuel, No Water, 25\% Oxygen........... B-109

B.139 Heat Release, No. 4 Fuel, Full Load, No Water, 25\% Oxygen ..................... B-110

B.140 Cumulative Heat Release, No. 4 Fuel, Full Load, No Water, 25\% Oxygen.......... B-110

B.141 Pressure, No. 4 Fuel, Half Load, No Water, 21\% Oxygen ...................... B-111

B.142 Slope of Cylinder Pressure Change, No. 4 Fuel, Half Load, No Water, $21 \%$ Oxygen .......................................................... B-111

B.143 Heat Release, No. 4 Fuel, Half Load, No Water, 21\% Oxygen ................... B-112

B.144 Cumulative Heat Release, No. 4 Fuel, Half Load, No Water, 21\% Oxygen ......... B-112

B.145 Pressure, No. 4 Fuel, Half Load, No Water, 27\% Oxygen ....................... B-113

B.146 Slope of Cylinder Pressure Change, No. 4 Fuel, Half Load, No Water, $27 \%$ Oxygen ............................................................. 113

B.147 Heat Release, No. 4 Fuel, Half Load, No Water, 27\% Oxygen .................... B-114

B.148 Cumulative Heat Release, No. 4 Fuel, Half Load, No Water, 27\% Oxygen .......... B-114

B.149 Pressure, No. 4 Fuel, Full Load, 5\% Water, 21\% Oxygen......................... B-115

B.150 Slope of Cylinder Pressure Change, No. 4 Fuel, Full Load, 5\% Water,

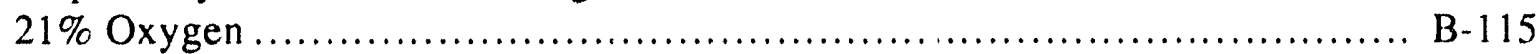

B.151 Heat Release, No. 4 Fuel, Full Load, 5\% Water, 21\% Oxygen..................... B-116

B.152 Cumulative Heat Release, No. 4 Fuel, Full Load, 5\% Water, 21\% Oxygen ......... B-116

B.153 Pressure, No. 4 Fuel, Full Load, 5\% Water, 25\% Oxygen.......................... B-117

B.154 Slope of Cylinder Pressure Change, No. 4 Fuel, Full Load, 5\% Water,

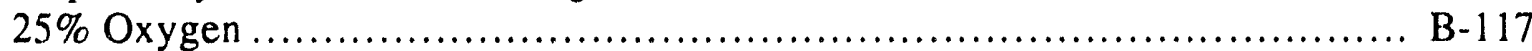

B.155 Heat Release, No. 4 Fuel, Full Load, 5\% Water, 25\% Oxygen....................... B-118

B.156 Cumulative Heat Release, No. 4 Fuel, Full Load, 5\% Water, 25\% Oxygen ......... B-118

B.157 Pressure, No. 4 Fuel, Half Load, 5\% Water, 21\% Oxygen ...................... B-119

B.158 Slope of Cylinder Pressure Change, No. 4 Fuel, Half Load, 5\% Water,

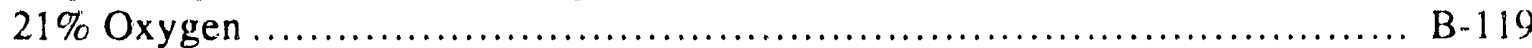

B.159 Heat Release, No. 4 Fuel, Half Load, 5\% Water, 25\% Oxygen ................... B-120

B.160 Cumulative Heat Release, No. 4 Fuel, Half Load, 5\% Water, 21\% Oxygen......... B-120) 


\section{Figures (Cont'd)}

B.161 Pressure, No. 4 Fuel, Half Load, 5\% Water, 25\% Oxygen ..................... B-121

B.162 Slope of Cylinder Pressure Change, No. 4 Fuel, Half Load, 5\% W'ater, $25 \%$ Oxygen .......................................................... 121

B.163 Heat Release, No. 4 Fuel, Half Load, 5\% Water, 21\% Oxygen ................. B-122

B.164 Cumulative Heat Release, No. 4 Fuel, Half Load, 5\% Water, 25\% Oxygen.......... B-122 


\section{Foreword}

This report contains the data gathered from tests conducted on a single-cylinder diesel engine to study the benefits and problems of oxygen-enriched diesel combustion and the use of water-emulsified and low-grade diesel fuels. This research, funded by the Office of Industrial Technologies (OI $\Gamma$ ) in the United States Department of Energy, is being conducted in support of the Industrial Cogeneration Program. The report is made up of two volumes. Volume 1 contains the description of the experiments, selected data points, discussion of rends, and conclusions and recommendations; Volume 2 contains the data sets. With the two-volume approach, readers can find information at the desired level of detail, depending on individual interest or need. 


\section{Appendix A: \\ Data Package of Diesel Engine Tests with Oxygen-Enriched Air and Water-Emulsified Fuels: Series I Tests}




$$
\text { A. } 2
$$


A.1 Tabulation of Test Data in the Order of Test Sequence 


\begin{tabular}{|c|c|c|c|c|c|c|c|}
\hline $\begin{array}{c}\text { Run } \\
\#\end{array}$ & Fuel & $\begin{array}{l}\text { Load } \\
\%\end{array}$ & $\begin{array}{l}\text { Power } \\
\text { BHP }\end{array}$ & $\begin{array}{l}H \geq Q \\
w t-\%\end{array}$ & $\begin{array}{c}\text { do Intake } \\
\text { vol-\% }\end{array}$ & $\begin{array}{r}\text { Exhaust } \\
\text { vol }-\%\end{array}$ & $\begin{array}{c}\text { Q己 Bottl } \\
\text { lb/min }\end{array}$ \\
\hline $\begin{array}{l}001 \\
002 \\
003 \\
004 \\
004 A\end{array}$ & $\begin{array}{l}2 \\
2 \\
2 \\
2 \\
2\end{array}$ & $\begin{array}{r}100 \\
50 \\
50 \\
50 \\
50\end{array}$ & $\begin{array}{l}50.1 \\
25.0 \\
25.0 \\
47.5 \\
33.5\end{array}$ & $\begin{array}{l}0.0 \\
0.0 \\
0.0 \\
0.0 \\
0.0\end{array}$ & $\begin{array}{l}20.31 \\
20.59 \\
25.13 \\
25.17 \\
25.07\end{array}$ & $\begin{array}{l}10.97 \\
13.52 \\
18.32 \\
13.50 \\
16.58\end{array}$ & $\begin{array}{l}0.000 \\
0.000 \\
0.456 \\
0.459 \\
0.459\end{array}$ \\
\hline $\begin{array}{l}005 \\
006 \\
007\end{array}$ & $\begin{array}{l}2 \\
2 \\
2\end{array}$ & $\begin{array}{l}50 \\
50 \\
50\end{array}$ & $\begin{array}{l}24.3 \\
24.3 \\
31.8\end{array}$ & $\begin{array}{l}4.0 \\
5.0 \\
5.1\end{array}$ & $\begin{array}{l}20.27 \\
24.98 \\
25.00\end{array}$ & $\begin{array}{l}13.63 \\
18.37 \\
16.78\end{array}$ & $\begin{array}{l}0.000 \\
0.459 \\
0.459\end{array}$ \\
\hline $\begin{array}{l}\text { OOBA } \\
\text { OOSA } \\
\text { O1OA } \\
011 A\end{array}$ & $\begin{array}{l}2 \\
2 \\
2 \\
2\end{array}$ & $\begin{array}{l}50 \\
50 \\
50 \\
50\end{array}$ & $\begin{array}{l}25.0 \\
25.0 \\
32.0 \\
43.8\end{array}$ & $\begin{array}{r}20.2 \\
18.9 \\
12.4 \\
7.0\end{array}$ & $\begin{array}{l}20.78 \\
25.02 \\
25.02 \\
25.00\end{array}$ & $\begin{array}{l}13.53 \\
17.67 \\
16.23 \\
13.54\end{array}$ & $\begin{array}{l}0.000 \\
0.423 \\
0.421 \\
0.421\end{array}$ \\
\hline $\begin{array}{l}012 \\
013 \\
014 \\
015\end{array}$ & $\begin{array}{l}4 \\
4 \\
4 \\
4\end{array}$ & $\begin{array}{l}50 \\
50 \\
50 \\
50\end{array}$ & $\begin{array}{l}25.0 \\
24.9 \\
32.8 \\
46.6\end{array}$ & $\begin{array}{l}0.0 \\
0.0 \\
0.0 \\
0.0\end{array}$ & $\begin{array}{l}20.22 \\
25.01 \\
25.04 \\
25.02\end{array}$ & $\begin{array}{l}13.25 \\
17.87 \\
16.35 \\
13.25\end{array}$ & $\begin{array}{l}0.000 \\
0.459 \\
0.459 \\
0.459\end{array}$ \\
\hline $\begin{array}{l}016 \\
017 \\
018 \\
019\end{array}$ & $\begin{array}{l}4 \\
4 \\
4 \\
4\end{array}$ & $\begin{array}{l}50 \\
50 \\
50 \\
50\end{array}$ & $\begin{array}{l}24.1 \\
24.0 \\
31.3 \\
45.3\end{array}$ & $\begin{array}{l}3.2 \\
3.7 \\
5.3 \\
4.2\end{array}$ & $\begin{array}{l}20.21 \\
25.01 \\
25.00 \\
24.99\end{array}$ & $\begin{array}{l}13.69 \\
18.43 \\
16.88 \\
13.68\end{array}$ & $\begin{array}{l}0.000 \\
0.462 \\
0.462 \\
0.462\end{array}$ \\
\hline $\begin{array}{l}020 \\
021 \\
023 \\
023\end{array}$ & $\begin{array}{l}4 \\
4 \\
4 \\
4\end{array}$ & $\begin{array}{l}50 \\
50 \\
50 \\
50\end{array}$ & $\begin{array}{l}24.7 \\
24.7 \\
31.7 \\
43.4\end{array}$ & $\begin{array}{l}10.2 \\
10.6 \\
10.9 \\
11.2\end{array}$ & $\begin{array}{l}20.62 \\
25.09 \\
25.03 \\
25.01\end{array}$ & $\begin{array}{l}13.52 \\
19.05 \\
16.30 \\
13.61\end{array}$ & $\begin{array}{l}0.000 \\
0.411 \\
0.413 \\
0.423\end{array}$ \\
\hline $\begin{array}{l}0 \geq 4 \\
0 \geq 5 \\
0 \geq 6 \\
0 \geqq 7\end{array}$ & $\begin{array}{l}4 \\
4 \\
4 \\
4\end{array}$ & $\begin{array}{l}100 \\
100 \\
100 \\
100\end{array}$ & $\begin{array}{l}42.8 \\
42.6 \\
52.5 \\
62.3\end{array}$ & $\begin{array}{l}11.7 \\
1 \geq .3 \\
11.7 \\
1 \geq .1\end{array}$ & $\begin{array}{l}20.44 \\
24.98 \\
25.05 \\
25.02\end{array}$ & $\begin{array}{l}11.03 \\
15.74 \\
13.57 \\
11.10\end{array}$ & $\begin{array}{l}0.000 \\
0.463 \\
0.496 \\
0.476\end{array}$ \\
\hline $\begin{array}{l}025 \\
027 \\
030 \\
031 \\
032\end{array}$ & $\begin{array}{l}4 \\
4 \\
4 \\
4 \\
4\end{array}$ & $\begin{array}{l}100 \\
100 \\
100 \\
100 \\
100\end{array}$ & $\begin{array}{l}49.0 \\
40.9 \\
47.1 \\
58.4 \\
65.3\end{array}$ & $\begin{array}{l}0.0 \\
0.0 \\
0.0 \\
0.0 \\
0.0\end{array}$ & $\begin{array}{l}20.35 \\
25.05 \\
25.02 \\
25.05 \\
25.00\end{array}$ & $\begin{array}{l}11.00 \\
16.03 \\
15.77 \\
13.54 \\
11.08\end{array}$ & $\begin{array}{l}0.000 \\
0.527 \\
0.456 \\
0.456 \\
0.474\end{array}$ \\
\hline $\begin{array}{l}033 \\
034 \\
035 \\
035\end{array}$ & $\begin{array}{l}4 \\
4 \\
4 \\
4\end{array}$ & $\begin{array}{l}100 \\
100 \\
100 \\
100\end{array}$ & $\begin{array}{l}47.5 \\
47.1 \\
57.3 \\
67.0\end{array}$ & $\begin{array}{l}5.9 \\
6.0 \\
6.3 \\
t .1\end{array}$ & $\begin{array}{l}20.52 \\
25.10 \\
24.99 \\
25.00\end{array}$ & $\begin{array}{l}11.03 \\
15.67 \\
13.37 \\
11.05\end{array}$ & $\begin{array}{l}0.000 \\
0.458 \\
0.492 \\
0.488\end{array}$ \\
\hline
\end{tabular}




\begin{tabular}{|c|c|c|c|c|c|c|c|}
\hline $\begin{array}{l}\text { Run } \\
\#\end{array}$ & $\begin{array}{l}\text { Air Flow } \\
\text { lb/min }\end{array}$ & $\begin{array}{c}\text { Net Fuel } \\
1 \mathrm{~b} / \mathrm{min}\end{array}$ & oe/Fuel & $\begin{array}{l}\text { P Intake } \\
\text { in. Hg abs }\end{array}$ & $\begin{array}{c}P \text { Exh. } \\
\text { in. Hg abs }\end{array}$ & $\begin{array}{l}\text { P Peak } \\
\text { Psi }\end{array}$ & $\begin{array}{l}\text { T Exh. } \\
\text { deg } F\end{array}$ \\
\hline $\begin{array}{l}001 \\
002 \\
003 \\
004 \\
004 A\end{array}$ & $\begin{array}{l}8.94 \\
6.94 \\
6.55 \\
6.49 \\
6.50\end{array}$ & $\begin{array}{l}0.300 \\
0.174 \\
0.168 \\
0.295 \\
0.215\end{array}$ & $\begin{array}{r}6.85 \\
9.17 \\
11.68 \\
6.62 \\
9.09\end{array}$ & $\begin{array}{l}53.9 \\
43.6 \\
44.8 \\
44.8 \\
44.7\end{array}$ & $\begin{array}{l}32.8 \\
32.2 \\
32.6 \\
32.8 \\
32.6\end{array}$ & $\begin{array}{l}1665 \\
1333 \\
1384 \\
1586 \\
1464\end{array}$ & $\begin{array}{l}794 \\
571 \\
560 \\
880 \\
659\end{array}$ \\
\hline $\begin{array}{l}005 \\
006 \\
007\end{array}$ & $\begin{array}{l}6.73 \\
6.56 \\
6.51\end{array}$ & $\begin{array}{l}0.161 \\
0.161 \\
0.200\end{array}$ & $\begin{array}{r}9.64 \\
12.24 \\
9.79\end{array}$ & $\begin{array}{l}43.8 \\
45.0 \\
45.1\end{array}$ & $\begin{array}{l}31.6 \\
31.9 \\
32.3\end{array}$ & $\begin{array}{l}1438 \\
1445 \\
1512\end{array}$ & $\begin{array}{l}573 \\
553 \\
\leq 52\end{array}$ \\
\hline $\begin{array}{l}\text { OOBA } \\
\text { OOAA } \\
010 A \\
\text { O11A }\end{array}$ & $\begin{array}{l}6.85 \\
6.53 \\
6.52 \\
6.48\end{array}$ & $\begin{array}{l}0.186 \\
0.170 \\
0.209 \\
0.271\end{array}$ & $\begin{array}{r}8.48 \\
11.36 \\
9.18 \\
7.04\end{array}$ & $\begin{array}{l}44.7 \\
44.7 \\
44.7 \\
44.8\end{array}$ & $\begin{array}{l}32.6 \\
32.6 \\
32.6 \\
32.4\end{array}$ & $\begin{array}{l}1355 \\
1374 \\
1423 \\
1472\end{array}$ & $\begin{array}{l}579 \\
580 \\
678 \\
875\end{array}$ \\
\hline $\begin{array}{l}012 \\
013 \\
014 \\
015\end{array}$ & $\begin{array}{l}6.85 \\
6.62 \\
6.60 \\
6.52\end{array}$ & $\begin{array}{l}0.189 \\
0.188 \\
0.229 \\
0.310\end{array}$ & $\begin{array}{r}8.34 \\
10.54 \\
8.63 \\
6.32\end{array}$ & $\begin{array}{l}44.3 \\
45.5 \\
45.5 \\
45.6\end{array}$ & $\begin{array}{l}32.5 \\
32.7 \\
32.7 \\
32.6\end{array}$ & $\begin{array}{l}1389 \\
1442 \\
1556 \\
1757\end{array}$ & $\begin{array}{l}600 \\
573 \\
674 \\
890\end{array}$ \\
\hline $\begin{array}{l}016 \\
017 \\
018 \\
019\end{array}$ & $\begin{array}{l}6.69 \\
6.51 \\
6.49 \\
6.49\end{array}$ & $\begin{array}{l}0.177 \\
0.183 \\
0.216 \\
0.299\end{array}$ & $\begin{array}{r}8.68 \\
10.71 \\
9.03 \\
6.55\end{array}$ & $\begin{array}{l}44.4 \\
45.4 \\
45.5 \\
45.5\end{array}$ & $\begin{array}{l}32.5 \\
32.6 \\
32.6 \\
32.6\end{array}$ & $\begin{array}{l}1347 \\
1477 \\
1485 \\
1695\end{array}$ & $\begin{array}{l}58 \leqslant \\
561 \\
660 \\
871\end{array}$ \\
\hline $\begin{array}{l}020 \\
021 \\
021 \\
0 \geq 2 \\
0 \geq 3\end{array}$ & $\begin{array}{l}6.50 \\
6.28 \\
6.26 \\
6.38\end{array}$ & $\begin{array}{l}0.199 \\
0.190 \\
0.221 \\
0.297\end{array}$ & $\begin{array}{l}7.79 \\
9.76 \\
8.38 \\
6.58\end{array}$ & $\begin{array}{l}42.2 \\
43.4 \\
43.0 \\
43.9\end{array}$ & $\begin{array}{l}32.0 \\
32.1 \\
32.1 \\
32.1\end{array}$ & $\begin{array}{l}1369 \\
1446 \\
1454 \\
1602\end{array}$ & $\begin{array}{l}612 \\
586 \\
693 \\
858\end{array}$ \\
\hline $\begin{array}{l}024 \\
025 \\
025 \\
027\end{array}$ & $\begin{array}{l}8.16 \\
7.86 \\
7.81 \\
7.73\end{array}$ & $\begin{array}{l}0.273 \\
0.272 \\
0.338 \\
0.409\end{array}$ & $\begin{array}{l}6.87 \\
8.34 \\
6.79 \\
5.51\end{array}$ & $\begin{array}{l}50.0 \\
51.5 \\
51.3 \\
51.4\end{array}$ & $\begin{array}{l}33.0 \\
33.0 \\
33.0 \\
33.3\end{array}$ & $\begin{array}{l}1669 \\
1696 \\
1705 \\
1767\end{array}$ & $\begin{array}{r}773 \\
742 \\
903 \\
1056\end{array}$ \\
\hline $\begin{array}{l}028 \\
029 \\
030 \\
031 \\
032\end{array}$ & $\begin{array}{l}8.86 \\
8.41 \\
8.59 \\
8.64 \\
8.60\end{array}$ & $\begin{array}{l}0.323 \\
0.307 \\
0.303 \\
0.378 \\
0.458\end{array}$ & $\begin{array}{l}6.31 \\
8.17 \\
8.03 \\
6.46 \\
5.35\end{array}$ & $\begin{array}{l}55.0 \\
55.5 \\
56.5 \\
56.7 \\
57.2\end{array}$ & $\begin{array}{l}33.2 \\
33.2 \\
33.1 \\
33.2 \\
33.9\end{array}$ & $\begin{array}{l}1717 \\
1821 \\
1721 \\
1831 \\
1931\end{array}$ & $\begin{array}{r}799 \\
762 \\
754 \\
918 \\
1037\end{array}$ \\
\hline $\begin{array}{l}03.3 \\
034 \\
035 \\
036\end{array}$ & $\begin{array}{l}8.59 \\
8.28 \\
8.27 \\
8.21\end{array}$ & $\begin{array}{l}0.299 \\
0.296 \\
0.362 \\
0.438\end{array}$ & $\begin{array}{l}6.61 \\
8.09 \\
6.59 \\
5.43\end{array}$ & $\begin{array}{l}53.2 \\
54.8 \\
54.8 \\
55.1\end{array}$ & $\begin{array}{l}33.1 \\
33.2 \\
33.2 \\
33.8\end{array}$ & $\begin{array}{l}1672 \\
1701 \\
1785 \\
1771\end{array}$ & $\begin{array}{r}909 \\
768 \\
927 \\
1095\end{array}$ \\
\hline
\end{tabular}




\begin{tabular}{|c|c|c|c|c|c|c|c|}
\hline $\begin{array}{l}\text { Run } \\
\#\end{array}$ & $\begin{array}{r}\text { Fuel } \\
\#\end{array}$ & $\begin{array}{c}\text { Load } \\
\%\end{array}$ & $\begin{array}{l}\text { Power } \\
\text { BHP }\end{array}$ & $\begin{array}{l}H \geq 0 \\
w t-\%\end{array}$ & $\begin{array}{c}\text { oe Intake } \\
\text { vol }-\%\end{array}$ & $\begin{array}{r}\text { Oe Exhaust } \\
\text { Yol-\% }\end{array}$ & $\begin{array}{l}\text { oe Bottle } \\
\text { ib/min }\end{array}$ \\
\hline $\begin{array}{l}037 \\
038 \\
039 \\
040\end{array}$ & $\begin{array}{l}2 \\
2 \\
2 \\
5\end{array}$ & $\begin{array}{l}100 \\
100 \\
100 \\
100\end{array}$ & $\begin{array}{l}49.9 \\
50.1 \\
60.6 \\
70.7\end{array}$ & $\begin{array}{l}0.0 \\
0.0 \\
0.0 \\
0.0\end{array}$ & $\begin{array}{l}20.52 \\
25.01 \\
25.02 \\
25.00\end{array}$ & $\begin{array}{l}11.01 \\
15.71 \\
13.36 \\
10.99\end{array}$ & $\begin{array}{l}0.000 \\
0.510 \\
0.504 \\
0.527\end{array}$ \\
\hline $\begin{array}{l}041 \\
042 \\
043 \\
044\end{array}$ & $\begin{array}{l}2 \\
2 \\
2 \\
2\end{array}$ & $\begin{array}{l}100 \\
100 \\
100 \\
100\end{array}$ & $\begin{array}{l}49.9 \\
50.1 \\
60.5 \\
70.0\end{array}$ & $\begin{array}{l}2.8 \\
4.2 \\
4.6 \\
4.7\end{array}$ & $\begin{array}{l}20.60 \\
25.05 \\
25.01 \\
25.02\end{array}$ & $\begin{array}{l}10.95 \\
15.66 \\
13.31 \\
11.00\end{array}$ & $\begin{array}{l}0.000 \\
0.507 \\
0.504 \\
0.493\end{array}$ \\
\hline $\begin{array}{l}045 \\
046 \\
047 \\
048\end{array}$ & $\begin{array}{l}2 \\
2 \\
2 \\
2\end{array}$ & $\begin{array}{l}100 \\
100 \\
100 \\
100\end{array}$ & $\begin{array}{l}50.0 \\
49.9 \\
61.0 \\
71.2\end{array}$ & $\begin{array}{l}25.7 \\
21.1 \\
18.3 \\
14.7\end{array}$ & $\begin{array}{l}20.38 \\
25.00 \\
24.96 \\
25.01\end{array}$ & $\begin{array}{l}10.93 \\
15.76 \\
13.33 \\
10.94\end{array}$ & $\begin{array}{l}0.000 \\
0.533 \\
0.533 \\
0.533\end{array}$ \\
\hline $\begin{array}{l}053 \\
054 \\
055\end{array}$ & $\begin{array}{l}2 \\
2 \\
2\end{array}$ & $\begin{array}{l}50 \\
50 \\
50\end{array}$ & $\begin{array}{l}25.1 \\
39.3 \\
60.3\end{array}$ & $\begin{array}{l}0.0 \\
0.0 \\
0.0\end{array}$ & $\begin{array}{l}29.53 \\
29.98 \\
29.96\end{array}$ & $\begin{array}{l}22.27 \\
19.57 \\
13.80\end{array}$ & $\begin{array}{l}0.817 \\
0.848 \\
0.934\end{array}$ \\
\hline $\begin{array}{l}056 \\
057 \\
058\end{array}$ & $\begin{array}{l}2 \\
2 \\
2\end{array}$ & $\begin{array}{l}50 \\
50 \\
50\end{array}$ & $\begin{array}{l}24.9 \\
44.7 \\
60.2\end{array}$ & $\begin{array}{l}0.0 \\
0.0 \\
0.0\end{array}$ & $\begin{array}{l}35.07 \\
35.04 \\
35.05\end{array}$ & $\begin{array}{l}27.72 \\
23.02 \\
18.67\end{array}$ & $\begin{array}{l}1.336 \\
1.353 \\
1.328\end{array}$ \\
\hline $\begin{array}{l}059 \\
060 \\
061 \\
062 \\
063 \\
064\end{array}$ & $\begin{array}{l}2 \\
2 \\
2 \\
2 \\
2 \\
2\end{array}$ & $\begin{array}{l}50 \\
50 \\
50 \\
50 \\
50 \\
50\end{array}$ & $\begin{array}{l}25.1 \\
25.1 \\
38.5 \\
45.7 \\
58.3 \\
58.1\end{array}$ & $\begin{array}{r}14.4 \\
7.1 \\
5.1 \\
3.9 \\
2.9 \\
2.4\end{array}$ & $\begin{array}{l}29.94 \\
34.98 \\
30.02 \\
35.08 \\
29.98 \\
34.98\end{array}$ & $\begin{array}{l}22.74 \\
27.99 \\
19.74 \\
23.04 \\
14.02 \\
19.04\end{array}$ & $\begin{array}{l}0.888 \\
1.370 \\
0.905 \\
1.328 \\
0.896 \\
1.311\end{array}$ \\
\hline $\begin{array}{l}0 \leq 5 \\
066 \\
0 \leqslant 7 \\
068 \\
0 \leqslant 9 \\
070\end{array}$ & $\begin{array}{l}2 \\
2 \\
2 \\
2 \\
2 \\
2\end{array}$ & $\begin{array}{l}50 \\
50 \\
50 \\
50 \\
50 \\
50\end{array}$ & $\begin{array}{l}25.0 \\
25.0 \\
37.4 \\
43.9 \\
59.0 \\
59.7\end{array}$ & $\begin{array}{r}23.8 \\
22.2 \\
14.9 \\
7.5 \\
8.2 \\
8.2\end{array}$ & $\begin{array}{l}30.03 \\
34.98 \\
29.97 \\
35.01 \\
30.01 \\
35.02\end{array}$ & $\begin{array}{l}22.76 \\
26.98 \\
19.70 \\
23.01 \\
13.52 \\
18.24\end{array}$ & $\begin{array}{l}0.910 \\
1.340 \\
0.586 \\
1.354 \\
0.897 \\
1.360\end{array}$ \\
\hline $\begin{array}{l}071 \\
072 \\
073 \\
074 \\
075 \\
076\end{array}$ & $\begin{array}{l}4 \\
4 \\
4 \\
4 \\
4 \\
4\end{array}$ & $\begin{array}{l}50 \\
50 \\
50 \\
50 \\
50 \\
50\end{array}$ & $\begin{array}{l}25.1 \\
25.0 \\
38.1 \\
44.3 \\
55.9 \\
55.9\end{array}$ & $\begin{array}{l}0.0 \\
0.0 \\
0.0 \\
0.0 \\
0.0 \\
0.0\end{array}$ & $\begin{array}{l}30.02 \\
35.00 \\
29.97 \\
35.02 \\
29.97 \\
35.04\end{array}$ & $\begin{array}{l}23.68 \\
27.56 \\
19.85 \\
23.05 \\
14.00 \\
19.65\end{array}$ & $\begin{array}{l}0.946 \\
1.410 \\
0.922 \\
1.497 \\
0.970 \\
1.422\end{array}$ \\
\hline
\end{tabular}




\begin{tabular}{|c|c|c|c|c|c|c|c|}
\hline$\underset{\#}{\text { Run }}$ & $\begin{array}{l}\text { Air Flow } \\
\text { lb/min }\end{array}$ & $\begin{array}{l}\text { Net Fuel } \\
1 b / \mathrm{min}\end{array}$ & $\begin{array}{c}\text { oe/Fuel } \\
\text {-- }\end{array}$ & $\begin{array}{l}\text { P Intake } \\
\text { in. Hg abs }\end{array}$ & $\begin{array}{c}P \text { Exh. } \\
\text { in. } H_{g} \text { abs }\end{array}$ & $\underset{\text { Psi }}{\text { Peak }}$ & $\begin{array}{l}\text { T Exh. } \\
\text { deg F }\end{array}$ \\
\hline $\begin{array}{l}037 \\
038 \\
039 \\
040\end{array}$ & $\begin{array}{l}9.01 \\
8.49 \\
8.33 \\
8.33\end{array}$ & $\begin{array}{l}0.310 \\
0.305 \\
0.371 \\
0.443\end{array}$ & $\begin{array}{l}6.69 \\
8.07 \\
6.52 \\
5.51\end{array}$ & $\begin{array}{l}55.6 \\
55.3 \\
55.1 \\
55.3\end{array}$ & $\begin{array}{l}33.5 \\
33.3 \\
33.5 \\
34.1\end{array}$ & $\begin{array}{l}1644 \\
1725 \\
1742 \\
1771\end{array}$ & $\begin{array}{r}827 \\
800 \\
963 \\
1131\end{array}$ \\
\hline $\begin{array}{l}041 \\
042 \\
043 \\
044\end{array}$ & $\begin{array}{l}8.93 \\
8.56 \\
8.56 \\
8.46\end{array}$ & $\begin{array}{l}0.339 \\
0.324 \\
0.378 \\
0.439\end{array}$ & $\begin{array}{l}6.07 \\
7.64 \\
6.55 \\
5.55\end{array}$ & $\begin{array}{l}55.0 \\
55.1 \\
55.2 \\
55.4\end{array}$ & $\begin{array}{l}33.0 \\
33.1 \\
33.3 \\
33.8\end{array}$ & $\begin{array}{l}1649 \\
1706 \\
1762 \\
1763\end{array}$ & $\begin{array}{r}831 \\
801 \\
957 \\
1113\end{array}$ \\
\hline $\begin{array}{l}045 \\
046 \\
047 \\
048\end{array}$ & $\begin{array}{l}9.14 \\
8.63 \\
8.61 \\
8.53\end{array}$ & $\begin{array}{l}0.292 \\
0.278 \\
0.348 \\
0.420\end{array}$ & $\begin{array}{l}7.19 \\
9.05 \\
7.22 \\
5.94\end{array}$ & $\begin{array}{l}56.6 \\
57.0 \\
56.4 \\
56.6\end{array}$ & $\begin{array}{l}33.6 \\
33.7 \\
33.8 \\
34.2\end{array}$ & $\begin{array}{l}1709 \\
1773 \\
1766 \\
1766\end{array}$ & $\begin{array}{r}783 \\
770 \\
941 \\
1109\end{array}$ \\
\hline $\begin{array}{l}053 \\
054 \\
055\end{array}$ & $\begin{array}{l}6.20 \\
6.15 \\
5.85\end{array}$ & $\begin{array}{l}0.189 \\
0.262 \\
0.384\end{array}$ & $\begin{array}{c}11.88 \\
8.64 \\
5.94\end{array}$ & $\begin{array}{l}44.4 \\
44.4 \\
43.1\end{array}$ & $\begin{array}{l}32.7 \\
32.7 \\
32.2\end{array}$ & $\begin{array}{l}1399 \\
1521 \\
1573\end{array}$ & $\begin{array}{r}592 \\
795 \\
1157\end{array}$ \\
\hline $\begin{array}{l}056 \\
057 \\
058\end{array}$ & $\begin{array}{l}5.70 \\
5.66 \\
5.60\end{array}$ & $\begin{array}{l}0.199 \\
0.288 \\
0.387\end{array}$ & $\begin{array}{r}13.30 \\
9.22 \\
6.76\end{array}$ & $\begin{array}{l}42.8 \\
42.9 \\
43.0\end{array}$ & $\begin{array}{l}32.0 \\
31.8 \\
32.0\end{array}$ & $\begin{array}{l}1343 \\
1549 \\
1611\end{array}$ & $\begin{array}{r}591 \\
891 \\
1159\end{array}$ \\
\hline $\begin{array}{l}059 \\
060 \\
061 \\
062 \\
063 \\
064\end{array}$ & $\begin{array}{l}5.79 \\
5.47 \\
5.67 \\
5.42 \\
5.68 \\
5.31\end{array}$ & $\begin{array}{l}0.173 \\
0.174 \\
0.232 \\
0.282 \\
0.360 \\
0.347\end{array}$ & $\begin{array}{r}12.86 \\
15.13 \\
9.53 \\
9.12 \\
6.11 \\
7.30\end{array}$ & $\begin{array}{l}40.6 \\
40.8 \\
40.3 \\
40.9 \\
40.9 \\
40.8\end{array}$ & $\begin{array}{l}32.1 \\
32.2 \\
31.9 \\
31.9 \\
32.1 \\
32.1\end{array}$ & $\begin{array}{l}1326 \\
1337 \\
1422 \\
1512 \\
1550 \\
1549\end{array}$ & $\begin{array}{r}\text { S05 } \\
611 \\
822 \\
933 \\
1161 \\
1157\end{array}$ \\
\hline $\begin{array}{l}065 \\
066 \\
067 \\
068 \\
069 \\
0.70\end{array}$ & $\begin{array}{l}5.65 \\
5.50 \\
5.78 \\
5.45 \\
5.69 \\
5.36\end{array}$ & $\begin{array}{l}0.160 \\
0.169 \\
0.241 \\
0.273 \\
0.383 \\
0.395\end{array}$ & $\begin{array}{c}13.79 \\
15.42 \\
9.18 \\
9.56 \\
5.76 \\
1.56\end{array}$ & $\begin{array}{l}40.9 \\
41.6 \\
41.9 \\
41.9 \\
42.0 \\
42.0\end{array}$ & $\begin{array}{l}31.8 \\
31.9 \\
32.0 \\
31.9 \\
32.1 \\
32.1\end{array}$ & $\begin{array}{l}1311 \\
1332 \\
1462 \\
1517 \\
1570 \\
1550\end{array}$ & $\begin{array}{r}594 \\
583 \\
766 \\
880 \\
1147 \\
1150\end{array}$ \\
\hline $\begin{array}{l}.71 \\
072 \\
073 \\
074 \\
075 \\
076\end{array}$ & $\begin{array}{l}5.39 \\
5.54 \\
5.88 \\
5.63 \\
5.64 \\
5.27\end{array}$ & $\begin{array}{l}0.199 \\
0.203 \\
0.271 \\
0.308 \\
0.391 \\
0.394\end{array}$ & $\begin{array}{r}11.56 \\
13.22 \\
8.39 \\
9.07 \\
5.80 \\
0.59\end{array}$ & $\begin{array}{l}42.3 \\
41.9 \\
42.3 \\
43.9 \\
41.8 \\
41.7\end{array}$ & $\begin{array}{l}32.0 \\
32.1 \\
31.9 \\
32.5 \\
32.5 \\
32.5\end{array}$ & $\begin{array}{l}1402 \\
1377 \\
1486 \\
1578 \\
1525 \\
1524\end{array}$ & $\begin{array}{l}608 \\
609 \\
807 \\
887 \\
1157 \\
11 \leq 1\end{array}$ \\
\hline
\end{tabular}




\begin{tabular}{|c|c|c|c|c|c|c|c|}
\hline $\begin{array}{c}\text { Run } \\
\#\end{array}$ & $\begin{array}{r}\text { Fuel } \\
\#\end{array}$ & $\begin{array}{c}\text { Load } \\
\%\end{array}$ & $\begin{array}{c}\text { Power } \\
\text { BHP }\end{array}$ & $\begin{array}{l}H \geq 0 \\
w t-\%\end{array}$ & $\begin{array}{c}\text { 口e Intake } \\
\text { vol-\% }\end{array}$ & $\begin{array}{r}\text { OE Exhaust } \\
\text { yol } 1-\%\end{array}$ & $\begin{array}{c}\text { oe Bottie } \\
\text { lb/min }\end{array}$ \\
\hline $\begin{array}{l}077 \\
078 \\
079 \\
080 \\
081 \\
082\end{array}$ & $\begin{array}{l}4 \\
4 \\
4 \\
4 \\
4 \\
4\end{array}$ & $\begin{array}{l}50 \\
50 \\
50 \\
50 \\
50 \\
50\end{array}$ & $\begin{array}{l}25.0 \\
25.0 \\
37.5 \\
43.5 \\
56.4 \\
56.5\end{array}$ & $\begin{array}{l}5.8 \\
5.8 \\
5.8 \\
6.5 \\
5.9 \\
7.3\end{array}$ & $\begin{array}{l}29.99 \\
35.05 \\
30.00 \\
34.98 \\
30.01 \\
35.04\end{array}$ & $\begin{array}{l}22.75 \\
27.68 \\
19.71 \\
23.06 \\
14.27 \\
19.05\end{array}$ & $\begin{array}{l}0.996 \\
1.506 \\
1.002 \\
1.497 \\
0.996 \\
1.497\end{array}$ \\
\hline $\begin{array}{l}083 \\
084 \\
085 \\
086 \\
087 \\
088\end{array}$ & $\begin{array}{l}4 \\
4 \\
4 \\
4 \\
4 \\
4\end{array}$ & $\begin{array}{l}50 \\
50 \\
50 \\
50 \\
50 \\
50\end{array}$ & $\begin{array}{l}25.1 \\
25.1 \\
35.7 \\
41.5 \\
58.5 \\
59.5\end{array}$ & $\begin{array}{l}10.0 \\
10.8 \\
10.3 \\
10.8 \\
11.3 \\
10.4\end{array}$ & $\begin{array}{l}29.95 \\
35.06 \\
30.03 \\
35.01 \\
30.04 \\
35.04\end{array}$ & $\begin{array}{l}22.48 \\
26.98 \\
19.81 \\
23.10 \\
13.37 \\
18.33\end{array}$ & $\begin{array}{l}0.970 \\
1.497 \\
0.989 \\
1.489 \\
0.989 \\
1.489\end{array}$ \\
\hline $\begin{array}{l}089 \\
090 \\
091 \\
092\end{array}$ & $\begin{array}{l}2 \\
2 \\
2 \\
2\end{array}$ & $\begin{array}{l}100 \\
100 \\
100 \\
100\end{array}$ & $\begin{array}{l}50.0 \\
50.0 \\
74.2 \\
75.4\end{array}$ & $\begin{array}{l}0.0 \\
0.0 \\
0.0 \\
0.0\end{array}$ & $\begin{array}{l}30.00 \\
34.99 \\
30.03 \\
34.97\end{array}$ & $\begin{array}{l}20.26 \\
25.31 \\
15.56 \\
20.26\end{array}$ & $\begin{array}{l}1.240 \\
1.800 \\
1.240 \\
1.800\end{array}$ \\
\hline $\begin{array}{l}093 \\
094 \\
095 \\
096\end{array}$ & $\begin{array}{l}2 \\
2 \\
2 \\
2\end{array}$ & $\begin{array}{l}100 \\
100 \\
100 \\
100\end{array}$ & $\begin{array}{l}50.0 \\
50.0 \\
74.2 \\
74.7\end{array}$ & $\begin{array}{l}3.2 \\
7.2 \\
5.6 \\
5.7\end{array}$ & $\begin{array}{l}30.03 \\
35.05 \\
29.99 \\
35.00\end{array}$ & $\begin{array}{l}20.44 \\
25.25 \\
15.34 \\
20.25\end{array}$ & $\begin{array}{l}1.240 \\
1.890 \\
1.240 \\
1.800\end{array}$ \\
\hline $\begin{array}{l}097 \\
098 \\
099 \\
100\end{array}$ & $\begin{array}{l}2 \\
2 \\
2 \\
2\end{array}$ & $\begin{array}{l}100 \\
100 \\
100 \\
100\end{array}$ & $\begin{array}{l}50.1 \\
50.0 \\
73.9 \\
74.8\end{array}$ & $\begin{array}{r}19.3 \\
4.5 \\
3.6 \\
6.9\end{array}$ & $\begin{array}{l}30.06 \\
34.50 \\
30.05 \\
35.05\end{array}$ & $\begin{array}{l}20.28 \\
24.75 \\
15.42 \\
20.39\end{array}$ & $\begin{array}{l}1.240 \\
1.710 \\
1.190 \\
1.800\end{array}$ \\
\hline $\begin{array}{l}101 \\
102 \\
103 \\
104\end{array}$ & $\begin{array}{l}4 \\
4 \\
4 \\
4\end{array}$ & $\begin{array}{l}100 \\
100 \\
100 \\
100\end{array}$ & $\begin{array}{l}49.9 \\
49.9 \\
74.9 \\
74.7\end{array}$ & $\begin{array}{l}0.0 \\
0.0 \\
0.0 \\
0.0\end{array}$ & $\begin{array}{l}30.07 \\
34.99 \\
29.99 \\
34.10\end{array}$ & $\begin{array}{l}20.23 \\
24.80 \\
14.83 \\
19.00\end{array}$ & $\begin{array}{l}1.250 \\
1.920 \\
1.240 \\
1.690\end{array}$ \\
\hline $\begin{array}{l}105 \\
106 \\
107 \\
108\end{array}$ & $\begin{array}{l}4 \\
4 \\
4 \\
4\end{array}$ & $\begin{array}{l}100 \\
100 \\
100 \\
100\end{array}$ & $\begin{array}{l}50.1 \\
49.9 \\
74.5 \\
74.2\end{array}$ & $\begin{array}{l}12.0 \\
11.1 \\
1 \Xi .5 \\
12.6\end{array}$ & $\begin{array}{l}30.03 \\
34.55 \\
30.05 \\
35.00\end{array}$ & $\begin{array}{l}19.96 \\
24.52 \\
15.00 \\
19.93\end{array}$ & $\begin{array}{l}1.230 \\
1.770 \\
1.210 \\
1.800\end{array}$ \\
\hline $\begin{array}{l}109 \\
110 \\
111 \\
112\end{array}$ & $\begin{array}{l}4 \\
4 \\
4 \\
4\end{array}$ & $\begin{array}{l}100 \\
100 \\
100 \\
100\end{array}$ & $\begin{array}{l}50.1 \\
50.1 \\
69.2 \\
67.5\end{array}$ & $\begin{array}{l}18.2 \\
17.3 \\
17.4 \\
16.9\end{array}$ & $\begin{array}{l}30.05 \\
34.95 \\
29.94 \\
34.90\end{array}$ & $\begin{array}{l}20.05 \\
24.97 \\
17.94 \\
24.20\end{array}$ & $\begin{array}{l}1.250 \\
1.930 \\
1.250 \\
1.800\end{array}$ \\
\hline
\end{tabular}




\begin{tabular}{|c|c|c|c|c|c|c|c|}
\hline Run & $\begin{array}{l}\text { Air Flow } \\
\text { lb/min }\end{array}$ & $\begin{array}{l}\text { Net Fuel } \\
\text { 1b/min }\end{array}$ & $02 /$ Fuel & $\begin{array}{l}\text { P Intake } \\
\text { in. Hg abs }\end{array}$ & $\begin{array}{c}\text { PExh. } \\
\text { in.Hg abs }\end{array}$ & $\begin{array}{l}\text { P Peak } \\
\text { Psi }\end{array}$ & $\begin{array}{l}\text { T Exh. } \\
\text { deg F }\end{array}$ \\
\hline $\begin{array}{l}077 \\
078 \\
079 \\
080 \\
081 \\
082\end{array}$ & $\begin{array}{l}6.09 \\
5.74 \\
6.06 \\
5.58 \\
5.85 \\
5.47\end{array}$ & $\begin{array}{l}0.182 \\
0.183 \\
0.254 \\
0.293 \\
0.386 \\
0.386\end{array}$ & $\begin{array}{r}13.19 \\
15.47 \\
9.42 \\
9.48 \\
6.06 \\
7.14\end{array}$ & $\begin{array}{l}44.1 \\
44.1 \\
44.2 \\
43.8 \\
43.7 \\
43.8\end{array}$ & $\begin{array}{l}32.0 \\
32.1 \\
32.0 \\
32.7 \\
33.0 \\
33.0\end{array}$ & $\begin{array}{l}1317 \\
1397 \\
1485 \\
1481 \\
1446 \\
1474\end{array}$ & $\begin{array}{r}591 \\
591 \\
784 \\
902 \\
1150 \\
1149\end{array}$ \\
\hline $\begin{array}{l}083 \\
084 \\
085 \\
086 \\
087 \\
088\end{array}$ & $\begin{array}{l}5.93 \\
5.59 \\
5.88 \\
5.53 \\
5.76 \\
5.42\end{array}$ & $\begin{array}{l}0.185 \\
0.188 \\
0.244 \\
0.282 \\
0.393 \\
0.401\end{array}$ & $\begin{array}{r}12.61 \\
14.81 \\
9.60 \\
9.78 \\
5.89 \\
6.82\end{array}$ & $\begin{array}{l}43.3 \\
43.2 \\
43.1 \\
43.2 \\
43.1 \\
43.3\end{array}$ & $\begin{array}{l}32.8 \\
32.8 \\
32.8 \\
32.7 \\
32.9 \\
32.9\end{array}$ & $\begin{array}{l}1470 \\
1492 \\
1559 \\
1607 \\
1648 \\
1691\end{array}$ & $\begin{array}{r}598 \\
598 \\
759 \\
854 \\
1156 \\
1146\end{array}$ \\
\hline $\begin{array}{l}089 \\
090 \\
091 \\
092\end{array}$ & $\begin{array}{l}8.08 \\
7.65 \\
8.03 \\
7.53\end{array}$ & $\begin{array}{l}0.300 \\
0.303 \\
0.452 \\
0.463\end{array}$ & $\begin{array}{r}10.33 \\
11.75 \\
6.83 \\
7.63\end{array}$ & $\begin{array}{l}55.7 \\
55.6 \\
55.6 \\
55.6\end{array}$ & $\begin{array}{l}32.5 \\
31.7 \\
31.8 \\
31.8\end{array}$ & $\begin{array}{l}1842 \\
1890 \\
1929 \\
1970\end{array}$ & $\begin{array}{r}782 \\
760 \\
1100 \\
1100\end{array}$ \\
\hline $\begin{array}{l}193 \\
094 \\
095 \\
096\end{array}$ & $\begin{array}{l}8.06 \\
7.59 \\
7.91 \\
7.41\end{array}$ & $\begin{array}{l}0.299 \\
0.295 \\
0.451 \\
0.453\end{array}$ & $\begin{array}{r}10.36 \\
12.34 \\
6.78 \\
7.73\end{array}$ & $\begin{array}{l}55.6 \\
55.6 \\
55.4 \\
55.5\end{array}$ & $\begin{array}{l}33.1 \\
33.1 \\
33.2 \\
33.2\end{array}$ & $\begin{array}{l}1772 \\
1867 \\
1863 \\
1907\end{array}$ & $\begin{array}{l}760 \\
756 \\
1102 \\
1100\end{array}$ \\
\hline $\begin{array}{l}097 \\
098 \\
099 \\
100\end{array}$ & $\begin{array}{l}8.03 \\
7.67 \\
7.90 \\
7.42\end{array}$ & $\begin{array}{l}0.284 \\
0.298 \\
0.440 \\
0.429\end{array}$ & $\begin{array}{r}10.88 \\
11.67 \\
6.84 \\
8.17\end{array}$ & $\begin{array}{l}55.4 \\
55.4 \\
55.3 \\
55.4\end{array}$ & $\begin{array}{l}33.2 \\
33.2 \\
33.4 \\
33.4\end{array}$ & $\begin{array}{l}1822 \\
1852 \\
1879 \\
1934\end{array}$ & $\begin{array}{r}746 \\
748 \\
1102 \\
1098\end{array}$ \\
\hline $\begin{array}{l}101 \\
102 \\
103 \\
104\end{array}$ & $\begin{array}{l}8.19 \\
7.69 \\
8.01 \\
7.53\end{array}$ & $\begin{array}{l}0.339 \\
0.347 \\
0.522 \\
0.526\end{array}$ & $\begin{array}{r}9.24 \\
10.62 \\
5.91 \\
6.51\end{array}$ & $\begin{array}{l}56.9 \\
56.9 \\
56.7 \\
56.7\end{array}$ & $\begin{array}{l}33.7 \\
33.7 \\
34.0 \\
33.8\end{array}$ & $\begin{array}{l}1920 \\
1935 \\
1938 \\
1919\end{array}$ & $\begin{array}{l}747 \\
753 \\
1107 \\
1096\end{array}$ \\
\hline $\begin{array}{l}105 \\
105 \\
107 \\
108\end{array}$ & $\begin{array}{l}8.18 \\
7.76 \\
7.99 \\
7.59\end{array}$ & $\begin{array}{l}0.317 \\
0.321 \\
0.479 \\
0.482\end{array}$ & $\begin{array}{r}9.83 \\
11.08 \\
6.36 \\
7.36\end{array}$ & $\begin{array}{l}56.6 \\
56.8 \\
56.6 \\
56.7\end{array}$ & $\begin{array}{l}33.4 \\
33.5 \\
33.9 \\
33.9\end{array}$ & $\begin{array}{l}1884 \\
1879 \\
1875 \\
1920\end{array}$ & $\begin{array}{r}759 \\
750 \\
1107 \\
1101\end{array}$ \\
\hline $\begin{array}{l}109 \\
110 \\
111 \\
1: \equiv\end{array}$ & $\begin{array}{l}8.13 \\
7.73 \\
8.10 \\
7.68\end{array}$ & $\begin{array}{l}0.315 \\
0.319 \\
0.448 \\
0.436\end{array}$ & $\begin{array}{r}9.90 \\
11.61 \\
6.95 \\
8.18\end{array}$ & $\begin{array}{l}57.1 \\
57.2 \\
57.1 \\
57.0\end{array}$ & $\begin{array}{l}33.5 \\
33.7 \\
33.8 \\
33.8\end{array}$ & $\begin{array}{l}1867 \\
1577 \\
1951 \\
1863\end{array}$ & $\begin{array}{r}758 \\
751 \\
1042 \\
1016\end{array}$ \\
\hline
\end{tabular}


A-11

A.2 Grouping of Test Data 
No. 2 Diesel Fuel -- 50\% Load

\begin{tabular}{|c|c|c|c|c|c|c|c|c|c|c|c|}
\hline $\begin{array}{c}H 2 D \\
\%\end{array}$ & 02 & $\begin{array}{c}D Z / F \\
--\end{array}$ & Eq. $R$ & $\begin{array}{l}\text { Power } \\
\text { BHP }\end{array}$ & $\begin{array}{c}\text { P Peak } \\
\text { psi }\end{array}$ & $\begin{array}{l}\text { Ig Del } \\
\text { deg }\end{array}$ & $\begin{array}{c}\text { Eff } \\
\%\end{array}$ & $\begin{array}{c}N O x \\
\mathrm{pPm} / \mathrm{BHP}\end{array}$ & $\begin{array}{l}\text { Part. } \\
\text { mg/hph }\end{array}$ & $\begin{array}{c}\text { Smoke } \\
\%\end{array}$ & Run \\
\hline $\begin{array}{r}0.0 \\
4.0 \\
20.2\end{array}$ & $\begin{array}{l}21 \\
21 \\
21\end{array}$ & $\begin{array}{l}9.17 \\
9.64 \\
8.48\end{array}$ & $\begin{array}{l}0.36 \\
0.35 \\
0.39\end{array}$ & $\begin{array}{l}25.0 \\
24.3 \\
25.0\end{array}$ & $\begin{array}{l}1333 \\
1438 \\
1355\end{array}$ & $\begin{array}{l}19.0 \\
19.0 \\
22.0\end{array}$ & $\begin{array}{l}33.3 \\
35.0 \\
31.1\end{array}$ & $\begin{array}{l}26.89 \\
21.81 \\
10.80\end{array}$ & $\begin{array}{l}145.5 \\
123.3 \\
157.8\end{array}$ & $\begin{array}{l}37 \\
16 \\
11\end{array}$ & $\begin{array}{r}2 \\
5 \\
9 A\end{array}$ \\
\hline $\begin{array}{r}0.0 \\
5.0 \\
18.9\end{array}$ & $\begin{array}{l}25 \\
25 \\
25\end{array}$ & $\begin{array}{l}11.68 \\
12.20 \\
11.35\end{array}$ & $\begin{array}{l}0.29 \\
0.27 \\
0.29\end{array}$ & $\begin{array}{l}25.0 \\
24.3 \\
25.0\end{array}$ & $\begin{array}{l}1384 \\
1445 \\
1374\end{array}$ & $\begin{array}{l}17.3 \\
17.5 \\
19.1\end{array}$ & $\begin{array}{l}34.4 \\
34.7 \\
34.1\end{array}$ & $\begin{array}{l}63.20 \\
46.90 \\
29.00\end{array}$ & $\begin{array}{r}75.0 \\
187.9 \\
159.3\end{array}$ & $\begin{array}{l}8 \\
2 \\
3\end{array}$ & $\begin{array}{r}3 \\
6 \\
9 A\end{array}$ \\
\hline $\begin{array}{r}0.0 \\
5.1 \\
12.4\end{array}$ & $\begin{array}{l}25 \\
25 \\
25\end{array}$ & $\begin{array}{l}9.10 \\
9.80 \\
9.17\end{array}$ & $\begin{array}{l}0.37 \\
0.34 \\
0.36\end{array}$ & $\begin{array}{l}33.5 \\
31.8 \\
32.0\end{array}$ & $\begin{array}{l}1464 \\
1512 \\
1423\end{array}$ & $\begin{array}{l}17.2 \\
17.2 \\
18.7\end{array}$ & $\begin{array}{l}36.0 \\
36.2 \\
35.4\end{array}$ & $\begin{array}{l}53.88 \\
43.24 \\
27.50\end{array}$ & & $\begin{array}{r}16 \\
3 \\
3\end{array}$ & $\begin{array}{r}4 A \\
7 \\
10 A\end{array}$ \\
\hline $\begin{array}{l}0.0 \\
7.0\end{array}$ & $\begin{array}{l}25 \\
25\end{array}$ & $\begin{array}{l}6.62 \\
7.04\end{array}$ & $\begin{array}{l}0.50 \\
0.47\end{array}$ & $\begin{array}{l}47.5 \\
43.8\end{array}$ & $\begin{array}{l}1586 \\
1472\end{array}$ & $\begin{array}{l}16.9 \\
17.4\end{array}$ & $\begin{array}{l}37.2 \\
37.3\end{array}$ & $\begin{array}{l}48.90 \\
25.70\end{array}$ & & $\begin{array}{r}16 \\
4\end{array}$ & $\begin{array}{r}4 \\
11 A\end{array}$ \\
\hline $\begin{array}{r}0.0 \\
14.4 \\
23.9\end{array}$ & $\begin{array}{l}30 \\
30 \\
30\end{array}$ & $\begin{array}{l}11.88 \\
12.90 \\
13.79\end{array}$ & $\begin{array}{l}0.28 \\
0.25 \\
0.24\end{array}$ & $\begin{array}{l}25.1 \\
25.1 \\
25.0\end{array}$ & $\begin{array}{l}1399 \\
1326 \\
1311\end{array}$ & $\begin{array}{l}16.7 \\
18.3 \\
18.3\end{array}$ & $\begin{array}{l}30.7 \\
33.6 \\
36.1\end{array}$ & $\begin{array}{l}34.46 \\
63.94 \\
57.00\end{array}$ & $\begin{array}{l}116.7 \\
243.9 \\
145.3\end{array}$ & $\begin{array}{l}6 \\
5 \\
5\end{array}$ & $\begin{array}{l}53 \\
59 \\
55\end{array}$ \\
\hline $\begin{array}{r}0.0 \\
5.1 \\
14.9\end{array}$ & $\begin{array}{l}30 \\
30 \\
30\end{array}$ & $\begin{array}{l}8.64 \\
9.52 \\
9.18\end{array}$ & $\begin{array}{l}0.39 \\
0.35 \\
0.36\end{array}$ & $\begin{array}{l}39.3 \\
38.5 \\
37.4\end{array}$ & $\begin{array}{l}1521 \\
1422 \\
1462\end{array}$ & $\begin{array}{l}16.2 \\
16.7 \\
17.2\end{array}$ & $\begin{array}{l}34.7 \\
38.4 \\
35.9\end{array}$ & $\begin{array}{l}24.66 \\
55.32 \\
38.50\end{array}$ & $\begin{array}{r}54.3 \\
105.0 \\
361.5\end{array}$ & $\begin{array}{l}5 \\
7 \\
9\end{array}$ & $\begin{array}{l}54 \\
61 \\
67\end{array}$ \\
\hline $\begin{array}{l}0.0 \\
2.0 \\
E . \equiv\end{array}$ & $\begin{array}{l}30 \\
30 \\
30\end{array}$ & $\begin{array}{l}5.94 \\
6.11 \\
5.76\end{array}$ & $\begin{array}{l}0.56 \\
0.55 \\
0.58\end{array}$ & $\begin{array}{l}60.3 \\
58.3 \\
59.0\end{array}$ & $\begin{array}{l}1573 \\
1550 \\
1570\end{array}$ & $\begin{array}{l}15.6 \\
16.0 \\
16.1\end{array}$ & $\begin{array}{l}36.3 \\
37.4 \\
35.6\end{array}$ & $\begin{array}{c}13.18 \\
20.24 \\
1.51\end{array}$ & $\begin{array}{r}.4 .4 \\
88.3 \\
111.7\end{array}$ & $\begin{array}{r}14 \\
7 \\
9\end{array}$ & $\begin{array}{l}55 \\
63 \\
69\end{array}$ \\
\hline $\begin{array}{l}0.0 \\
7.1 \\
2 \equiv . \Xi\end{array}$ & $\begin{array}{l}35 \\
35 \\
35\end{array}$ & $\begin{array}{l}13.30 \\
15.13 \\
15.42\end{array}$ & $\begin{array}{l}0.25 \\
0 . \equiv 2 \\
0.23\end{array}$ & $\begin{array}{l}24.9 \\
25.1 \\
25.0\end{array}$ & $\begin{array}{l}1343 \\
1337 \\
1332\end{array}$ & $\begin{array}{l}15.7 \\
16.2 \\
17.6\end{array}$ & $\begin{array}{l}28.9 \\
33.4 \\
34.2\end{array}$ & $\begin{array}{l}32.73 \\
79.08 \\
71.80\end{array}$ & $\begin{array}{l}214.1 \\
159.8 \\
207.5\end{array}$ & $\begin{array}{l}3 \\
5 \\
4\end{array}$ & $\begin{array}{l}\text { Et } \\
60 \\
66\end{array}$ \\
\hline $\begin{array}{l}0.0 \\
3.0 \\
-.5\end{array}$ & $\begin{array}{l}35 \\
35 \\
35\end{array}$ & $\begin{array}{l}5.22 \\
0.11 \\
0.50\end{array}$ & $\begin{array}{l}0.36 \\
0.37 \\
0.35\end{array}$ & $\begin{array}{l}44.7 \\
45.7 \\
43.9\end{array}$ & $\begin{array}{l}1549 \\
15: 2 \\
: 517\end{array}$ & $\begin{array}{l}15.0 \\
15.1 \\
15.3\end{array}$ & $\begin{array}{l}35.9 \\
37.4 \\
37.2\end{array}$ & $\begin{array}{l}46.42 \\
43.54 \\
16.17\end{array}$ & $\begin{array}{r}99.9 \\
34.3 \\
117.5\end{array}$ & $\begin{array}{r}10 \\
6 \\
10\end{array}$ & $\begin{array}{l}57 \\
62 \\
68\end{array}$ \\
\hline $\begin{array}{l}0.0 \\
\equiv .4 \\
\Xi . \Xi\end{array}$ & $\begin{array}{l}35 \\
35 \\
25\end{array}$ & $\begin{array}{l}6.76 \\
7.30 \\
6.56\end{array}$ & $\begin{array}{l}0.49 \\
0.46 \\
0.51\end{array}$ & $\begin{array}{l}60.2 \\
58.1 \\
50.7\end{array}$ & $\begin{array}{l}1611 \\
1549 \\
1550\end{array}$ & $\begin{array}{l}14.3 \\
14.9 \\
15.1\end{array}$ & $\begin{array}{l}36.0 \\
38.8 \\
35.0\end{array}$ & $\begin{array}{r}22.43 \\
23.06 \\
0.65\end{array}$ & $\begin{array}{r}94.0 \\
62.9 \\
127.2\end{array}$ & $\begin{array}{r}10 \\
6 \\
7\end{array}$ & $\begin{array}{l}58 \\
64 \\
70\end{array}$ \\
\hline
\end{tabular}


No. 2 Diesel Fuel -- 100\% Load

\begin{tabular}{|c|c|c|c|c|c|c|c|c|c|c|c|}
\hline $\begin{array}{c}\text { HED } \\
\%\end{array}$ & o. & $\begin{array}{c}D E / F \\
--\end{array}$ & Eq. $R$ & $\begin{array}{l}\text { Power } \\
\text { BHP }\end{array}$ & $\begin{array}{l}\text { P Pea } \\
\text { psi }\end{array}$ & $\begin{array}{c}\text { Ig DeI } \\
\text { deg }\end{array}$ & $\begin{array}{c}\text { Eff } \\
\%\end{array}$ & $\begin{array}{c}N O x \\
P R m / B H P\end{array}$ & $\begin{array}{l}\text { Part. } \\
\mathrm{mg} / \mathrm{hph}\end{array}$ & $\begin{array}{l}\text { Smoke } \\
\%\end{array}$ & Ruin \\
\hline & -- & ---- & --- & $-\cdots$ & $\ldots-$ & & ---- & ------ & - - & --- & \\
\hline $\begin{array}{r}0 \\
2.8 \\
25.6\end{array}$ & $\begin{array}{l}21 \\
21 \\
21\end{array}$ & $\begin{array}{l}6.68 \\
6.07 \\
7.19\end{array}$ & $\begin{array}{l}0.50 \\
0.55 \\
0.46\end{array}$ & $\begin{array}{l}49.9 \\
49.9 \\
50.0\end{array}$ & $\begin{array}{l}1644 \\
1649 \\
1709\end{array}$ & $\begin{array}{l}17.1 \\
17.1 \\
20.0\end{array}$ & $\begin{array}{l}37.2 \\
34.1 \\
39.6\end{array}$ & $\begin{array}{l}9.12 \\
9.82 \\
8.10\end{array}$ & $\begin{array}{l}121.4 \\
113.1 \\
134.7\end{array}$ & $\begin{array}{r}15 \\
12 \\
2\end{array}$ & $\begin{array}{l}37 \\
41 \\
45\end{array}$ \\
\hline $\begin{array}{r}0 \\
4.2 \\
21.0\end{array}$ & $\begin{array}{l}25 \\
25 \\
25\end{array}$ & $\begin{array}{l}8.07 \\
7.64 \\
9.05\end{array}$ & $\begin{array}{l}0.4 ! \\
0.44 \\
0.37\end{array}$ & $\begin{array}{l}50.1 \\
50.1 \\
49.9\end{array}$ & $\begin{array}{l}1725 \\
1706 \\
1773\end{array}$ & $\begin{array}{l}16.0 \\
16.1 \\
17.0\end{array}$ & $\begin{array}{l}38.0 \\
35.7 \\
41.5\end{array}$ & $\begin{array}{l}20.96 \\
22.26 \\
23.55\end{array}$ & $\begin{array}{r}55.0 \\
67.4 \\
131.8\end{array}$ & $\begin{array}{l}5 \\
3 \\
1\end{array}$ & $\begin{array}{l}38 \\
42 \\
46\end{array}$ \\
\hline $\begin{array}{r}0 \\
4.6 \\
18.3\end{array}$ & $\begin{array}{l}25 \\
25 \\
25\end{array}$ & $\begin{array}{l}6.52 \\
6.55 \\
7.22\end{array}$ & $\begin{array}{l}0.51 \\
0.51 \\
0.46\end{array}$ & $\begin{array}{l}60.6 \\
60.5 \\
61.0\end{array}$ & $\begin{array}{l}1742 \\
1762 \\
1766\end{array}$ & $\begin{array}{l}15.6 \\
16.0 \\
16.5\end{array}$ & $\begin{array}{l}37.8 \\
37.1 \\
40.5\end{array}$ & $\begin{array}{l}19.14 \\
19.92 \\
20.74\end{array}$ & $\begin{array}{l}87.0 \\
82.0 \\
92.0\end{array}$ & $\begin{array}{l}2 \\
3 \\
2\end{array}$ & $\begin{array}{l}39 \\
43 \\
47\end{array}$ \\
\hline $\begin{array}{r}0 \\
4.7 \\
14.7\end{array}$ & $\begin{array}{l}25 \\
25 \\
25\end{array}$ & $\begin{array}{l}5.51 \\
5.55 \\
5.93\end{array}$ & $\begin{array}{l}0.61 \\
0.60 \\
0.56\end{array}$ & $\begin{array}{l}70.7 \\
70.0 \\
71.2\end{array}$ & $\begin{array}{l}1771 \\
1763 \\
1766\end{array}$ & $\begin{array}{l}15.3 \\
16.0 \\
16.0\end{array}$ & $\begin{array}{l}36.9 \\
36.8 \\
39.2\end{array}$ & $\begin{array}{l}17.54 \\
18.29 \\
18.82\end{array}$ & $\begin{array}{r}118.0 \\
98.0 \\
143.0\end{array}$ & $\begin{array}{l}3 \\
3 \\
3\end{array}$ & $\begin{array}{l}40 \\
44 \\
48\end{array}$ \\
\hline $\begin{array}{r}0 \\
8.2 \\
19.3\end{array}$ & $\begin{array}{l}30 \\
30 \\
30\end{array}$ & $\begin{array}{l}10.30 \\
10.40 \\
10.90\end{array}$ & $\begin{array}{l}0.32 \\
0.32 \\
0.31\end{array}$ & $\begin{array}{l}50.0 \\
50.0 \\
50.1\end{array}$ & $\begin{array}{l}1842 \\
1772 \\
1822\end{array}$ & $\begin{array}{l}15.2 \\
16.3 \\
16.2\end{array}$ & $\begin{array}{l}38.6 \\
38.7 \\
36.3\end{array}$ & $\begin{array}{l}54.20 \\
60.20 \\
58.70\end{array}$ & $\begin{array}{r}181.7 \\
112.8 \\
91.9\end{array}$ & $\begin{array}{r}25 \\
2 \\
5\end{array}$ & $\begin{array}{l}89 \\
93 \\
97\end{array}$ \\
\hline $\begin{array}{r}0 \\
3.6 \\
5.6\end{array}$ & $\begin{array}{l}30 \\
30 \\
30\end{array}$ & $\begin{array}{l}6.83 \\
6.84 \\
6.78\end{array}$ & $\begin{array}{l}0.49 \\
0.49 \\
0.49\end{array}$ & $\begin{array}{l}74.2 \\
73.9 \\
74.2\end{array}$ & $\begin{array}{l}1929 \\
1879 \\
1863\end{array}$ & $\begin{array}{l}15.0 \\
15.2 \\
15.3\end{array}$ & $\begin{array}{l}38.0 \\
38.9 \\
38.0\end{array}$ & $\begin{array}{l}42.70 \\
37.10 \\
47.70\end{array}$ & $\begin{array}{l}144.3 \\
297.1 \\
120.0\end{array}$ & $\begin{array}{r}11 \\
10 \\
4\end{array}$ & $\begin{array}{l}91 \\
99 \\
95\end{array}$ \\
\hline $\begin{array}{r}0 \\
4.5 \\
7.2\end{array}$ & $\begin{array}{r}35 \\
34.5 \\
35\end{array}$ & $\begin{array}{l}11.70 \\
11.70 \\
12.30\end{array}$ & $\begin{array}{l}0.29 \\
0.29 \\
0.27\end{array}$ & $\begin{array}{l}50.0 \\
50.0 \\
50.0\end{array}$ & $\begin{array}{l}1890 \\
1852 \\
1867\end{array}$ & $\begin{array}{l}14.4 \\
14.7 \\
14.6\end{array}$ & $\begin{array}{l}38.2 \\
38.8 \\
39.2\end{array}$ & $\begin{array}{l}58.40 \\
60.40 \\
64.60\end{array}$ & $\begin{array}{r}95.9 \\
74.3 \\
100.0\end{array}$ & $\begin{array}{r}10 \\
6 \\
2\end{array}$ & $\begin{array}{l}90 \\
98 \\
94\end{array}$ \\
\hline $\begin{array}{r}0 \\
5.7 \\
6.9\end{array}$ & $\begin{array}{l}35 \\
35 \\
35\end{array}$ & $\begin{array}{l}7.63 \\
7.73 \\
8.17\end{array}$ & $\begin{array}{l}0.44 \\
0.43 \\
0.41\end{array}$ & $\begin{array}{l}75.4 \\
74.7 \\
74.8\end{array}$ & $\begin{array}{l}1907 \\
1934\end{array}$ & $\begin{array}{l}13.6 \\
14.2 \\
13.5\end{array}$ & $\begin{array}{l}37.7 \\
38.1 \\
40.3\end{array}$ & $\begin{array}{l}43.00 \\
50.60 \\
37.40\end{array}$ & $\begin{array}{r}97.8 \\
100.0 \\
90.0\end{array}$ & $\begin{array}{r}11 \\
2 \\
7\end{array}$ & $\begin{array}{r}92 \\
96 \\
100\end{array}$ \\
\hline
\end{tabular}


No. 4 Diesel Fuel - 50 \% Load

\begin{tabular}{|c|c|c|c|c|c|c|c|c|c|c|c|}
\hline $\begin{array}{c}\mathrm{H} 2 \mathrm{O} \\
\%\end{array}$ & $0 ?$ & $\begin{array}{c}D 2 / F \\
--\end{array}$ & $\underset{--}{E q .} R$ & $\begin{array}{c}\text { Power } \\
\text { BHP }\end{array}$ & $\begin{array}{c}P \text { Peak } \\
\text { psi }\end{array}$ & $\begin{array}{l}\text { Ig } D e l \\
\text { deg }\end{array}$ & $\begin{array}{c}\text { Eff } \\
\%\end{array}$ & $\begin{array}{c}N O x \\
p R / B H P\end{array}$ & $\begin{array}{l}\text { Part. } \\
\text { mg/hph }\end{array}$ & $\begin{array}{c}\text { Smoke } \\
\%\end{array}$ & $\underset{\ddagger}{R}$ \\
\hline--- & -- & ---- & $--\cdots$ & ---- & ---- & ------ & --- & -ーーーーー & ------ & -- & -- \\
\hline $\begin{array}{r}0.0 \\
3.2 \\
10.2\end{array}$ & $\begin{array}{l}\equiv 1 \\
\equiv 1 \\
\equiv 1\end{array}$ & $\begin{array}{l}8.34 \\
8.68 \\
7.79\end{array}$ & $\begin{array}{l}0.40 \\
0.38 \\
0.43\end{array}$ & $\begin{array}{l}25.0 \\
24.1 \\
24.7\end{array}$ & $\begin{array}{l}1389 \\
1347 \\
1369\end{array}$ & $\begin{array}{l}21.2 \\
21.4 \\
22.2\end{array}$ & $\begin{array}{l}31.9 \\
32.8 \\
31.1\end{array}$ & $\begin{array}{l}24.72 \\
24.31 \\
25.30\end{array}$ & $\begin{array}{l}463.5 \\
596.2 \\
543.0\end{array}$ & $\begin{array}{l}14 \\
14 \\
20\end{array}$ & $\begin{array}{l}12 \\
16 \\
20\end{array}$ \\
\hline $\begin{array}{r}0.0 \\
3.7 \\
10.6\end{array}$ & $\begin{array}{l}25 \\
25 \\
25\end{array}$ & $\begin{array}{r}10.54 \\
10.71 \\
9.76\end{array}$ & $\begin{array}{l}0.31 \\
0.31 \\
0.34\end{array}$ & $\begin{array}{l}24.9 \\
24.0 \\
24.7\end{array}$ & $\begin{array}{l}1442 \\
1477 \\
1446\end{array}$ & $\begin{array}{l}19.3 \\
19.9 \\
18.9\end{array}$ & $\begin{array}{l}31.9 \\
31.6 \\
31.4\end{array}$ & $\begin{array}{l}56.43 \\
53.75 \\
59.11\end{array}$ & $\begin{array}{l}471.9 \\
495.3 \\
629.8\end{array}$ & $\begin{array}{r}5 \\
10 \\
9\end{array}$ & $\begin{array}{l}13 \\
17 \\
21\end{array}$ \\
\hline $\begin{array}{r}0.0 \\
5.3 \\
10.5\end{array}$ & $\begin{array}{l}25 \\
25 \\
25\end{array}$ & $\begin{array}{l}9.63 \\
9.03 \\
9.39\end{array}$ & $\begin{array}{l}0.38 \\
0.37 \\
0.40\end{array}$ & $\begin{array}{l}32.8 \\
31.3 \\
31.7\end{array}$ & $\begin{array}{l}1553 \\
1485 \\
1454\end{array}$ & $\begin{array}{l}19.3 \\
19.3 \\
19.1\end{array}$ & $\begin{array}{l}34.6 \\
34.9 \\
34.6\end{array}$ & $\begin{array}{l}52.13 \\
48.40 \\
55.99\end{array}$ & $\begin{array}{l}423.3 \\
444.7 \\
456.9\end{array}$ & $\begin{array}{l}5 \\
0 \\
9\end{array}$ & $\begin{array}{l}14 \\
13 \\
\equiv 2\end{array}$ \\
\hline $\begin{array}{r}0.0 \\
4.2 \\
11.2\end{array}$ & $\begin{array}{l}25 \\
\equiv 5 \\
25\end{array}$ & $\begin{array}{l}6.32 \\
6.55 \\
6.58\end{array}$ & $\begin{array}{l}0.52 \\
0.51 \\
0.50\end{array}$ & $\begin{array}{l}46.6 \\
45.3 \\
43.4\end{array}$ & $\begin{array}{l}1757 \\
1695 \\
1602\end{array}$ & $\begin{array}{l}19.0 \\
19.3 \\
19.4\end{array}$ & $\begin{array}{l}36.3 \\
36.6 \\
36.5\end{array}$ & $\begin{array}{l}40.77 \\
40.73 \\
49.88\end{array}$ & $\begin{array}{l}321.9 \\
347.8 \\
497.7\end{array}$ & $\begin{array}{l}5 \\
8 \\
8\end{array}$ & $\begin{array}{l}15 \\
17 \\
23\end{array}$ \\
\hline $\begin{array}{r}5.0 \\
5.8 \\
10.0\end{array}$ & $\begin{array}{l}30 \\
30 \\
30\end{array}$ & $\begin{array}{l}11.56 \\
13.20 \\
12.60\end{array}$ & $\begin{array}{l}0.29 \\
0.25 \\
0.26\end{array}$ & $\begin{array}{l}25.1 \\
25.0 \\
25.1\end{array}$ & $\begin{array}{l}1402 \\
1317 \\
1470\end{array}$ & $\begin{array}{l}18.0 \\
18.4 \\
19.9\end{array}$ & $\begin{array}{l}30.4 \\
33.2 \\
32.7\end{array}$ & $\begin{array}{r}83.06 \\
74.00 \\
100.40\end{array}$ & $\begin{array}{l}553.9 \\
791.3 \\
696.1\end{array}$ & $\begin{array}{l}7 \\
10 \\
11\end{array}$ & $\begin{array}{l}71 \\
77 \\
83\end{array}$ \\
\hline $\begin{array}{r}0.0 \\
5.8 \\
10 .\end{array}$ & $\begin{array}{l}30 \\
30 \\
30\end{array}$ & $\begin{array}{l}8.39 \\
9.42 \\
9.60\end{array}$ & $\begin{array}{l}0.39 \\
0.35 \\
0.35\end{array}$ & $\begin{array}{l}38.1 \\
37.5 \\
35.7\end{array}$ & $\begin{array}{l}1486 \\
1485 \\
1559\end{array}$ & $\begin{array}{l}19.1 \\
18.7 \\
19.4\end{array}$ & $\begin{array}{l}33.9 \\
35.6 \\
35.3\end{array}$ & $\begin{array}{l}52.89 \\
56.40 \\
77.00\end{array}$ & $\begin{array}{l}529.3 \\
545.7 \\
518.0\end{array}$ & $\begin{array}{r}7 \\
19 \\
9\end{array}$ & $\begin{array}{l}73 \\
79 \\
85\end{array}$ \\
\hline $\begin{array}{r}0.5 \\
5.7 \\
1.3\end{array}$ & $\begin{array}{l}30 \\
30 \\
30\end{array}$ & $\begin{array}{l}5.80 \\
6.06 \\
5.89\end{array}$ & $\begin{array}{l}0.57 \\
0.55 \\
0.56\end{array}$ & $\begin{array}{l}55.8 \\
56.4 \\
58.5\end{array}$ & $\begin{array}{l}1525 \\
1446 \\
1643\end{array}$ & $\begin{array}{l}18.3 \\
: 9.0 \\
19.0\end{array}$ & $\begin{array}{l}34.4 \\
35.2 \\
35.9\end{array}$ & $\begin{array}{l}50.63 \\
57.45 \\
55.90\end{array}$ & $\begin{array}{l}440.7 \\
441.0 \\
473.3\end{array}$ & $\begin{array}{r}4 \\
20 \\
17\end{array}$ & $\begin{array}{l}75 \\
E 1 \\
87\end{array}$ \\
\hline $\begin{array}{r}6.8 \\
5 . \Xi \\
6.5\end{array}$ & $\begin{array}{l}\equiv 5 \\
35 \\
35\end{array}$ & $\begin{array}{l}1 \equiv . \equiv 0 \\
15.50 \\
14.50\end{array}$ & $\begin{array}{l}0.25 \\
0.21 \\
0.22\end{array}$ & $\begin{array}{l}25.0 \\
25.0 \\
25.1\end{array}$ & $\begin{array}{l}1377 \\
1397 \\
1492\end{array}$ & $\begin{array}{l}13.3 \\
1 \Xi .5 \\
1 E .0\end{array}$ & $\begin{array}{l}27.7 \\
33.0 \\
32.2\end{array}$ & $\begin{array}{r}11.60 \\
85.50 \\
108.20\end{array}$ & $\begin{array}{l}595.6 \\
603.9 \\
692.2\end{array}$ & $\begin{array}{r}6 \\
10 \\
8\end{array}$ & $\begin{array}{l}72 \\
73 \\
64\end{array}$ \\
\hline $\begin{array}{r}9.2 \\
6.5 \\
10 . \equiv\end{array}$ & $\begin{array}{l}35 \\
35 \\
35\end{array}$ & $\begin{array}{l}0.06 \\
5.46 \\
=.79\end{array}$ & $\begin{array}{l}5.37 \\
6.35 \\
5.34\end{array}$ & $\begin{array}{l}44.3 \\
45.5 \\
41.5\end{array}$ & $\begin{array}{l}1575 \\
1401 \\
1507\end{array}$ & $\begin{array}{l}17.1 \\
10.1 \\
17.6\end{array}$ & $\begin{array}{l}34.7 \\
35.5 \\
35.5\end{array}$ & $\begin{array}{l}64.11 \\
73.10 \\
71.44\end{array}$ & $\begin{array}{l}445.6 \\
502.6 \\
612.0\end{array}$ & $\begin{array}{l}10 \\
12 \\
12\end{array}$ & $\begin{array}{l}74 \\
80 \\
85\end{array}$ \\
\hline $\begin{array}{l}\because \cdot 0 \\
7.5 \\
\because .6\end{array}$ & $\begin{array}{l}35 \\
35 \\
35\end{array}$ & $\begin{array}{l}6.6 \Xi \\
7.14 \\
=.5 \Xi\end{array}$ & $\begin{array}{l}0.50 \\
5.46 \\
\therefore .40\end{array}$ & $\begin{array}{l}55.9 \\
56.5 \\
5.5 .5\end{array}$ & $\begin{array}{l}1524 \\
1474 \\
1 \therefore 91\end{array}$ & 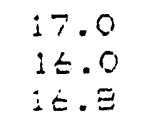 & $\begin{array}{l}34.2 \\
35.3 \\
35.5\end{array}$ & $\begin{array}{l}50.90 \\
64.60 \\
5 \equiv .50\end{array}$ & $\begin{array}{l}350.1 \\
429.4 \\
=15.0\end{array}$ & $\begin{array}{l}5 \\
11 \\
14\end{array}$ & $\begin{array}{l}75 \\
82 \\
28\end{array}$ \\
\hline
\end{tabular}


Ho. 4 Diesel Fuel - 100 \% Load

\begin{tabular}{|c|c|c|c|c|c|c|c|c|c|c|c|}
\hline $\begin{array}{c}H \geq D \\
\%\end{array}$ & $\begin{array}{r}\text { वe } \\
\%\end{array}$ & $\begin{array}{c}0 \geq / F \\
--\end{array}$ & Eq. $R$ & $\begin{array}{c}\text { Power } \\
\text { BHP }\end{array}$ & $\begin{array}{c}P \text { Peak } \\
\text { Psi }\end{array}$ & $\begin{array}{l}\text { Ig } D e 1 \\
\text { deg }\end{array}$ & $\begin{array}{c}E f f \\
\%\end{array}$ & $\begin{array}{c}N D x \\
p p m / B H P\end{array}$ & $\begin{array}{l}\text { Part. } \\
\text { mg/hph }\end{array}$ & $\begin{array}{c}\text { Smoke } \\
\%\end{array}$ & Pinn \\
\hline--- & -- & ---- & $--m$ & ---- & $-\infty$ & $-\cdots---m$ & --- & $--m-\infty-\infty$ & $---\infty$ & --- & --- \\
\hline $\begin{array}{r}0.0 \\
5.9 \\
11.7\end{array}$ & $\begin{array}{l}21 \\
21 \\
21\end{array}$ & $\begin{array}{l}6.31 \\
6.61 \\
6.87\end{array}$ & $\begin{array}{l}0.52 \\
0.50 \\
0.48\end{array}$ & $\begin{array}{l}49.0 \\
47.5 \\
42.8\end{array}$ & $\begin{array}{l}1717 \\
1672 \\
1669\end{array}$ & $\begin{array}{l}20.1 \\
21.0 \\
21.7\end{array}$ & $\begin{array}{l}36.6 \\
38.3 \\
37.9\end{array}$ & $\begin{array}{l}15.31 \\
11.05 \\
19.16\end{array}$ & $\begin{array}{l}233.9 \\
426.9 \\
360.4\end{array}$ & $\begin{array}{r}5 \\
14 \\
10\end{array}$ & $\begin{array}{l}29 \\
33 \\
24\end{array}$ \\
\hline $\begin{array}{r}0.0 \\
6.0 \\
12.3\end{array}$ & $\begin{array}{l}25 \\
25 \\
25\end{array}$ & $\begin{array}{l}\text { 日. } 03 \\
\text { 日.09 } \\
8.34\end{array}$ & $\begin{array}{l}0.41 \\
0.41 \\
0.40\end{array}$ & $\begin{array}{l}47.1 \\
47.1 \\
42.6\end{array}$ & $\begin{array}{l}1721 \\
1701 \\
1696\end{array}$ & $\begin{array}{l}19.3 \\
20.1 \\
20.4\end{array}$ & $\begin{array}{l}37.5 \\
38.4 \\
37.8\end{array}$ & $\begin{array}{l}28.45 \\
27.18 \\
46.95\end{array}$ & $\begin{array}{l}394.2 \\
437.4 \\
363.9\end{array}$ & $\begin{array}{l}6 \\
6 \\
5\end{array}$ & $\begin{array}{l}30 \\
34 \\
25\end{array}$ \\
\hline $\begin{array}{r}0.0 \\
6.3 \\
11.7\end{array}$ & $\begin{array}{l}25 \\
25 \\
25\end{array}$ & $\begin{array}{l}6.46 \\
6.59 \\
6.78\end{array}$ & $\begin{array}{l}0.51 \\
0.50 \\
0.49\end{array}$ & $\begin{array}{l}58.4 \\
57.3 \\
52.5\end{array}$ & $\begin{array}{l}1831 \\
1785 \\
1705\end{array}$ & $\begin{array}{l}19.0 \\
19.1 \\
20.4\end{array}$ & $\begin{array}{l}37.3 \\
38.2 \\
37.5\end{array}$ & $\begin{array}{l}24.33 \\
24.17 \\
40.38\end{array}$ & $\begin{array}{l}411.0 \\
232.7 \\
336.4\end{array}$ & $\begin{array}{r}4 \\
12 \\
5\end{array}$ & $\begin{array}{l}31 \\
35 \\
25\end{array}$ \\
\hline $\begin{array}{r}0.0 \\
3.1 \\
:=.1\end{array}$ & $\begin{array}{l}25 \\
25 \\
25\end{array}$ & $\begin{array}{l}5.35 \\
5.43 \\
5.51\end{array}$ & $\begin{array}{l}0.62 \\
0.61 \\
0.60\end{array}$ & $\begin{array}{l}69.2 \\
67.0 \\
62.3\end{array}$ & $\begin{array}{l}1931 \\
1771 \\
1.767\end{array}$ & $\begin{array}{l}18.1 \\
19.2 \\
20.0\end{array}$ & $\begin{array}{l}36.5 \\
36.9 \\
36.7\end{array}$ & $\begin{array}{l}22.40 \\
21.94 \\
35.63\end{array}$ & $\begin{array}{l}314.3 \\
368.1 \\
356.0\end{array}$ & $\begin{array}{r}6 \\
15 \\
9\end{array}$ & $\begin{array}{l}32 \\
36 \\
27\end{array}$ \\
\hline $\begin{array}{l}0.0 \\
i \equiv .0 \\
13.2\end{array}$ & $\begin{array}{l}30 \\
30 \\
30\end{array}$ & $\begin{array}{l}9.24 \\
7.82 \\
9.90\end{array}$ & $\begin{array}{l}0.35 \\
0.34 \\
0.33\end{array}$ & $\begin{array}{l}49.9 \\
50.1 \\
50.1\end{array}$ & $\begin{array}{l}1920 \\
1884 \\
1867\end{array}$ & $\begin{array}{l}18.1 \\
18.1 \\
18.3\end{array}$ & $\begin{array}{l}35.5 \\
38.2 \\
38.4\end{array}$ & $\begin{array}{l}47.49 \\
48.10 \\
46.81\end{array}$ & $\begin{array}{l}564.9 \\
631.9 \\
543.3\end{array}$ & $\begin{array}{l}17 \\
12 \\
30\end{array}$ & $\begin{array}{l}10 ! \\
105 \\
109\end{array}$ \\
\hline $\begin{array}{r}0.0 \\
12.5 \\
17.4\end{array}$ & $\begin{array}{l}30 \\
30 \\
30\end{array}$ & $\begin{array}{l}5.90 \\
6.36 \\
6.95\end{array}$ & $\begin{array}{l}0.56 \\
0.52 \\
0.48\end{array}$ & $\begin{array}{l}74.9 \\
74.5 \\
69.2\end{array}$ & $\begin{array}{l}1938 \\
1875 \\
1851\end{array}$ & $\begin{array}{l}18.0 \\
18.2 \\
18.3\end{array}$ & $\begin{array}{l}34.6 \\
37.5 \\
37.3\end{array}$ & $\begin{array}{l}45.66 \\
37.18 \\
38.29\end{array}$ & $\begin{array}{l}582.5 \\
580.3 \\
665.8\end{array}$ & $\begin{array}{l}21 \\
21 \\
42\end{array}$ & $\begin{array}{l}103 \\
107 \\
111\end{array}$ \\
\hline $\begin{array}{l}0.0 \\
: 1.1 \\
17.3\end{array}$ & $\begin{array}{r}35 \\
34 . \\
35\end{array}$ & $\begin{array}{l}10.60 \\
11.10 \\
11.60\end{array}$ & $\begin{array}{l}0.31 \\
0.30 \\
0.29\end{array}$ & $\begin{array}{l}48.9 \\
49.9 \\
50.1\end{array}$ & $\begin{array}{l}1935 \\
1879 \\
1877\end{array}$ & $\begin{array}{l}17.0 \\
17.2 \\
17.2\end{array}$ & $\begin{array}{l}34.7 \\
37.5 \\
37.8\end{array}$ & $\begin{array}{l}57.70 \\
49.60 \\
49.00\end{array}$ & $\begin{array}{l}345.1 \\
677.2 \\
616.7\end{array}$ & $\begin{array}{l}16 \\
15 \\
32\end{array}$ & $\begin{array}{l}102 \\
106 \\
110\end{array}$ \\
\hline $\begin{array}{r}0.0 \\
: 5.3 \\
15.9\end{array}$ & $\begin{array}{l}35 \\
35 \\
35\end{array}$ & $\begin{array}{l}6.50 \\
7.35 \\
8.18\end{array}$ & $\begin{array}{l}0.51 \\
0.45 \\
0.40\end{array}$ & $\begin{array}{l}74.7 \\
74.2 \\
67.5\end{array}$ & $\begin{array}{l}1719 \\
1920 \\
1863\end{array}$ & $\begin{array}{l}17.1 \\
17.2 \\
17.4\end{array}$ & $\begin{array}{l}34.3 \\
3 . \cdot 1 \\
37.3\end{array}$ & $\begin{array}{l}40.65 \\
36.63 \\
40.44\end{array}$ & $\begin{array}{l}523.4 \\
563.3 \\
527.4\end{array}$ & $\begin{array}{l}E \equiv \\
N A \\
37\end{array}$ & $\begin{array}{l}104 \\
103 \\
112\end{array}$ \\
\hline
\end{tabular}


A.3 Graphic Display of Test Data for No. 2 Fuel 
A-18 


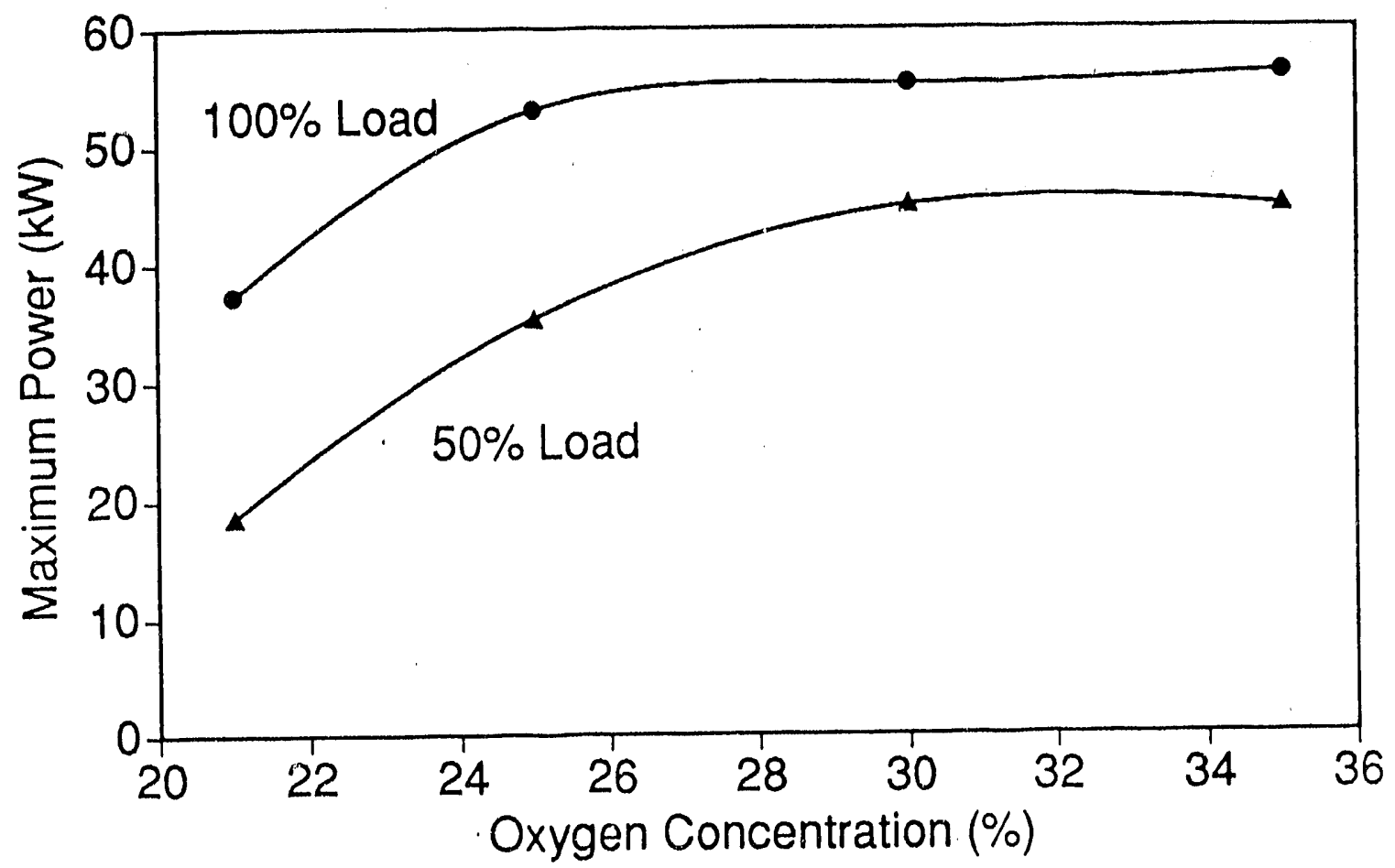

FIGURE A.1 Maximum Attainable Power vs. Oxygen Concentration, No. 2 Fuel

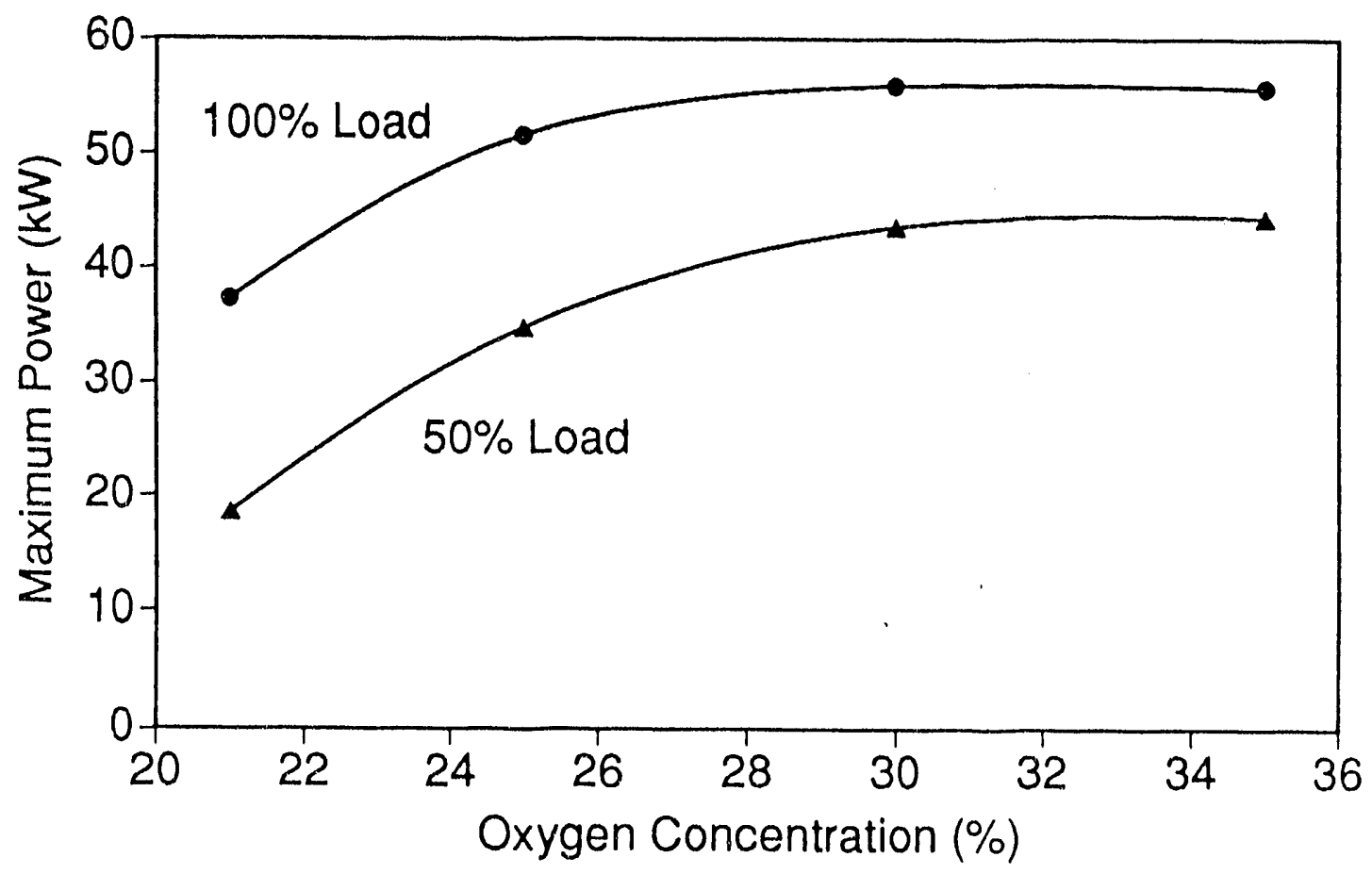

FIGURE A.2 Maximum Attainable Power vs. Oxygen Concentration, No. 4 Fuel 


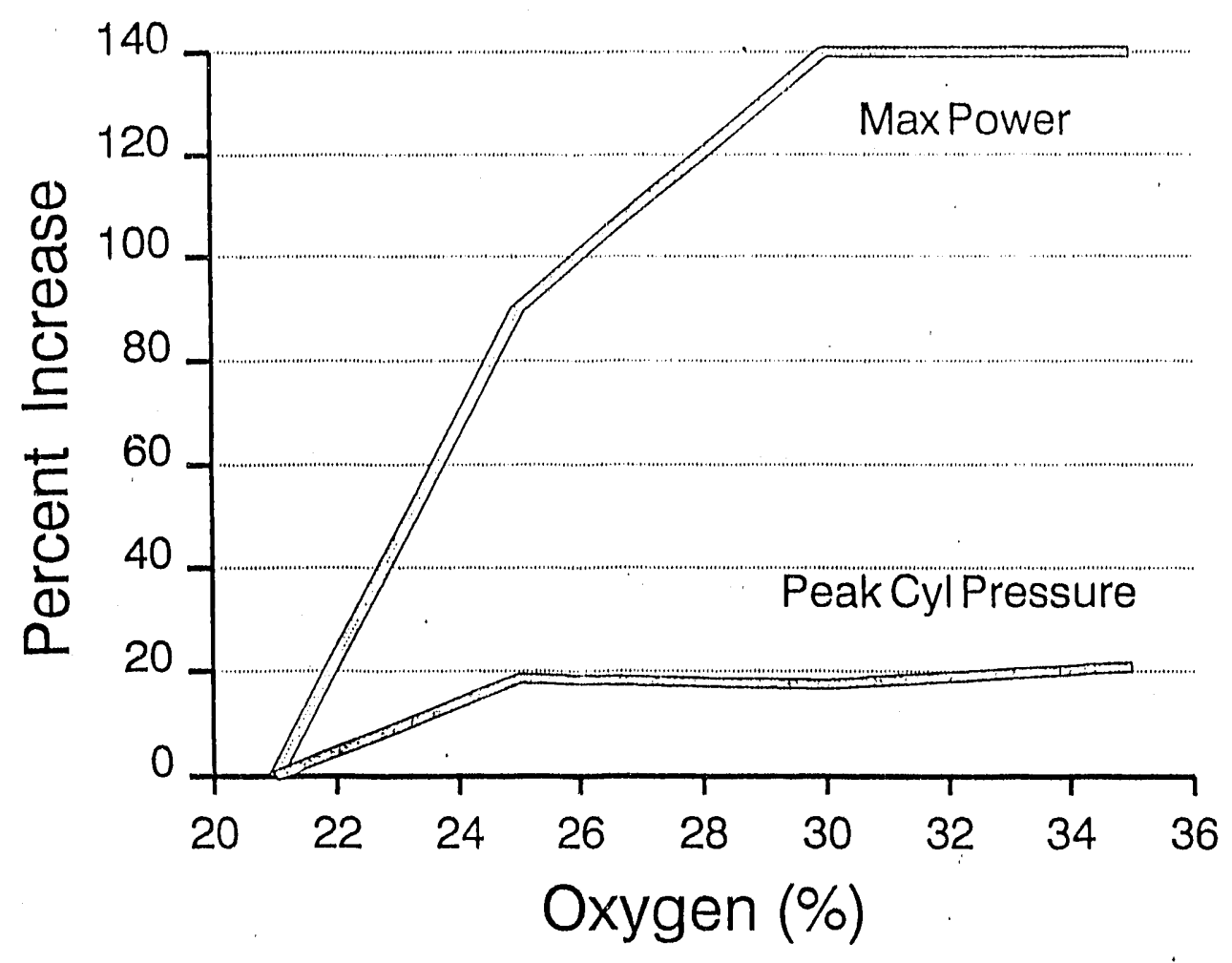

FIGURE A.3 Effect of Oxygen Level on Maximum Power Output and Peak Cylinder Pressure, Half Load

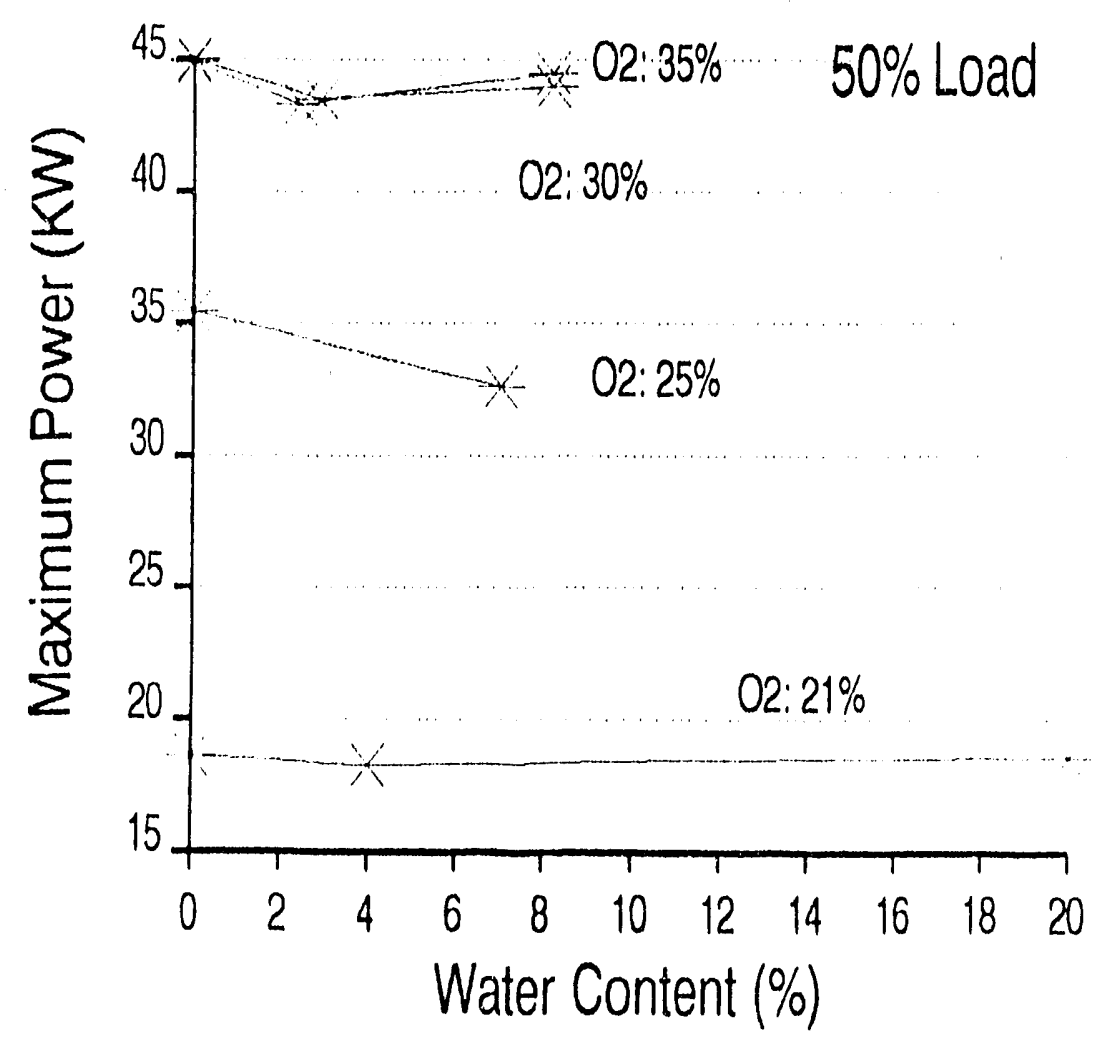

FIGURE A.4 Maximum Attainable Power, No. 2 Fuel-Water Emulsion 


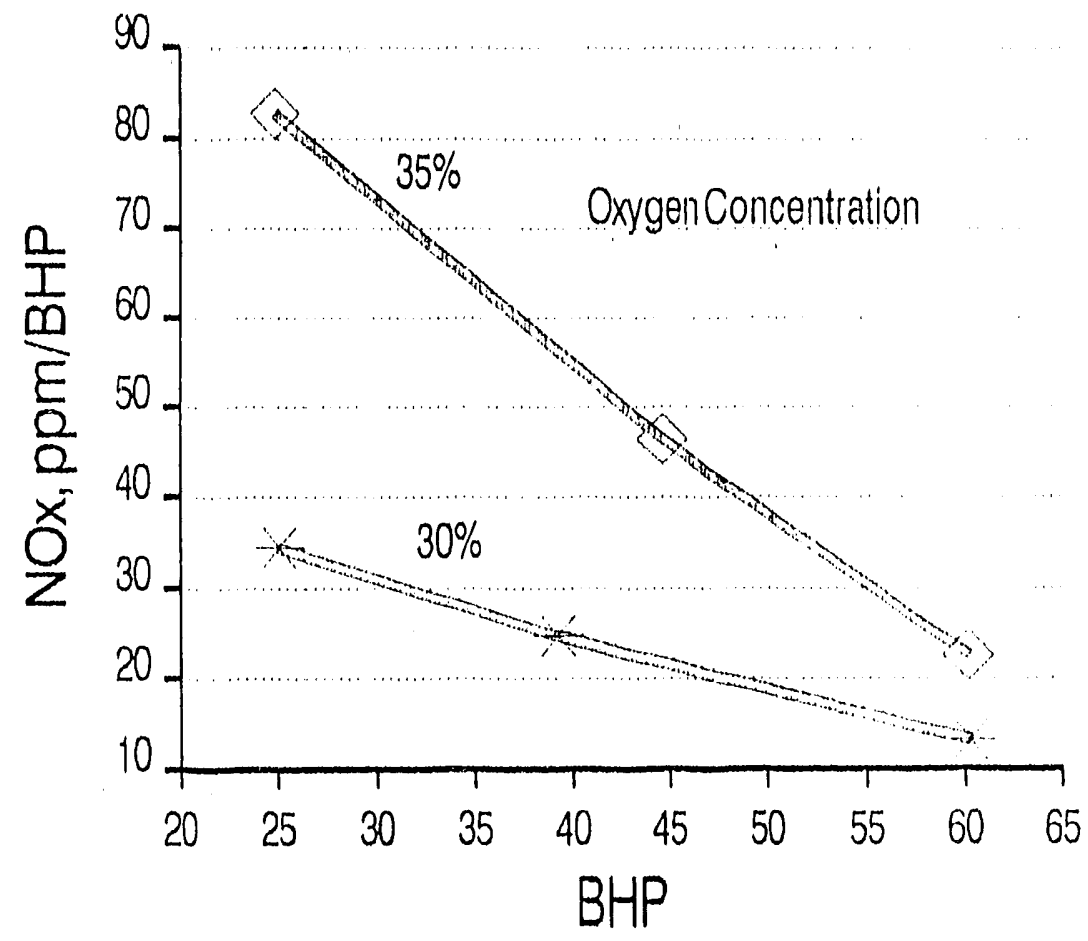

FIGURE A.5 Nitrogen Oxides vs. Brake Horsepower, No. 2 Fuel, $50 \%$ Load, No Water

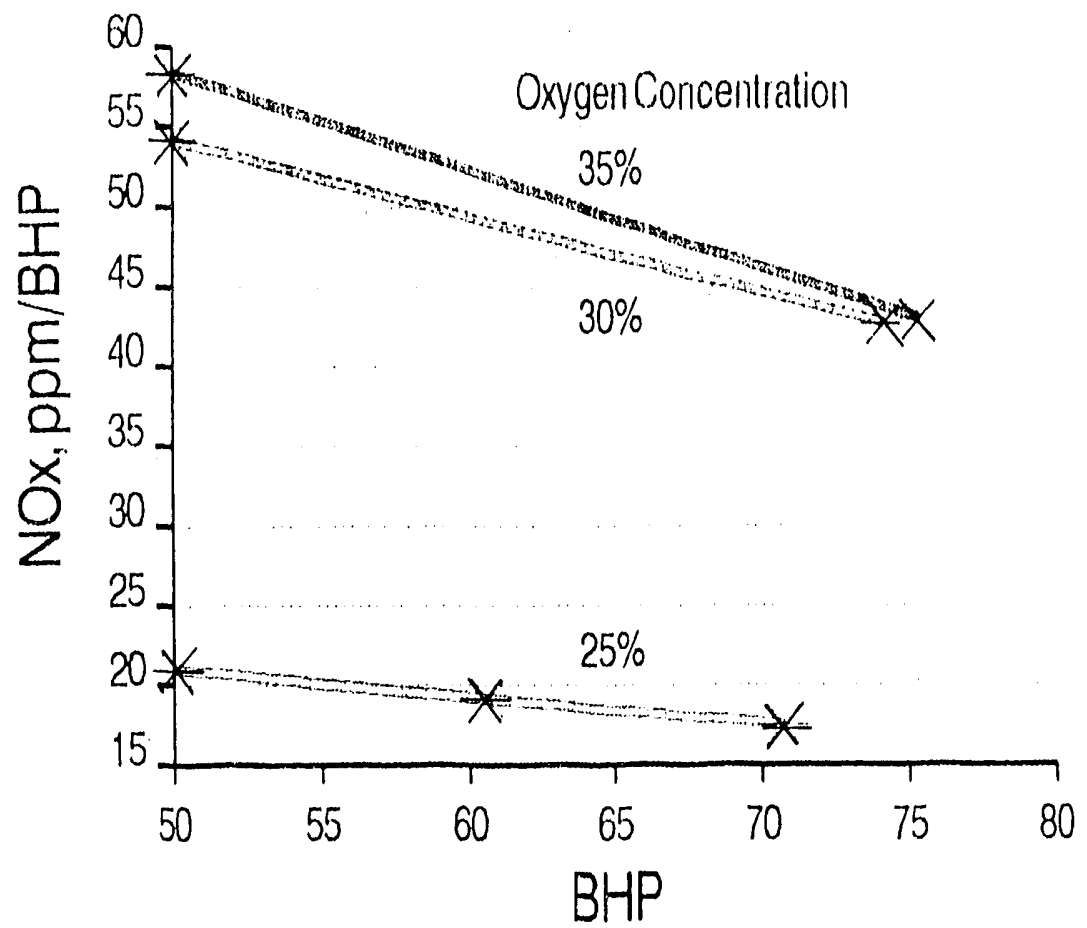

FIGURE A.6 Nitrogen Oxides vs. Brake Horsepower, No. 2 Fuel, $100 \%$ Load, No Water 


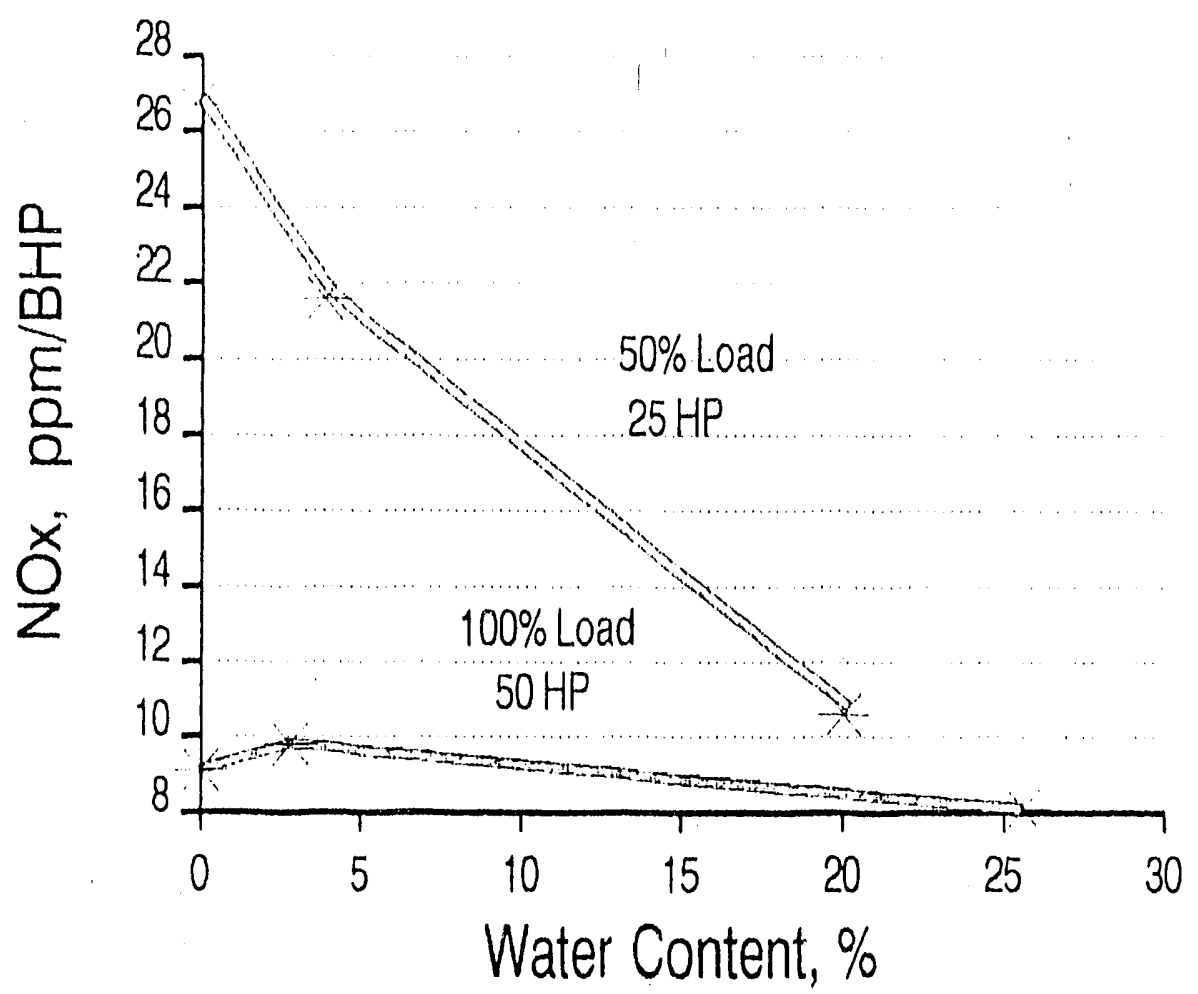

FIGURE A.7 Nit:ogen Oxides vs. Water Content, No. 2 Fuel, $21 \%$ Oxygen

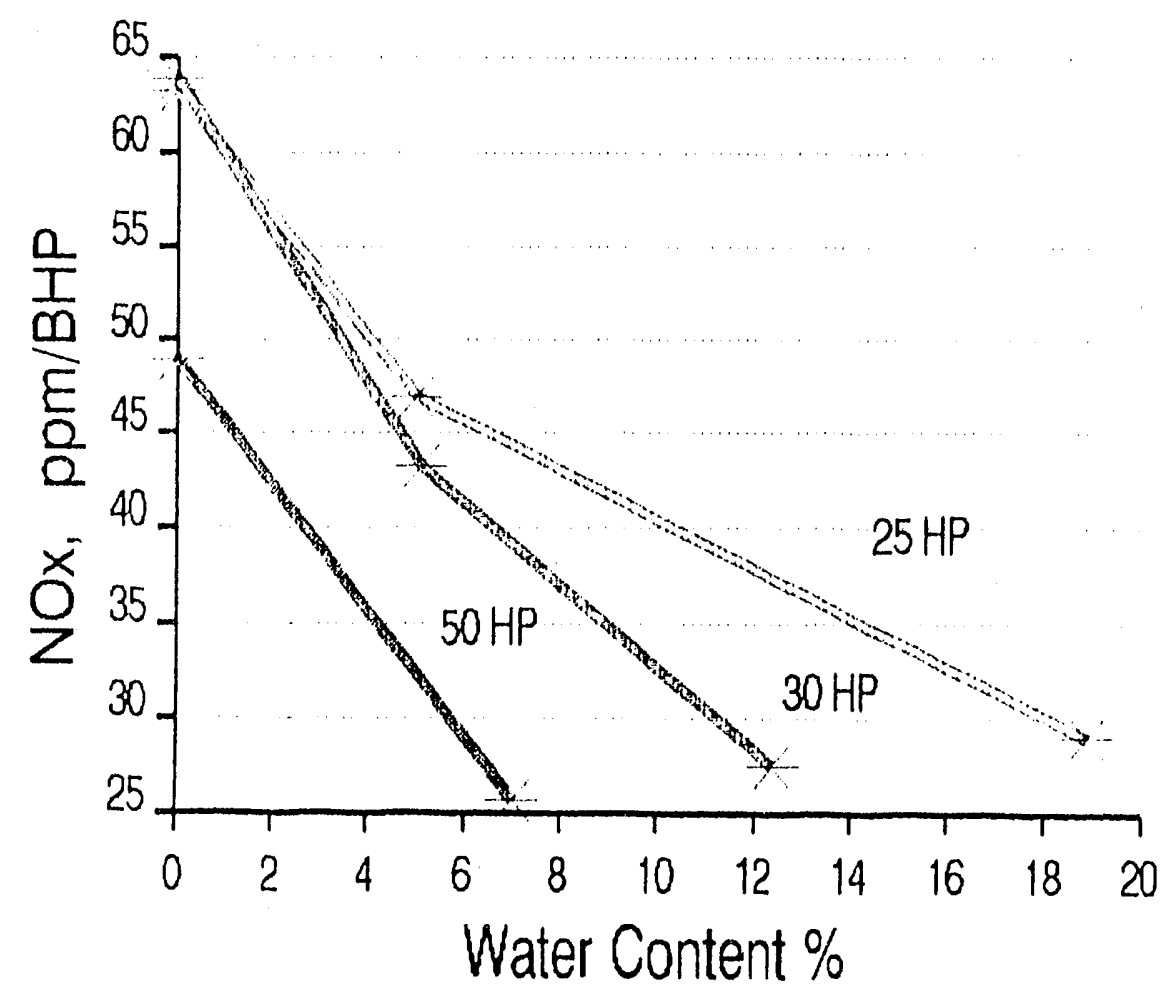

FIGURE A.8 Nitrogen Oxides vs. Water Content, No. 2 Fuel, 50\% Load, $25 \%$ Oxygen 


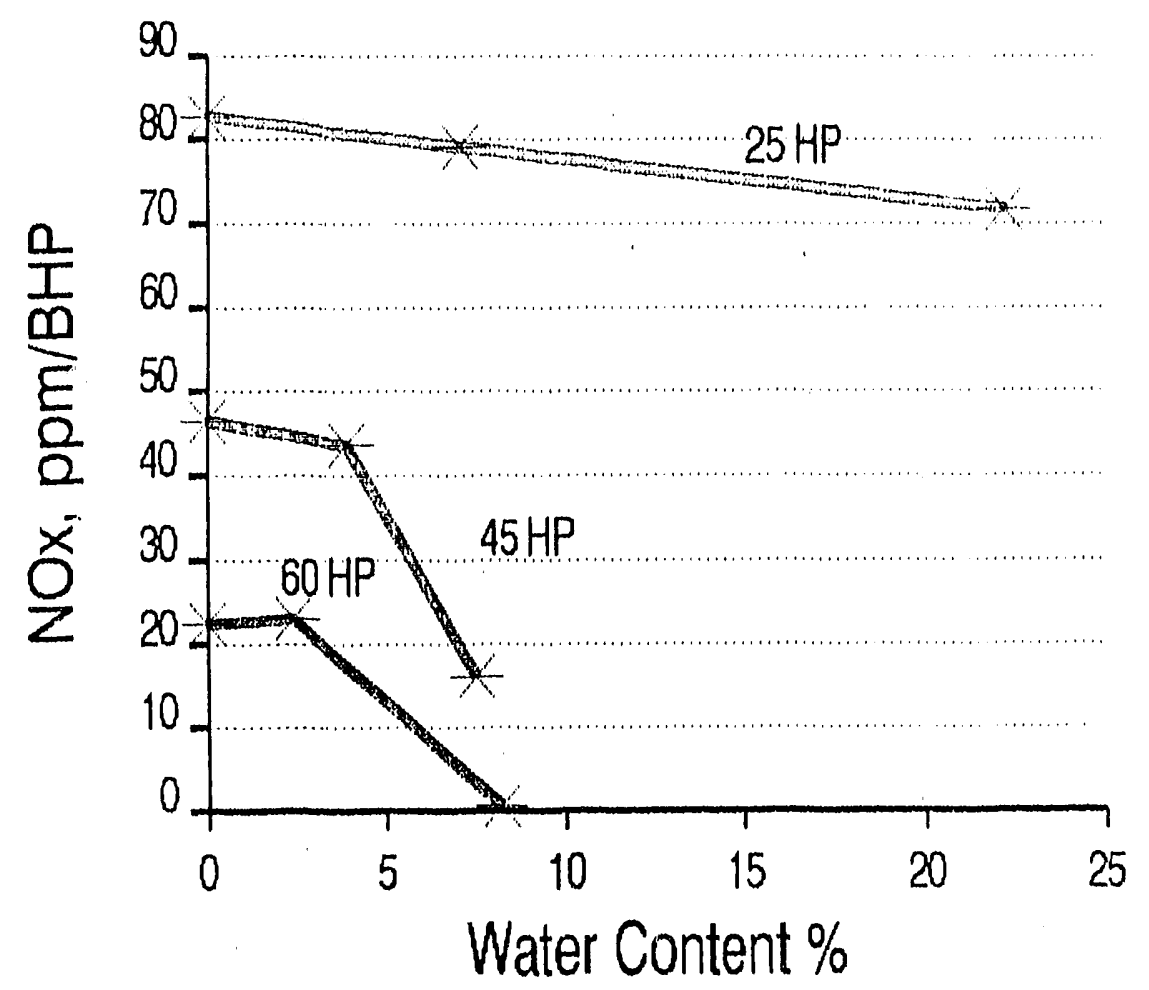

FIGURE A.9 Nitrogen Oxides vs. Water Content, No. 2 Fuel, $50 \%$ Load, $35 \%$ Oxygen

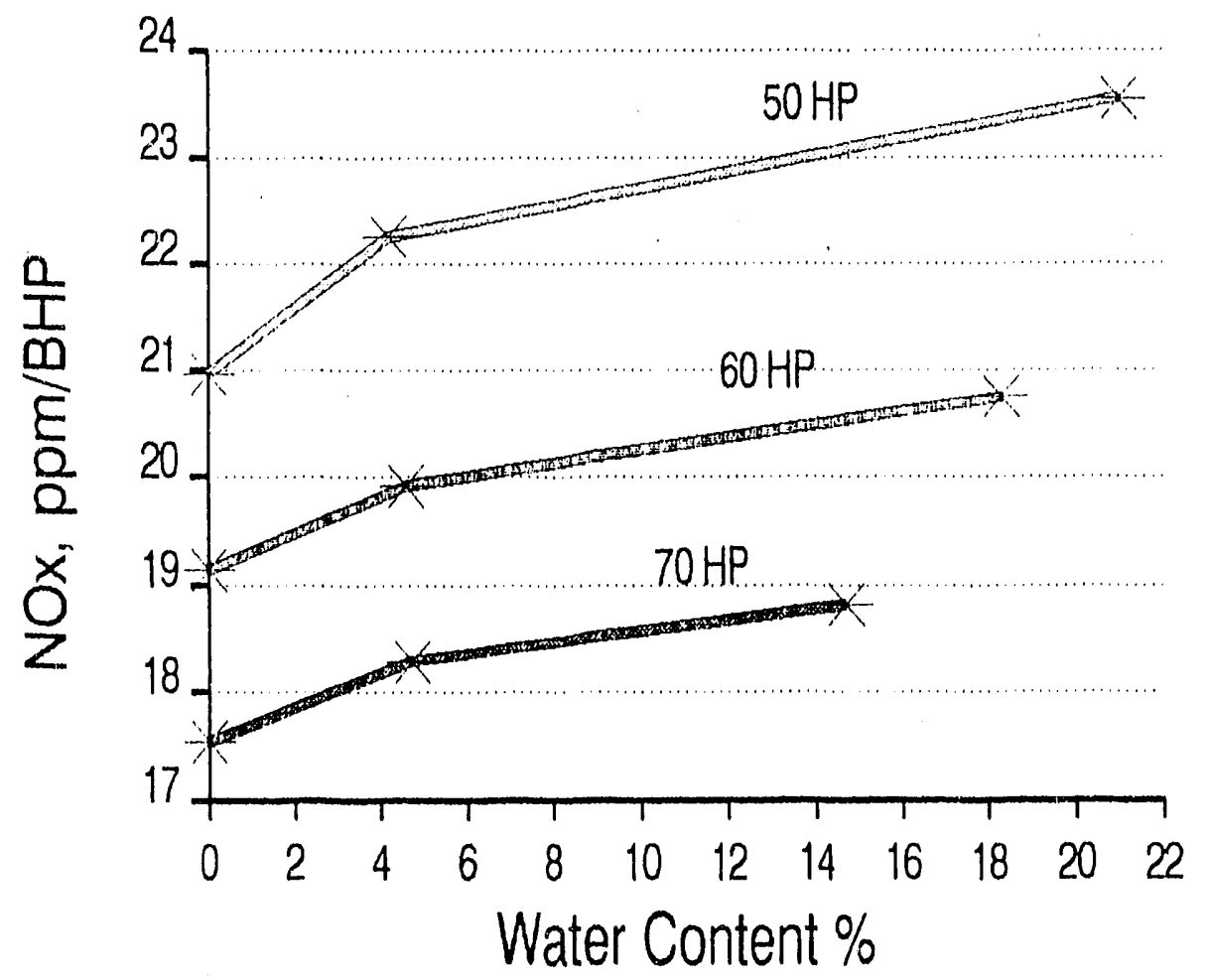

FIGURE A.10 Nitrogen Oxides vs. Water Content, No. 2 Fuel, 50\% Load, $30 \%$ Oxygen 


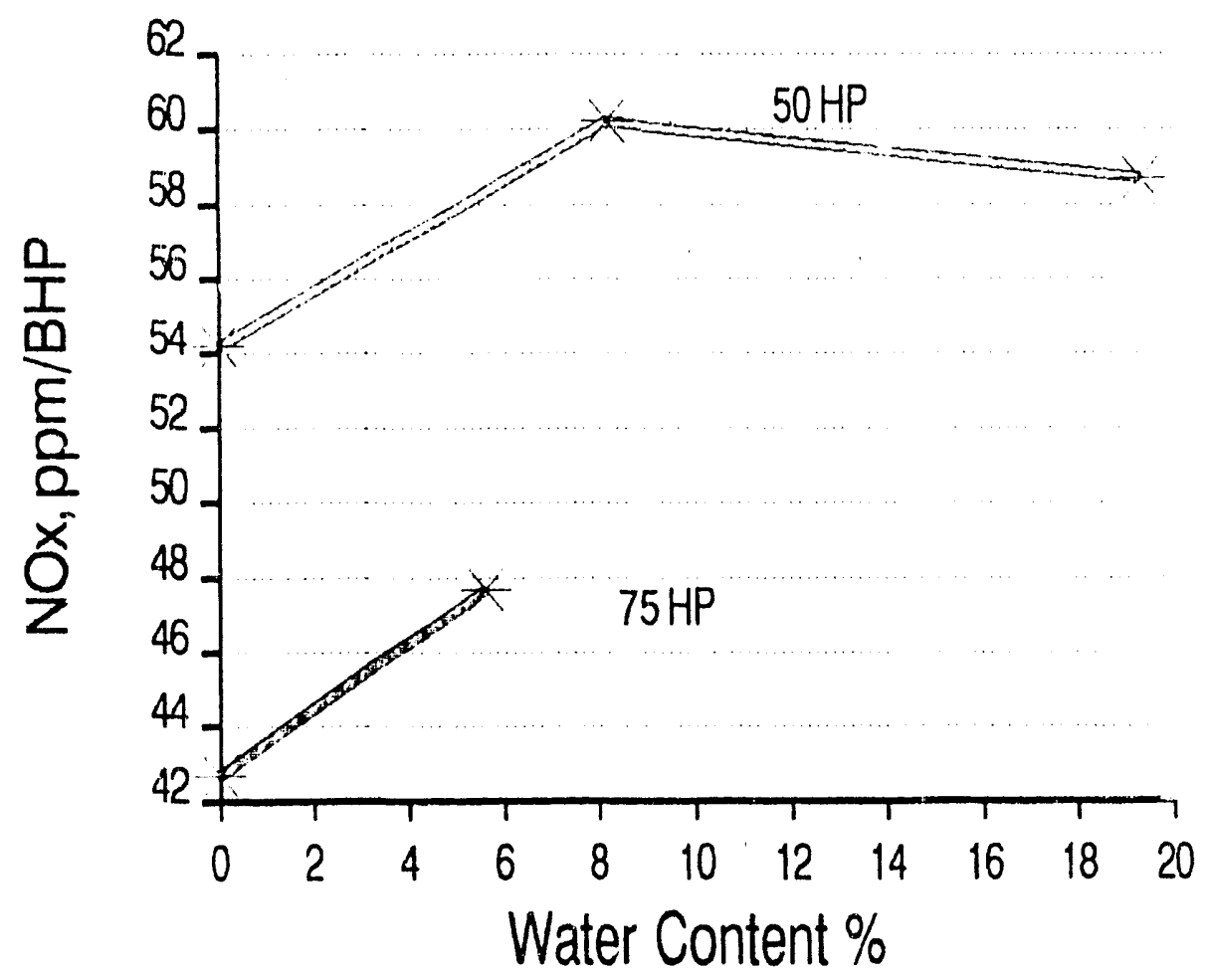

FIGURE A.11 Nitrogen Oxides vs. Water Content, No. 2 Fuel, 100\% Load, $30 \%$ Oxygen

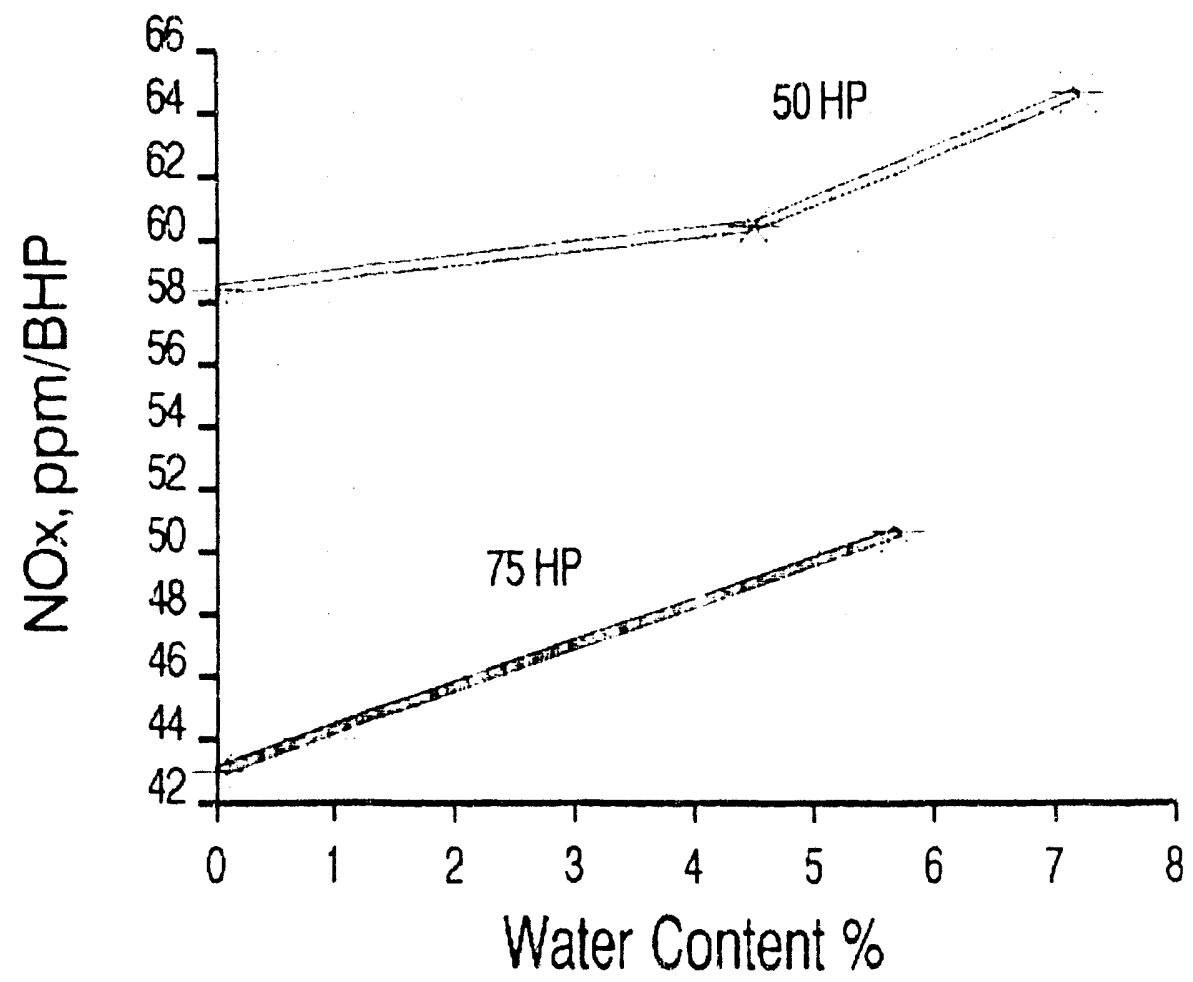

FIGURE A.12 Nitrogen Oxides vs. Water Contem, No. 2 Fuel, 100\% Load, $35 \%$ Oxygen 


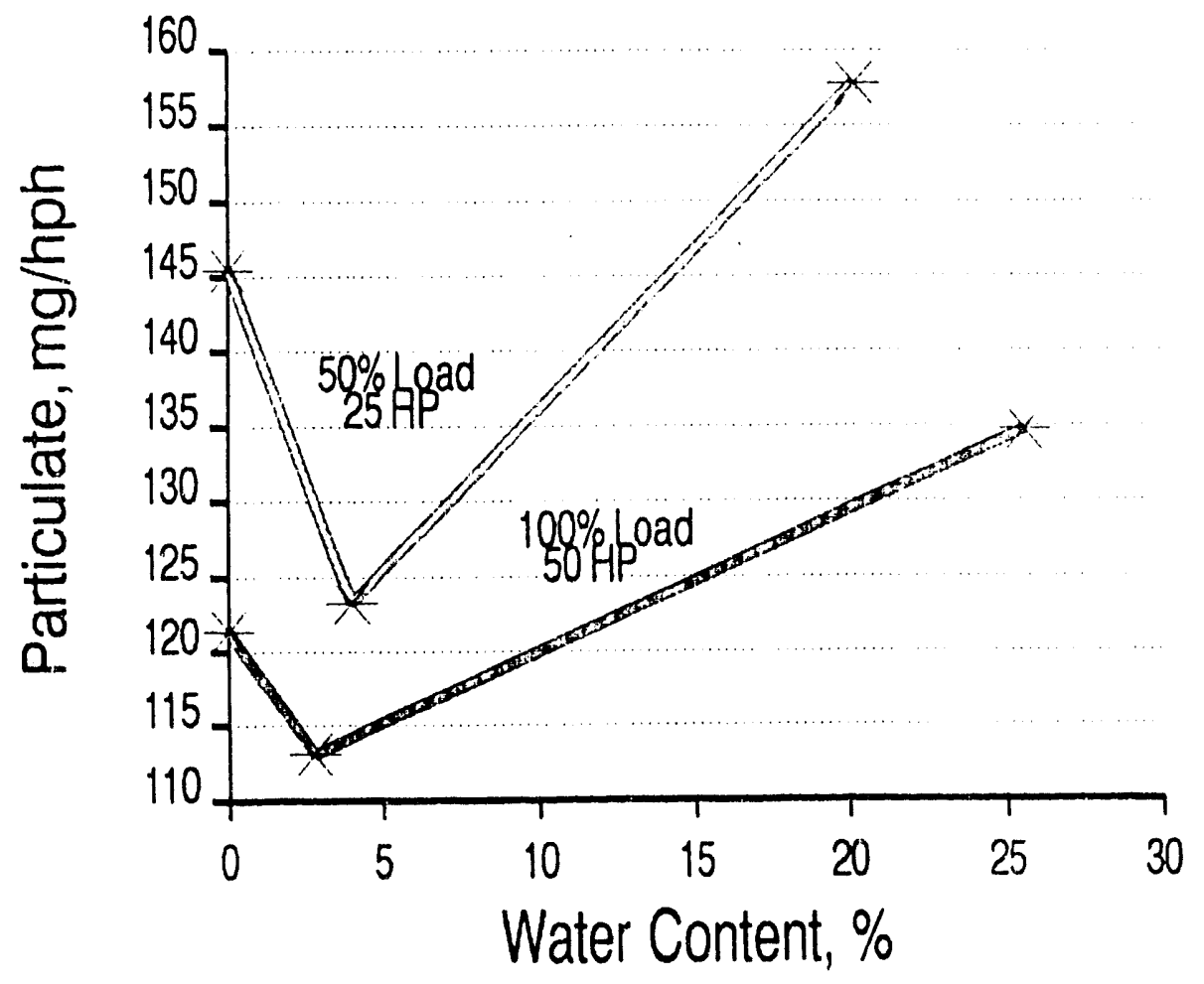

FIGURE A.13 Particulate Matter vs. Water Content, No. 2 Fuel, 21\% Oxygen

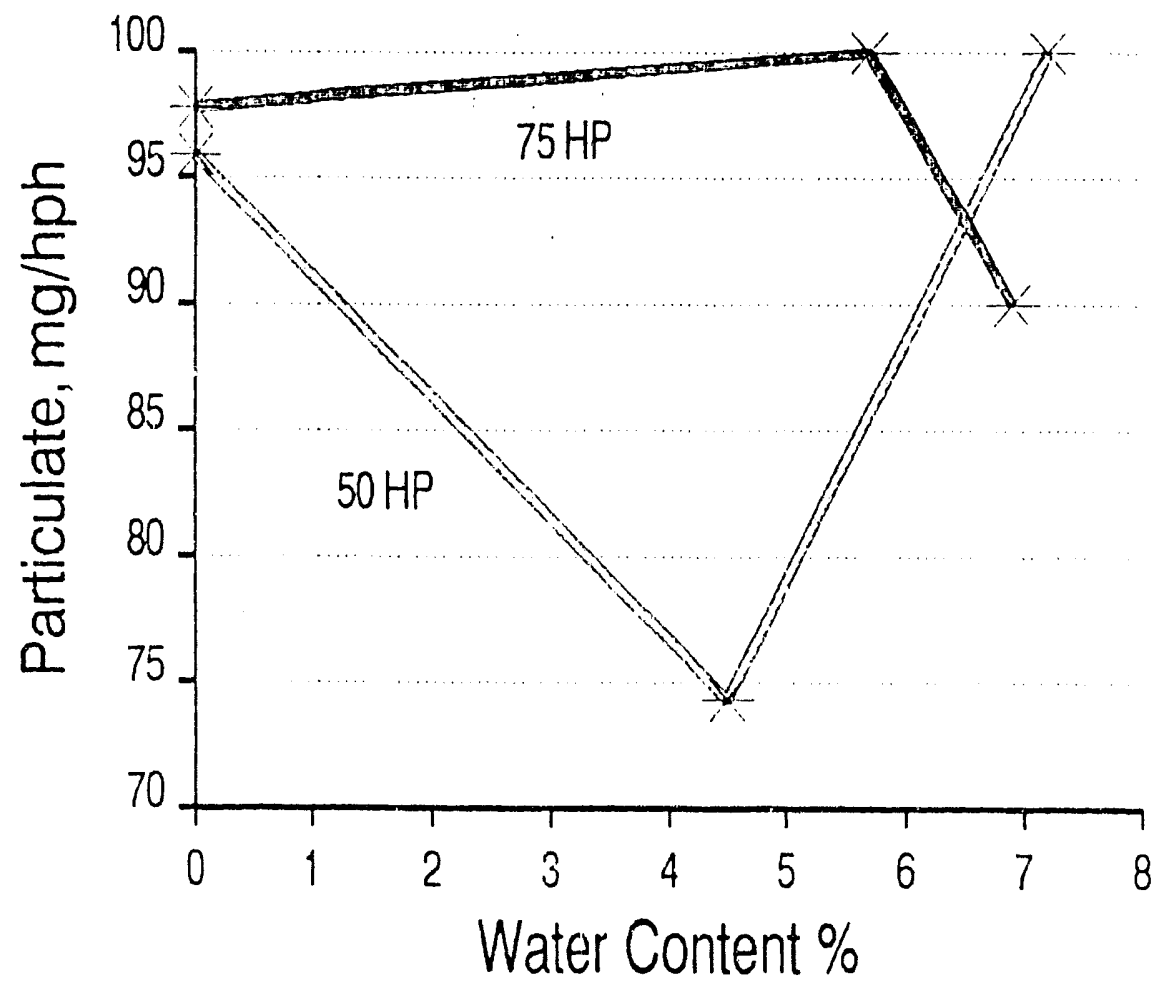

FIGURE A.14 Particulate Matter vs. Water Content, No. 2 Fuel, $100 \%$ Load, $35 \%$ Oxygen 


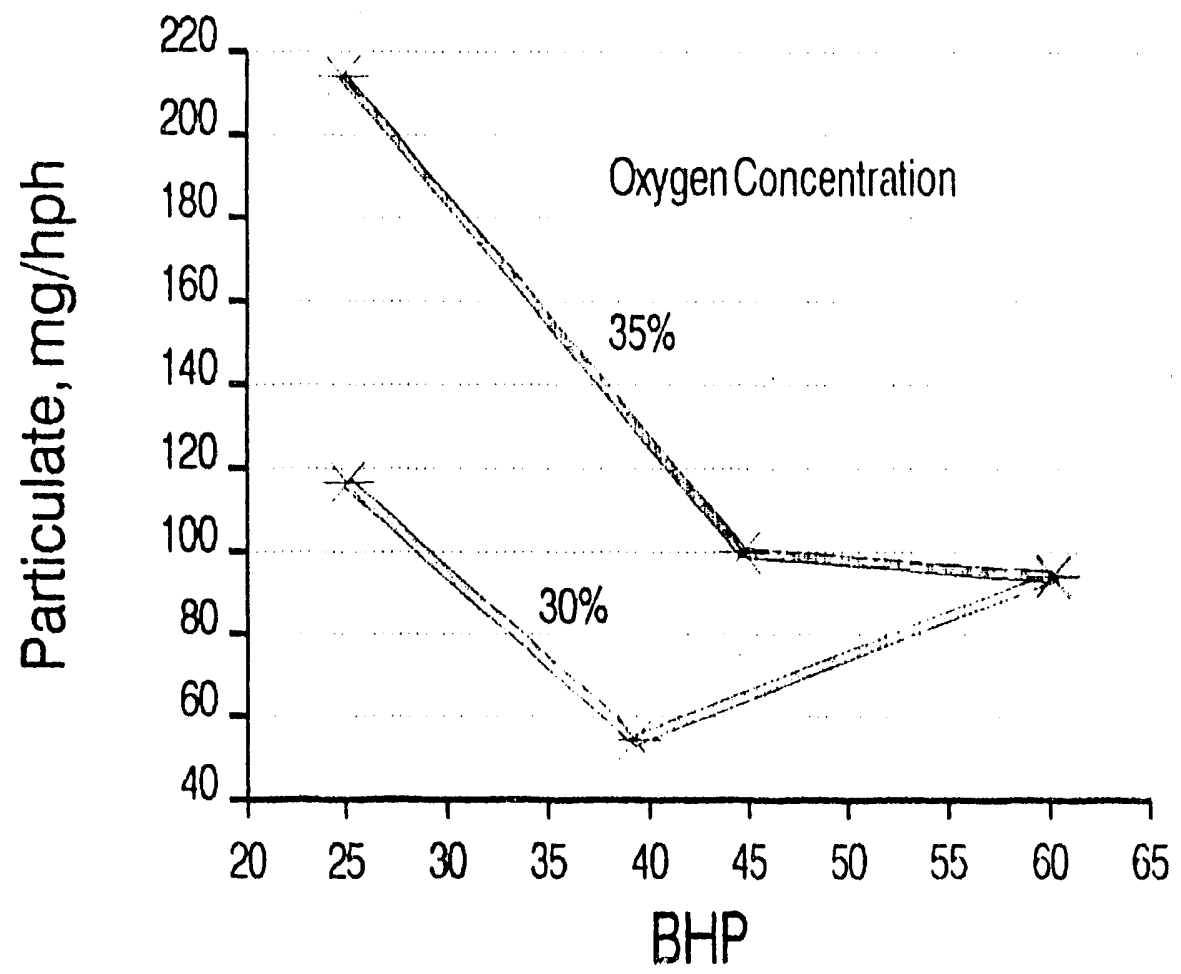

FIGURE A.15 Particulate Matter vs. Brake Horsepower, No. 2 Fuel, $50 \%$ Load, No Water

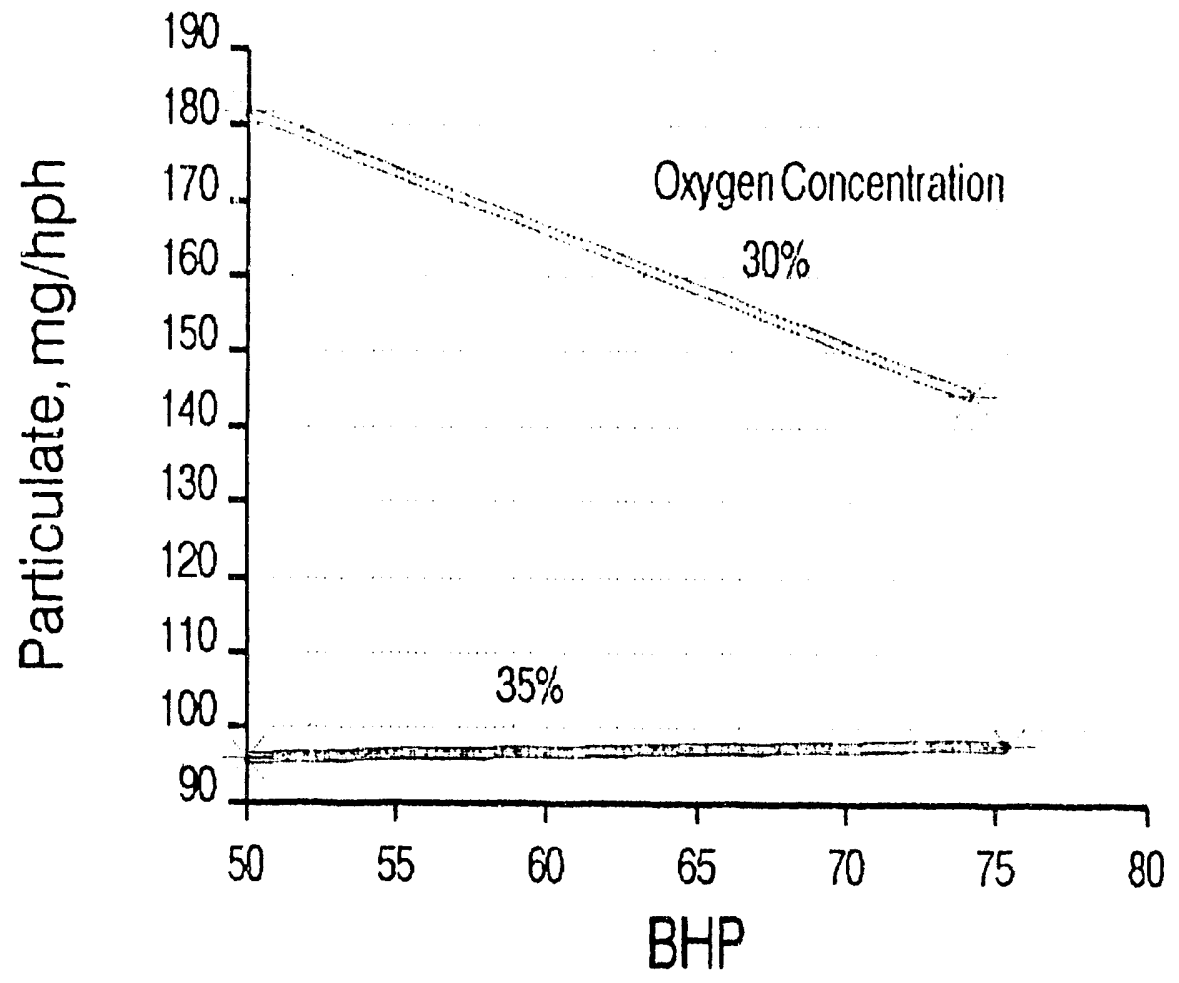

FIGURE A.16 Particulate Matter vs. Brake Horsepower, No. 2 Fuel, $100 \%$ Load, No Water 


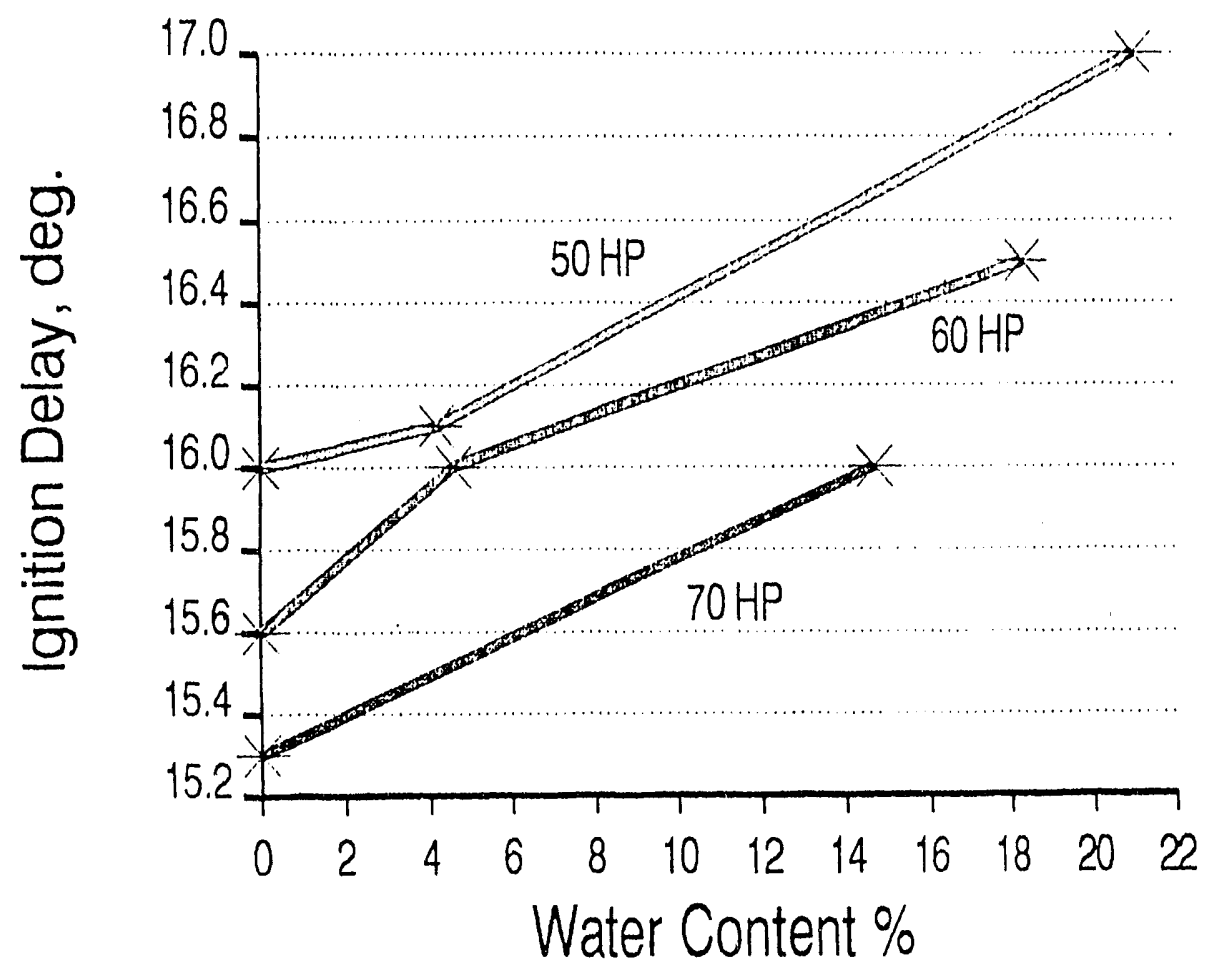

FIGURE A.17 Ignition Delay vs. Water Content, No. 2 Fuel, $100 \%$ Load, $25 \%$ Oxygen 
A-29

A.4 Graphic Display of Test Data for No. 4 Fuel 
A-30 


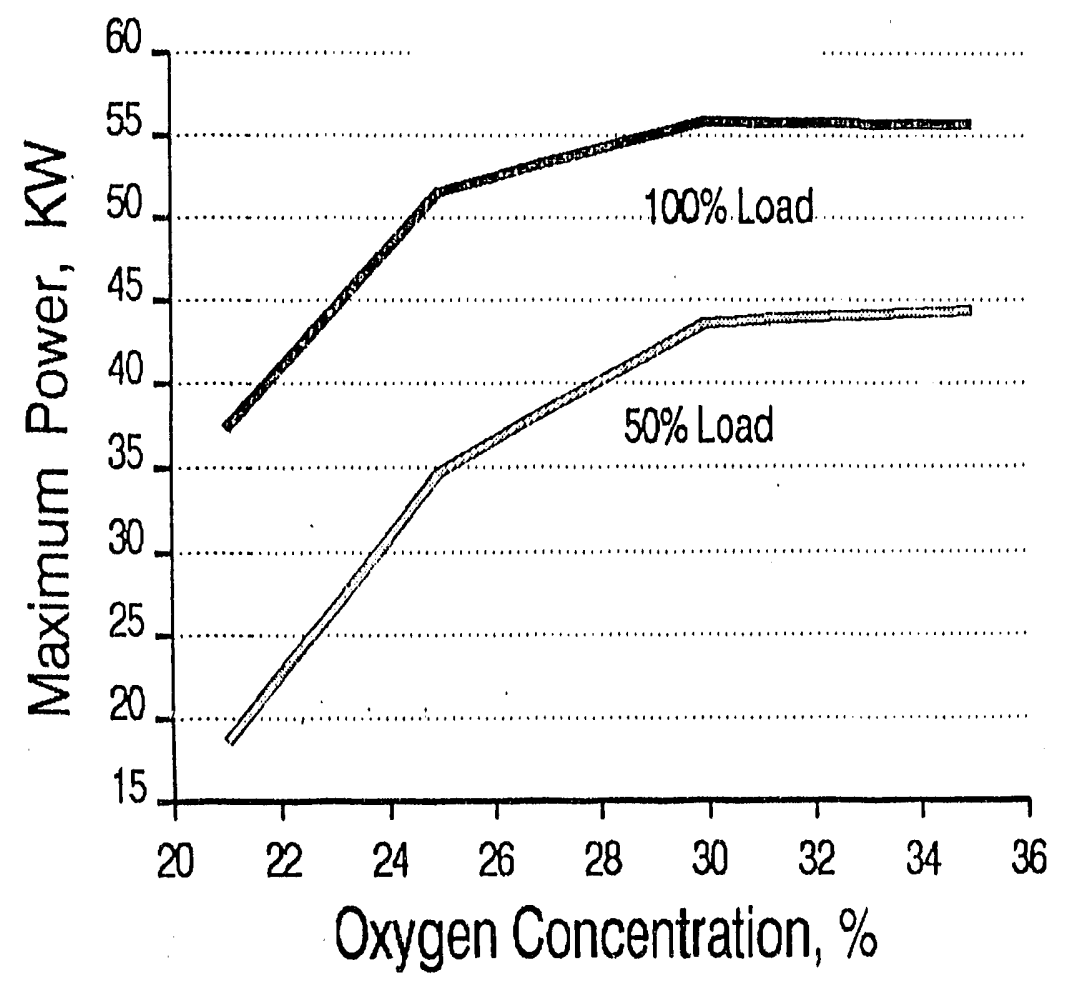

FIGURE A.18 Maximum Attainable Power, No. 4 Diesel Fuel

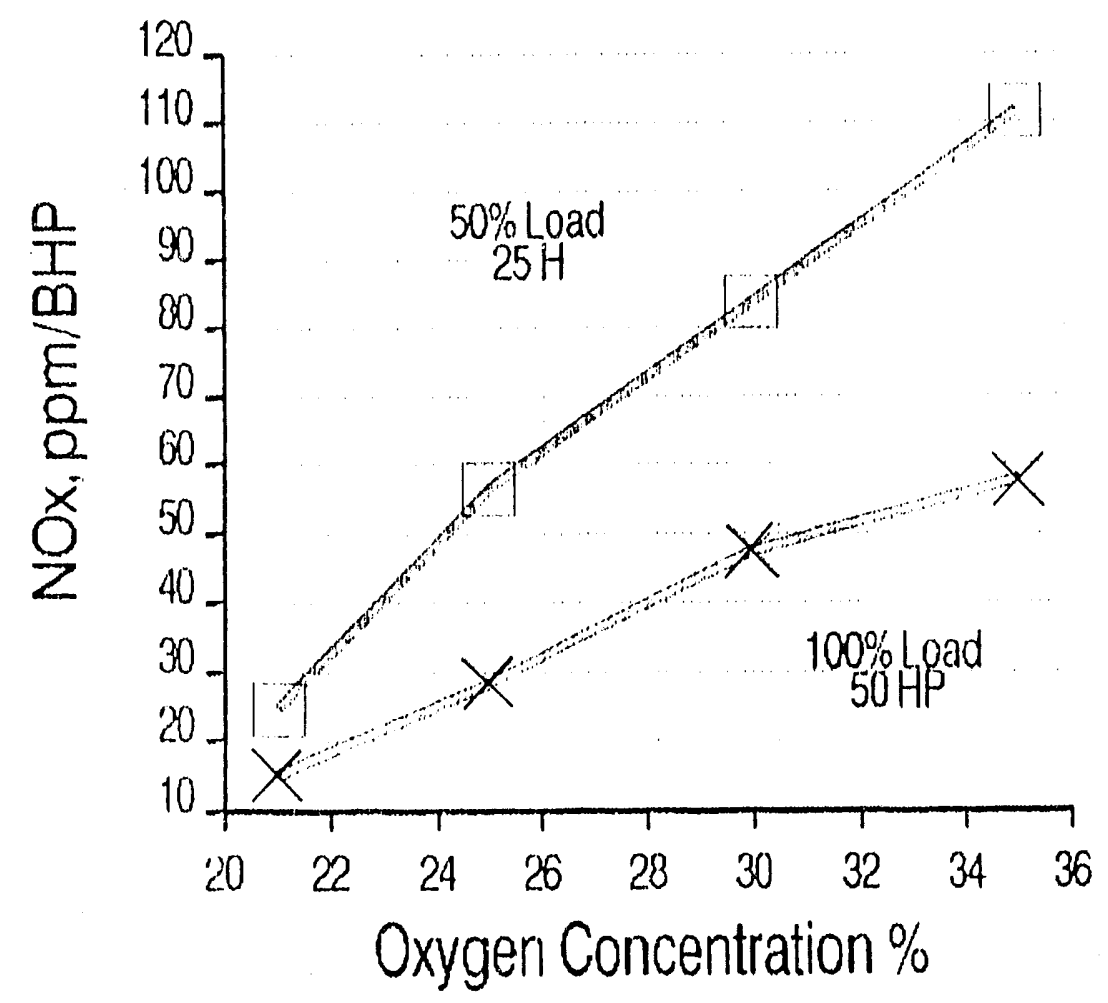

FIGURE A.19 Nitrogen Oxides vs. Oxygen Concentration, No. 4 Fuel, No Water 


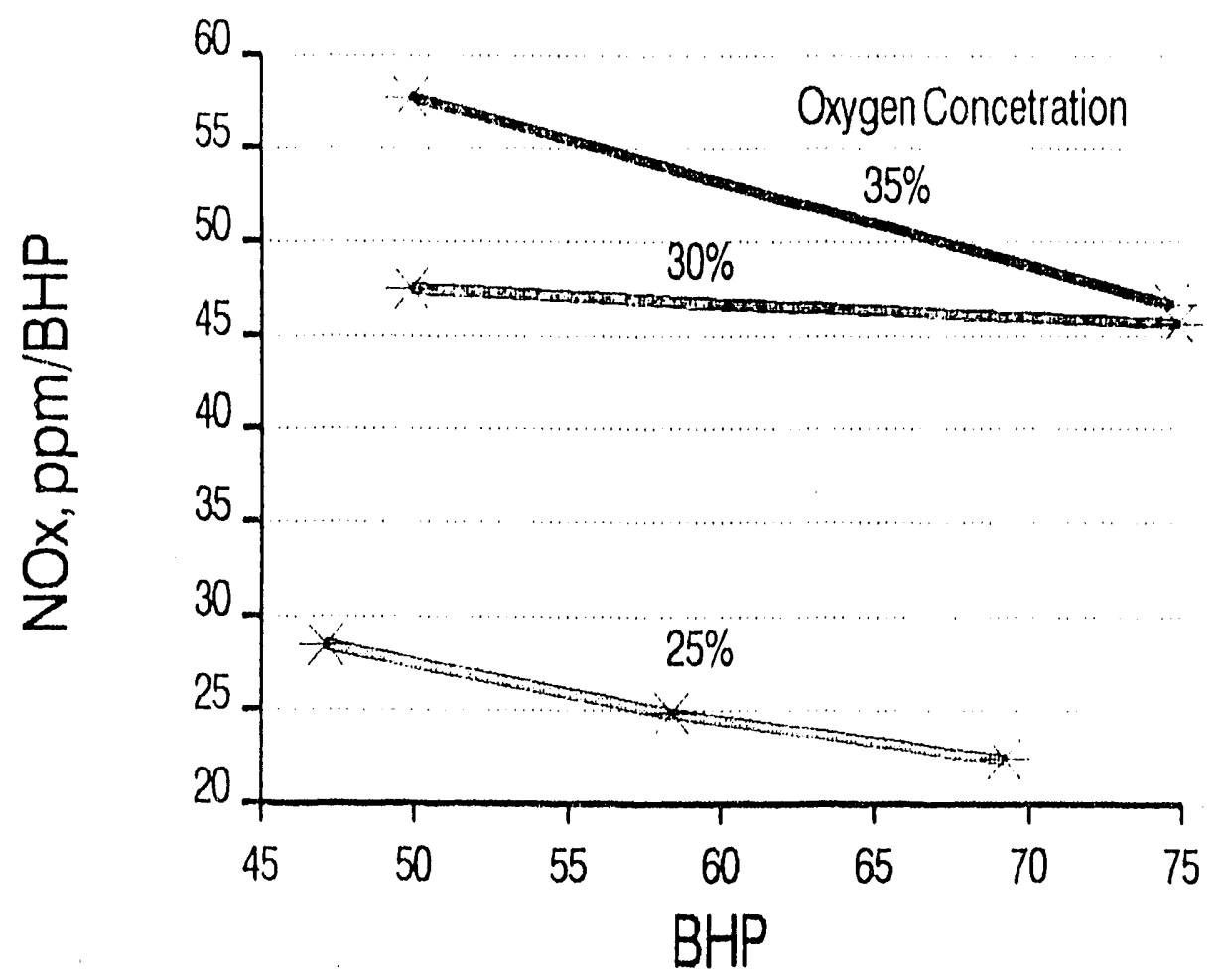

FIGURE A.20 Nitrogen Oxides vs. Brake Horsepower, No. 4 Fuel, $100 \%$ Load, No Water

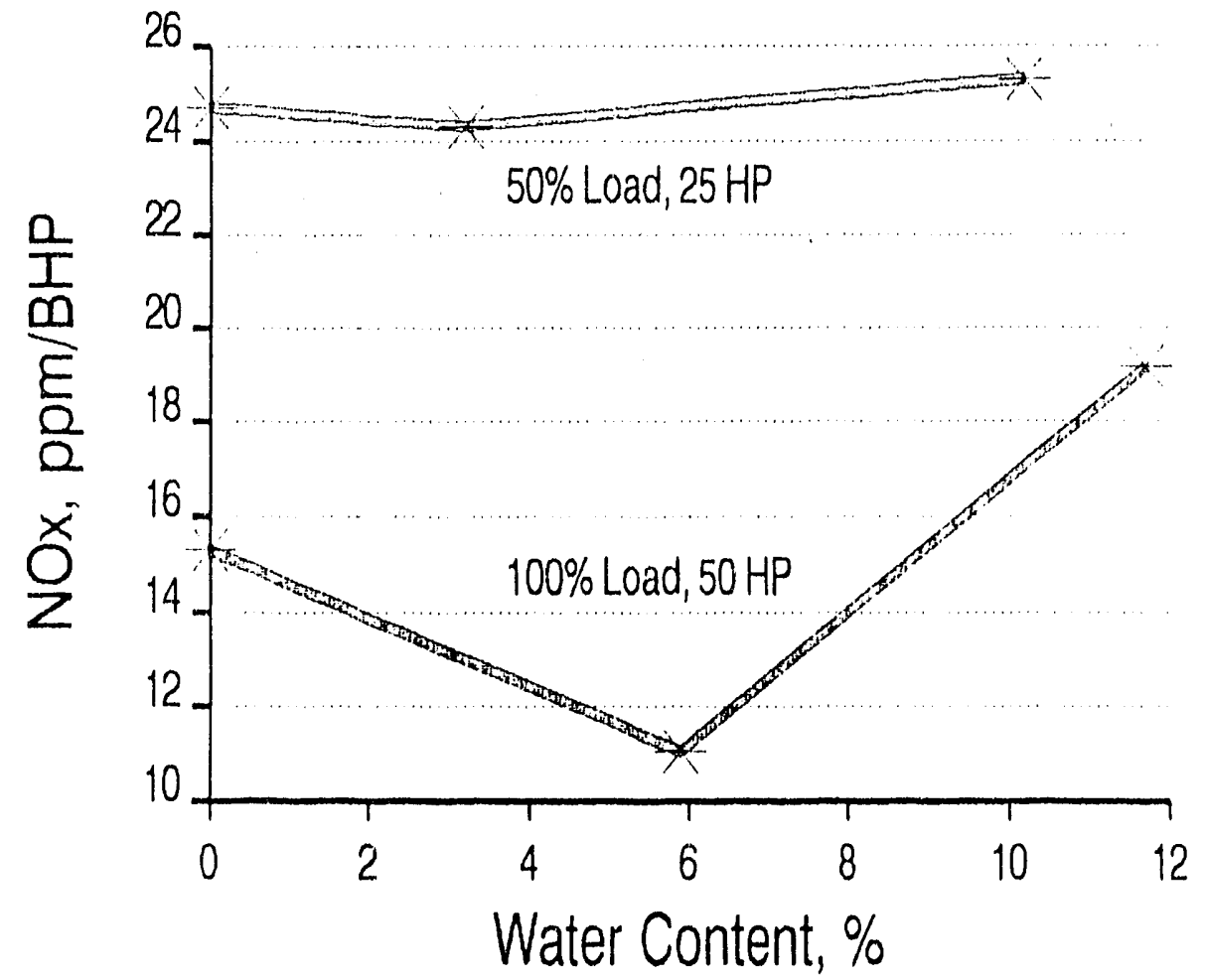

FIGURE A.21 Nitrogen Oxides vs. Water Content, No. 4 Fuel, 21\% Oxygen 


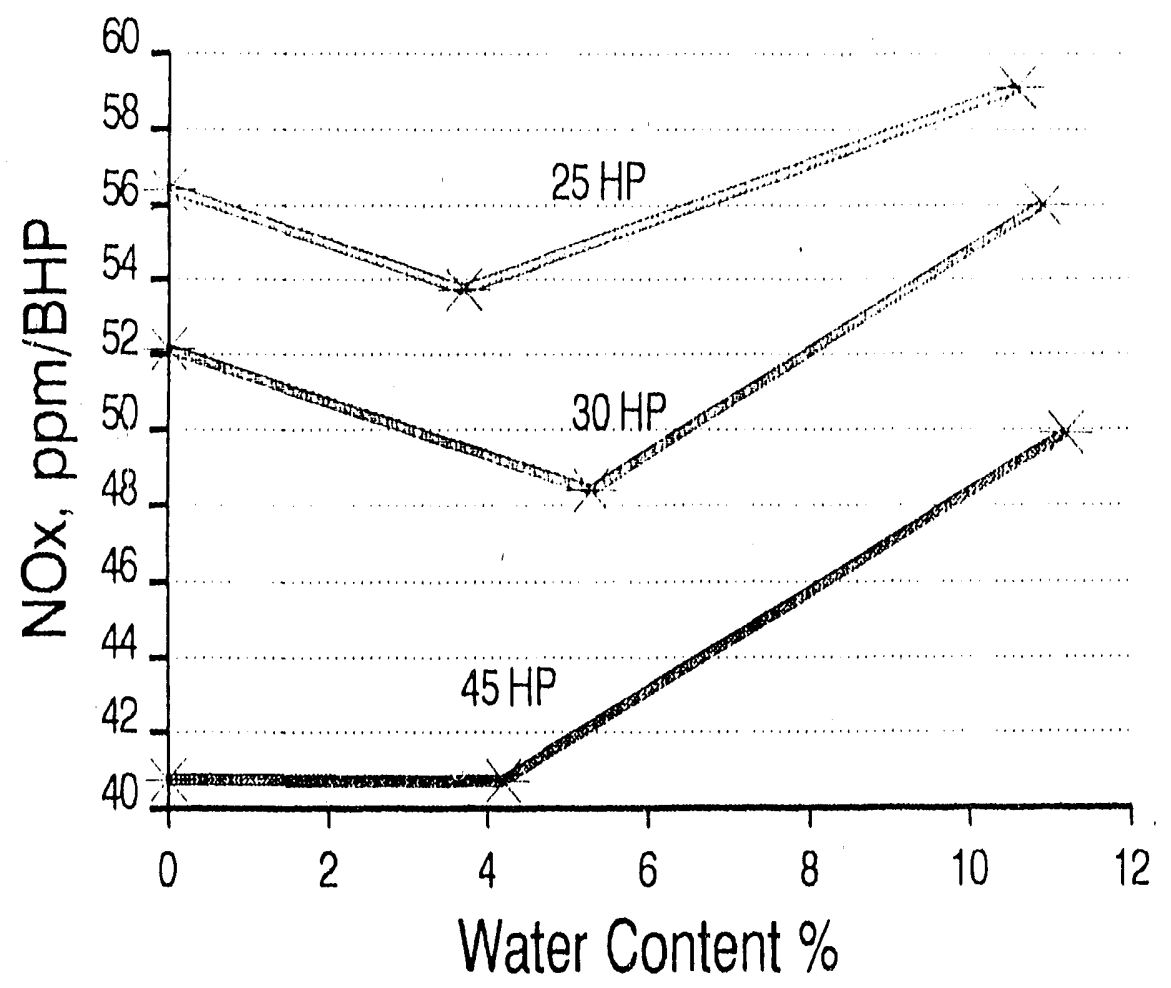

FIGURE A.22 Nitrogen Oxides vs. Water Content, No. 4 Fuel, $50 \%$ Load, $25 \%$ Oxygen

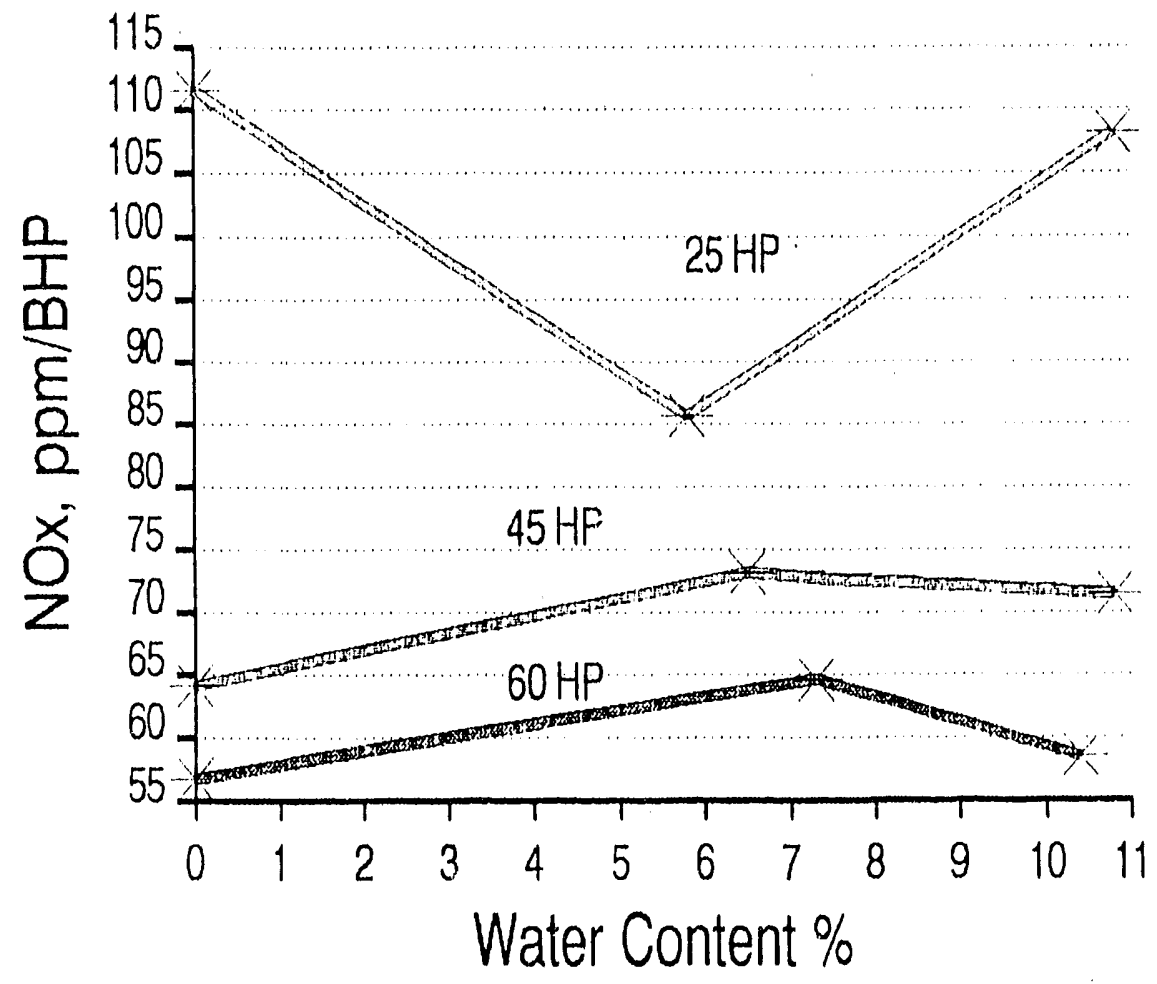

FIGURE A.23 Nitrogen Oxides vs. Water Content, No. 4 Fuel, $50 \%$ Load, $35 \%$ Oxygen 


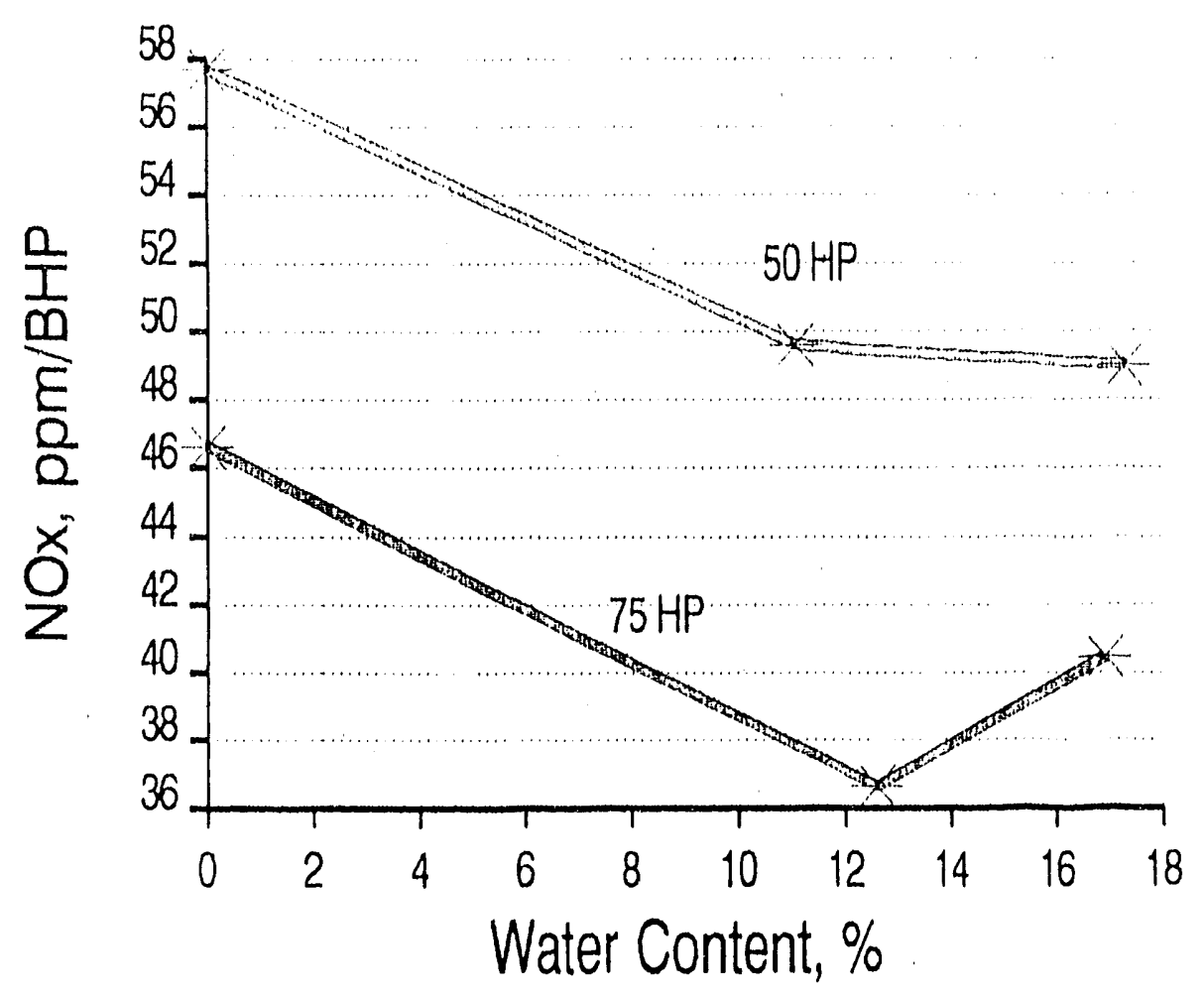

FIGURE A.24 Nitrogen Oxides vs. Water Content, No. 4 Fuel, 100\% Load, $35 \%$ Oxygen

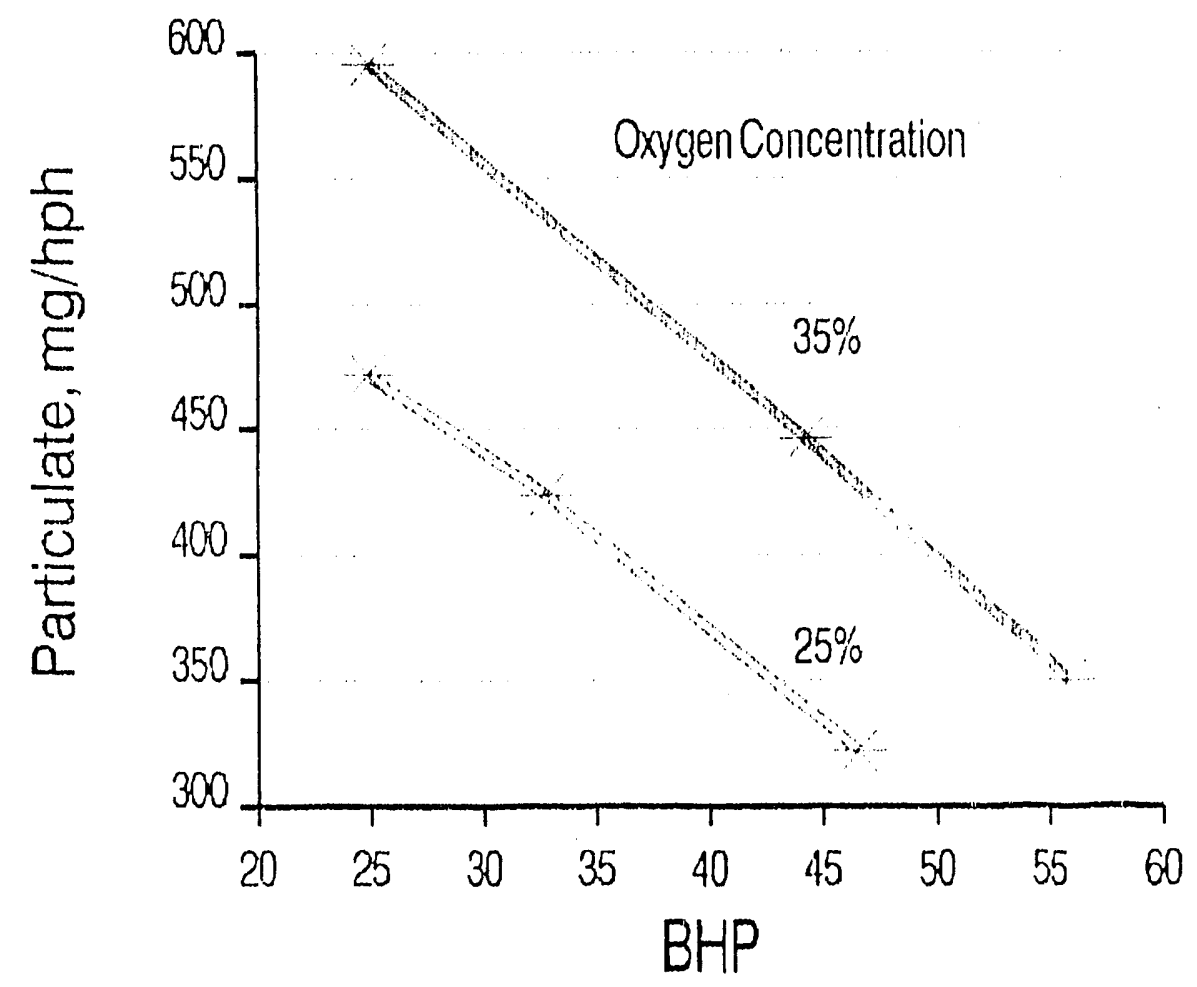

FIGURE A.25 Particulate Matter vs. Brake Horsepower, No. 4 Fuel, $50 \%$ Load, No Water 
A-35

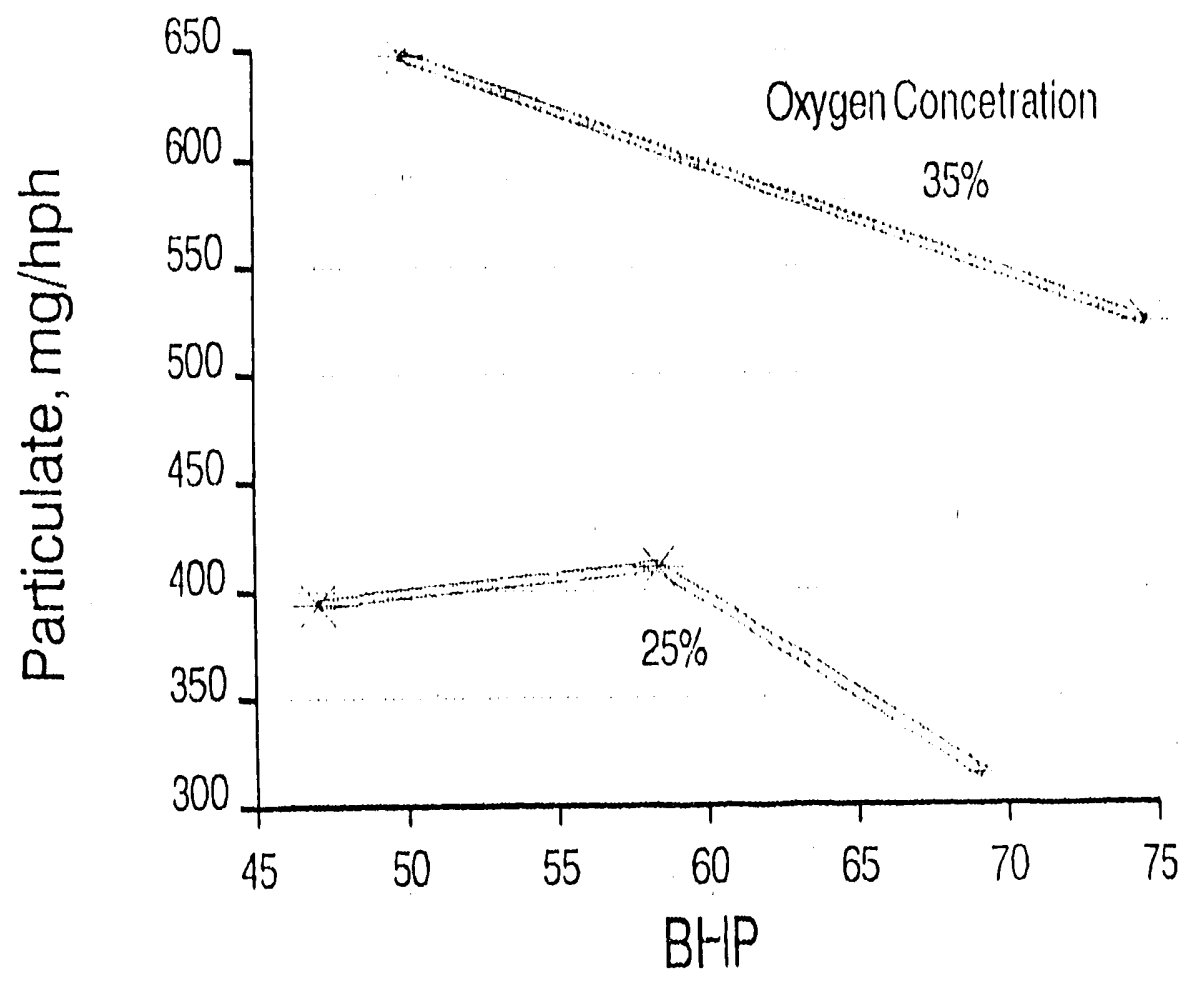

FIGURE A.26 Particulate Matter vs. Brake Horsepower, No. 4 Fuel, $100 \%$ Load, No Water

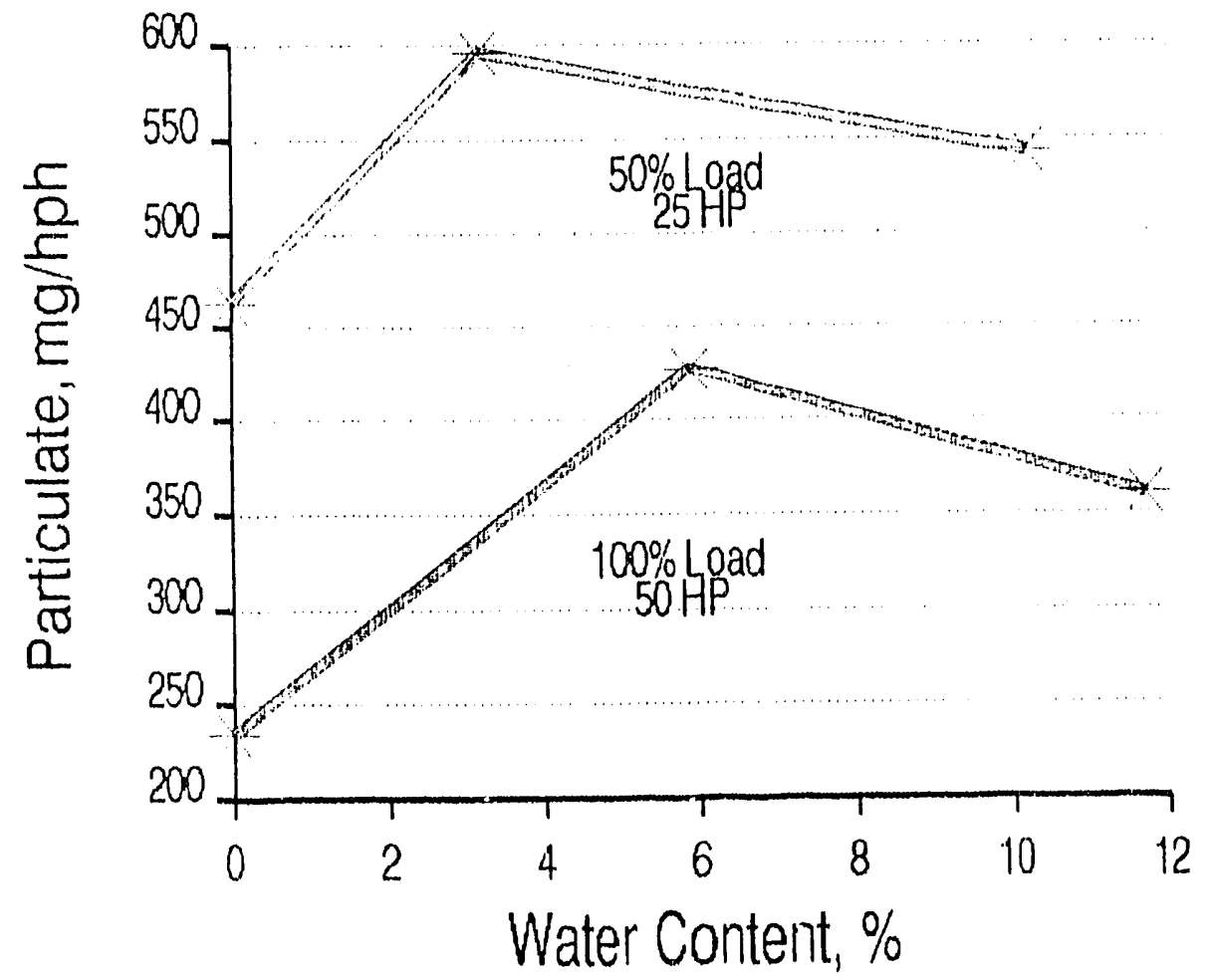

FIGURE A.27 Particulate Matter vs. Water Content, No. 4 Fuel, $21 \%$ Oxygen 


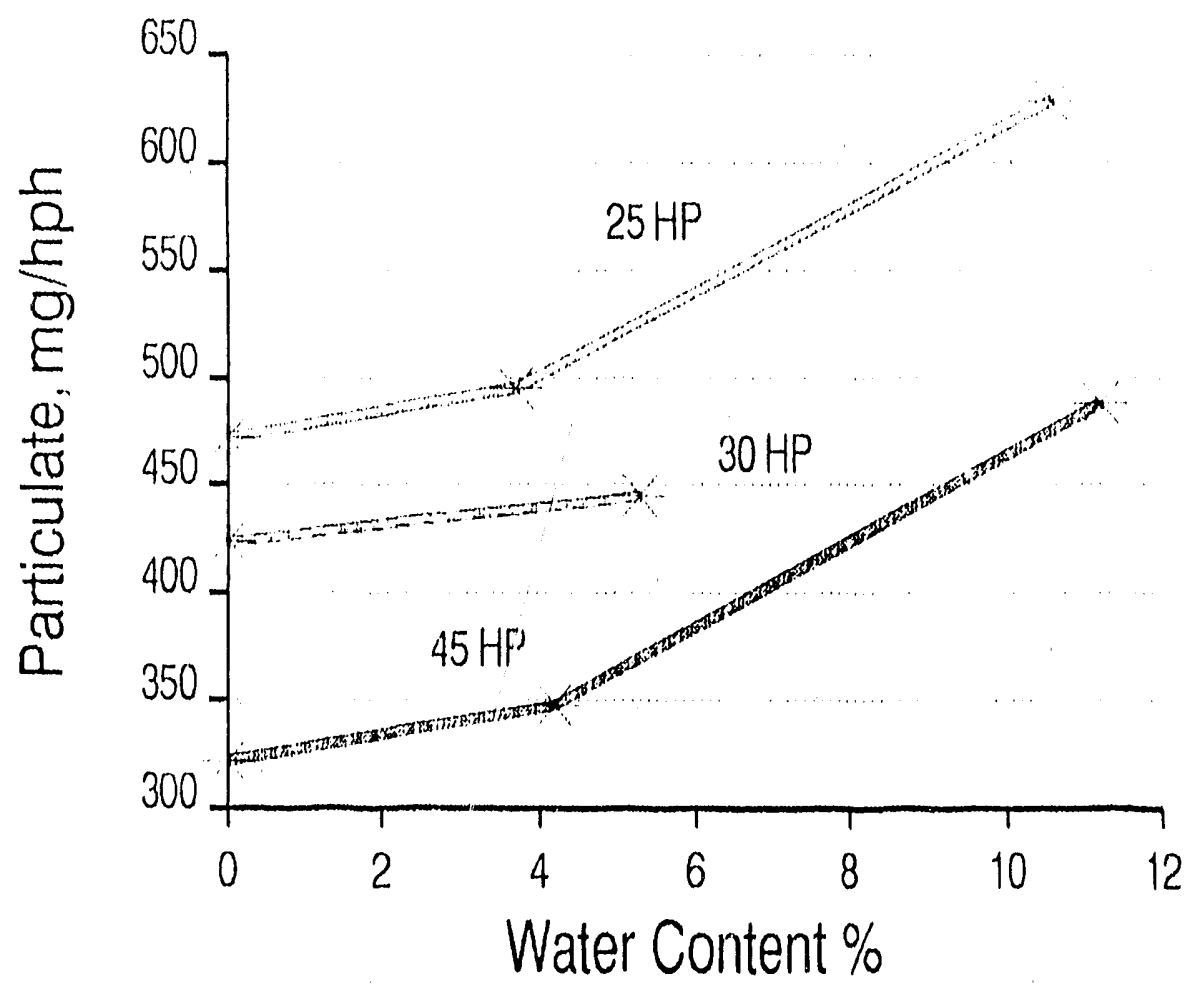

FIGURE A.28 Particulate Matter vs. Water Content, No. 4 Fuel, 50\% Load, $25 \%$ Oxygen

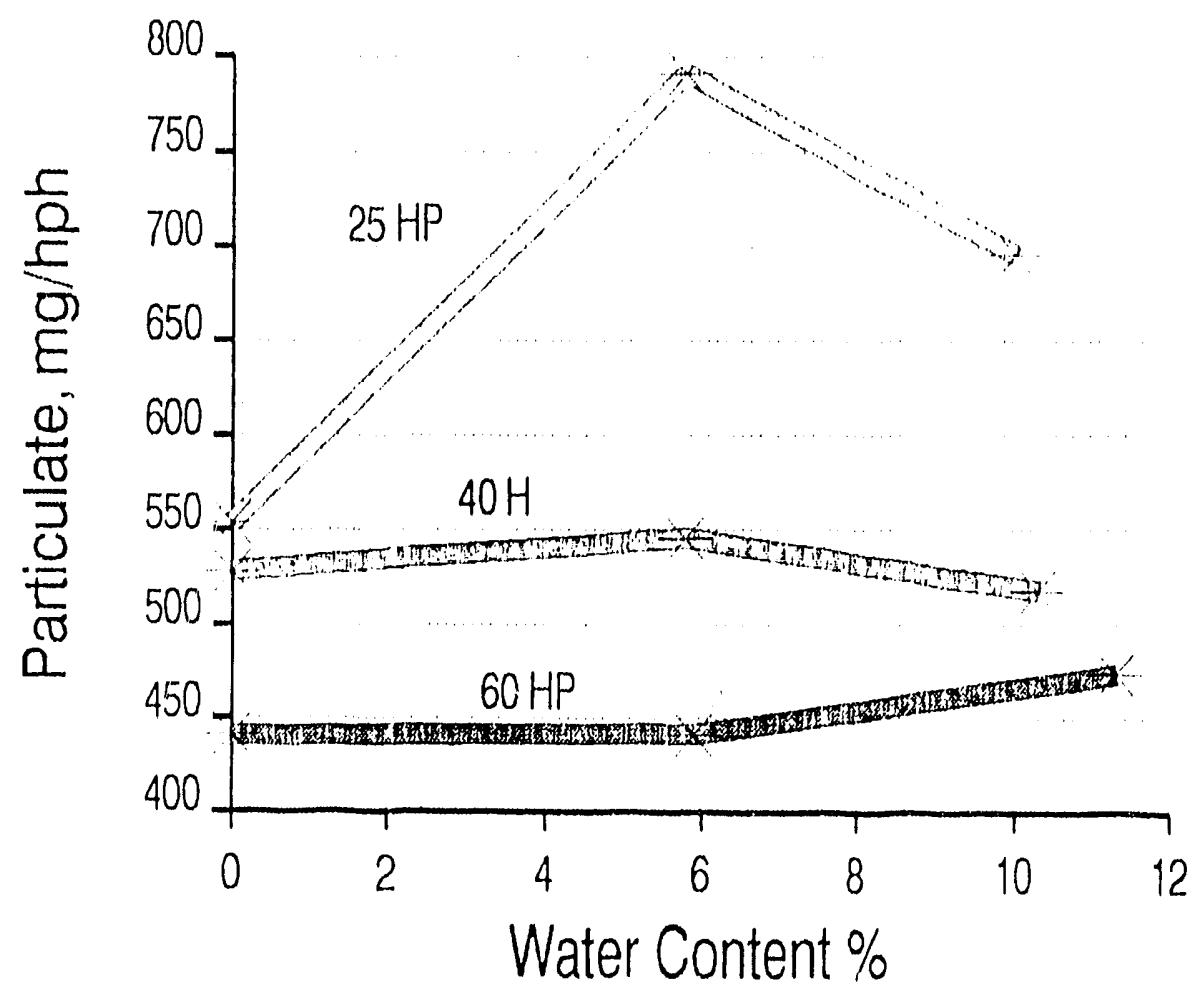

FIGURE A.29 Particulate Matter vs. Water Content, No. 4 Fuel, 50\% Load, $30 \%$ Oxygen 


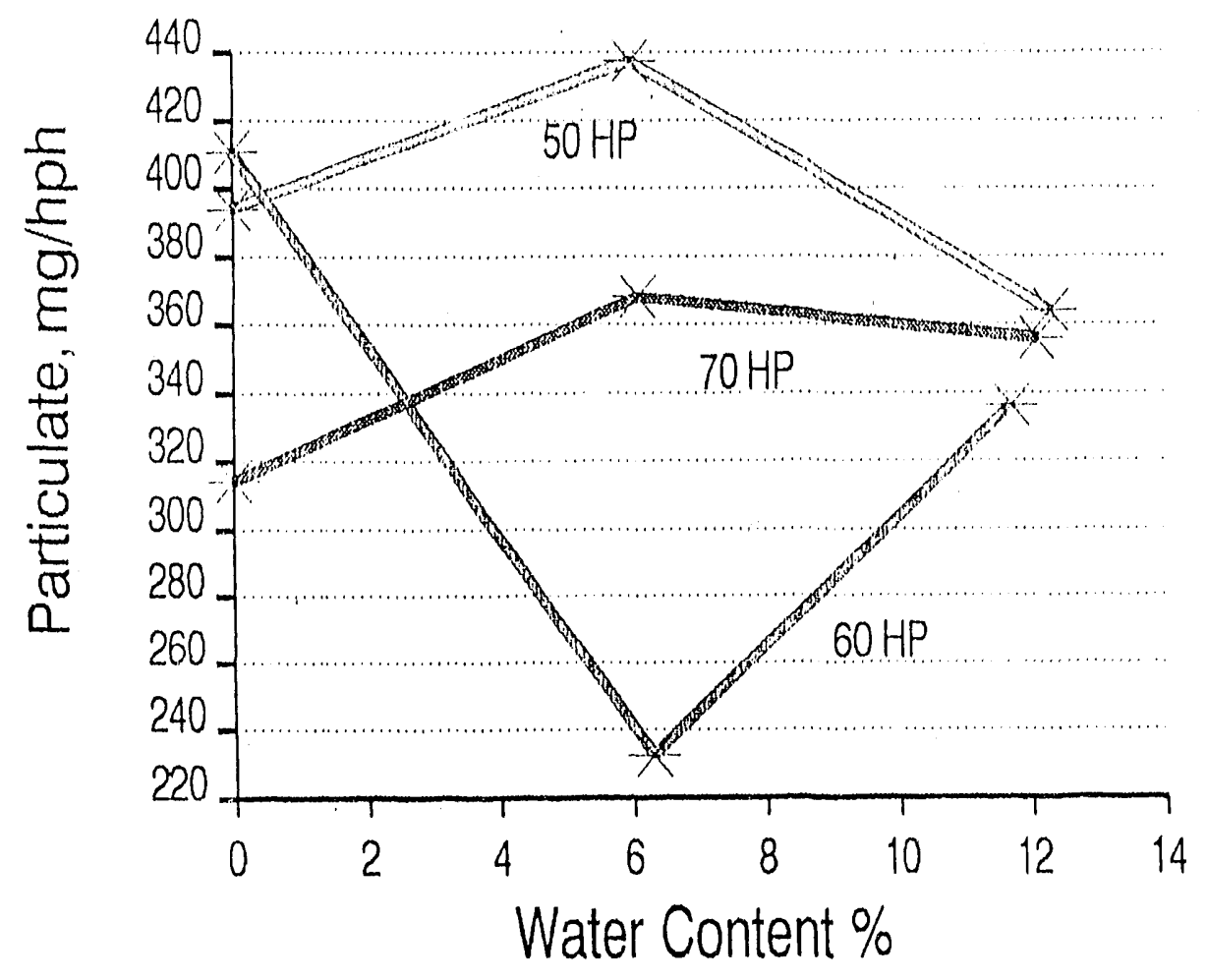

FIGURE A.30 Particulate Matter vs. Water Content, No. 4 Fuel, 100\% Load, $25 \%$ Oxygen

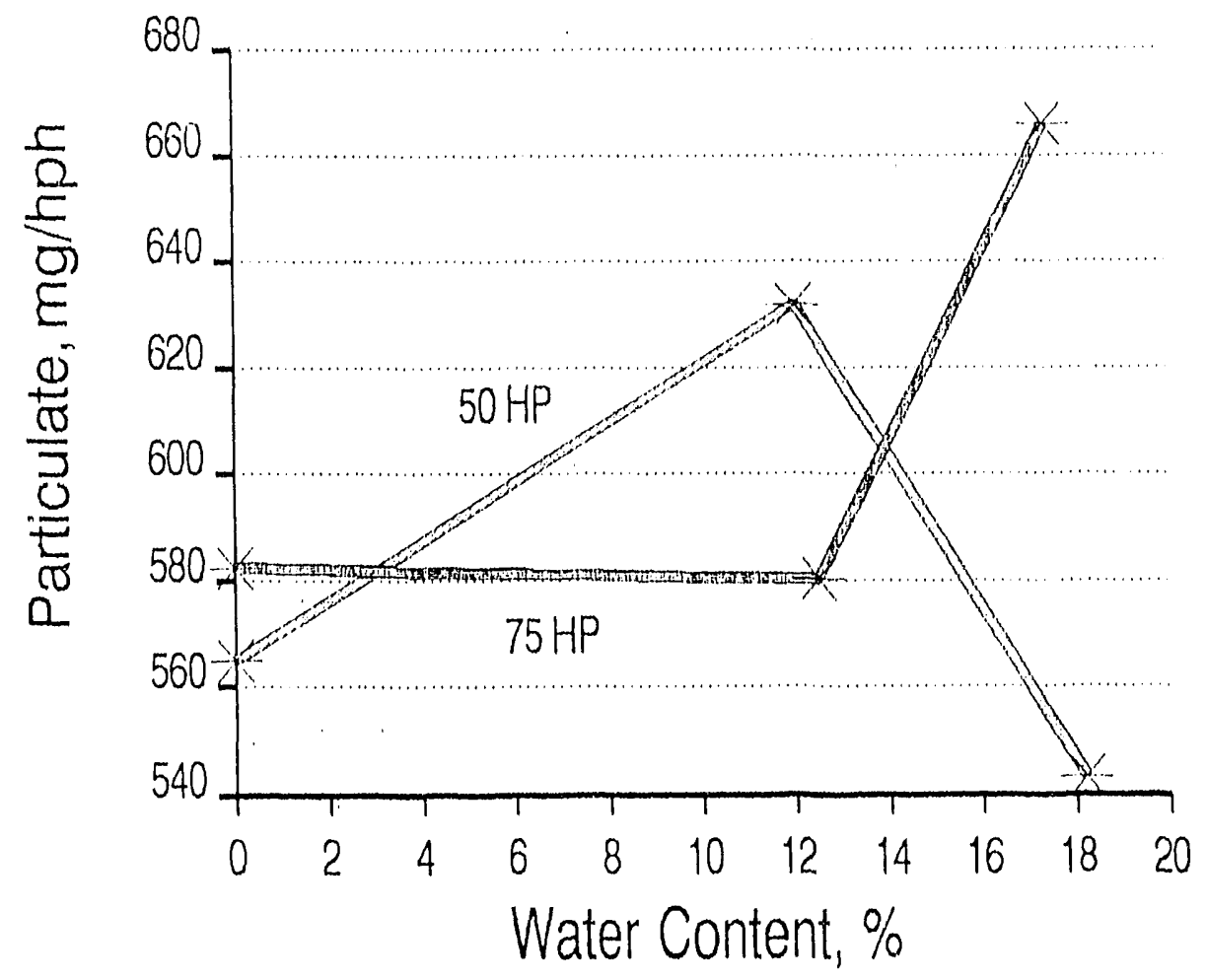

FIGURE A.31 Particulate Matter vs. Water Content, No. 4 Fuel, 100\% Load, $30 \%$ Oxygen 


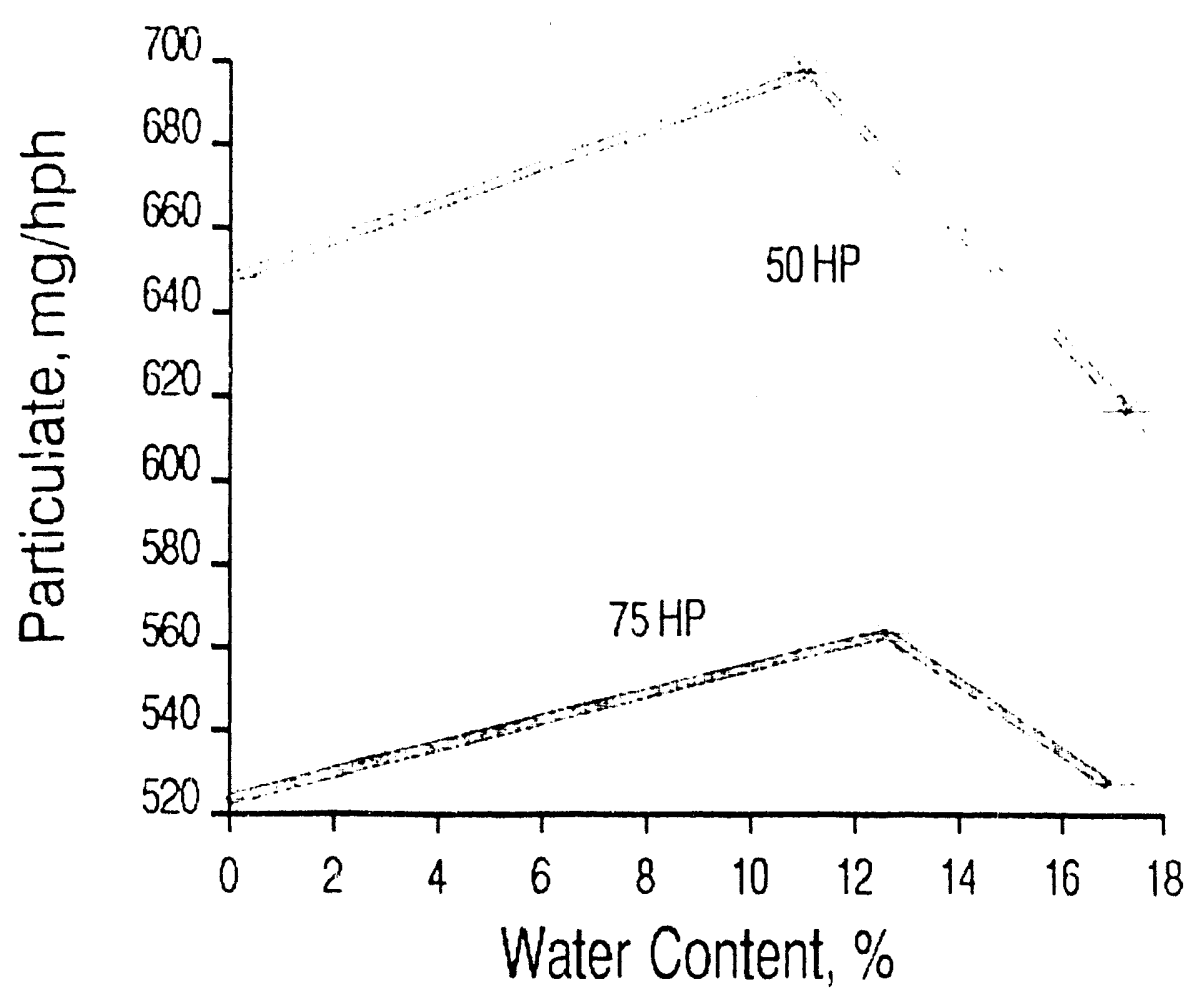

FIGURE A.32 Particulate Matter vs. Water Content, No. 4 Fuel, 100\% Loac, $35 \%$ Oxygen

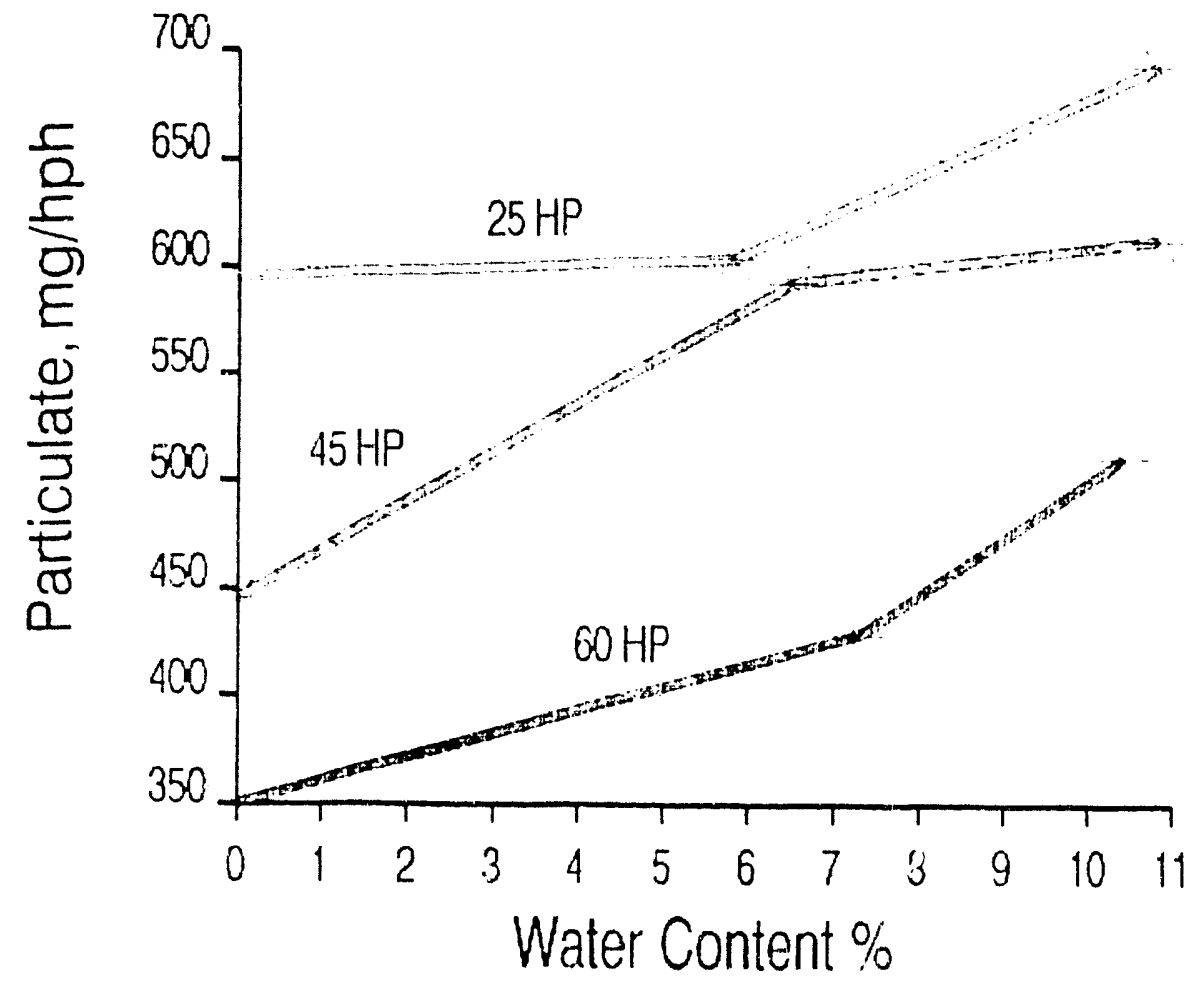

FIGURE A.33 Particulate Matter vs. Water Content, No. 4 Fuel, 50\% Load, $35 \%$ Oxygen 


\section{A.5 Comparison of Test Data for No. 2 and No. 4 Fuels}


$A-41$

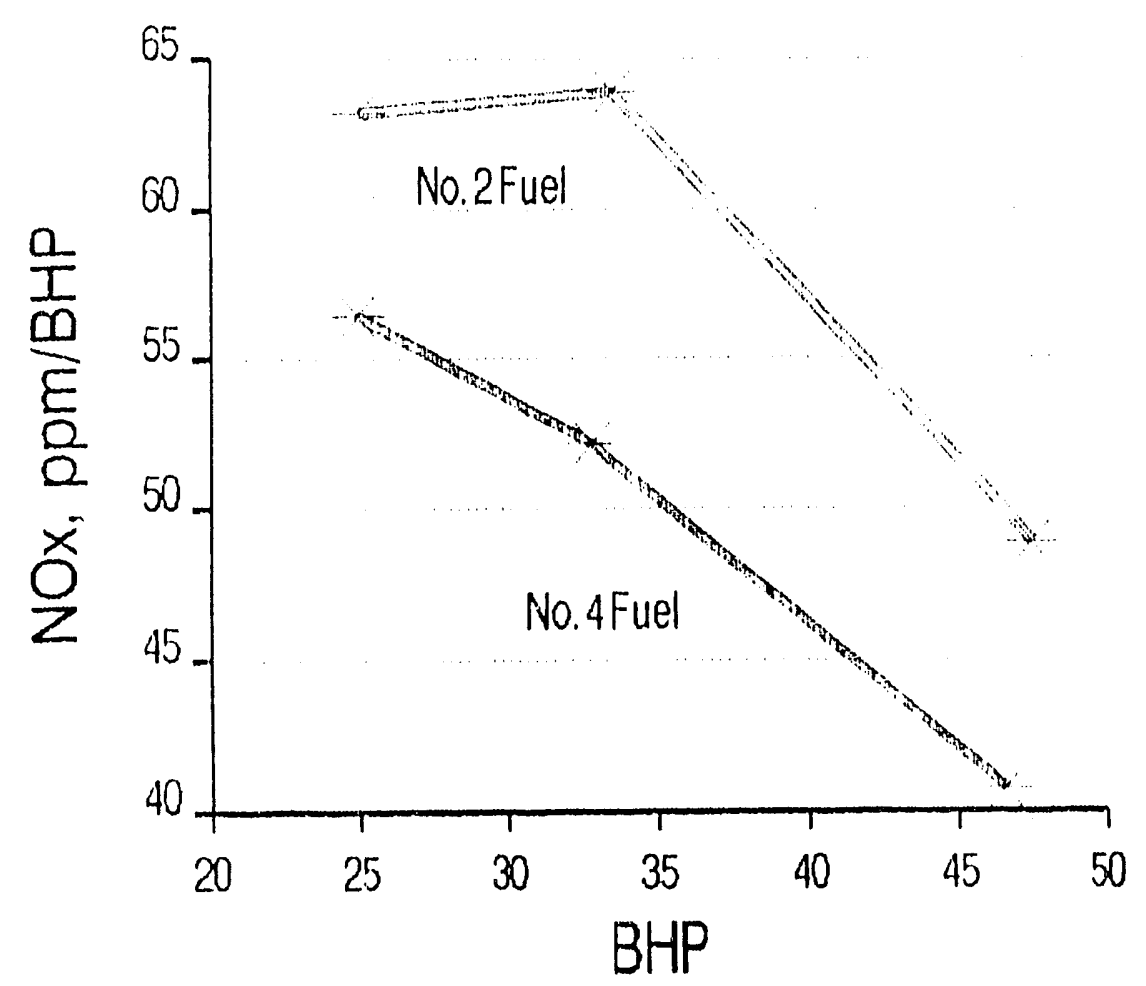

FIGURE A.34 Nitrogen Oxides vs. Brake Horsepower, 50\% Load, No Water, $25 \%$ Oxygen

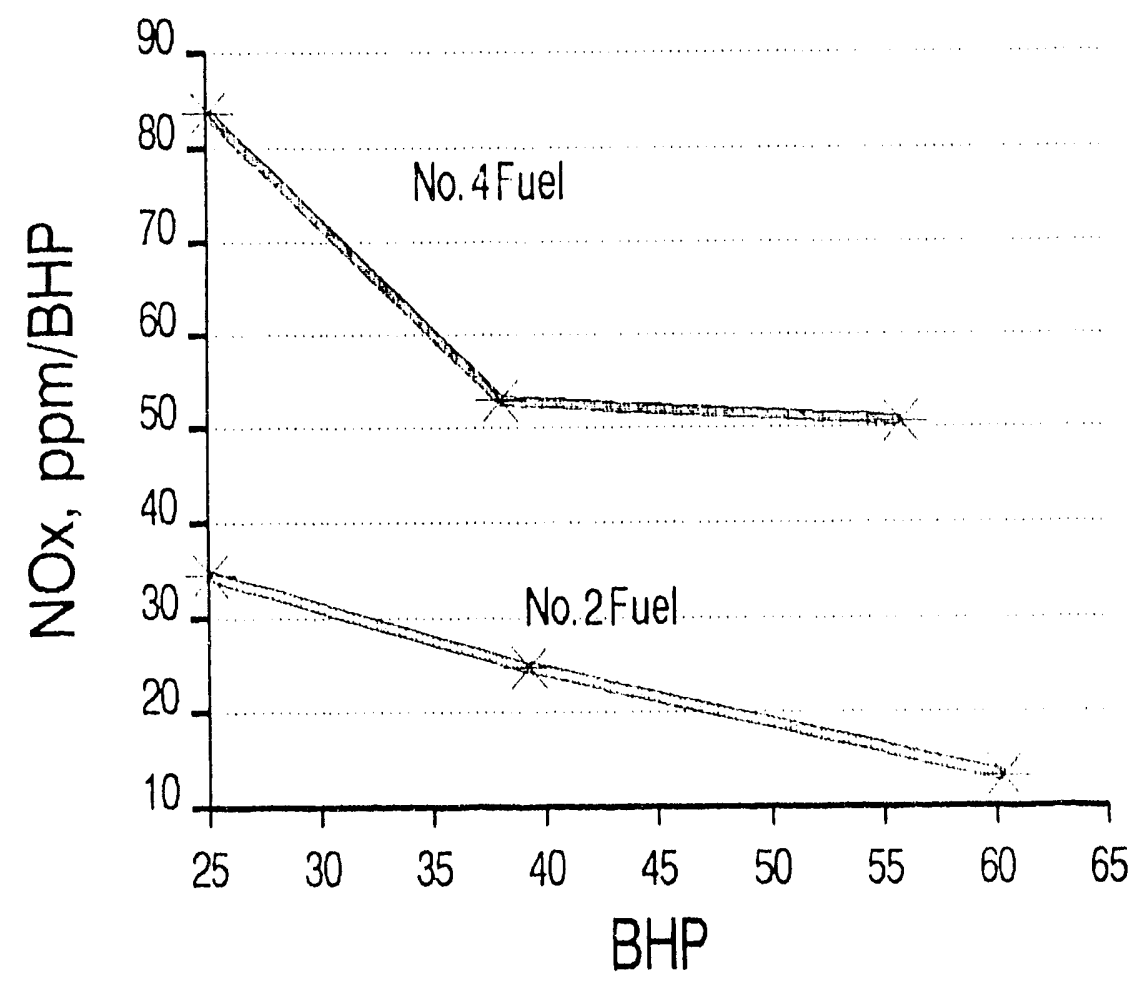

FIGURE A.35 Nitrogen Oxides vs. Brake Horsepower, $50 \%$ Load, No Water, $30 \%$ Oxygen 


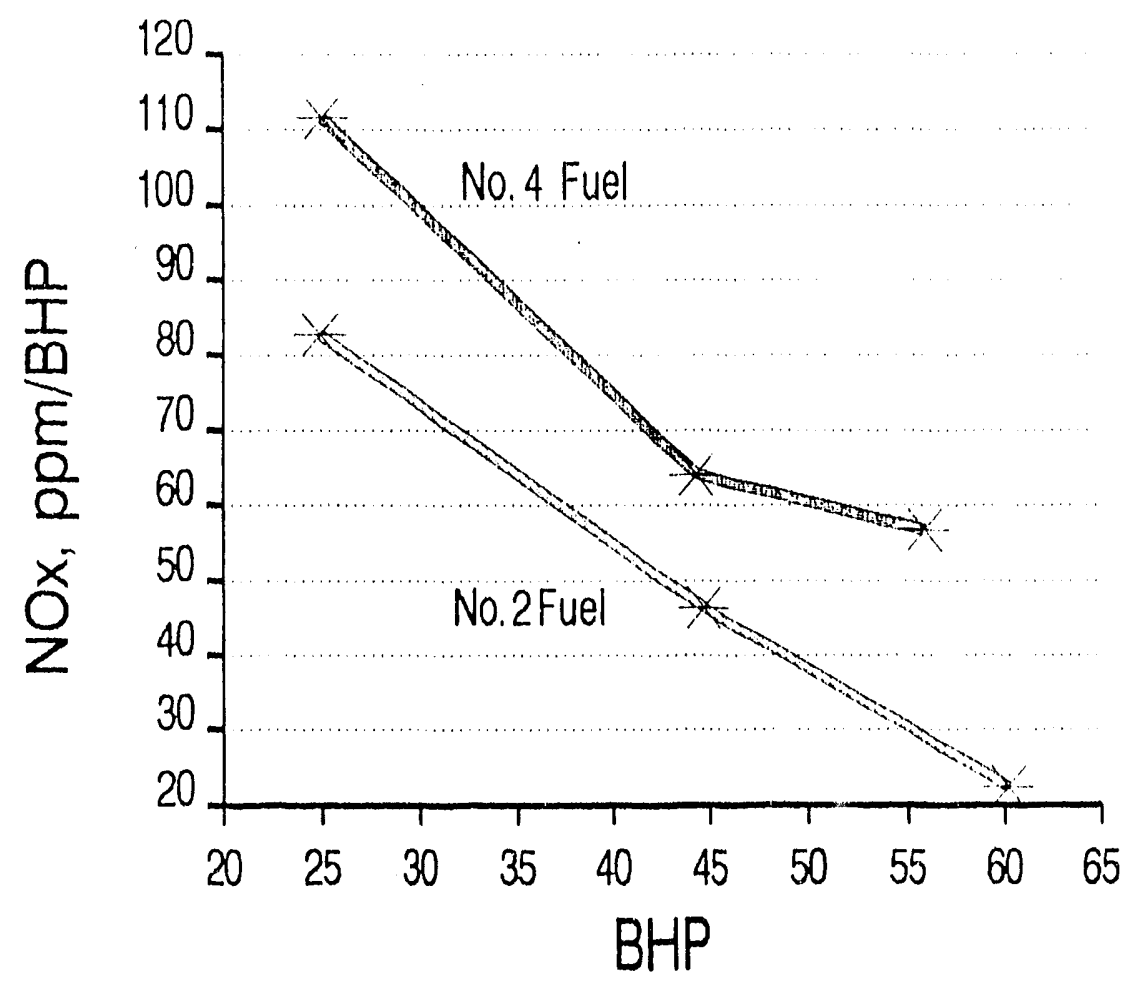

FIGURE A.36 Nitrogen Oxides vs. Brake Horsepower, 50\% Load, No Water, $35 \%$ Oxygen

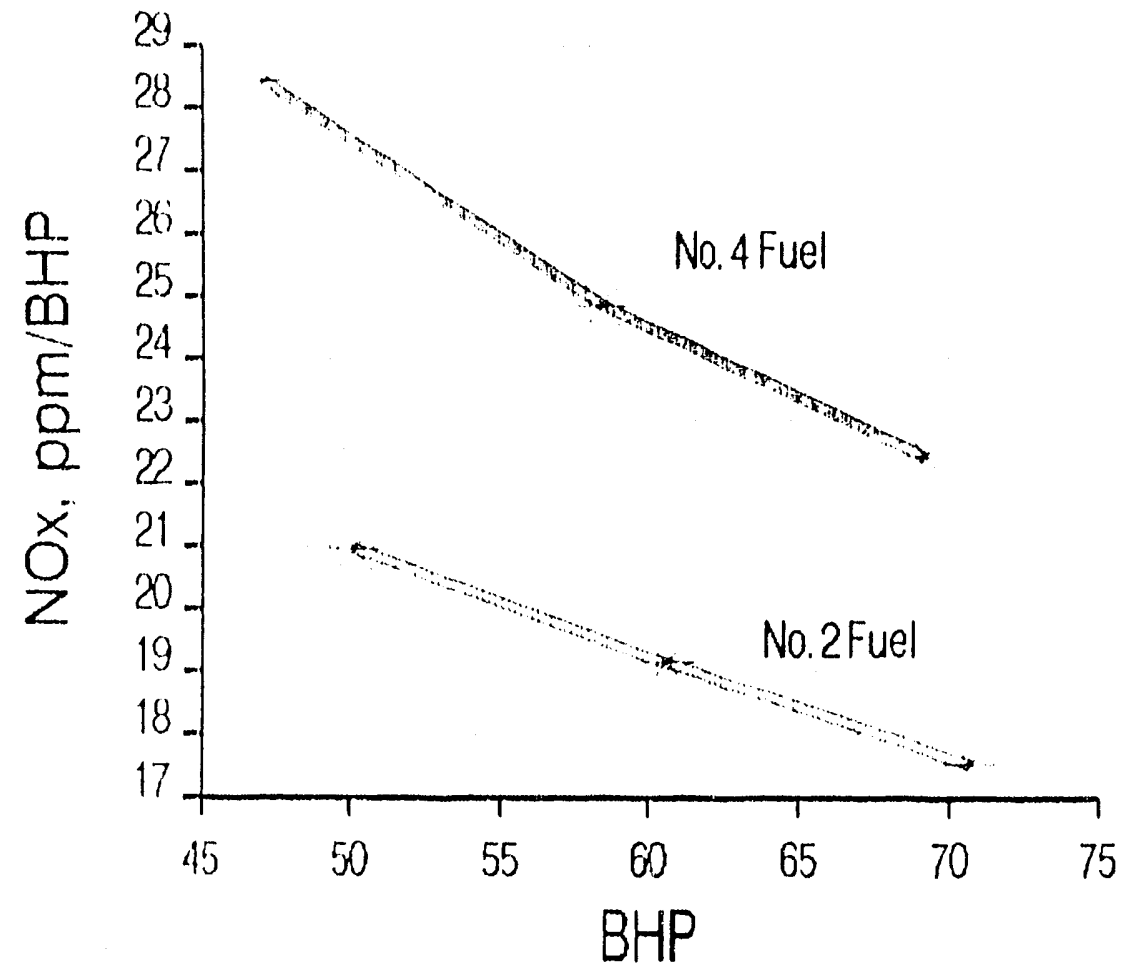

FIGURE A.37 Nitrogen Oxides vs. Brake Horsepower, 100\% Load, No Water, $25 \%$ Oxygen 


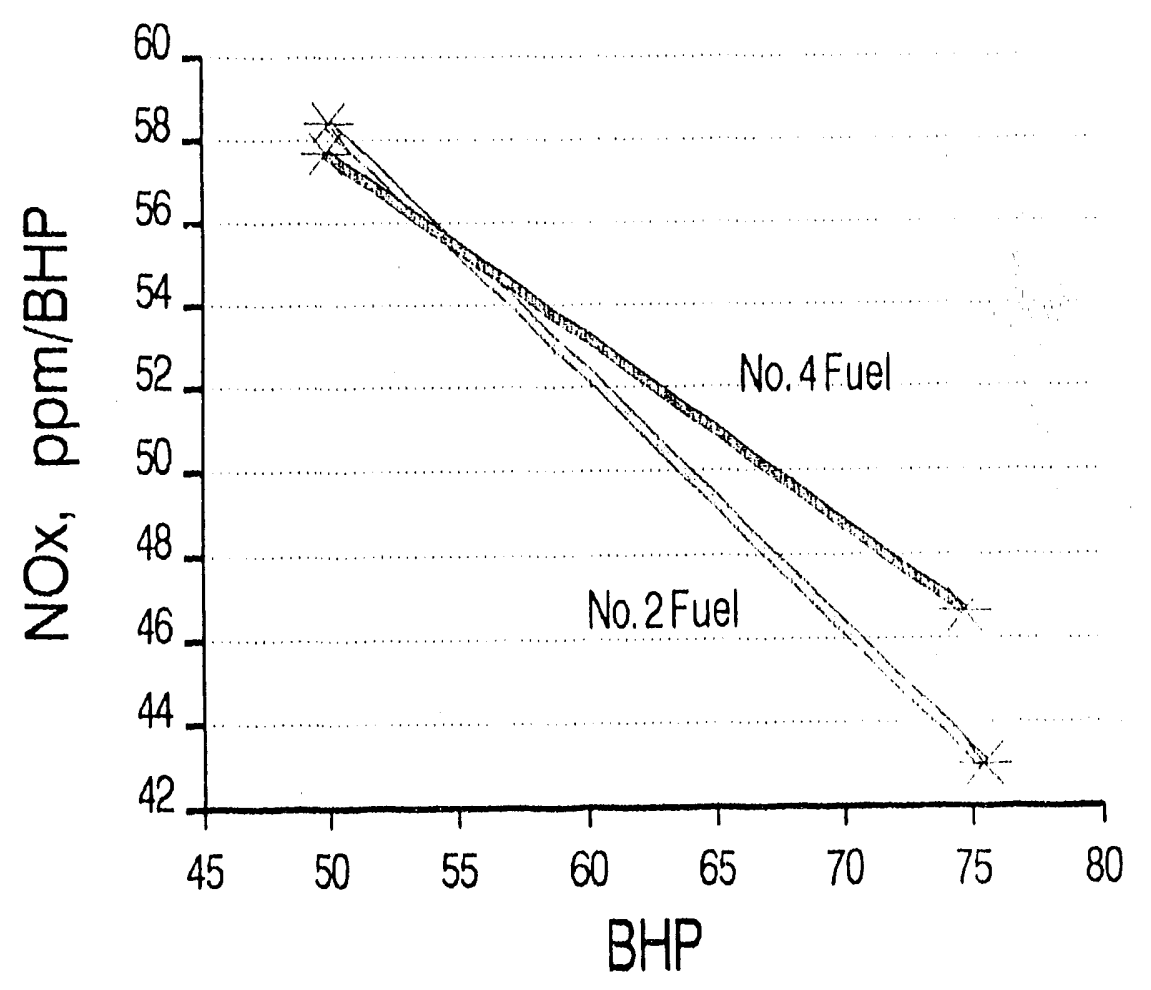

FIGURE A.38 Nitrogen Oxides vs. Brake Horsepower, 100\% Load, No Water, $35 \%$ Oxygen

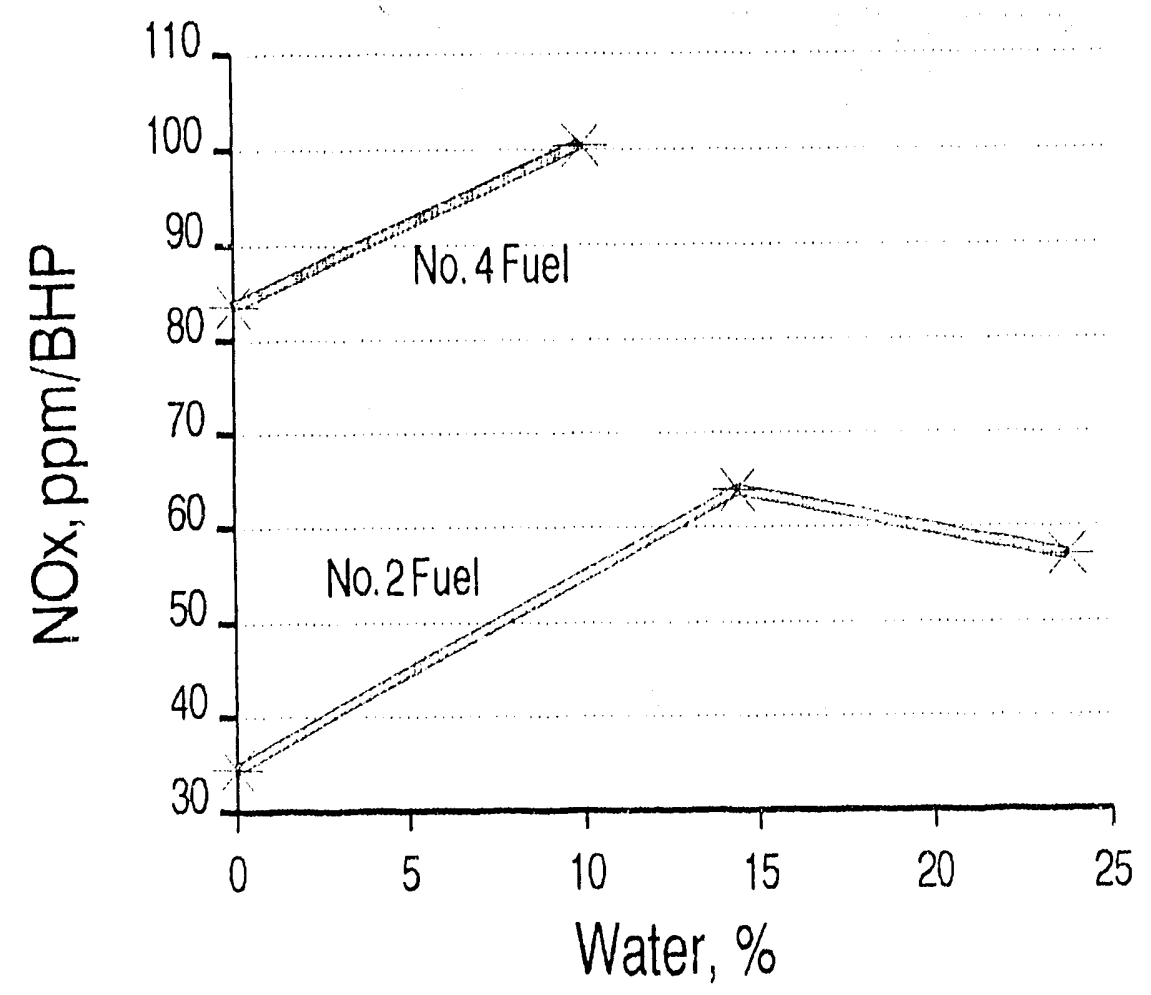

FIGURE A.39 Nitrogen Oxides vs. Water Content, $50 \%$ Load, 25 Horsepower, 30\% Oxygen 


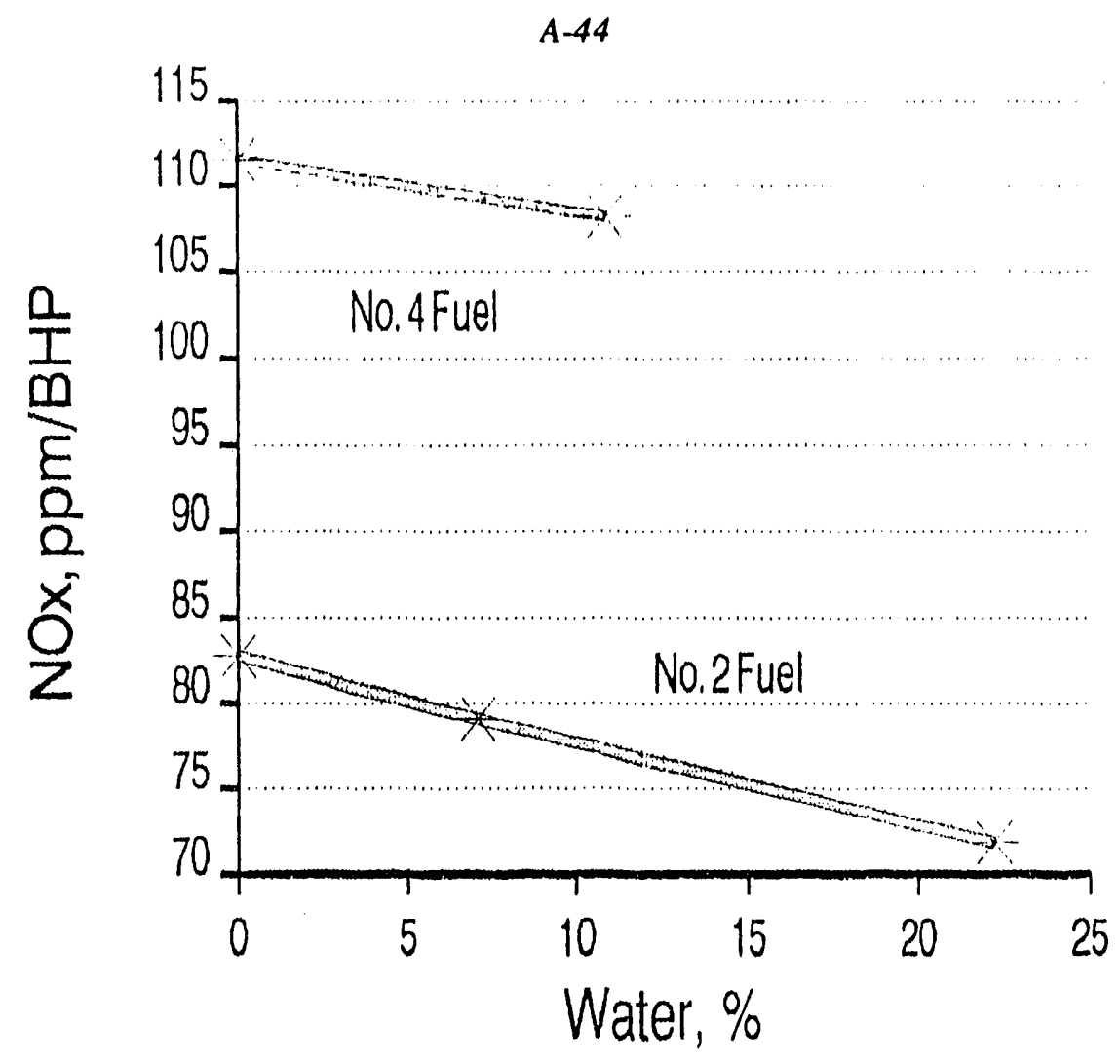

FIGURE A.40 Nitrogen Oxides vs. Water Content, $50 \%$ Load, 25 Horsepower, 35\% Oxygen

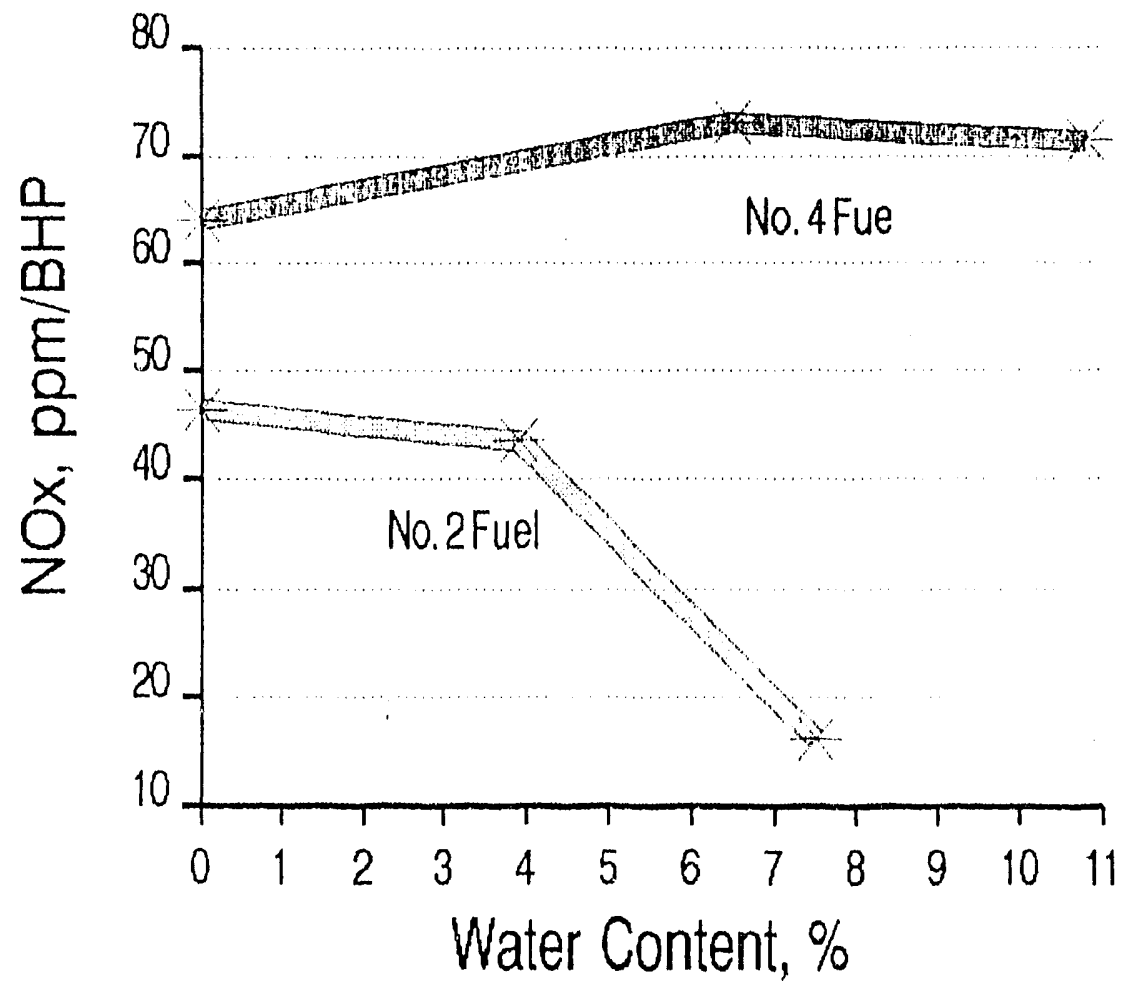

FIGURE A.41 Nitrogen Oxides vs. Water Content, 50\% Load, 45 Horsepower, $35 \%$ Oxygen 


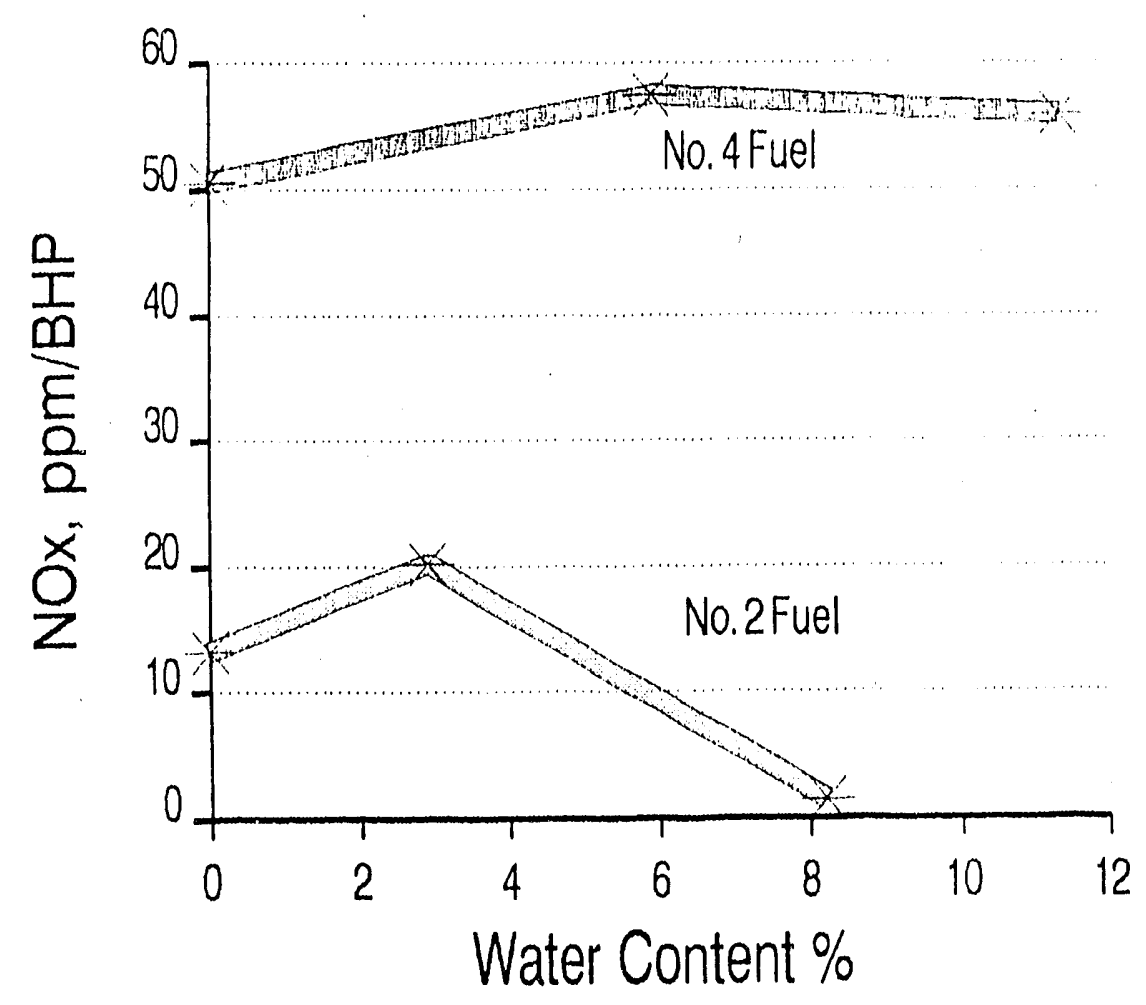

FIGURE A.42 Nitrogen Oxides vs. Water Content, $50 \%$ Load, 60 Horsepower, $30 \%$ Oxygen

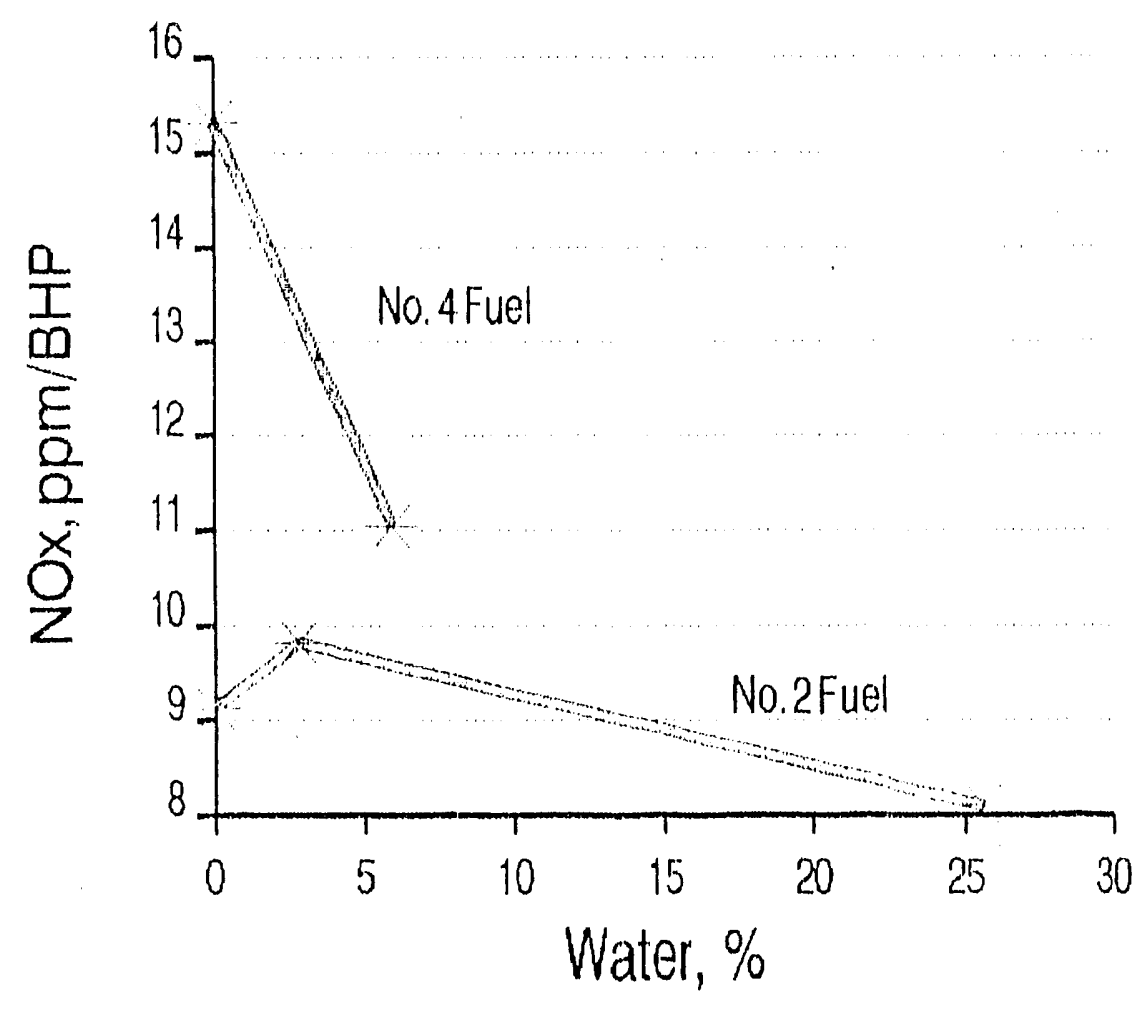

FIGURE A.43 Nitrogen Oxides vs. Water Content, 100\% Load, 50 Horsepower, $21 \%$ Oxygen 


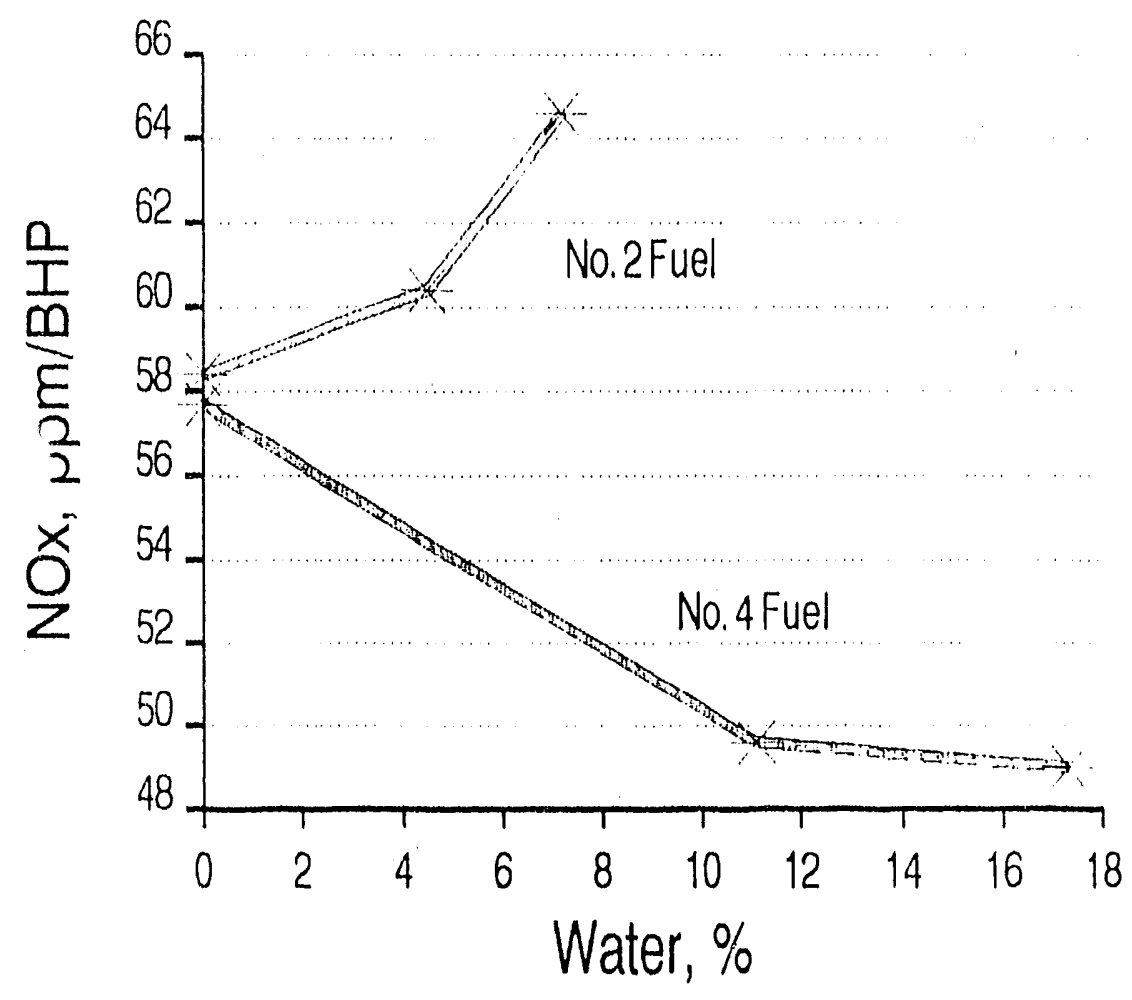

FIGURE A.44 Nitrogen Oxides vs. Water Content, 100\% Load, 50 Horsepower, $35 \%$ Oxygen

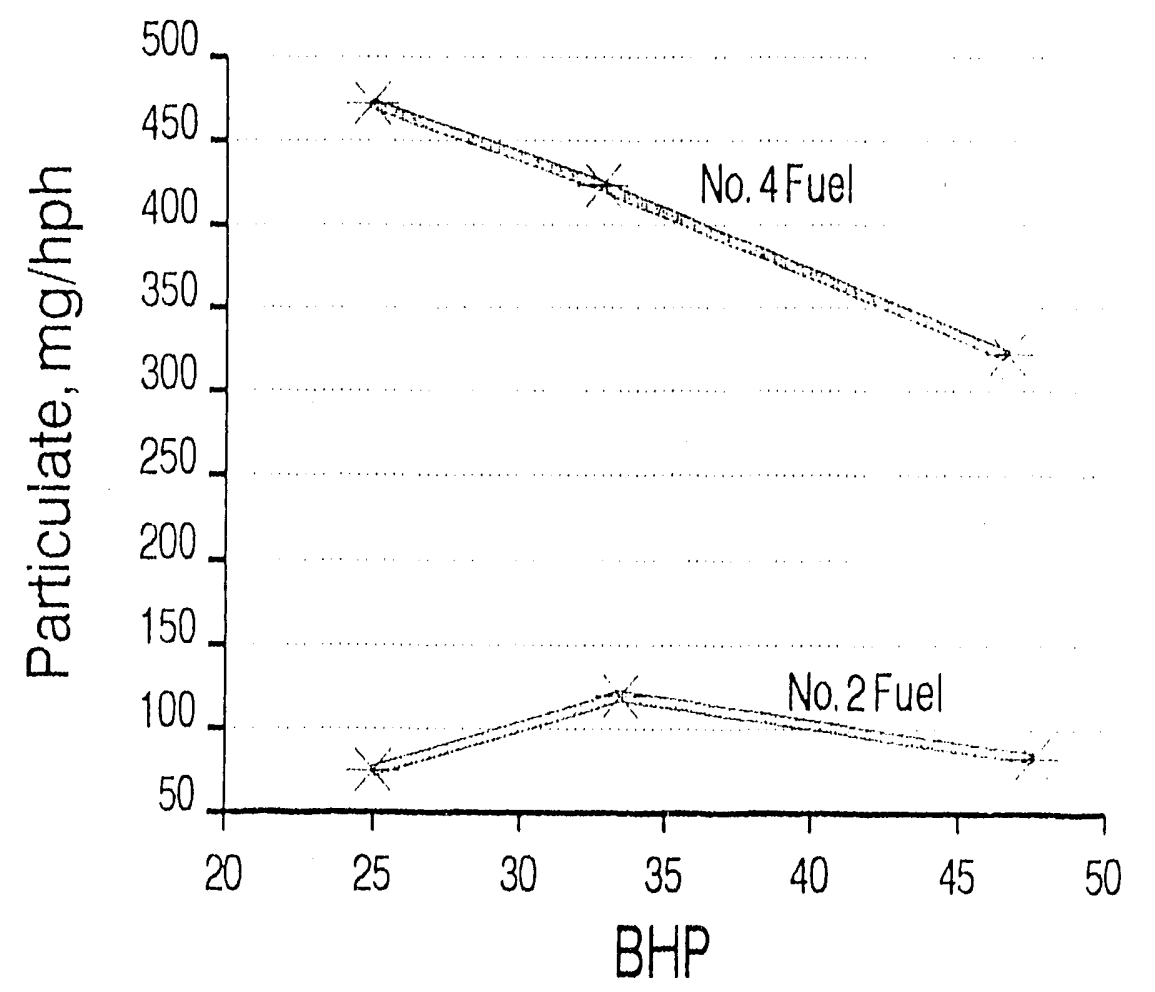

FIGURE A.45 Particulate Matter vs. Brake Horsepower, 50\% Load, No Water, $25 \%$ Oxygen 


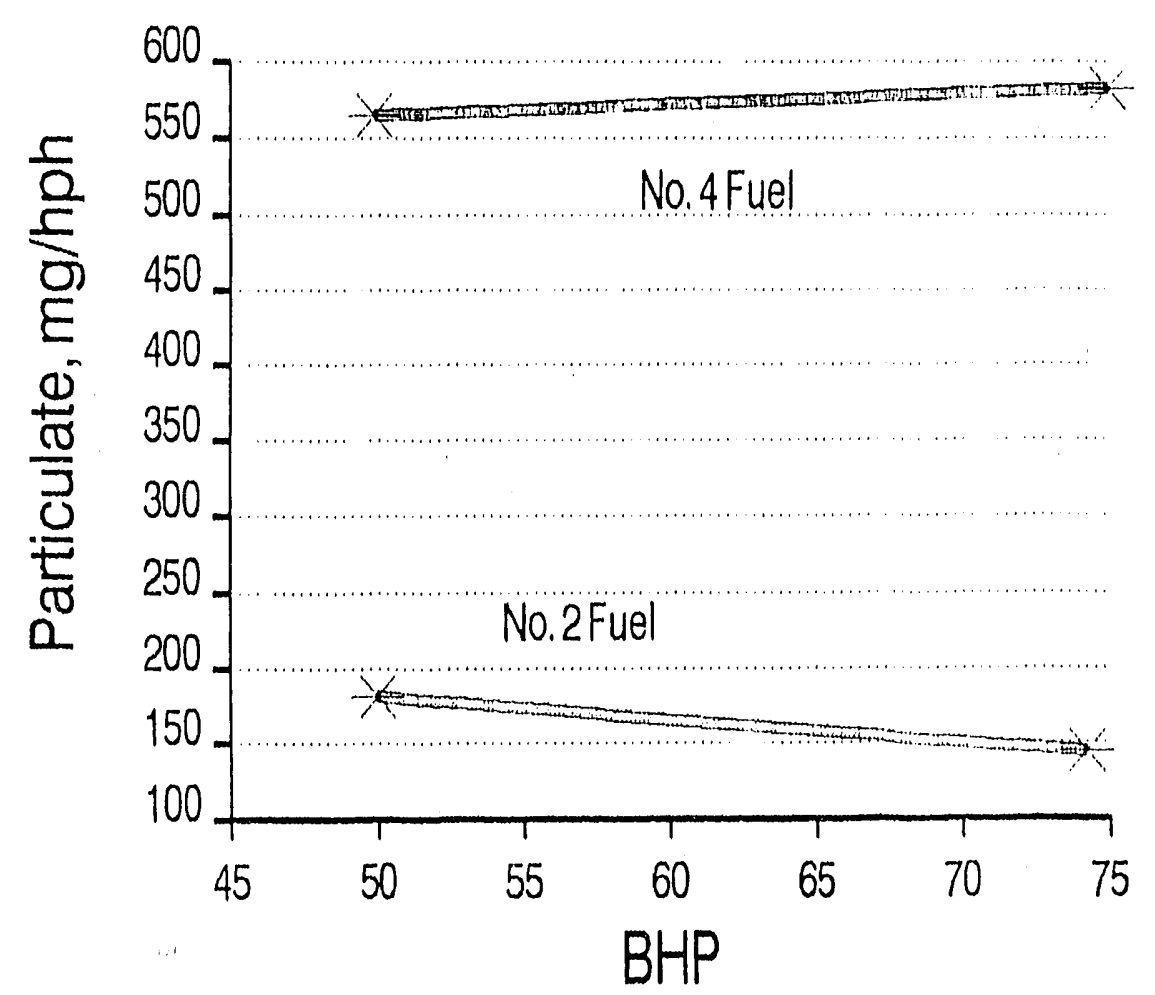

FIGURE A.46 Particulate Matter vs. Brake Horsepower, 100\% Load, No Water, $30 \%$ Oxygen

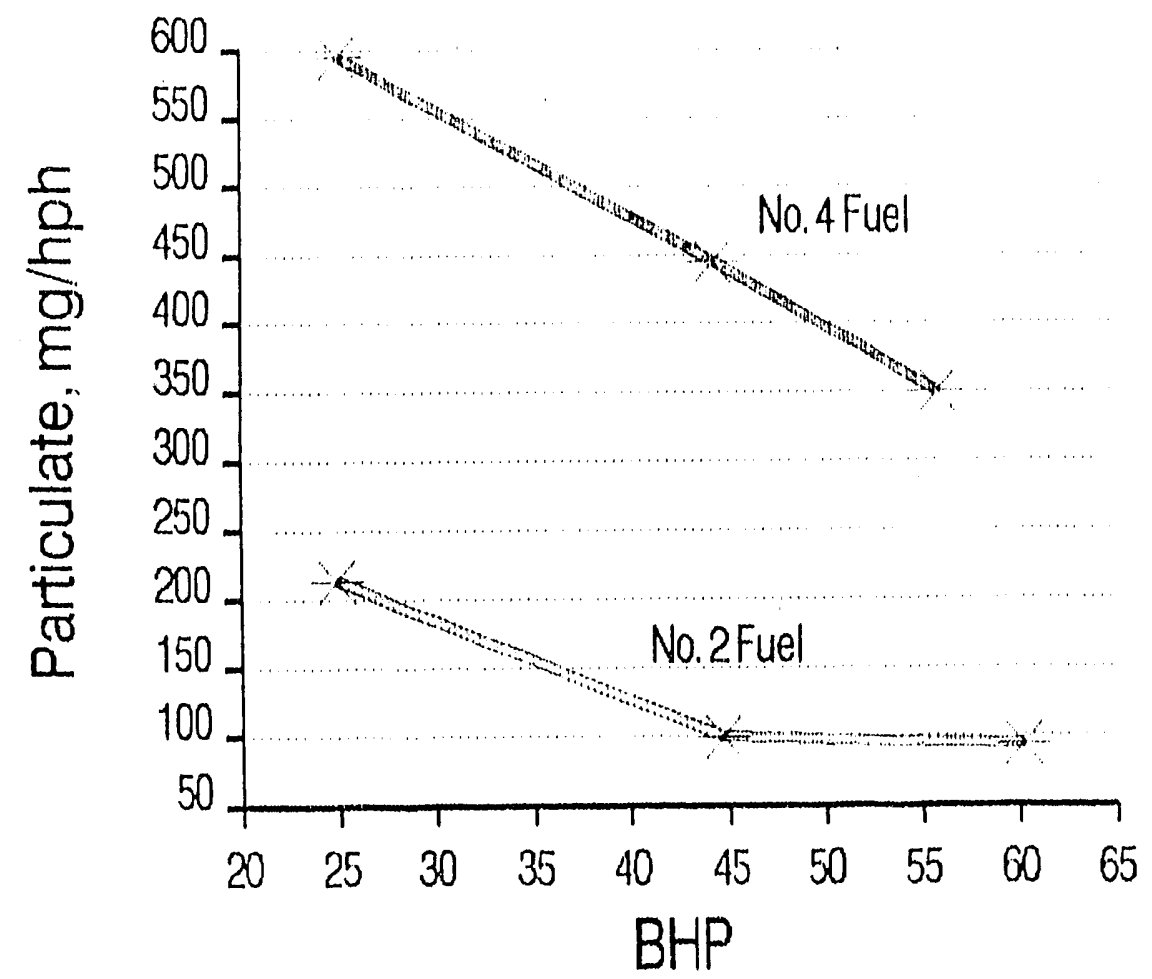

FIGURE A.47 Particulate Matter vs. Brake Horsepower, 50\% Load, No Water, $35 \%$ Oxygen 


\section{$A-48$}

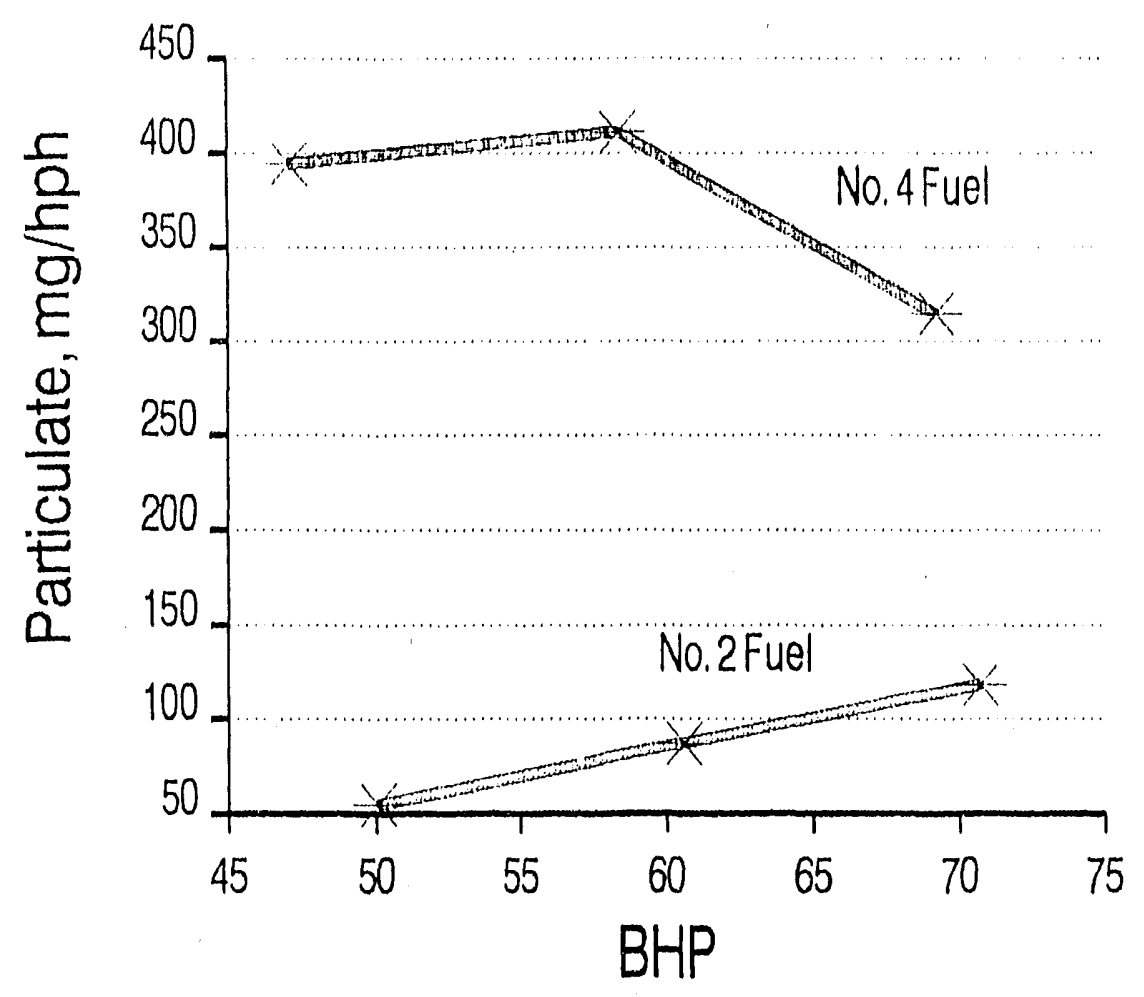

FIGURE A.48 Particulate Matter vs. Brake Horsepower, 100\% Load, No Water, $25 \%$ Oxygen

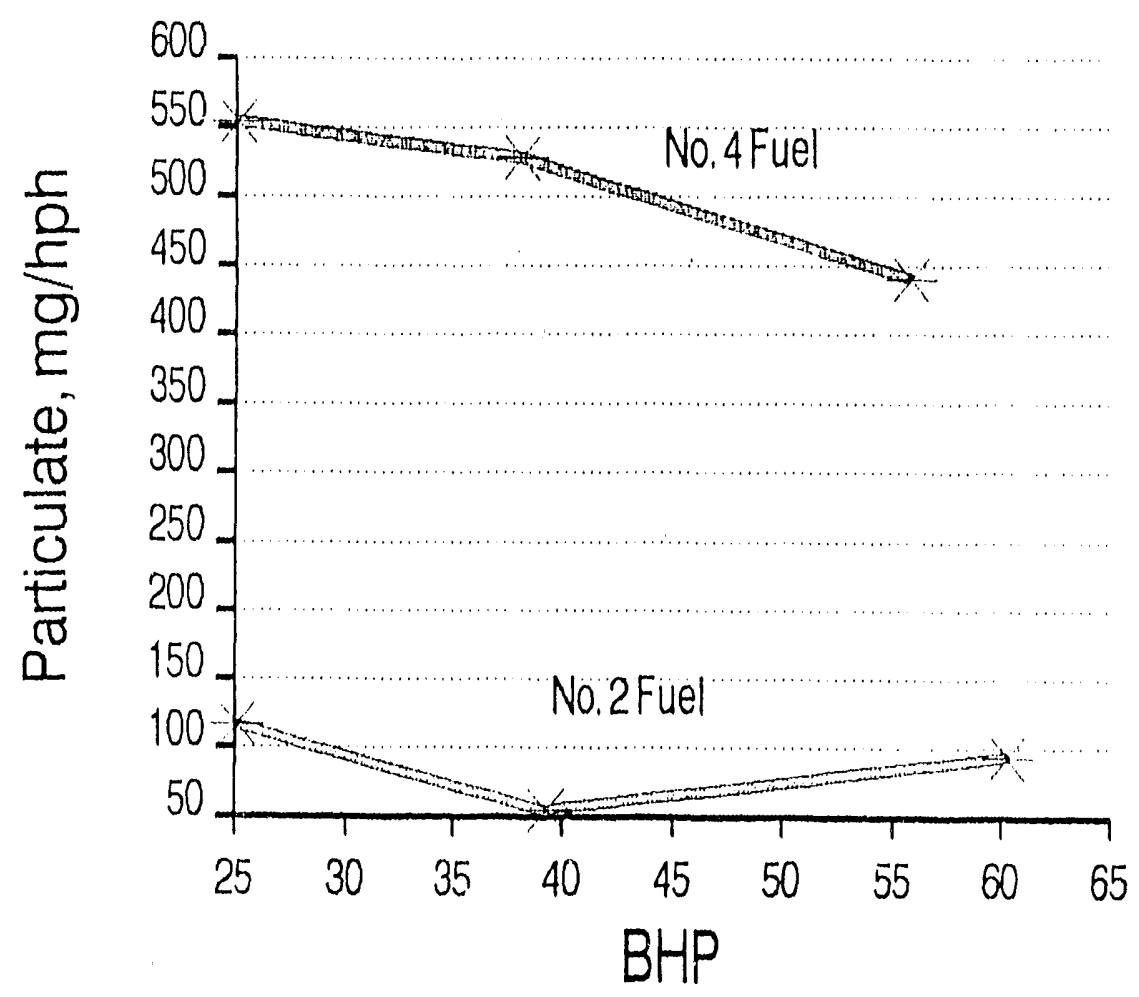

FIGURE A.49 Particulate Matter vs. Brake Hiorsepower, 50\% Load, No Water, $30 \%$ Oxygen 


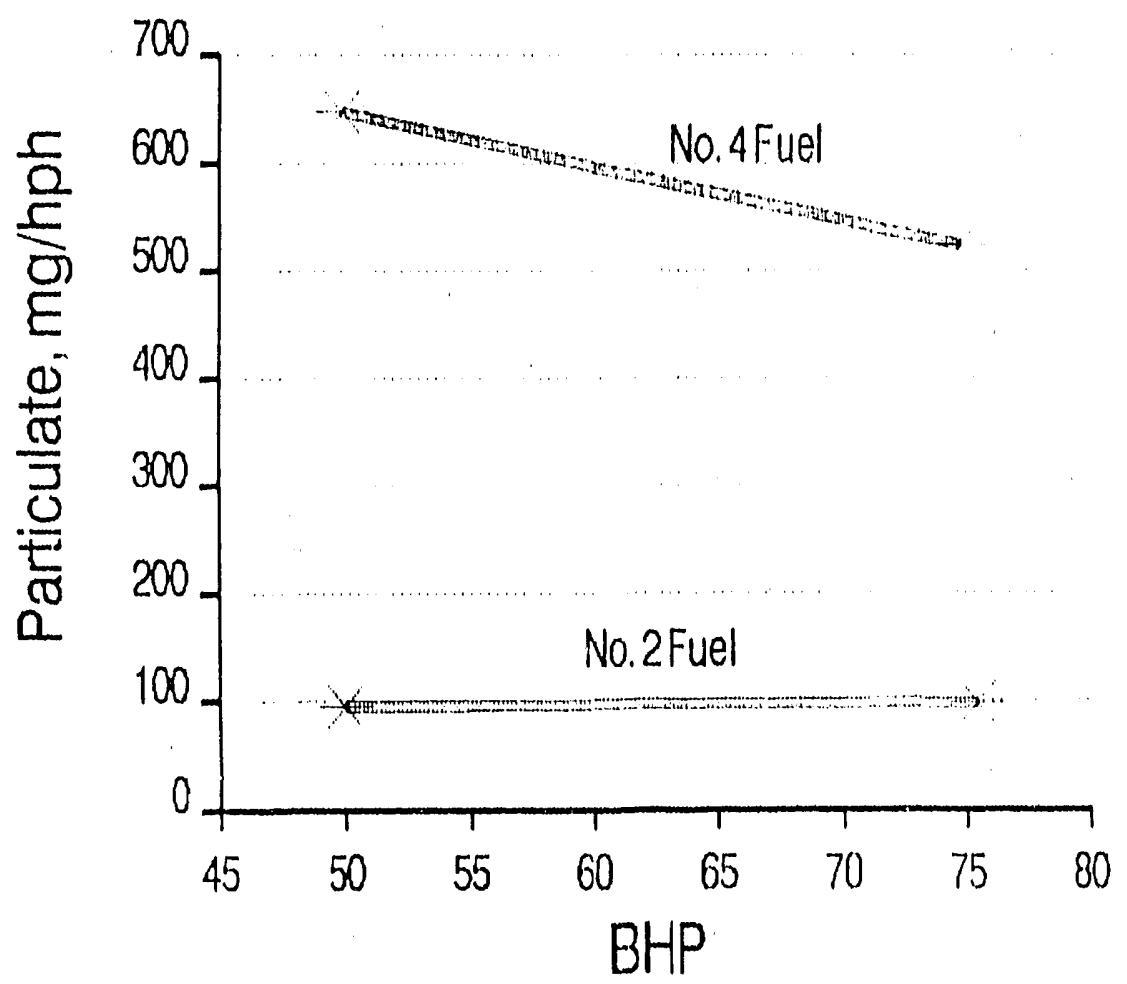

FIGURE A.50 Particulate Matter vs. Brake Horsepower, 100\% Load, No Water, $35 \%$ Oxygen

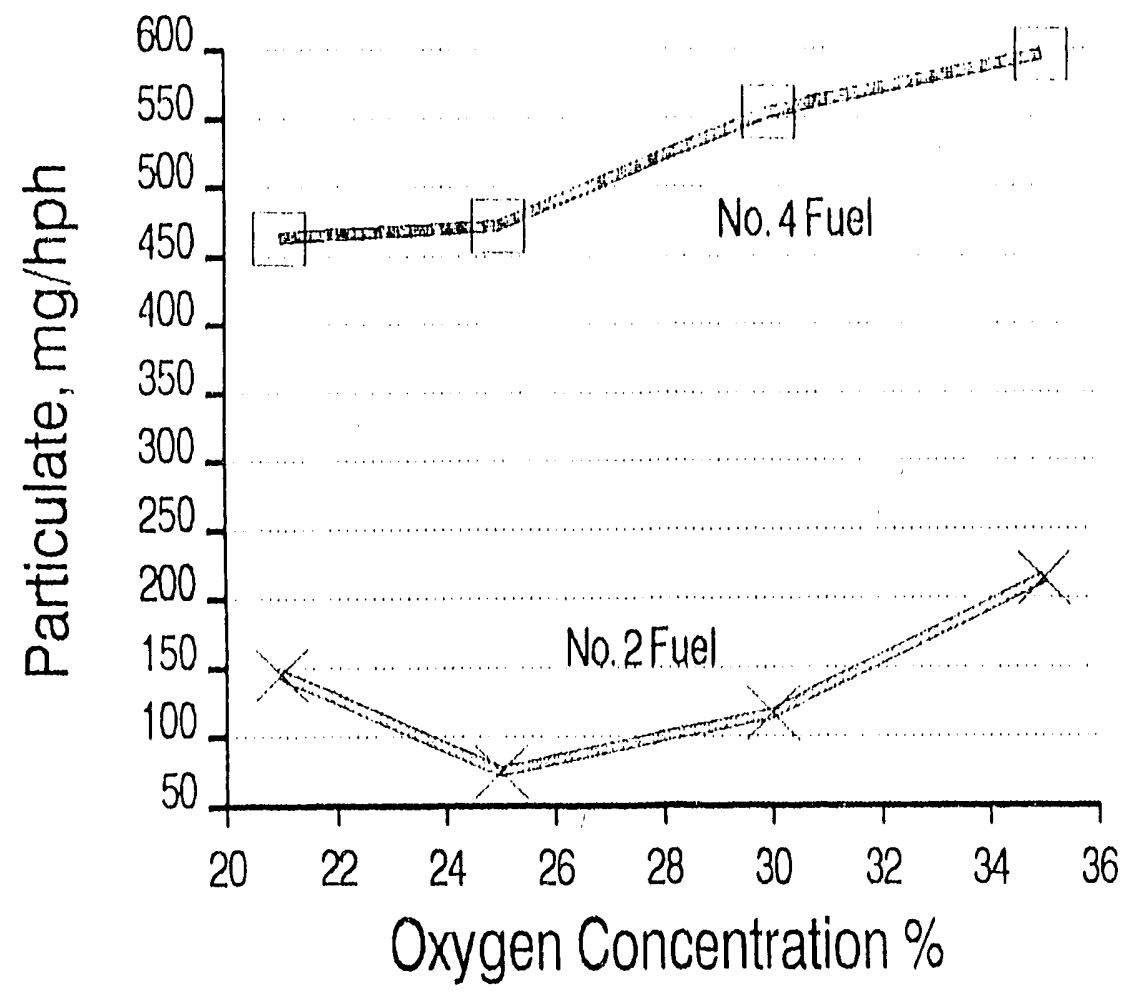

FIGURE A.51 Particulate-Matter Emissions, 50\% Load, 25 Brake Horsepower, No Water 


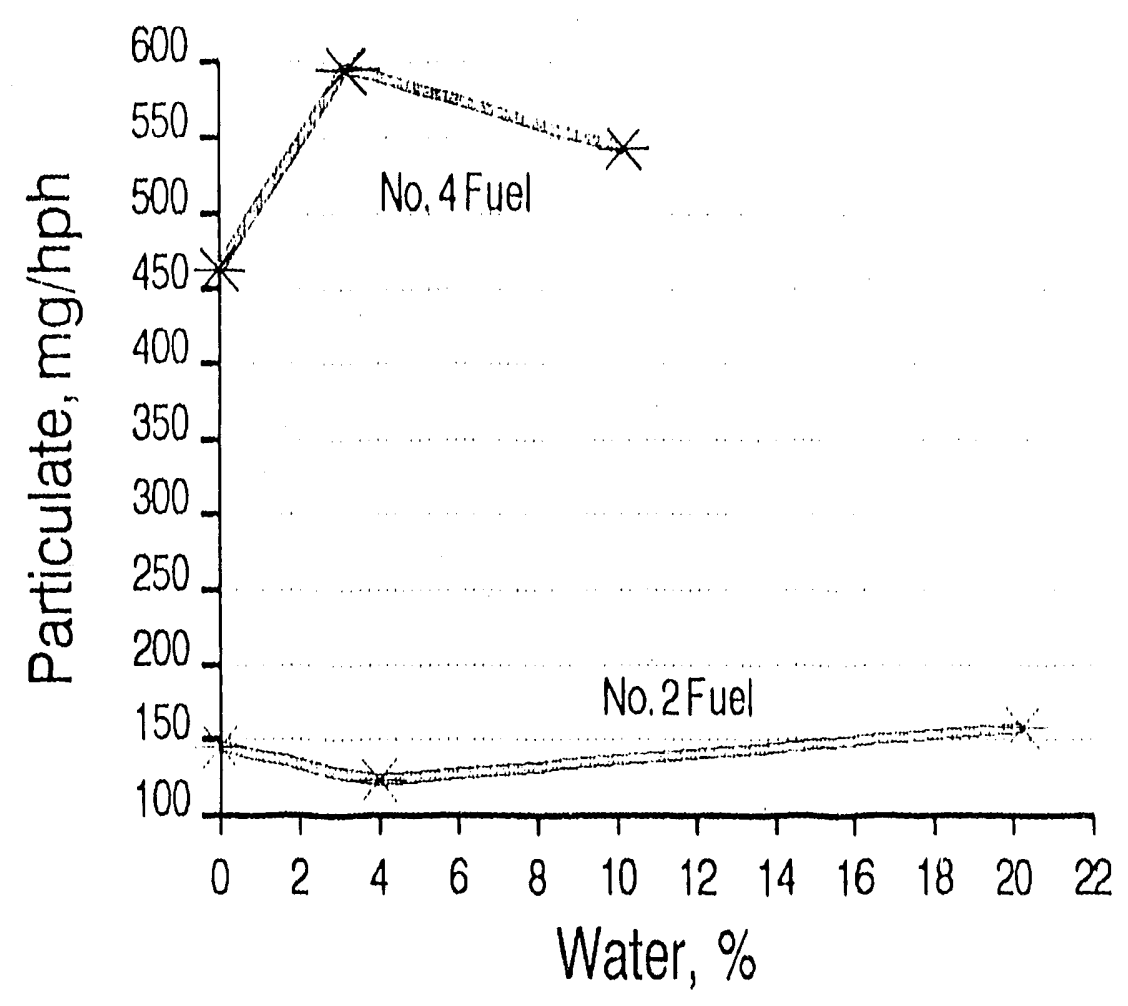

FIGURE A.52 Particulate Matter vs. Water Content, 50\% Load, 25 Horsepower, $21 \%$ Oxygen

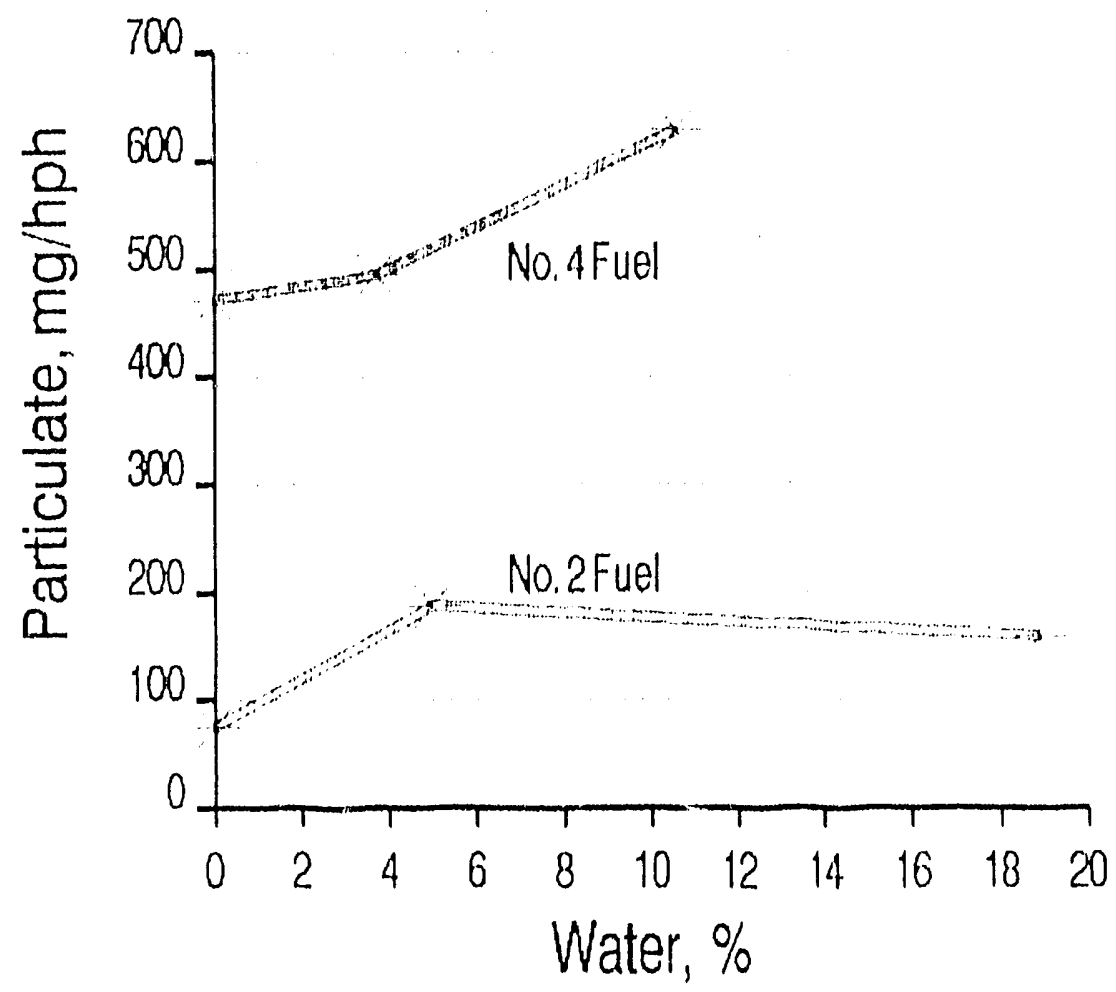

FIGURE A.53 Particulate Matter vs. Water Content, 50\% Load, 25 Horsepower, 25\% Oxygen 


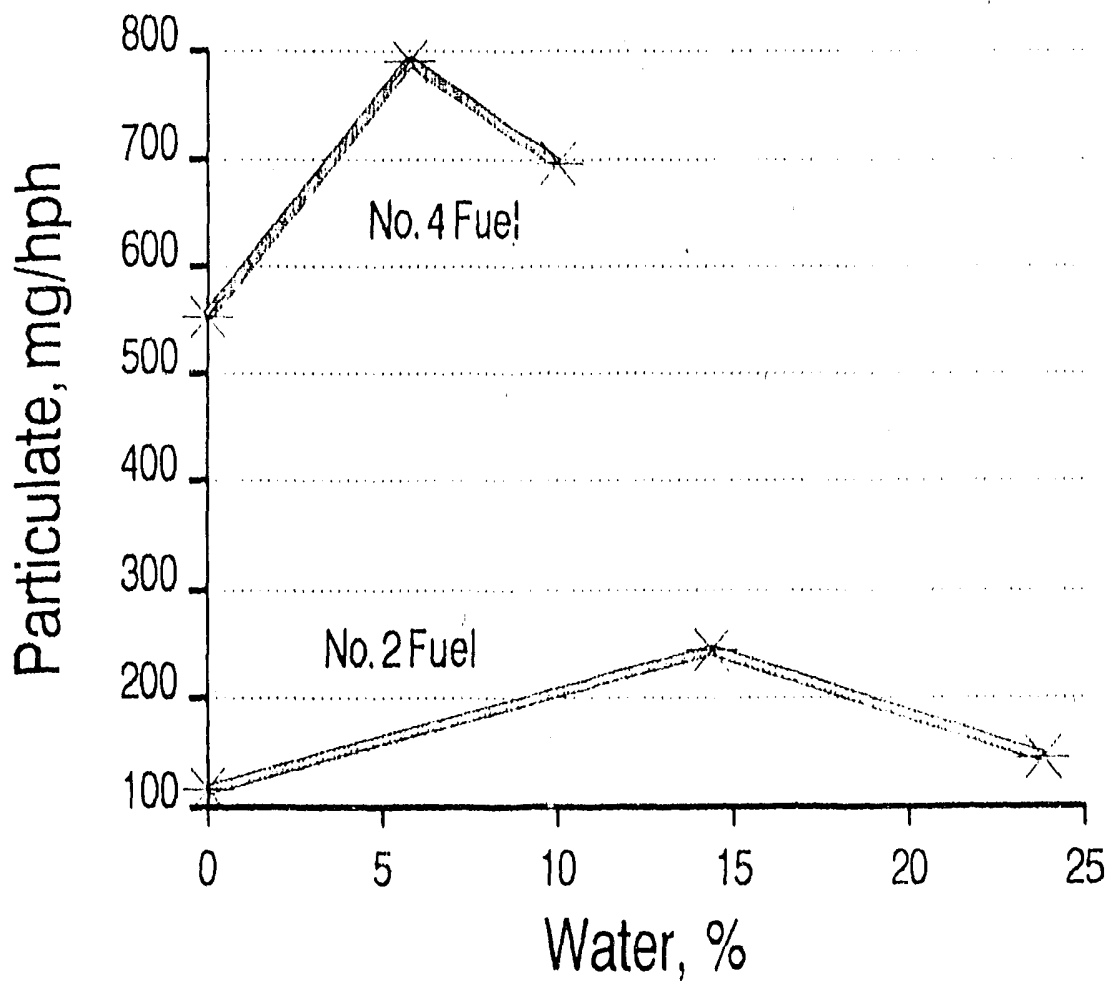

FIGURE A.54 Particulate Matter vs. Water Content, 50\% Load, 25 Horsepower, $30 \%$ Oxygen

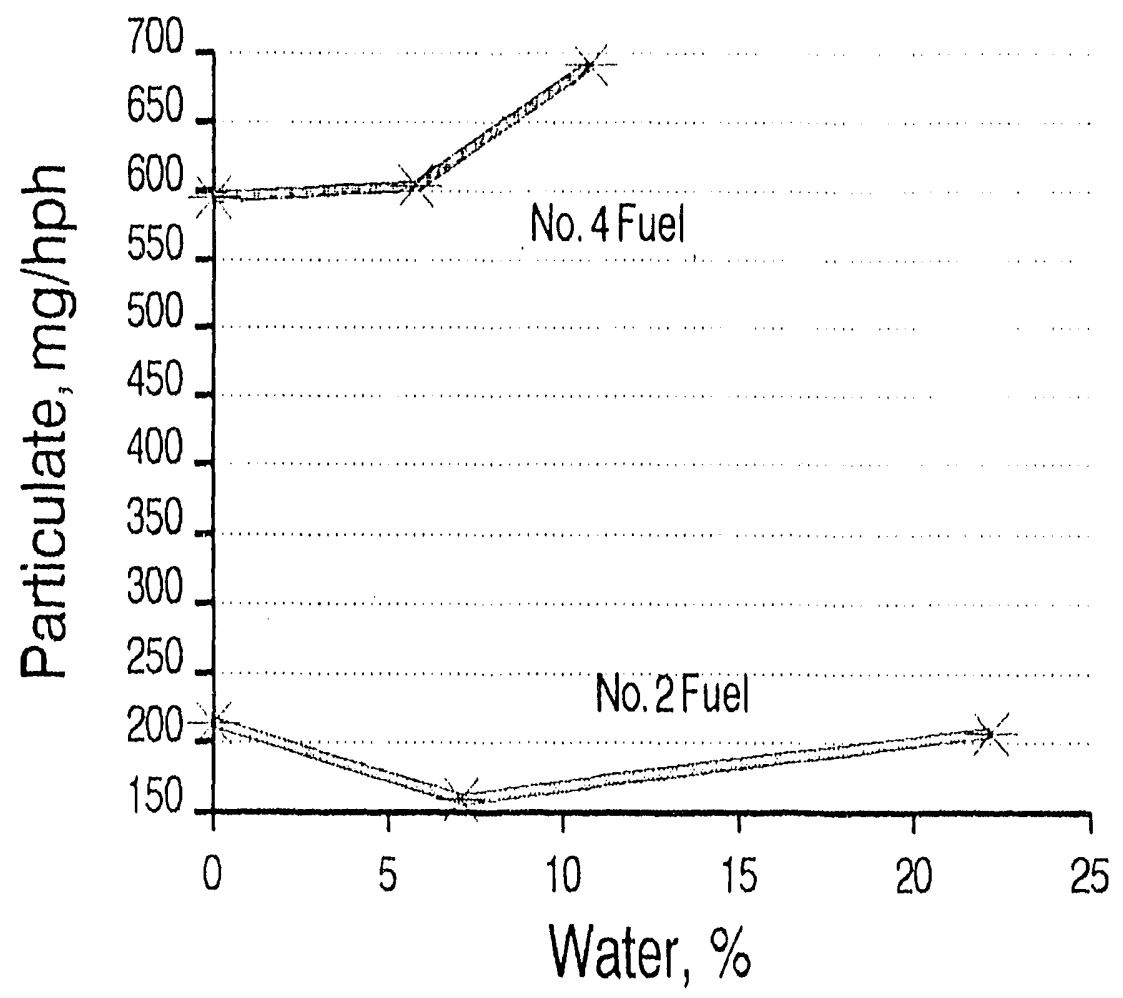

FIGURE A.55 Particulate Matter vs. Water Content, 50\% Load, 25 Horsepower, 35\% Oxygen 


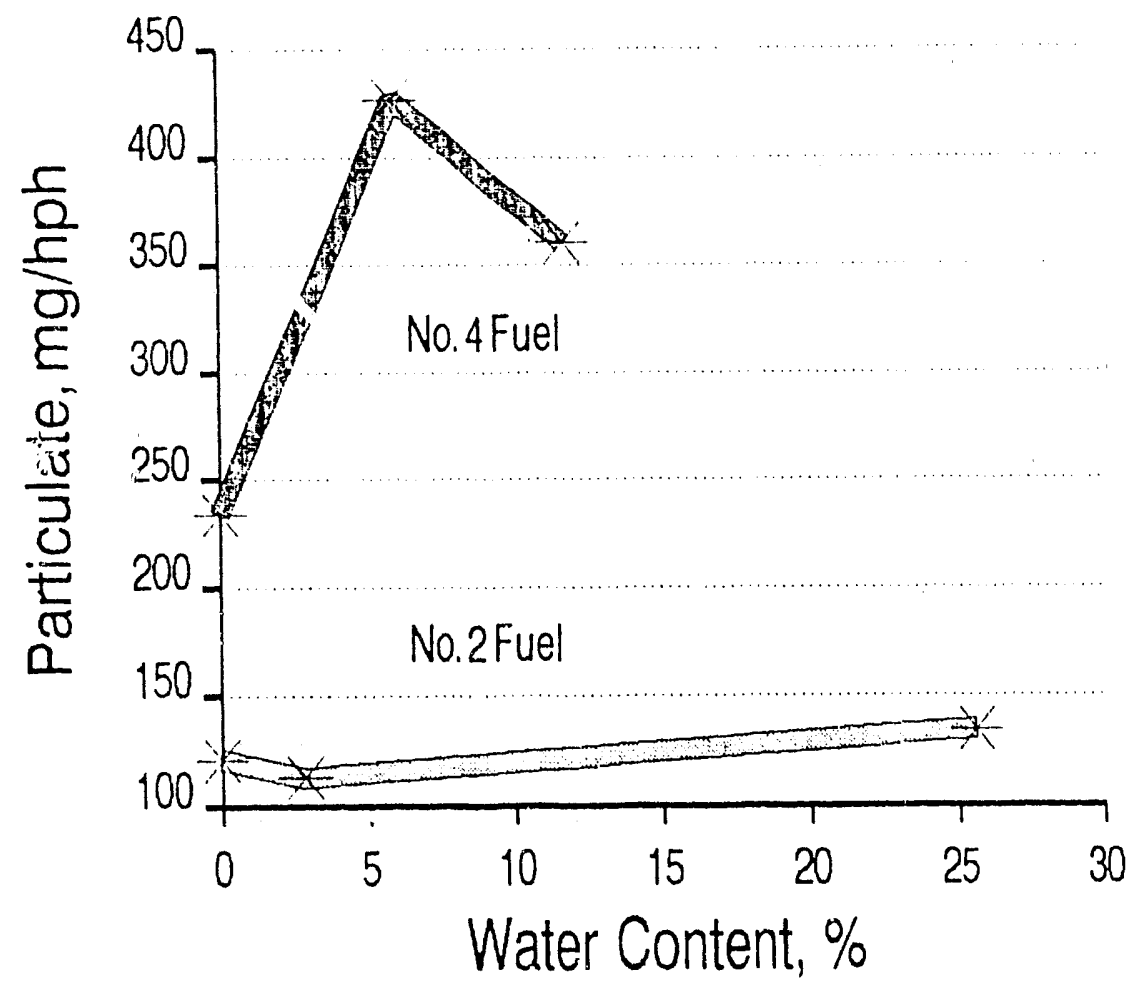

FIGURE A.56 Particulate Matter vs. Water Content, 100\% Load, 50 Horsepower, $21 \%$ Oxygen

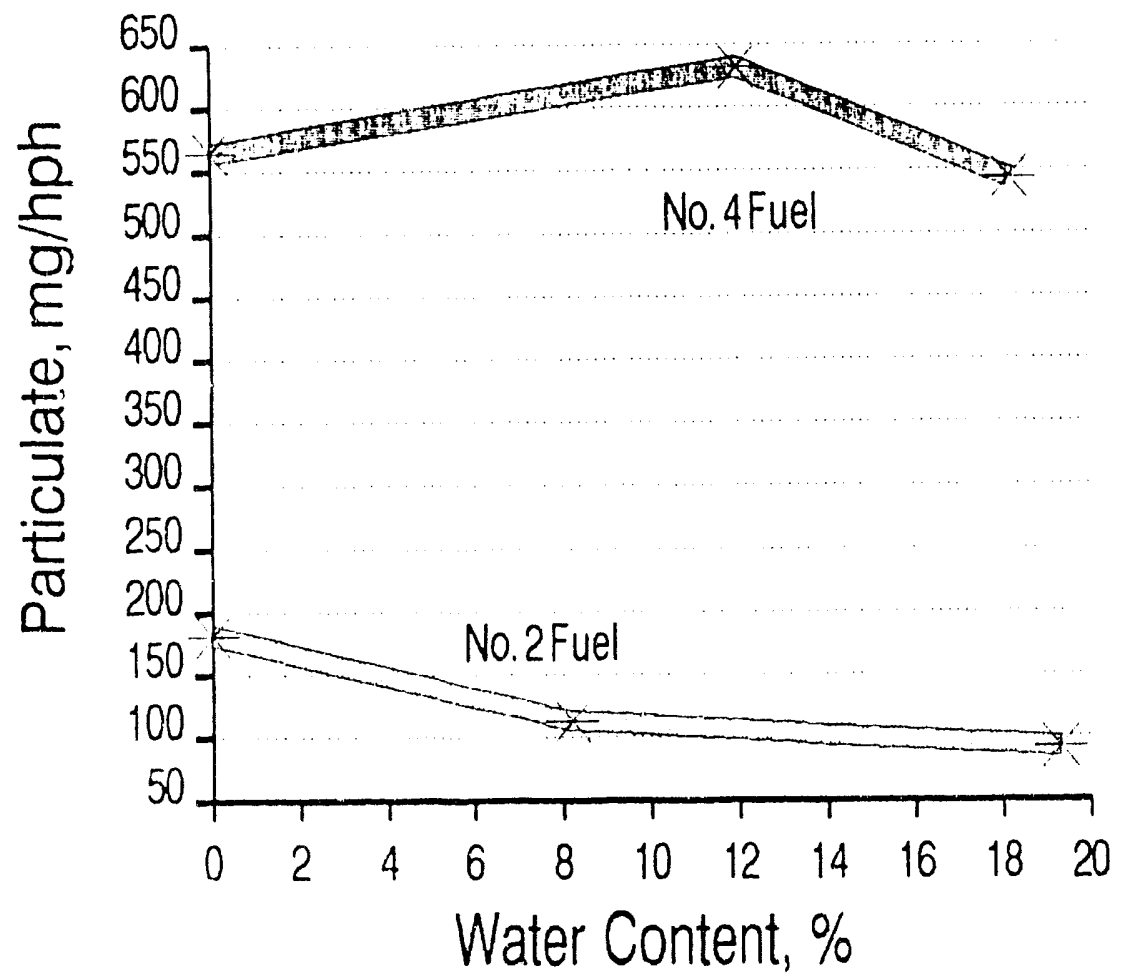

FIGURE A.57 Particulate Matter vs. Water Content, 100\% l.oad, 50 Horsepower, $30 \%$ Oxygen 
A-53

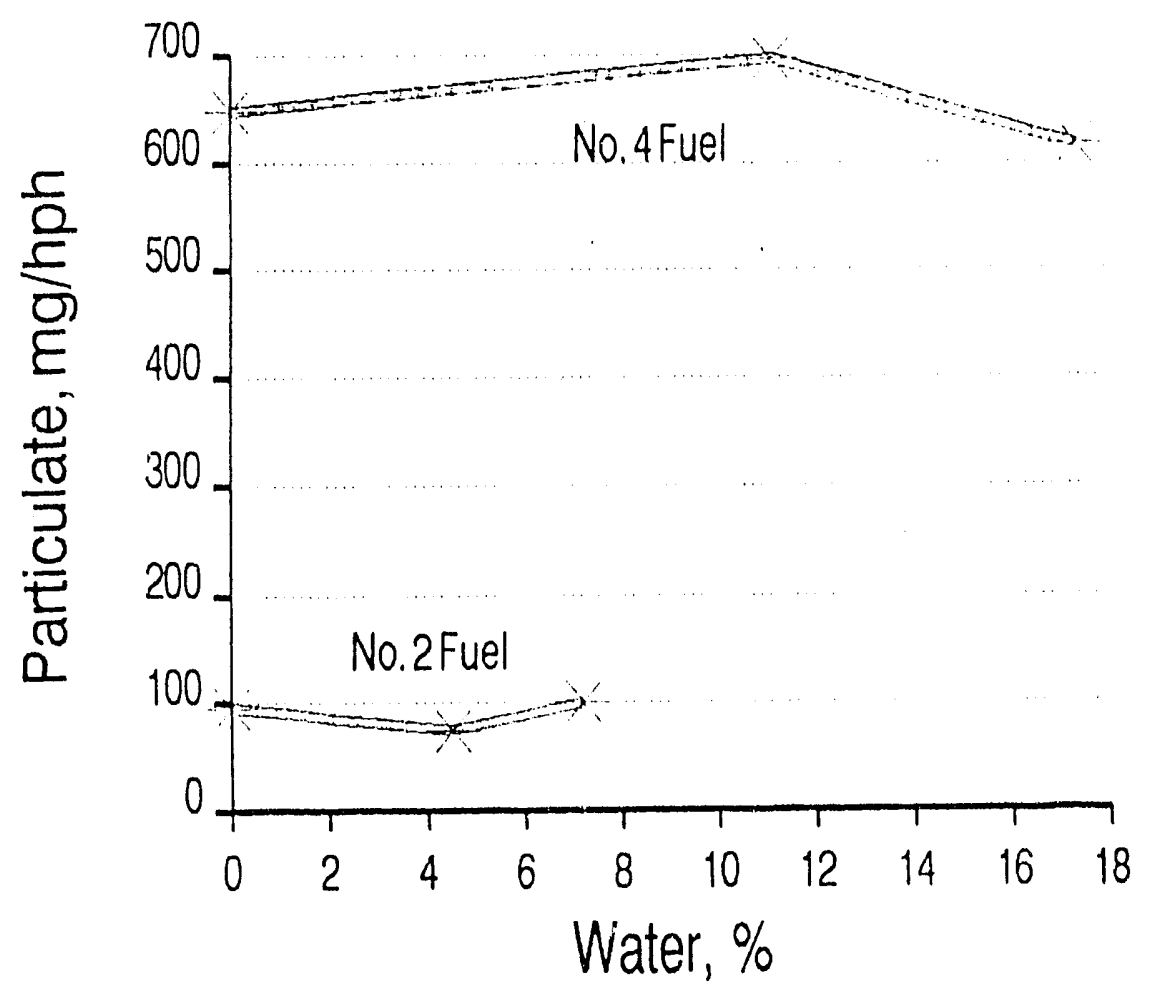

FIGURE A.58 Particulate-Matter Emissions, 100\% Load, 50 Horsepower, $35 \%$ Oxygen

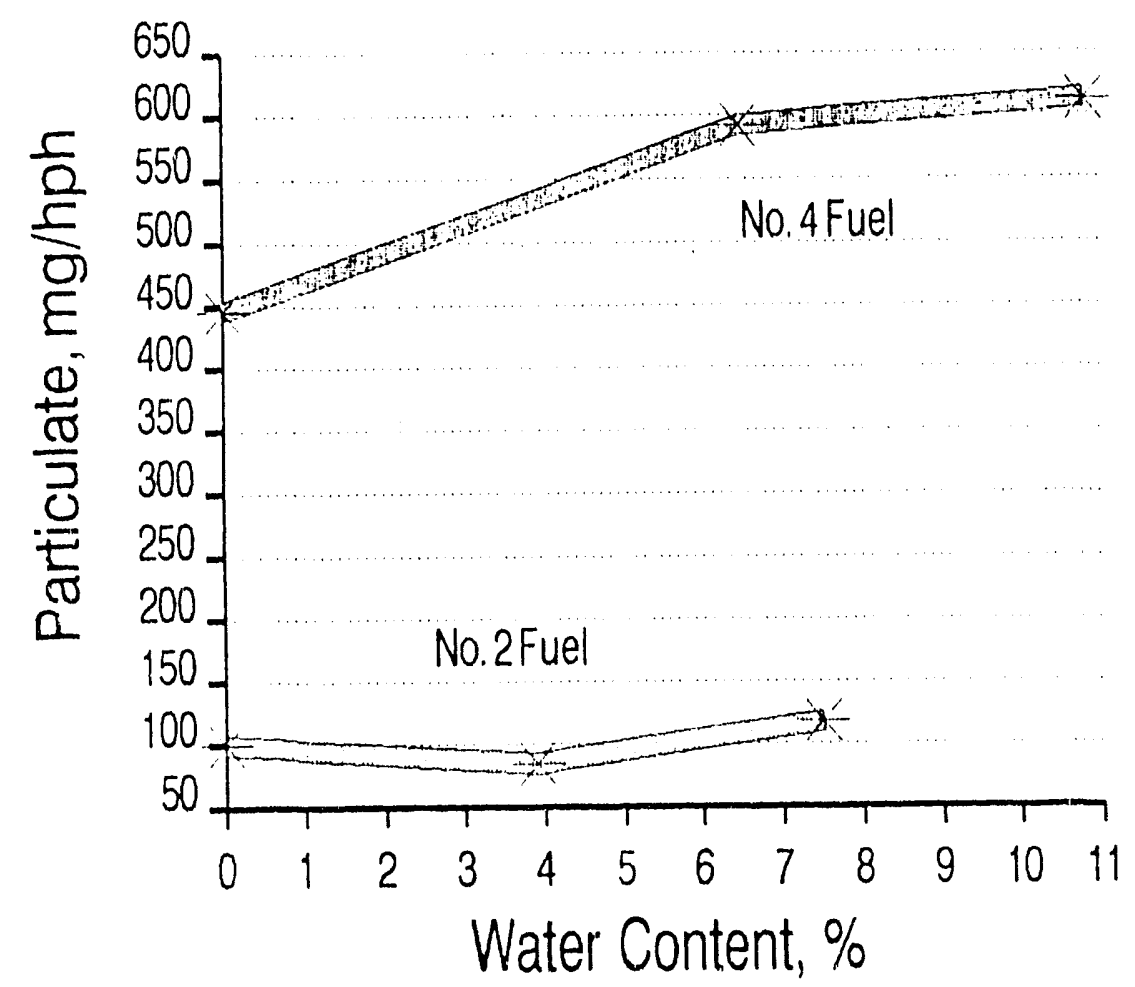

FIGURE A.59 Part. 'slate Matter vs. Water Content, $50 \%$ Load, 45 Horsepower, $35 \%$ Oxygen 


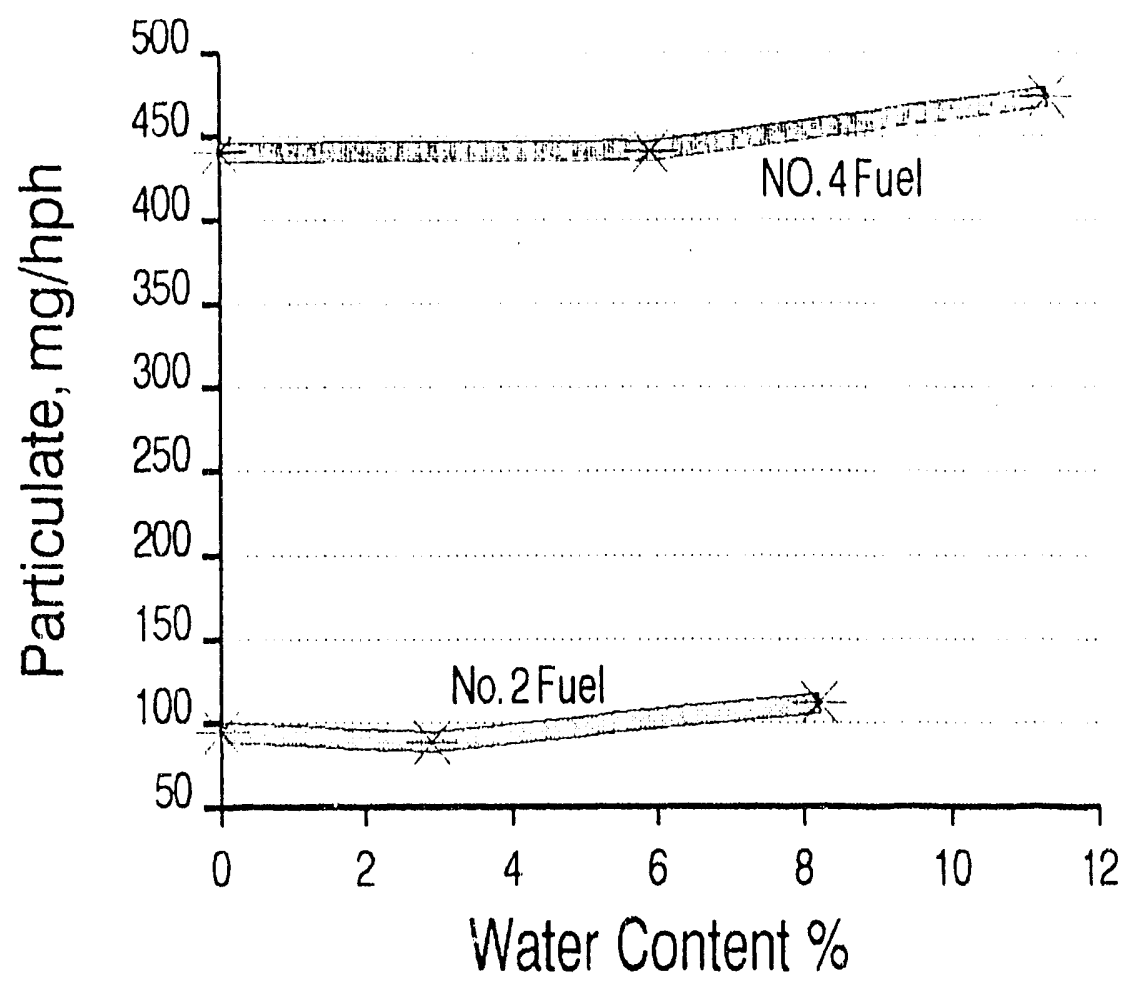

FIGURE A.60 Particulate Matter vs. Water Content, 50\% Load, 60 Horsepower, $30 \%$ Oxygen

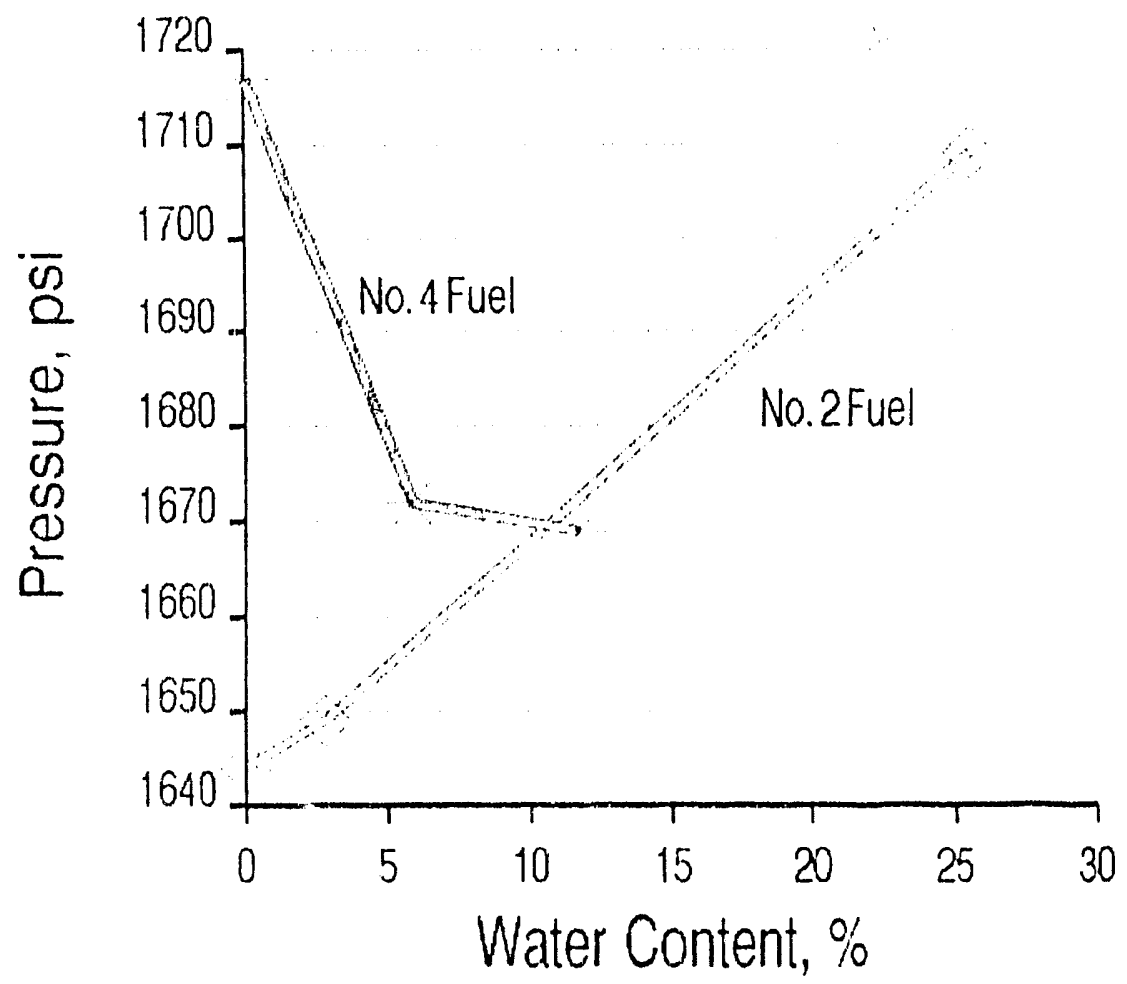

FIGURE A.61 Peak Pressure, 100\% Load, 50 Horsepower, $21 \%$ Oxygen 
A.6 Graphic Display of Cylinder Pressures and Results of Heat-Release Analyses for No. 2 Fuel 
A-56 


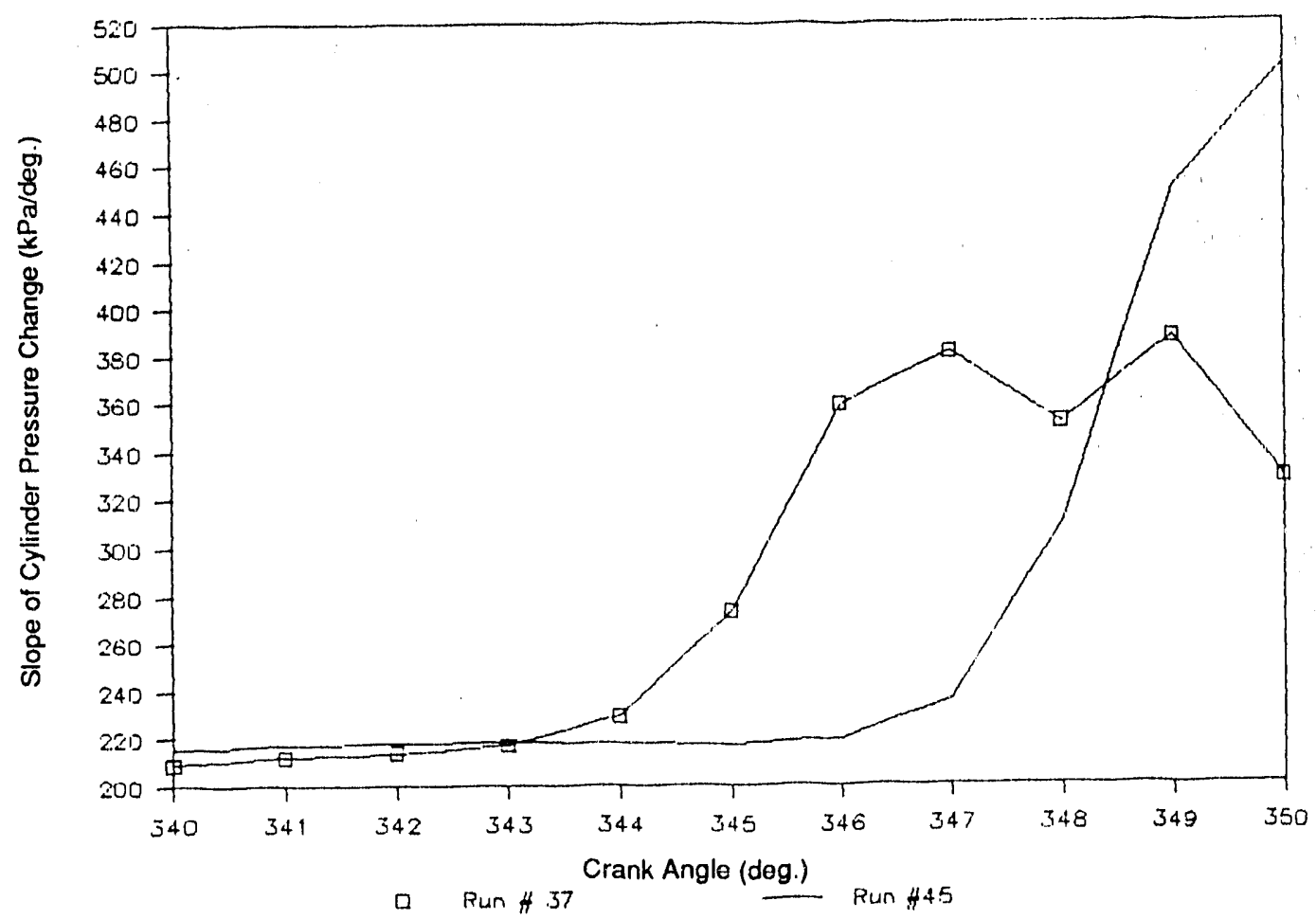

FIGURE A.62 Effect of Water Content on Slope of Cylinder Pressure Change, No. 2 Fuel, $100 \%$ Load, $21 \%$ Oxygen, \#37 vs. \#45

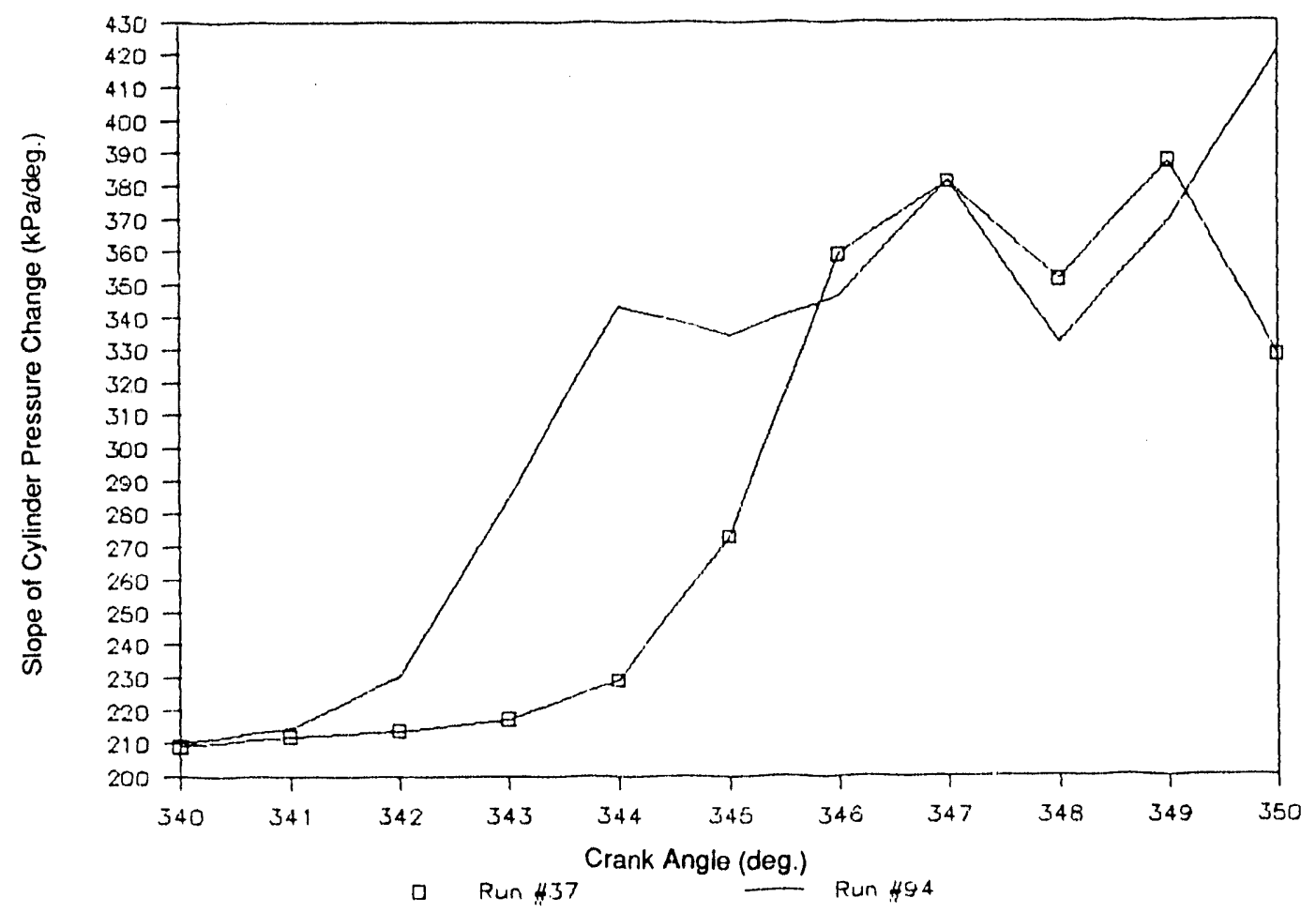

FIGURE A.63 Effect of Oxygen Level and Water Content on Slope of Cylinder Pressure Change, No. 2 Fuel, $100 \%$ Load, \#37 vs. \#94 


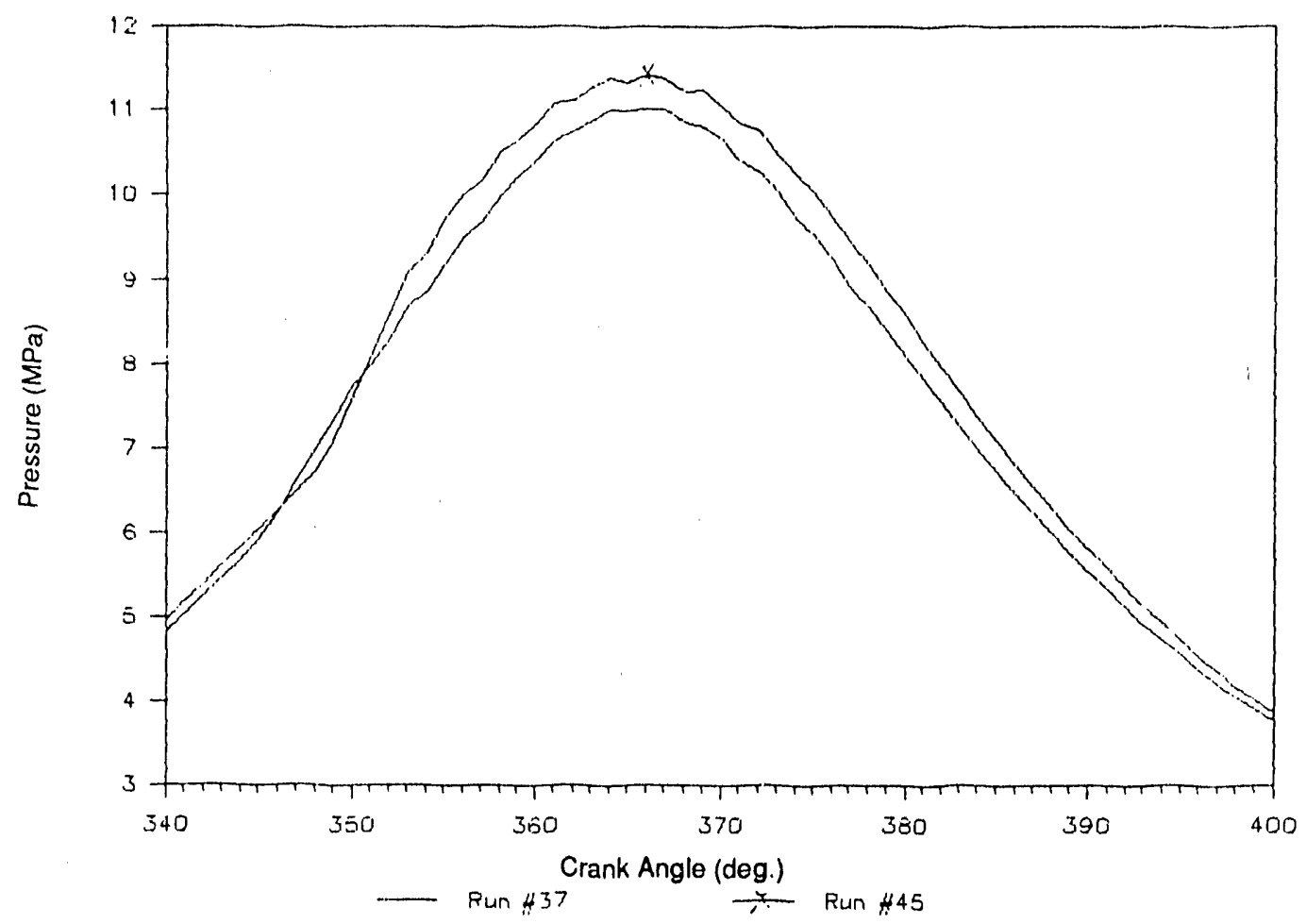

FIGURE A.64 Effect of Water Content on Cylinder Pressure, No. 2 Fuel, 100\% Load, $21 \%$ Oxygen, $\$ 37$ vs. \#45

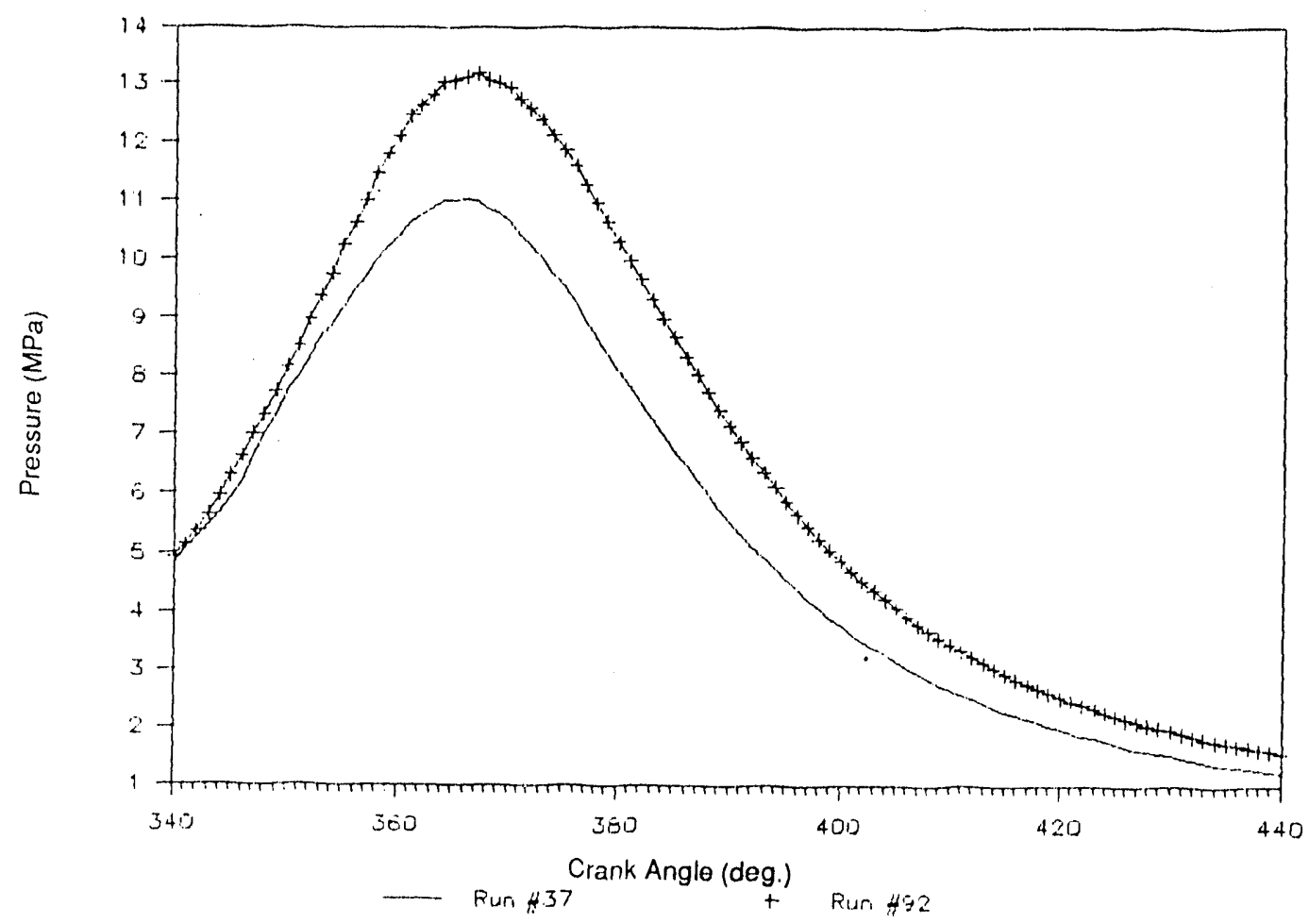

FIGURE A.65 Effect of Oxygen Level and Power on Cylinder Pressure, No. 2 Fuel, $100 \%$ Load, No Water, \#37 vs. \#92 


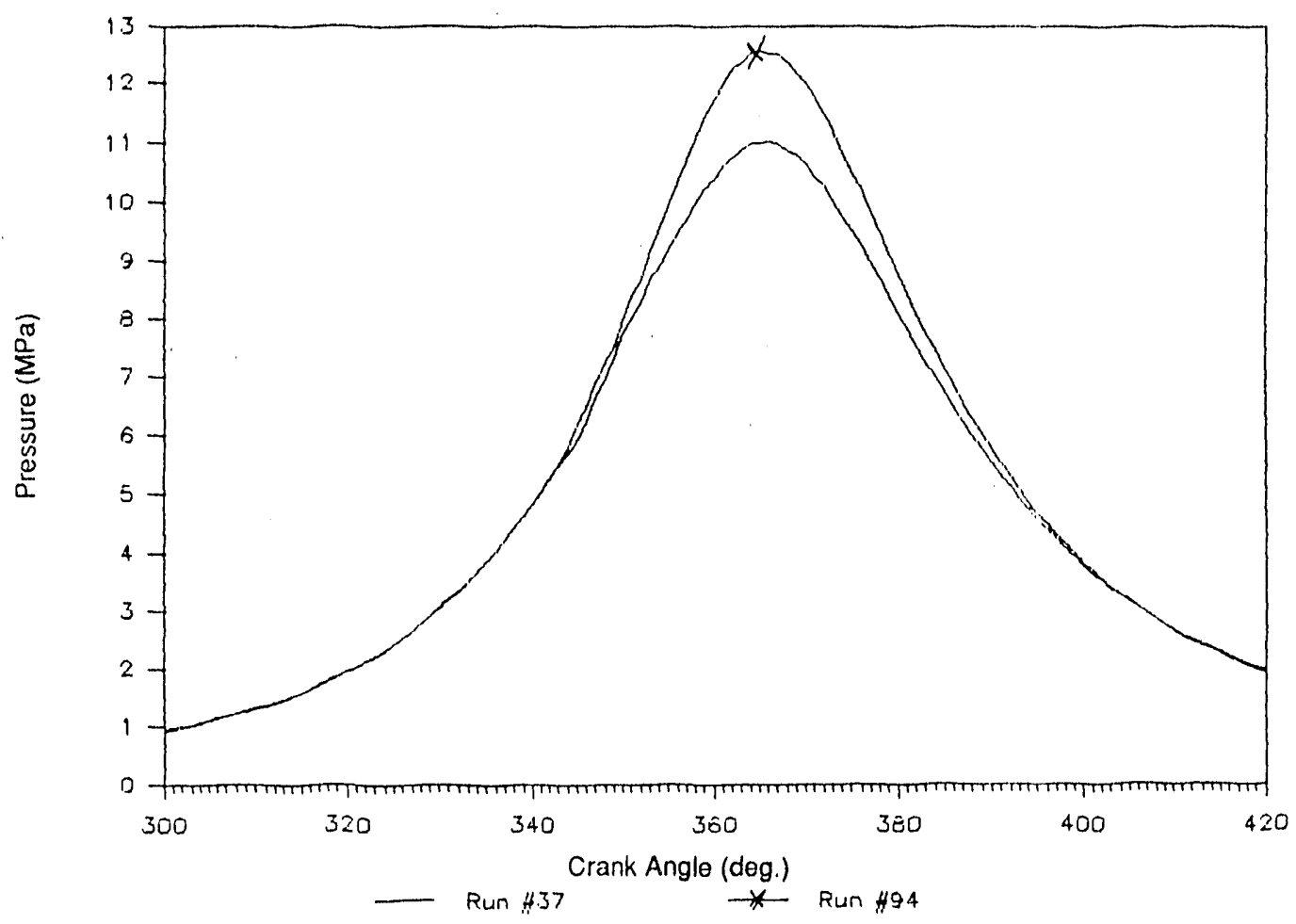

FIGURE A.66 Effect of Water Content and Oxygen Level on Cylinder Pressure, No. 4 Fuel, 100\% Load, $21 \%$ Oxygen, \#28 vs. \#24

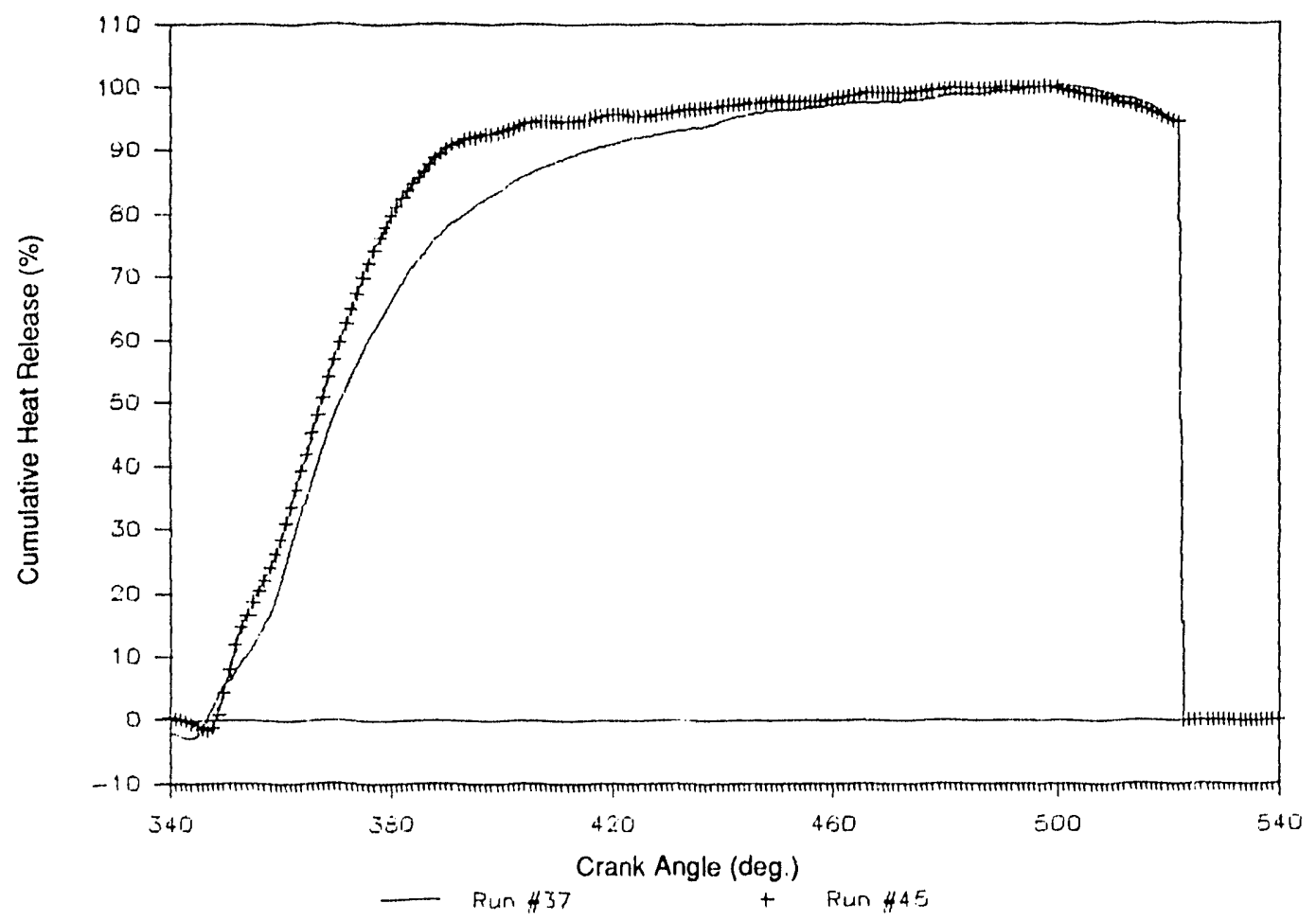

FIGURE A.67 Effect of Water Content on Cumulative Heat Release, No. 2 Fuel, $100 \%$ Load, 50 Horsepower, $21 \%$ Oxygen 


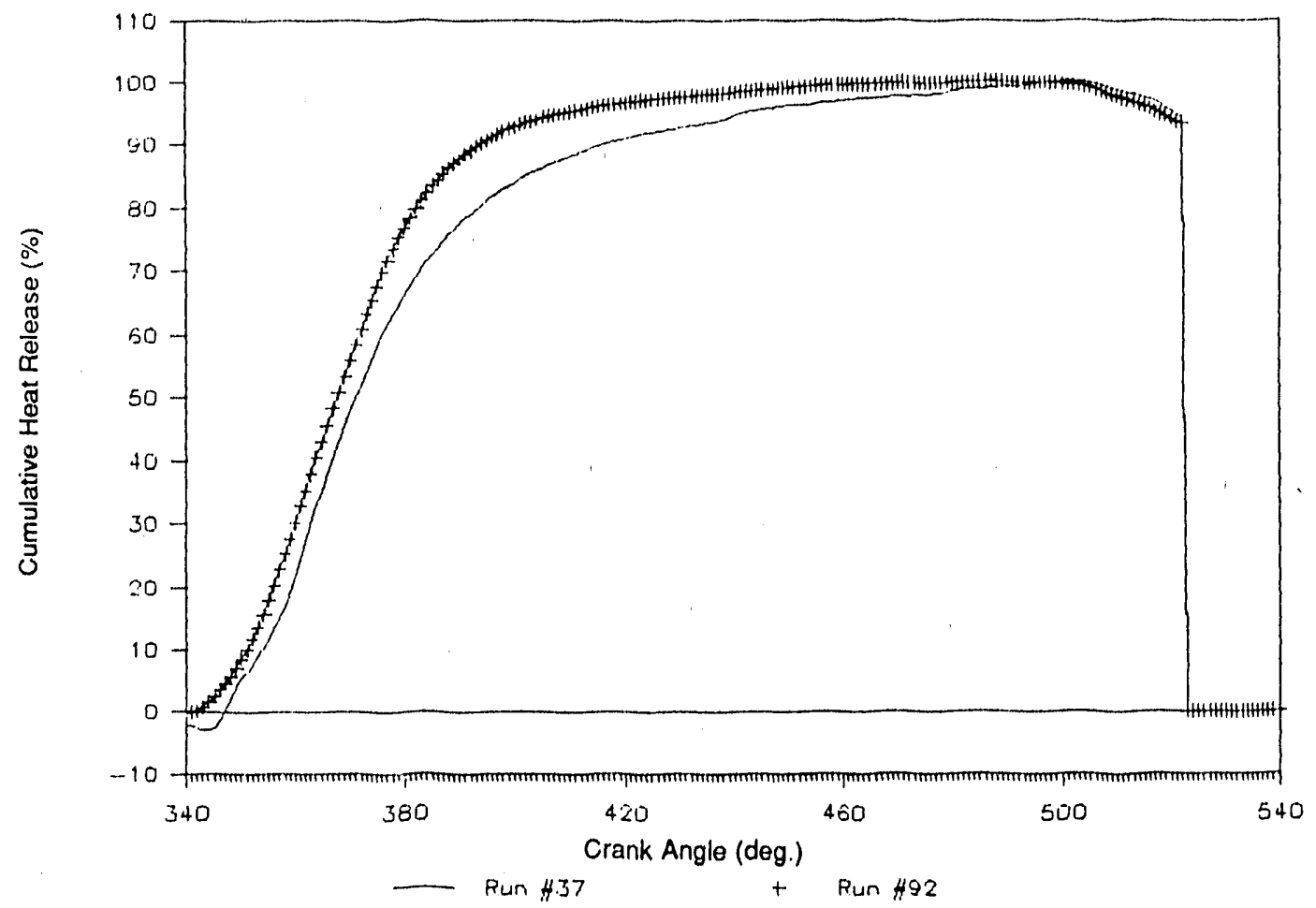

FIGURE A.68 Effect of Oxygen Level and Power on Cumulativt Heat Release, No. 2 Fuel, $100 \%$ Load, No Water

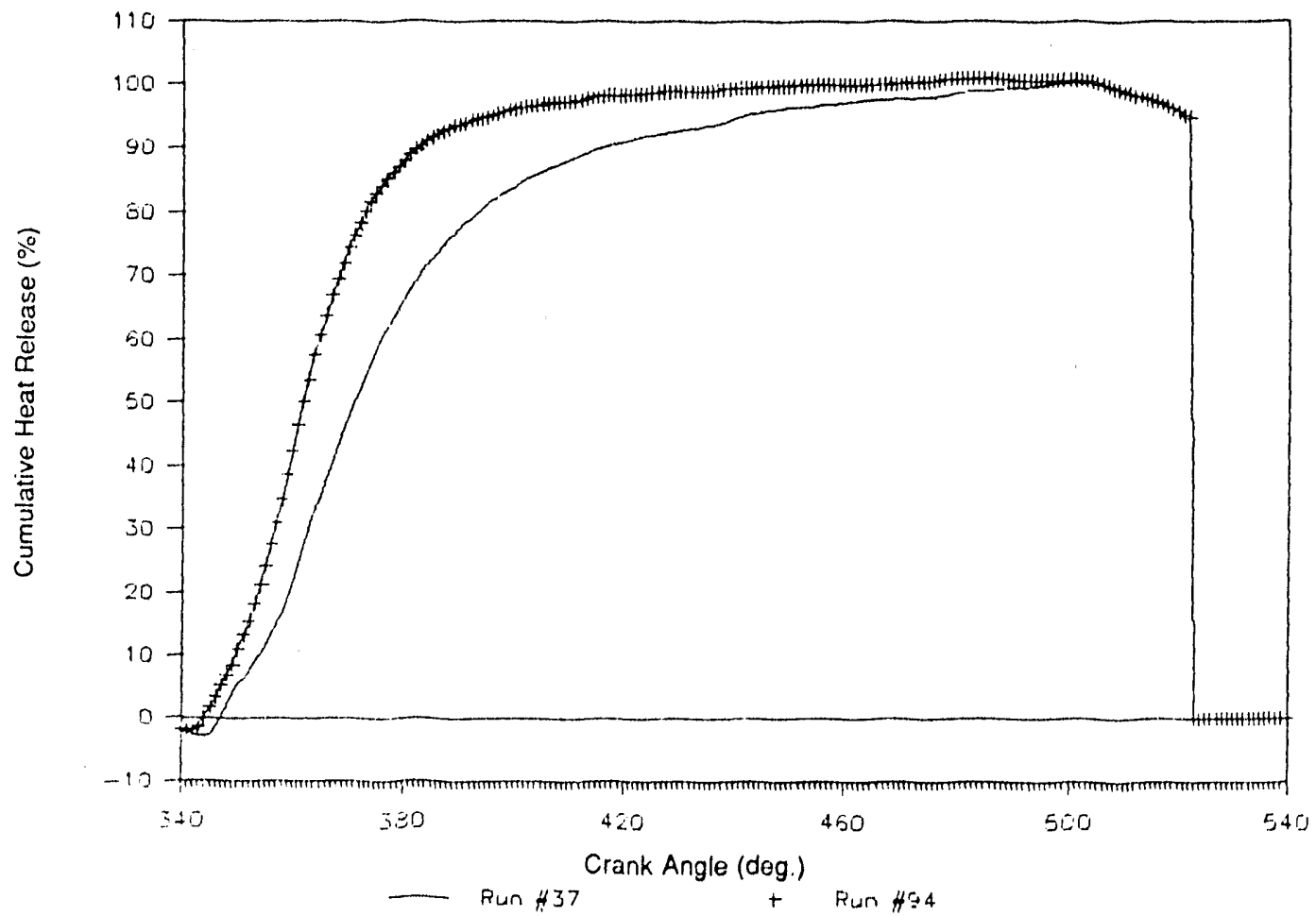

FIGURE A.69 Effect of Water Content and Oxygen Level on Cumulative Heat Release, No. 2 Fuel, $100 \%$ Load, 50 Horsepower 


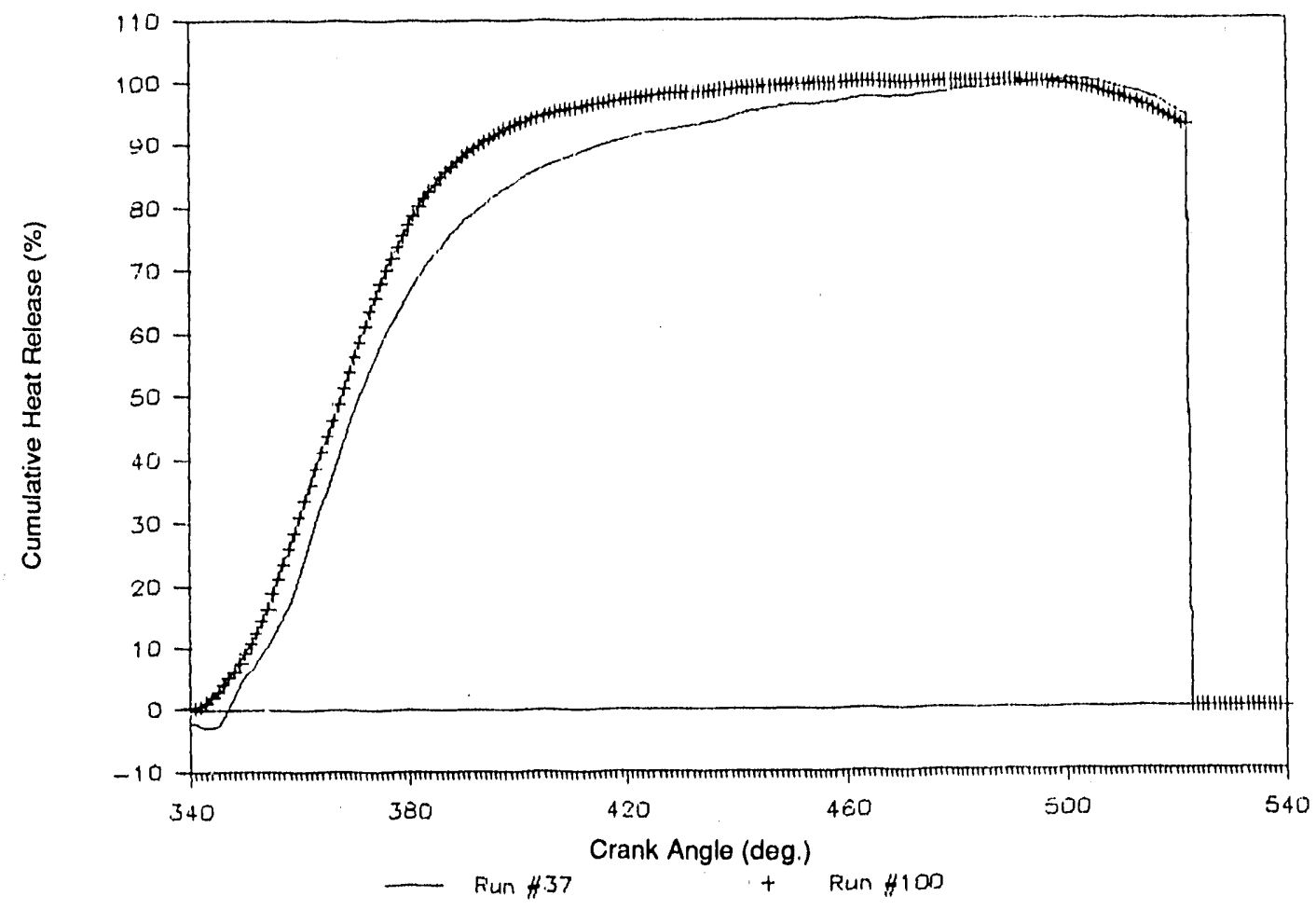

FIGURE A.70 Effect of Oxygen Level, Water Content, and Power on Cumulative Heat Release, No. 2 Fuel, 100\% Load

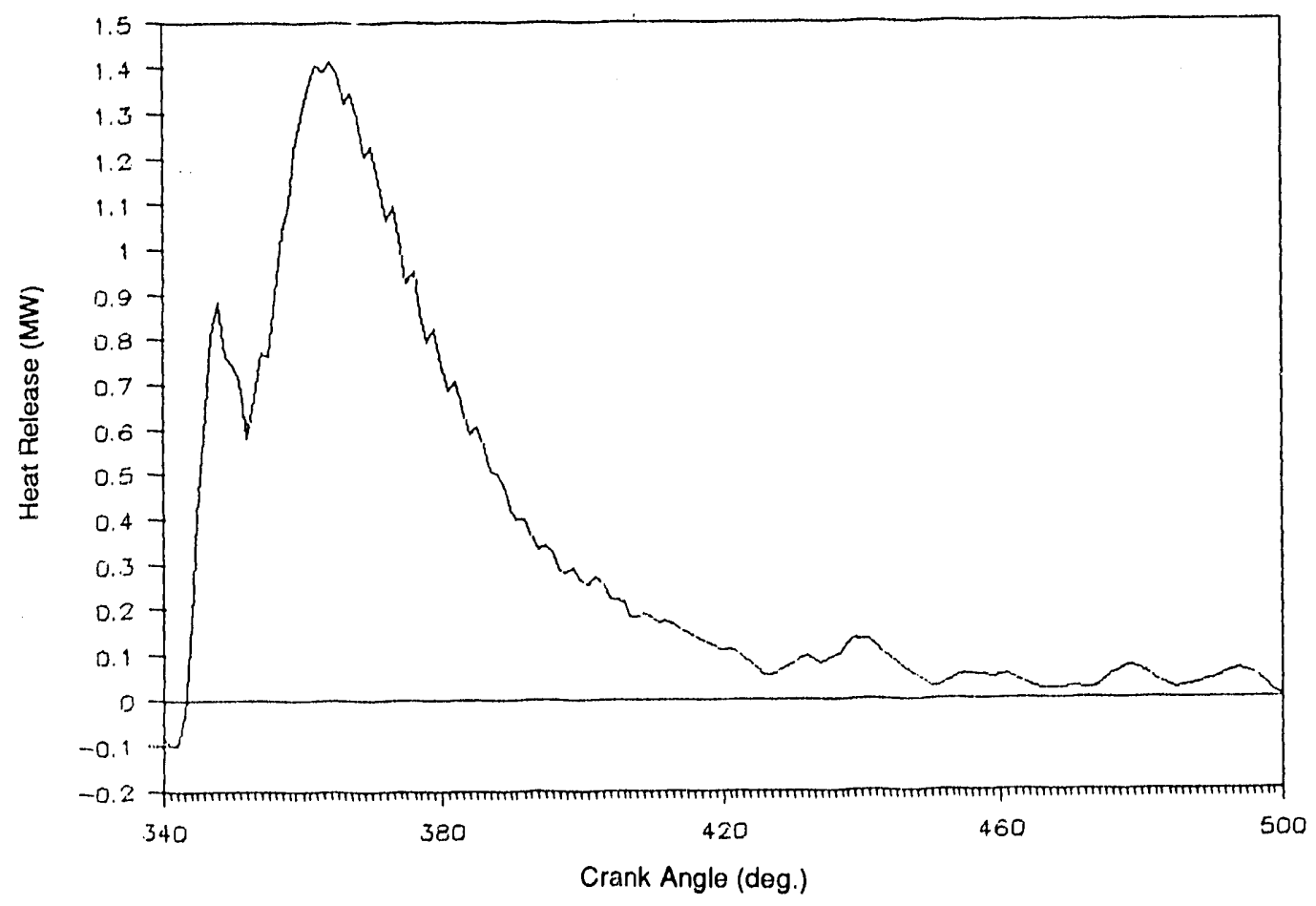

FIGURE A.71 Heat Release, No. 2 Fuel, 100\% Load, 50 Horsepower 


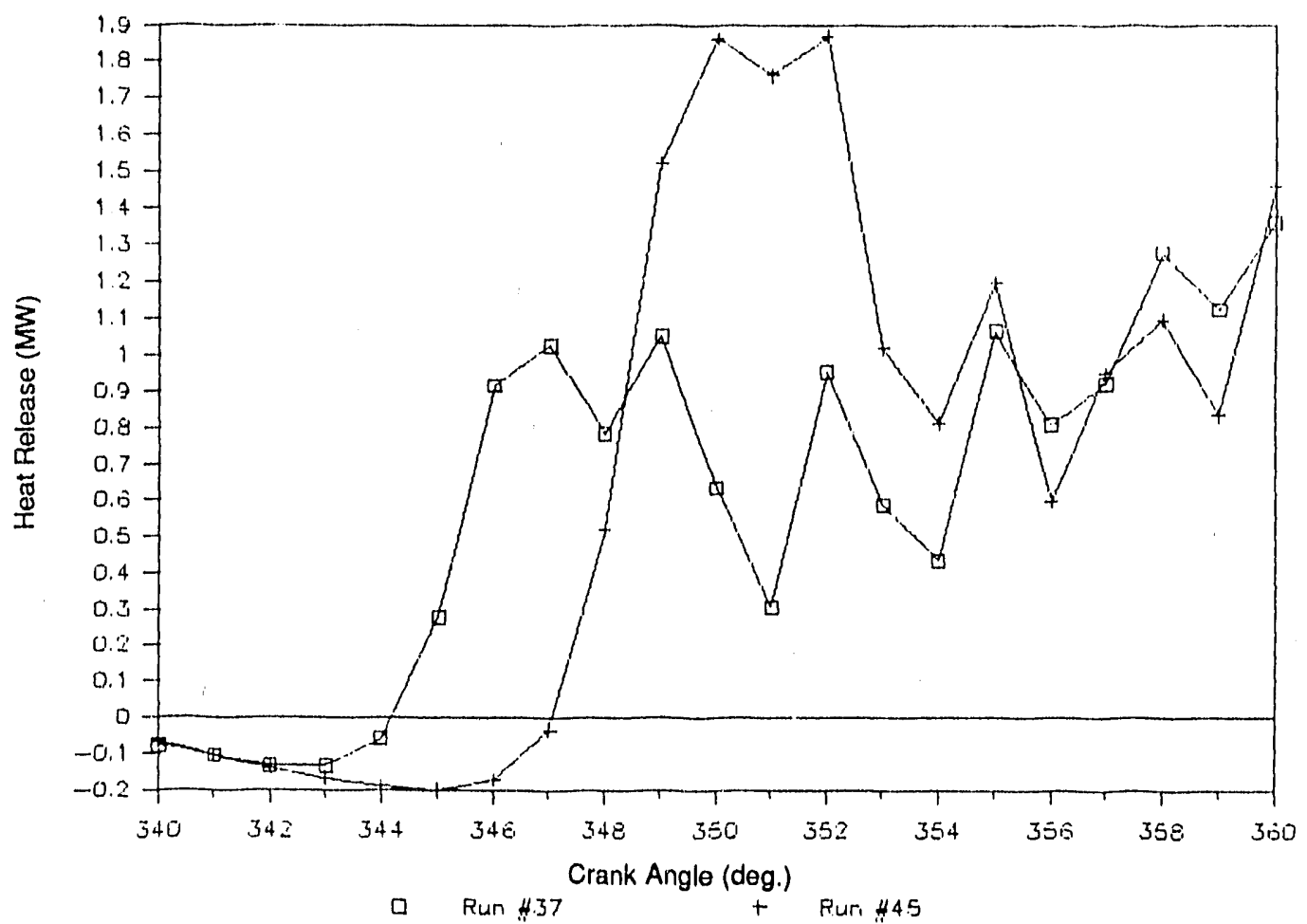

FIGURE A.72 Heat Release, No. 2 Fuel, 100\% Load, 21\% Oxygen

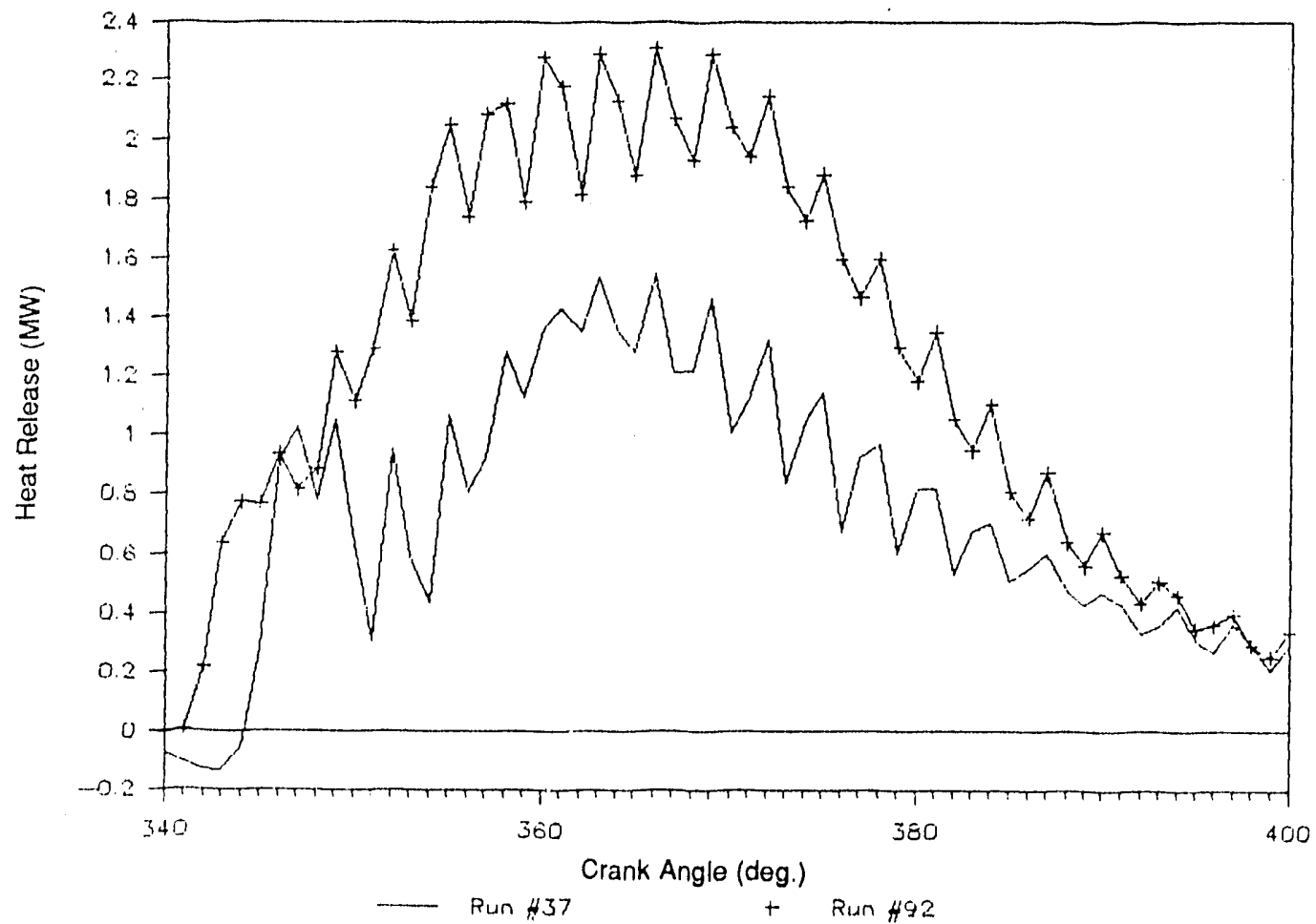

FIGURE A.73 Heat Release, No. 2 Fuel, 100\% Load, No Water 


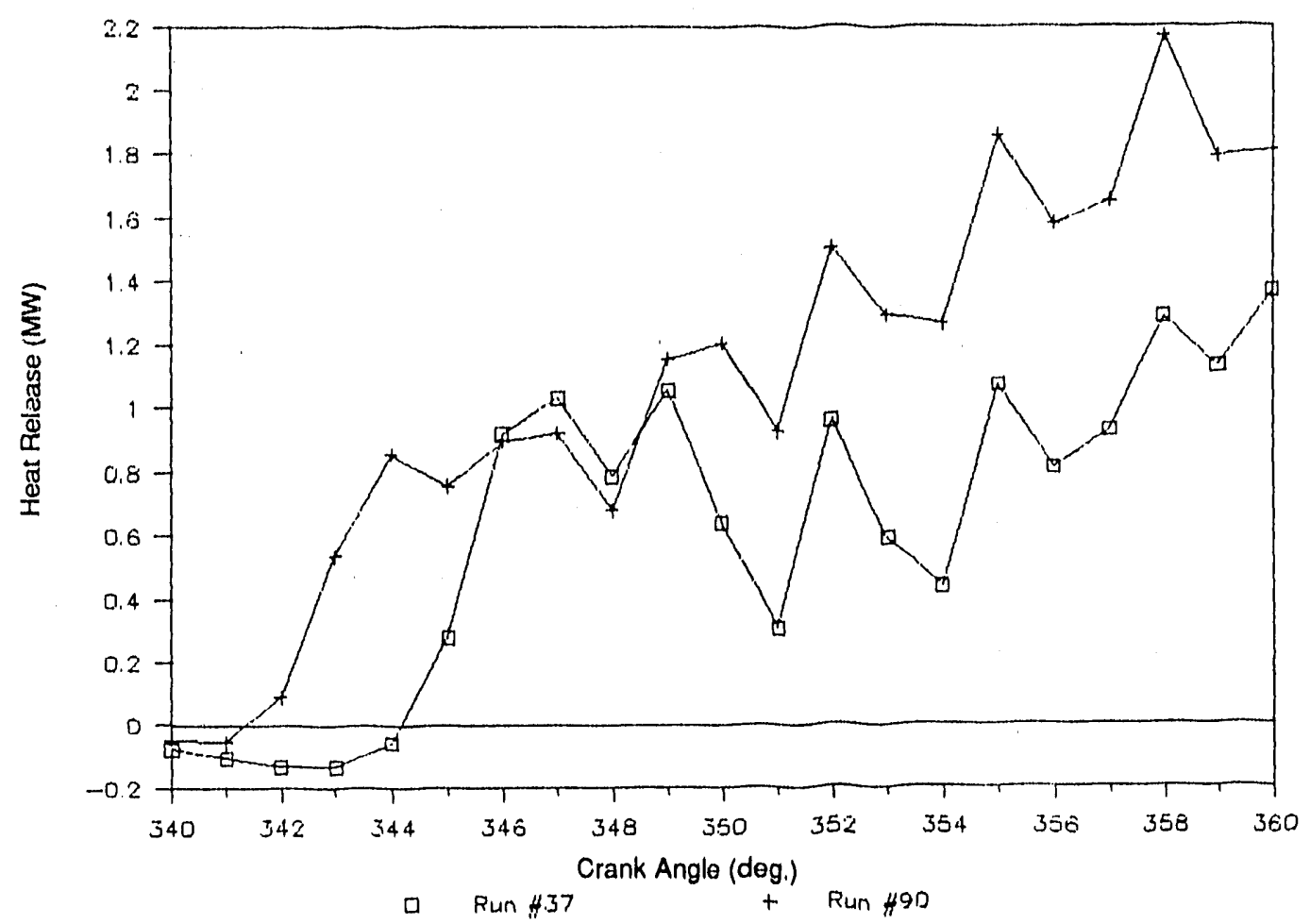

FIGURE A.74 Heat Release, No. 2. Fuel, 100\% Load, 50 Horsepower, No Water

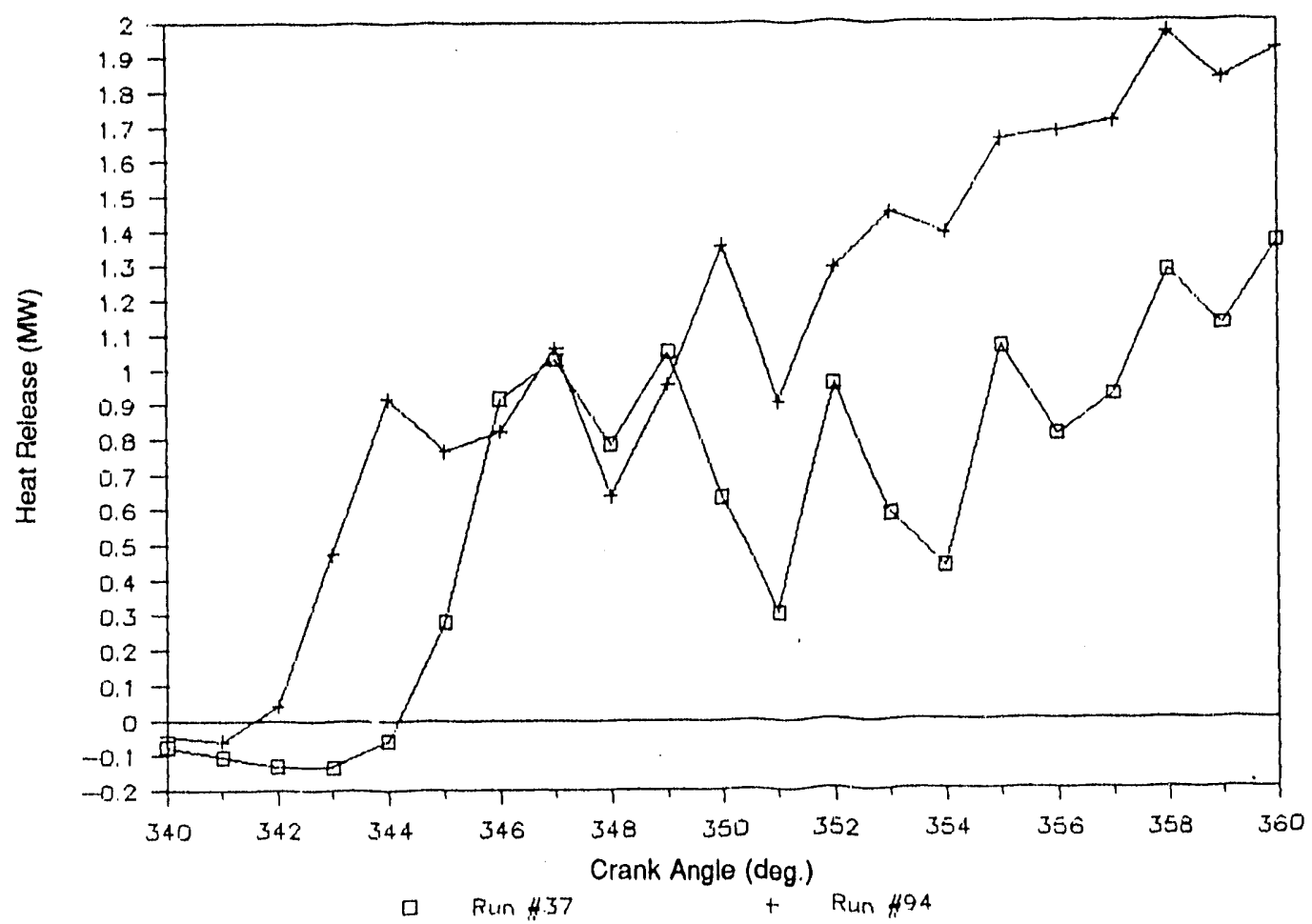

FIGURE A.75 Heat Release, No. 2 Fuel, 100\% Load, 50 Horsepower 


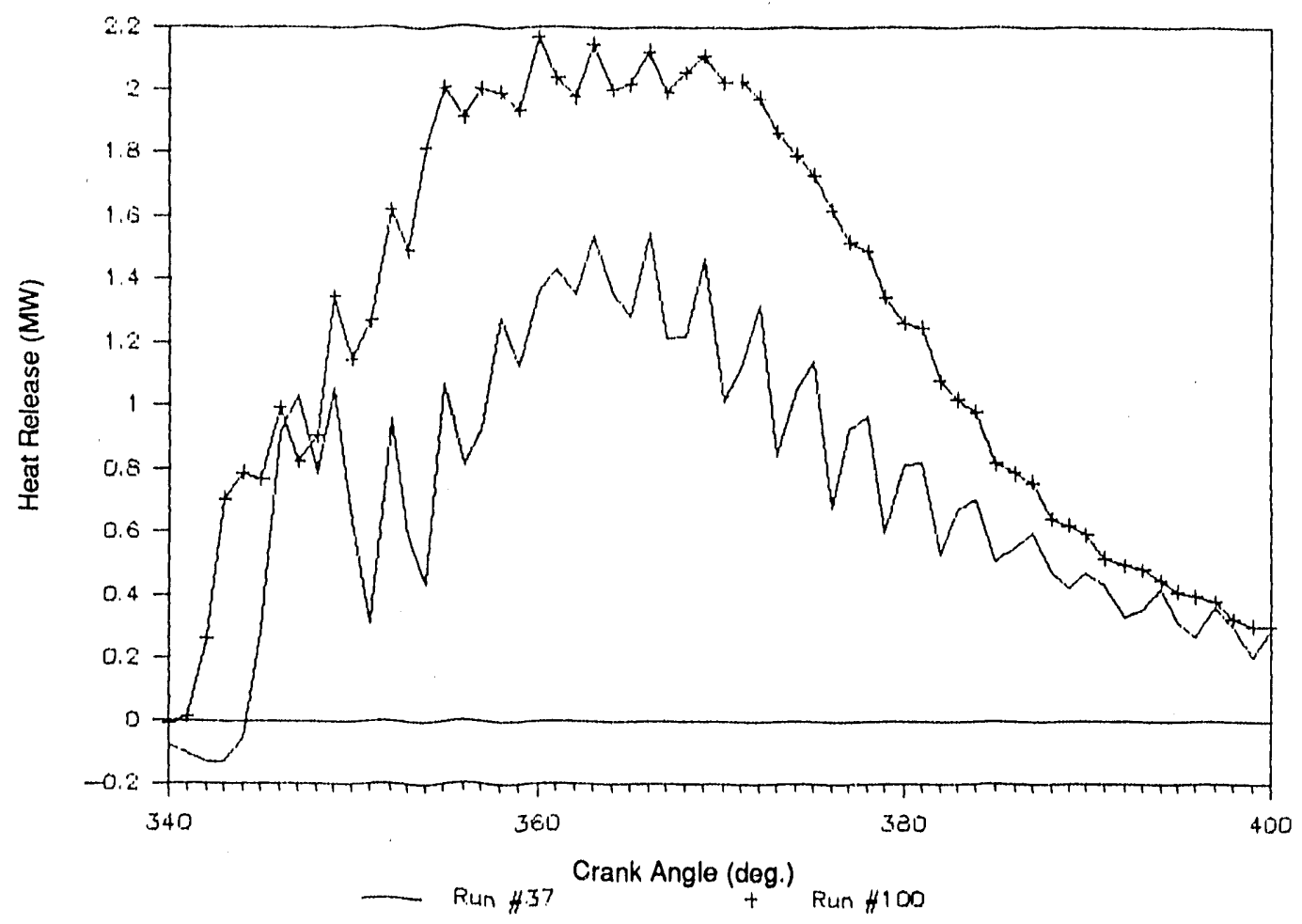

FIGURE A.76 Heat Release, No. 2 Fuel, 100\% Load

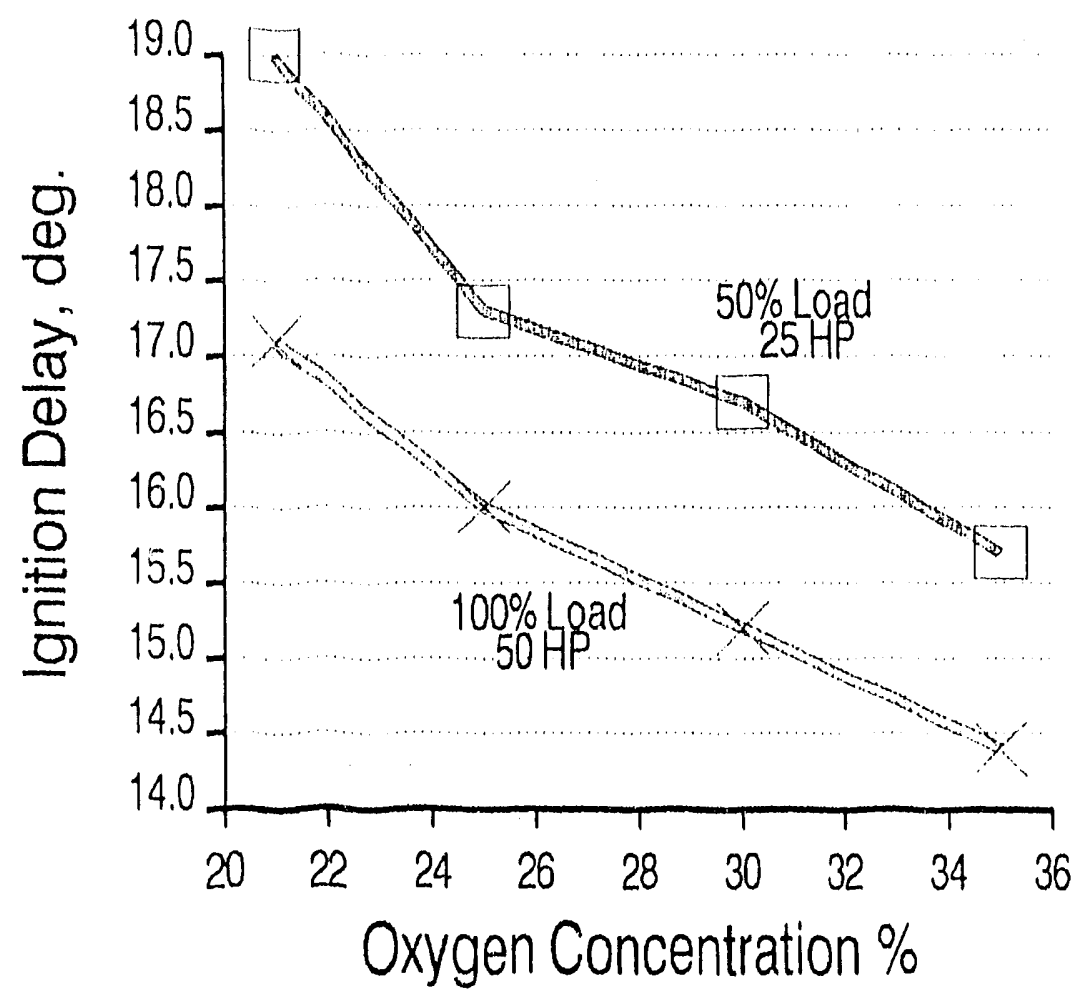

FIGURE A.77 Igrition Delay vs. Oxygen Concentration, No. 2 Fuel, No Water 
A.65

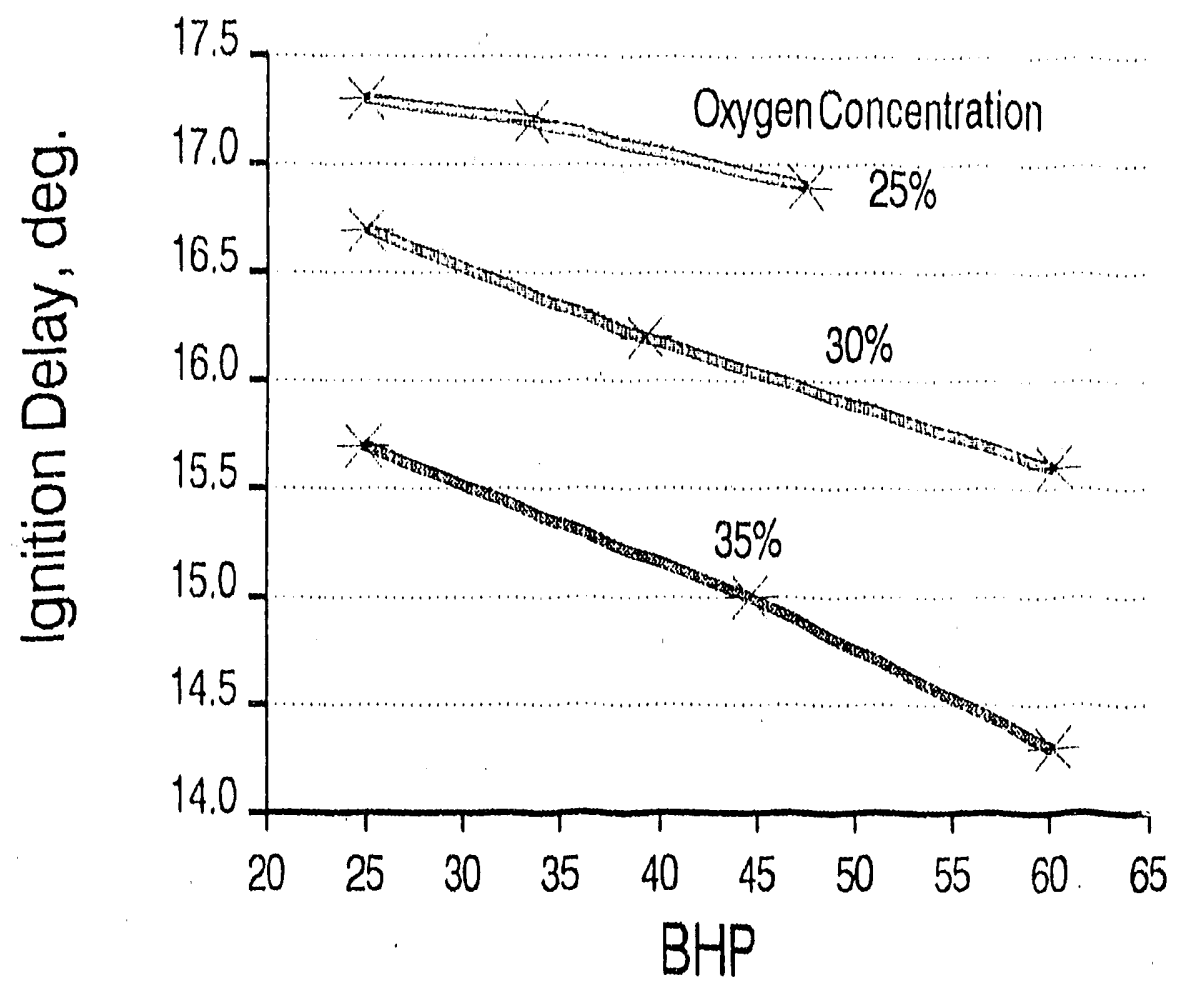

FIGURE A.78 Ignition Delay vs. Brake Horsepower, No. 2 Fuel, 50\% Load, No Water

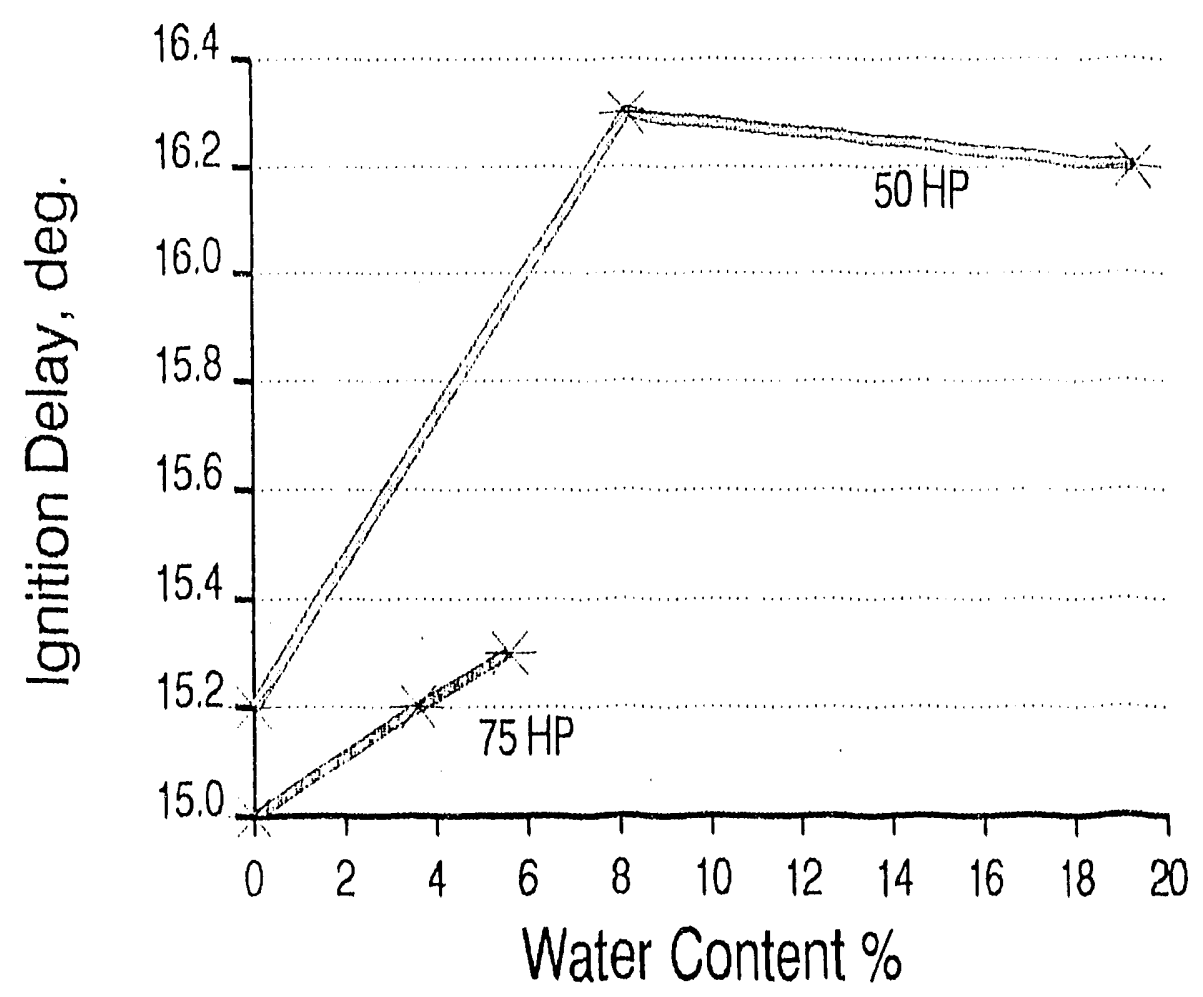

FIGURE A.79 Ignition Delay vs. Water Content, No. 2 Fuel, $100 \%$ Load, $30 \%$ Oxygen 


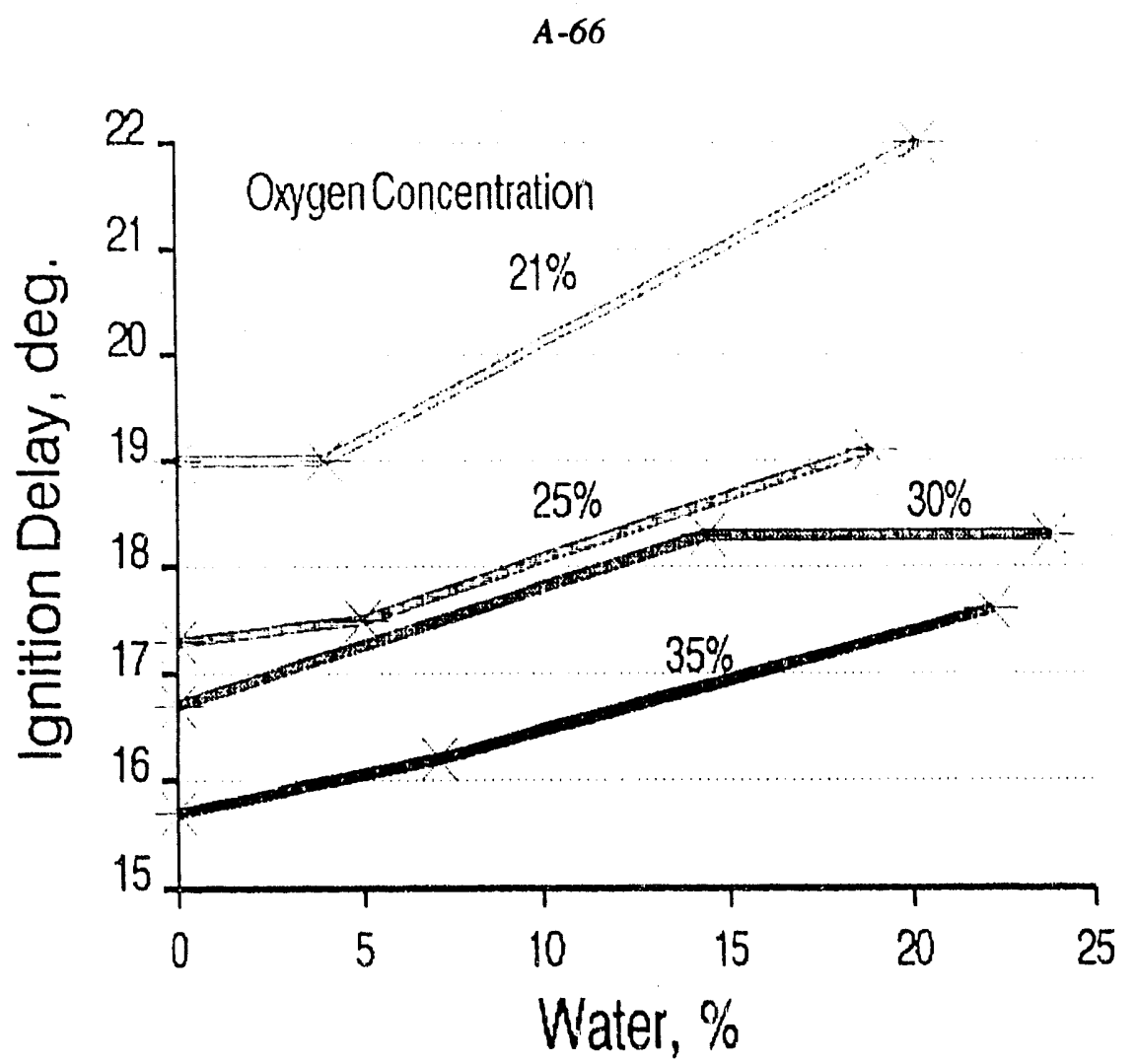

FIGURE A.80 Ignition Delay vs. Water Content, No. 2 Fuel, $50 \%$ Load, 25 Horsepower

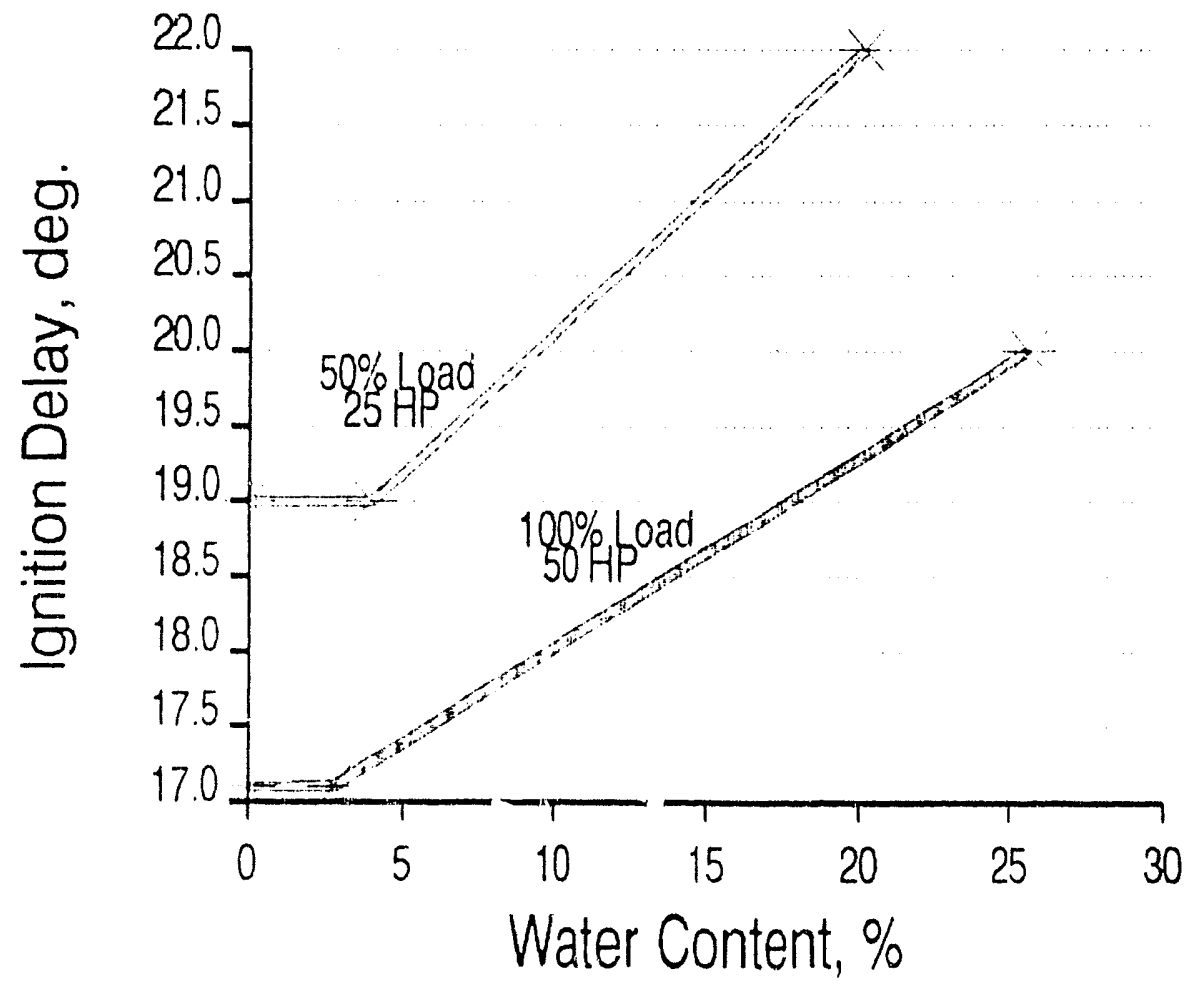

FIGURE A.81 Ignition Delay vs. Wate. Content, No. 2 Fuel, $21 \%$ Oxygen 


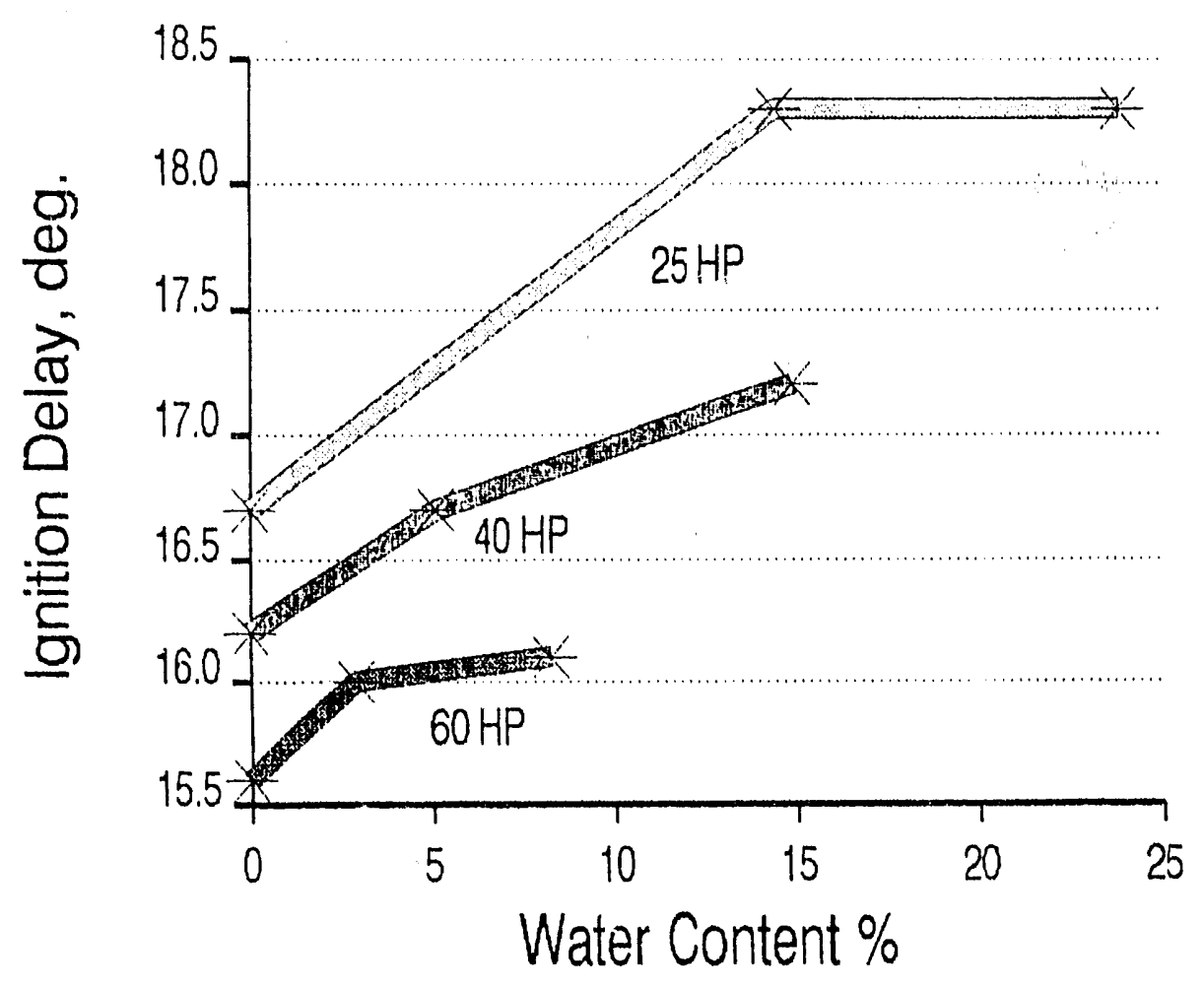

FIGURE A.82 Ignition Delay vs. Water Content, No. 2 Fuel, $50 \%$ Load, $30 \%$ Oxygen

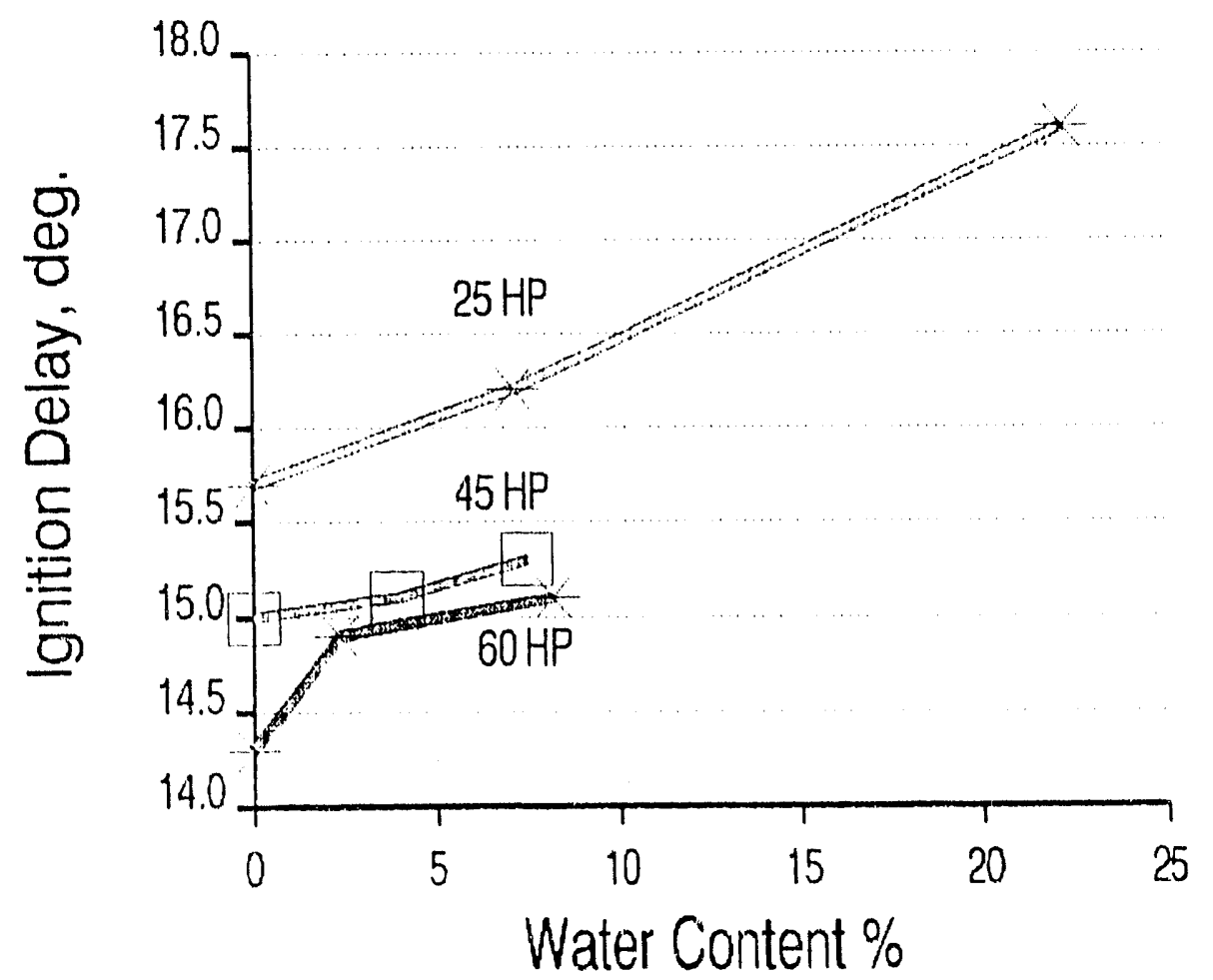

FIGURE A.83 Ignition Delay vs. Water Content, No. 2 Fuel, $50 \%$ Load, $35 \%$ Oxygen 


\section{A.7 Graphic Display of Cylinder Pressures and Results of Heat-Release Analyses for No. 4 Fuel}


$A-71$

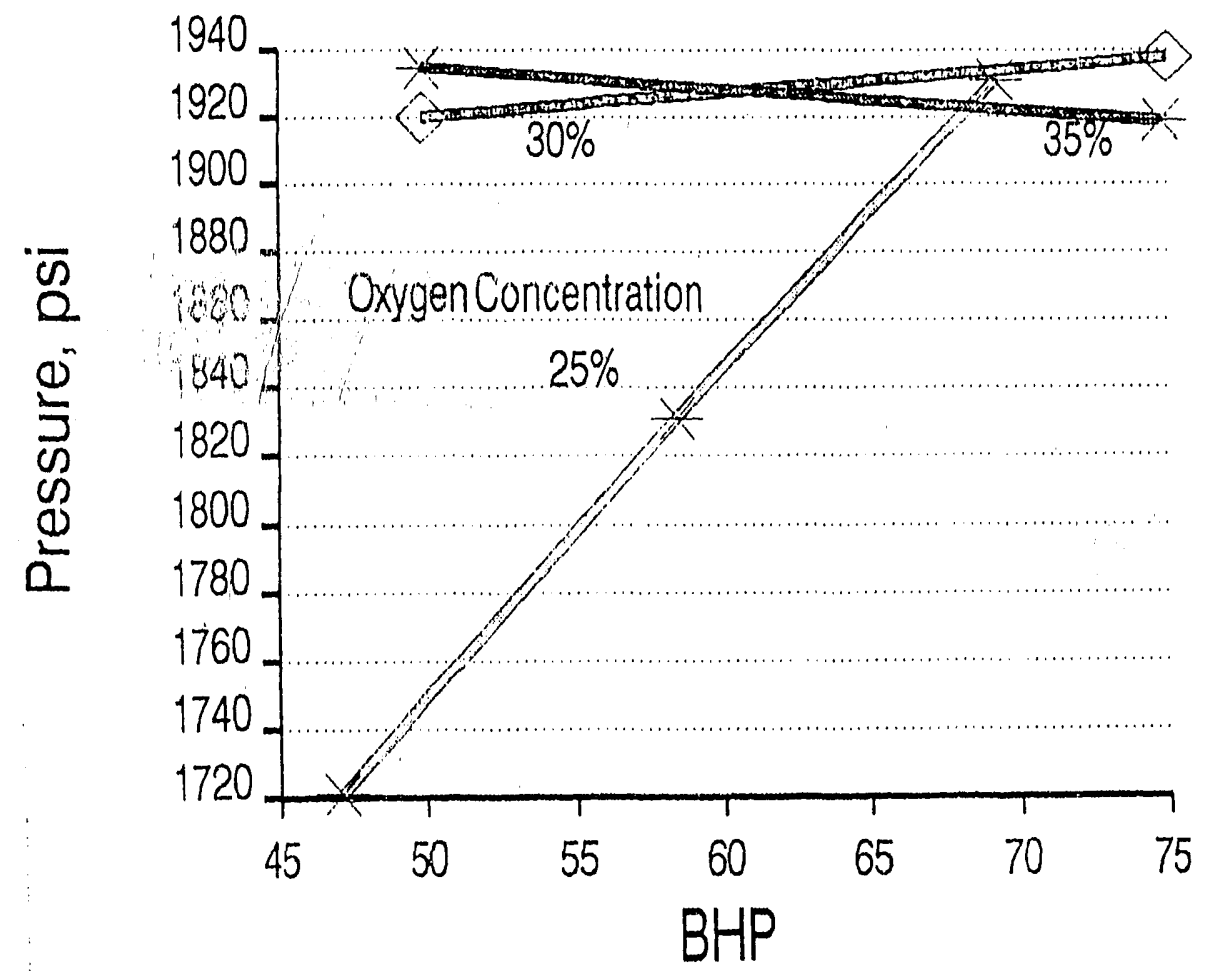

FIGURE A.84 Peak Cylinder Pressure, No. 4 Fuel, 100\% Load, No Water

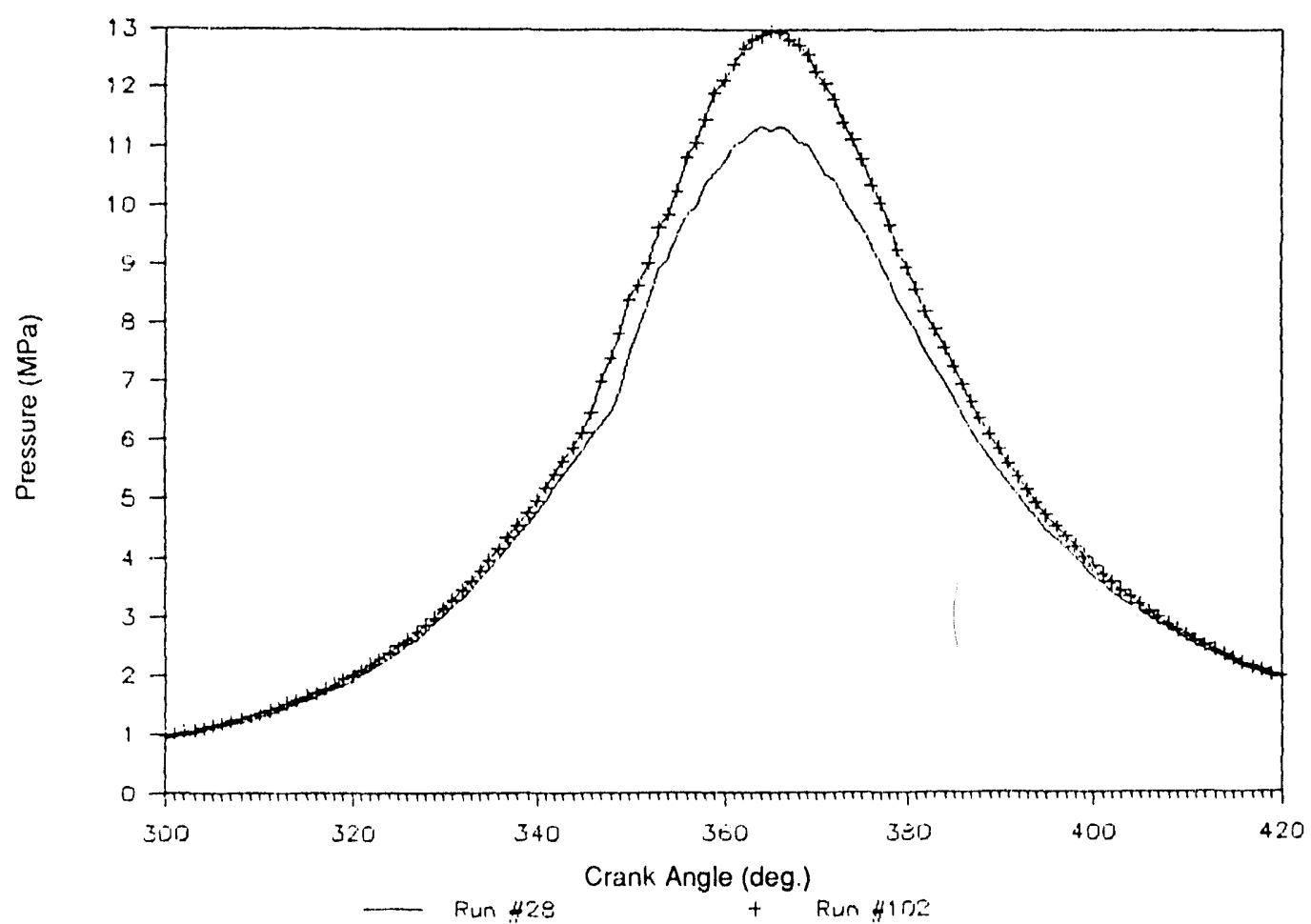

FIGURE A.85 Pressure, No. 4 Fuel, $100 \%$ Load, No Water, \#28 vs. \#102 


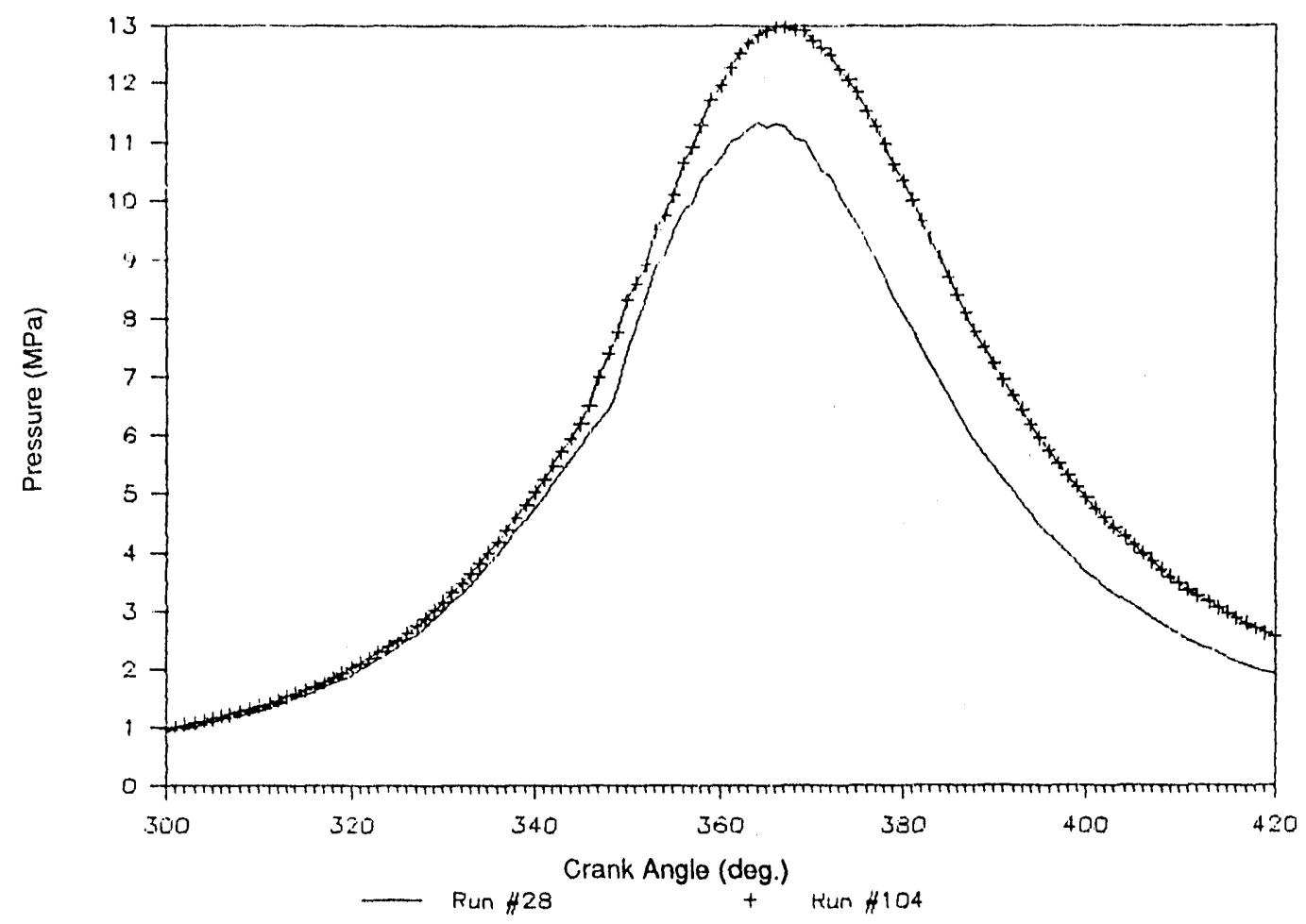

FIGURE A.86 Pressure, No. 4 Fuel, 100\% Load, No Water, \#28 vs. \#104

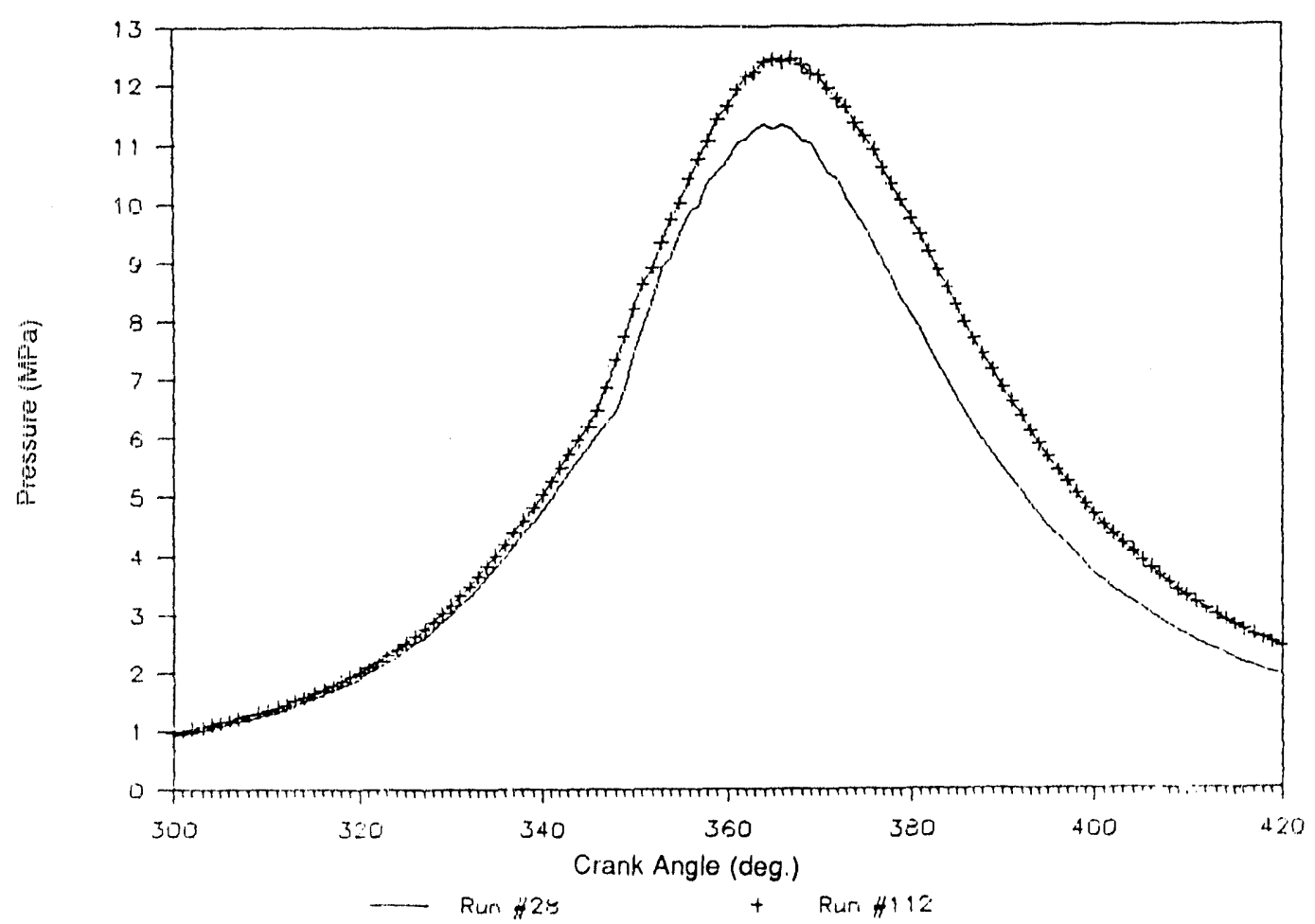

FIGURE A.87 Pressure, No. 4 Fuel, 100\% Load, \#28 vs. \#112 


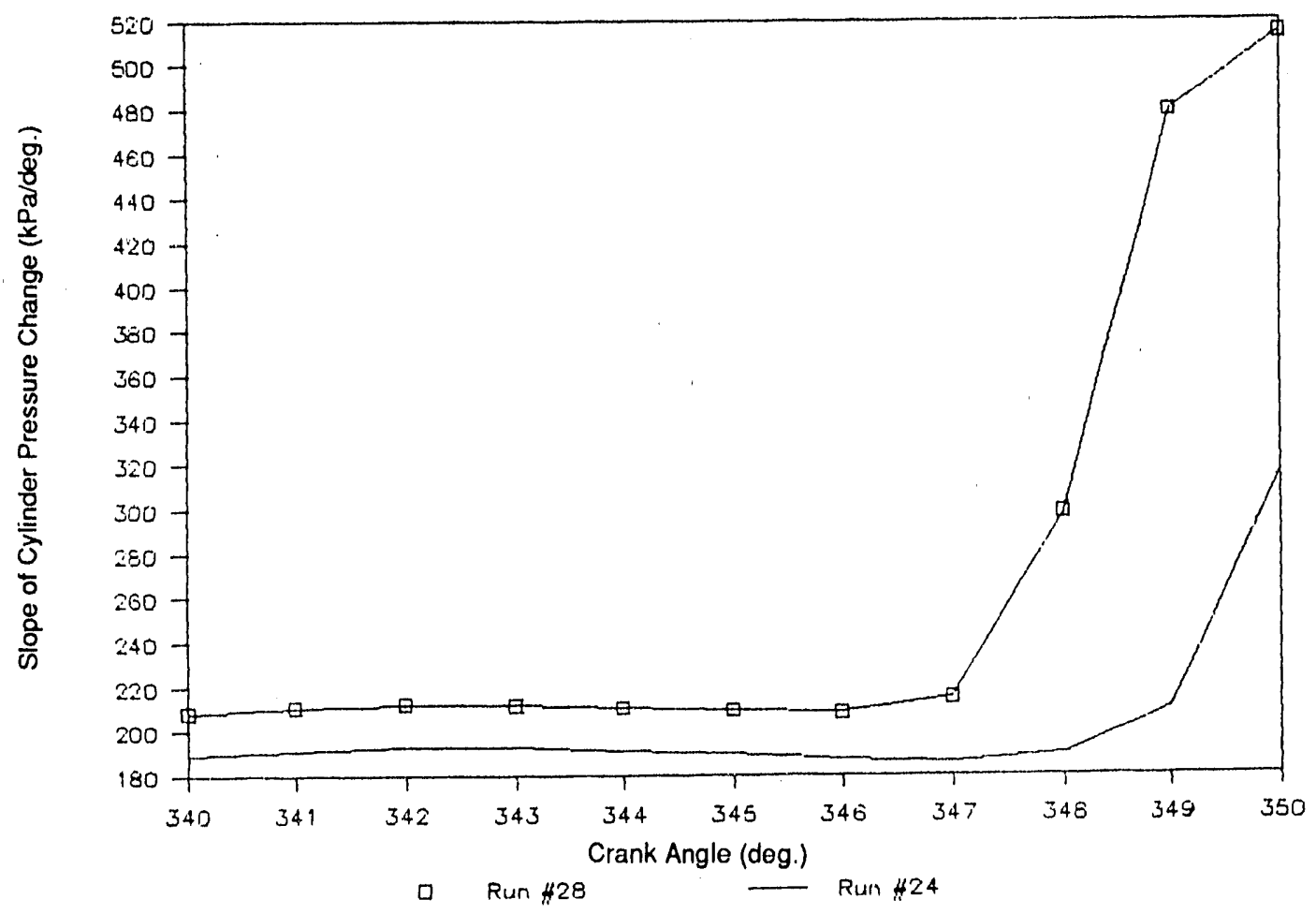

FIGURE A.88 Slope of Cylinder Pressure Change, No. 4 Fuel, 100\% Load, $21 \%$ Oxygen, \#28 vs. \#24

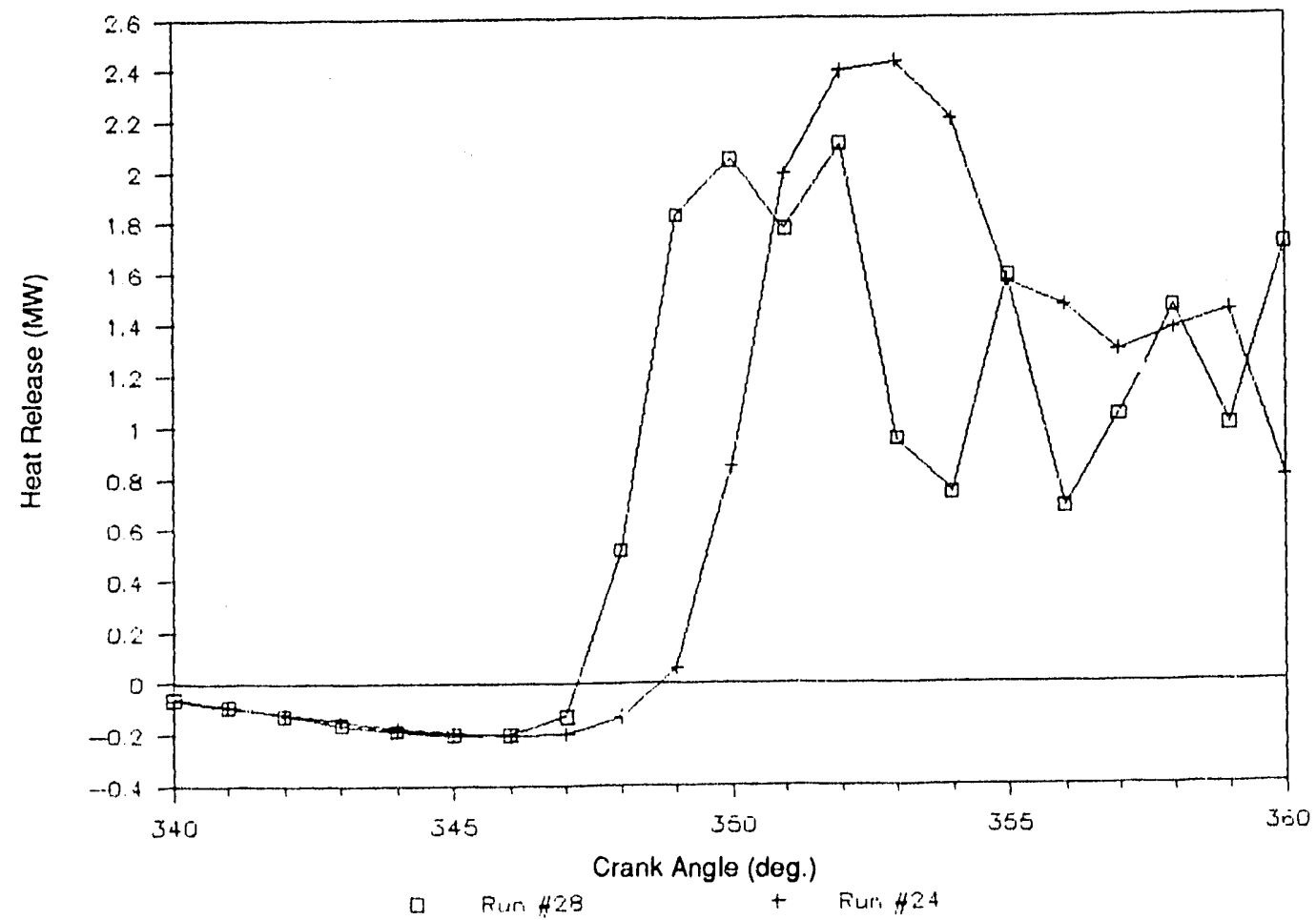

FIGURE A.89 Heat Release, No. 4 Fuel, 100\% Load, 21\% Oxygen 


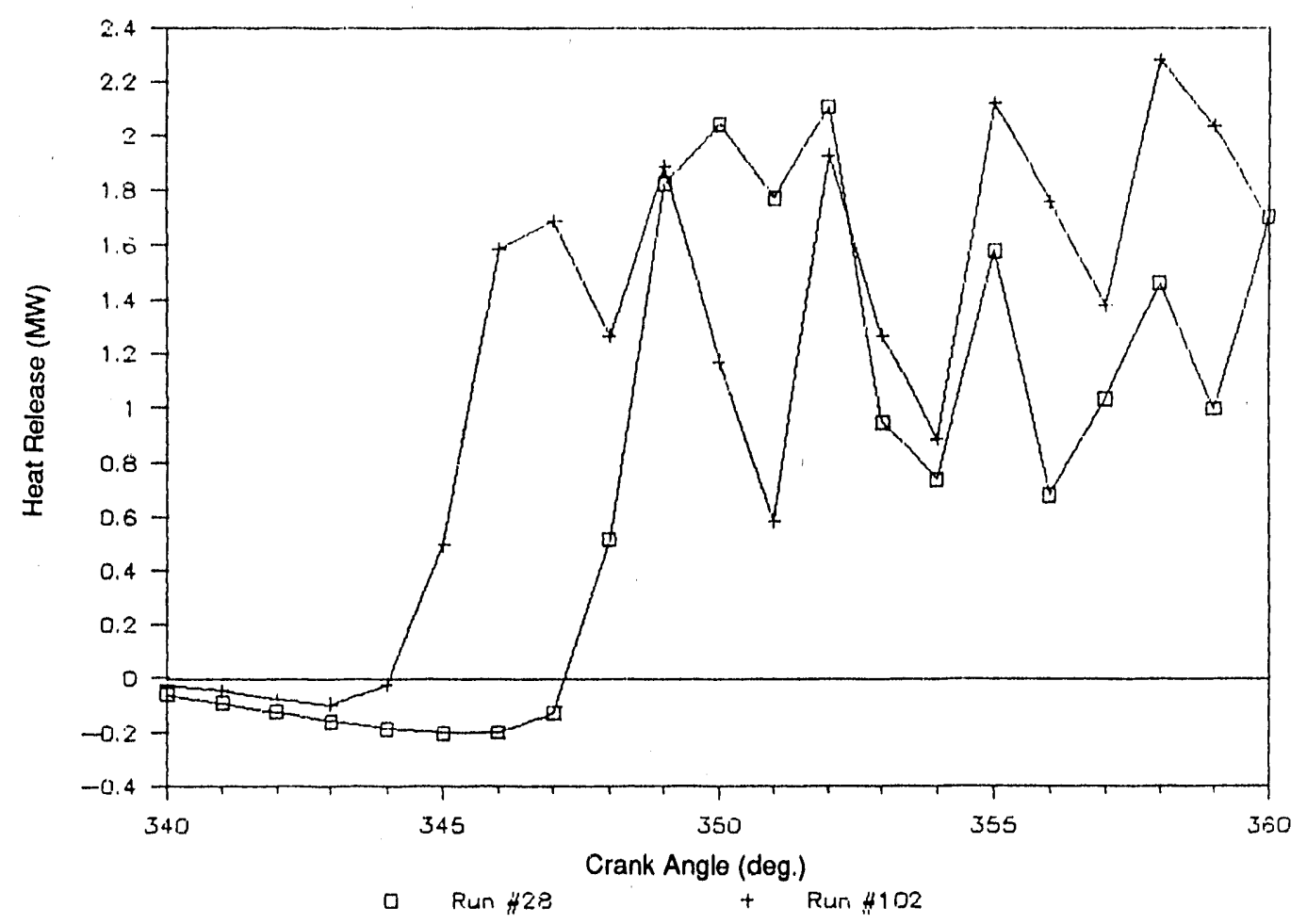

FIGURE A.90 Heat Release, No. 4 Fuel, 100\% Load, No Water, 50 Horsepower

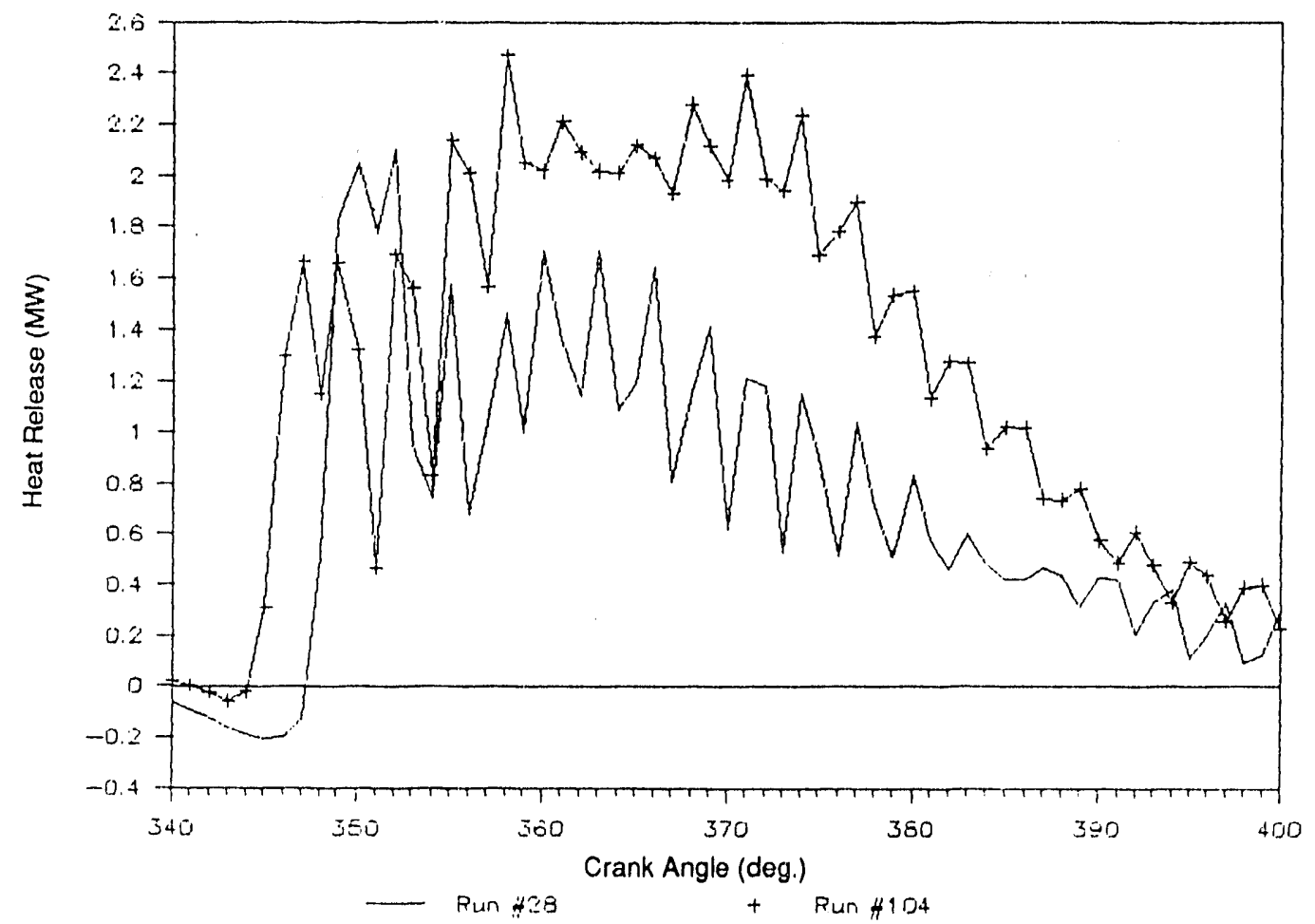

FIGURE A.91 Heat Release, No. 4 Fuel, 100\% Load, No Water 


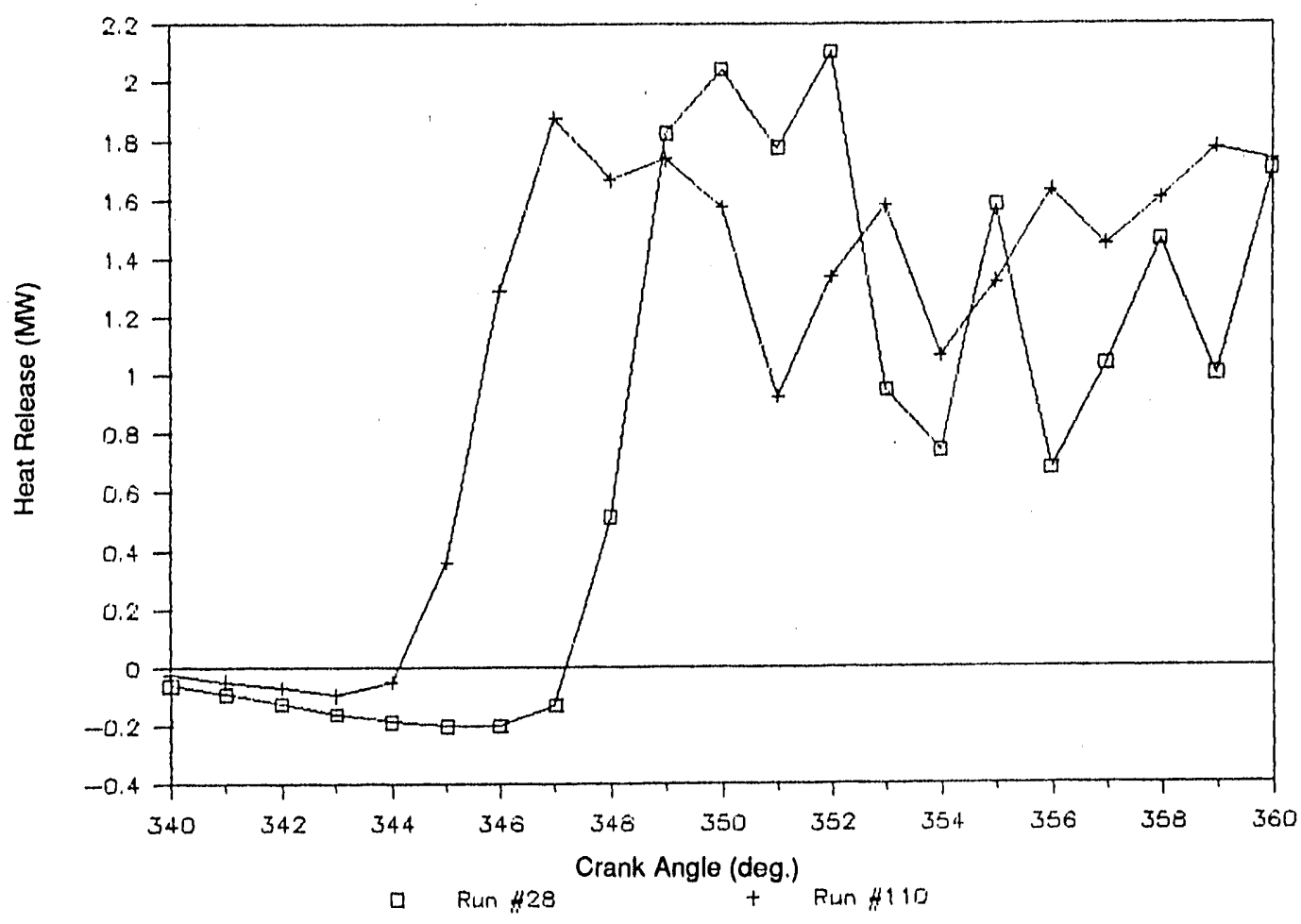

FIGURE A.92 Heat Release, No. 4 Fuel, 100\% Load, 50 Horsepower

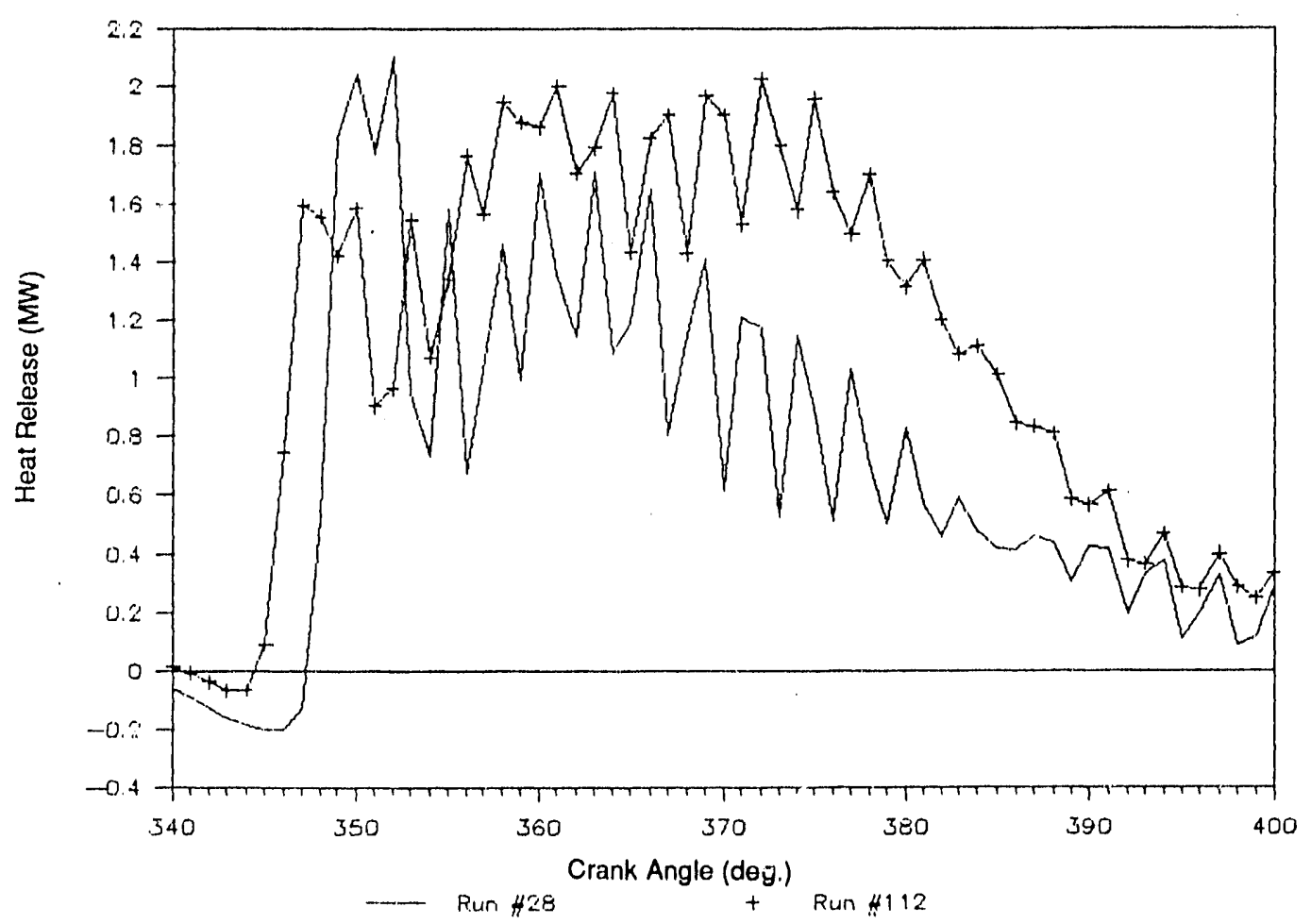

FIGURE A.93 Heat Release, No. 4 Fuel, 100\% Load 


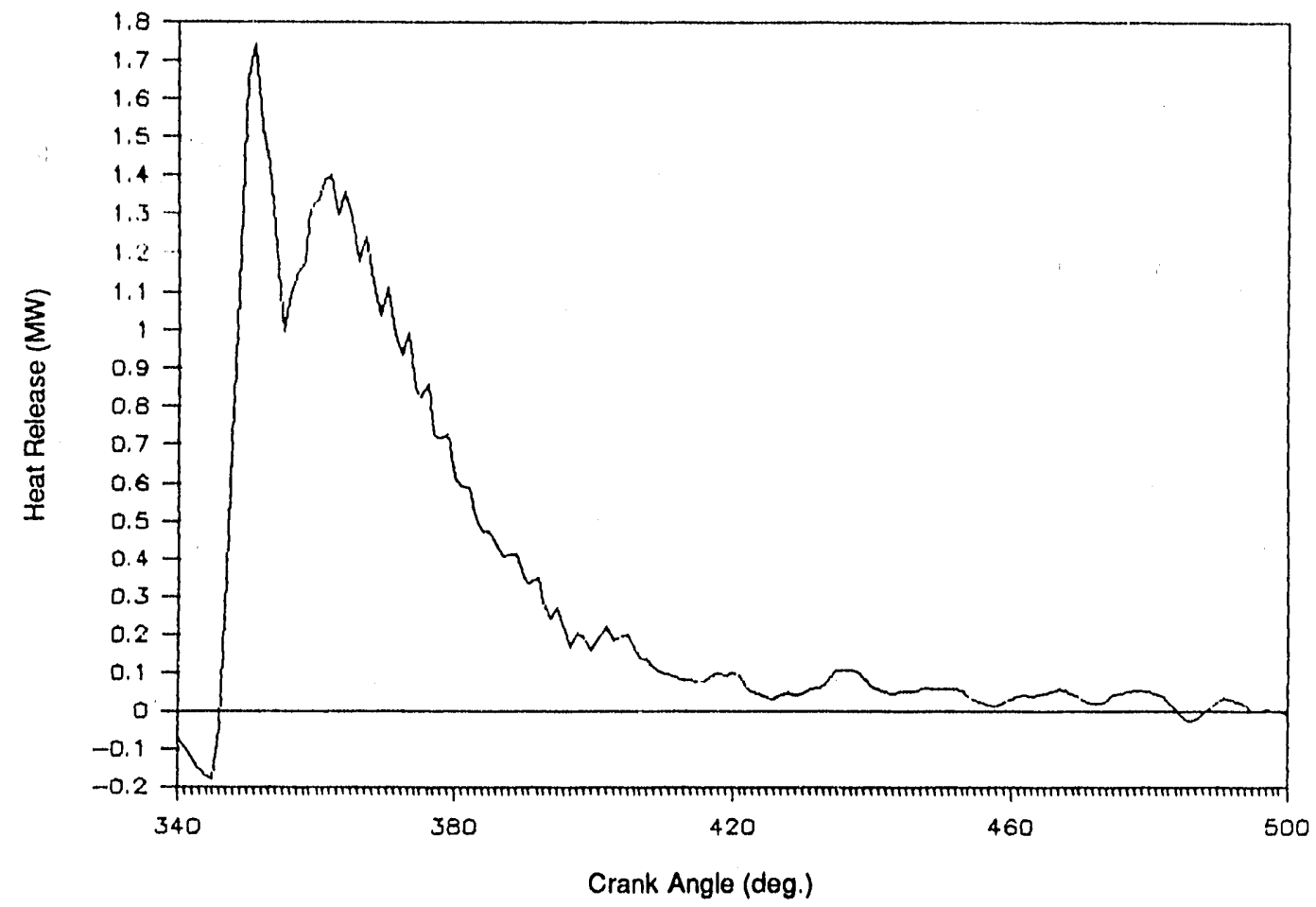

FIGURE A.94 Heat Release, No. 4 Fuel, 100\% Load, 50 Horsepower

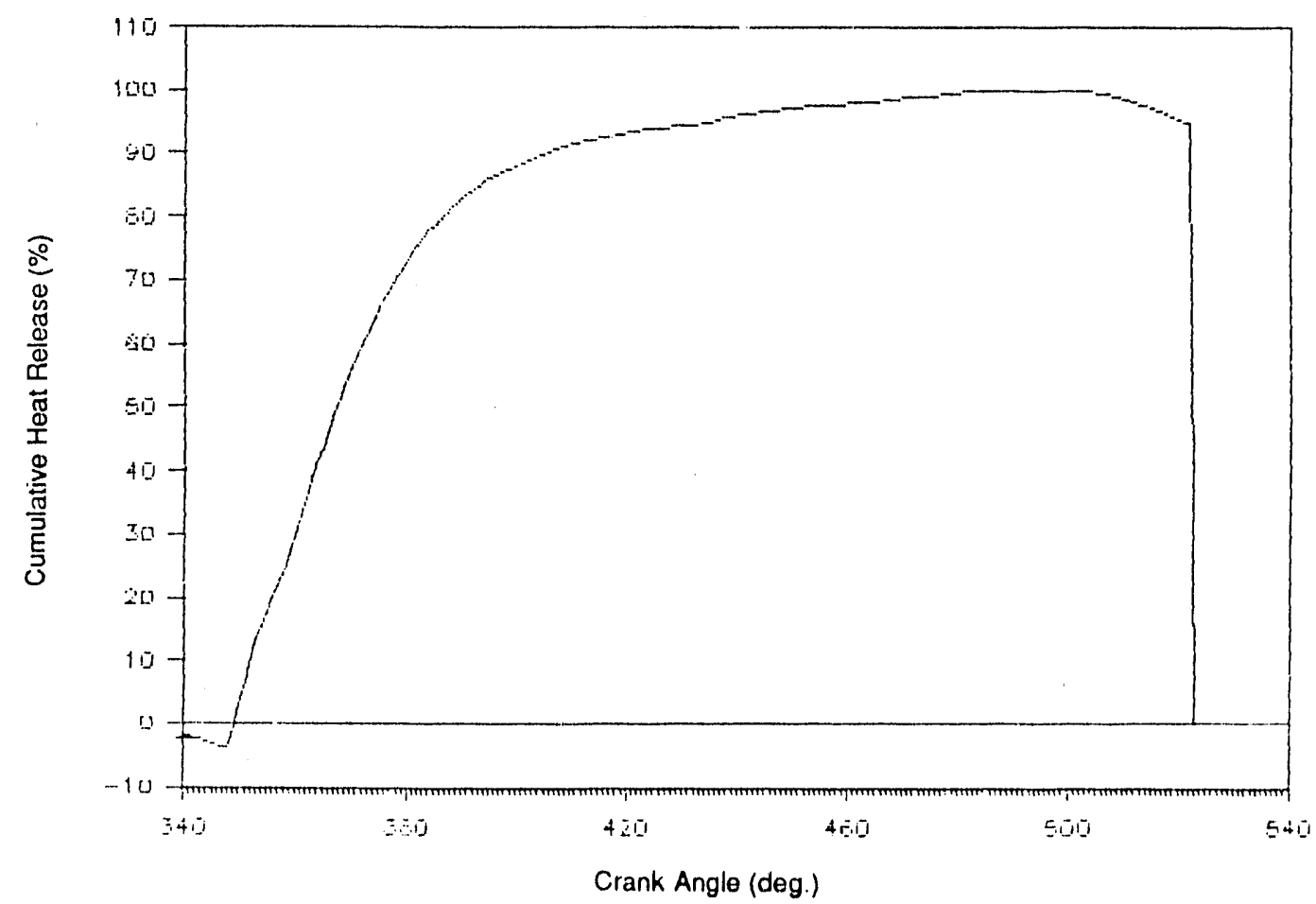

FIGURE A.95 Cumulative Heat Release, No. 4 Fuel, 100\% Load, 50 Horsepower, $21 \%$ Oxygen 


\section{A-77}

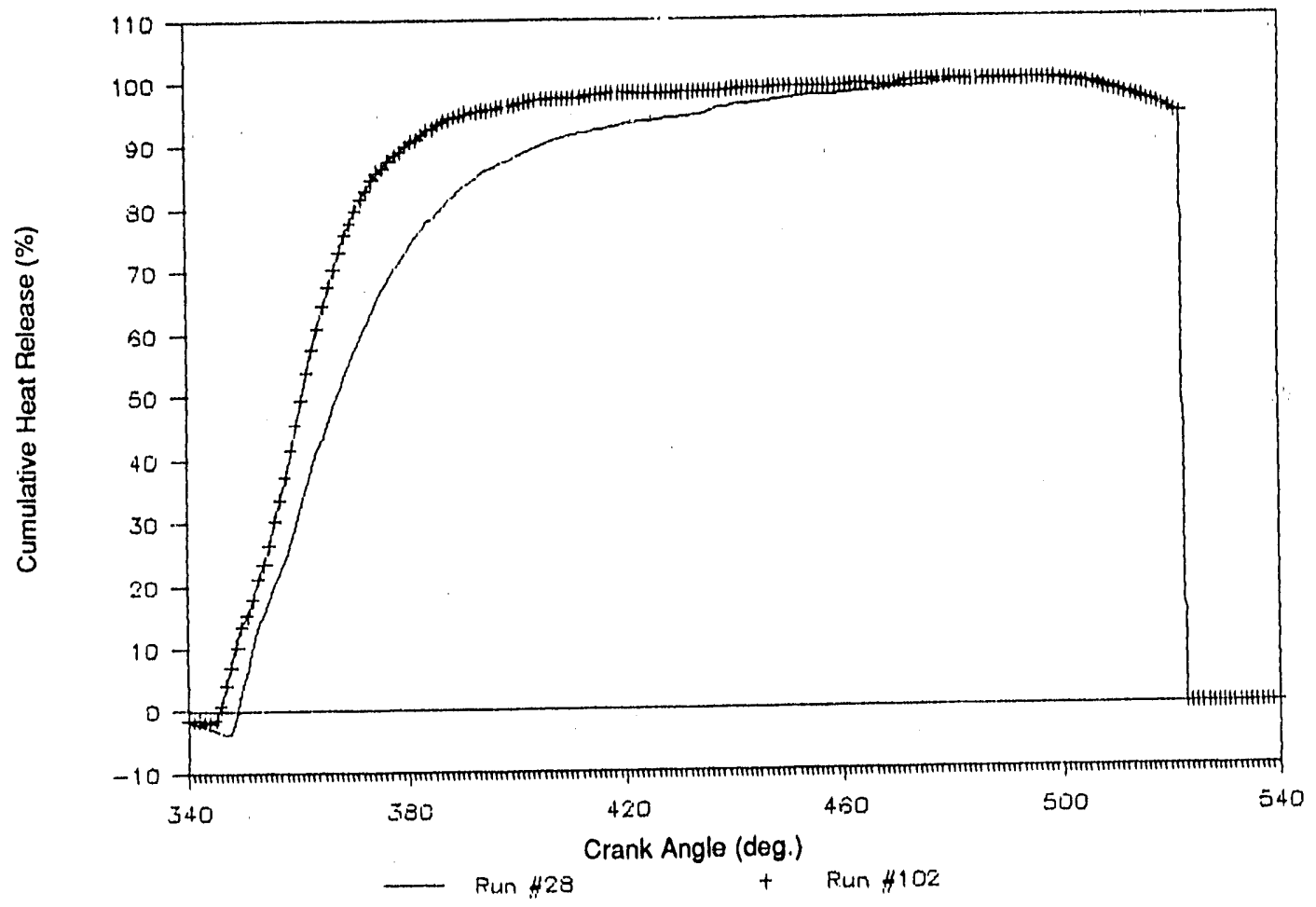

FIGURE A.96 Cumulative Heat Release, No. 4 Fuel, 100\% Load, 50 Horsepower, No Water

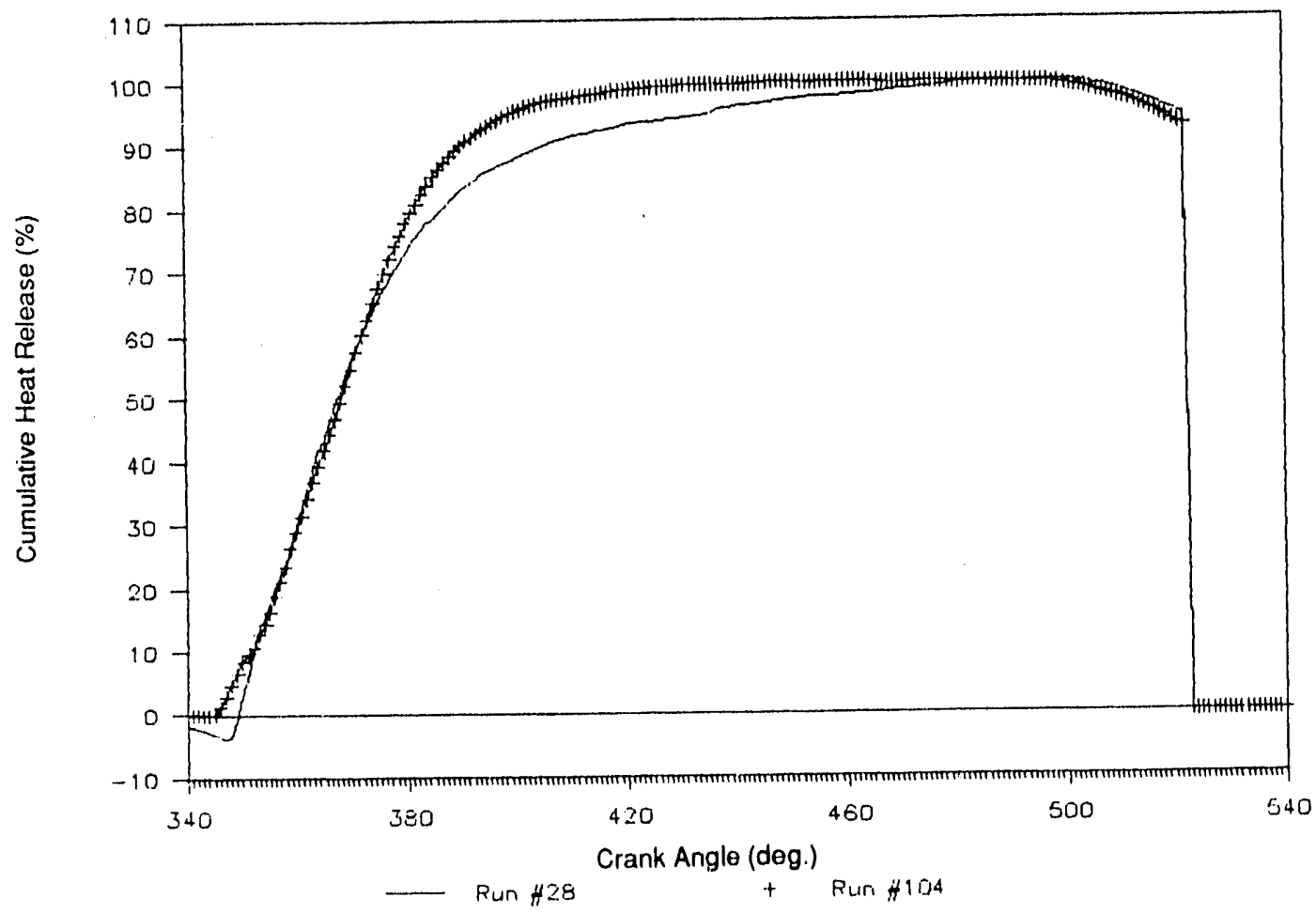

FIGURE A.97 Cumulative Heat Release, No. 4 Fuel, 100\% Load, No Water 


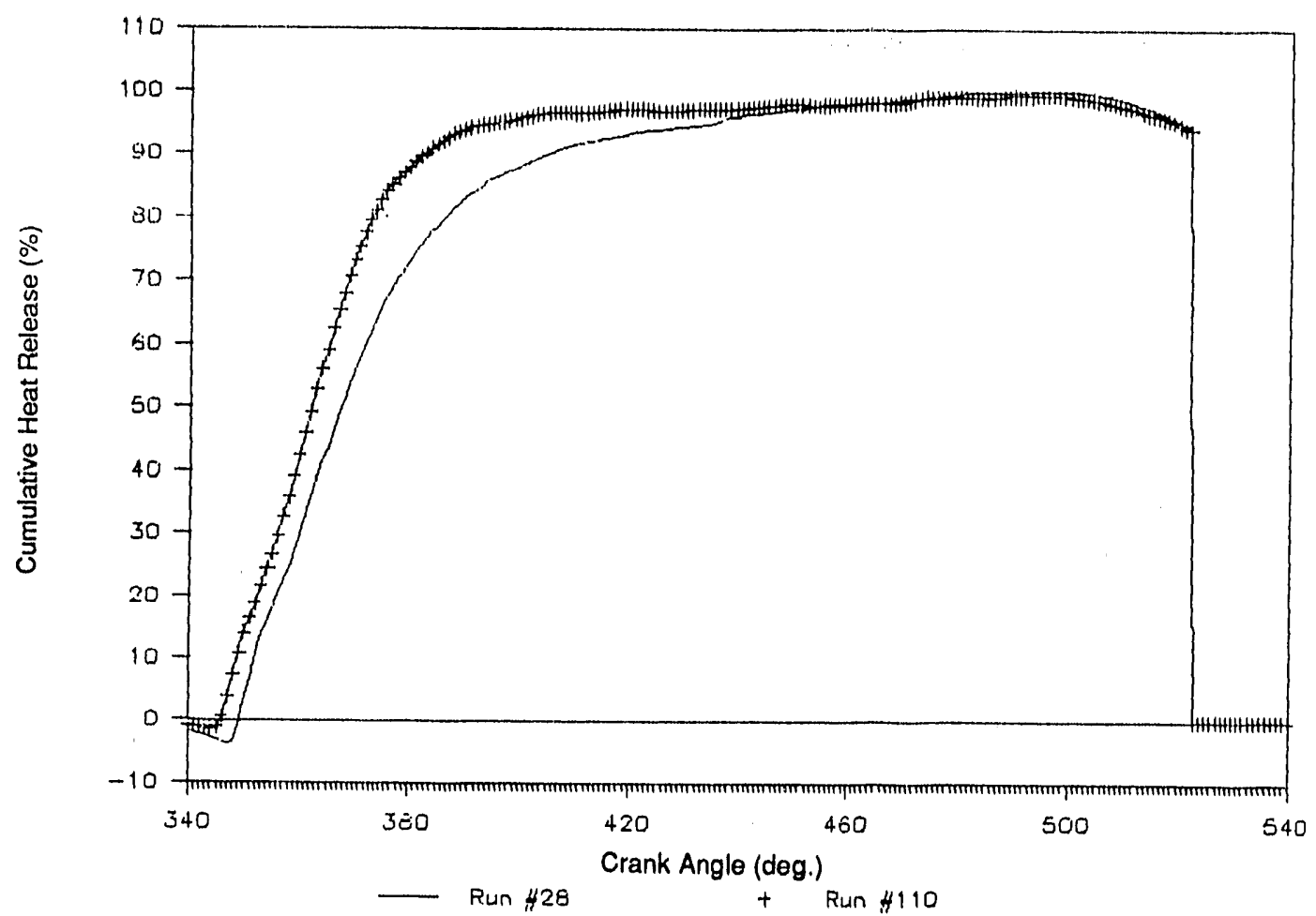

FIGURE A.98 Cumulative Heat Release, No. 4 Fuel, 100\% Load, 50 Horsepower

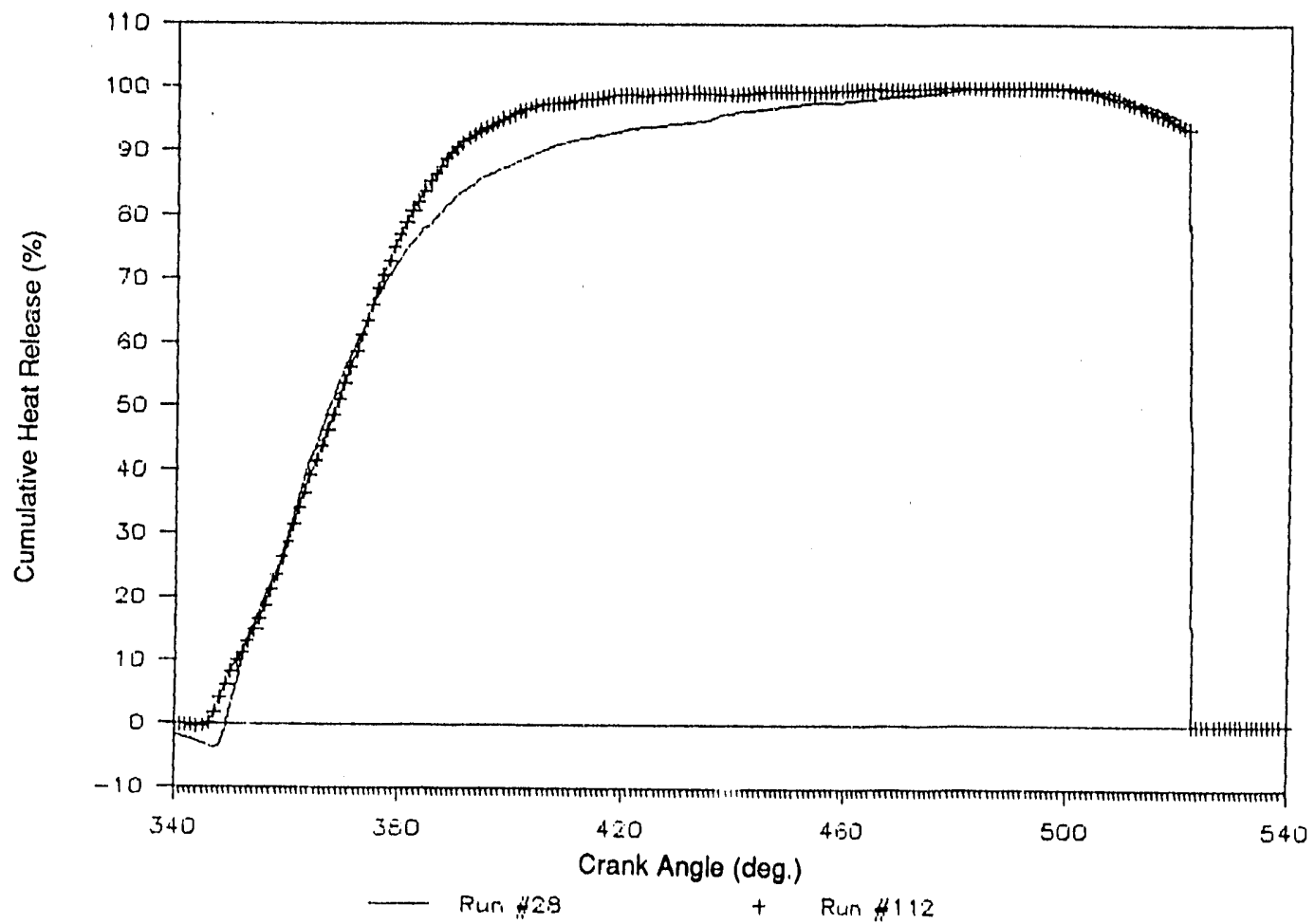

FIGURE A.99 Cumulative Heat Release, No. 4 Fuel, 100\% Load 


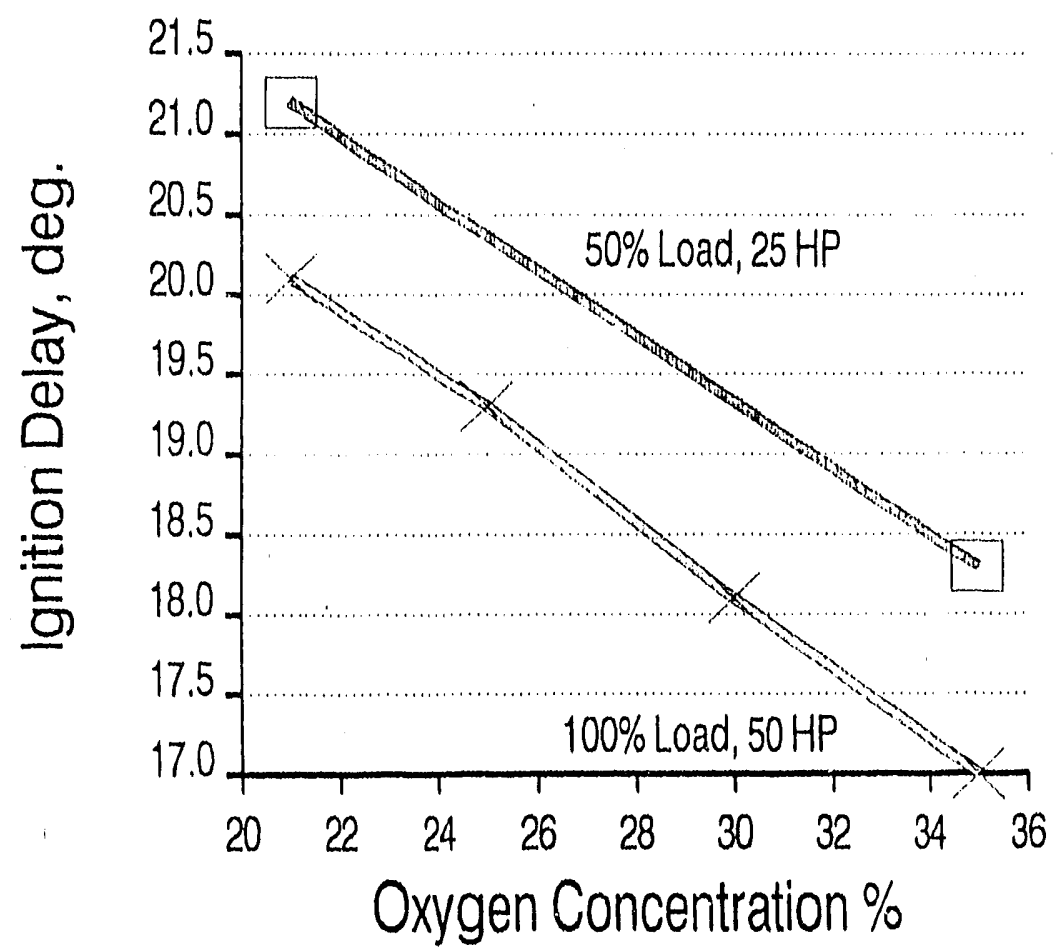

FIGURE A.100 Ignition Delay vs. Oxygen Concentration, No. 4 Fuel, No Water

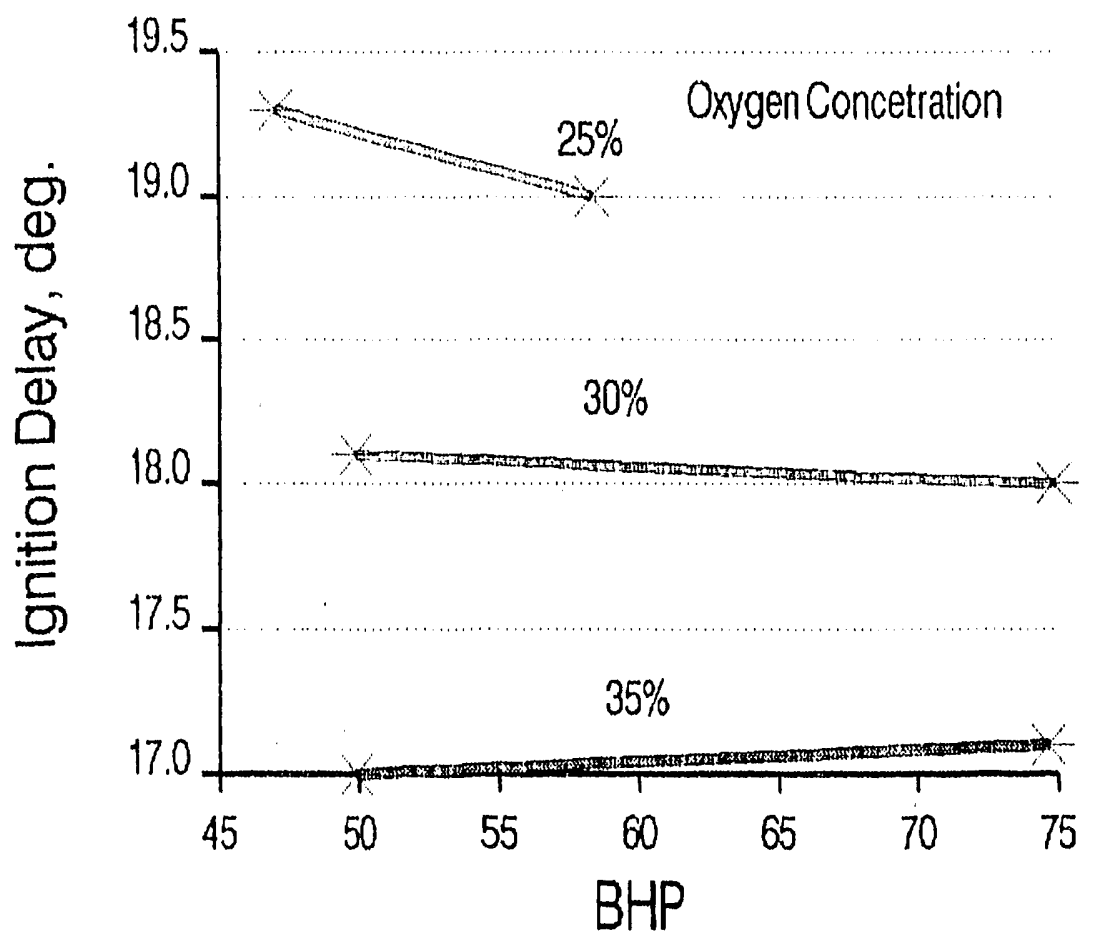

FIGURE A.101 Ignition Delay vs. Brake Horsepower, No. 4 Fuel, $100 \%$ Load, No Water 


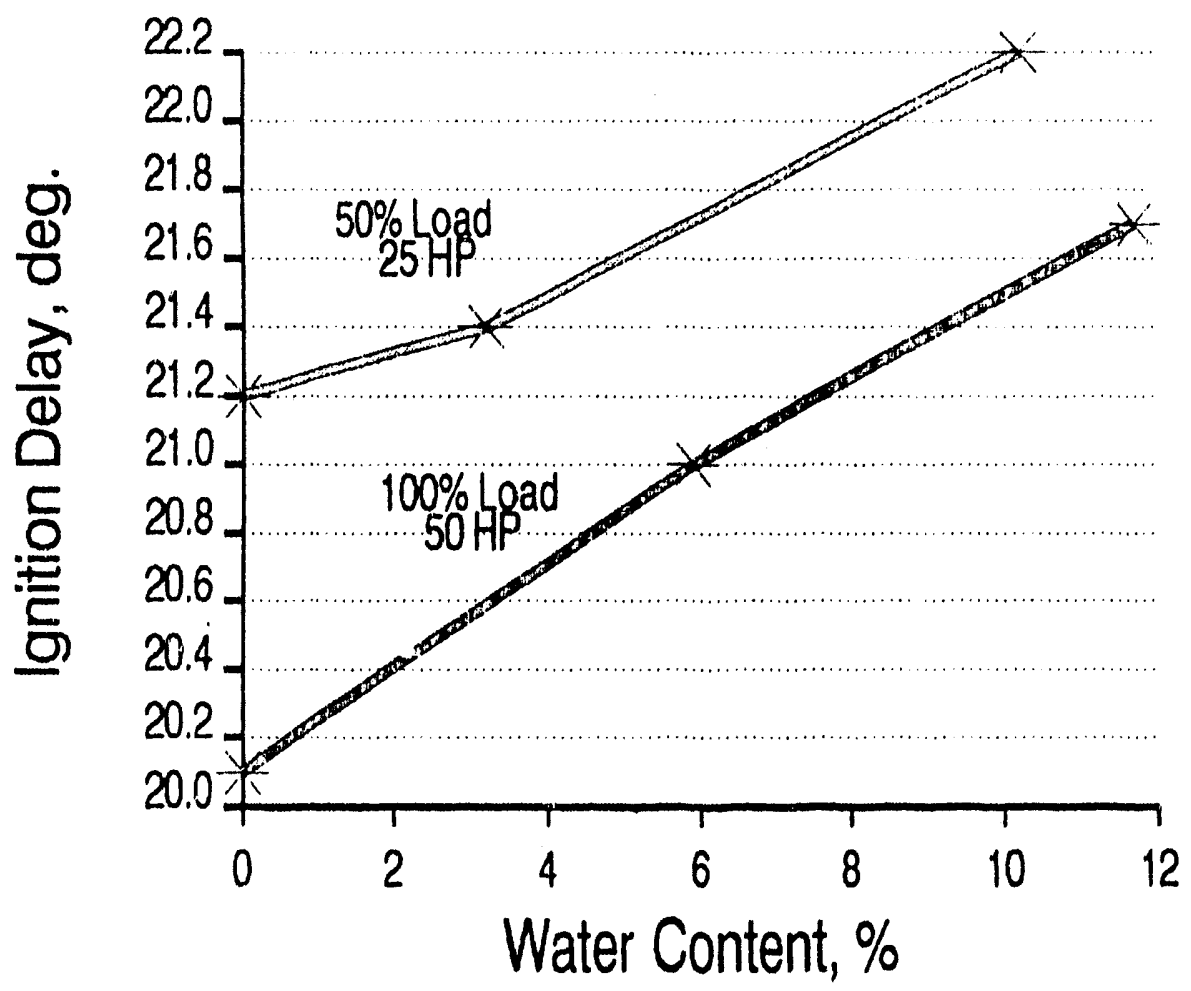

FIGURE A.102 Ignition Delay vs. Water Content, No. 4 Fuel, $21 \%$ Oxygen 
A.8 Comparison of Calculated Ignition Delays for No. 2 and No. 4 Fuels 
A-82 
$A-83$

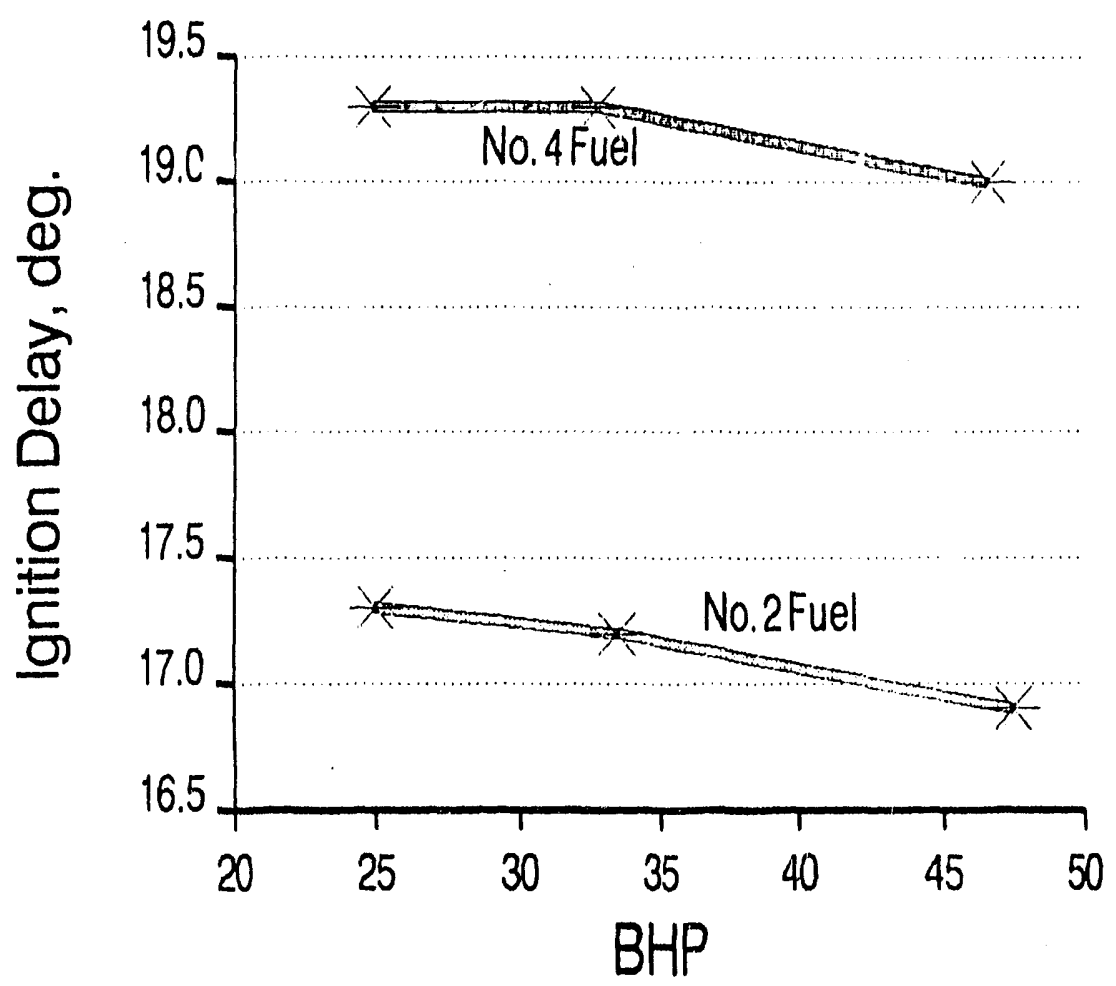

FIGURE A.103 Ignition Delay vs. Brake Horsepower, 50\% Load, No Water, $25 \%$ Oxygen

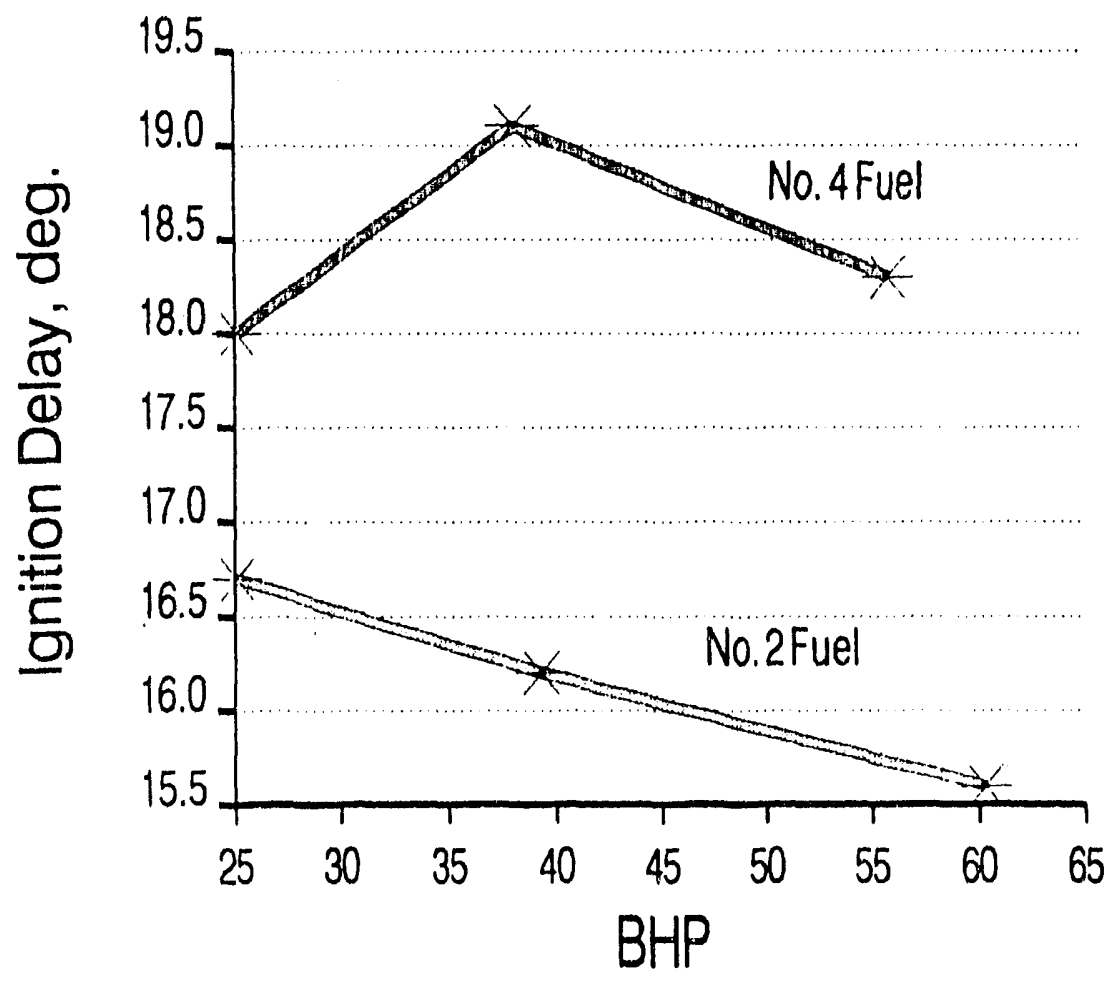

FIGURE A.104 Ignition Delay vs. Brake Horsepower, 50\% Load, No Water, $30 \%$ Oxygen 


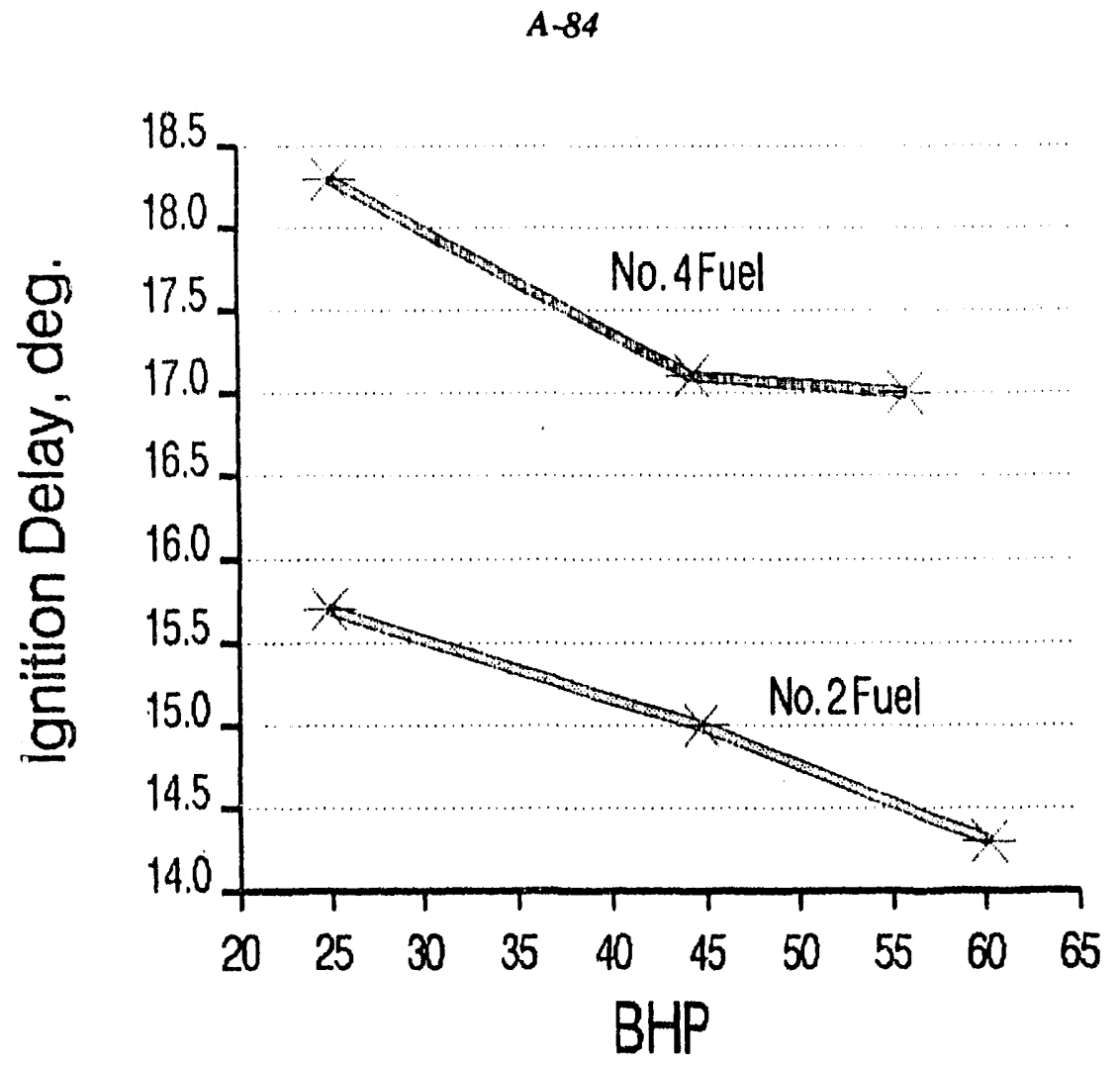

FIGURE A.105 Ignition Delay vs. Brake Horsepower, $50 \%$ Load, No Water, 35\% Oxygen

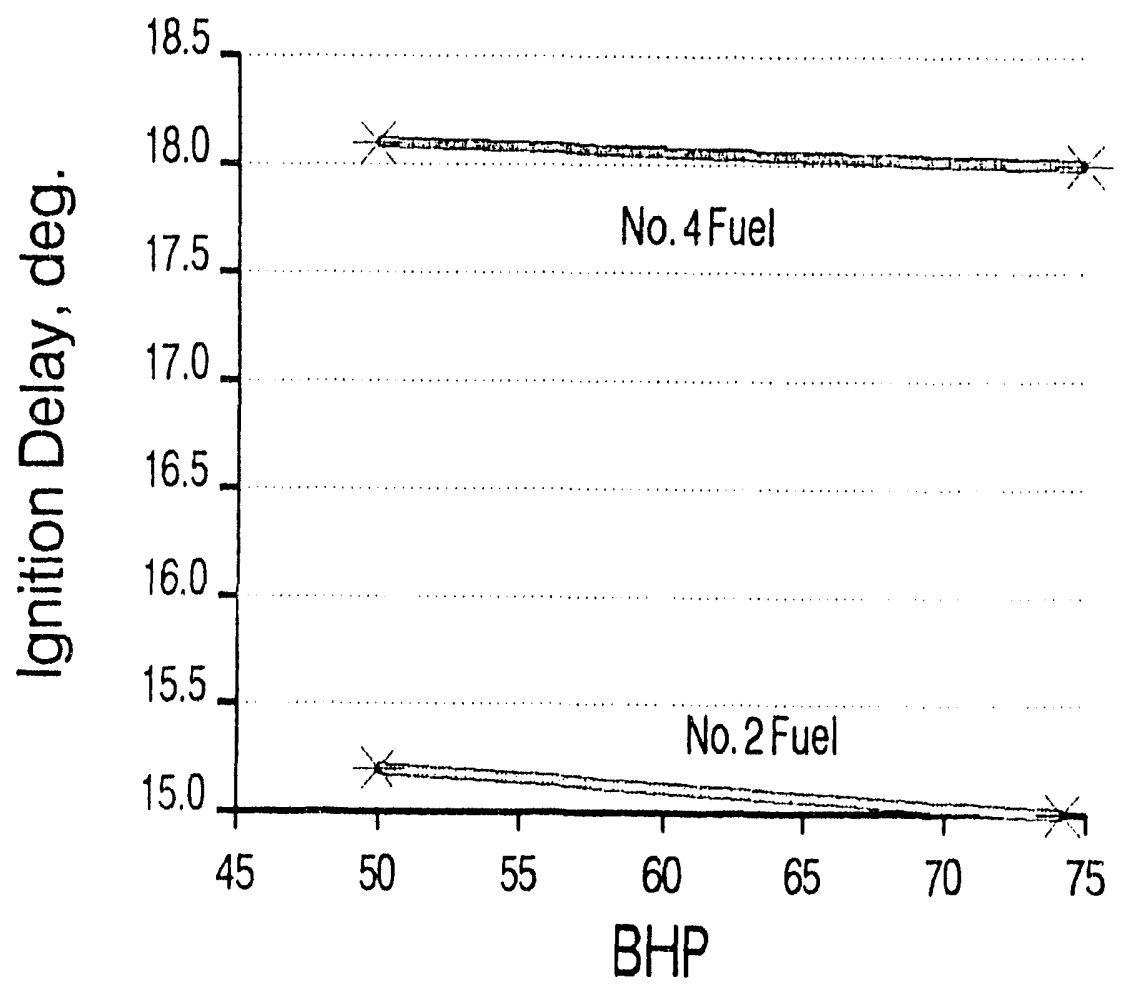

FIGURE A.106 Ignition Delay vs. Brake Horsepower, 100\% Load, No Water, $30 \%$ Oxygen 


\section{$A-85$}

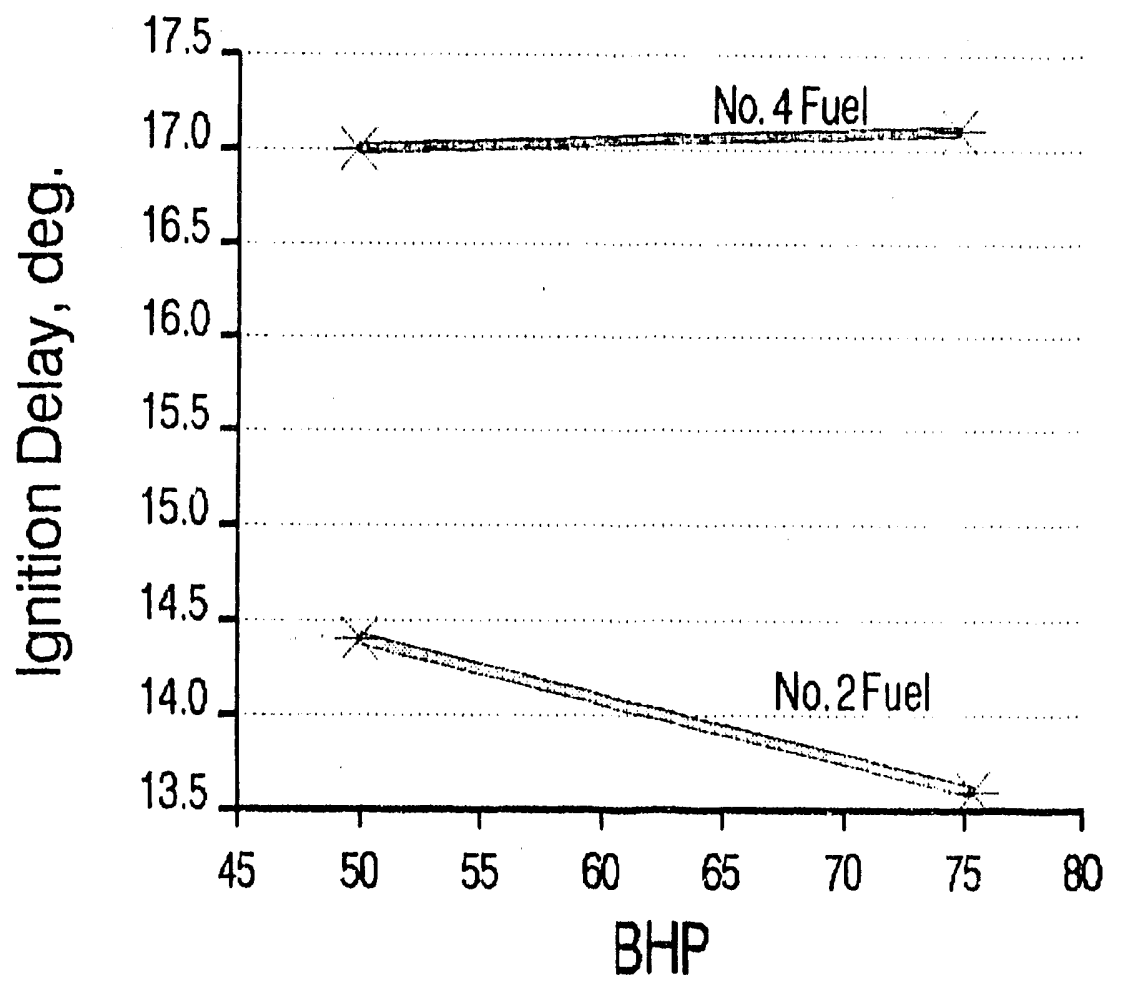

FIGURE A.107 Ignition Delay vs. Brake Horsepower, 100\% Load, No Water, 35\% Oxygen

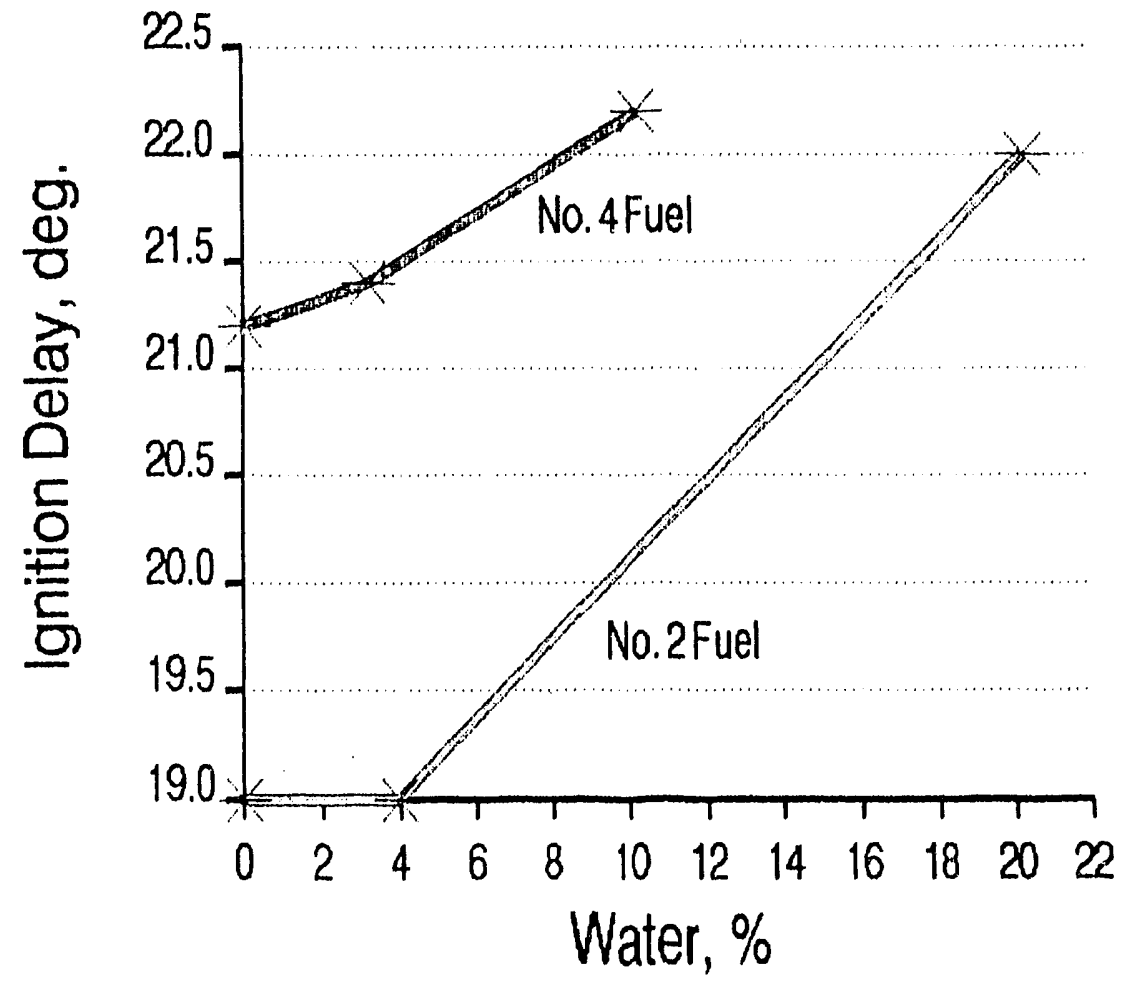

FIGURE A.108 Ignition Delay vs. Water Content, $50 \%$ Load, 25 Horsepower, $21 \%$ Oxygen 


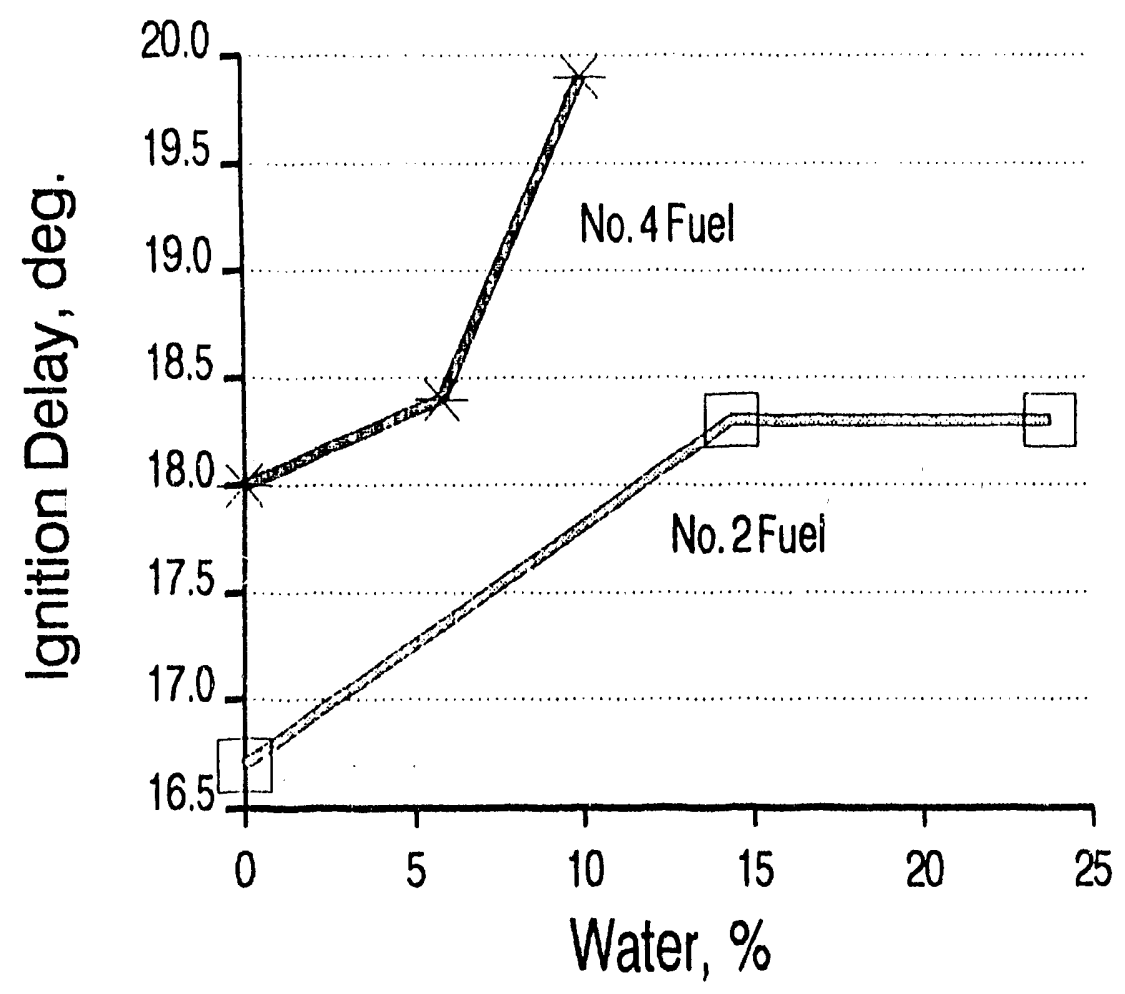

FIGURE A.109 Ignition Delay vs. Water Content, 50\% Load, 25 Horsepower, $30 \%$ Oxygen

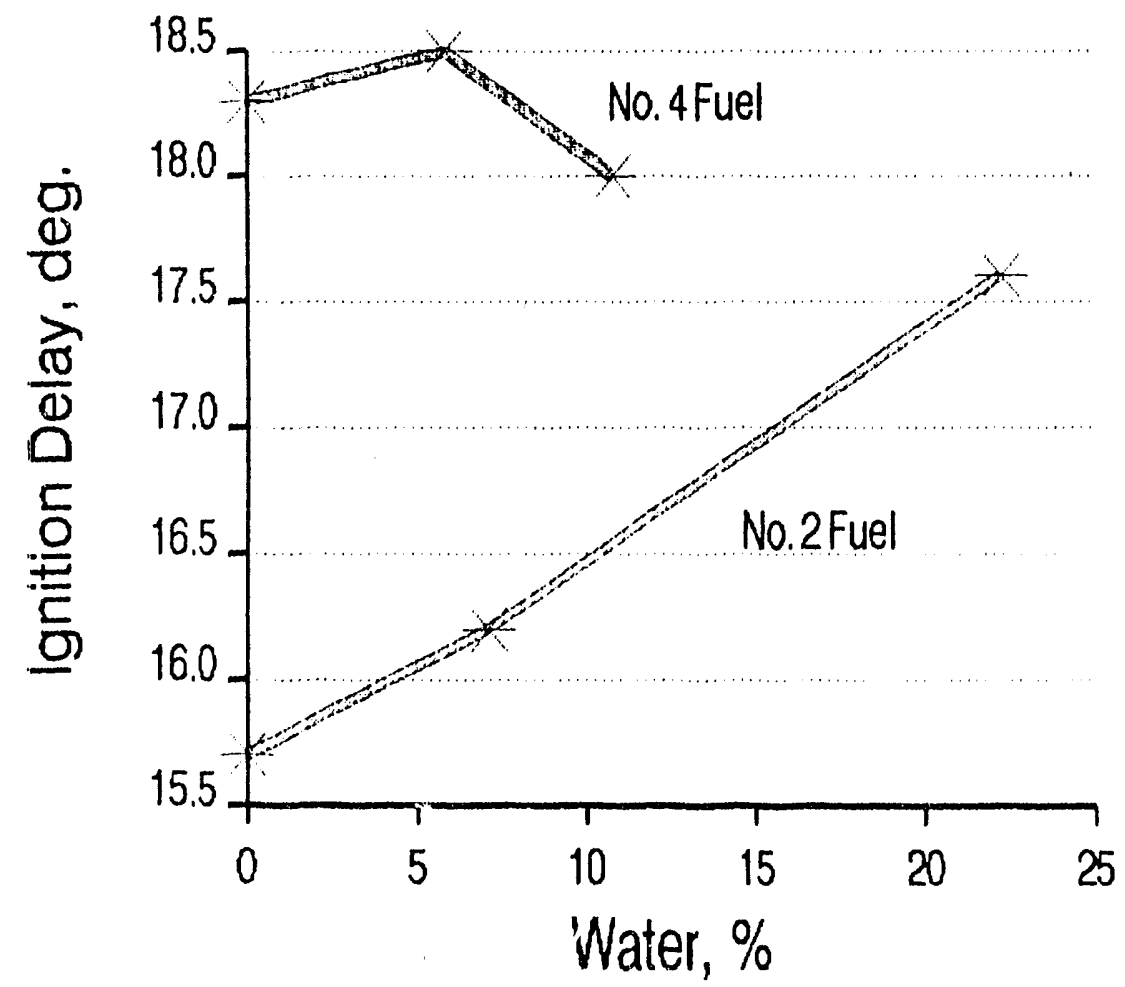

FIGURE A.110 Ignition Delay vs. Water Content, 50\% Load, 25 Horsepower, 35\% Oxygen 


\section{$A-87$}

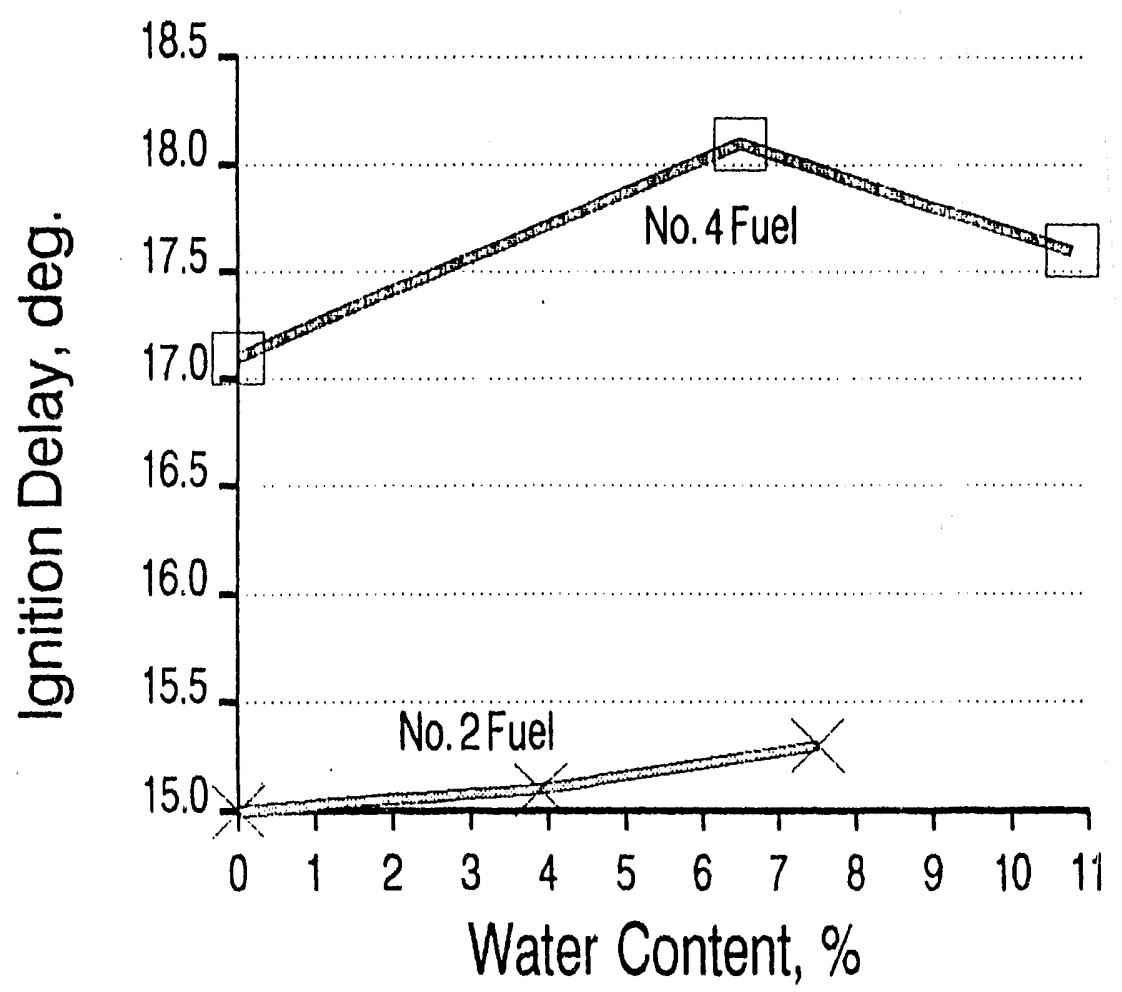

FIGURE A.111 Ignition Delay vs. Water Content, 50\% Load, 45 Horsepower, $35 \%$ Oxygen

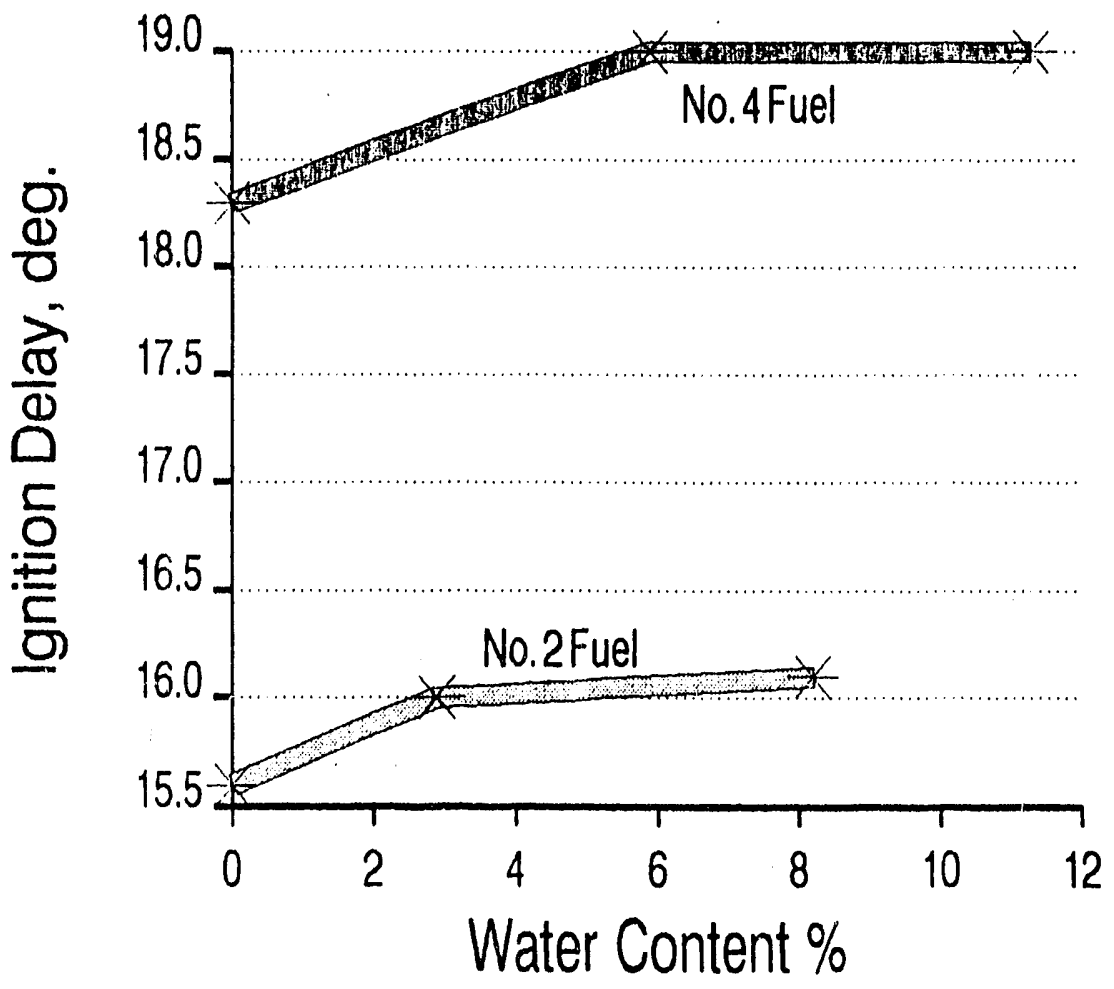

FIGURE A.112 Ignition Delay vs. Water Content, 50\% Load, 60 Horsepower, $30 \%$ Oxygen 


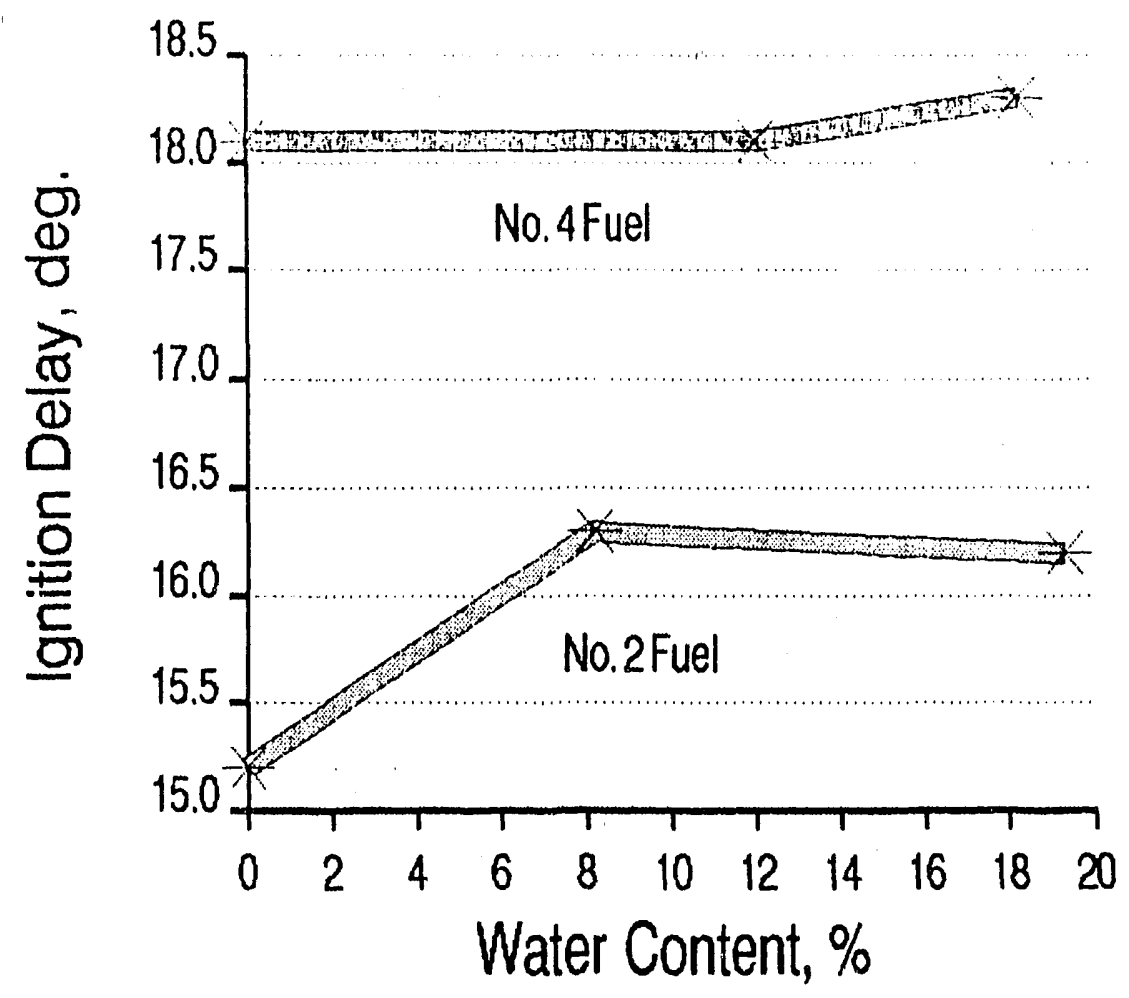

FIGURE A.113 Ignition Delay vs. Water Content, 100\% Load, 50 Horsepower, $30 \%$ Oxygen

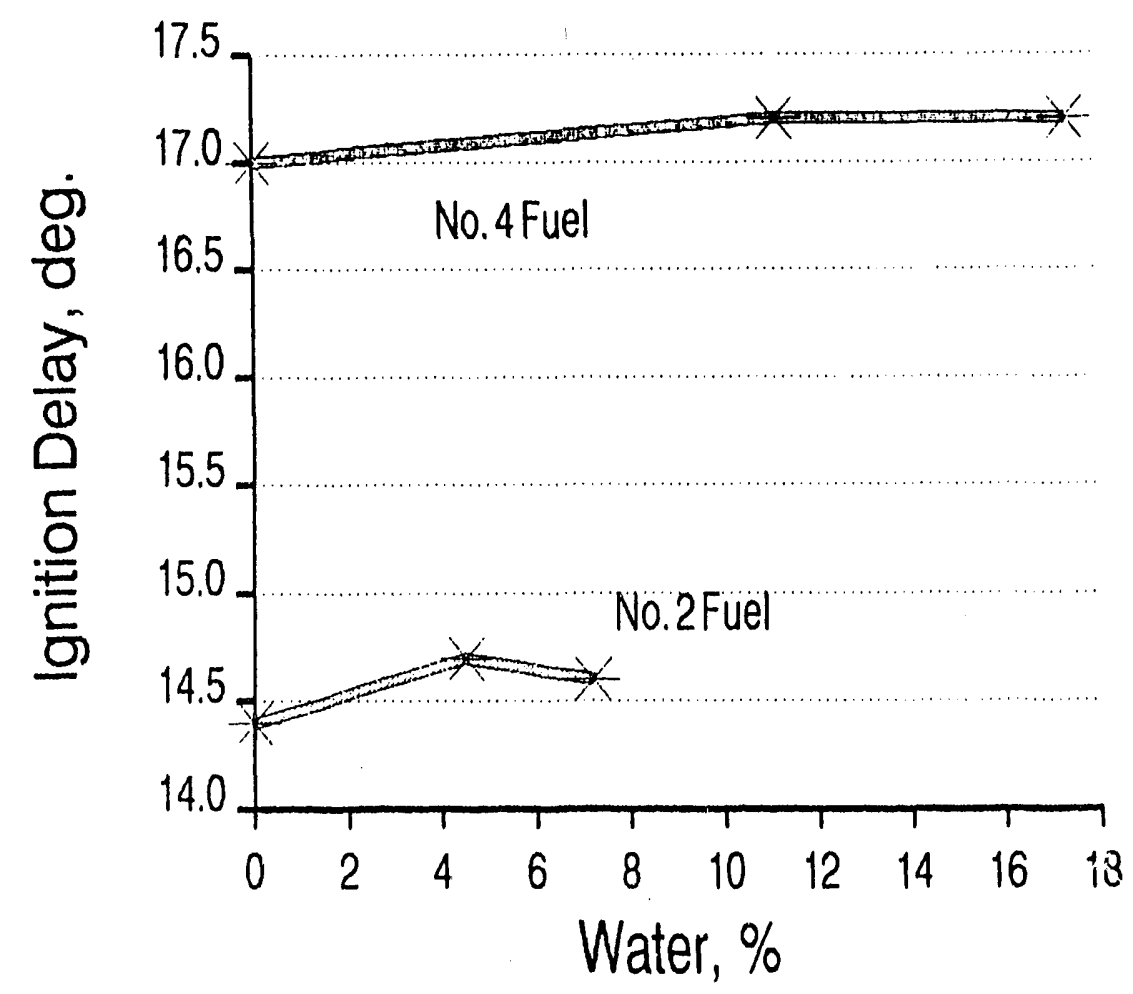

FIGURE A.114 Ignition Delay vs. Water Content, 100\% Load, 50 Horsepower, $35 \%$ Oxygen 
A.9 Graphic Display of Thermal Efficiency for No. 2 Fuel 


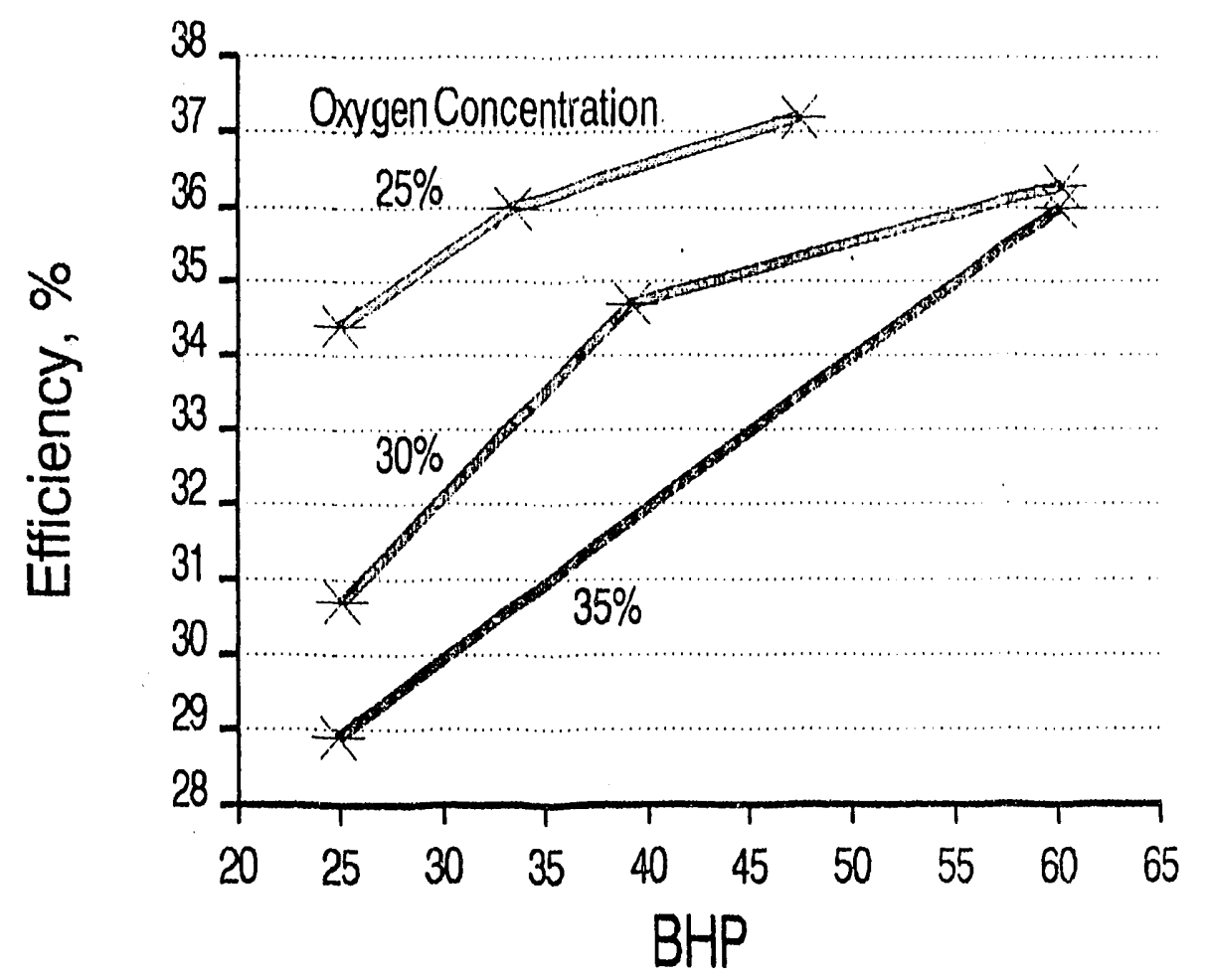

FIGURE A.115 Thermal Efficiency vs. Brake Horsepower, No. 2 Fuel, $50 \%$ Load, No Water

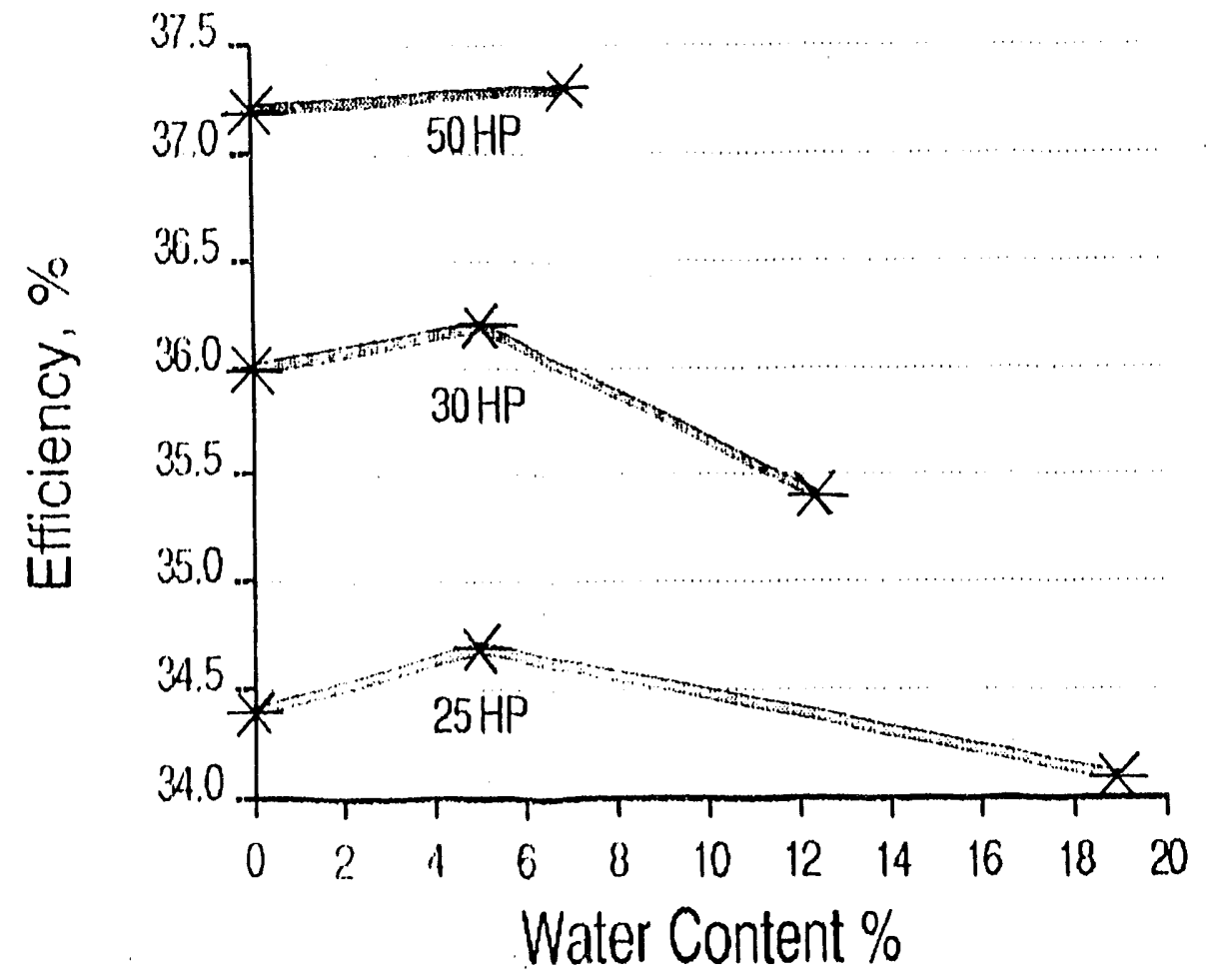

FIGURE A.116 Thermal Efficiency vs. Water Content, No. 2 Fuel, $50 \%$ Load, $25 \%$ Oxygen 


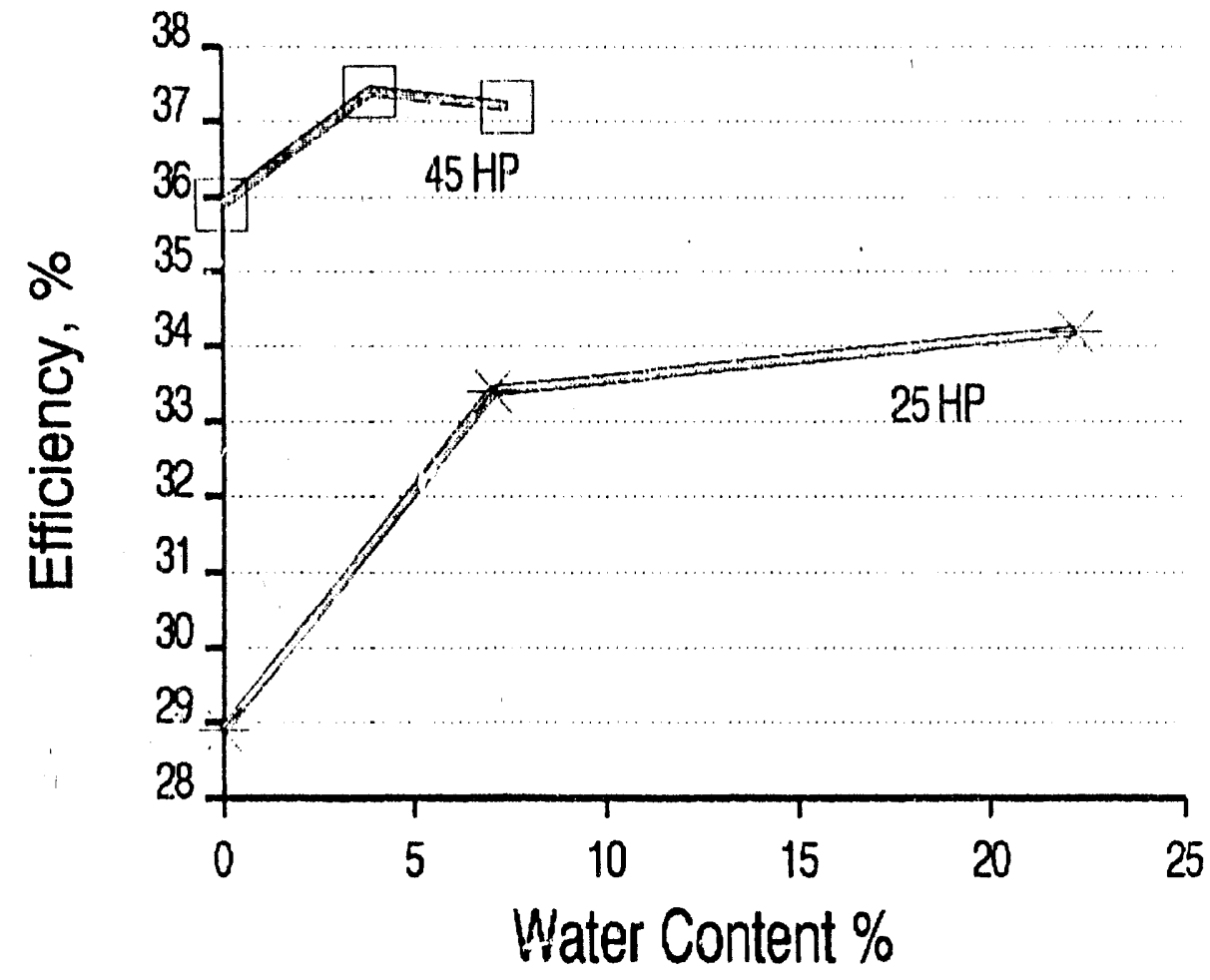

FIGURE A.117 Thermal Efficiency ys. Water Content, No. 2 Fuel, $50 \%$ Load, $35 \%$ Oxygen

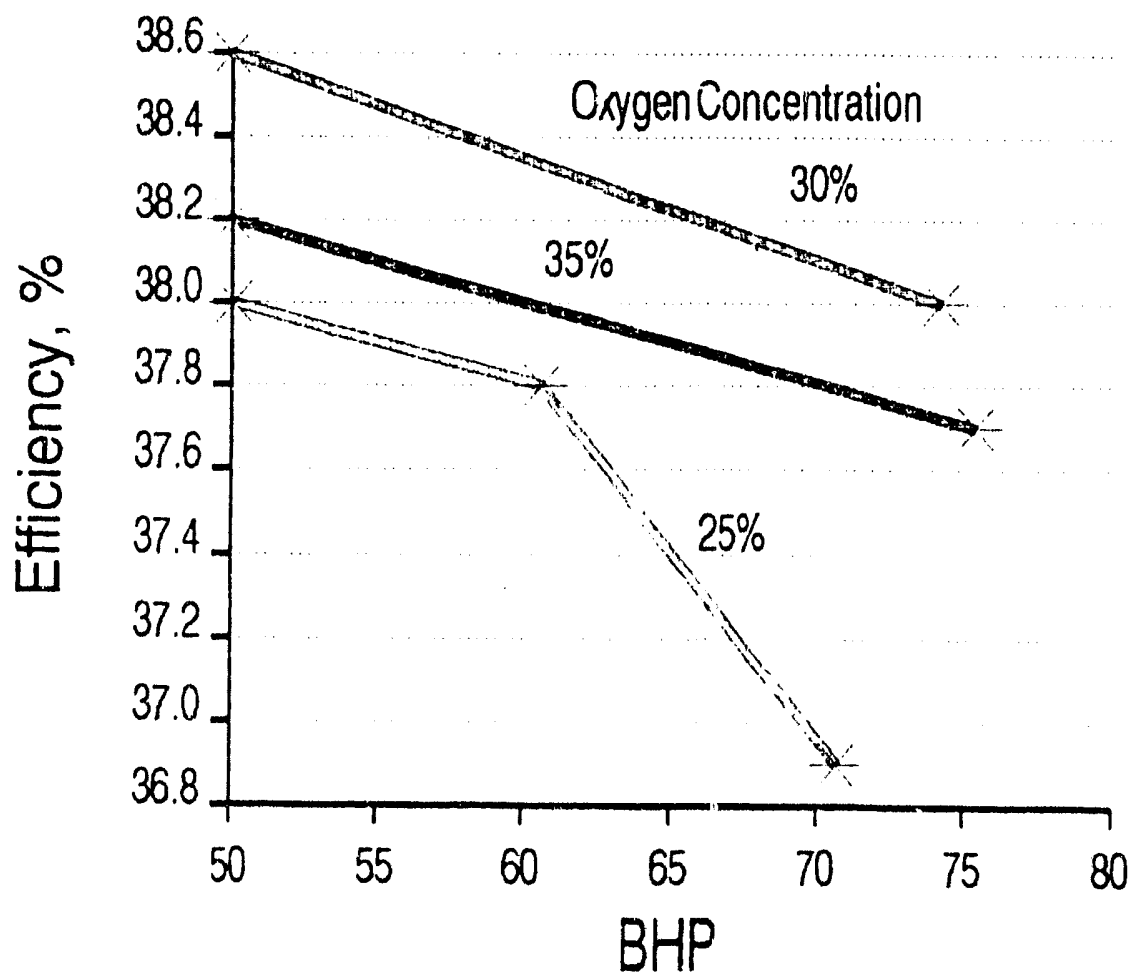

FIGURE A.118 Thermal Efficiency vs. Brake Horsepower, No. 2 Fuel, $100 \%$ Load, No Water 


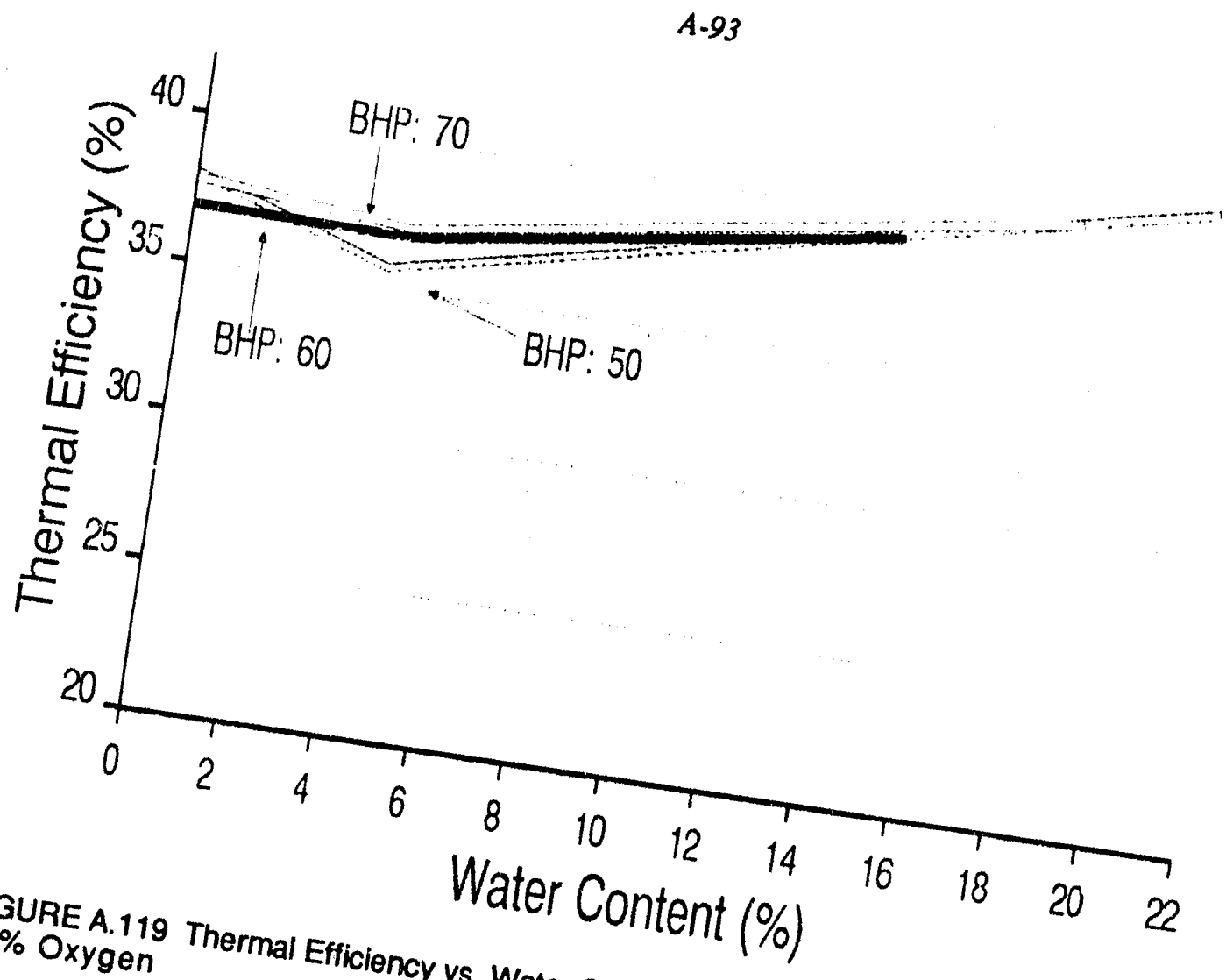

FIGURE A.119 Thermal Efficiency vs. Water Content, No. 2 Fuel, $100 \%$ Load,
Oxygen

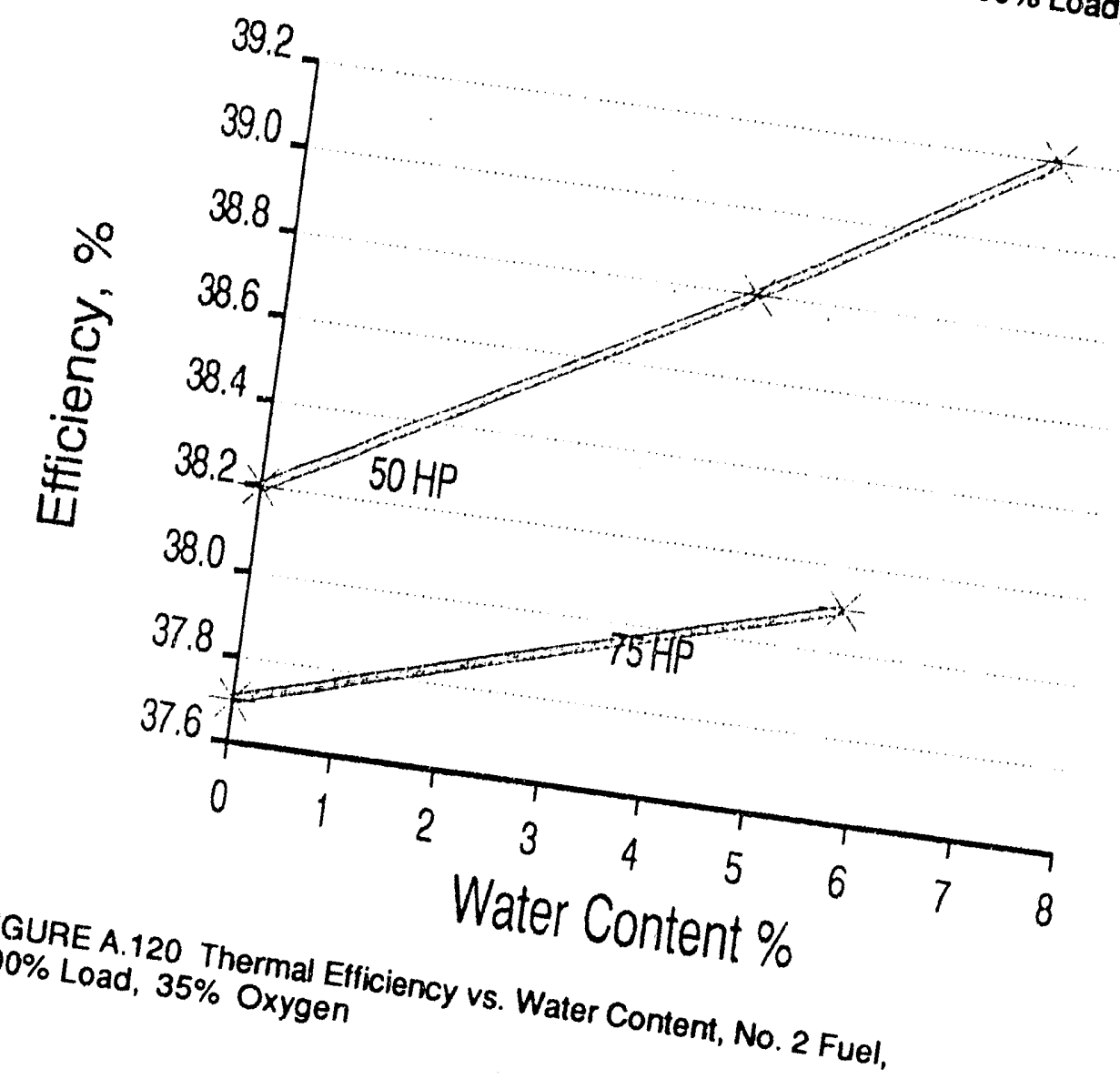




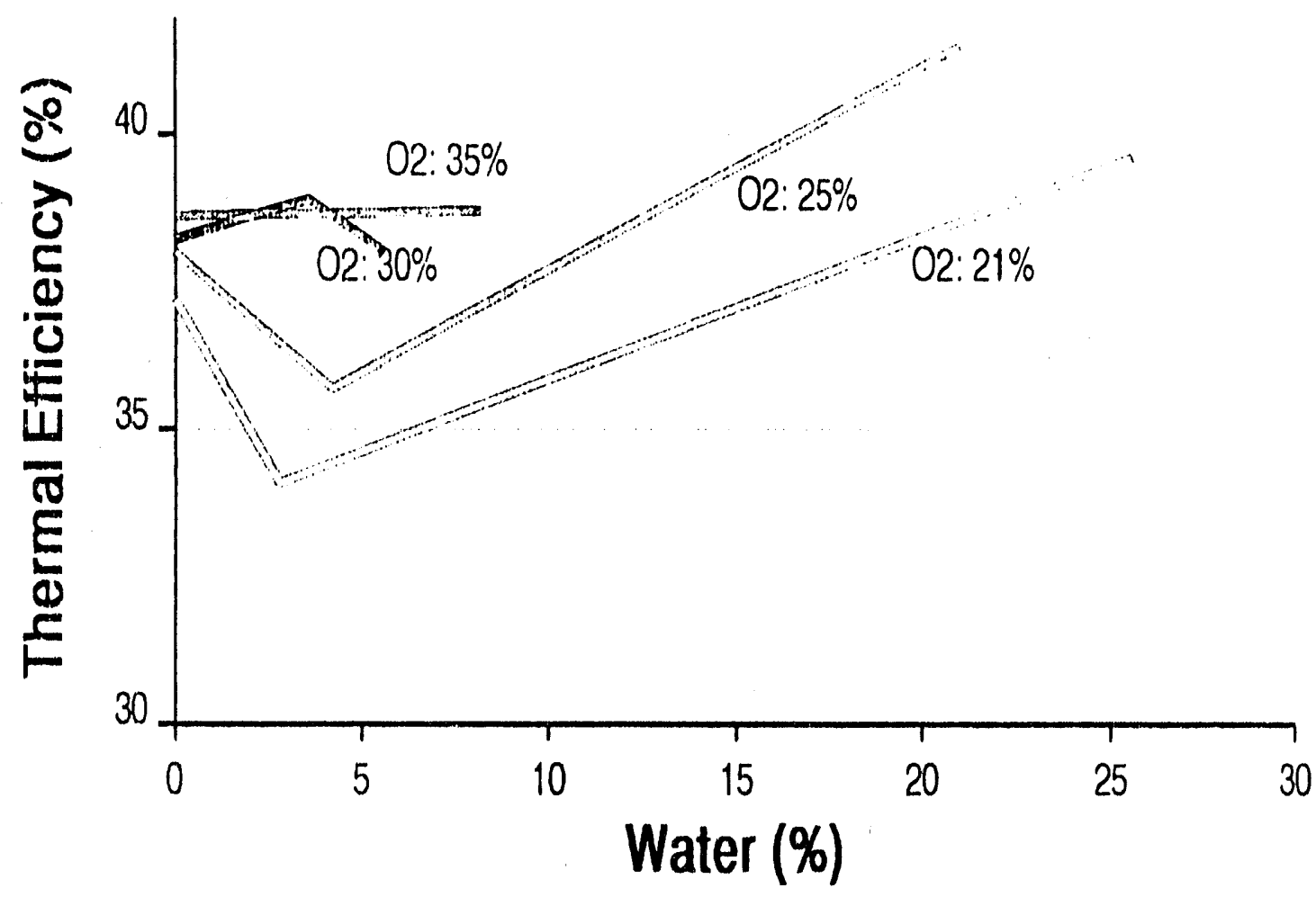

FIGURE A.121 Thermal Efficiency vs. Water Content, No. 2 Fuel, 100\% Load, 50 Horsepower 
A.10 Graphlc Display of Thermal Efficiency for No. 4 Fuel 
A-96 


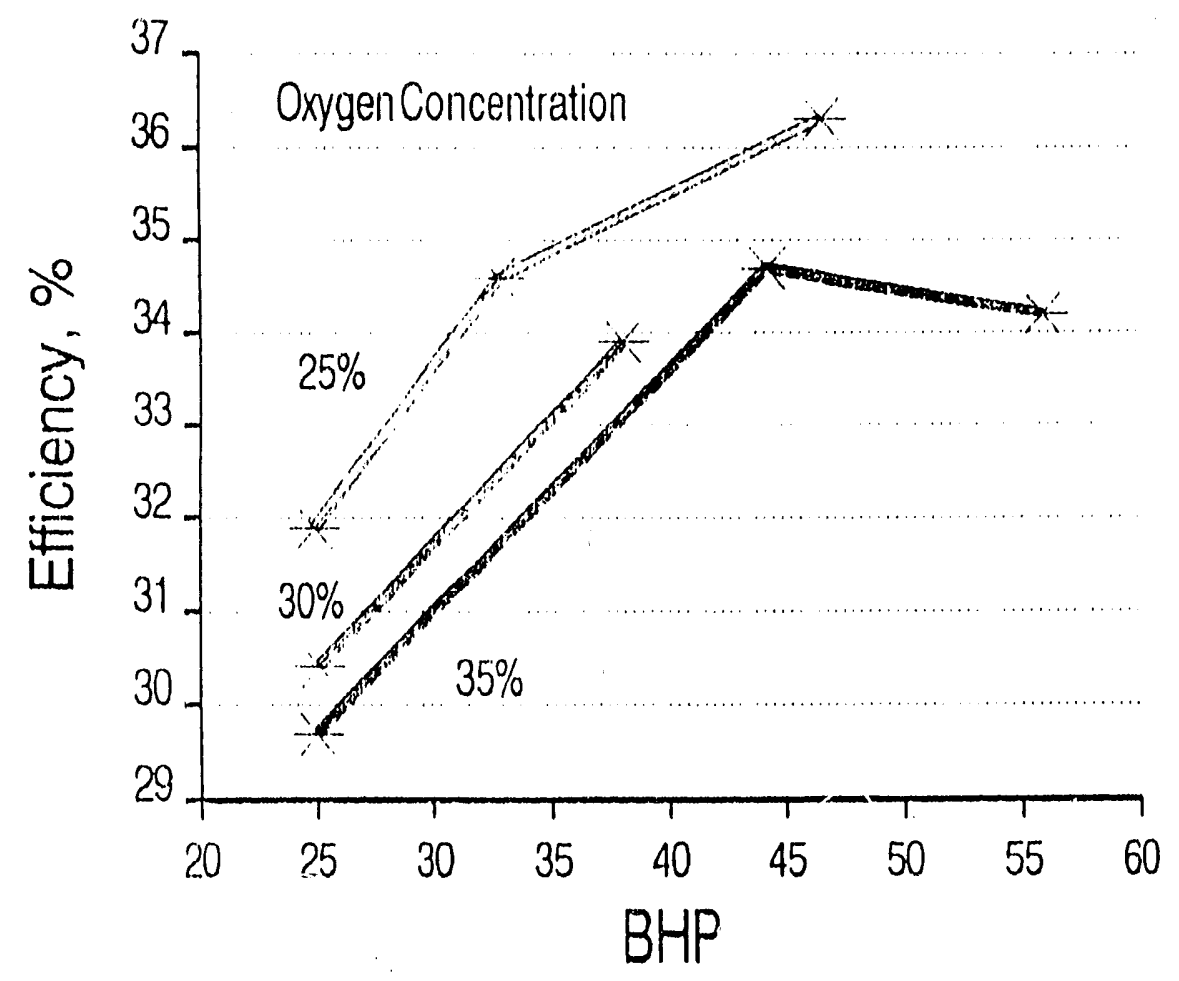

FIGURE A.122 Thermal Efficiency vs. Brake Horsepower, No. 4 Fuel, $50 \%$ Load, No Water

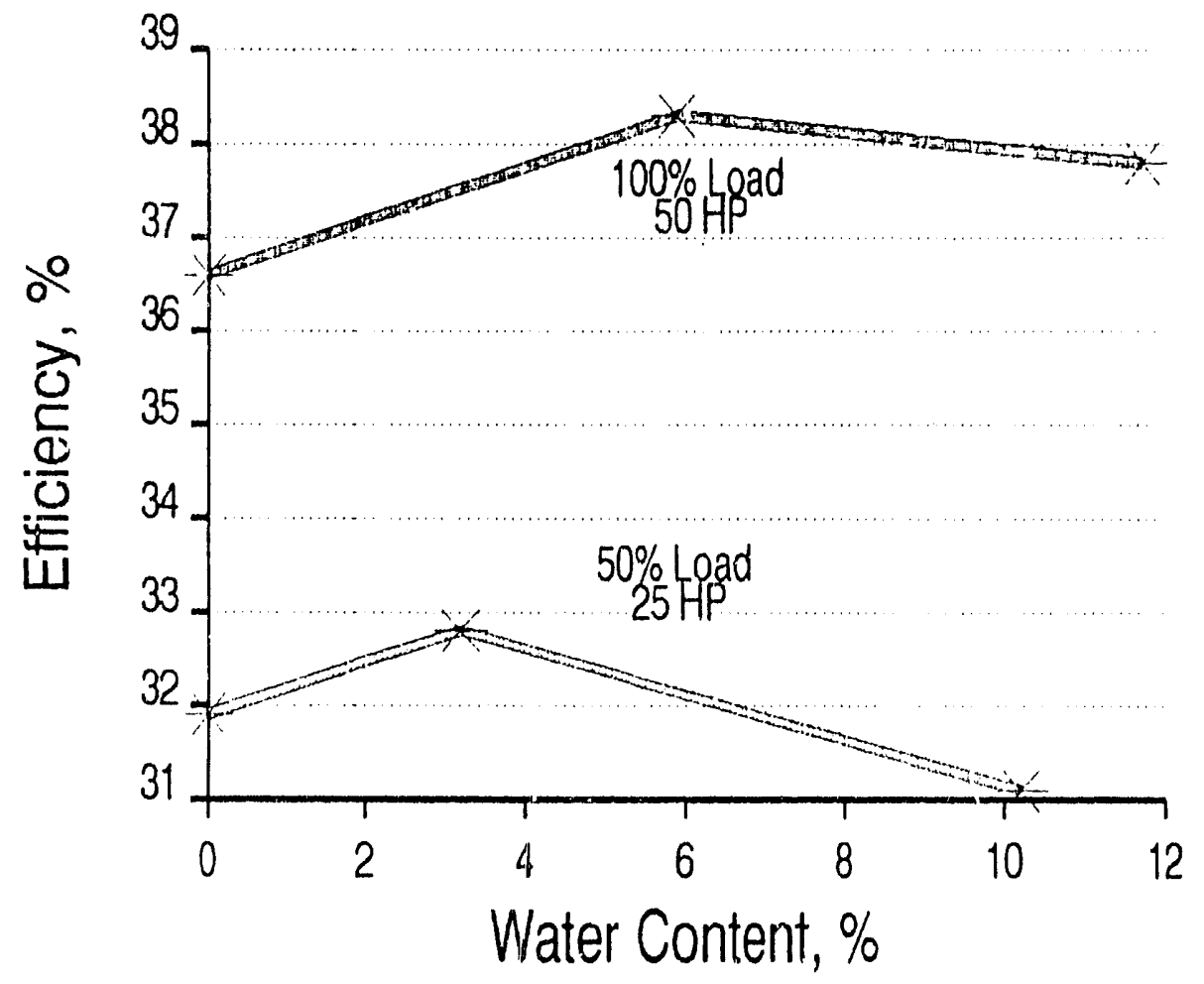

FIGURE A.123 Thermal Efficiency vs. Water Content, No. 4 Fuel, $21 \%$ Oxygen 


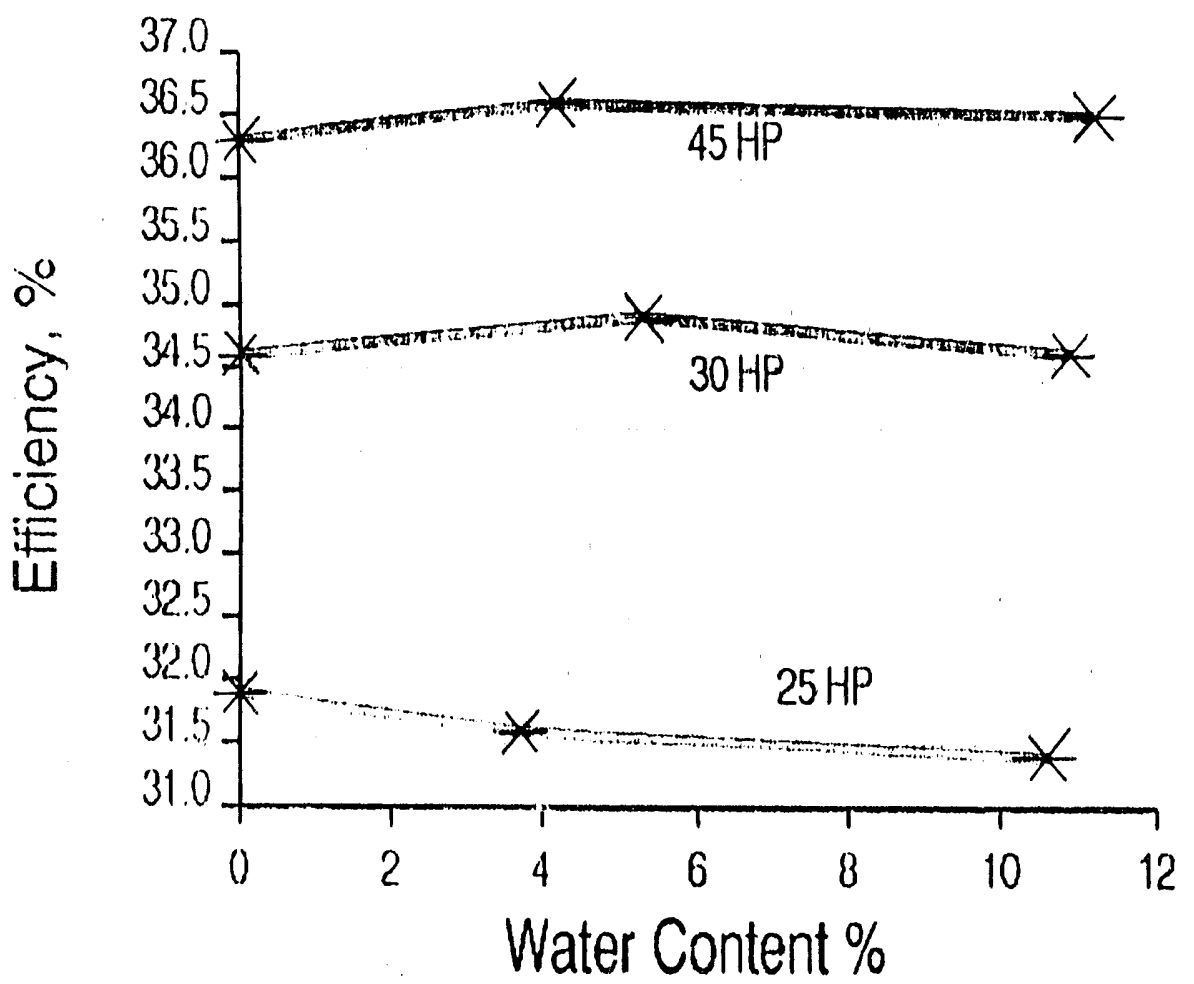

FIGURE A.124 Thermal Efficiency vs. Water Content, No. 4 Fuel, $50 \%$ Load, $25 \%$ Oxygen

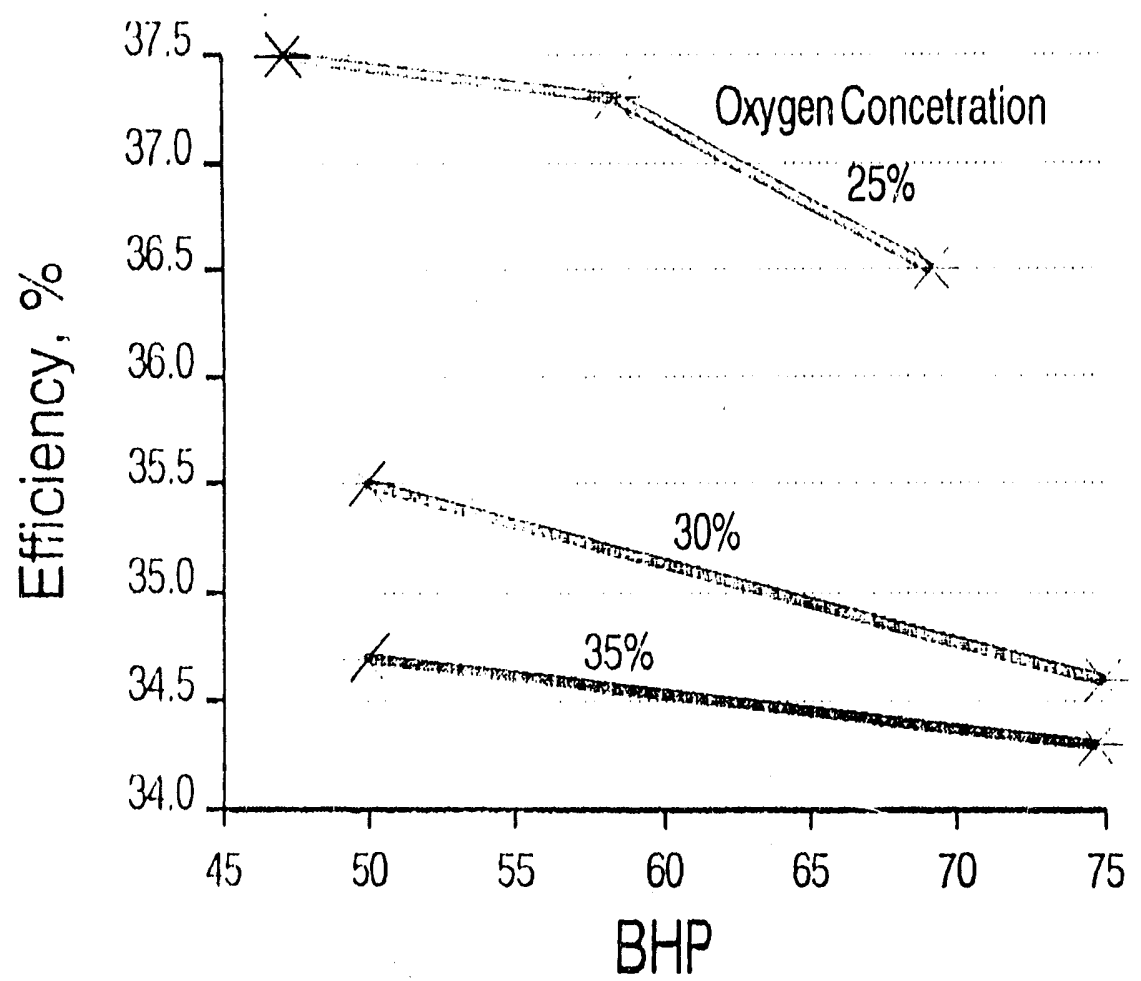

FIGURE A.125 Thermal Efficiency vs. Brake Horsepower, No. 4 Fuel, $100 \%$ Load, No Water 


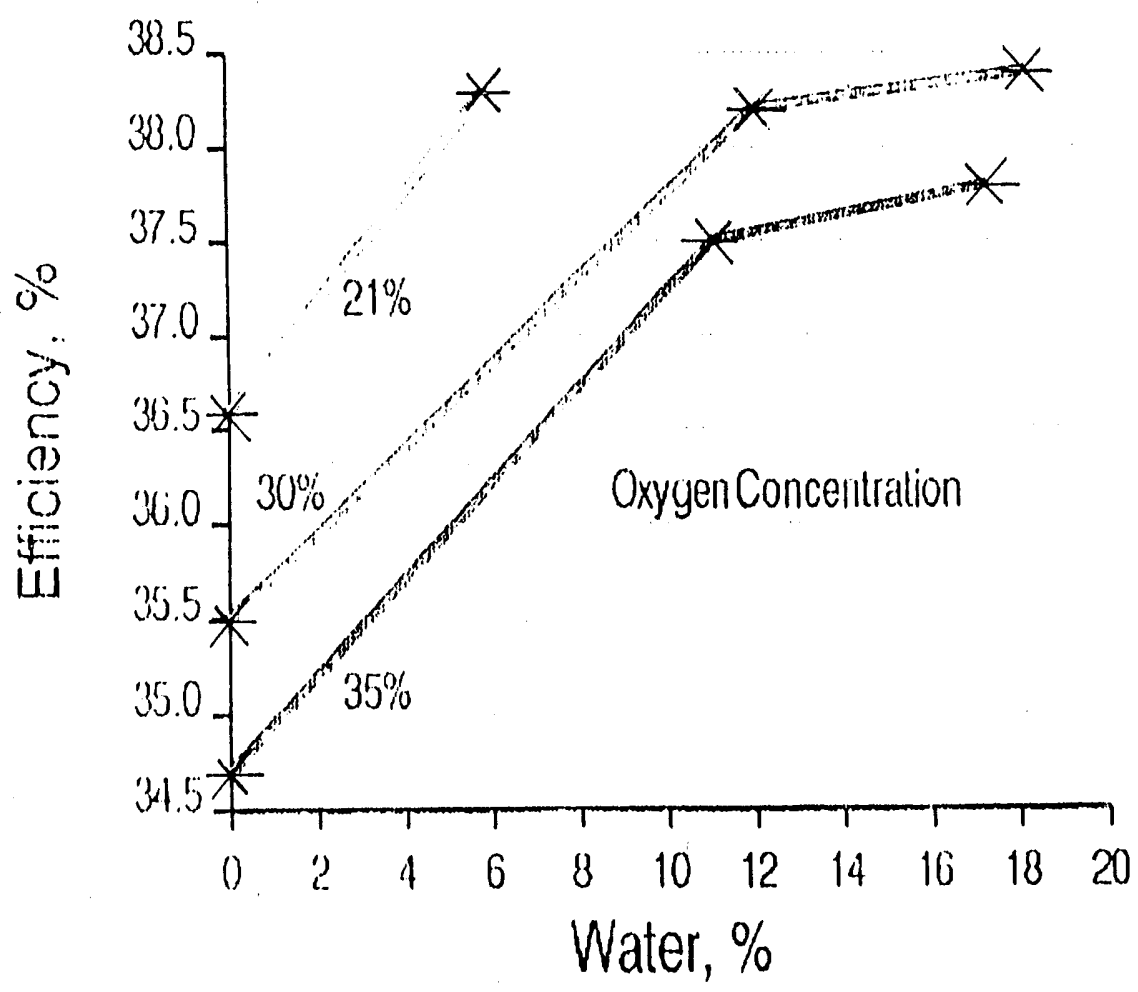

FIGURE A.126 Thermal Efficiency vs. Water Content, No. 4 Fuel, $100 \%$ Load, 50 Horsepower

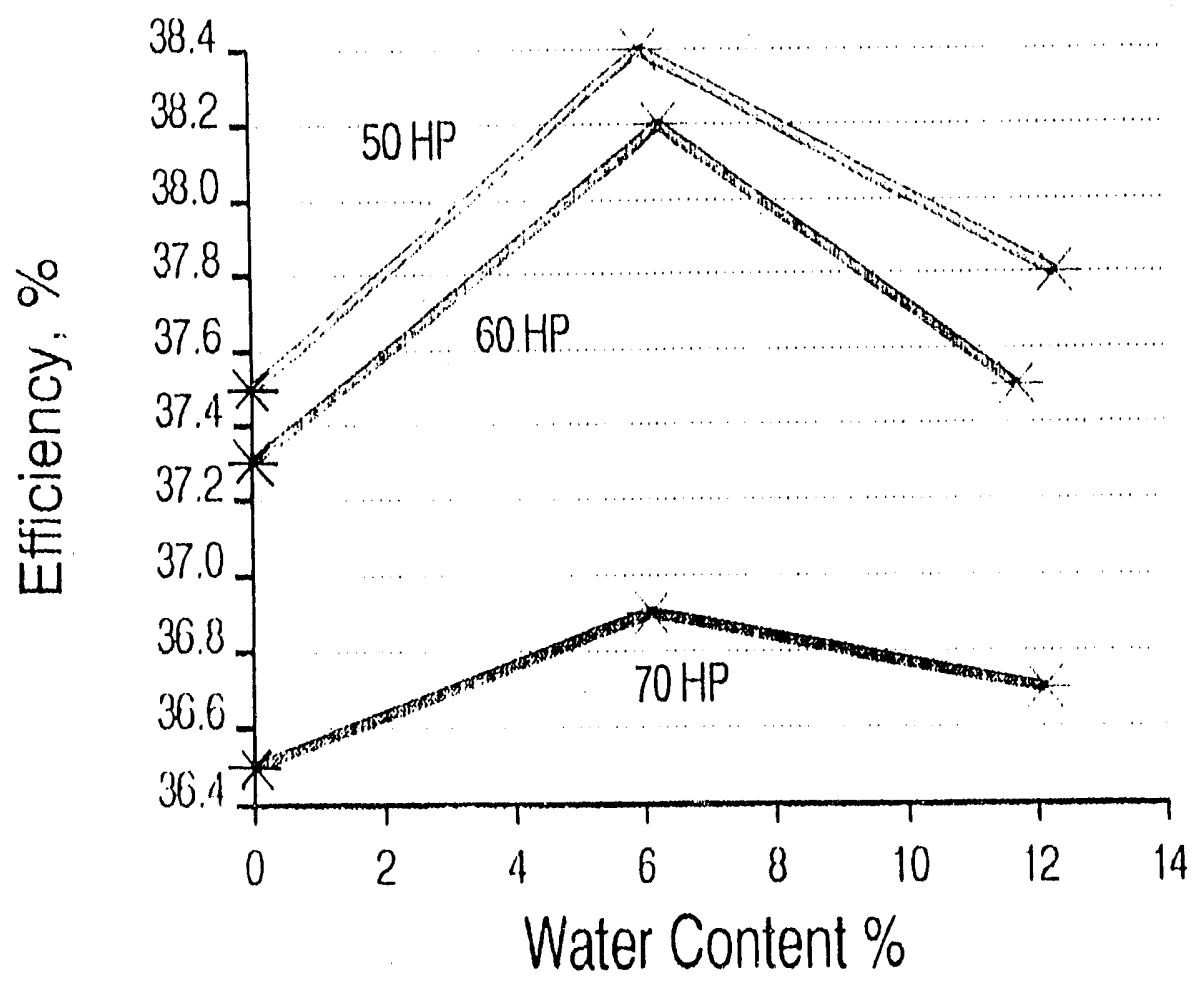

FIGURE A.127 Thermal Efficiency vs. Water Content, No. 4 Fuel, $100 \%$ Load, $25 \%$ Oxygen 


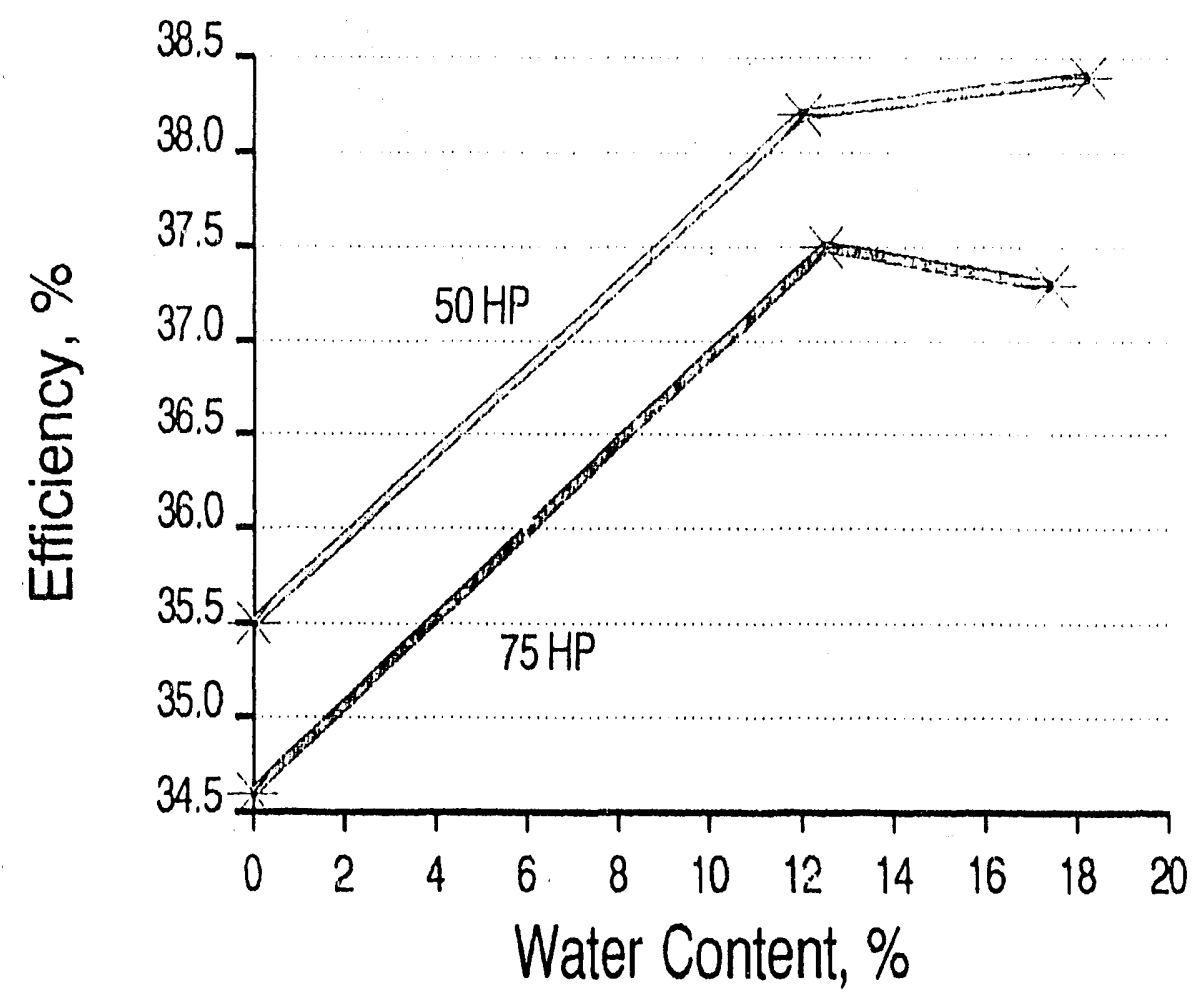

FIGURE A.128 Thermal Efficiency vs. Water Content, No. 4 Fuel, $100 \%$ Load, $30 \%$ Oxygen

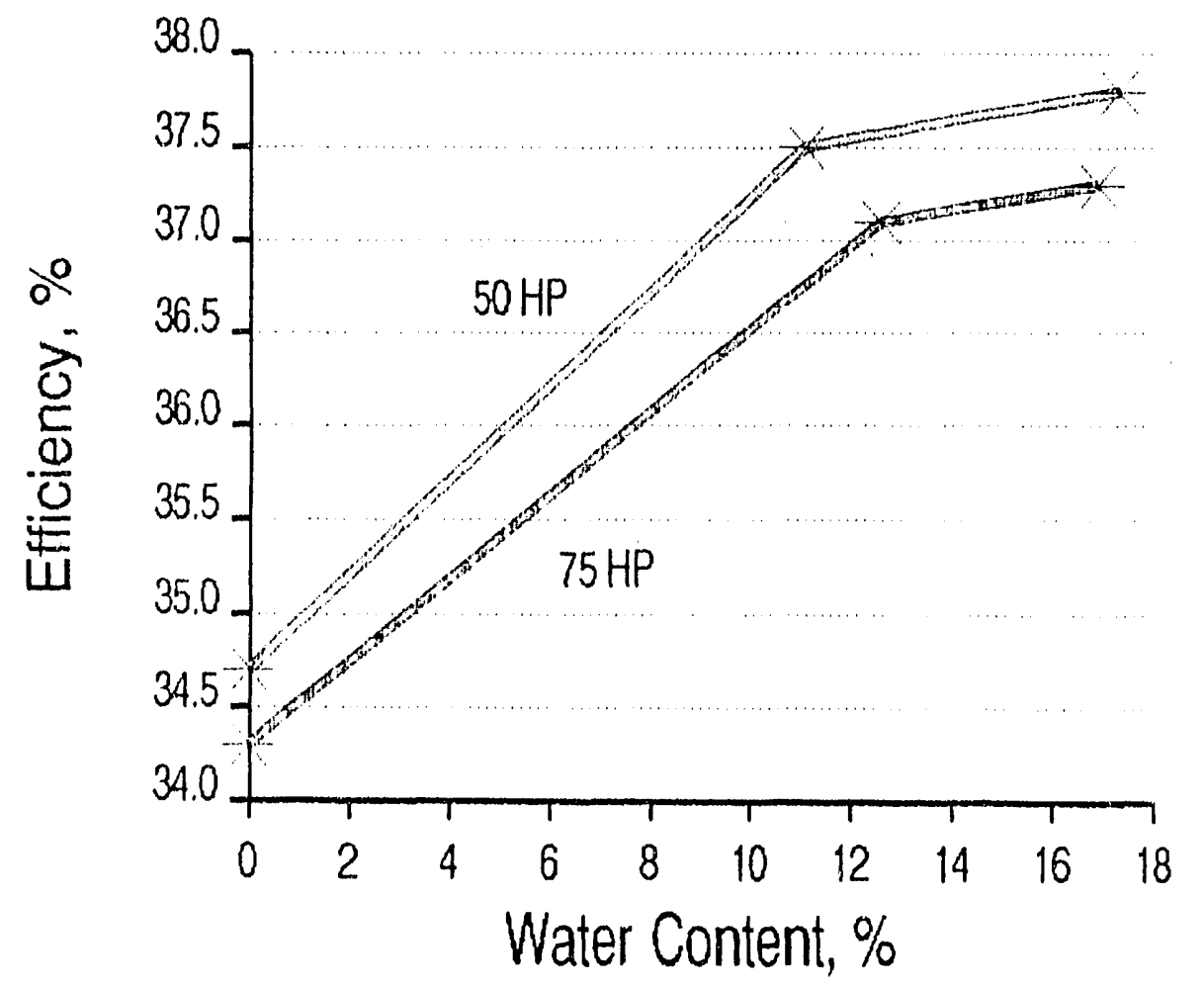

FIGURE A.129 Thermal Efficiency vs. Water Content, No. 4 Fuel, $100 \%$ Load, $35 \%$ Oxygen 
A.11 Comparison of Thermal Efficiency for No. 2 and No. 4 Fuels 


\section{A-102}




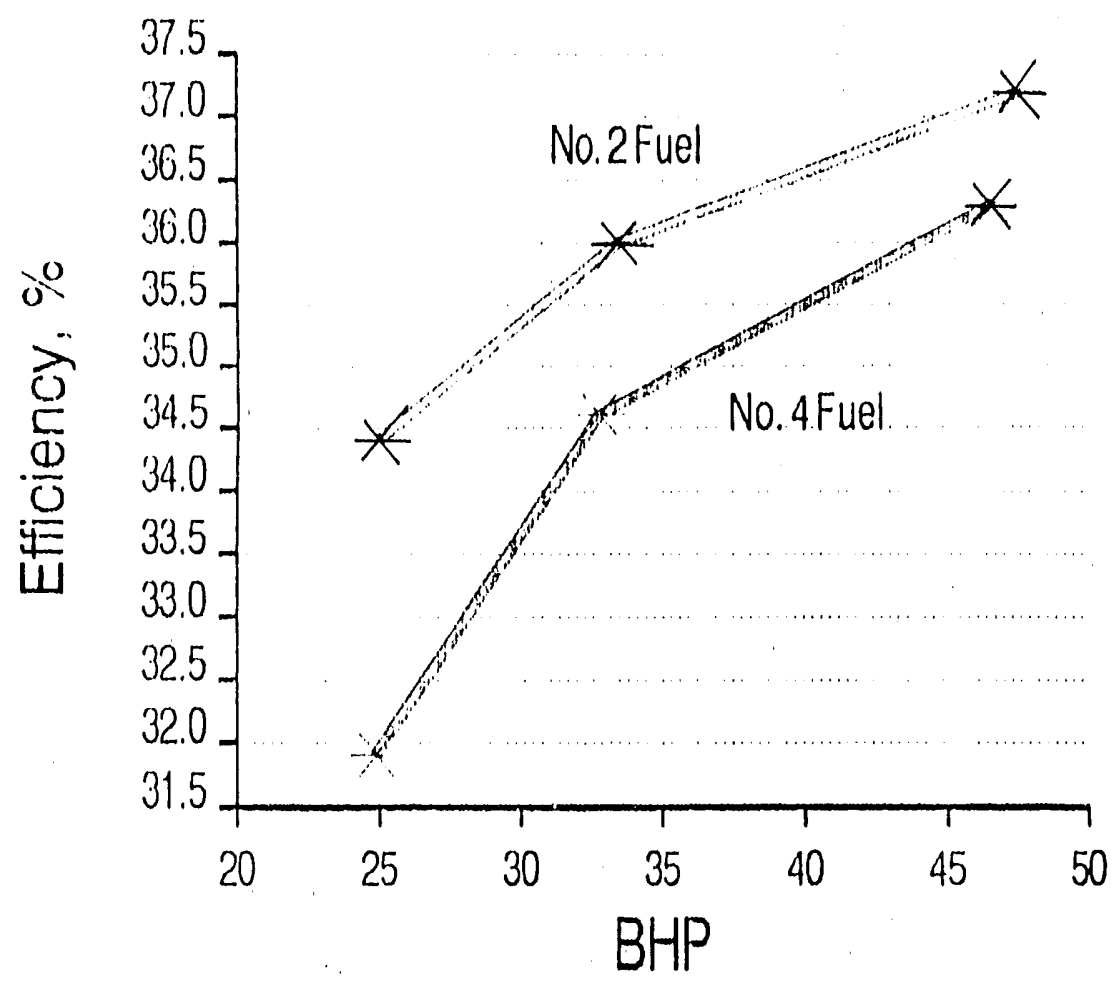

FIGURE A.130 Thermal Efficiency vs. Brake Horsepower, 50\% Load, No Water, $25 \%$ Oxygen

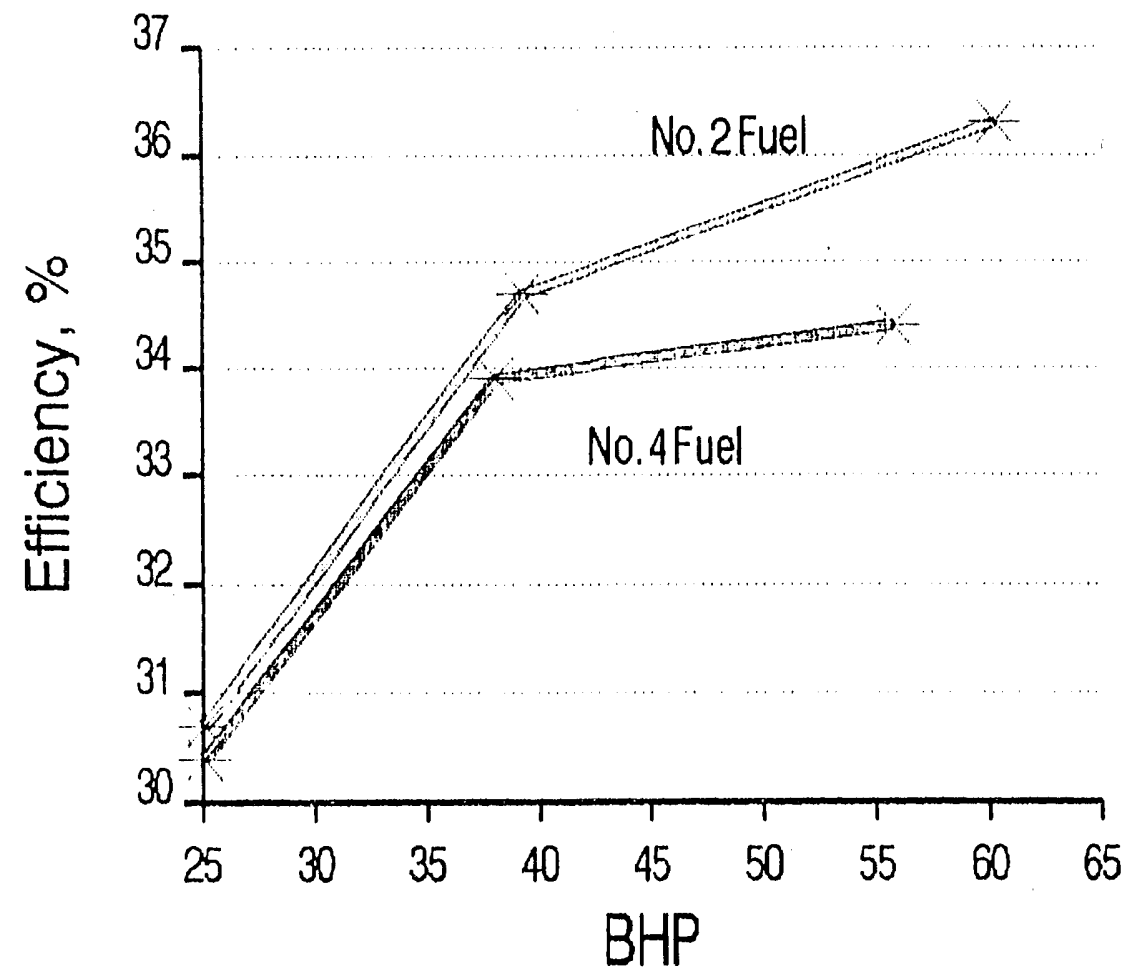

FIGURE A.131 Thermal Efficiency vs. Brake Horsepower, 50\% Load, No Water, $30 \%$ Oxygen 


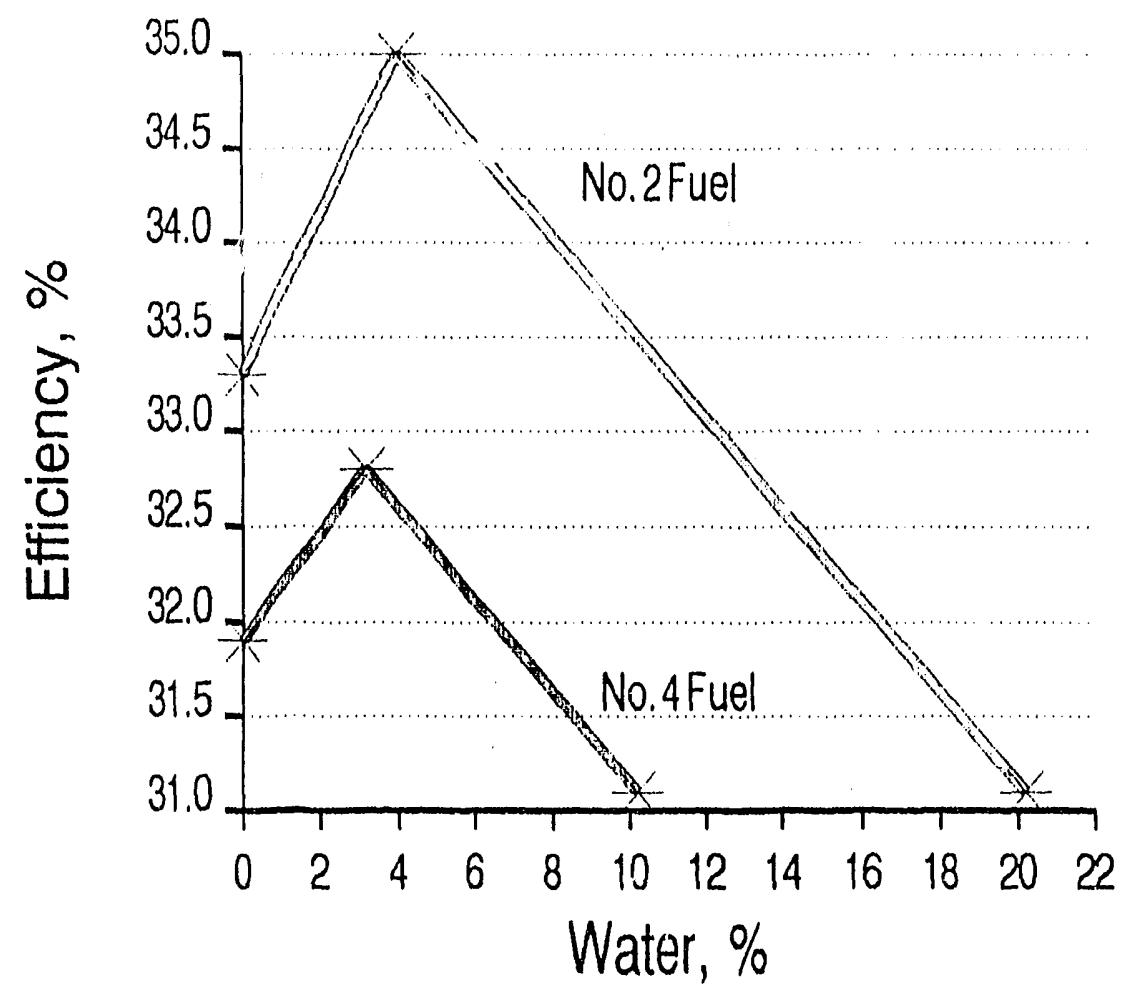

FIGURE A.132 Thermal Efficiency vs. Water Content, 50\% Load, 25 Horsepower, $21 \%$ Oxygen

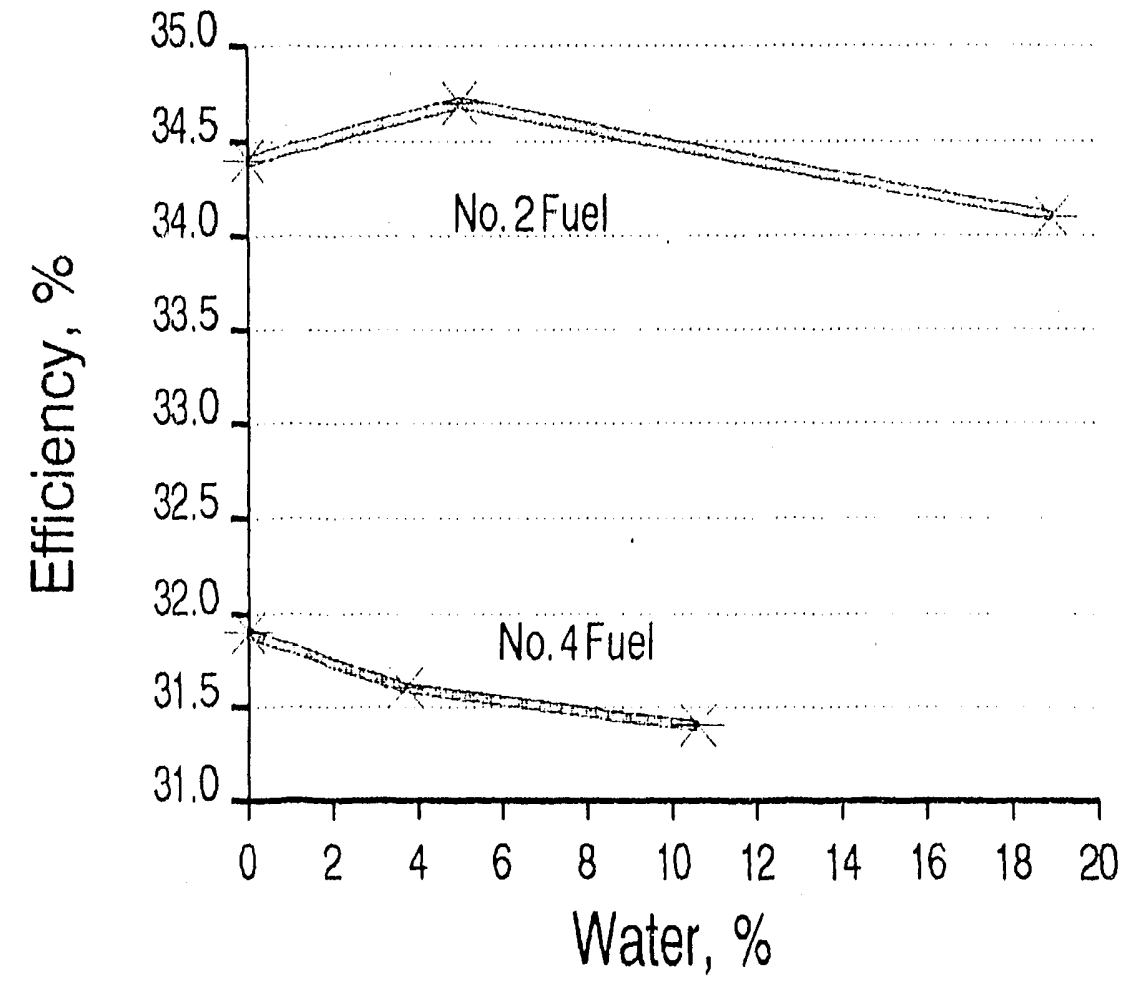

FIGURE A.133 Thermal Efficiency vs. Water Content, 50\% Load, 25 Horsepower, 25\% Oxygen 


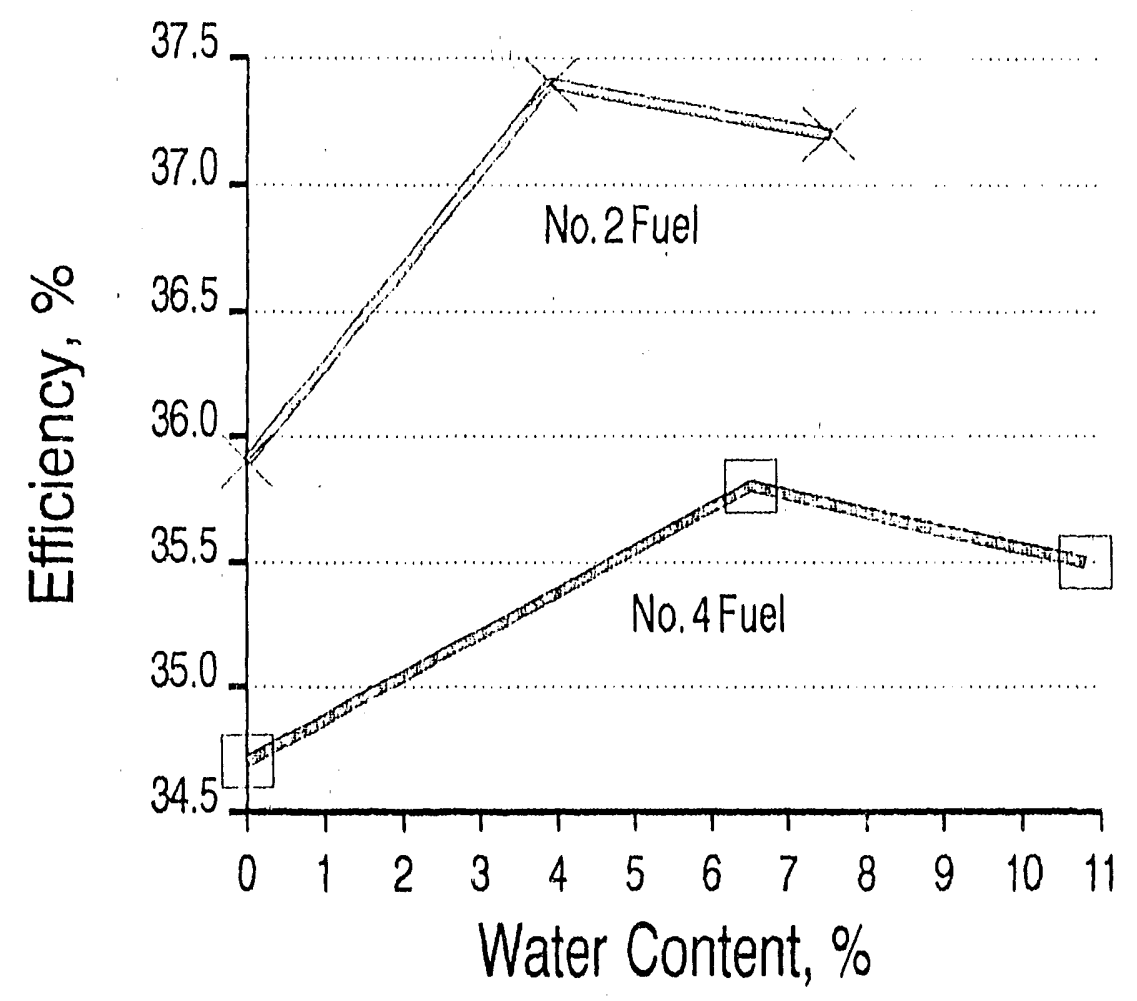

FIGURE A.134 Thrermal Efficiency vs. Water Content, 50\% Load, 45 Horsepower, $35 \%$ Oxygen

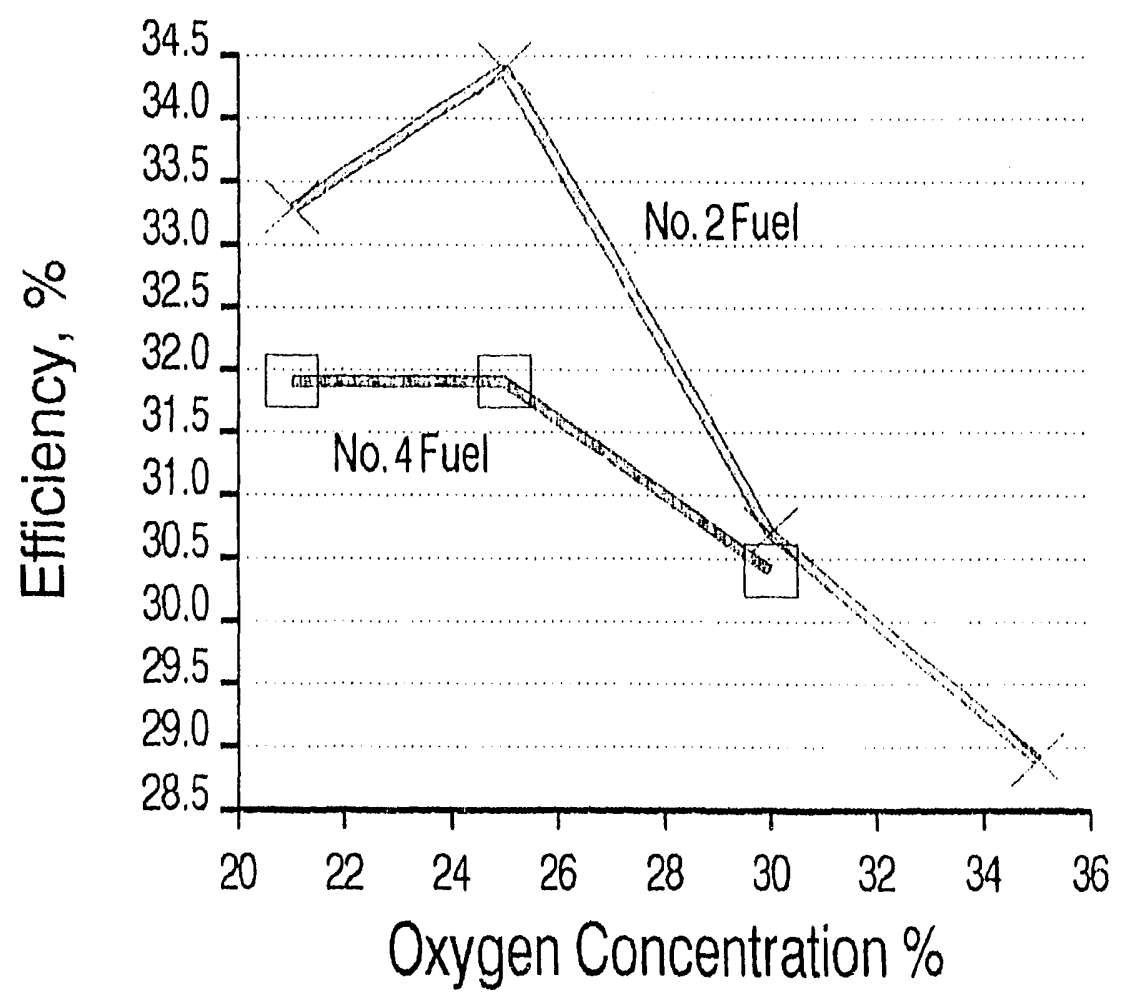

FIGURE A.135 Thermal Efficiency vs. Oxygen Concentration, $50 \%$ Load, 25 Horsepower, No Water 


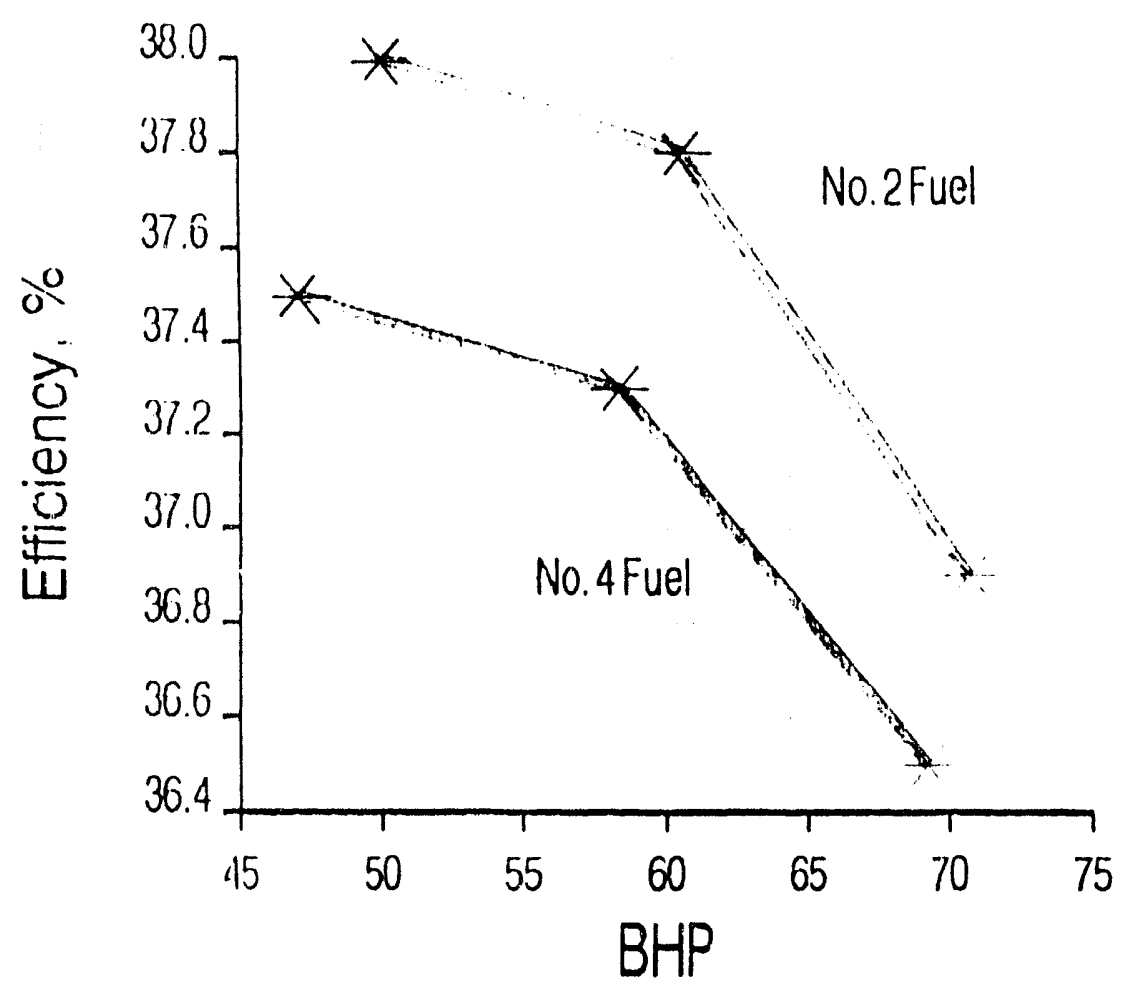

FIGURE A.136 Thermal Efficiency vs. Brake Horsepower, 100\% Load, No Water, $25 \%$ Oxygen

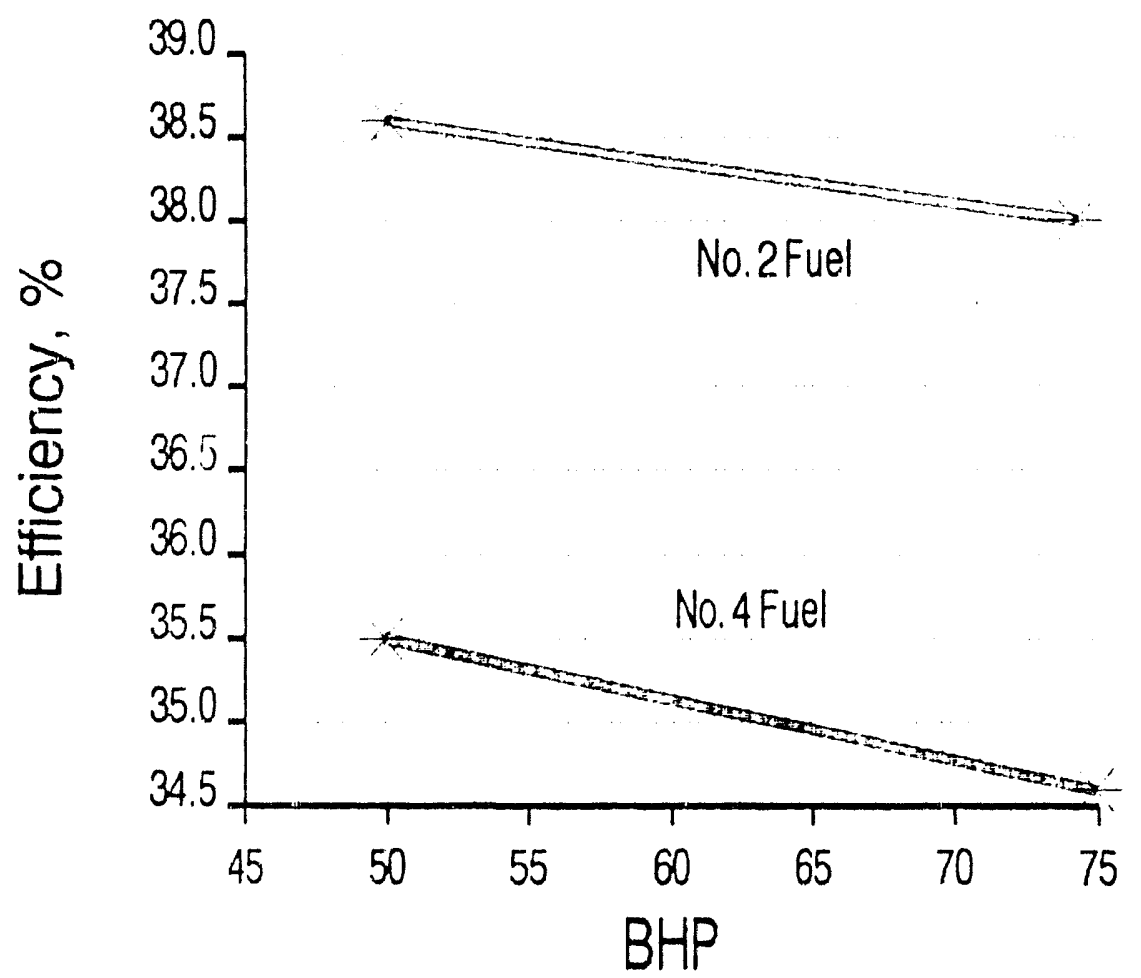

FIGURE A.137 Thermal Etficiency vs. Brake Horsepower, 100\% Load, No Water, $30 \%$ Oxygen 
A-107

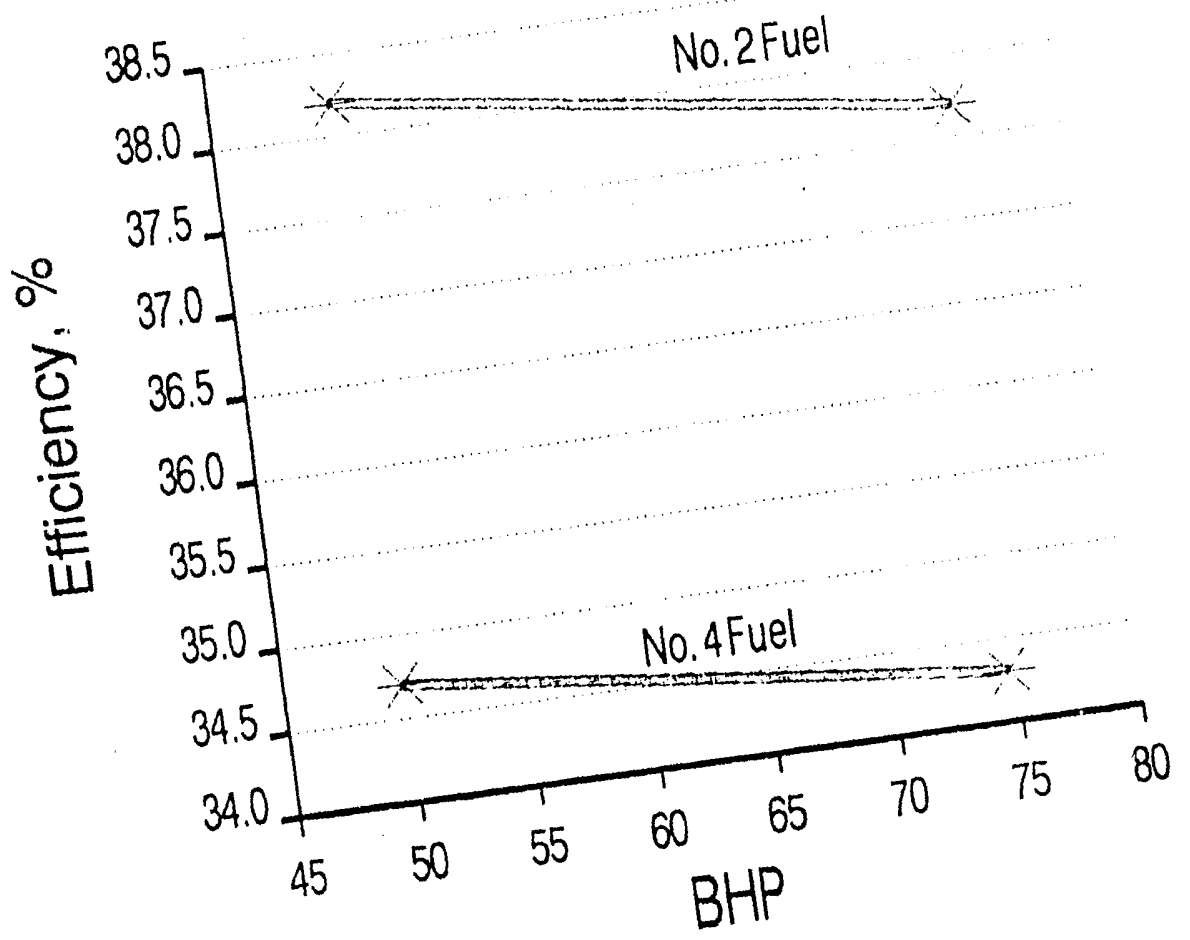

FIGURE A.138 Thermal Efficiency vs. Brake Horsepower,

$100 \%$ Load, No Water, $35 \%$ Oxygen

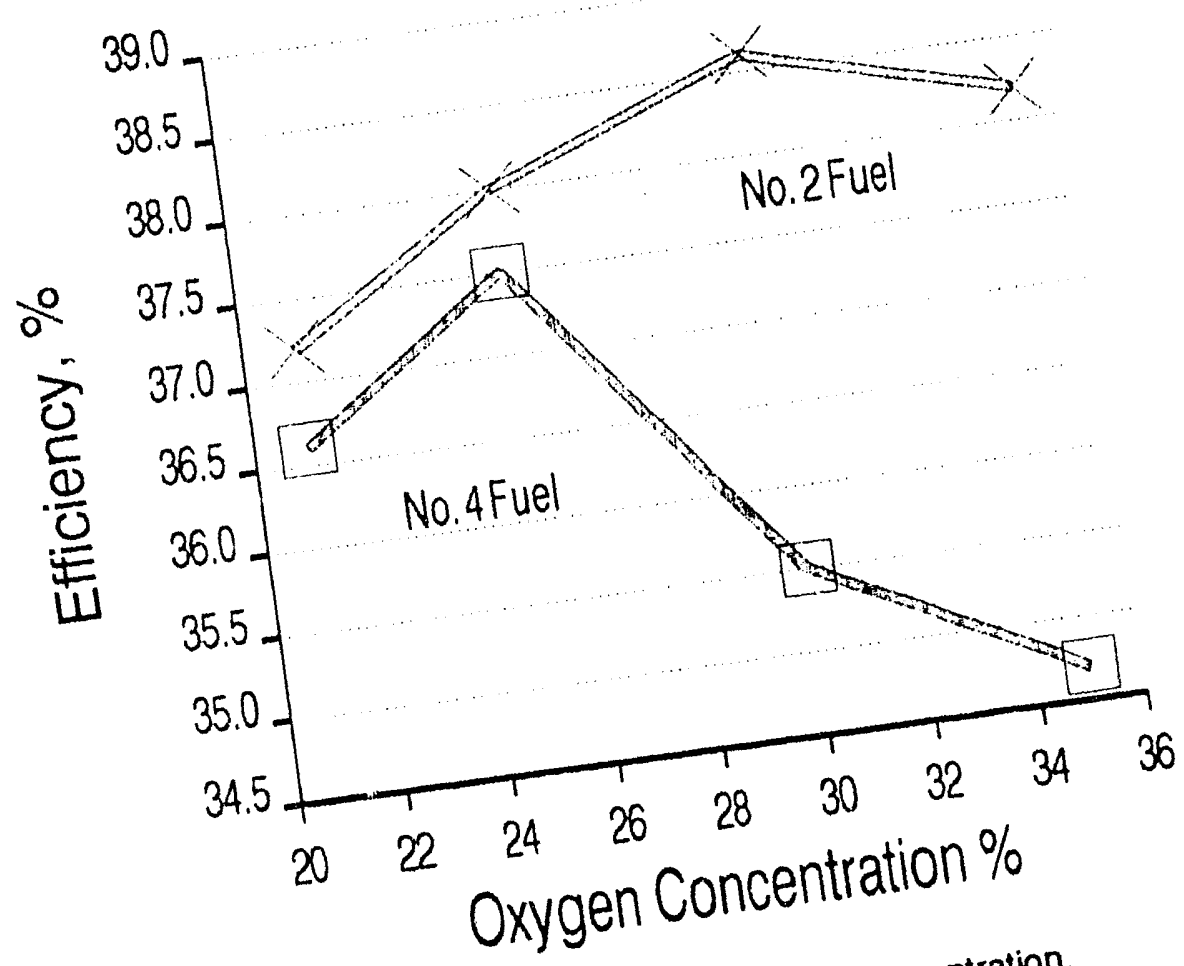

FIGURE A.139 Thermal Efficiency vs. Oxygen Concentration, $100 \%$ Load, 50 Horsepower, No Water 


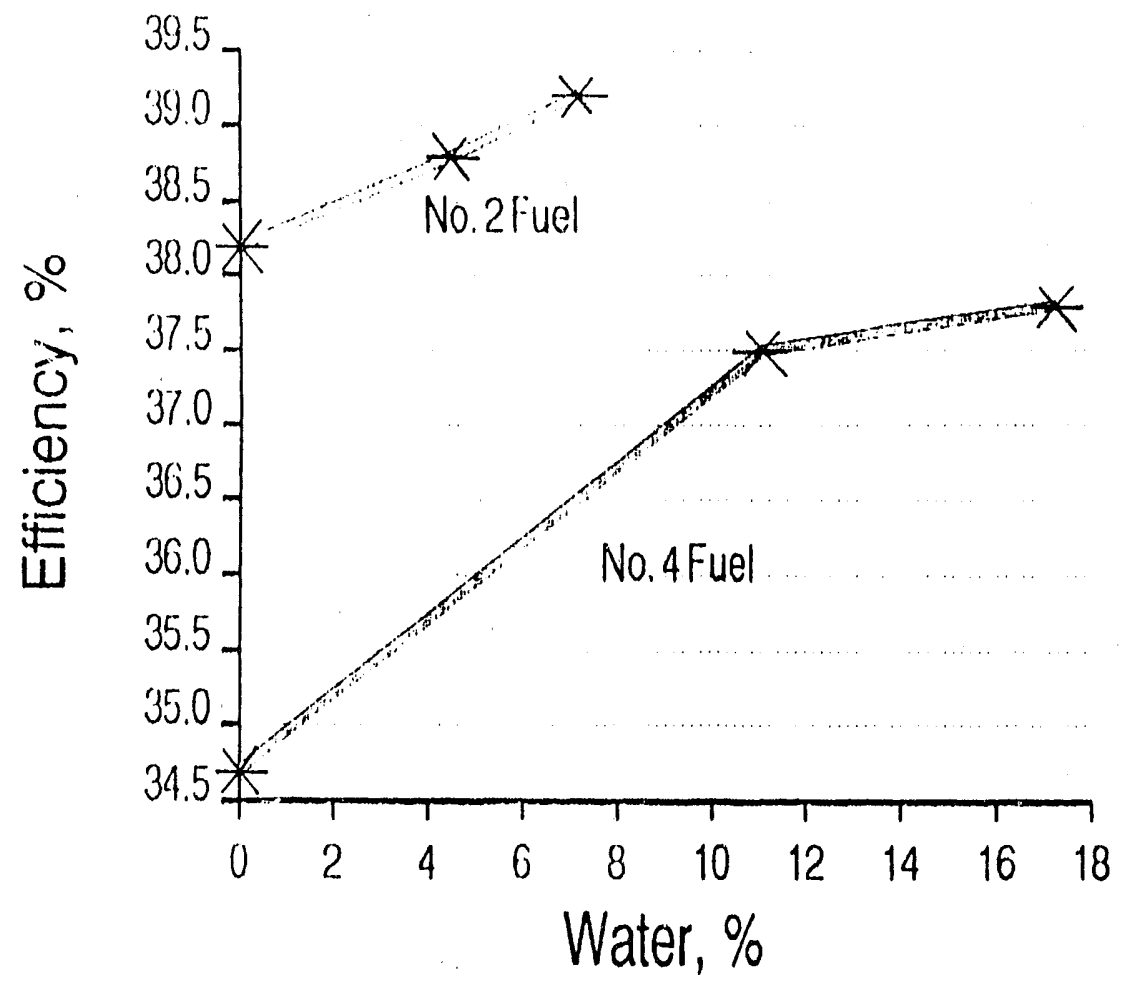

FIGURE A.140 Thermal Efficiency vs. Water Content, 100\% Load, 50 Horsepower, $35 \%$ Oxygen 
A.12 Graphic Display of Smoke Emissions 
A-11]

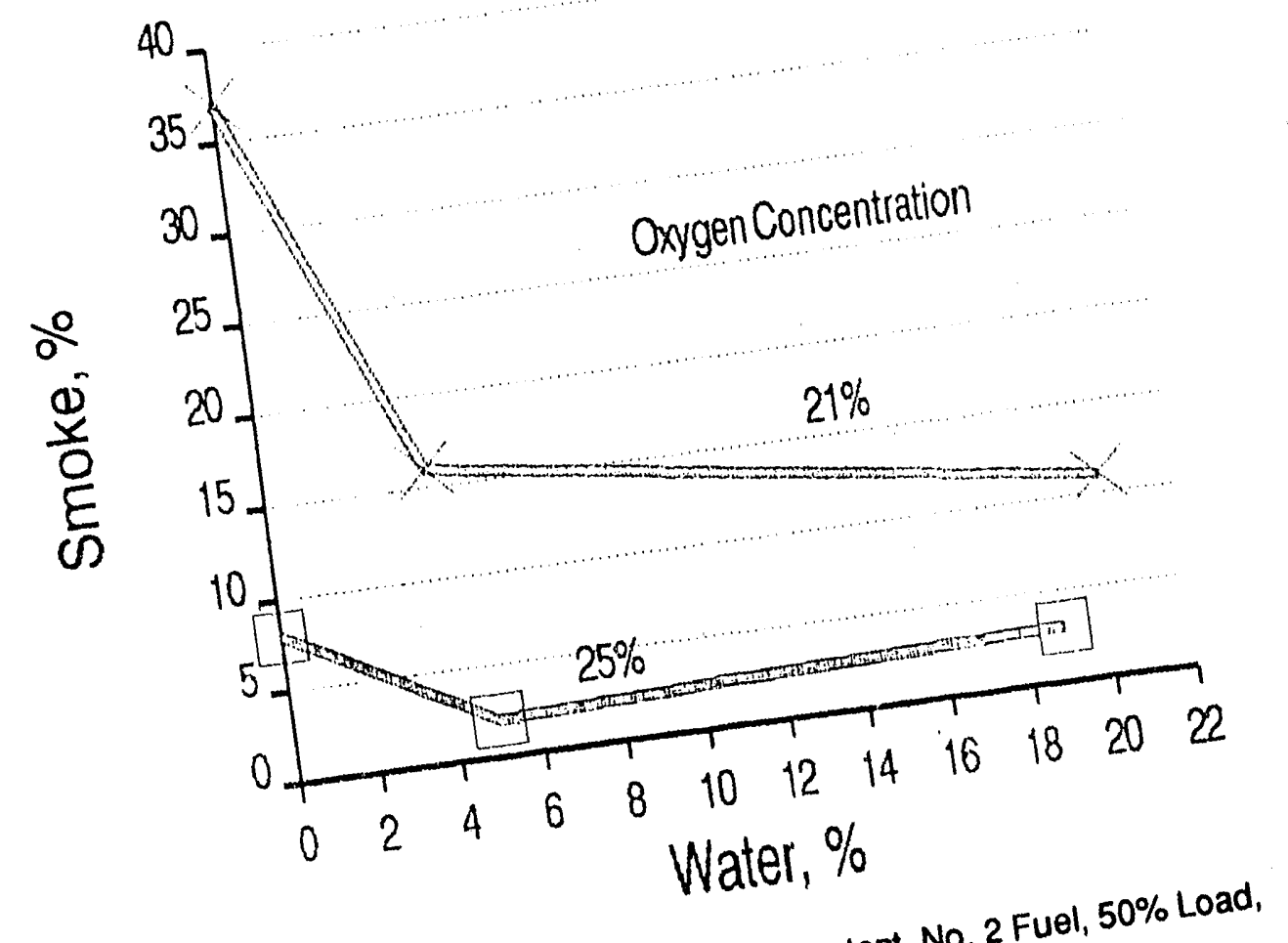

FIGURE A.141 Smoke Emission vs. Water Content, No. 2 Fuel, 25 Horsepower

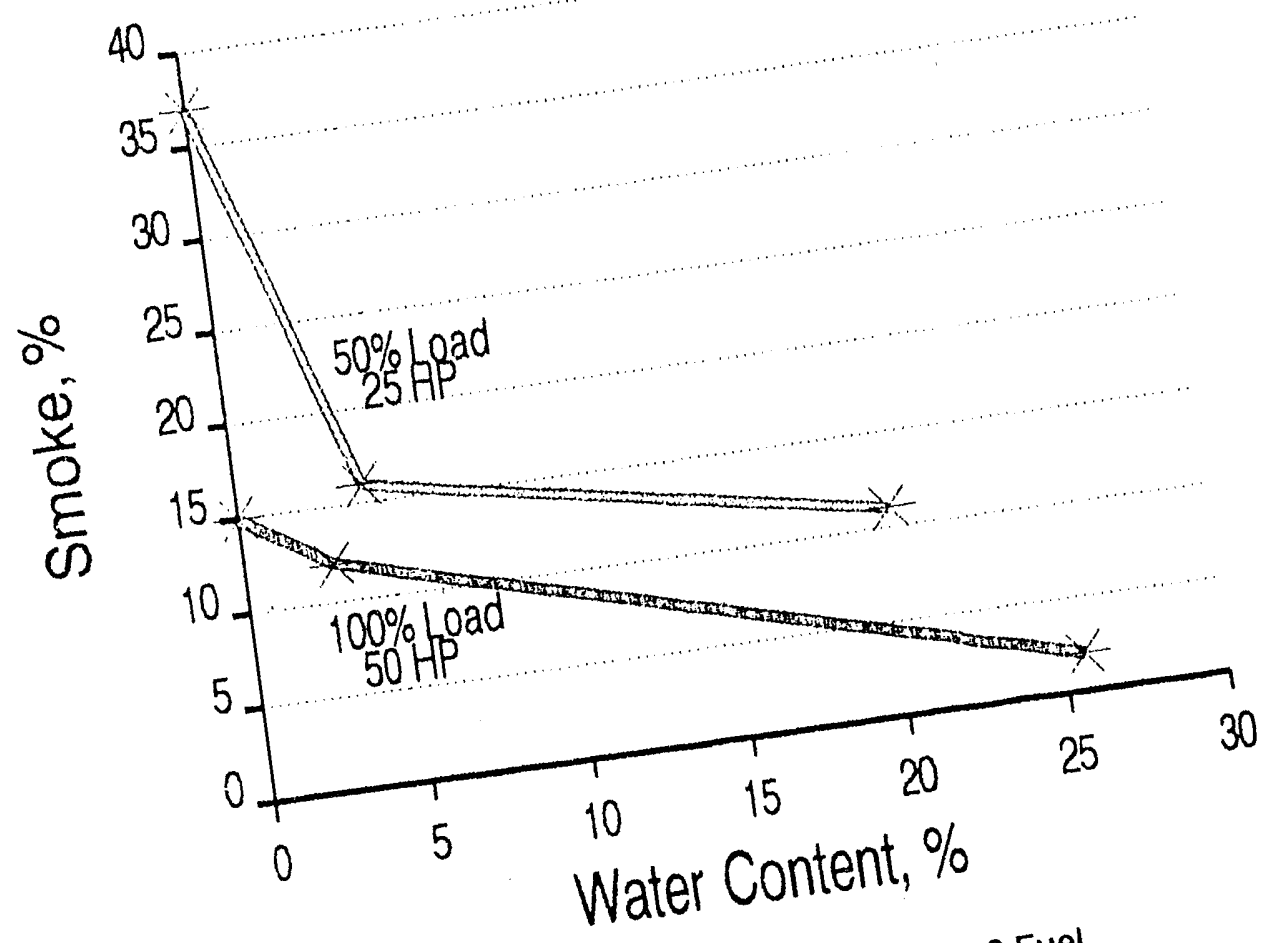

FIGURE A.142 Smoke Emission vs. Water Content, No. 2 Fuel, $21 \%$ Oxygen 


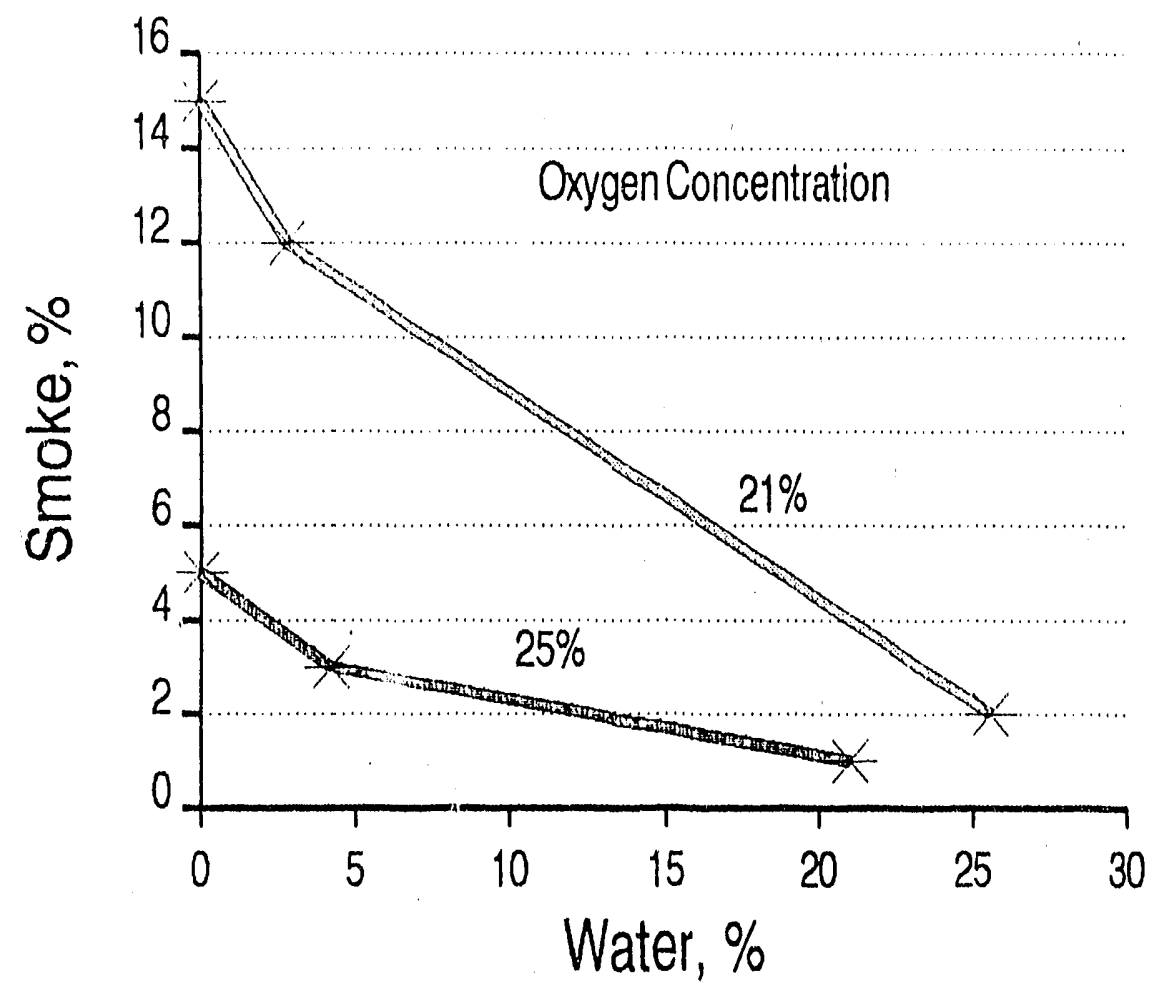

FIGURE A.143 Smoke Emission vs. Water Content, No. 2 Fuel, $100 \%$ Load, 50 Horsepower

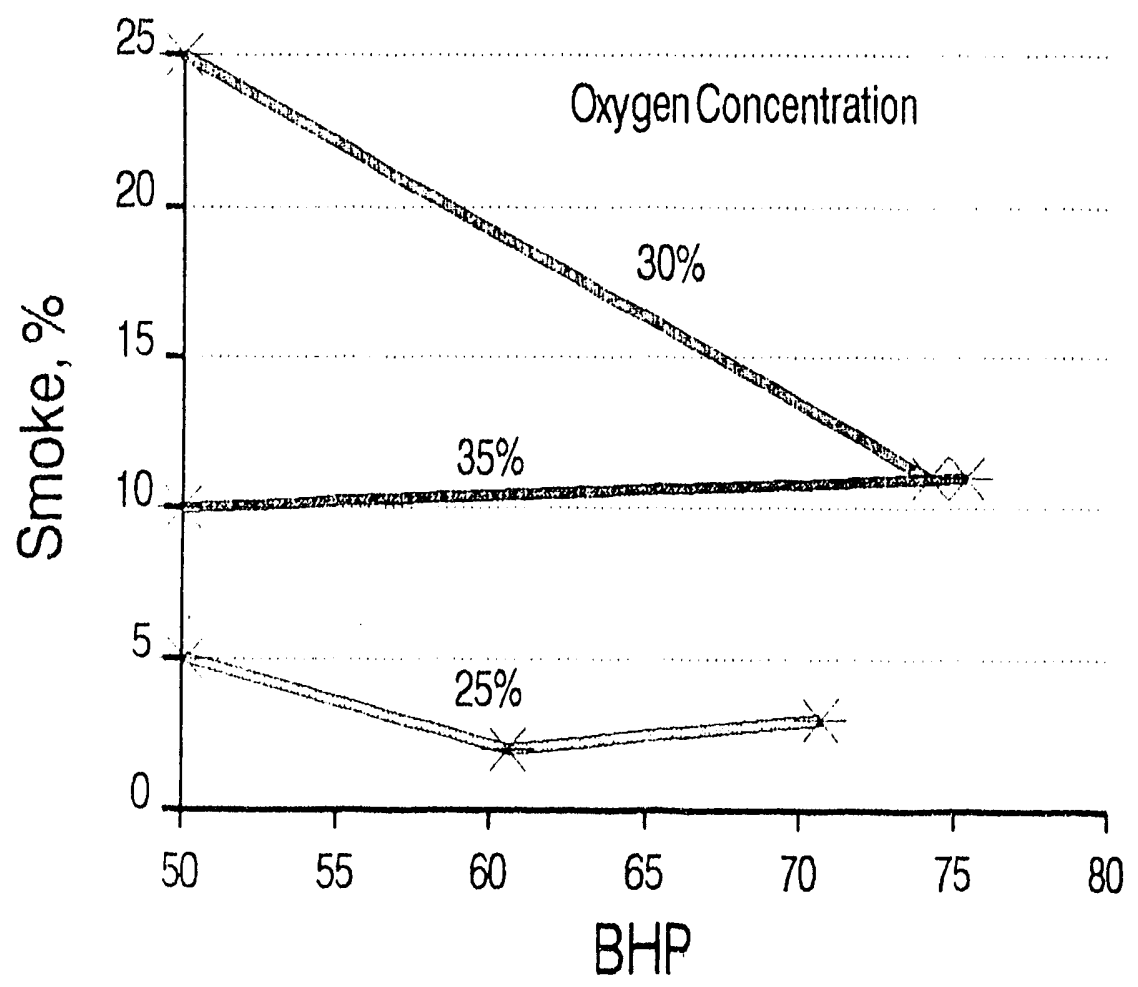

FIGURE A.144 Smoke Emission vs. Brake Horsepower, No. 2 Fuel, $100 \%$ Load, No Water 


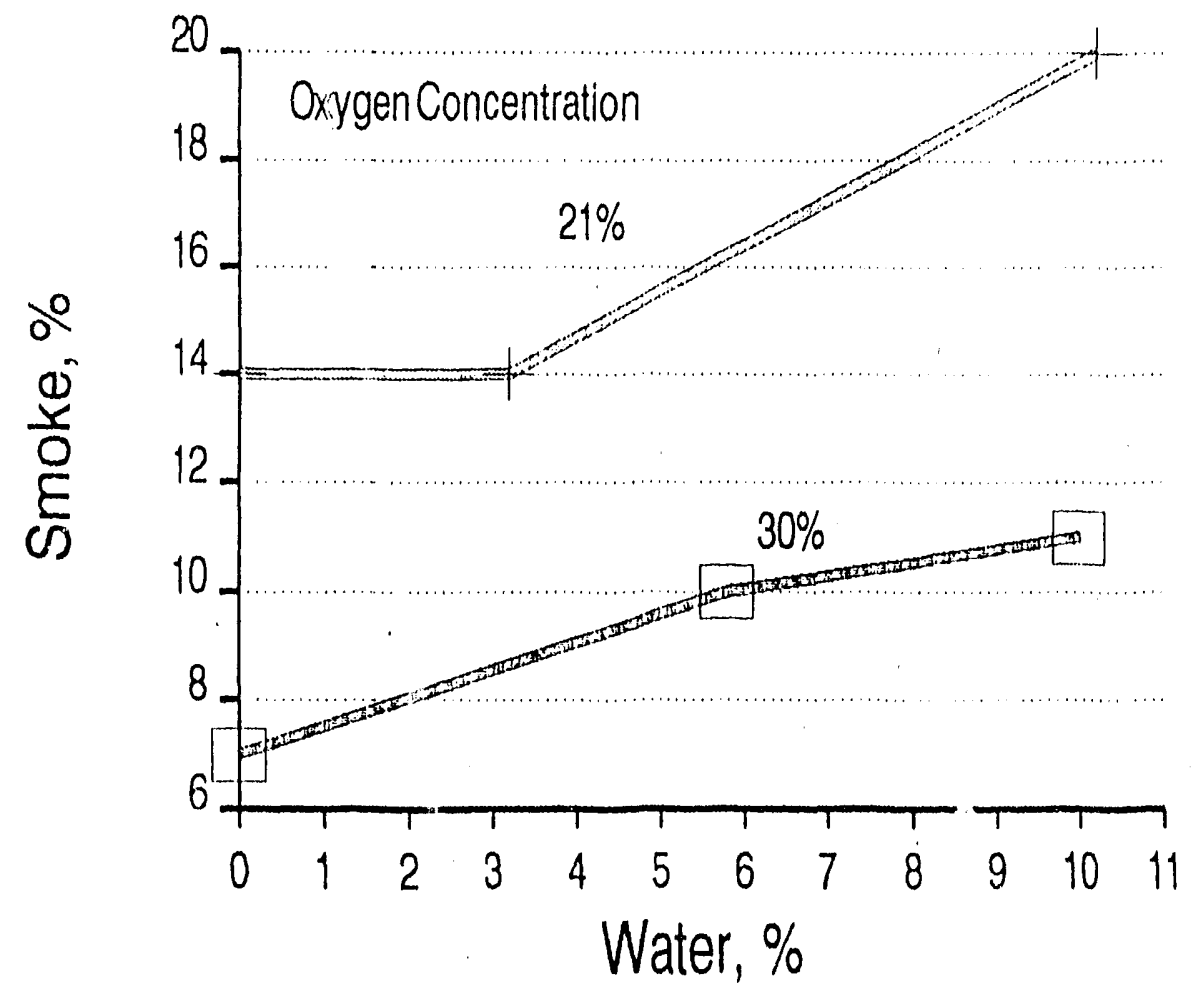

FIGURE A.145 Smoke Emission vs. Water Content, No. 4 Fuel, $50 \%$ Load, 25 Horsepower

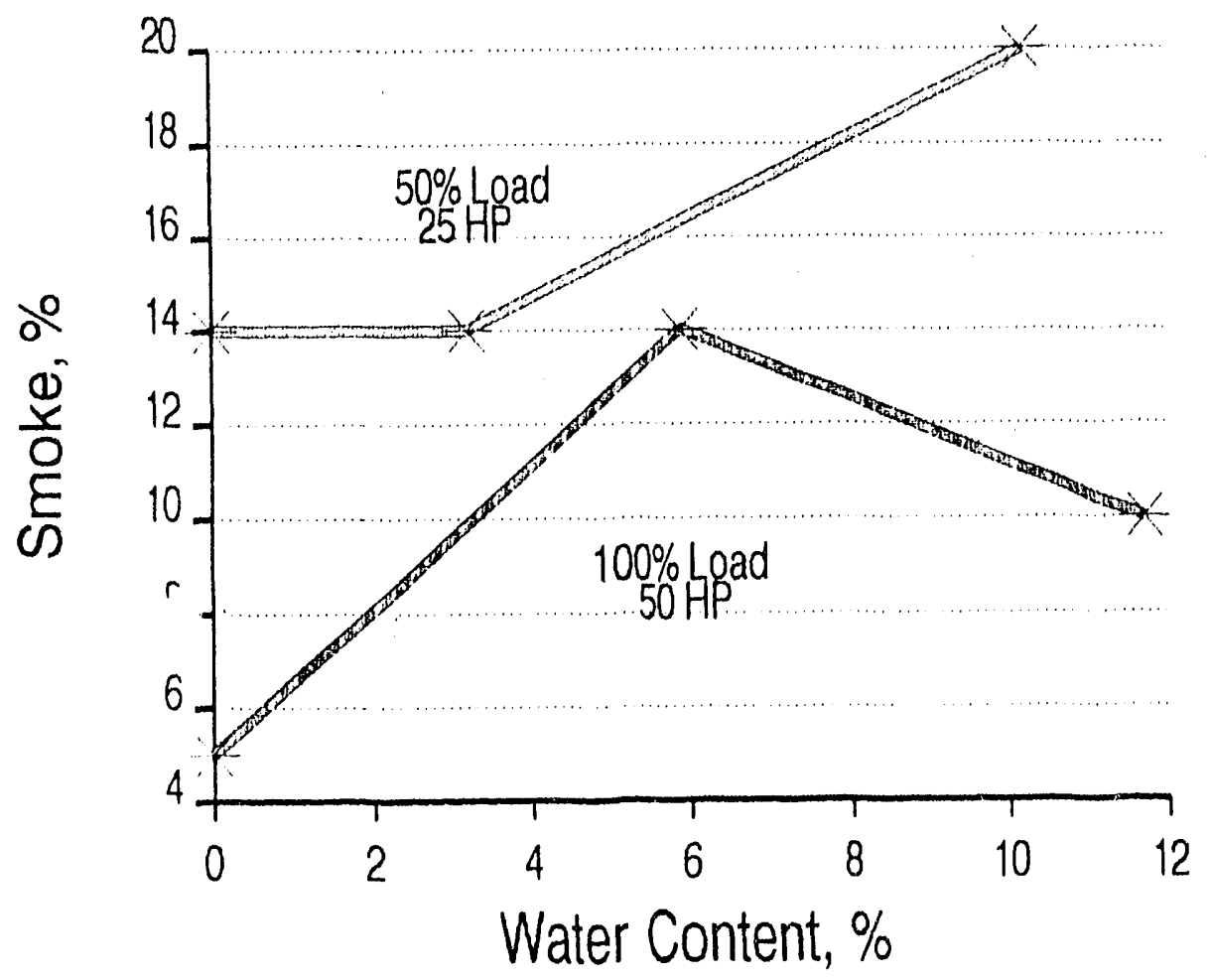

FIGURE A.146 Smoke Emission vs. Water Content, No. 4 Fuel, $21 \%$ Oxygen 


\section{$A .114$}

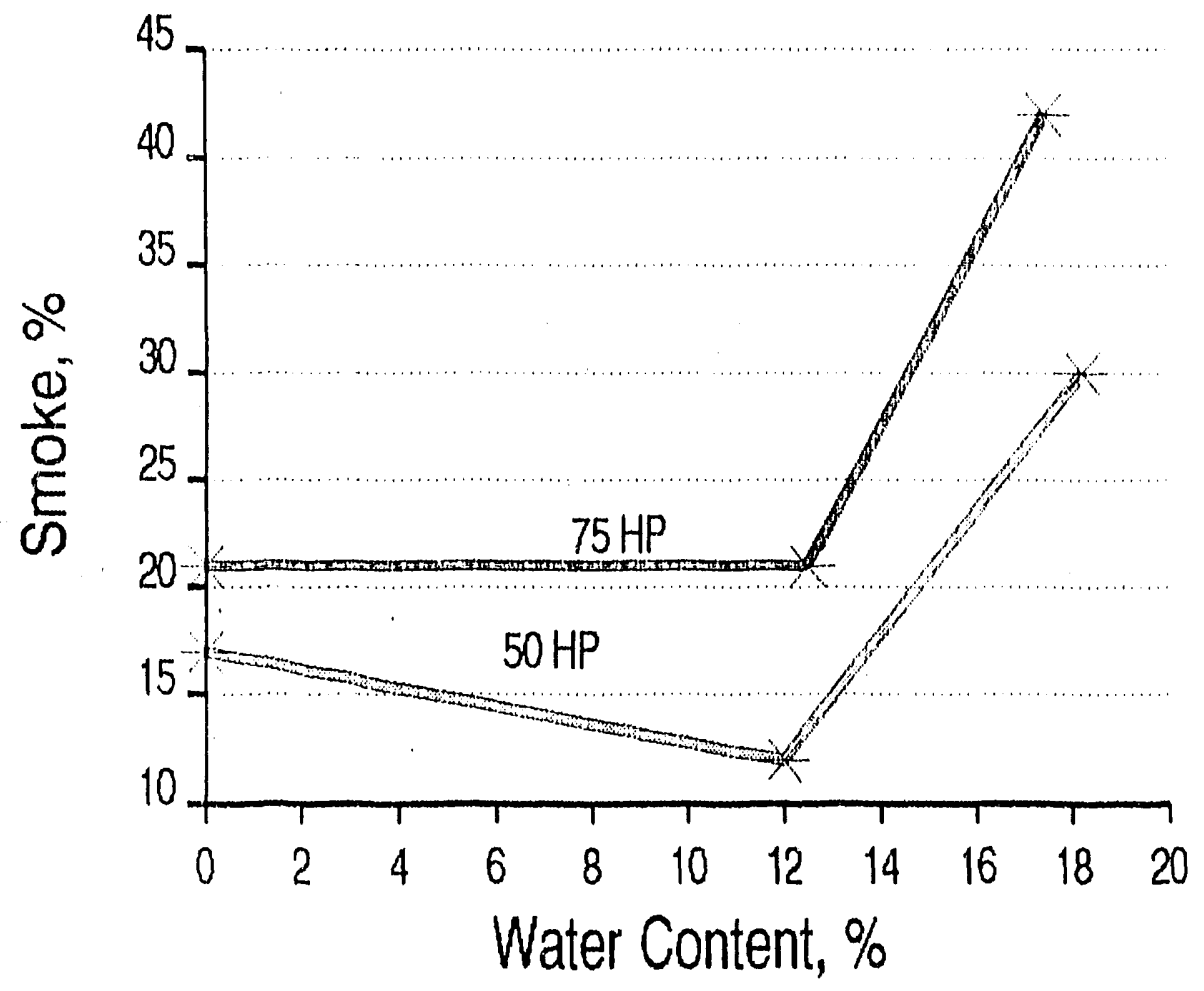

FIGURE A.147 Smoke Emicsion vs. Water Content, No. 4 Fuel, 100\% Load, $30 \%$ Oxygen

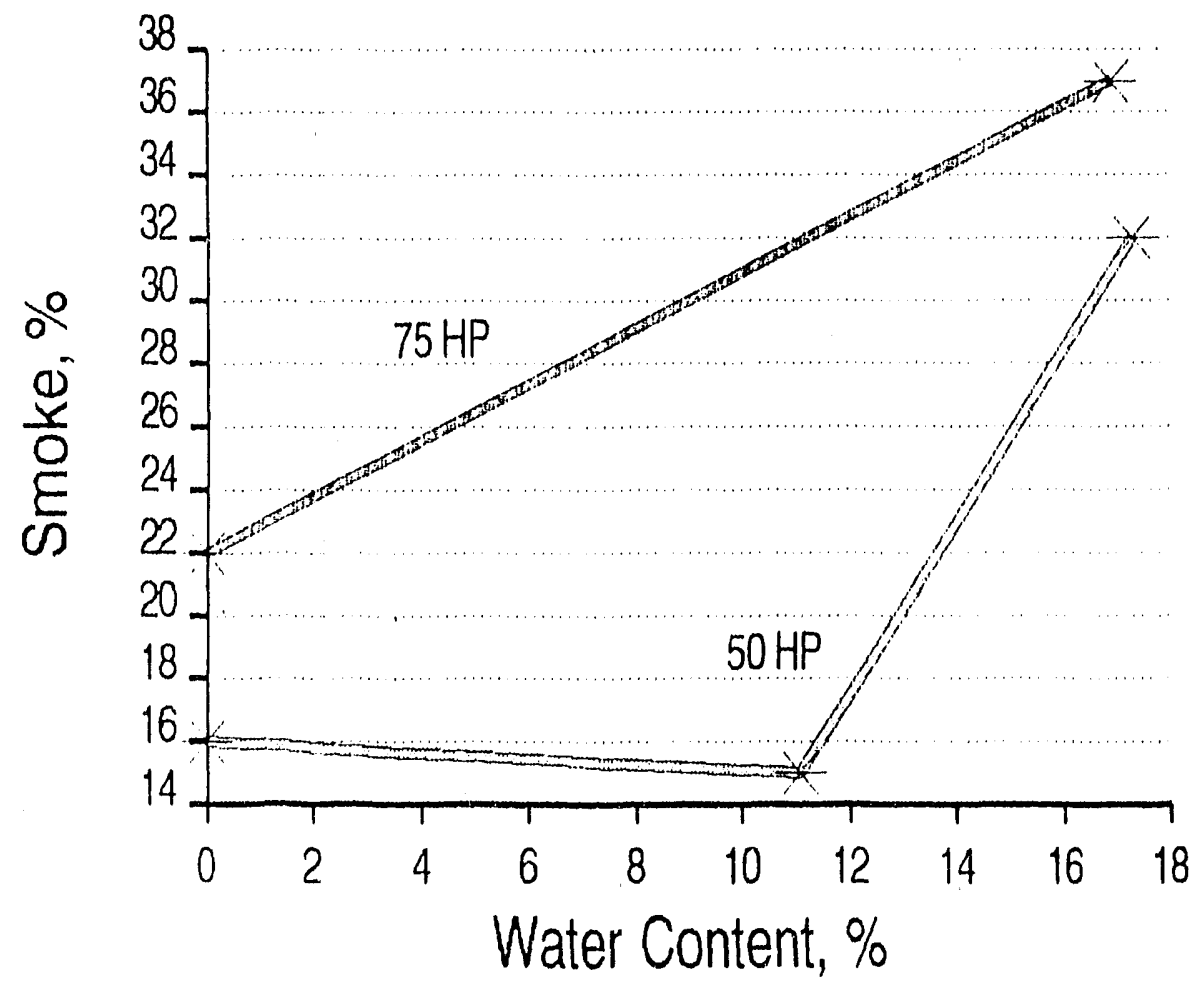

FIGURE A.148 Smoke Emission vs. Water Content, No. 4 Fuel, 100\% Load, $35 \%$ Oxygen 


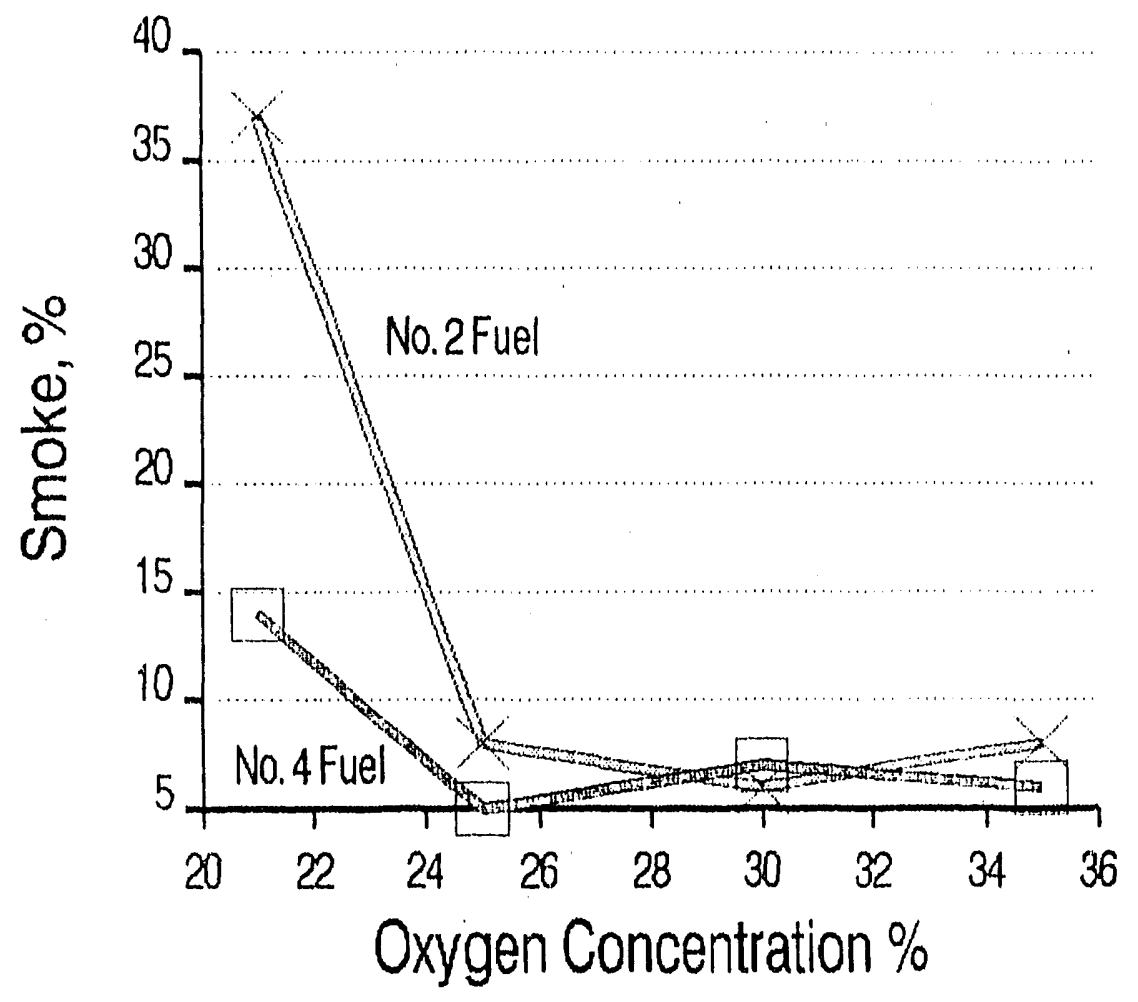

FIGURE A.149 Smoke Emission vs. Oxygen Concontration, $50 \%$ Load, 25 Horsepower, No Water

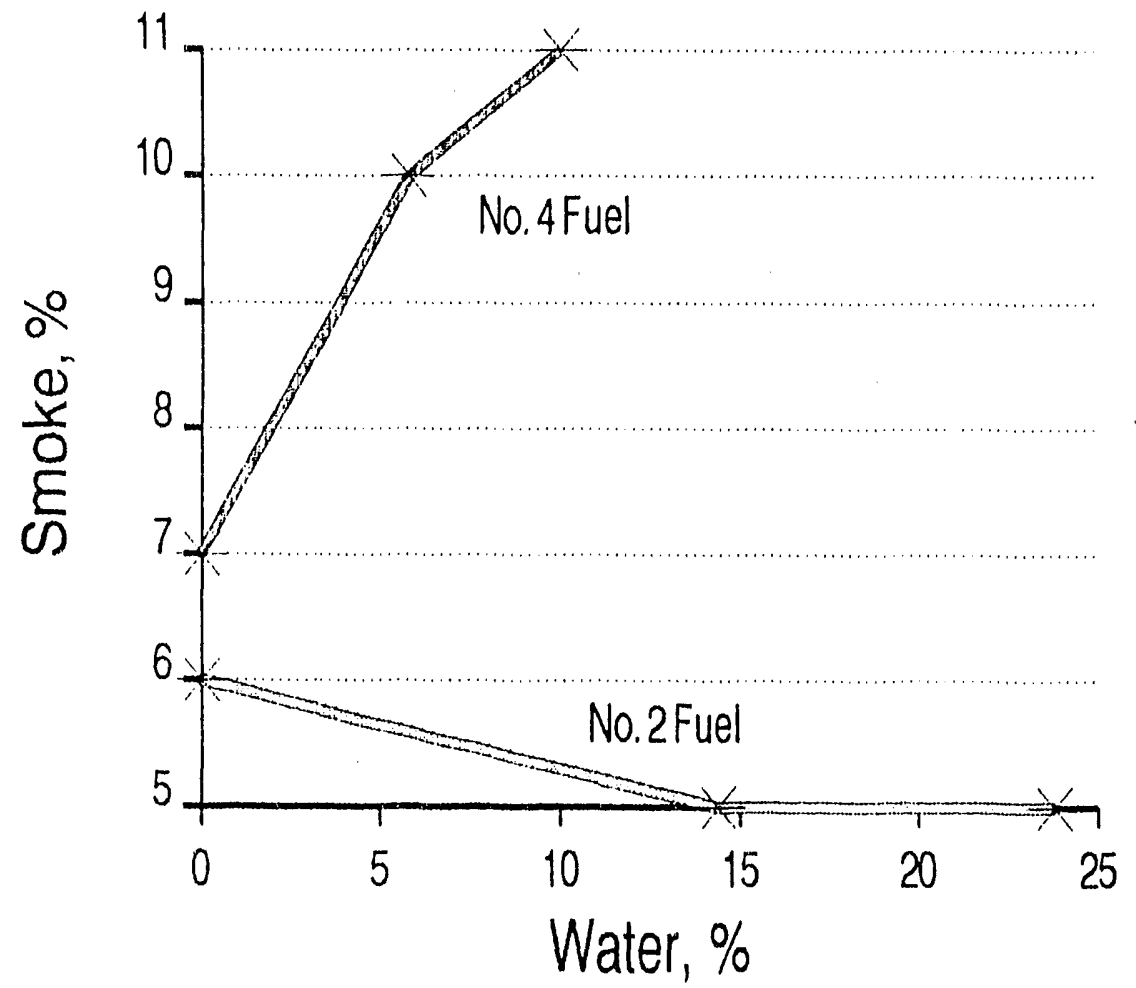

FIGURE A.150 Smoke Emission vs. Water Content, 50\% Load, 25 Horsepower, 30\% Oxygen 


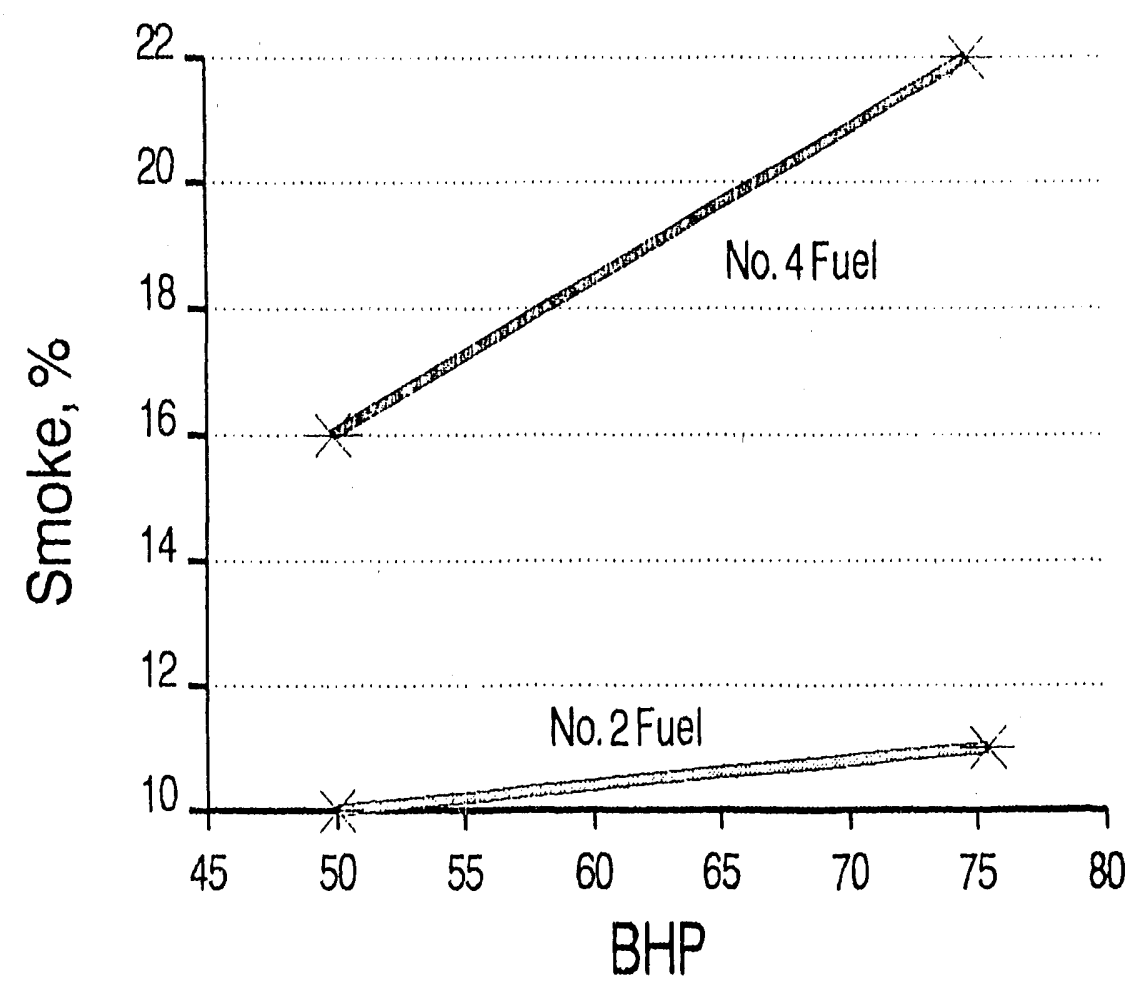

FIGURE A.151 Smoke Emission vs. Brake Horsepower, 100\% Load, No Water, 35\% Oxygen 


\section{Appendix B: \\ Data Package of Diesel Engine Tests with Oxygen-Enriched Air and Water-Emulsified Fuels: Series II Tests}


B-3

B.1 Tabulation of Test Data as a Function of Injection Time 


$$
B-4
$$


OXYGEN-ENRICHED DIESEL COMBUSTION TESTS -- SERIES II

No. 2 Fuel, Full Load, No Water, 218 oxygen Level

Injection Timing (deg btdc): $33,27,22$

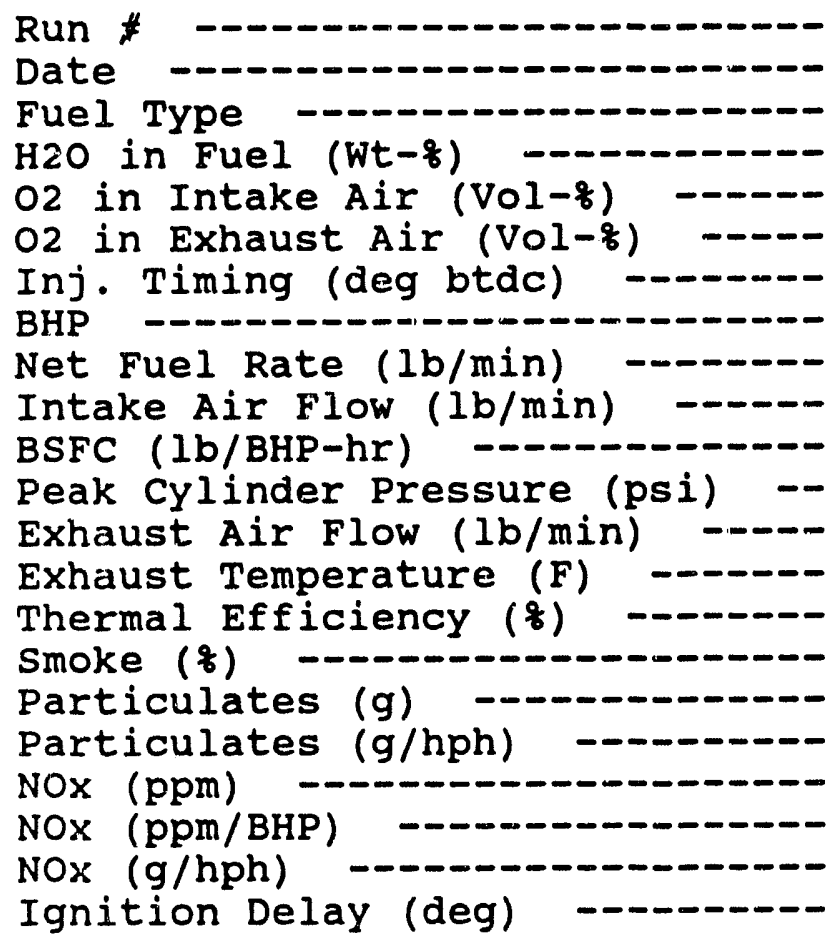

\begin{tabular}{|c|c|c|}
\hline $\begin{array}{l}217 \\
9 / 10 / 90 \\
* 2\end{array}$ & $\begin{array}{l}209 \\
9 / 5 / 90 \\
\# 2\end{array}$ & $\begin{array}{l}213 \\
9 / 6 / 90 \\
\# 2\end{array}$ \\
\hline 0 & 0 & 0 \\
\hline 20.79 & 20.8 & 20.74 \\
\hline 11.94 & 11.68 & 11.35 \\
\hline 33 & 27 & 22 \\
\hline 50 & 49.84 & 49.98 \\
\hline 0.286 & 0.297 & 0.316 \\
\hline 8.11 & 8.57 & 8.7 \\
\hline 0.3432 & 0.357544 & 0.379352 \\
\hline 1790 & 1519 & 1336 \\
\hline 8.396 & 8.867 & 9.016 \\
\hline 747 & 796 & 867 \\
\hline 0.43931 & 38.81694 & 36.58549 \\
\hline 25 & 47 & 52 \\
\hline 0.0066 & 0.0163 & 0.016 \\
\hline 0.130098 & 0.348181 & 0.372229 \\
\hline 850 & & 385 \\
\hline$\perp 7$ & & 7.703081 \\
\hline $\begin{array}{r}3.884508 \\
16.2\end{array}$ & $\begin{array}{r}2.590402 \\
15.1\end{array}$ & $\begin{array}{r}1.890136 \\
14.8\end{array}$ \\
\hline
\end{tabular}


OXYGEN-ENRICHED DIESEL COMBUSTION TESTS -- SERIES II

No. 2. Fuel, Full Load, No Water, $25 \%$ oxygen Level Injection Timing (deg btdc): $33,27,22$

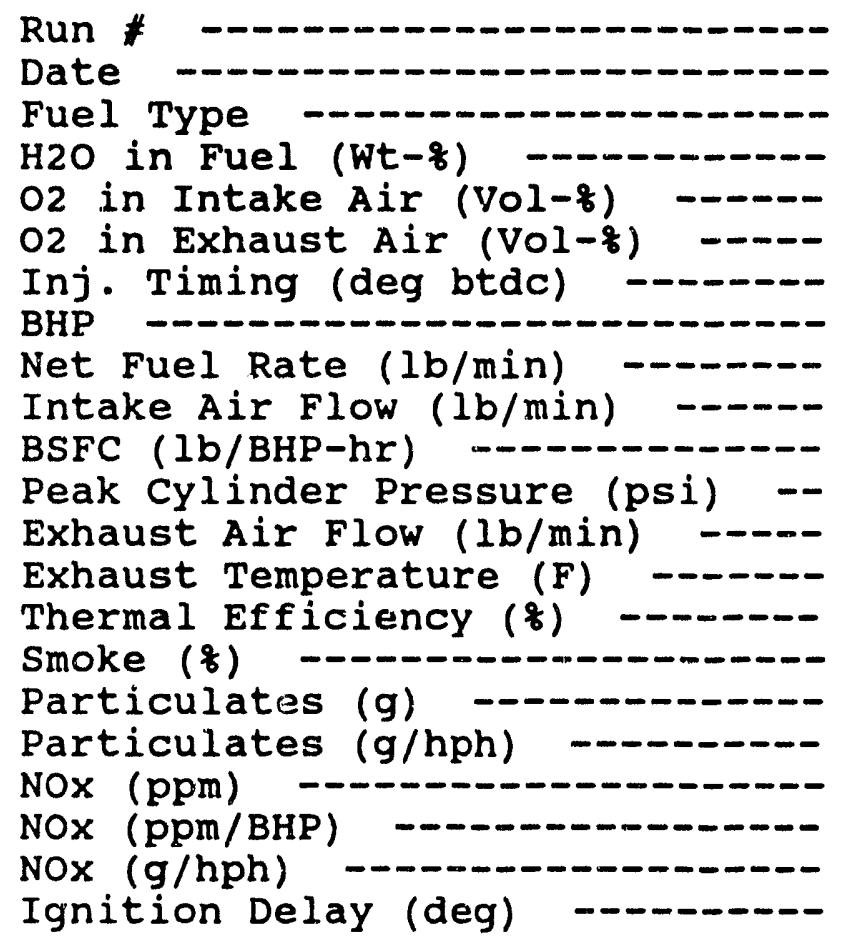

$$
\begin{array}{rrr}
218 & \multicolumn{1}{l}{265} & \multicolumn{1}{l}{214} \\
9 / 10 / 90 & 11 / 06 / 90 & 9 / 6 / 90 \\
\# 2 & \multicolumn{1}{r}{\# 2} & \multicolumn{1}{l}{\# 2} \\
24.98 & 23.36 & 25.01 \\
16.37 & 15.13 & 16 \\
33 & 27 & 22 \\
50.16 & 50.18 & 50.02 \\
0.287 & 0.293 & 0.305 \\
8.55 & 8.06 & 8.59 \\
0.343301 & 0.350339 & 0.365854 \\
1917 & 1630 & 1384 \\
8.837 & 8.353 & 8.895 \\
707 & 738 & 810 \\
40.42736 & 41.31912 & 37.93531 \\
7 & 26 & 14 \\
0.0062 & 0.0055 & 0.0073 \\
0.126864 & 0.102374 & 0.161066 \\
1670 & 1350 & 1035 \\
33.29346 & 26.90315 & 20.69172 \\
8.00716 & 6.115899 & 5.009073 \\
15.4 & 14.3 & 14.3
\end{array}
$$


OXYGEN-ENRICHED DIESEL COMBUSTION TESTS -- SERIES II No. 2 Fuel, Half Load, No Water, 218 Oxygen Level Injection Timing (deg btdc): 33, 27, 22

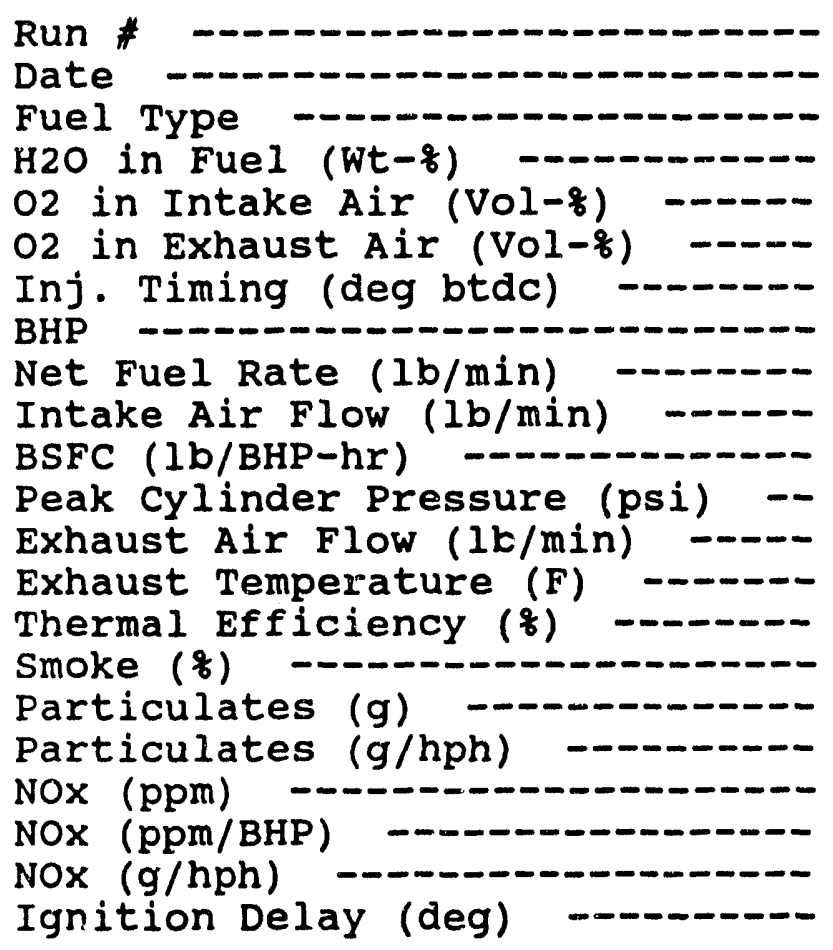

\begin{tabular}{|c|c|c|}
\hline $\begin{array}{l}219 \\
9 / 11 / 90 \\
\# 2\end{array}$ & $\begin{array}{l}211 \\
9 / 5 / 90 \\
\# 2\end{array}$ & $\begin{array}{l}215 \\
9 / 7 / 90 \\
\# 2\end{array}$ \\
\hline 0 & 0 & 0 \\
\hline 20.9 & 20.9 & 20.4 \\
\hline 14.42 & 14 & 14.57 \\
\hline 33 & 27 & 22 \\
\hline 24.93 & 25.02 & 25.01 \\
\hline 0.162 & 0.167 & 0.172 \\
\hline 6.35 & 6.66 & 6.72 \\
\hline 0.389892 & 0.40048 & 0.412635 \\
\hline 1357 & 1158.6 & 1149 \\
\hline 6.512 & 6.827 & 6.892 \\
\hline 537 & 586 & 573 \\
\hline 35.59648 & 34.65537 & 33.6345 \\
\hline 16 & 54 & 66 \\
\hline 0.0037 & 0.0152 & 0.0169 \\
\hline 0.109 & 0.476098 & 0.55313 \\
\hline 643 & 383 & 235 \\
\hline 25.79222 & 15.30775 & 9.396242 \\
\hline $\begin{array}{r}4.571069 \\
18.1\end{array}$ & $\begin{array}{r}2.844174 \\
16.3\end{array}$ & $\begin{array}{r}1.762439 \\
15.6\end{array}$ \\
\hline
\end{tabular}


OXYGEN-ENRICHED DIESEL COMBUSTION TESTS -- SERIES II No. 2 Fuel, Half Load, No Water, $25 q$ Oxygen Level Injection Timing (deg btdc): 33, 27, 22

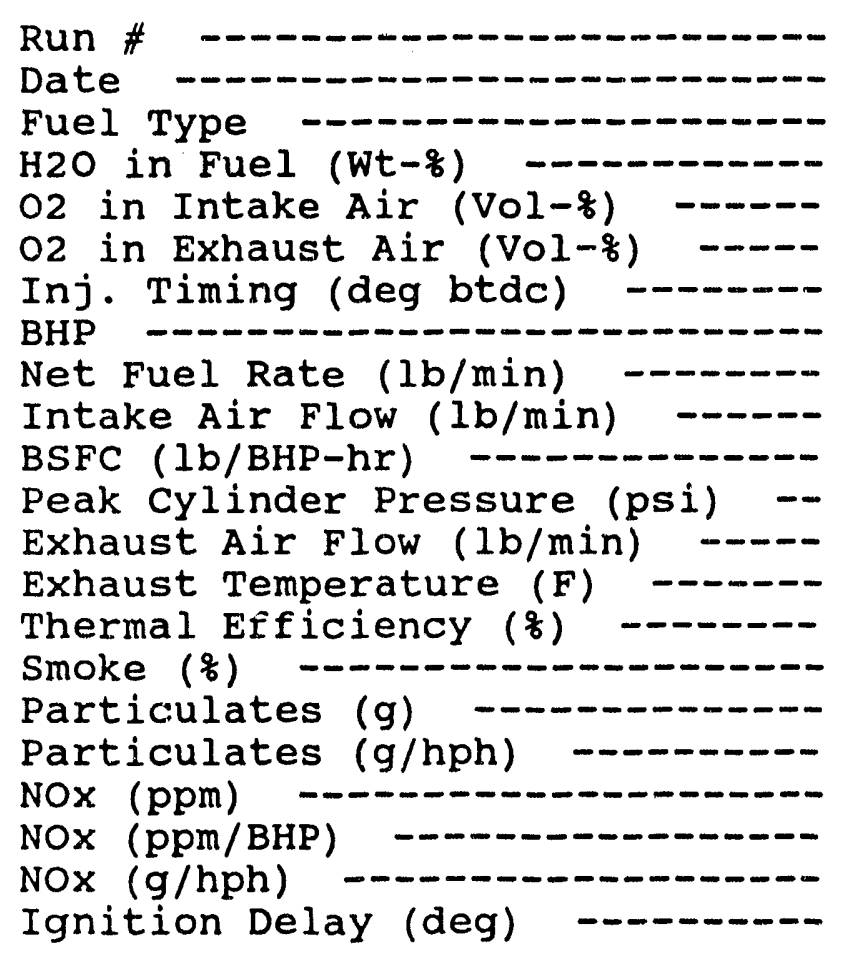

$$
\begin{aligned}
& 220 \quad 212 \quad 216 \\
& 9 / 11 / 90 \quad 9 / 5 / 90 \quad 9 / 7 / 90 \\
& \# 2 \# 2 \quad \# 2 \\
& \begin{array}{rrr}
0 & 0 & 0 \\
27.03 & 26.81 & 26.58
\end{array} \\
& 20.34 \quad 20.08 \quad 20.96 \\
& 33 \quad 27 \quad 22 \\
& \begin{array}{lll}
24.7 & 24.96 & 24.76
\end{array} \\
& 0.164 \quad 0.166 \quad 0.167 \\
& 7 \quad 6.59 \quad 7.54 \\
& 0.3983810 .3990380 .404685 \\
& \begin{array}{lll}
1469 & 1239.7 & 1221
\end{array} \\
& \begin{array}{lll}
7.164 & 6.756 & 7.707
\end{array} \\
& 513 \quad 552 \quad 520 \\
& 34.83797 \quad 34.78053 \quad 34.29525 \\
& \begin{array}{lll}
0.0049 & 0.008 & 0.006
\end{array} \\
& \begin{array}{llll}
0.148228 & 0.240426 & 0.200071
\end{array} \\
& \begin{array}{lll}
1926 & 1160 & 885
\end{array} \\
& 77.97571 \quad 46.47436 \quad 35.74313 \\
& 15.203018 .545112 \quad 7.49709 \\
& \begin{array}{lll}
17 & 15.2 & 14.8
\end{array}
\end{aligned}
$$


OXYGEN-ENRICHED DIESEL COMBUSTION TESTS -- SERIES II No. 2 Fuel, Full Load, 58 Water, 218 Oxygen Level Injection Timing (deg btdc): 33, 27, 22

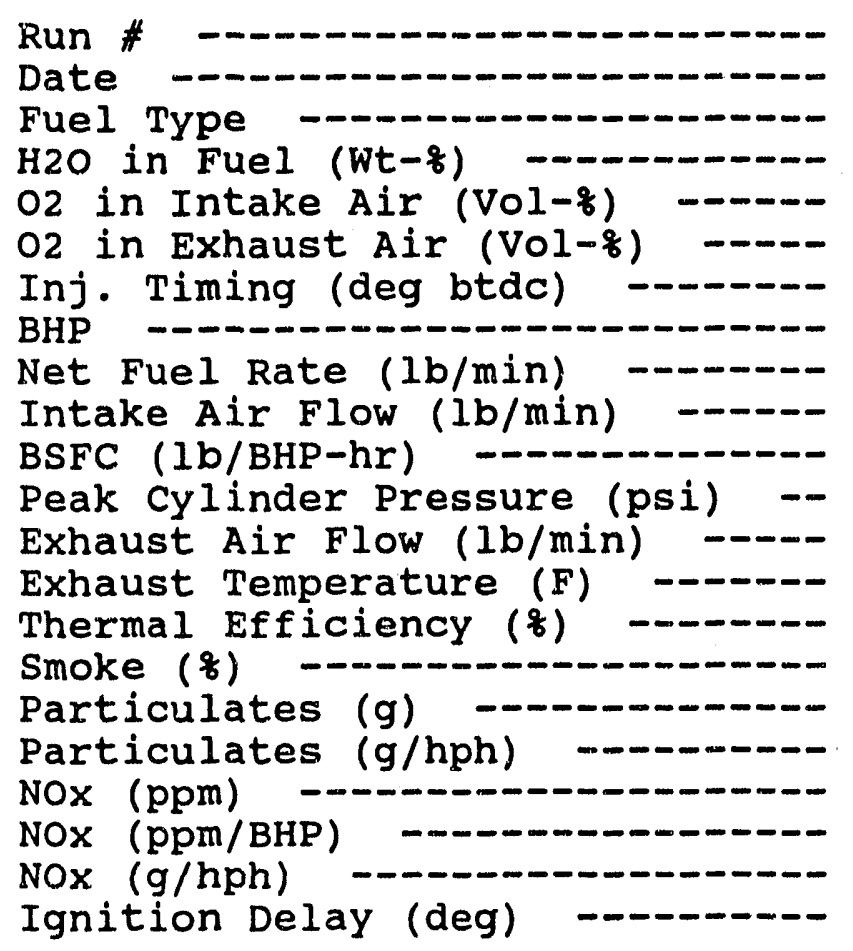

\begin{tabular}{rr}
221 & \multicolumn{2}{l}{232} \\
$9 / 20 / 90$ & $10 / 3 / 90$ \\
$\# 2$ & \multicolumn{2}{l}{$\# 2$} \\
3.3 & 3.85 \\
20.45 & 20.3 \\
11.59 & 12.03 \\
33 & 27 \\
50.23 & 50.34 \\
0.289971 & 0.294014 \\
7.96 & 8.62 \\
0.361935 & 0.368296 \\
1845 & 1518 \\
8.263 & 8.929 \\
749 & 779 \\
40.06941 & 39.60466 \\
18 & 14 \\
0.0066 & 0.0064 \\
0.123784 & 0.133602 \\
433 & 620 \\
8.620346 & 12.31625 \\
1.938551 & 2.992926 \\
16.3 & 15.6
\end{tabular}


OXYGEN-ENRICHED DIESEL COMBUSTION TESTS -- SERIES II No. 2 Fuel, Full Load, 5\% Water, 25\% oxygen Level Injection Timing (deg btdc): $33,27,22$

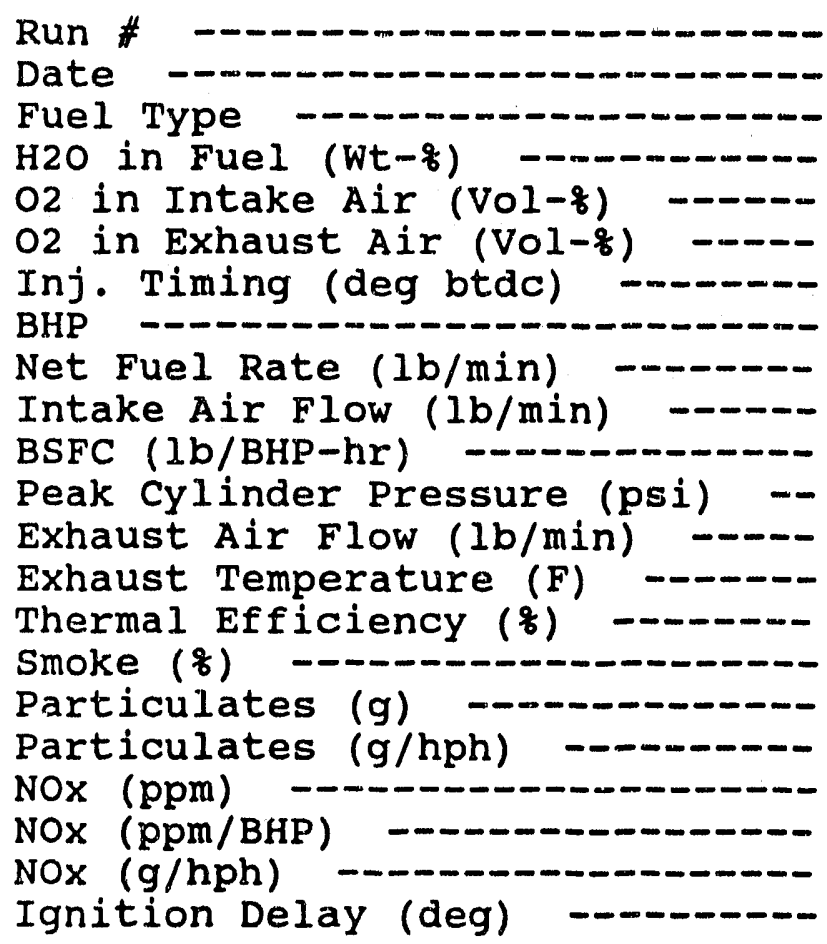

$$
\begin{aligned}
& 222 \quad 231 \quad 226 \\
& 9 / 20 / 90 \quad 10 / 3 / 90 \quad 9 / 26 / 90 \\
& \text { \#2 \#2 \#2 } \\
& \begin{array}{lll}
3.4 & 4 & 4.25
\end{array} \\
& 24.75 \quad 24.15 \quad 24.73 \\
& \begin{array}{lll}
16.32 & 16.08 & 16.38
\end{array} \\
& 33 \quad 27 \quad 22 \\
& \begin{array}{lll}
49.75 & 50.41 & 50.12
\end{array} \\
& \begin{array}{llll}
0.283932 & 0.2888 & 0.300358
\end{array} \\
& 8.67 \quad 8.63 \quad 8.2 \\
& \begin{array}{llll}
0.358191 & 0.361833 & 0.379489
\end{array} \\
& \begin{array}{lll}
1913 & 1634 & 1388
\end{array} \\
& \begin{array}{lll}
8.967 & 8.934 & 8.517
\end{array} \\
& \begin{array}{lll}
705 & 736 & 792
\end{array} \\
& 40.53024 \quad 40.37568 \quad 38.59904 \\
& \begin{array}{lll}
0.0069 & 0.0068 & 0.0049
\end{array} \\
& \begin{array}{llll}
0.146751 & 0.135865 & 0.096897
\end{array} \\
& 1090 \quad 1380 \quad 1170 \\
& \begin{array}{llll}
21.90955 & 27.37552 & 23.34397
\end{array} \\
& 5.3468176 .6561495 .410983 \\
& 16 \\
& 15 \\
& 14.3
\end{aligned}
$$


OXYGEN-ENRICHED DIESEL COMBUSTION TESTS -- SERIES II OXYGEN-ENRCHED DIESEL COMBUSION TESTS -No. 2 Fuel, Half Load, 58 Water, $21 \%$ Oxygen Level Injection Timing (deg btdc): $33,27,22$

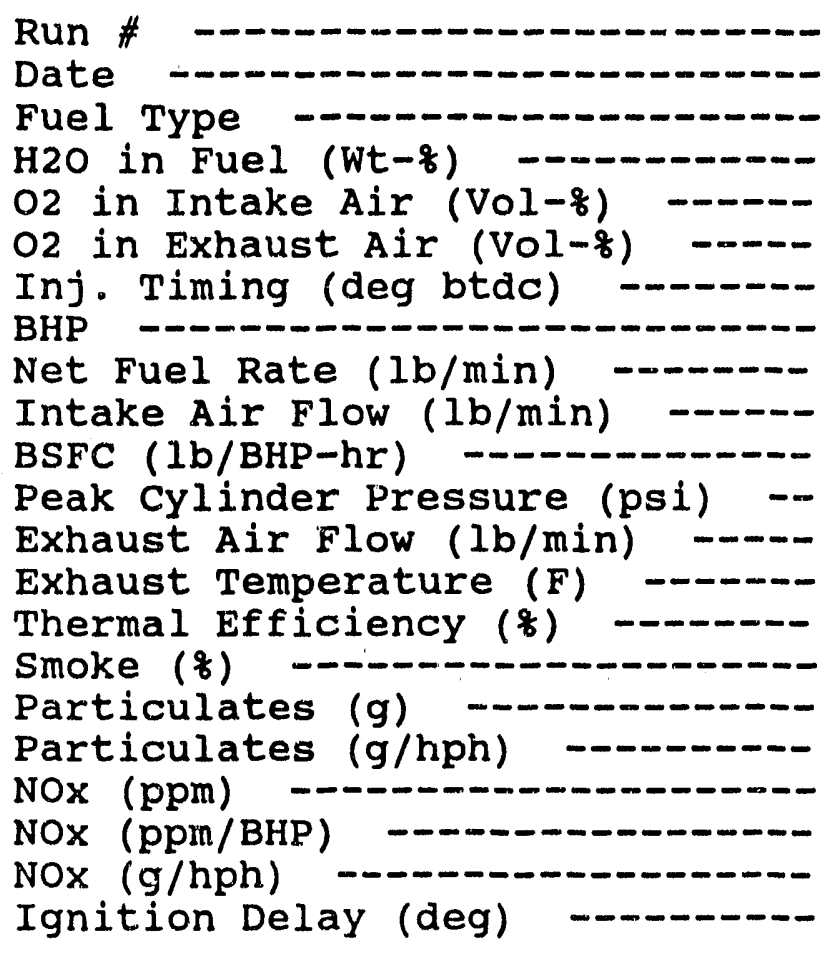

$$
\begin{array}{rrr}
223 & \multicolumn{1}{l}{229} & \multicolumn{1}{l}{227} \\
9 / 21 / 90 & 10 / 3 / 90 & \multicolumn{1}{l}{10 / 2 / 90} \\
\# 2 & \multicolumn{2}{l}{\# 2} \\
3.3 & 3.9 & 4.7 \\
20.48 & 20.35 & 20.72 \\
14.35 & 13.94 & 14.39 \\
33 & 27 & 22 \\
25.04 & 24.99 & 24.82 \\
0.16269 & 0.163572 & 0.166911 \\
6.45 & 6.6 & 6.8 \\
0.407348 & 0.412965 & 0.427881 \\
1472 & 1164 & 1123 \\
6.62 & 6.772 & 6.977 \\
519 & 568 & 564 \\
35.60254 & 35.33986 & 34.39701 \\
24 & 23 & 31 \\
0.0149 & 0.0128 & 0.0092 \\
0.449159 & 0.402364 & 0.311017 \\
200 & 320 & 268 \\
7.98722 & 12.80512 & 10.79774 \\
1.439025 & 2.360018 & 2.050296 \\
18.2 & 17.4 & 16
\end{array}
$$


OXYGEN-ENRICHED DIESEL COMBUSTION TESTS -- SERIES II No. 2 Fuel, Half Load, $5 \%$ Water, $26 \%$ oxygen Level
Injection Timing (deg btdc): $33,27,22$

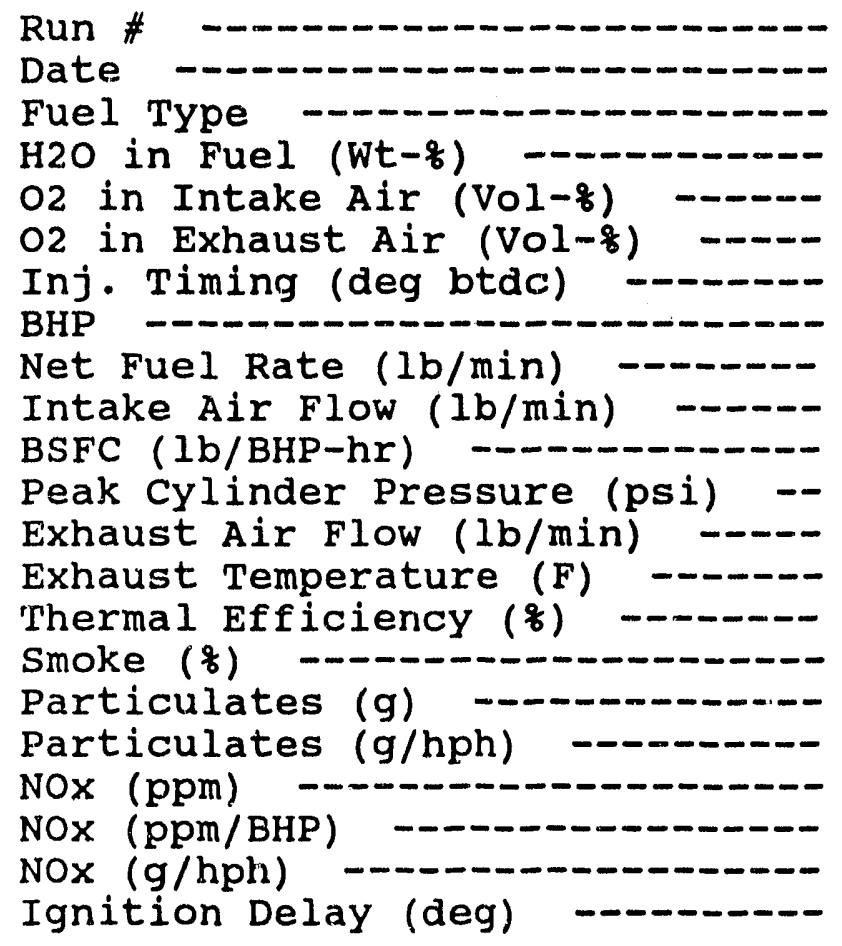

$$
\begin{aligned}
& 224 \quad 263 \quad 228 \\
& 9 / 21 / 90 \quad 11 / 01 / 90 \quad 10 / 2 / 90 \\
& \text { \#2 } \\
& \begin{array}{lll}
3.75 & 5.8 & 5.3
\end{array} \\
& 26.08 \quad 26.4 \quad 26.15 \\
& \begin{array}{lll}
19.8 & 19.45 & 19.7
\end{array} \\
& 33 \quad 27 \quad 22 \\
& \begin{array}{lll}
25.08 & 25.2 & 24.98
\end{array} \\
& \begin{array}{llll}
0.164783 & 0.165896 & 0.164912
\end{array} \\
& \begin{array}{rrr}
7.18 & 6.91 & 6.84
\end{array} \\
& \begin{array}{llll}
0.413876 & 0.42381 & 0.422738
\end{array} \\
& \begin{array}{lll}
1498 & 1168 & 1163
\end{array} \\
& \begin{array}{lll}
7.353 & 7.088 & 7.016
\end{array} \\
& \begin{array}{lll}
517 & 564 & 543
\end{array} \\
& 35.20633 \quad 36.6482 \quad 35.03837 \\
& 10 \quad 16 \quad 8 \\
& \begin{array}{lll}
0.0064 & 0.0041 & 0.004
\end{array} \\
& \begin{array}{llll}
0.212769 & 0.14081 & 0.133609
\end{array} \\
& \begin{array}{lll}
660 & 1100 \quad 975
\end{array} \\
& 26.31579 \quad 43.6507939 .03122 \\
& 5.26618 \quad 8.420358 \quad 7.452749 \\
& \begin{array}{lll}
17.3 & 16.3 & 14.9
\end{array}
\end{aligned}
$$


OXYGEN-ENRICHED DIESEL COMBUSTION TESTS -- SERIES II

No. 4 Fuel, Full Load, No Water, $21 \%$ oxygen Level Injection Timing (deg btdc): 33, 27, 22

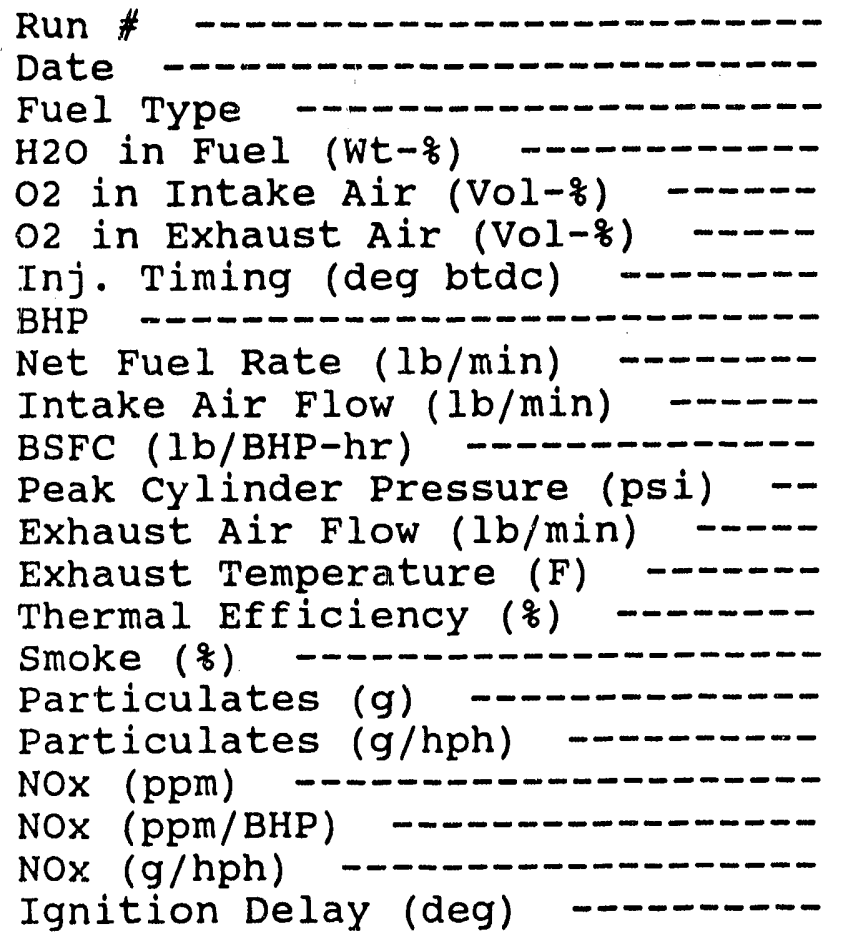

\begin{tabular}{rrr}
242 & 236 & \multicolumn{2}{l}{241} \\
$10 / 12 / 90$ & $10 / 8 / 90$ & \multicolumn{2}{c}{$10 / 11 / 90$} \\
$\# 4$ & 0 & \multicolumn{2}{c}{$\# 4$} \\
20.4 & 20.53 & 20.9 \\
12.18 & 12.45 & 11.96 \\
33 & 27 & 22 \\
49.93 & 50.14 & 49.91 \\
0.302 & 0.308 & 0.32 \\
8.7 & 8.52 & 8.5 \\
0.362908 & 0.368568 & 0.384692 \\
1913 & 1633 & 1471 \\
9.002 & 8.828 & 8.82 \\
722 & 760 & 803 \\
39.88804 & 39.2755 & 37.62926 \\
13 & 32 & 32 \\
0.0112 & 0.0166 & 0.0132 \\
0.229488 & 0.30725 & 0.277844 \\
860 & 630 & 480 \\
17.22411 & 12.56482 & 9.617311 \\
4.219788 & 3.018793 & 2.308538 \\
18.1 & 16.7 & 15.4
\end{tabular}


OXYGEN-ENRICHED DIESEL COMBUSTION TESTS -- SERIES II

No. 4 Fuel, Full Load, No Water, 25\% Oxygen Level Injection Timing (deg btdc): 33, 27, 22

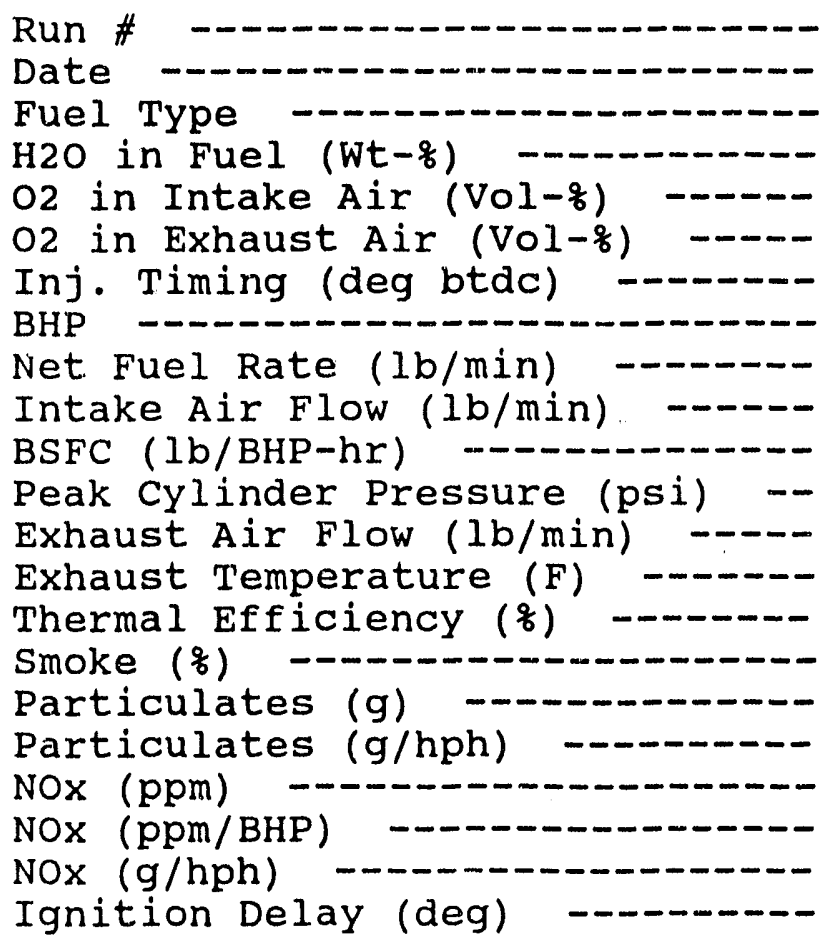

$$
\begin{aligned}
& 243 \\
& 10 / 12 / 90 \\
& \# 4 \\
& 237 \quad 240 \\
& 10 / 8 / 90 \quad 10 / 11 / 90 \\
& \text { \#4 } \\
& \begin{array}{rrrr}
0 & 0 & 0
\end{array} \\
& \begin{array}{lll}
15.75 & 15.87 & 15.6
\end{array} \\
& \begin{array}{lll}
33 & 27 & 22
\end{array} \\
& \begin{array}{lll}
49.78 & 50 & 49.91
\end{array} \\
& 0.303 \quad 0.307 \quad 0.316 \\
& \begin{array}{lll}
8.8 & 8.65 & 7.52
\end{array} \\
& \begin{array}{llll}
0.365207 & 0.3684 & 0.379884
\end{array} \\
& \begin{array}{lll}
1988 & 1679 & 1520
\end{array} \\
& \begin{array}{lll}
9.103 & 8.957 & 7.836
\end{array} \\
& 697 \quad 735 \quad 784 \\
& 39.6369639 .2934138 .10558 \\
& \begin{array}{rrr}
5 & 15 & 18
\end{array} \\
& \begin{array}{lll}
0.0117 & 0.0174 & 0.0107
\end{array} \\
& \begin{array}{llll}
0.173179 & 0.369108 & 0.196033
\end{array} \\
& \begin{array}{lll}
1800 & 1440 & 1130
\end{array} \\
& \begin{array}{lll}
36.1591 & 28.8 \quad 22.64075
\end{array} \\
& 8.95812 \quad 7.020528 \quad 4.828364 \\
& \begin{array}{lll}
17.1 & 15.8 & 14.5
\end{array}
\end{aligned}
$$


OXYGEN-ENRICHED DIESEL COMBUSTION TESTS -- SERIES II No. 4 Fuel, Half Load, No Water, $21 \%$ Oxygen Level Injection Timing (deg btdc): 33, 27, 22

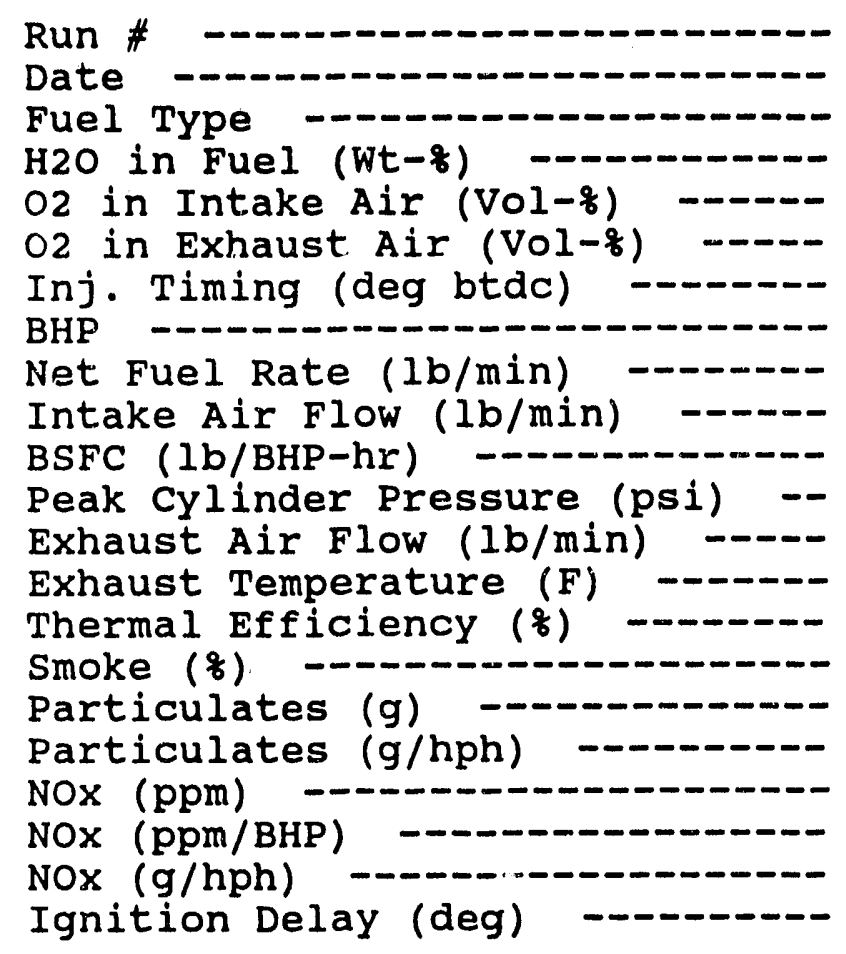

\begin{tabular}{|c|c|c|}
\hline $\begin{array}{c}244 \\
10 / 17 / 90 \\
\# 4\end{array}$ & $\begin{array}{c}234 \\
10 / 5 / 90 \\
\# 4\end{array}$ & $\begin{array}{c}238 \\
10 / 11 / 90 \\
\# 4\end{array}$ \\
\hline 0 & 0 & 0 \\
\hline $\begin{array}{r}20.5 \\
14.15\end{array}$ & $\begin{array}{r}20.5 \\
13.91\end{array}$ & $\begin{array}{l}20.46 \\
13.84\end{array}$ \\
\hline 33 & 27 & 22 \\
\hline 24.87 & 24.95 & 24.89 \\
\hline 0.172 & 0.177 & 0.182 \\
\hline $\begin{array}{r}6.5 \\
0.414958\end{array}$ & $\begin{array}{r}6.8 \\
0.425651\end{array}$ & $\begin{array}{r}6.8 \\
0.43873\end{array}$ \\
\hline 1407 & 1222 & 1188 \\
\hline 6.672 & 6.977 & 6.982 \\
\hline 554 & 586 & 612 \\
\hline 34.88473 & 34.00833 & 32.9945 \\
\hline 35 & 45 & 50 \\
\hline 0.01 & 0.0156 & 0.0173 \\
\hline 0.301478 & 0.490476 & 0.560676 \\
\hline 640 & 390 & 270 \\
\hline 25.73382 & 15.63126 & 10.84773 \\
\hline 4.672776 & 2.968093 & 2.061263 \\
\hline $2 c$ & 19.4 & 18.1 \\
\hline
\end{tabular}


OXYGEN-ENRICHED DIESEL COMBUSTION TESTS -- SERIES II

No. 4 Fuel, Half Load, No Water, $27 \%$ oxygen Level Injection Timing (deg btdc): 33, 27, 22

Run \# -

Date

Fuel Type

H2O in Fuel (Wt-q)

02 in Intake Air (Vol-\%)

02 in Exhaust Air (Vol- $f$ ) ----

Inj. Timing (deg btdc) -

BHP -

Net Fuel Rate (1b/min) ------

Intake Air Flow ( $1 \mathrm{~b} / \mathrm{min})$

BSFC (lb/BHP-hr)

Peak Cylinder Pressure (psi) -

Exhaust Air Flow (1b/min)

Exhaust Temperature (F)

Thermal Efficiency ( $\%$ )

Smoke $(f)$ - $f$ -

Particulates (g)

Particulates (g/hph) --------

NOX (ppm) -

NOX (ppm/BHP) -

NOx (g/hph) -

Ignition Delay (deg) -------

\begin{tabular}{|c|c|c|}
\hline $\begin{array}{c}245 \\
10 / 17 / 90 \\
\# 4\end{array}$ & $\begin{array}{c}235 \\
10 / 5 / 90 \\
\# 4\end{array}$ & $\begin{array}{c}239 \\
10 / 11 / 90 \\
\# 4\end{array}$ \\
\hline 0 & 0 & 0 \\
\hline 26.85 & 26.68 & 26.53 \\
\hline 19.57 & 19.67 & 19.86 \\
\hline 33 & 27 & 22 \\
\hline 25.11 & 25.03 & 25.05 \\
\hline 0.176 & 0.176 & 0.18 \\
\hline 6.57 & 6.47 & 6.39 \\
\hline 0.42055 & 0.421894 & 0.431138 \\
\hline 1468 & 1288 & 1163 \\
\hline 6.746 & 6.646 & 6.5 \\
\hline 561 & 562 & 58 \\
\hline 4.42089 & 34.31123 & 33.5755 \\
\hline$\quad 19$ & 20 & \\
\hline 0.0099 & 0.0119 & 0.006 \\
\hline 294529 & 0.405549 & 0.19369 \\
\hline 1770 & 1500 & 100 \\
\hline 70.48984 & 59.92809 & 39.9201 \\
\hline 12.94159 & 10.83941 & 7.13793 \\
\hline 18.5 & 17.4 & \\
\hline
\end{tabular}


OXYGEN-ENRICHED DIESEL COMBUSTION TESTS -- SERIES II

No. 4 Fuel, Full Load, $5 \%$ Water, $21 \%$ Oxygen Level Injection Timing (deg btdc): 33, 27, 22

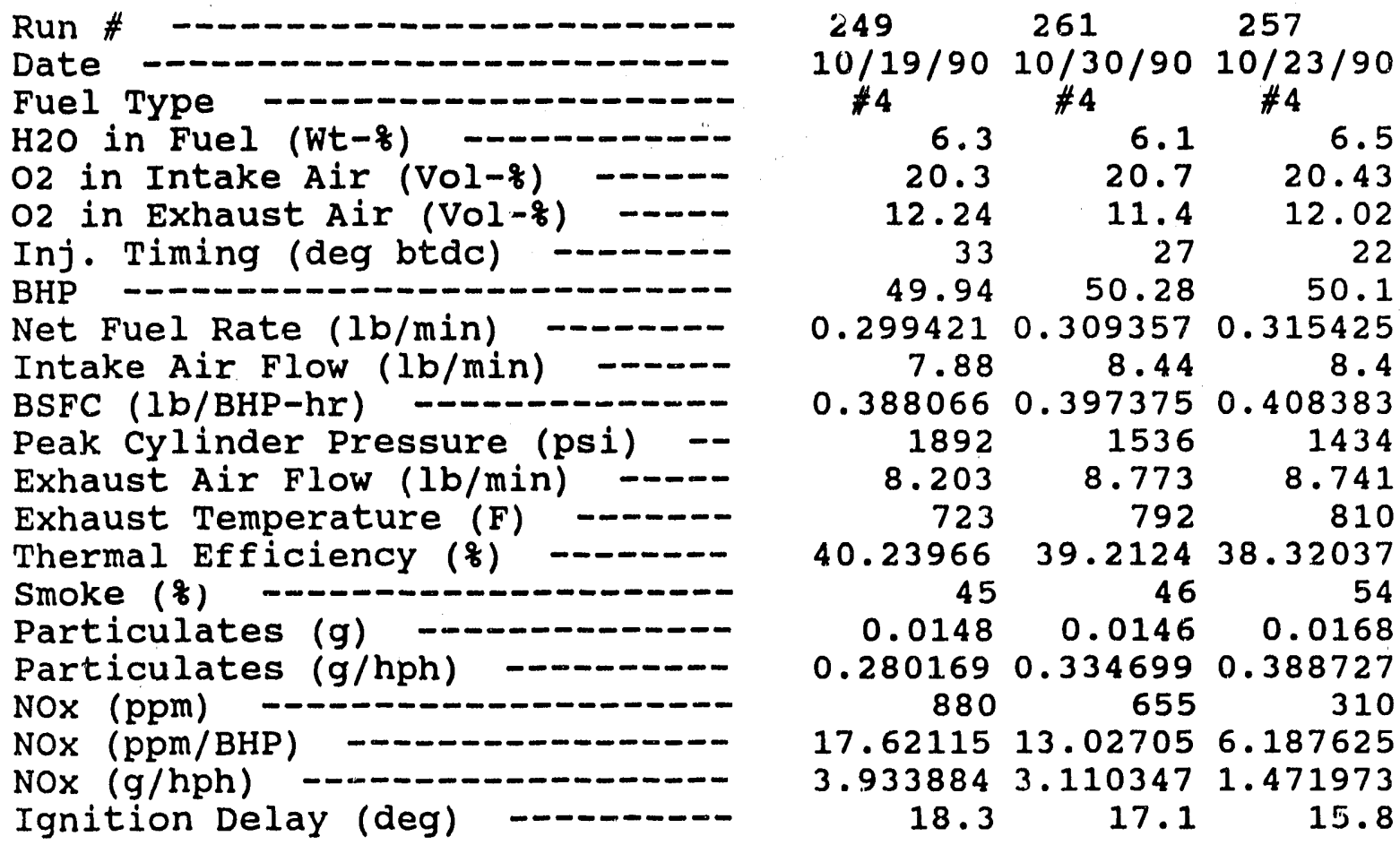


OXYGEN-ENRICHED DIESEL COMBUSTION TESTS -- SERIES II No. 4 Fuel, Full Load, 58 Water, $25 \%$ Oxygen Level Injection Timing (deg btdc): $33,27,22$

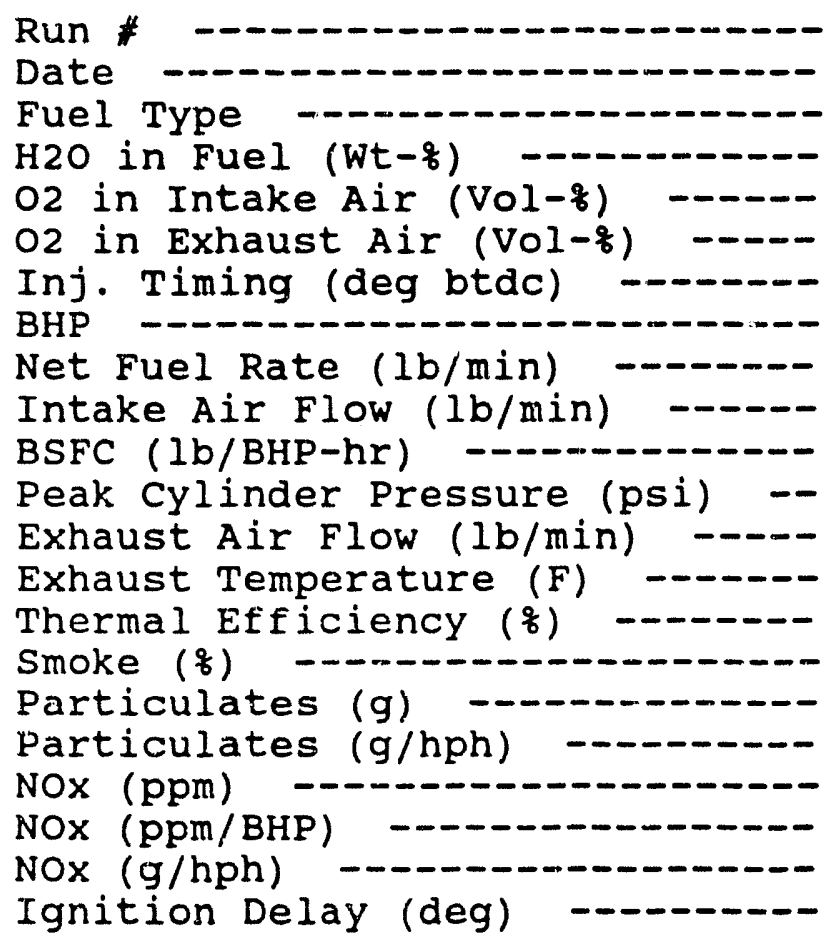

\begin{tabular}{|c|c|c|}
\hline $\begin{array}{l}248 \\
10 / 19 / 90 \\
\quad 4\end{array}$ & $\begin{array}{l}252 \\
10 / 22 / 90 \\
\# 4\end{array}$ & $\begin{array}{c}256 \\
10 / 23 / 90 \\
\# 4\end{array}$ \\
\hline $\begin{array}{r}6.5 \\
23.62 \\
15.24\end{array}$ & $\begin{array}{r}6.6 \\
23.78 \\
15.47\end{array}$ & $\begin{array}{r}6.7 \\
23.7 \\
15.59\end{array}$ \\
\hline 33 & 27 & 22 \\
\hline 49.78 & 49.93 & 50.06 \\
\hline $\begin{array}{r}0.2997 \\
7.68\end{array}$ & $\begin{array}{r}0.30492 \\
8.66\end{array}$ & $\begin{array}{r}0.312897 \\
8.64\end{array}$ \\
\hline .390518 & 0.396555 & 0.406312 \\
\hline 1935 & 1713 & 1464 \\
\hline 8.004 & 8.99 & 8.979 \\
\hline 707 & 724 & 764 \\
\hline 40.0734 & 39.50606 & 38.59913 \\
\hline 42 & 47 & 44 \\
\hline 0.0179 & 0.0115 & 0.0134 \\
\hline J. 32696 & 0.238361 & 0.704329 \\
\hline 1610 & 1110 & 720 \\
\hline 423 & 23112 & 38274 \\
\hline & 43921 & 4668 \\
\hline 17.2 & 15.3 & 15 \\
\hline
\end{tabular}


OXYGEN-ENRICHED DIESEL COMBUSTION TESTS -- SERIES II

-

No. 4 Fuel, Half Load, 5\% Water, $21 \%$ Oxygen Level Injection Timing (deg btdc): $33,27,22$

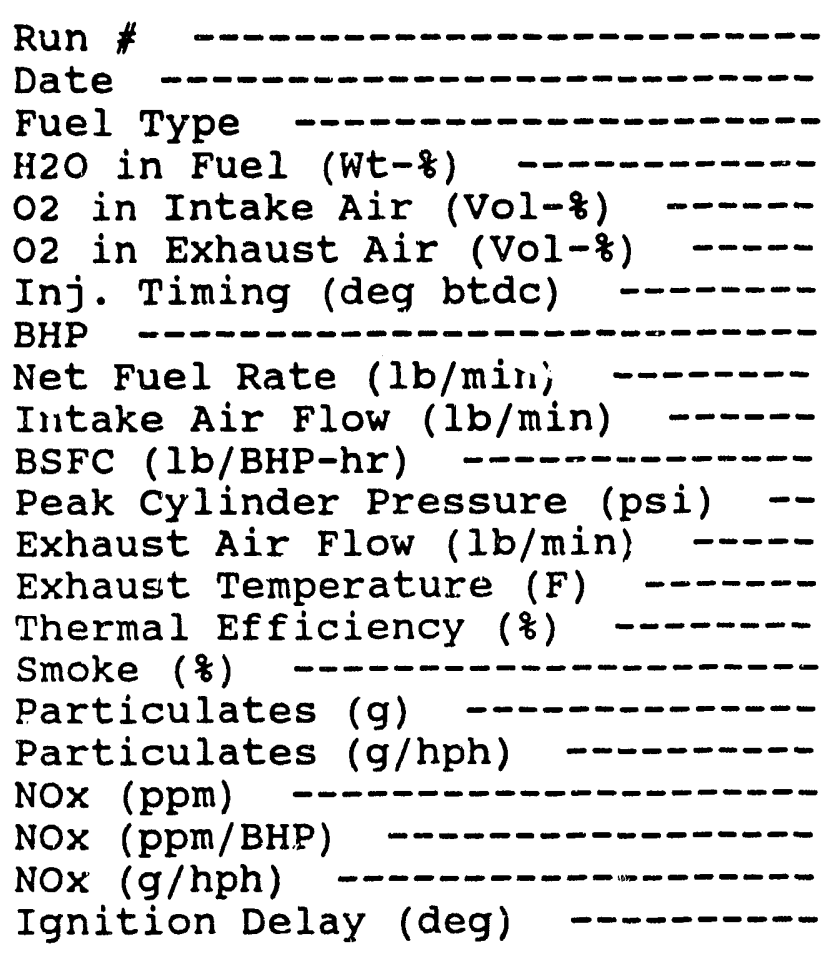

\begin{tabular}{|c|c|c|}
\hline $\begin{array}{l}246 \\
10 / 19 / 90 \\
\# 4\end{array}$ & $\begin{array}{l}250 \\
10 / 22 / 90 \\
\# 4\end{array}$ & $\begin{array}{l}254 \\
10 / 23 / 90 \\
\quad \# 4\end{array}$ \\
\hline 6 & 6.7 & 6.7 \\
\hline 20.56 & 20.34 & 20.28 \\
\hline 14.7 & 14.2 & 14.07 \\
\hline 33 & 27 & 22 \\
\hline 24.77 & 25.04 & 25.2 \\
\hline 0.17205 & 0.172601 & 0.179062 \\
\hline 6.4 & 6.6 & 6.75 \\
\hline 448123 & 0.448083 & 0.461905 \\
\hline 1473 & 1308 & 1141 \\
\hline 6.585 & 6.787 & 6.944 \\
\hline 521 & 563 & 587 \\
\hline 34.73437 & 35.00089 & 33.95355 \\
\hline 47 & 58 & 62 \\
\hline 0129 & 0.0132 & 0.0131 \\
\hline 0.38798 & 0.431061 & 0.460024 \\
\hline 555 & 335 & 175 \\
\hline & 13.37859 & 6.944444 \\
\hline & 2.471172 & 1.312387 \\
\hline 21 & 19 & 18.2 \\
\hline
\end{tabular}


OXYGEN-ENRICHED DIESEL COMBUSTION TESTS -- SERIES II

No. 4 Fuel, Half Load, $5 \%$ Water, $25 \%$ Oxygen Level Injection Timing (deg btdc): $33,27,22$

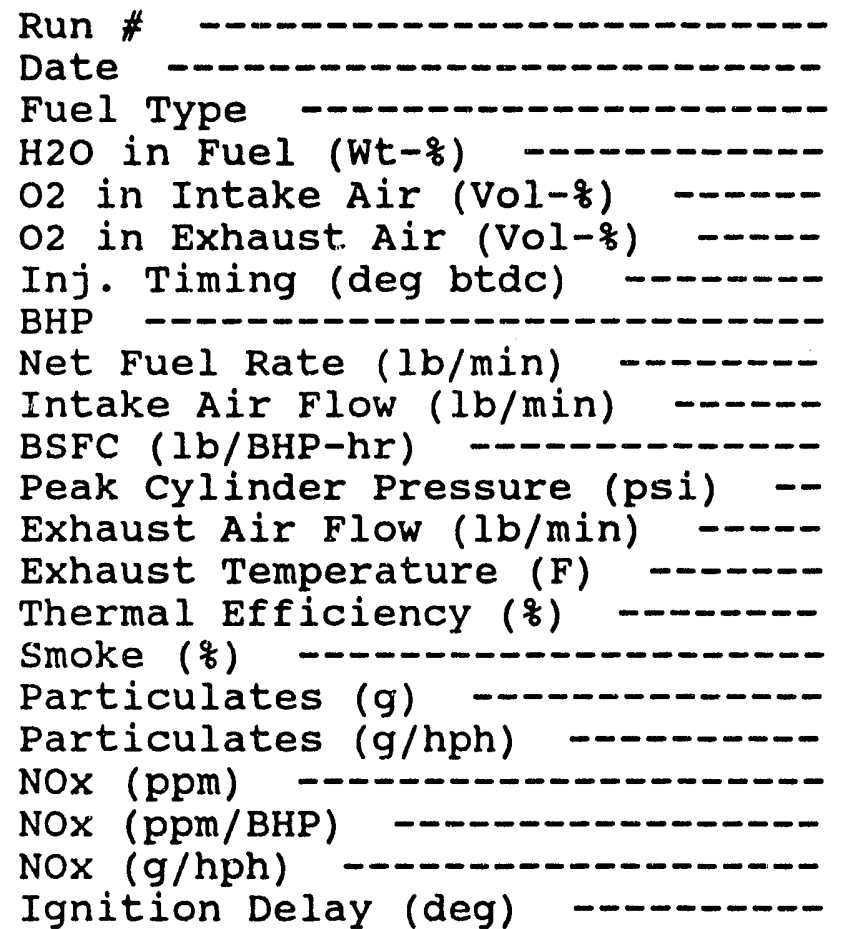

$$
\begin{aligned}
& 247 \quad 251 \quad 255 \\
& 10 / 19 / 90 \quad 10 / 22 / 90 \quad 10 / 23 / 90 \\
& \text { \#4 \#4 \#4 } \\
& \begin{array}{lll}
6.6 & 7 & 7.3
\end{array} \\
& 25.2 \quad 25.36 \quad 25.05 \\
& 19.12 \quad 19.07 \quad 19.21 \\
& 33 \quad 27 \quad 22 \\
& \begin{array}{lll}
24.71 & 25.06 & 24.94
\end{array} \\
& \begin{array}{llll}
0.17094 & 0.17112 & 0.171479
\end{array} \\
& \begin{array}{lll}
6.23 & 6.46 & 6.72
\end{array} \\
& \begin{array}{llll}
0.449211 & 0.445331 & 0.44988
\end{array} \\
& \begin{array}{lll}
1581 & 1373 & 1200
\end{array} \\
& \begin{array}{lll}
6.415 & 6.646 & 6.907
\end{array} \\
& 500 \quad 540 \quad 547 \\
& 34.87523 \quad 35.3320135 .08921 \\
& \begin{array}{rrr}
35 & 43 & 36 \\
0.0139 & 0.0104 & 0.011
\end{array} \\
& \begin{array}{llll}
0.407501 & 0.332908 & 0.379883
\end{array} \\
& 1290 \quad 940 \quad 660 \\
& 52.20558 \quad 37.50998 \quad 26.46351 \\
& 9.114406 \quad 6.784562 \quad 4.974526 \\
& 19.3 \quad 17.3 \quad 16.4
\end{aligned}
$$


$B-21$

B.2 Analyses of Data from Emissions Repeatability Tests 
B-22 


\section{OXYGEN-ENRICHED DIESEL COMBUSTION TESTS -- SERIES II}

\section{Emissions Repeatability Tests}

50 BHP, No. 2 Fuel, of Water, 22 deg btdc Inj. Timing

Parameter

Particulates ( $g / \mathrm{hph})$

NOX $(\mathrm{g} / \mathrm{hph})$

Smoke ( $\left(\frac{\circ}{6}\right)$

Exhaust Temperature (F)

02 Exhaust (\%)
one-Day Tests

Average std

$0.26185 \quad 0.03538$

$1.15 \quad 0.05$

64.30

3.56

$822.60 \quad 2.72$

11.99

0.06
11.80

1. 39

$56.28 \quad 12.26$

$832.22 \quad 22.27$

$0.27186 \quad 0.04390$

0.38

0.37

\begin{tabular}{|c|c|c|c|c|c|c|c|}
\hline Date & Time & O2 $\underset{f}{\text { Exh. }}$ & $\begin{array}{l}\text { Exh. T } \\
\operatorname{deg} F\end{array}$ & $\begin{array}{c}\text { Smoke } \\
\frac{q}{q}\end{array}$ & $\begin{array}{l}\text { Part. } \\
\text { g/hph }\end{array}$ & $\begin{array}{c}\text { NOx } \\
\text { g/hph }\end{array}$ & Run \# \\
\hline $10 / 25 / 90$ & $10: 10$ & 12.00 & 818.00 & 60.00 & 0.33 & 1.12 & \\
\hline $10 / 25 / 90$ & $10: 40$ & 11.90 & 821.00 & 58.00 & 0.30 & 1.13 & \\
\hline $10 / 25 / 90$ & $11: 10$ & 12.00 & 819.00 & 62.00 & 0.25 & 1.11 & \\
\hline $10 / 25 / 90$ & $11: 40$ & 12.00 & 826.00 & 64.00 & 0.25 & 1. 11 & \\
\hline $10 / 25 / 90$ & $12: 40$ & 12.00 & 824.00 & 63.00 & 0.30 & 1.09 & \\
\hline $10 / 25 / 90$ & $13: 10$ & 12.10 & 825.00 & 65.00 & 0.22 & 1.22 & \\
\hline $10 / 25 / 90$ & $13: 40$ & 12.00 & 825.00 & 67.00 & 0.22 & 1.22 & \\
\hline $10 / 25 / 90$ & $14: 25$ & 12.00 & 823.00 & 68.00 & 0.26 & 1.18 & \\
\hline $10 / 25 / 90$ & $14: 55$ & 11.90 & 824.00 & 68.00 & 0.24 & 1.18 & \\
\hline $10 / 25 / 90$ & $15: 25$ & 12.00 & 821.00 & 68.00 & 0.24 & 1.15 & \\
\hline $10 / 23 / 90$ & $11: 23$ & 12.23 & 825.00 & 64.00 & 0.24 & 1.35 & \\
\hline $10 / 11 / 90$ & $10: 20$ & 11.97 & 801.00 & 55.00 & 0.24 & 1.83 & \\
\hline $10 / 11 / 90$ & $15: 13$ & 11.96 & 803.00 & 32.00 & 0.28 & 2.31 & 241 \\
\hline $09 / 06 / 90$ & $13: 15$ & 11.35 & 867.00 & 52.00 & 0.37 & 1.89 & 213 \\
\hline $07 / 16 / 90$ & $14: 25$ & 11.00 & 867.00 & 35.00 & 0.32 & 1.16 & 205 \\
\hline $09 / 26 / 90$ & $14: 57$ & 11.74 & 853.00 & 36.00 & - & 1.93 & \\
\hline $09 / 06 / 90$ & $12: 45$ & 1.1 .35 & 868.00 & 56.00 & - & 1.86 & \\
\hline $07 / 16 / 90$ & $13: 40$ & 10.98 & 870.00 & 40.00 & - & 1.18 & \\
\hline
\end{tabular}




\section{$B-24$}

OXYGEN-ENRICHED DIESEL COMBUSTION TESTS -- BERIES II

Emissions Repeatability Tests

50 BHP, No. 2 Fuel, $0 \%$ Water, 27 deg btdc Inj. Timing

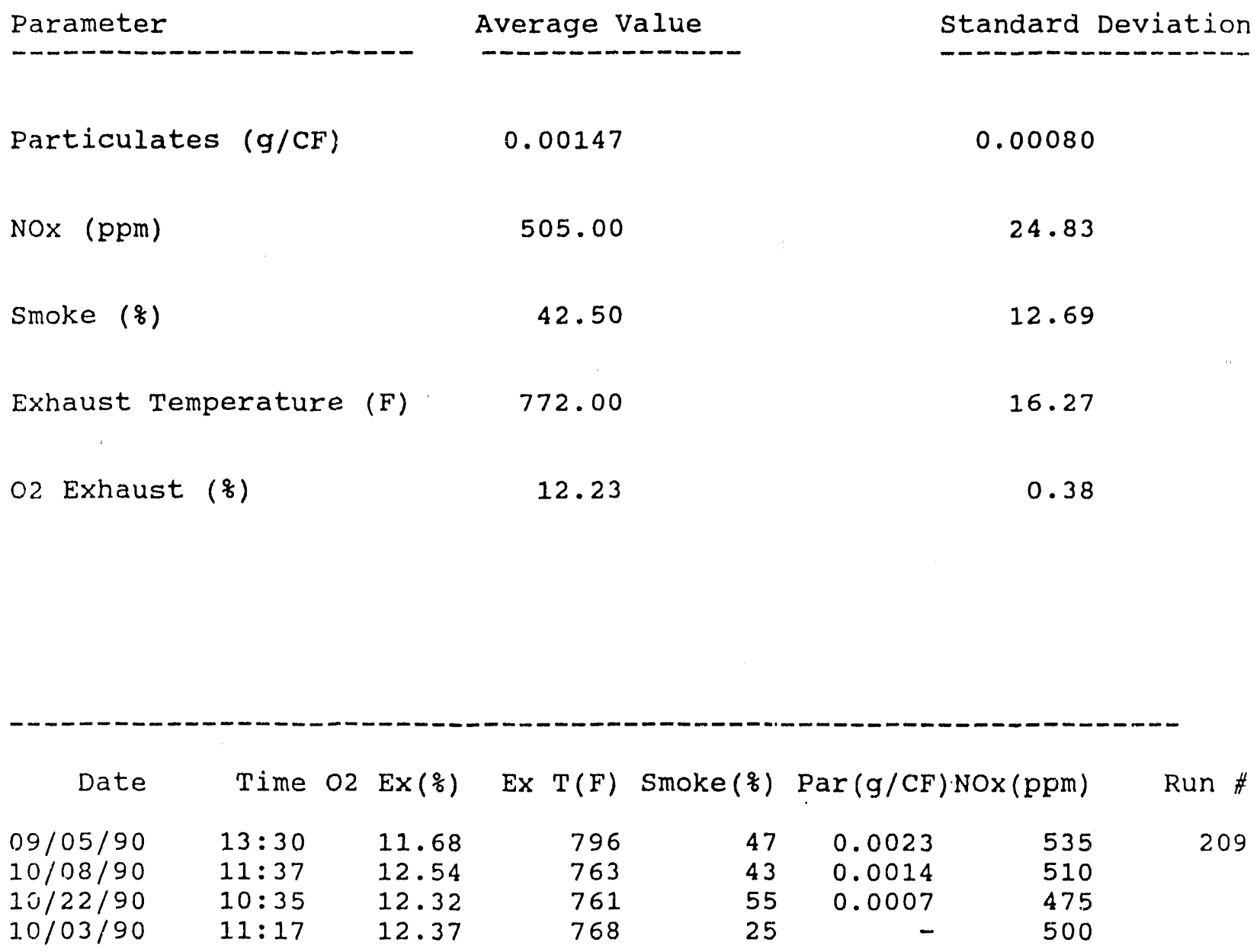


OXYGEN-ENRICHED DIESEL COMBUSTION TESTS -- SERIES II

Emissions Repeatability Tests

50 BHP, No. 2 Fuel, $0 \%$ Water, 33 deg btdc Inj. Timing

$\begin{array}{lcc}\text { Parameter } & \text { Average Value } & \text { Standard Deviation } \\ \text { Particulates (g/CF) } & 0.00093 & 0.00035 \\ \text { Nox (ppm) } & 699.57 & 128.96 \\ \text { Smoke (q) } & 22.57 & 8.08 \\ \text { Exhaust Temperature (F) } & 754.86 & 36.45 \\ \text { O2 Exhaust (q) } & 11.79 & 0.66\end{array}$

$\begin{array}{rrrrrrrr}\text { Date } & \text { Time } 02 & \text { Ex }(\%) & \text { Ex T }(F) & \text { Smoke }(q) & \text { Par }(g / C F) \text { NOx (ppm) } & \text { Run \# } \\ 07 / 12 / 90 & 14: 30 & 10.90 & 806 & 18 & 0.0013 & 575 & 201 \\ 09 / 10 / 90 & 11: 42 & 11.94 & 747 & 25 & 0.0009 & 850 & 217 \\ 10 / 12 / 90 & 13: 00 & 12.44 & 721 & 12 & 0.0006 & 750 & \\ 07 / 12 / 90 & 13: 20 & 10.98 & 800 & 19 & - & 590 & \\ 09 / 10 / 90 & 11: 07 & 11.83 & 755 & 34 & - & 540 \\ 09 / 20 / 90 & 13: 05 & 11.82 & 745 & 18 & - & 750 \\ 10 / 19 / 90 & 10: 54 & 12.64 & 710 & 32 & - & \end{array}$




\section{OXYGEN-ENRICHED DIESEL COMBUSTION TESTS -- SERIES II \\ Emissions Repeatability Tests \\ 25 BHP, No. 2 Fuel, $0 \%$ Water, 22 deg btdc Inj. Timing}

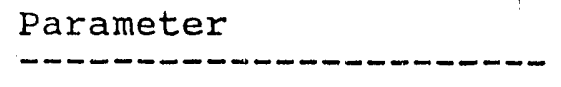

Particula es ( $g / C F)$

NOX (ppm)

Smoke (\%)

Exhaust Temperature (F)

02 Exhaust (\%)

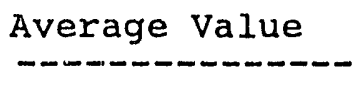

0.00210

226.25

58.50

582.25

14.32
Standard Deviation

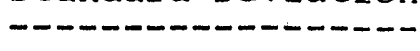

0.00030

46.79

15.80

10.11

0.21

Date Time 02 Ex $(q)$ Ex $T(F)$ Smoke $(q)$ Par $(g / C F)$ NOx $(\mathrm{ppm}) \quad$ Run \#

$\begin{array}{llllllll}09 / 07 / 90 & 12: 50 & 14.57 & 573 & 66 & 0.0024 & 235 & 215 \\ 10 / 11 / 90 & 11: 07 & 14.09 & 591 & 64 & 0.0018 & 240 & \\ 10 / 23 / 90 & 12: 54 & 14.22 & 591 & 69 & 0.0021 & 160 & \\ 10 / 02 / 90 & 12: 55 & 14.41 & 574 & 35 & - & 270 & \end{array}$


OXYGEN-ENRICHED DIESEL COMBUSTION TESTS -- SERIES II

Emissions Repeatability Tests

25 BHP, No. 2 Fuel, of Water, 27 deg btdc Inj. Timing

\begin{tabular}{|c|c|c|c|c|}
\hline \multirow{3}{*}{ Parameter } & \multicolumn{2}{|c|}{ One-Day Tests } & \multicolumn{2}{|c|}{ Multi-Day Tests } \\
\hline & Average & std & Average & std \\
\hline & $-\cdots-\cdots$ & $-\ldots-$ & $-\cdots-\cdots$ & ----- \\
\hline Particulates (g/CF) & 0.283822 & 0.03004 & 0.318657 & 0.093929 \\
\hline NOX (ppm) & 1.16 & 0.16 & 1.76 & 0.79 \\
\hline Smoke $(\%)$ & 56.78 & 1.48 & 54.40 & 8.77 \\
\hline Exhaust Temperature (F) & 559.78 & 4.41 & 567.47 & 12.09 \\
\hline 02 Exhaust $(\%)$ & 14.46 & 0.12 & 14.33 & 0.22 \\
\hline
\end{tabular}

$\begin{array}{cccrrrrr}\text { Date } & \text { Time } & \text { O2 Exh. } & \begin{array}{l}\text { Exh. T } \\ \text { deg F }\end{array} & \begin{array}{c}\text { Smoke } \\ \%\end{array} & \begin{array}{l}\text { Part. } \\ \text { g/hph }\end{array} & \begin{array}{c}\text { Nox } \\ \text { g/hph }\end{array} & \text { Run \# } \\ 10 / 26 / 90 & 10: 40 & 14.60 & 557 & 54 & 0.26 & 1.36 \\ 10 / 26 / 90 & 11: 10 & 14.60 & 555 & 57 & 0.26 & 1.34 \\ 10 / 26 / 90 & 11: 40 & 14.60 & 556 & 55 & 0.23 & 1.33 \\ 10 / 26 / 90 & 12: 40 & 14.40 & 559 & 57 & 0.29 & 1.20 \\ 10 / 26 / 90 & 13: 10 & 14.50 & 556 & 56 & 0.31 & 1.16 \\ 10 / 26 / 90 & 13: 40 & 14.40 & 559 & 58 & 0.33 & 1.04 \\ 10 / 26 / 90 & 14: 40 & 14.40 & 564 & 58 & 0.30 & 1.04 \\ 10 / 26 / 90 & 15: 00 & 14.30 & 566 & 58 & 0.31 & 1.00 \\ 10 / 26 / 90 & 15: 25 & 14.30 & 566 & 58 & 0.27 & 0.96 \\ 10 / 22 / 90 & 11: 38 & 14.32 & 563 & 63 & 0.18 & 2.33 \\ 10 / 05 / 90 & 11.48 & 14.10 & 585 & 60 & 0.44 & 2.59 \\ 10 / 05 / 90 & 13: 17 & 13.91 & 586 & 45 & 0.49 & 2.97 \\ 09 / 05 / 90 & 16: 55 & 14.00 & 586 & 54 & 0.48 & 2.84 \\ 10 / 03 / 90 & 12: 52 & 14.44 & 568 & 26 & - & 2.29 \\ 09 / 05 / 90 & 16: 20 & 14.08 & 586 & 57 & - & 2.87\end{array}$




\section{OXYGEN-ENRICHED DIESEL COMBUSTION TESTS -- SERIES II}

Emissions Repeatability Tests

25 BHP, No. 2 Fuel, $0 \%$ Water, 33 deg btdc Inj. Timing

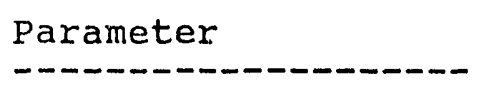

Particulates $(\mathrm{g} / \mathrm{CF})$

NOX (ppm)

Smoke (\%)

Exhaust Temperature (F)

02 Exhaust (\%)

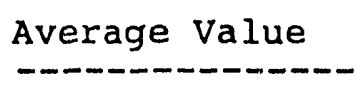

0.00070

494.50

25.00

544.50

14.33
Standard Deviation

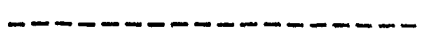

0.00032

183.30

7.16

32.99

0.40

\begin{tabular}{|c|c|c|c|c|c|c|c|c|}
\hline Date & Time & 02 & $\operatorname{Ex}\left(\frac{\circ}{6}\right)$ & $\mathrm{Ex} T(F)$ & Smoke $(q)$ & $\operatorname{Par}(g / C F$ & pm) & Run \# \\
\hline $07 / 12 / 90$ & $16: 20$ & & 13.57 & 608 & 21 & 0.0008 & 310 & 203 \\
\hline $09 / 11 / 90$ & $11: 42$ & & 14.42 & 537 & 16 & 0.0005 & 643 & 219 \\
\hline $10 / 17 / 90$ & $13: 16$ & & 14.32 & 546 & 28 & 0.0011 & 600 & \\
\hline $10 / 19 / 90$ & $13: 07$ & & 14.75 & 517 & 35 & 0.0004 & 550 & \\
\hline $09 / 21 / 90$ & $1.3: 05$ & & 14.45 & 521 & 30 & - & 220 & \\
\hline $09 / 11 / 90$ & $11: 15$ & & 14.48 & 538 & 20 & - & 644 & \\
\hline
\end{tabular}




\section{B.3 Graphic Display of Test Data for No. 2 Fuel}




\section{B-30}




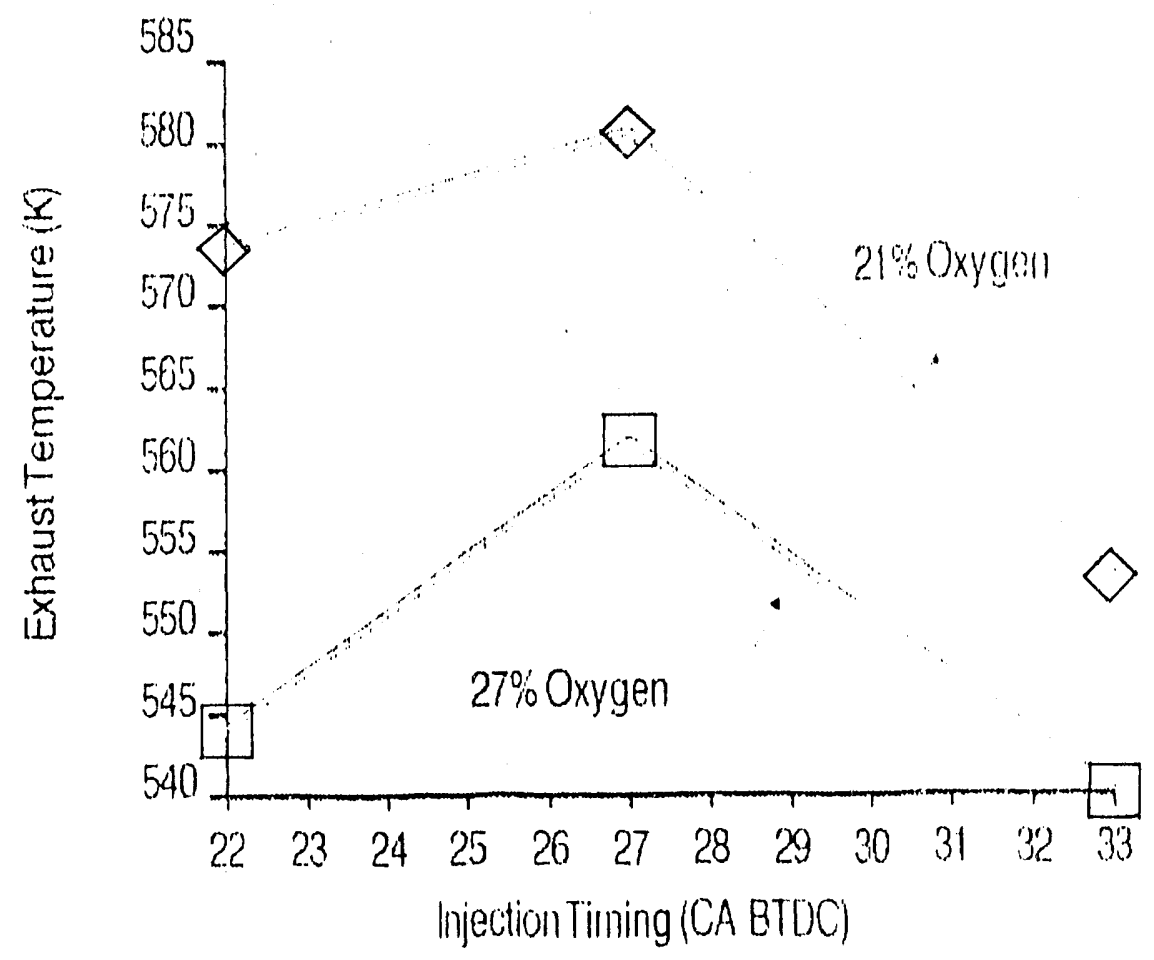

FIGURE B.1 Effect of Injection Timing on Exhaust Temperature, 25 Brake Horsepower, No. 2 Diesel, No Water

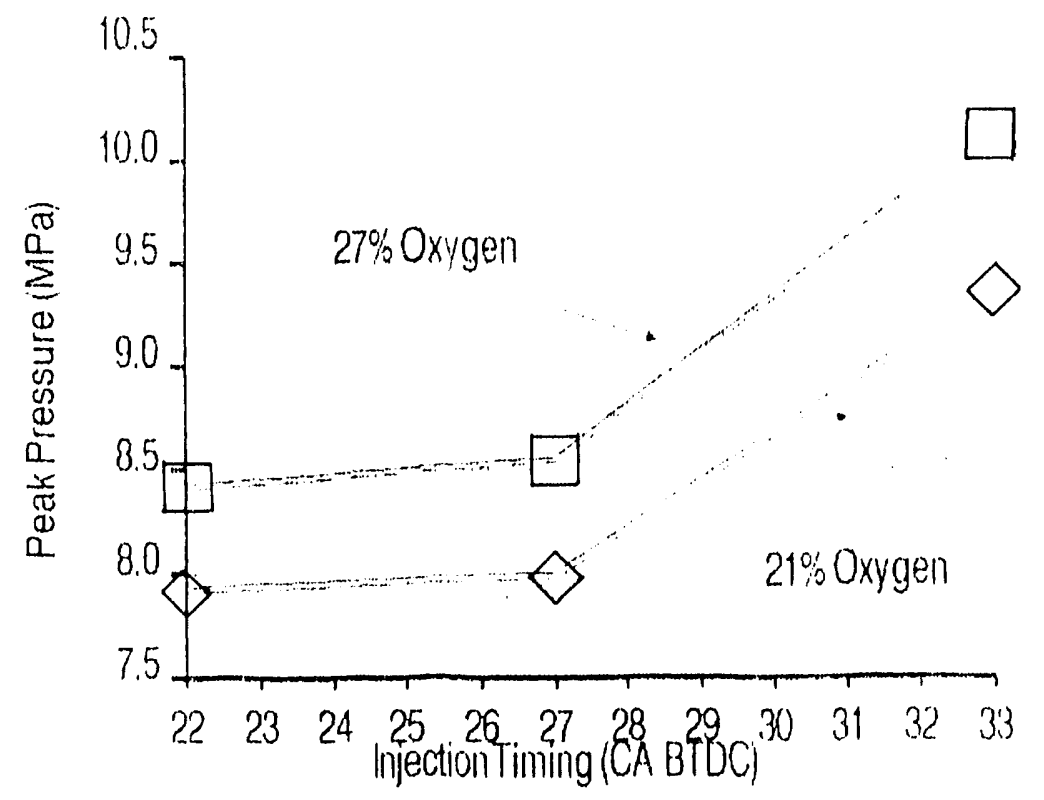

FIGURE B.2 Effect of Injection Timing on Peak Cylinder Pressure, 25 Brake Horsepower, No. 2 Diesel, No Water 


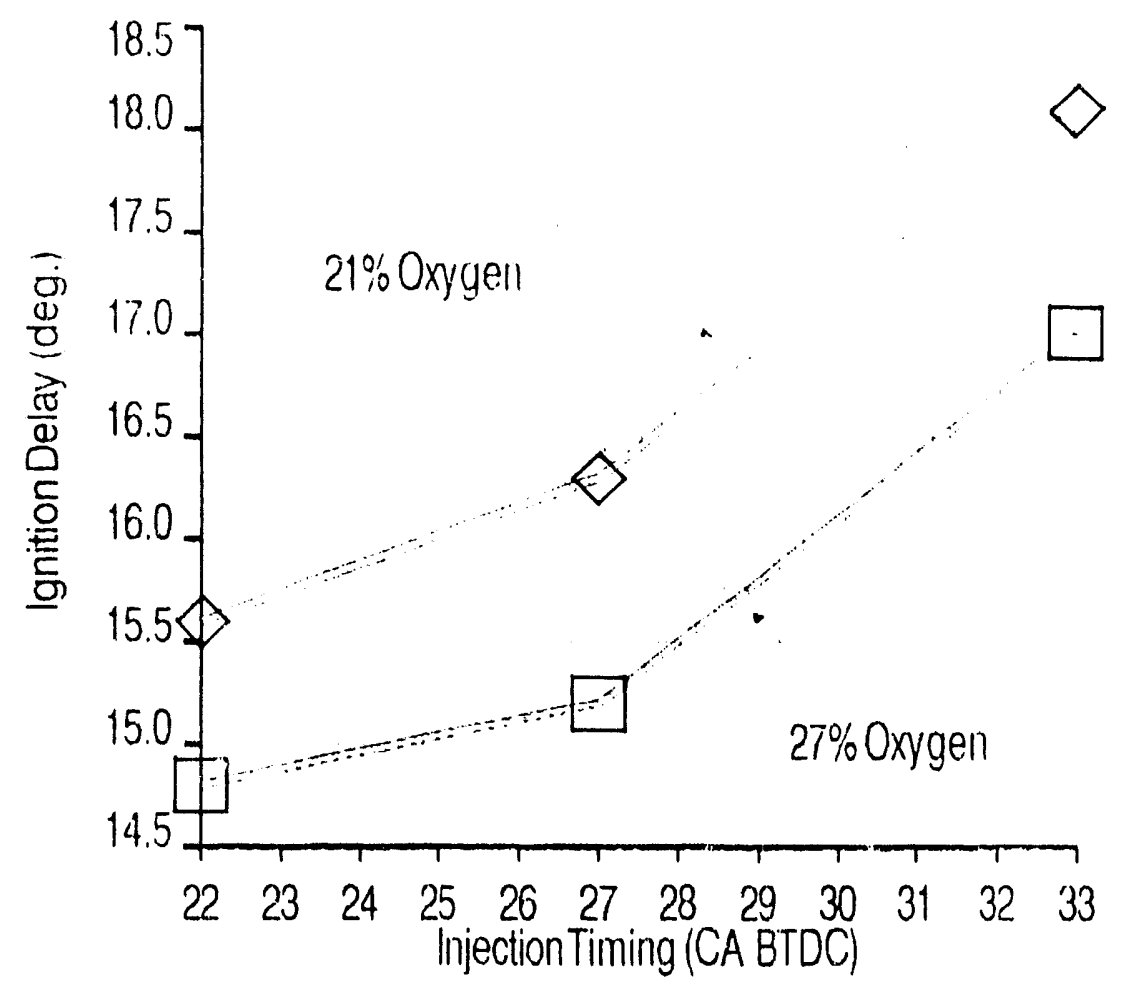

FIGURE B.3 Effect of Injection Timing on Ignition Delay, 25 Brake Horsepower, No. 2 Diesel, No Water

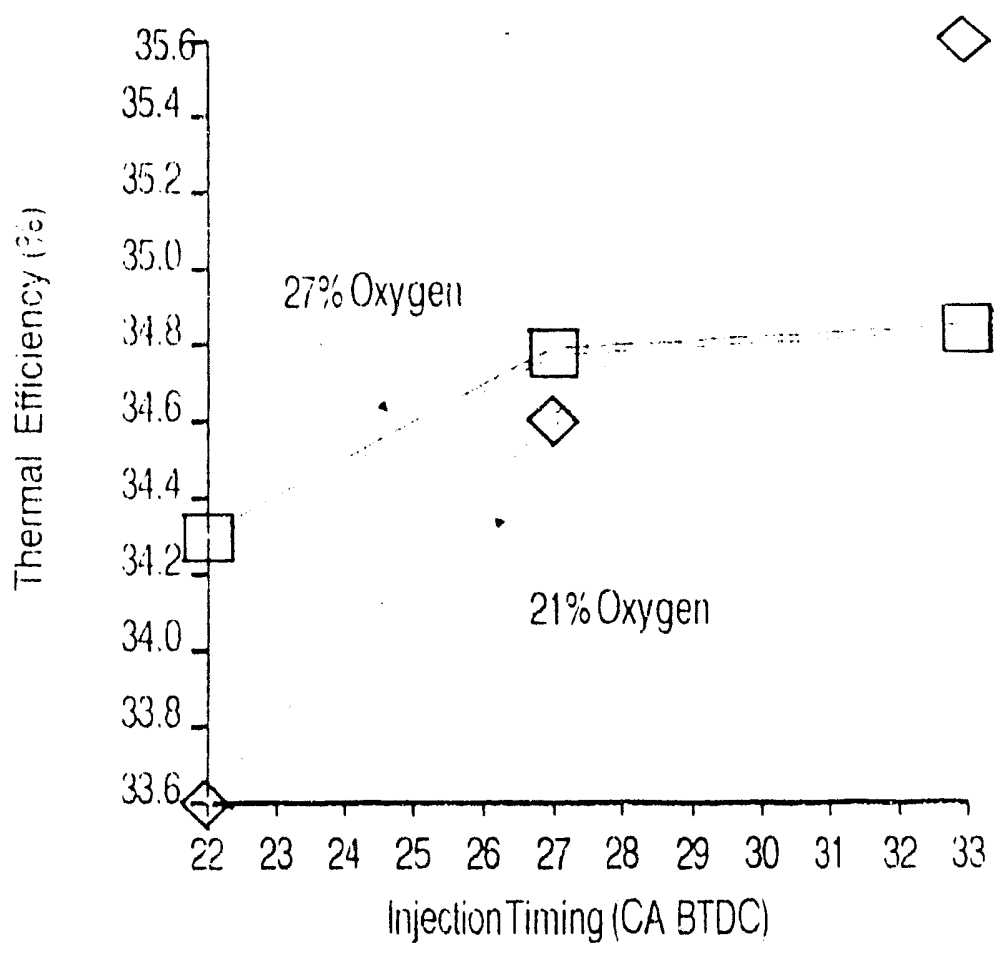

FIGURE B.4 Effect of Injection Timing on Thermal Efficiency, 25 Brake Horsepower, No. 2 Diesel, No Water 


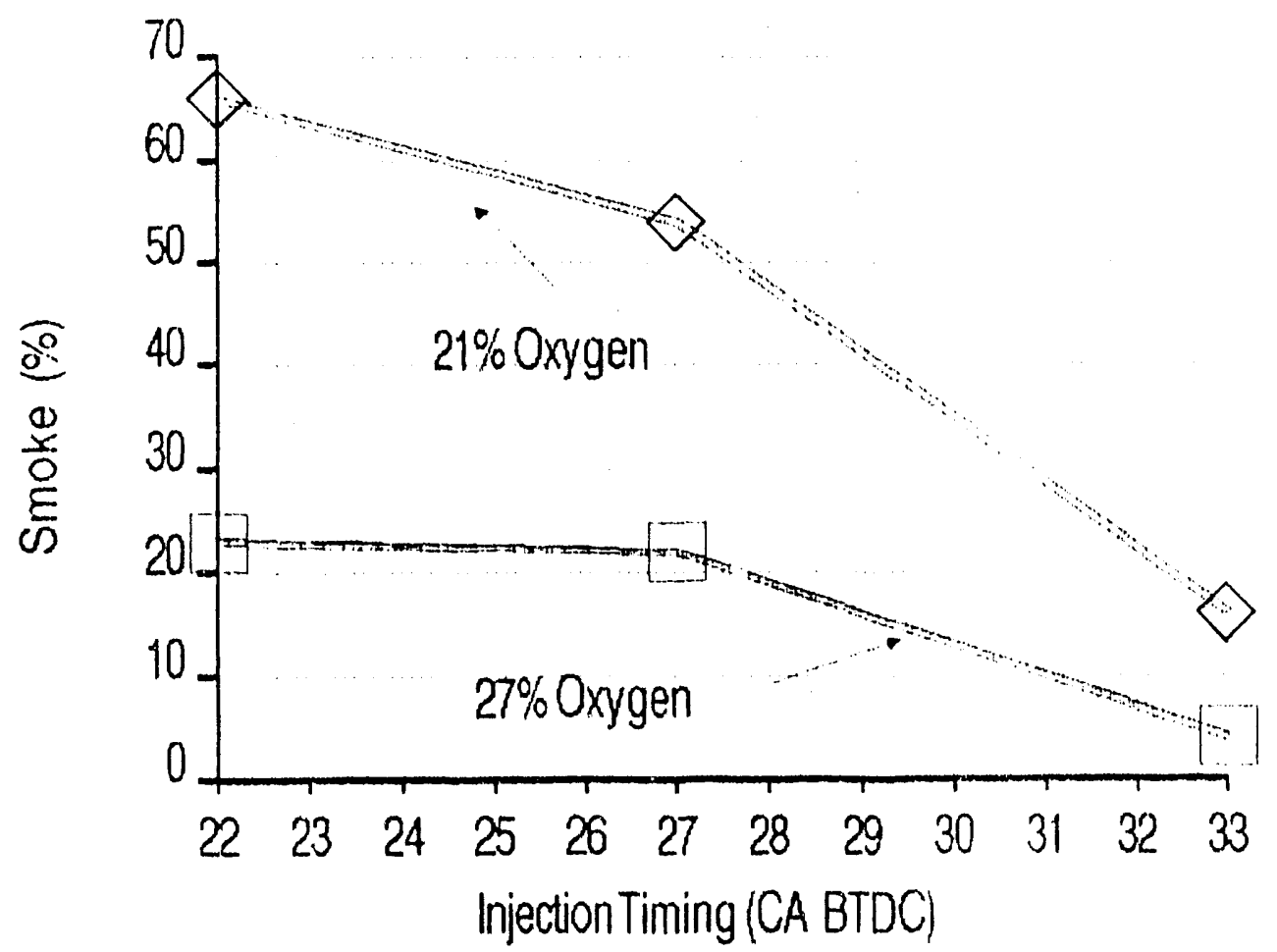

FIGURE B.5 Effect of Injection Timing on Smoke Emission, 25 Brake Horsepower, No. 2 Diesel, No Water

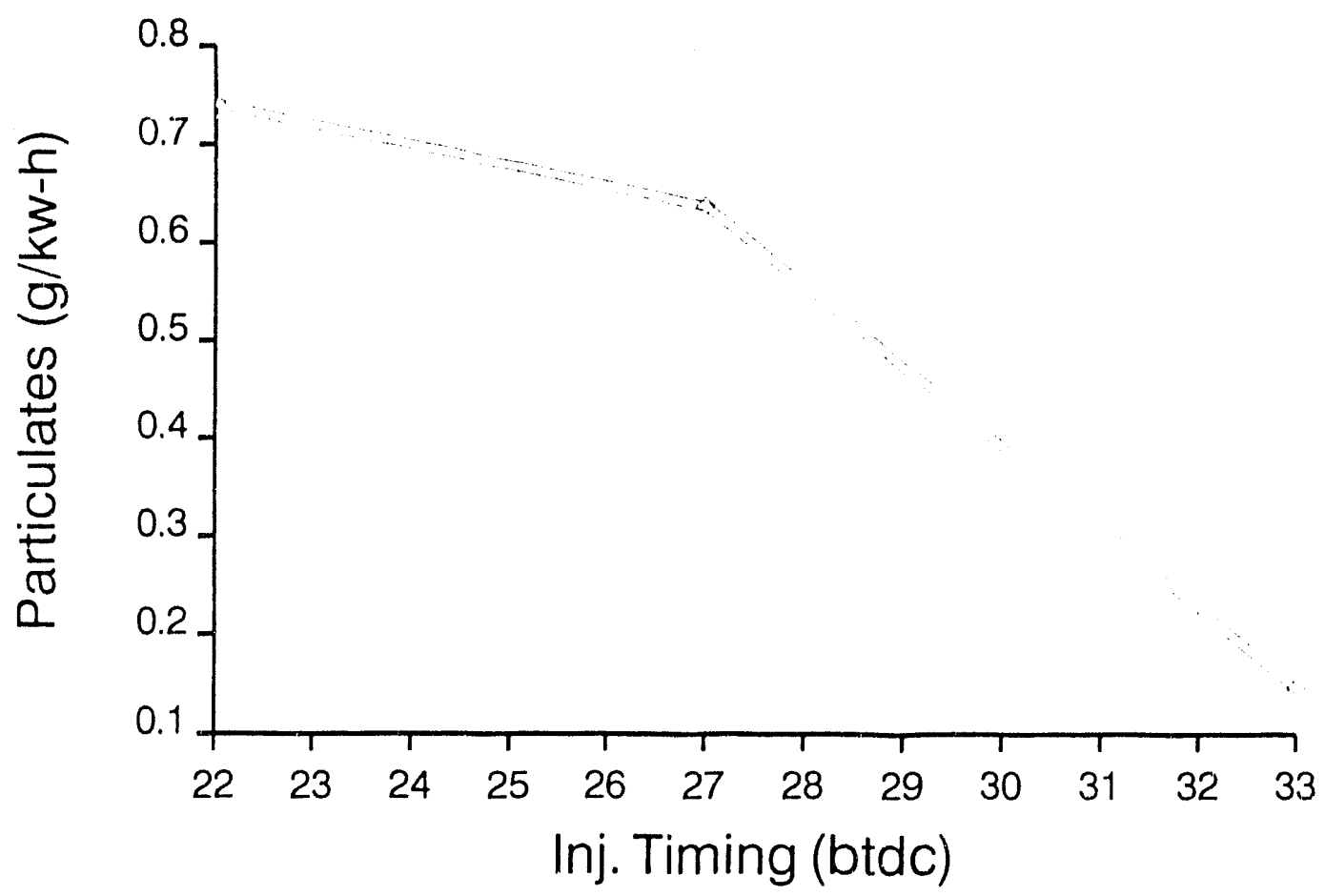

FIGURE B.6 Effect of In ?ction Timing on Particulate Matter, 25 Brake Horsepower, No. 2 Diesel, No Water, 21\% Oxygen 


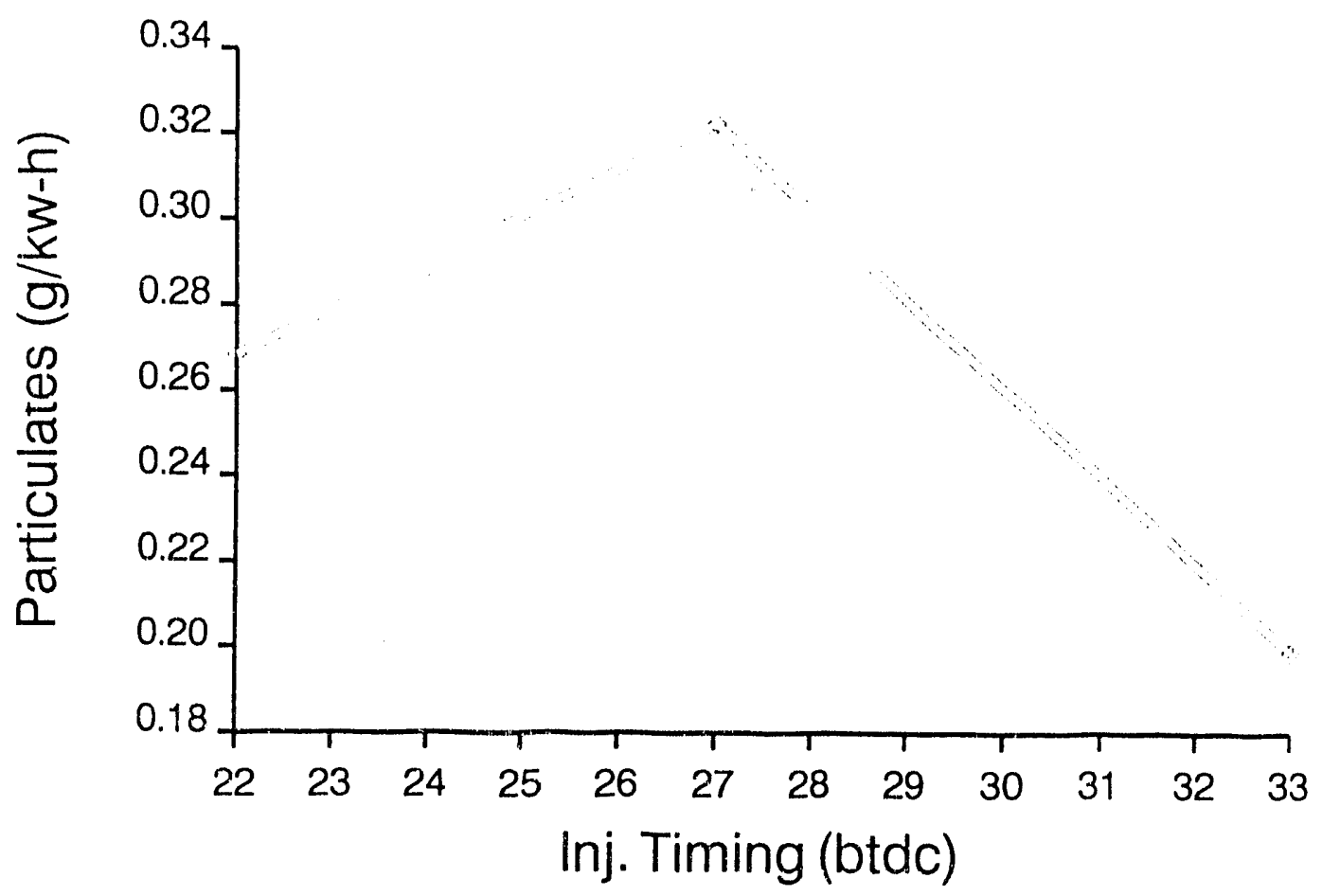

FIGURE B.7 Effect of Injection Timing on Partirulate Matter, 25 Brake Horsepower, No. 2 Diesel, No Water, $25 \%$ Oxygen

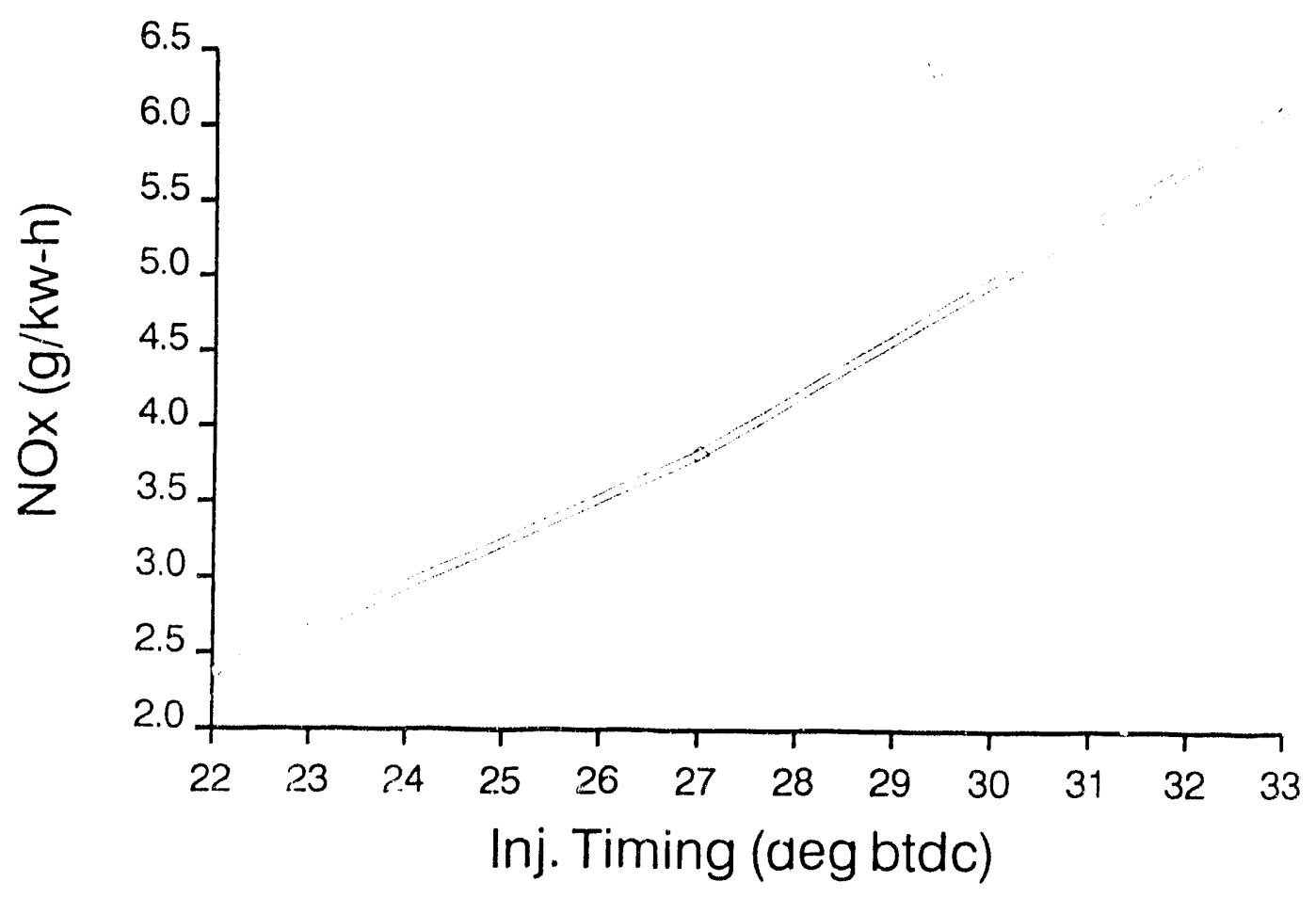

FIGURE B.8 Effect of injection Timing on NO, 25 Brake Horsepower, No. 2 Diesel, No Water, $21 \%$ Oxygen 


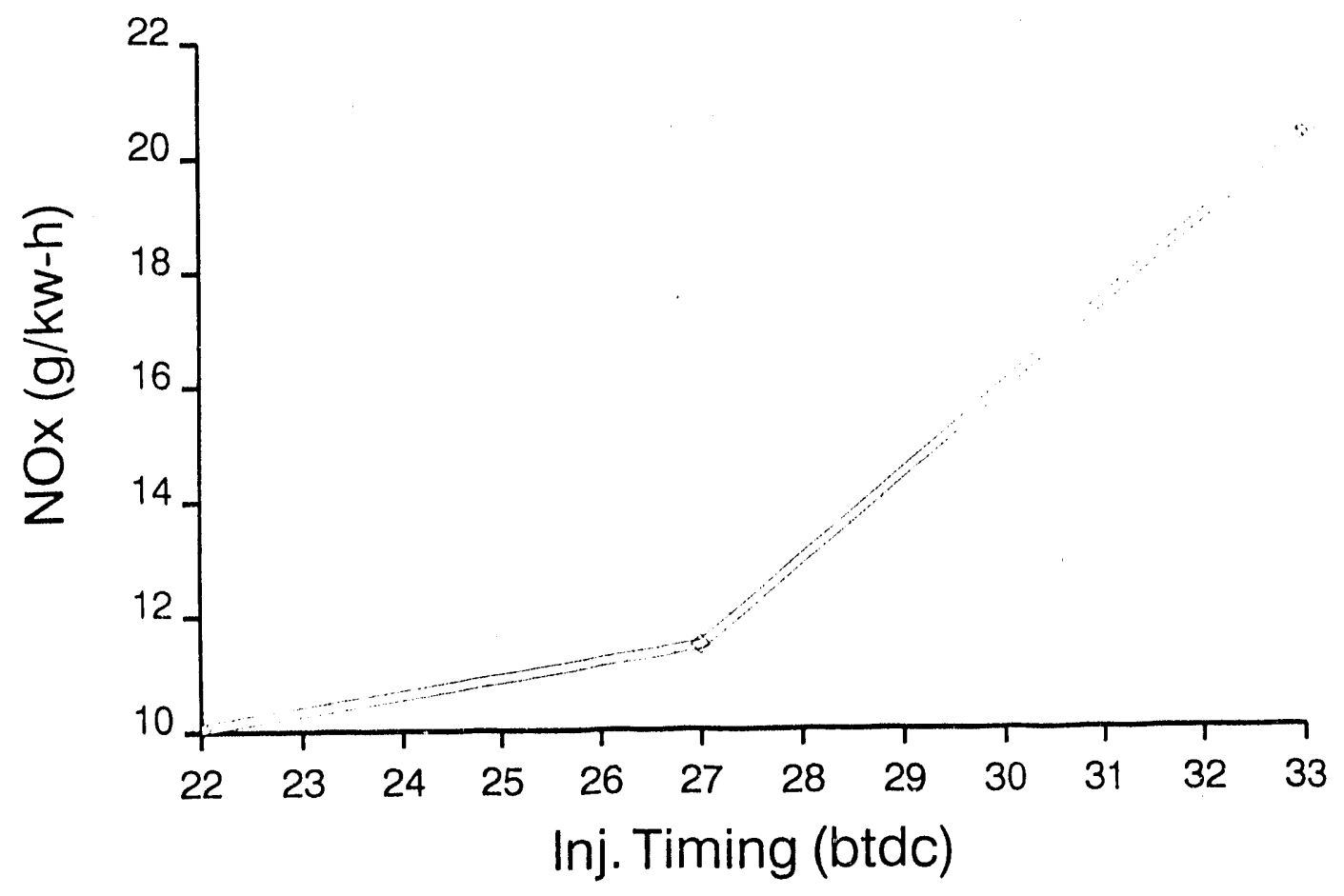

FIGURE B.9 Effect of Injection Timing on $\mathrm{NO}_{\mathrm{x}}, 25$ Brake Horsepower, No. 2 Diesel, No Water, $25 \%$ Oxygen

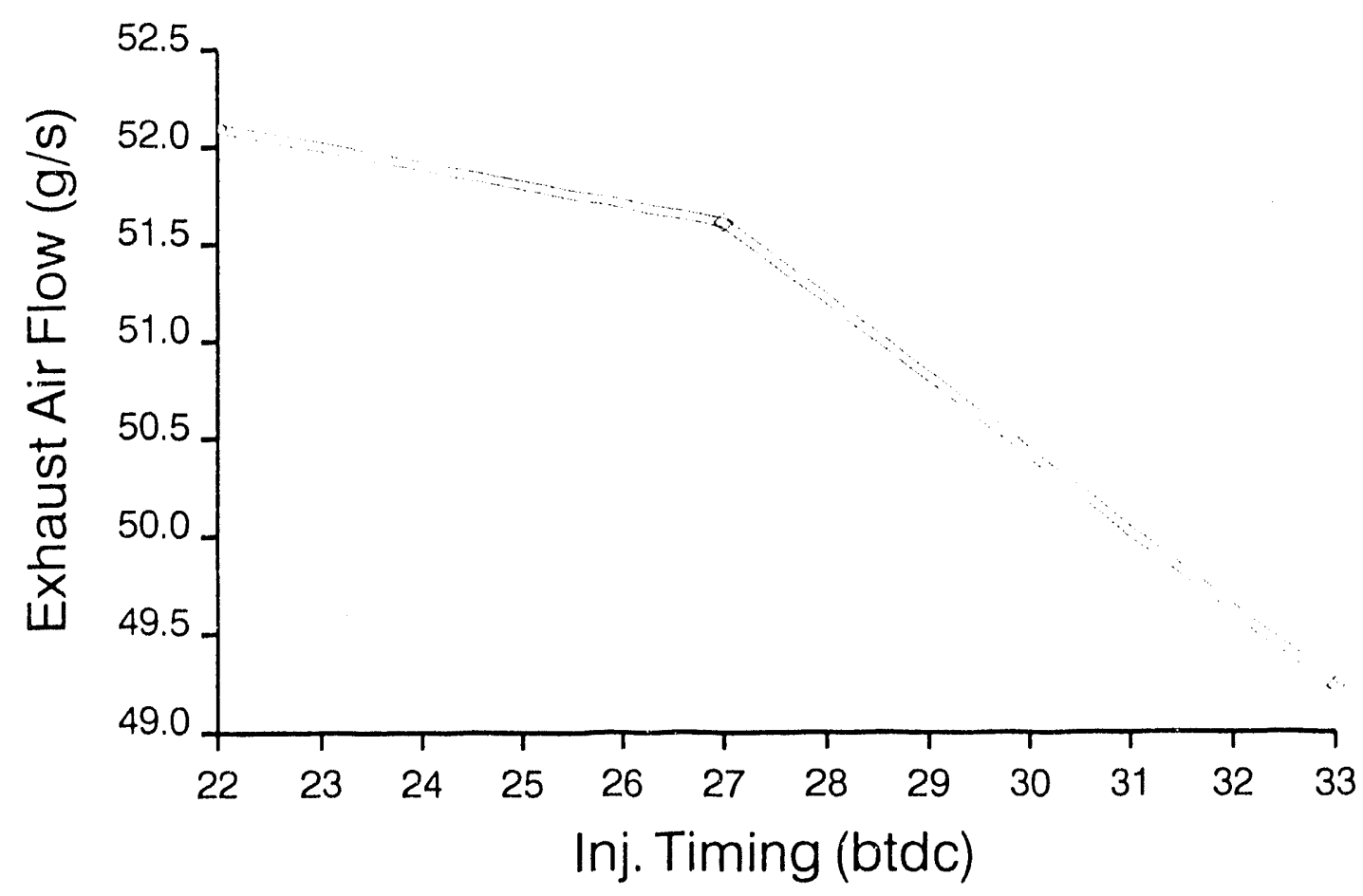

FIGURE B.10 Effect of Injection Timing on Exhaust Air Flow, 25 Brake Horsepower, No. 2 Diesel, No Water, $21 \%$ Oxygen 


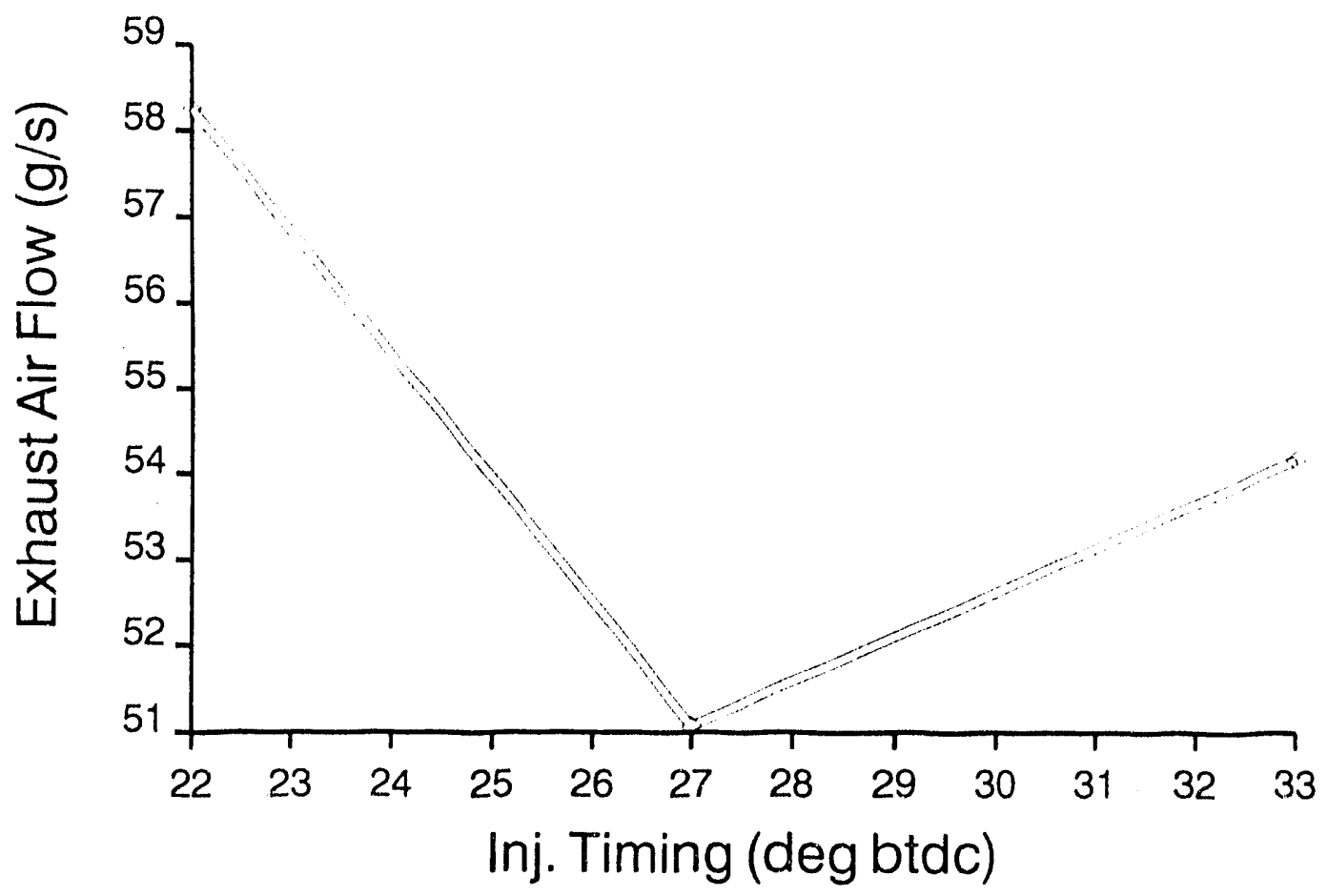

FIGURE B.11 Effect of Injection Timing on Exhaust Air Flow, 25 Brake Horsepower, №. 2 Diesel, No Water, 25\% Oxygen

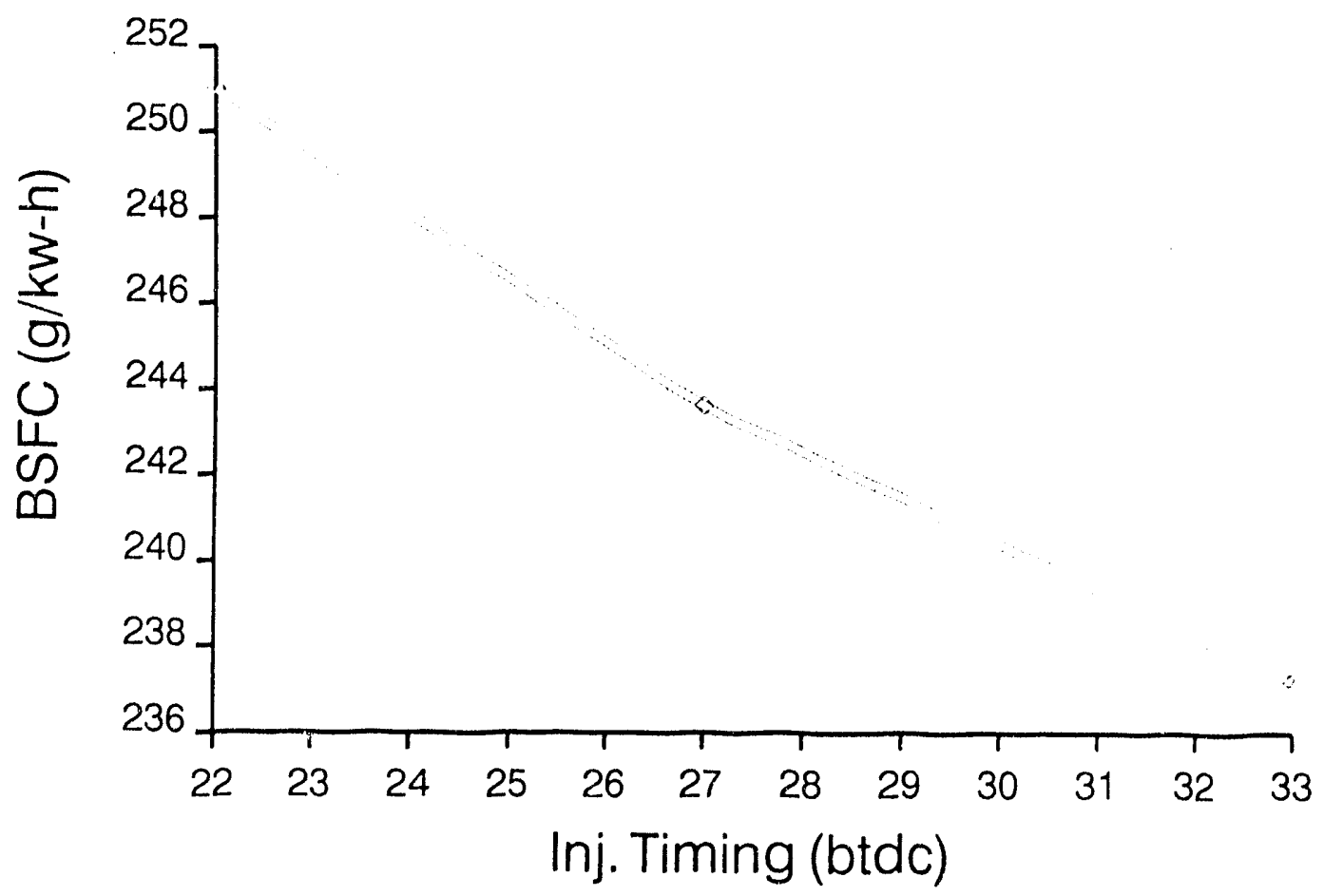

FIGURE B.12 Effect of Injection Timing on Brake-Specific Fuel Consumption, 25 Brake Horsepower, No. 2 Diesel, No Water, $21 \%$ Oxygen 


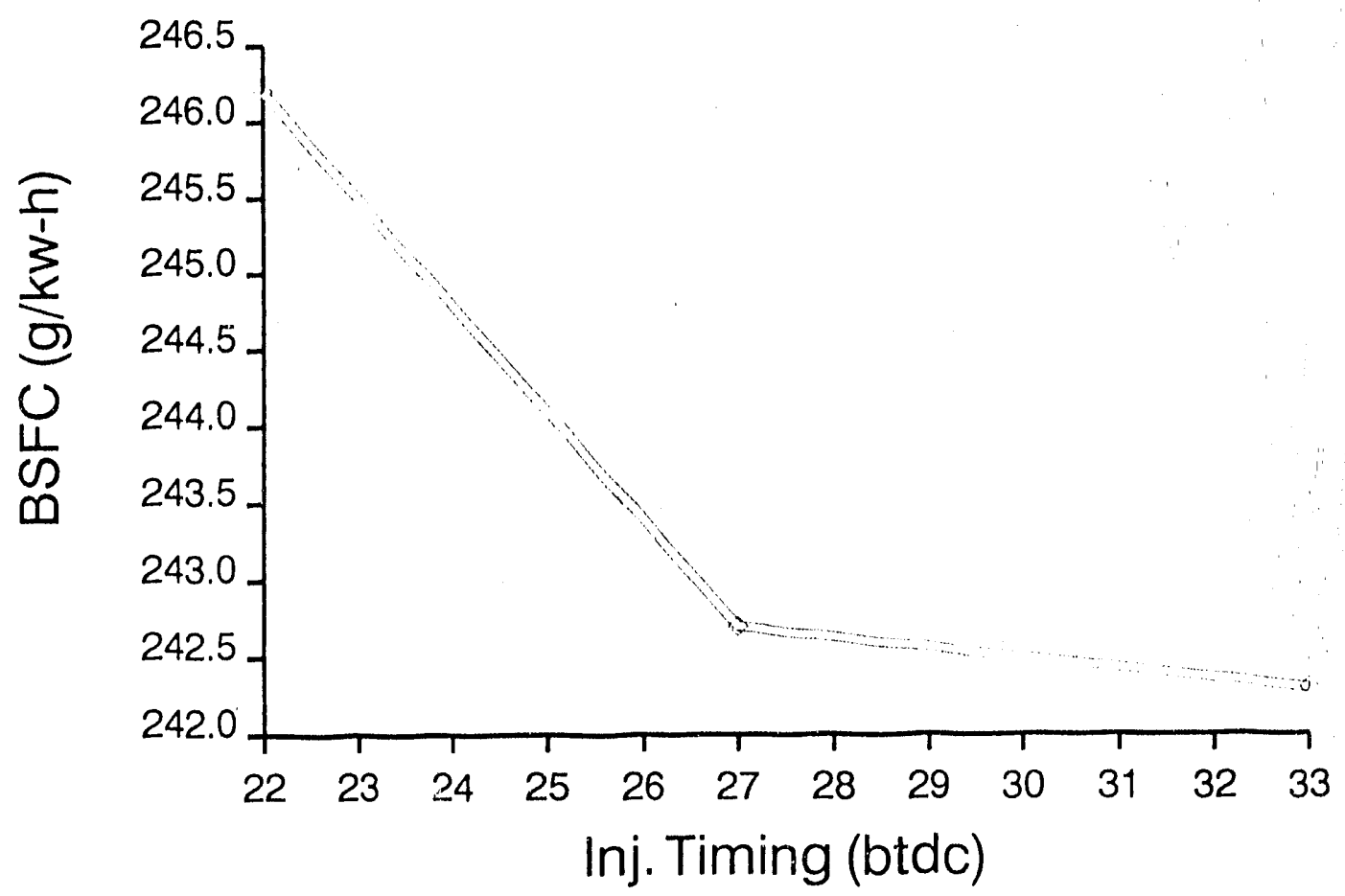

FIGURE B.13 Effect of Injection Timing on Brake-Specific Fuel Consumption, 25 Brake Horsepower, No. 2 Diesel, No Water, 25\% Oxygen

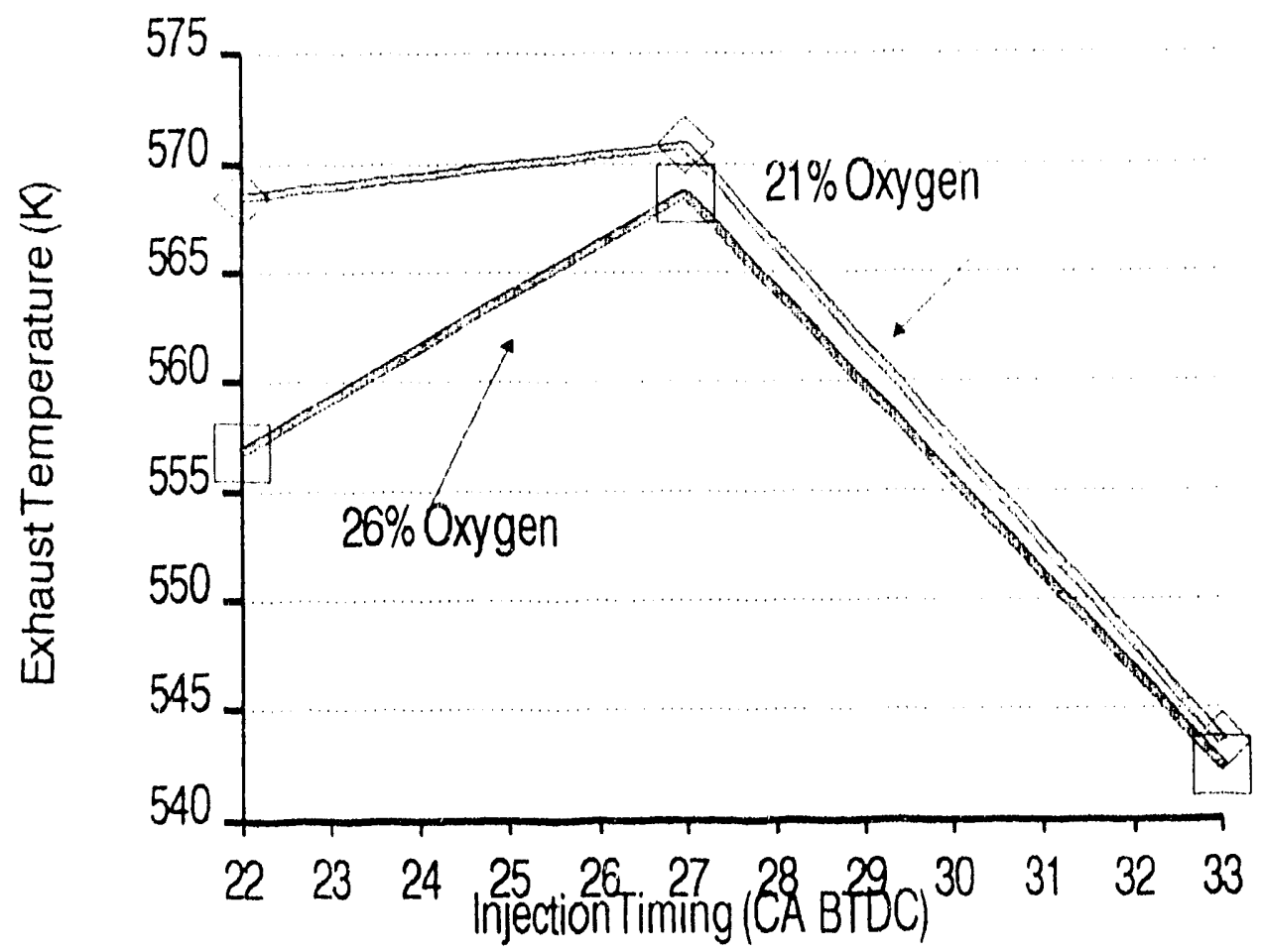

FIGURE B.14 Effect of Injection Timing on Exhaust Temperature, 25 Brake Horsepower, No. 2 Diesel, $5 \%$ Water 


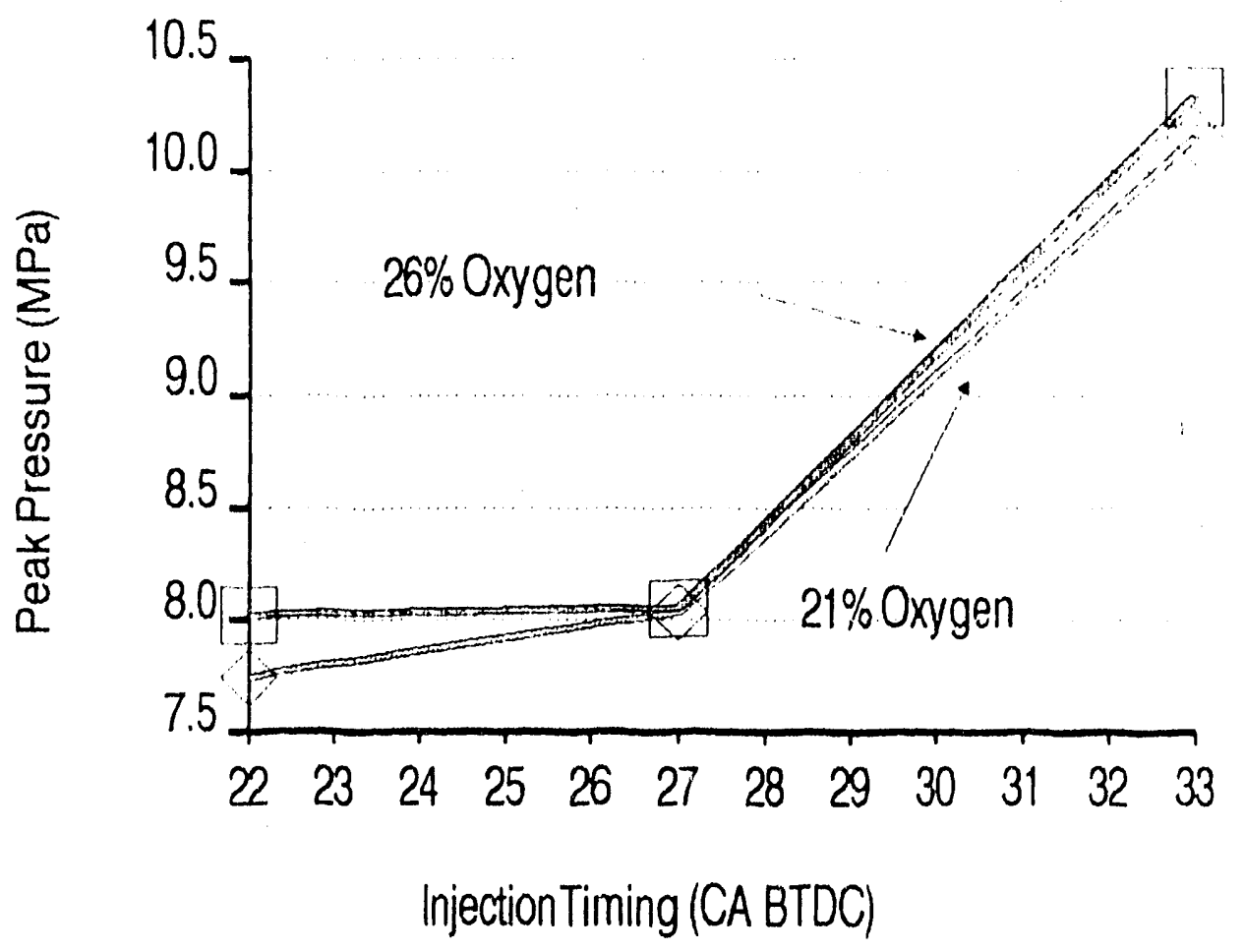

FIGURE B.15 Effect of Injection Timing on Peak Cylinder Pressure, 25 Brake Horsepower, No. 2 Diesel, $5 \%$ Water

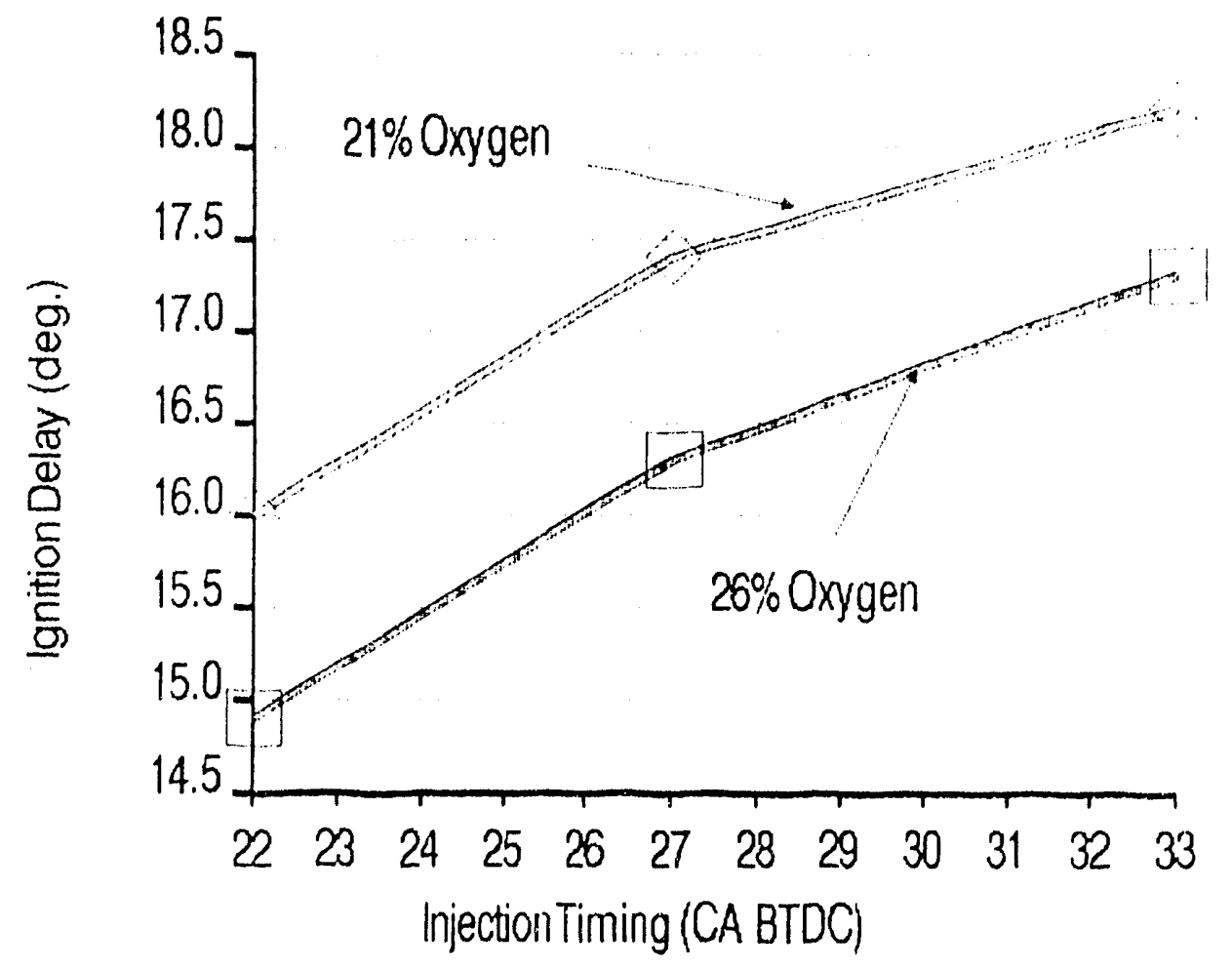

FIGURE B.16 Effect of Injection Timing on Ignition Delay, 25 Brake Horsepower, No. 2 Diesel, $5 \%$ Water 


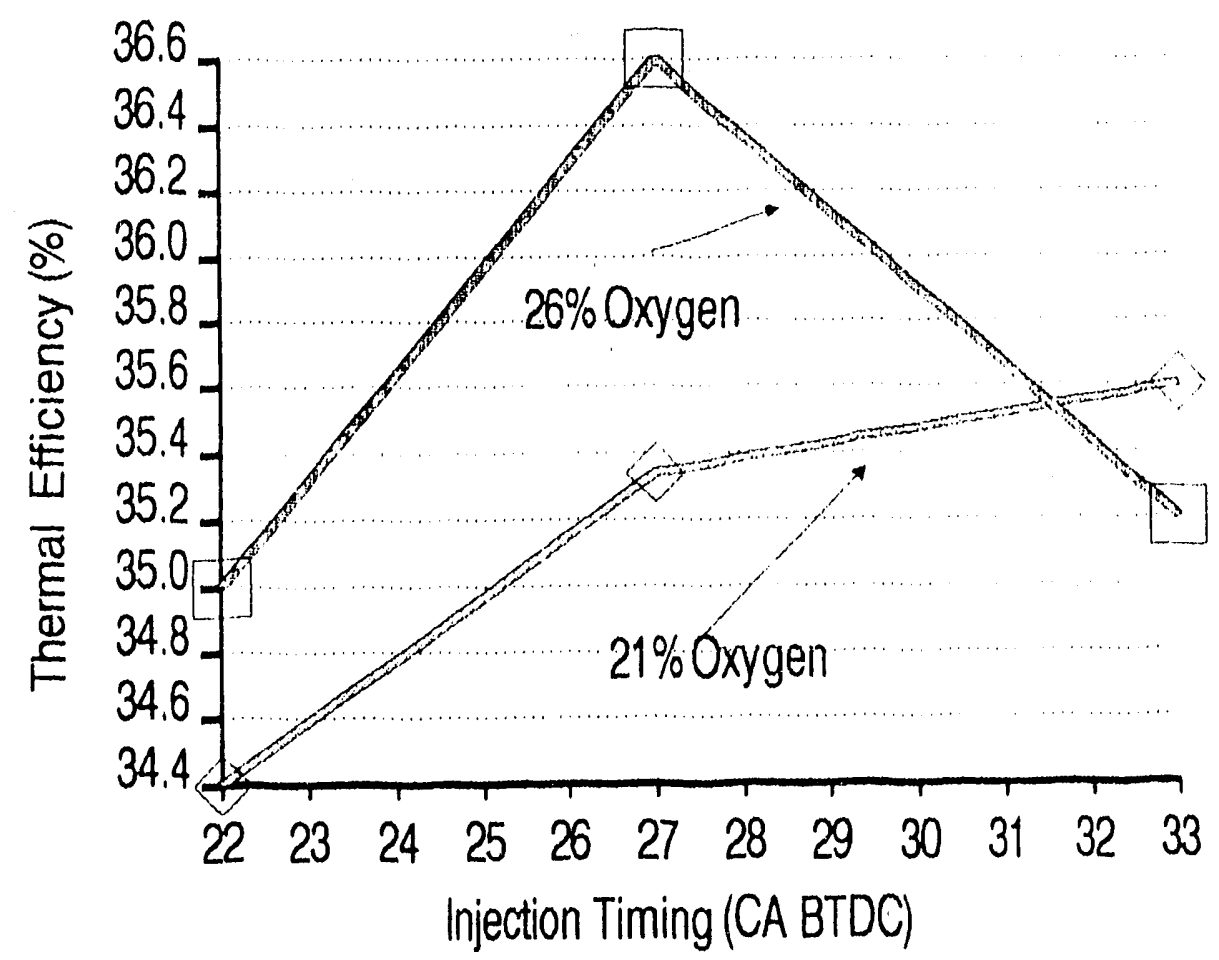

FIGURE B.17 Effect of Injection Tirning on Thermal Efficiency, 25 Brake Horsepower, No. 2 Diesel, $5 \%$ Water

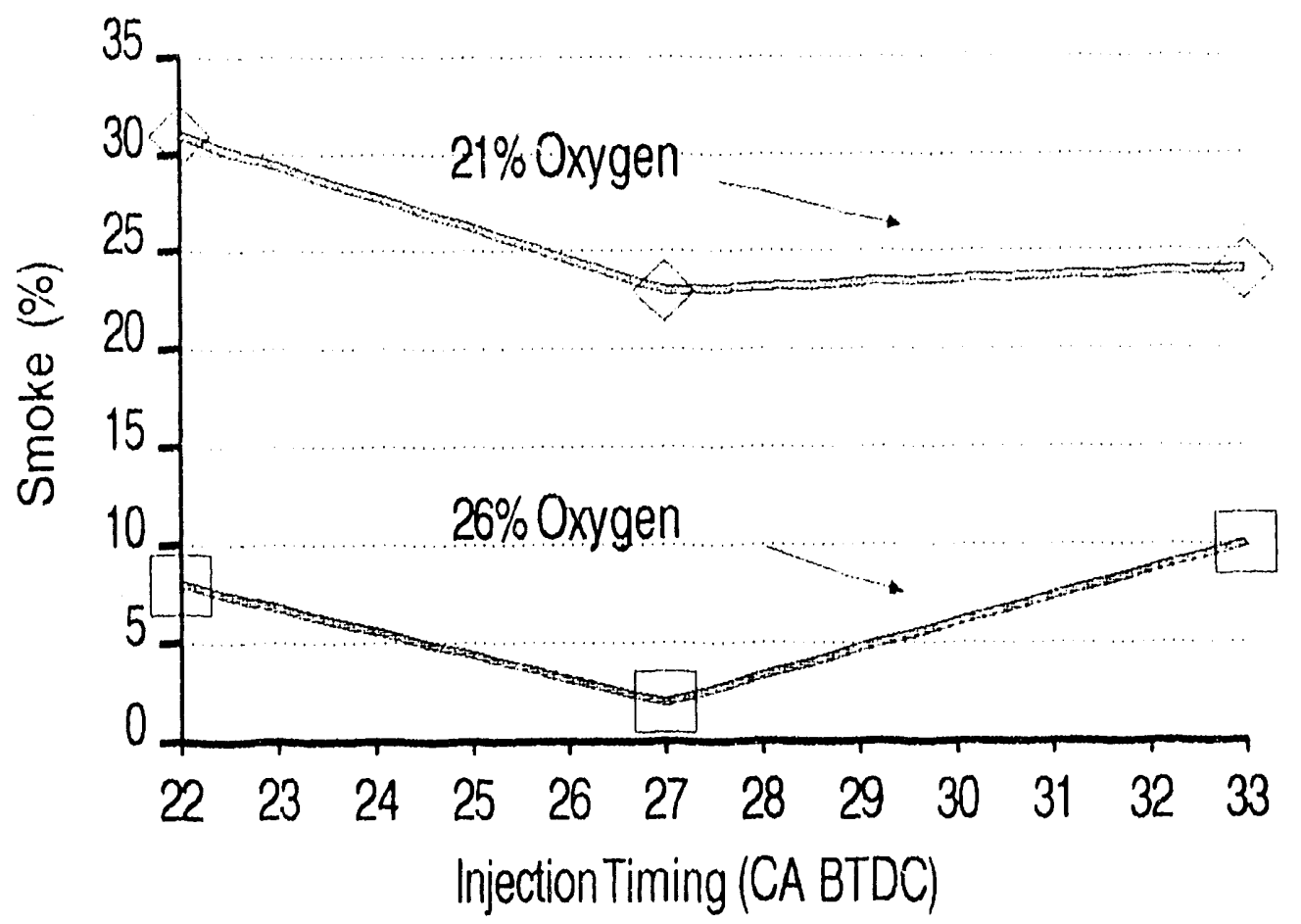

FIGURE B.18 Effect of Injection Timing on Smoke Emission, 25 Brake Horsepower, No. 2 Diesel, 5\% Water 


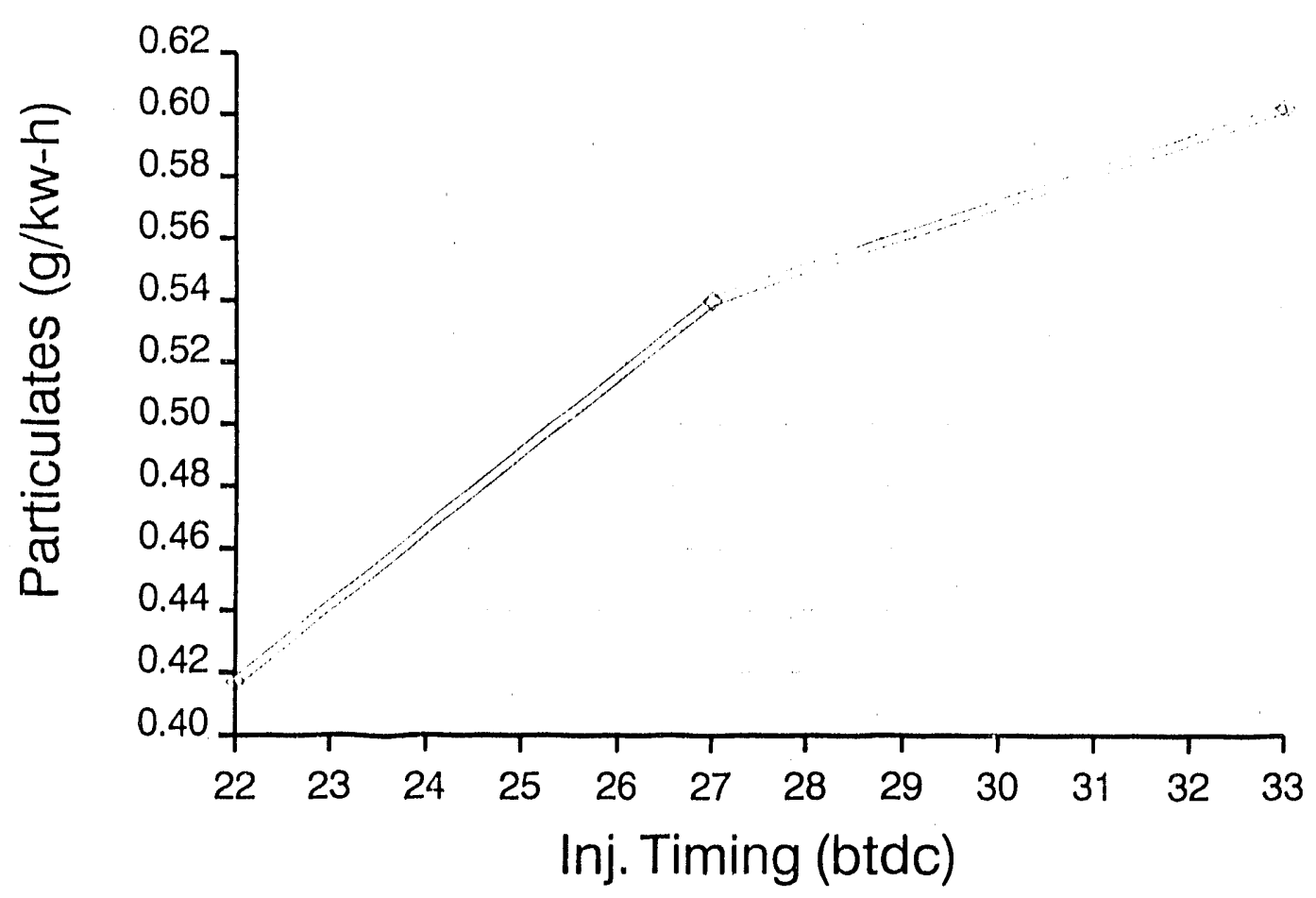

FIGURE B.19 Effuct of Injection Timing on Particulate Matter, 25 Brake Horsepower, No. 2 Diesel, $5 \%$ Water, $21 \%$ Oxygen

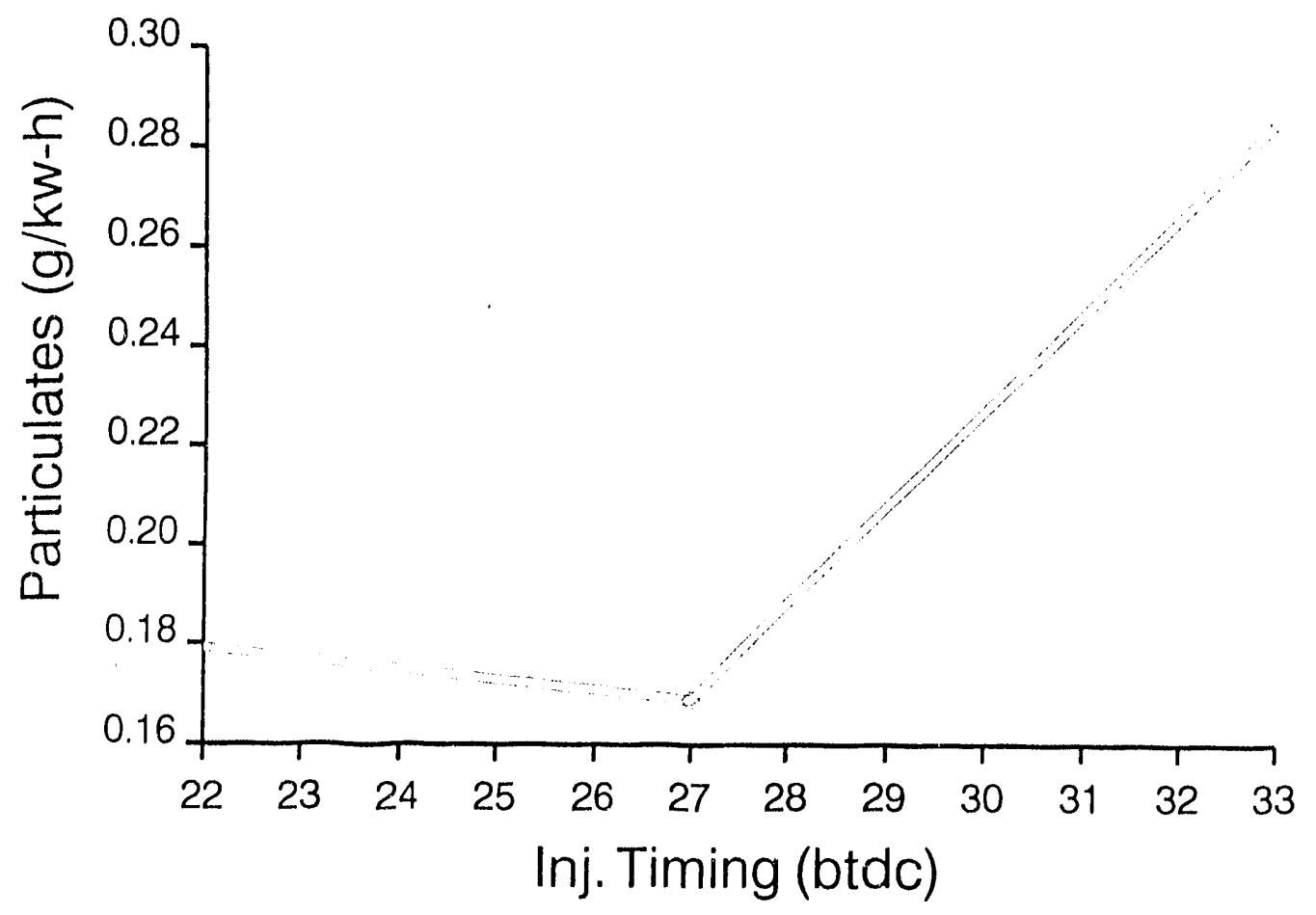

FIGURE B.20 Effect of Injection Timing on Particulate Matter, 25 Brake Horsepower, No. 2 Diesel, $5 \%$ Water, $26 \%$ Oxygen 


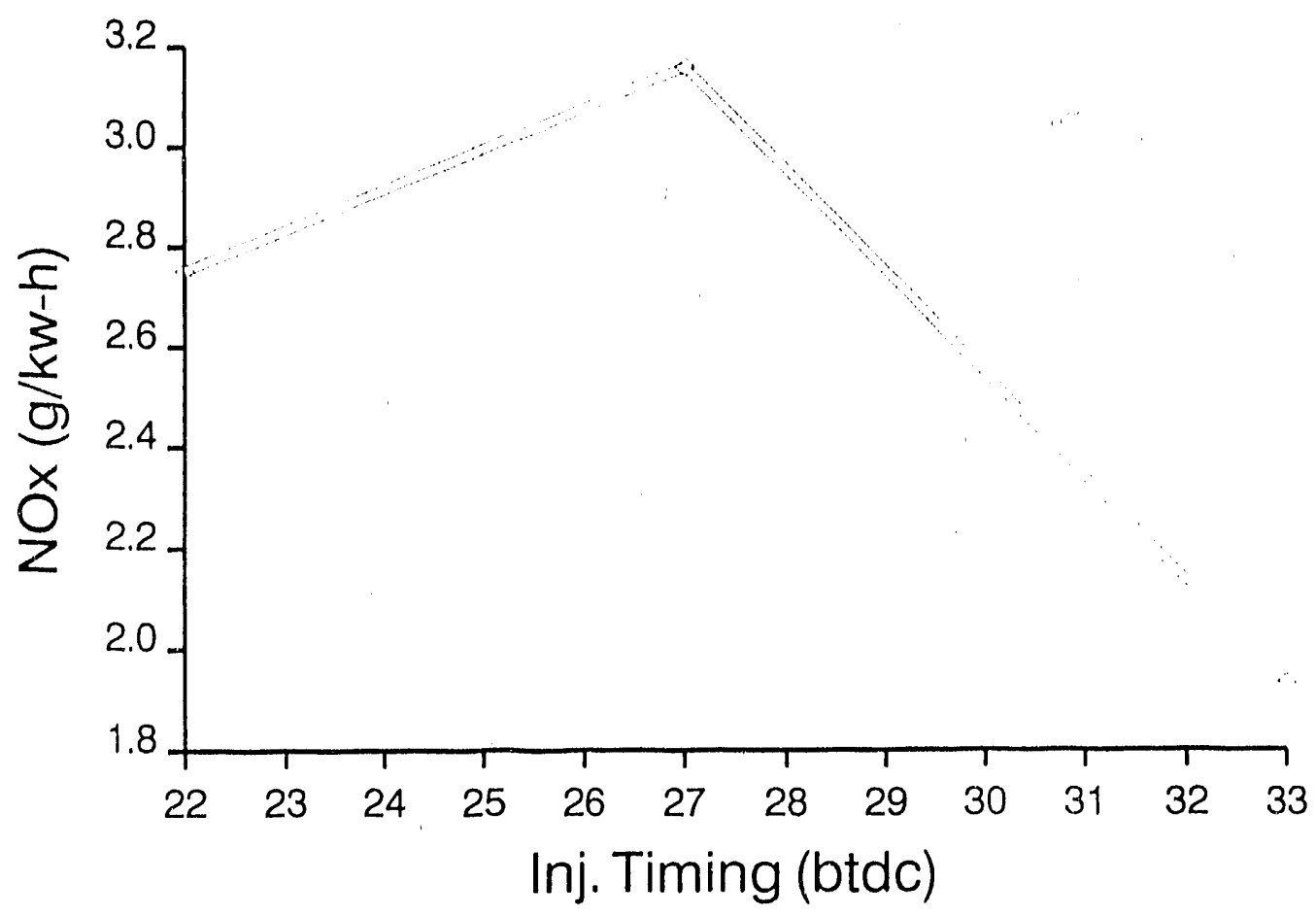

FIGURE B.21 Effect of Injection Timing on $\mathrm{NO}_{\mathrm{x}}, 25$ Brake Horsepower, No. 2 Diesel, $5 \%$ Water, $21 \%$ Oxygen

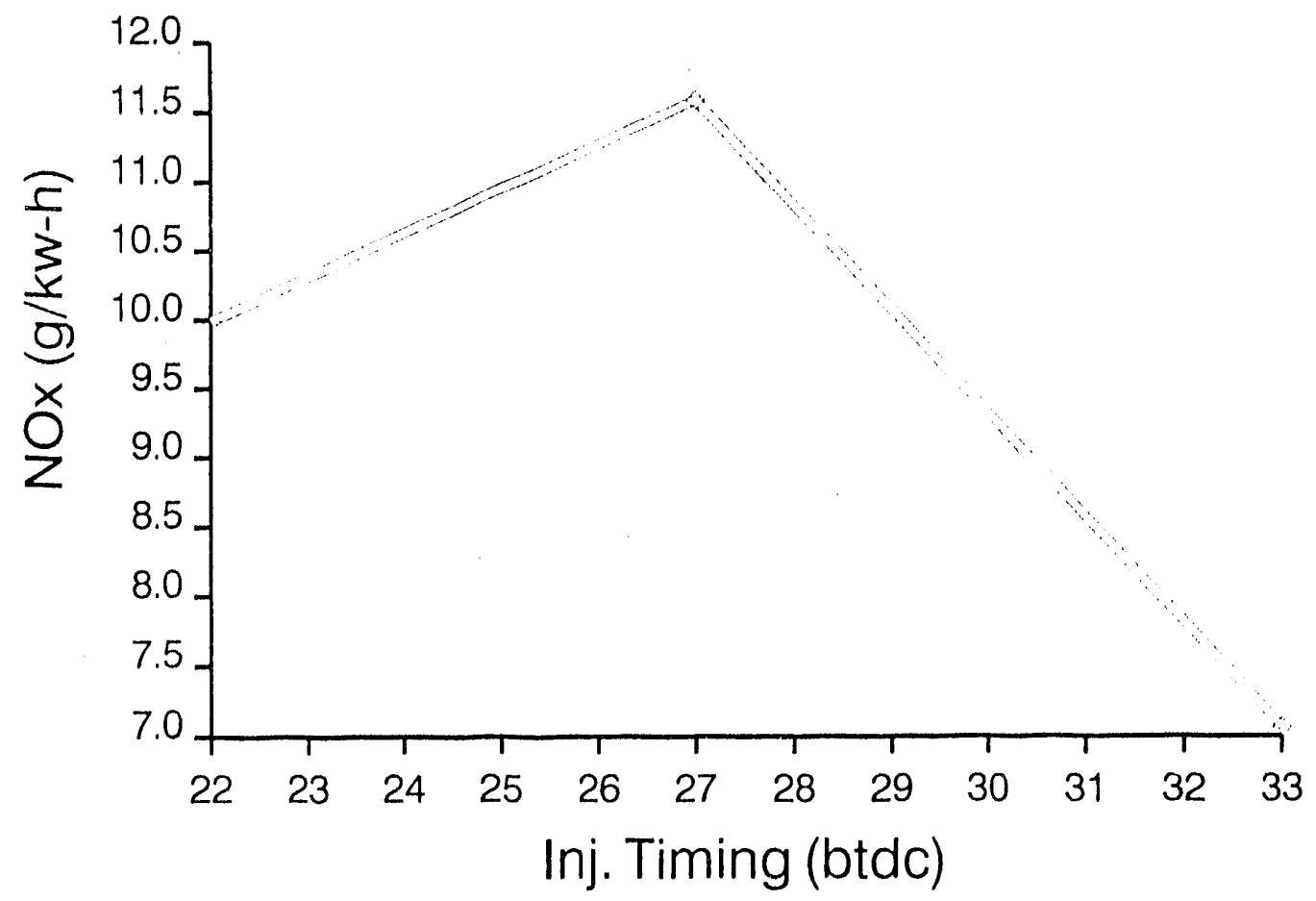

FIGURE B.22 Effect of Injection Timing on $\mathrm{NO}_{x}, 25$ Brake Horsepower, No. 2 Diesel, $5 \%$ Water, $26 \%$ Oxygen 


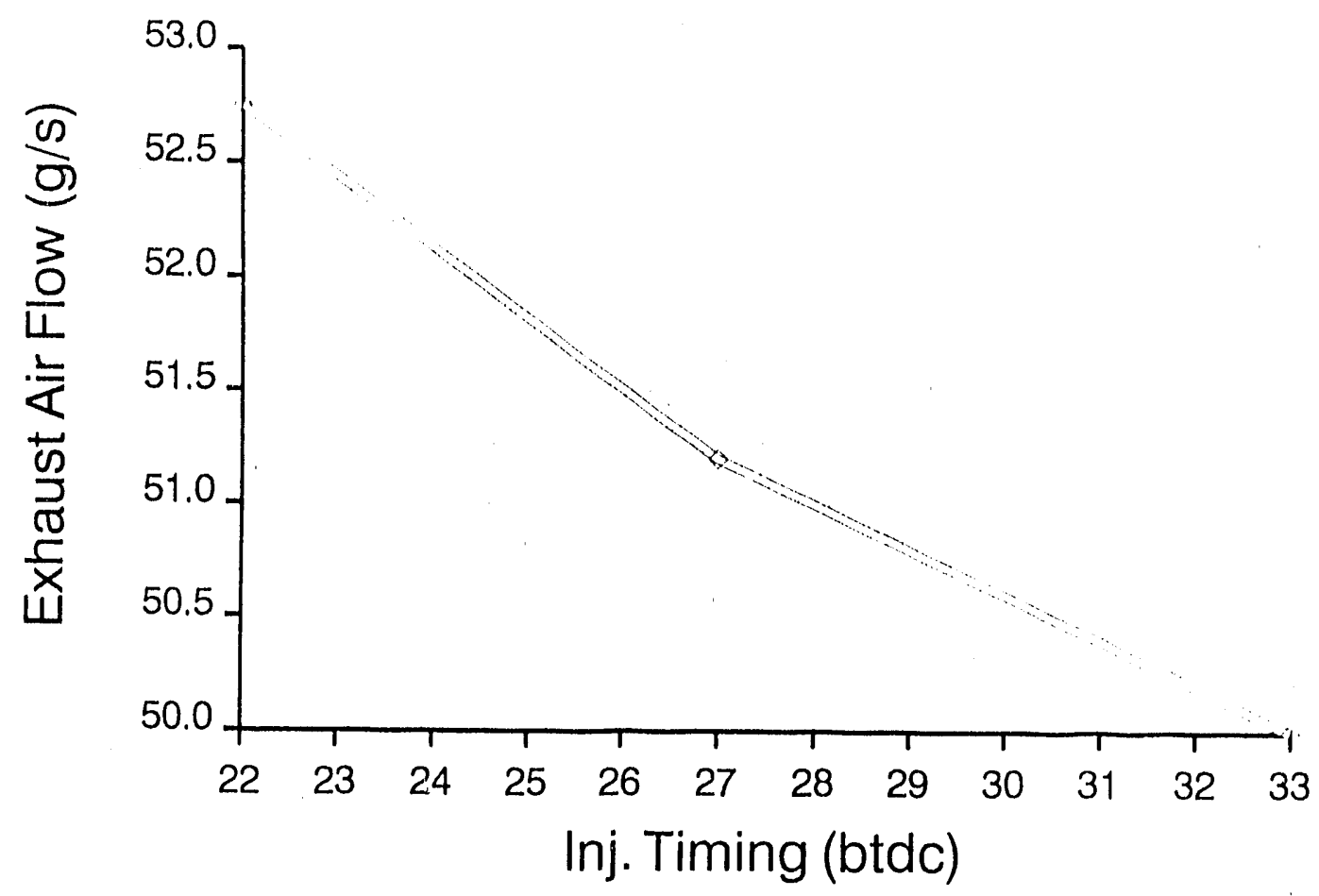

FIGURE B.23 Effect of Injection Timing on Exhaust Air Flow, 25 Brake Horsepower, No. 2 Diesel, $5 \%$ Water, $21 \%$ Oxygen

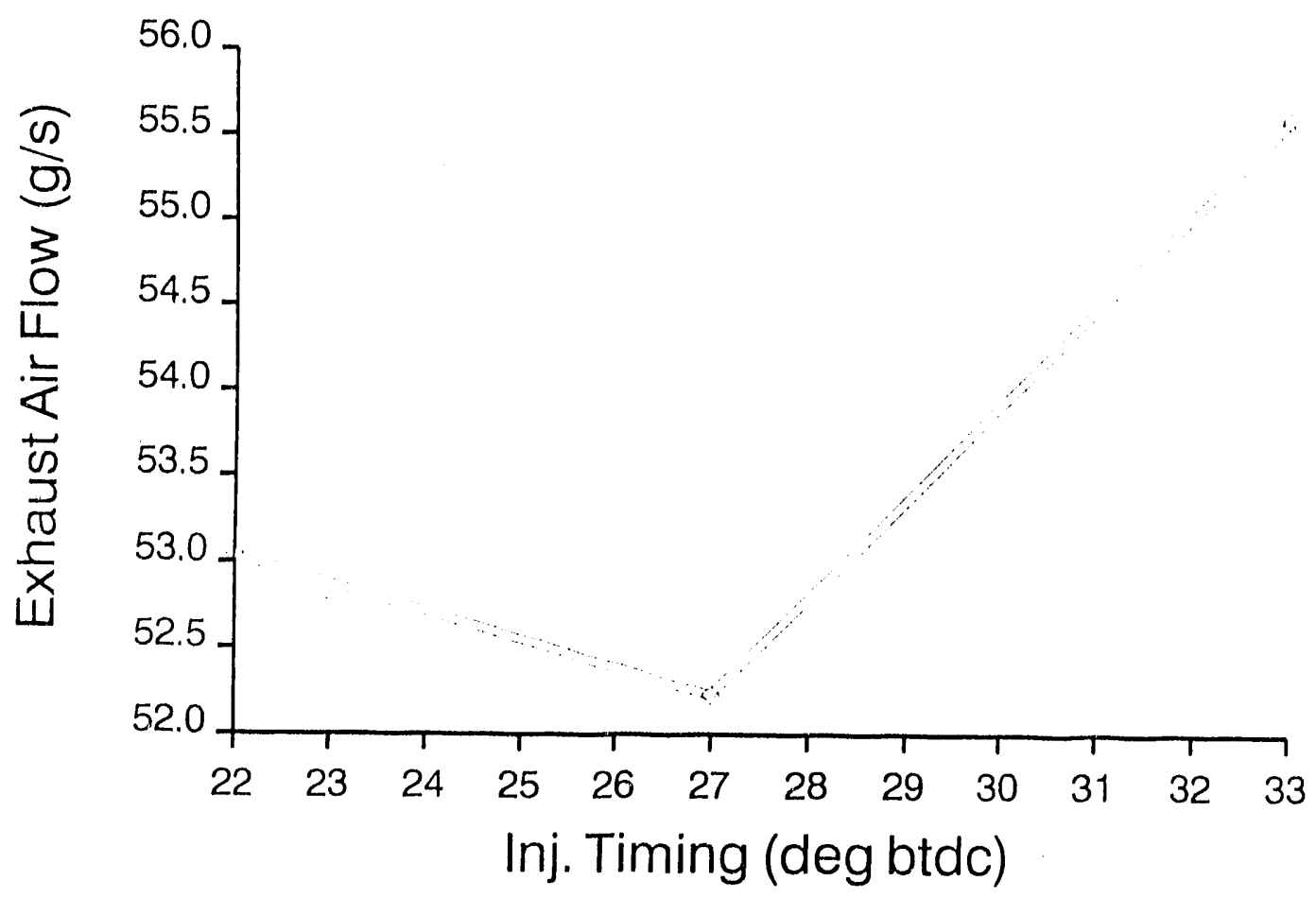

FIGURE B.24 Effect of Injection Timing on Exhaust Air Flow, 25 Brake Horsepower, No. 2 Diesel, $5 \%$ Water, $26 \%$ Oxygen 


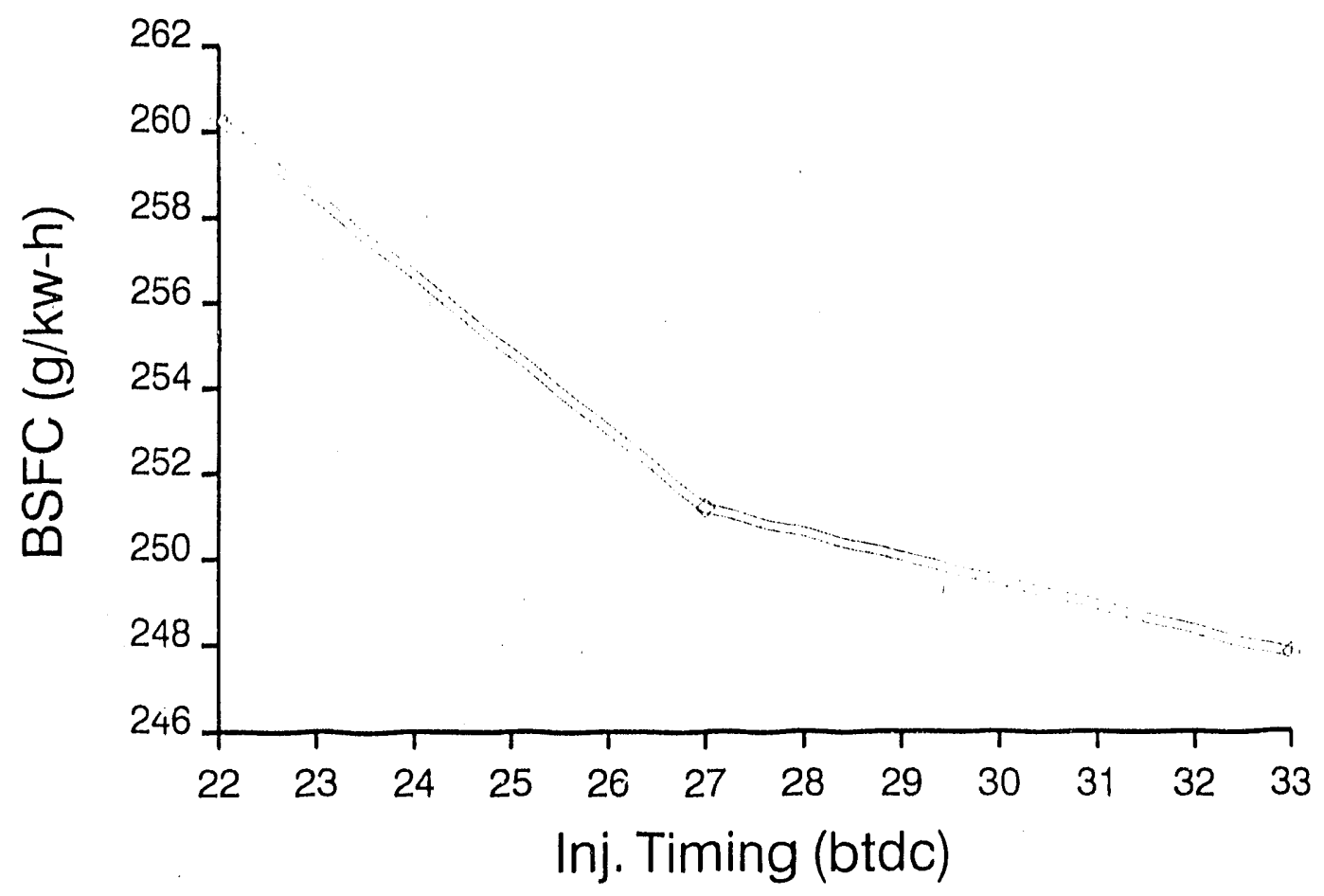

FIGURE B.25 Effect of Injection Timing on Brake-Specific Fuel Consumption, 25 Brake Horsepower, No. 2 Diesel, $5 \%$ Water, $21 \%$ Oxygeri

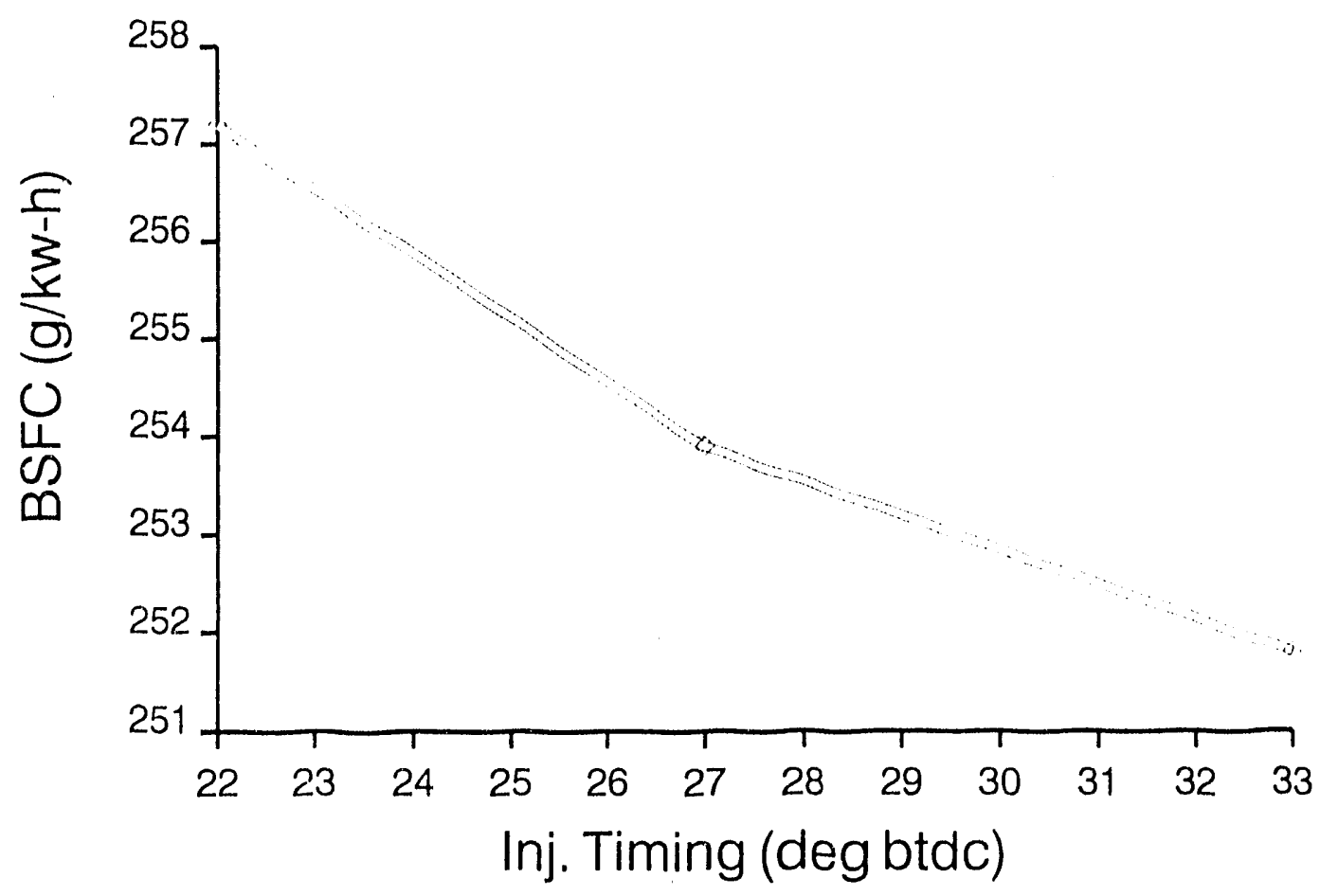

FIGURE B.26 Effect of Injection Timing on Brake-Specific Fuel Consumption, 25 Brake Horsepower, No. 2 Diesel, $5 \%$ Water, 26\% Oxygen 


\section{$B-44$}

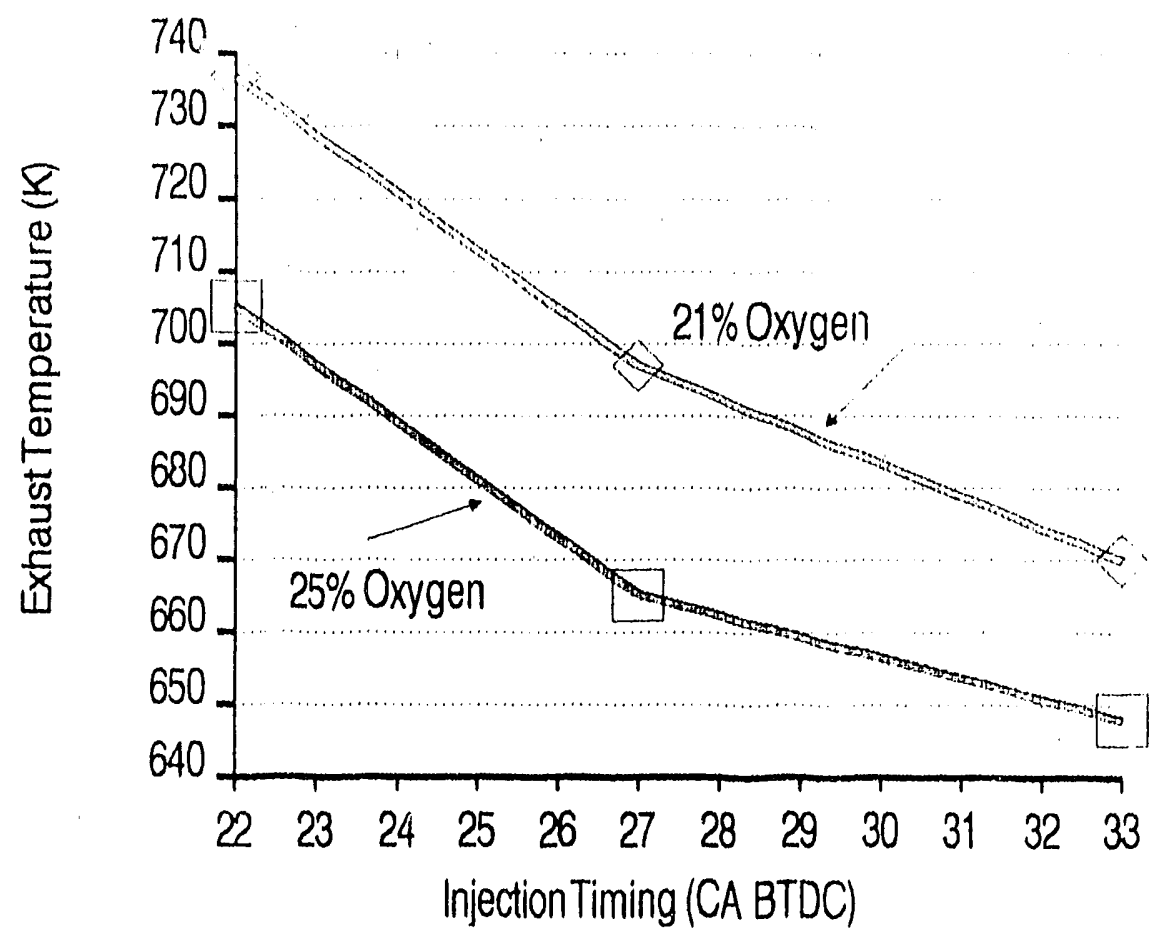

FIGURE B.27 Effect of Injection Timing on Exhaust Temperature, 50 Brake Horsepower, No. 2 Diesel, No Water

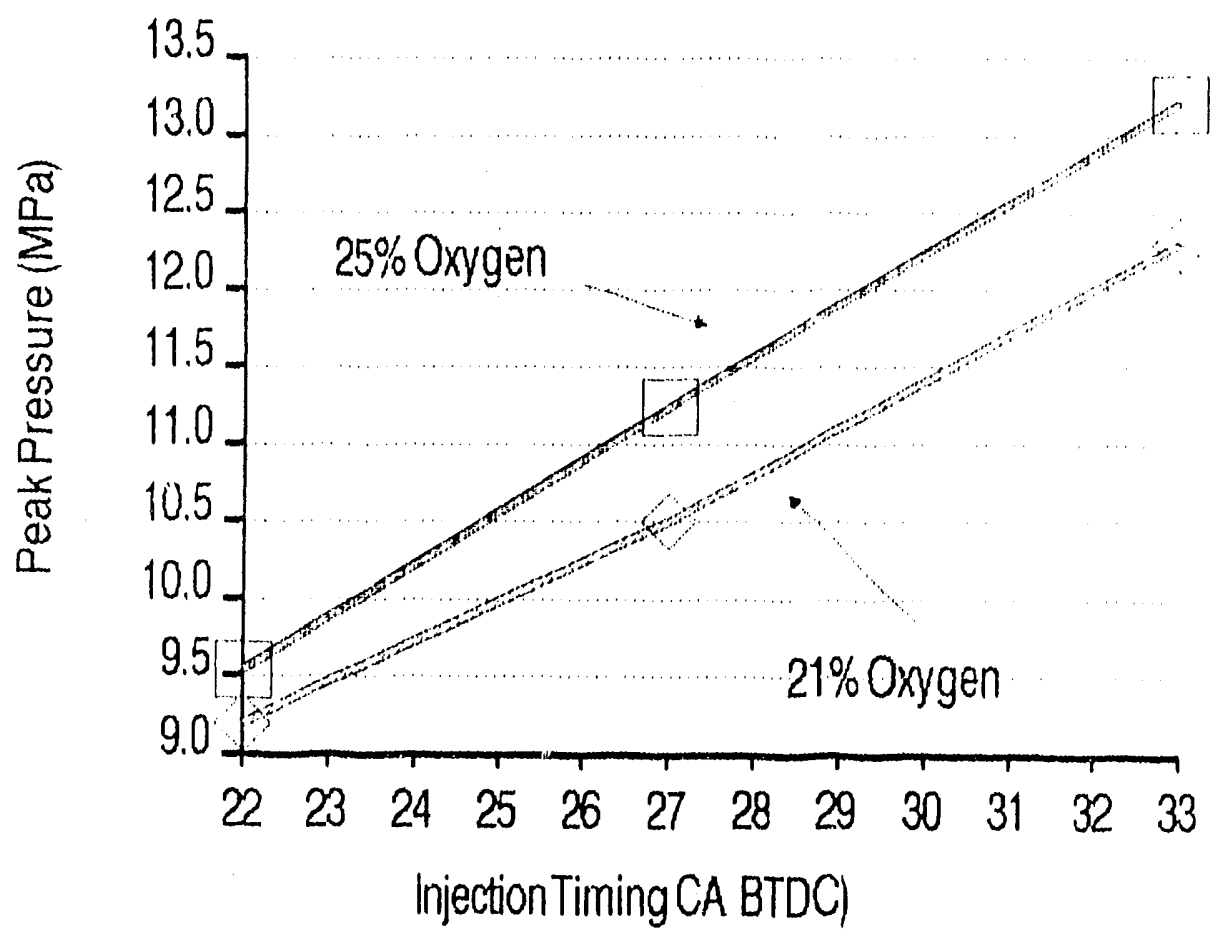

FIGURE B.28 Effect of Injection Timing on Peak Cylinder Pressure, 50 Brake Horsepower, No. 2 Diesel, No Water 


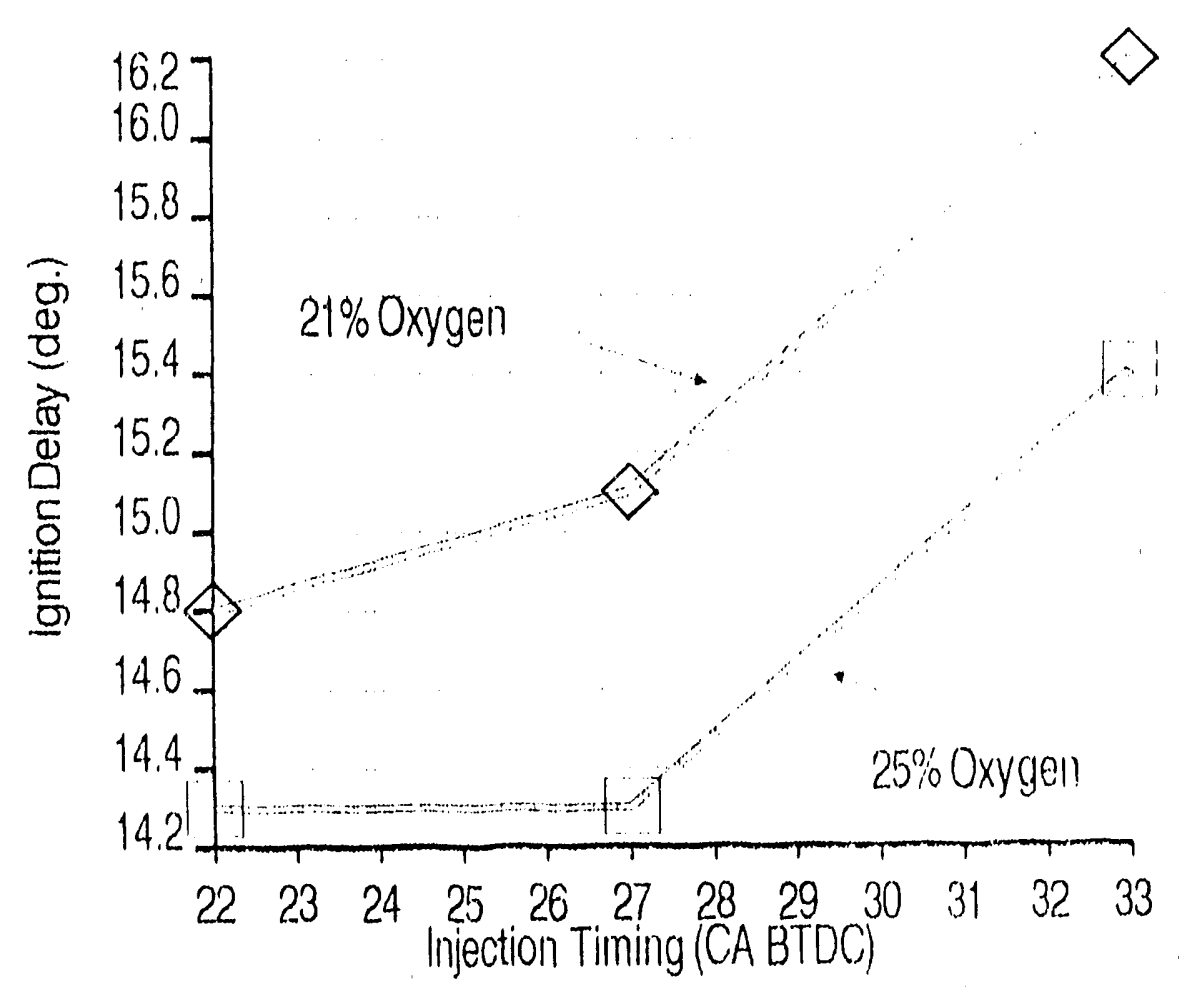

FIGURE B.29 Effect of Injection Timing on Ignition Delay, 50 Brake Horsepower, No. 2 Diesel, No Water

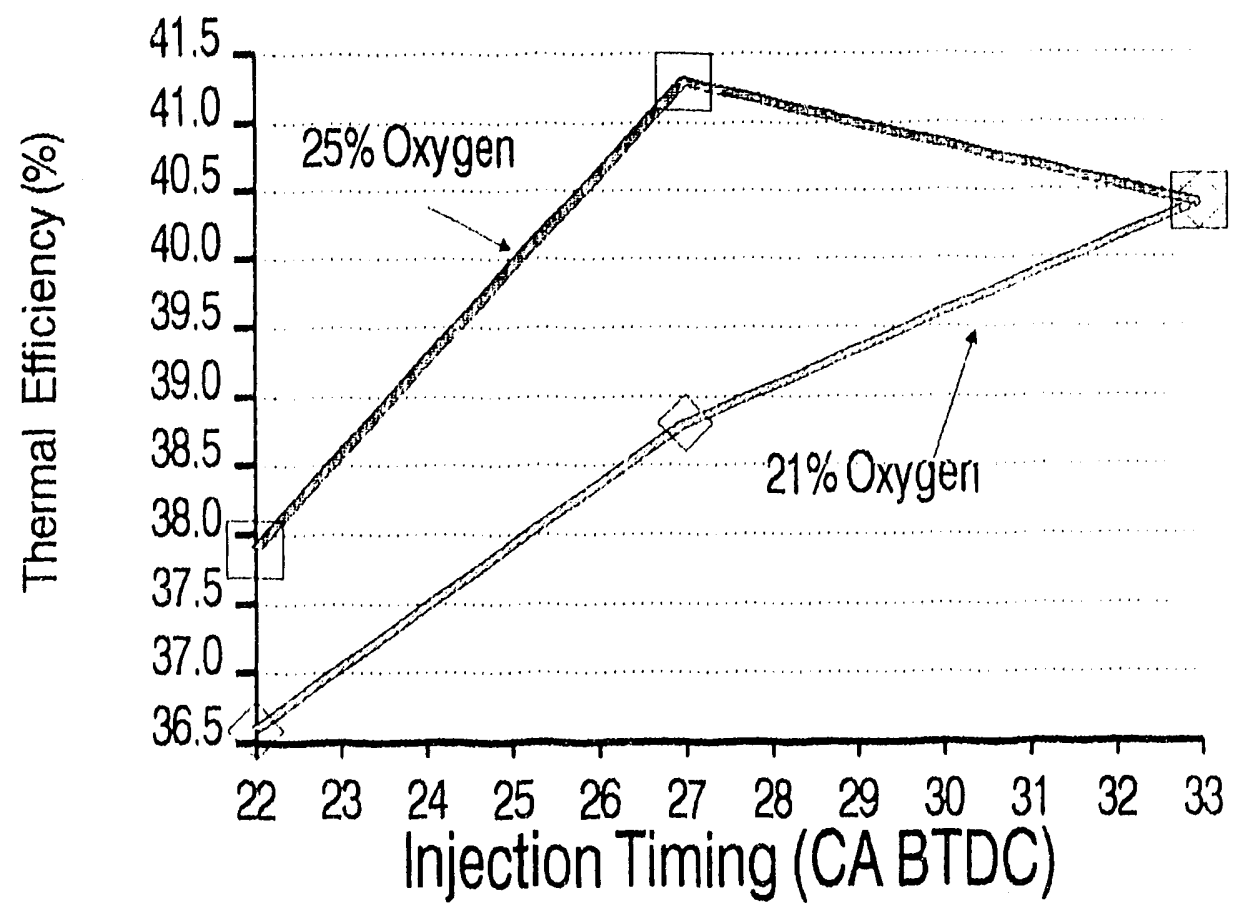

FIGURE B.30 Effect of Injection Timing on Thermal Efficiency, 50 Brake Horsepower, No. 2 Diesel, No Water 


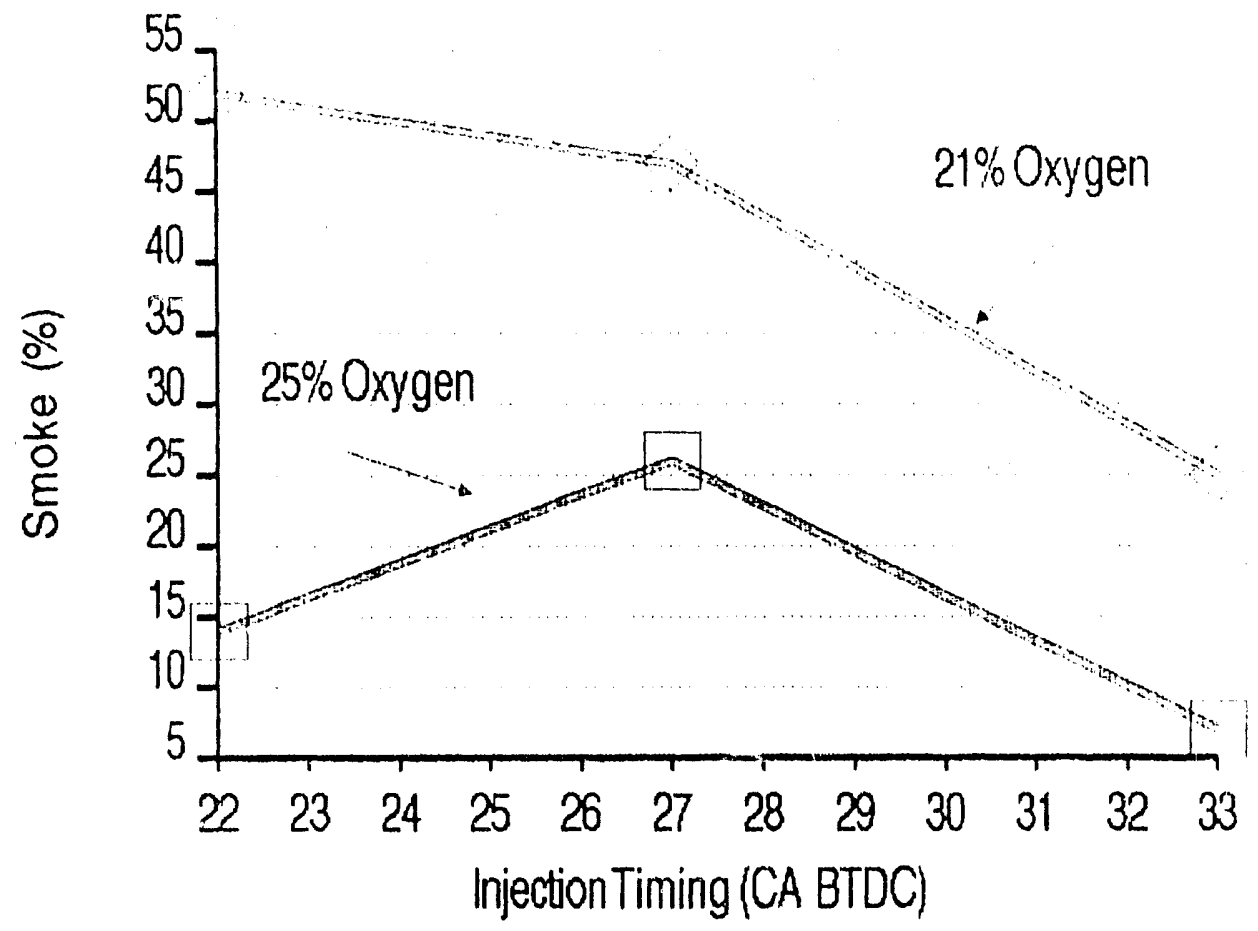

FIGURE B.31 Effect of Injection Timing on Smoke Emission, 50 Brake Horsepower, No. 2 Diesel, No Water

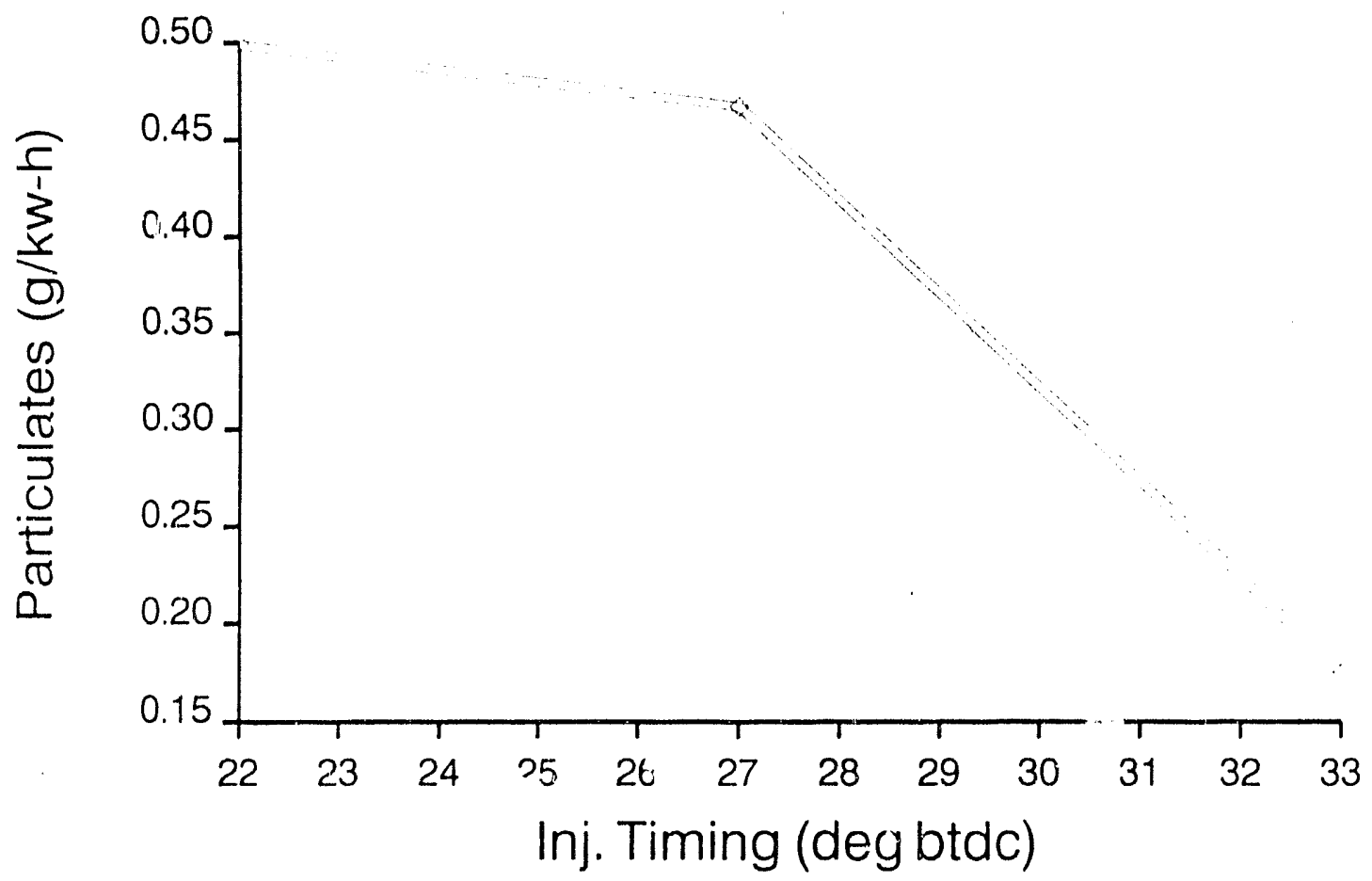

FIGURE B.32 Effect of Injection Timing on Particulate Matter, 50 Brake Horsepower, No. 2 Diesel, No Water, $21 \%$ Oxygen 


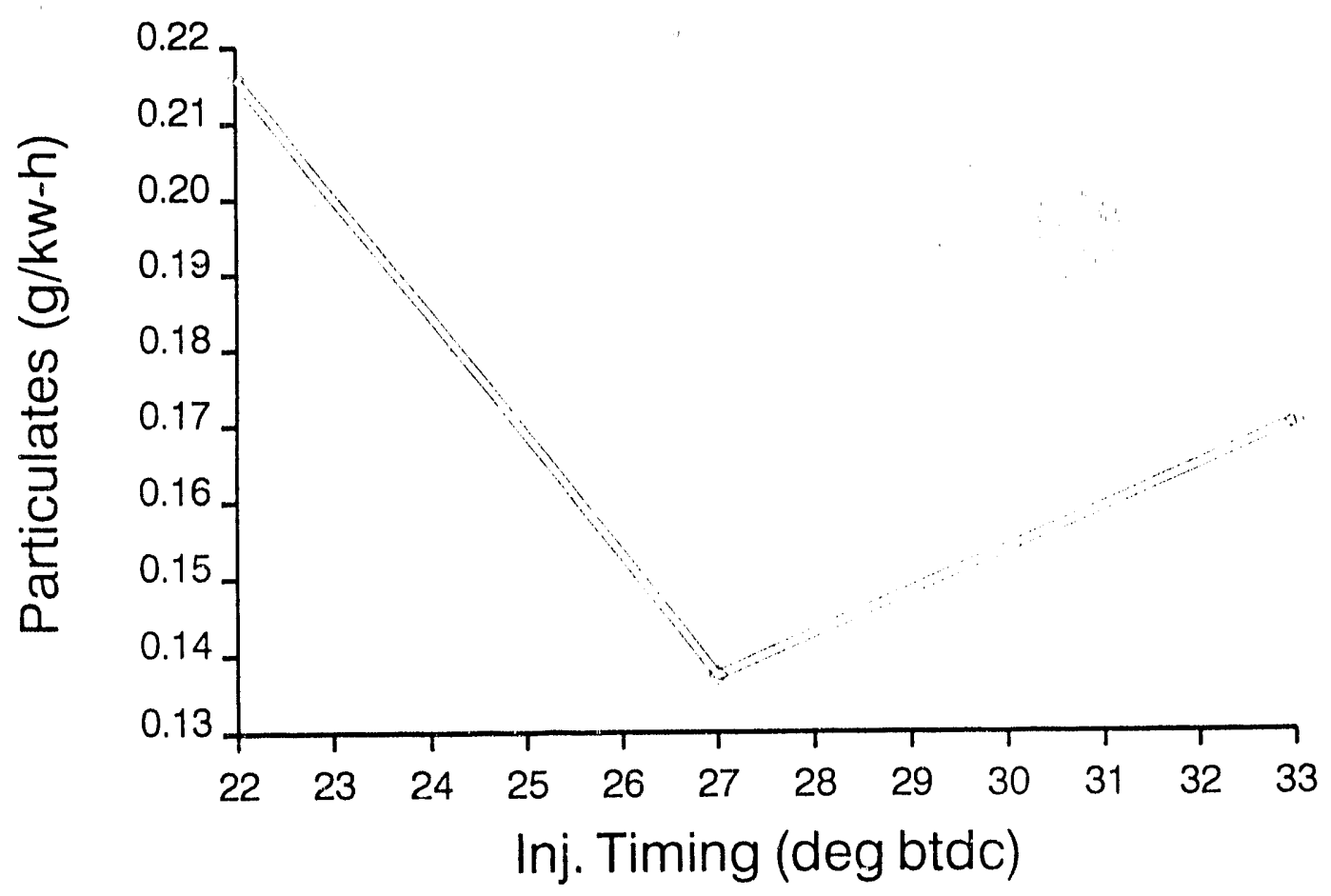

FIGURE B.33 Effect of Injection Timing on Particulate Matter, 50 Brake Horsepower, No. 2 Diesel, No Water, $25 \%$ Oxygen

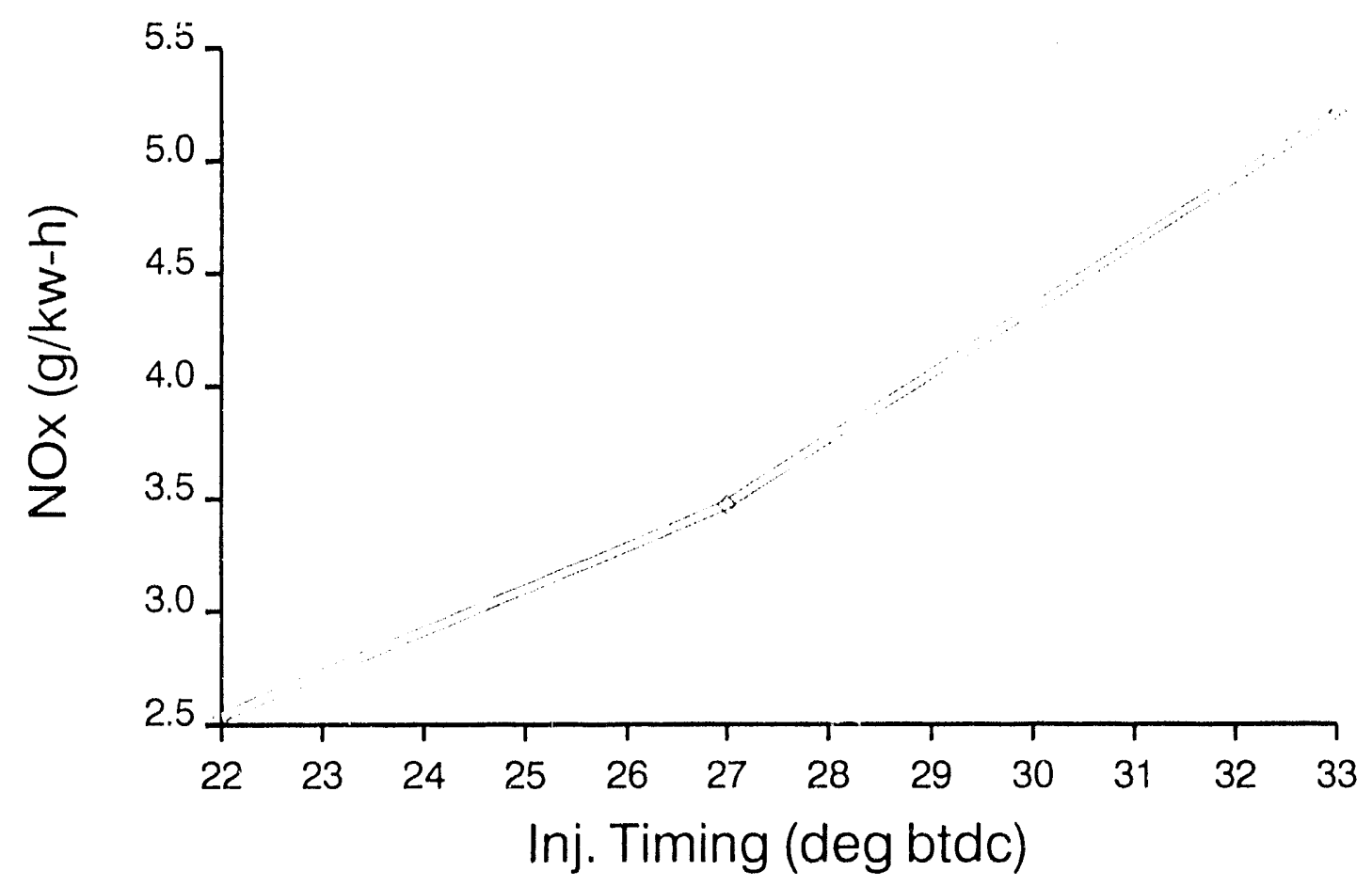

FIGURE B.34 Effect of Injection Timing on $\mathrm{NO}_{x}, 50$ Brake Horsepower, No. 2 Diesel, No Water, $21 \%$ Oxygen 


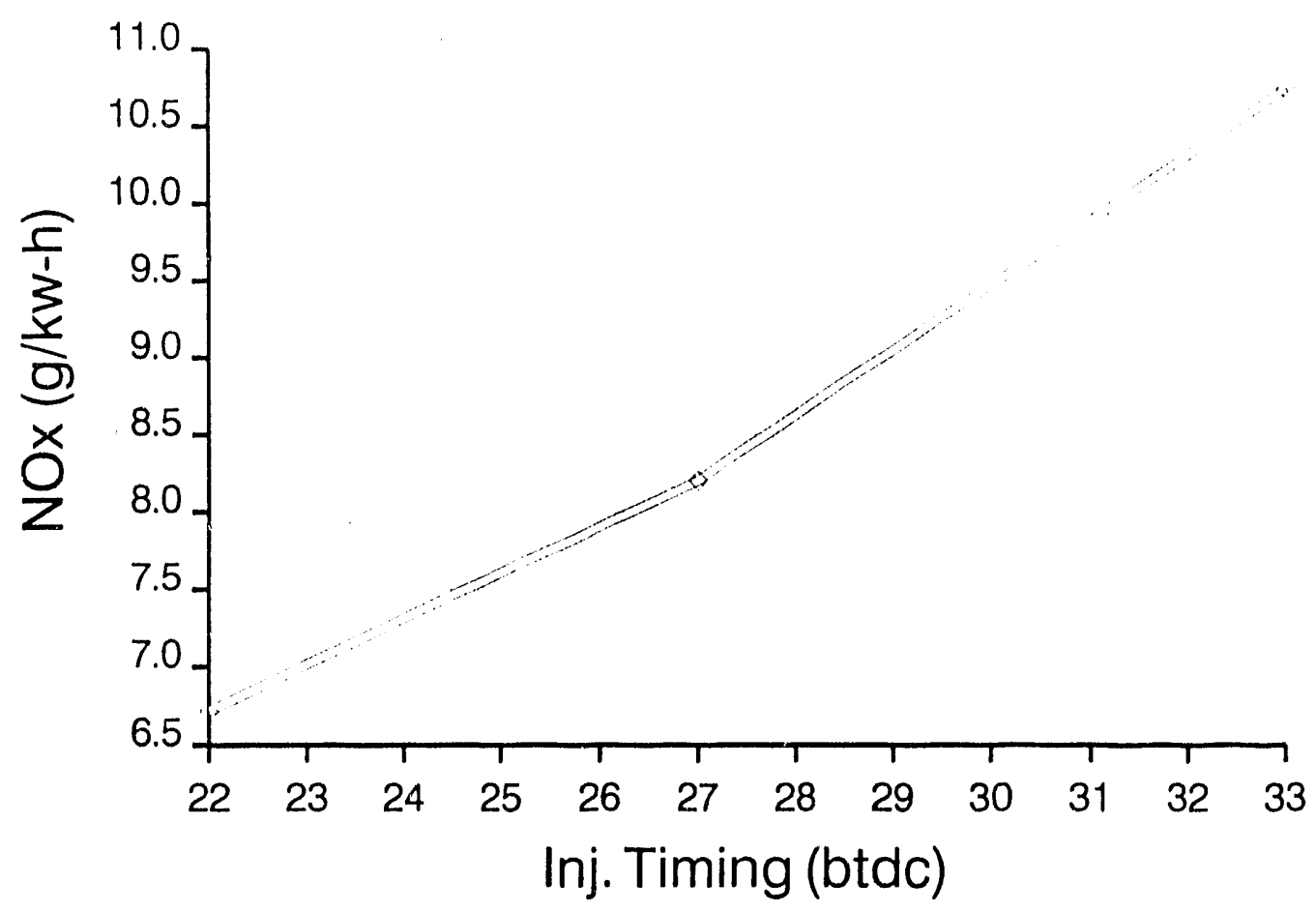

FIGURE B.35 Effect of Injection Timing on $\mathrm{NO}_{x}, 50$ Brake Horsepower, No. 2 Diesel, No Water, $25 \%$ Oxygen

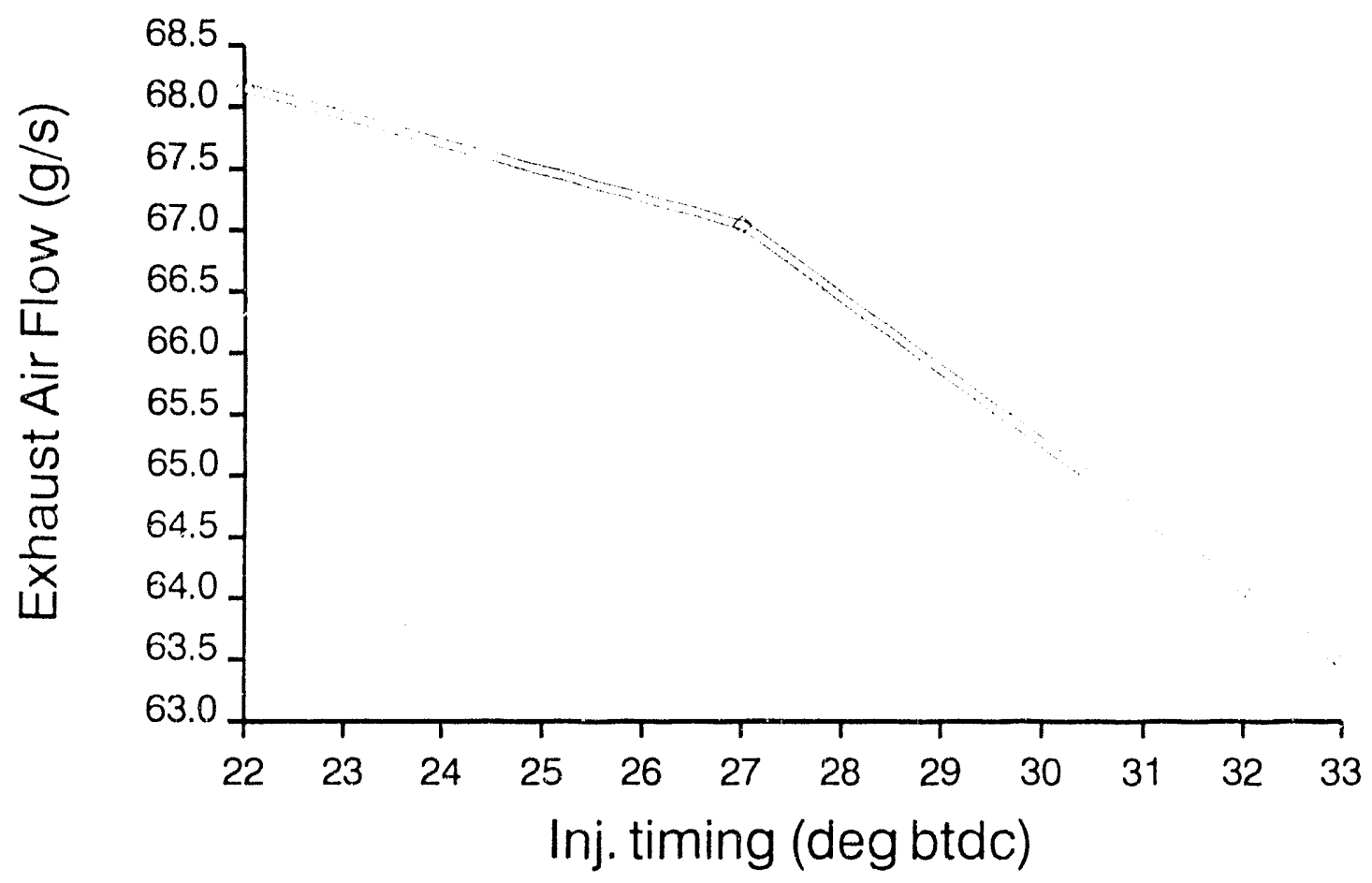

FIGURE B.36 Effect of Injection Timing on Exhaust Air Flow, 50 Brake Horsepower, No. 2 Diesel, No Water, $21 \%$ Oxygen 


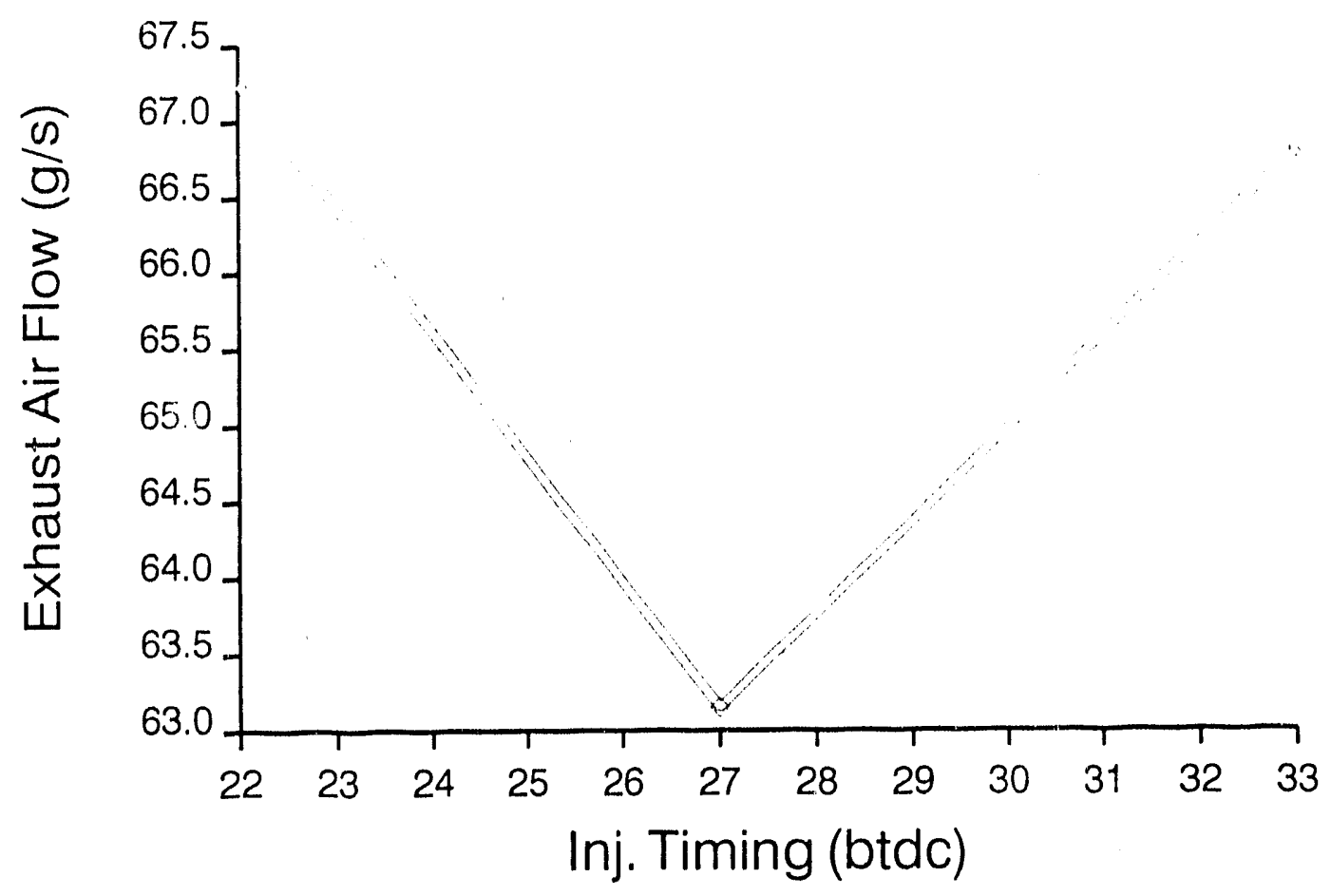

FIGURE B.37 Effect of Injection Timing on Exhaust Air Flow, 50 Brake Horsepower, No. 2 Diesel, No Water, 25\% Oxygen

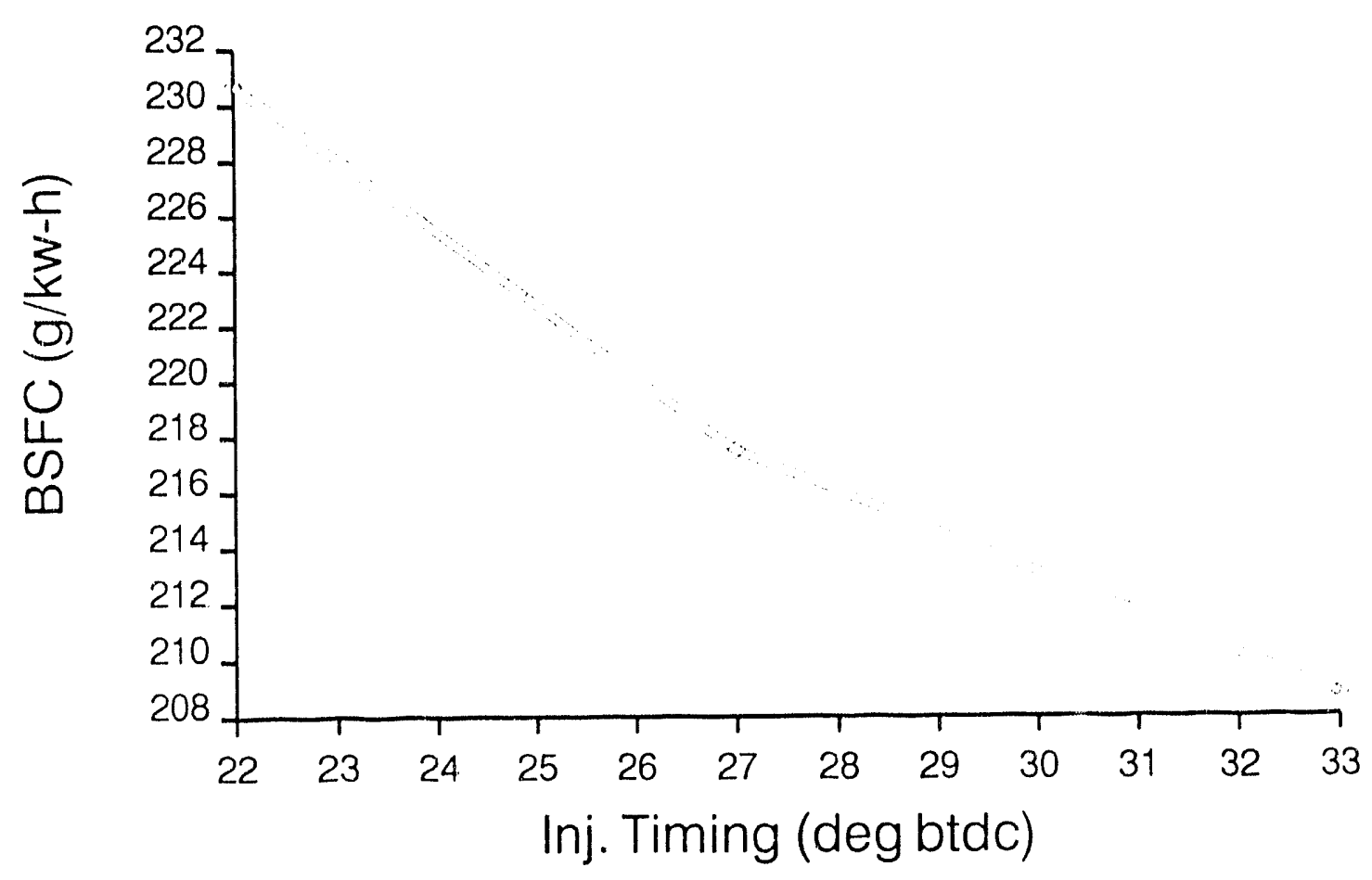

FIGURE B.38 Effect of Injection Timing on Brake-Specific Fuel Consumption, 50 Brake Horsepower, No. 2 [iesel, No Water, $21 \%$ Oxygen 


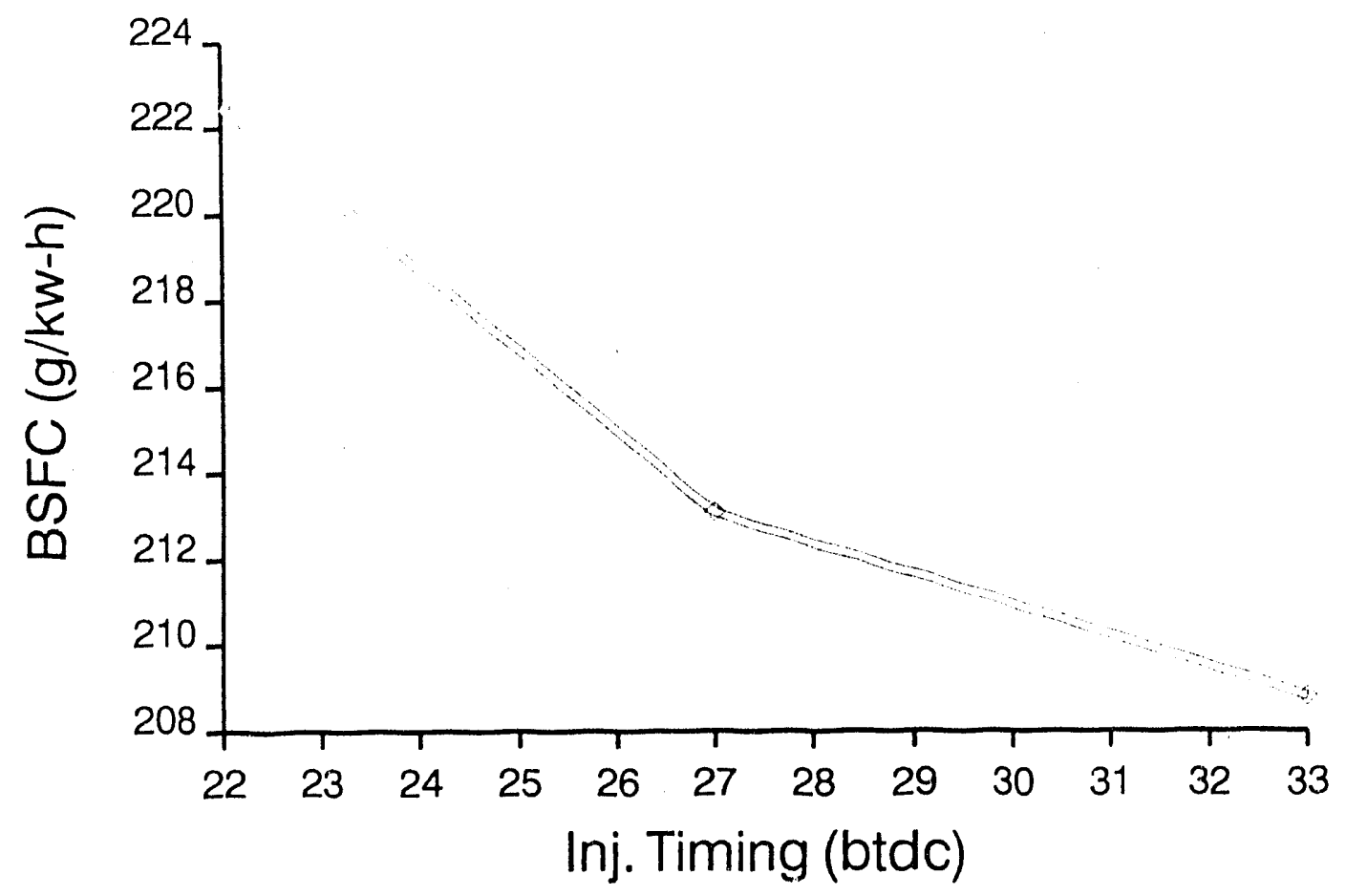

FIGURE B.39 Effect of Injection Timing on Brake-Specific Fuel Consumption, 50 Brake Horsepower, No. 2 Diesel, No Water, 25\% Oxygen

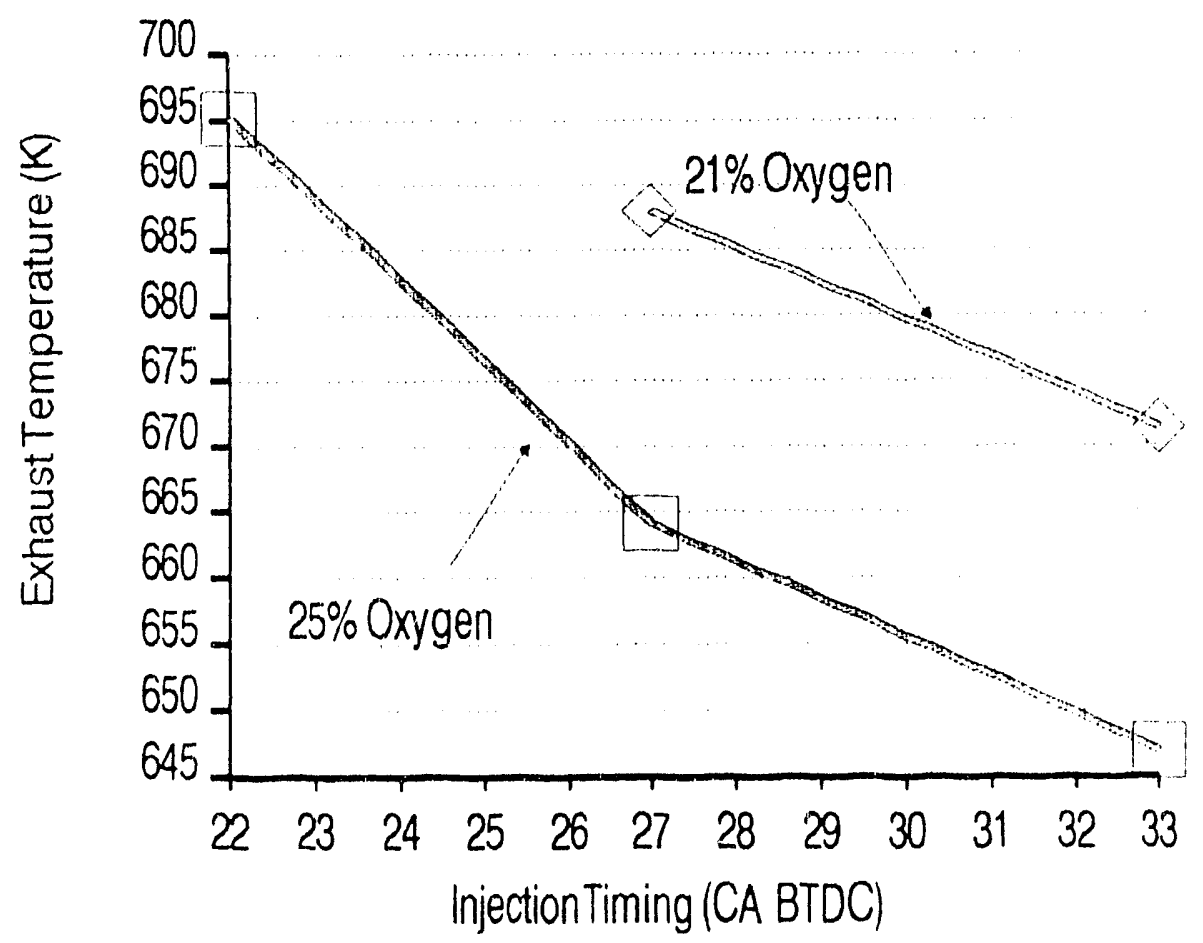

FIGURE B.40 Effect of Injection Timing on Exhaust Temperature, 50 Brake Horsepower, No. 2 Diesel, 5\% Water 


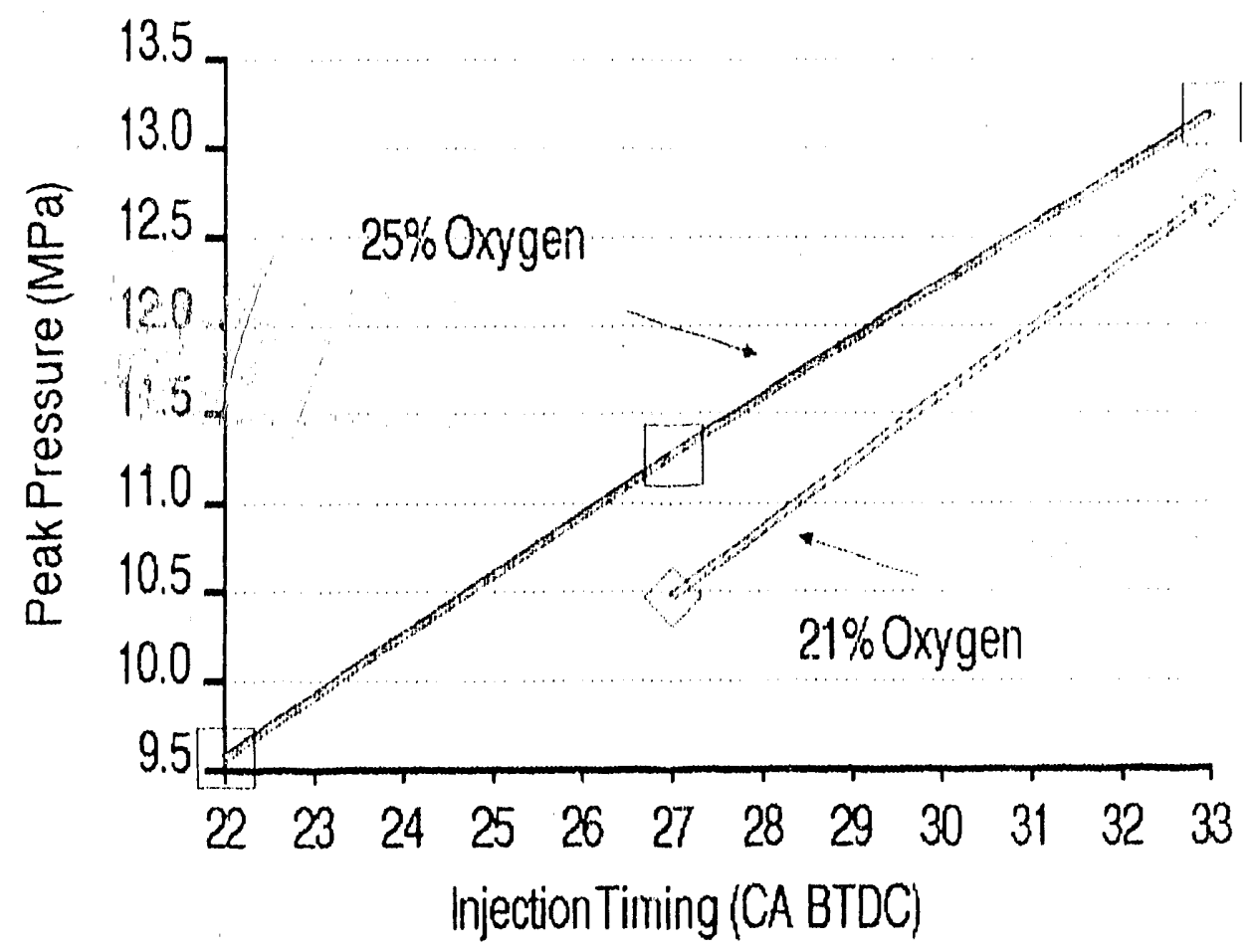

FIGURE B.41 Effect of Injection Timing on Peak Cylinder Pressure, 50 Brake Horsepower, No. 2 Diesel, $5 \%$ Water

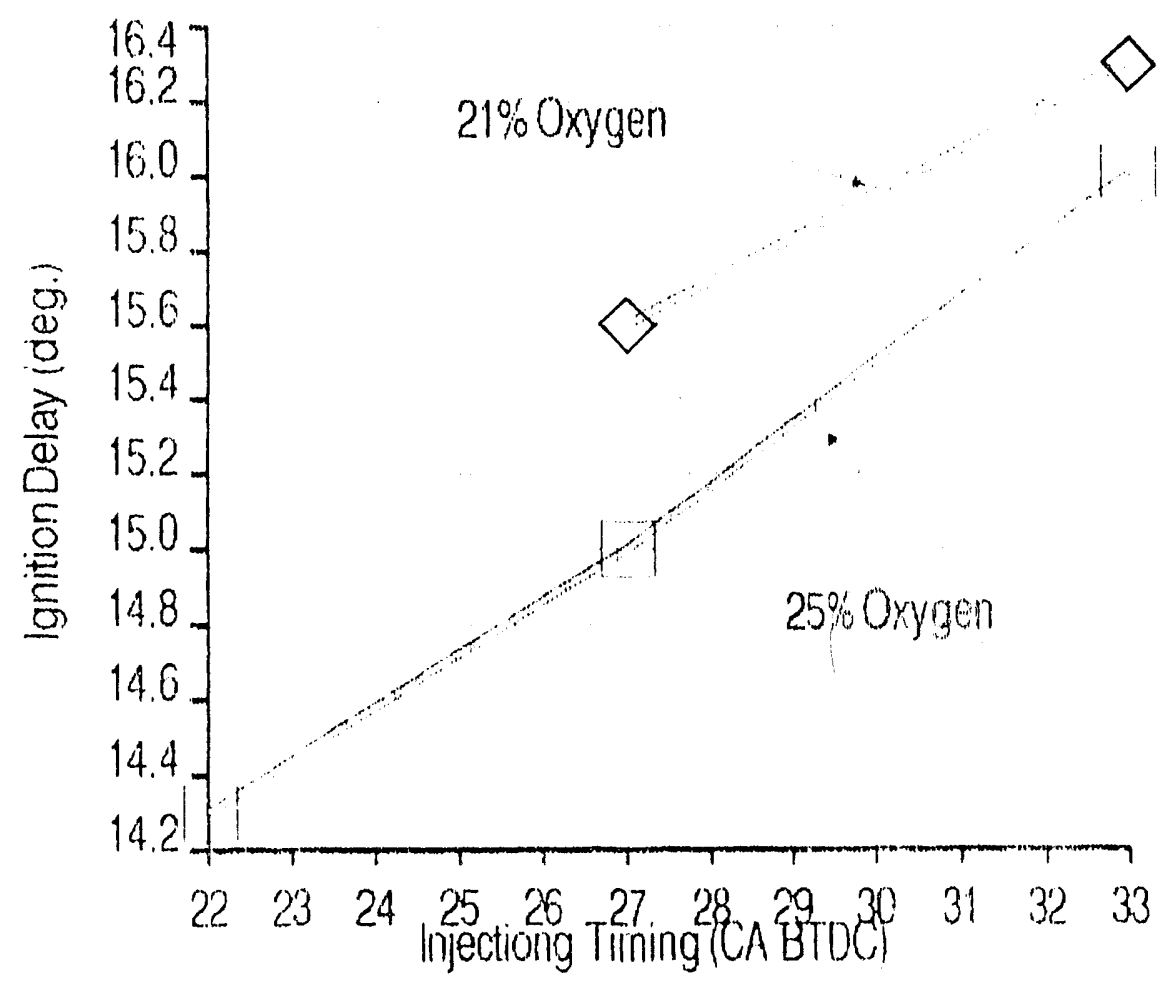

FIGURE B.42. Effect of Injection Timing on Ignition Delay, 50 Brake Horsepower, No. 2 Diesel, $5 \%$ Water 


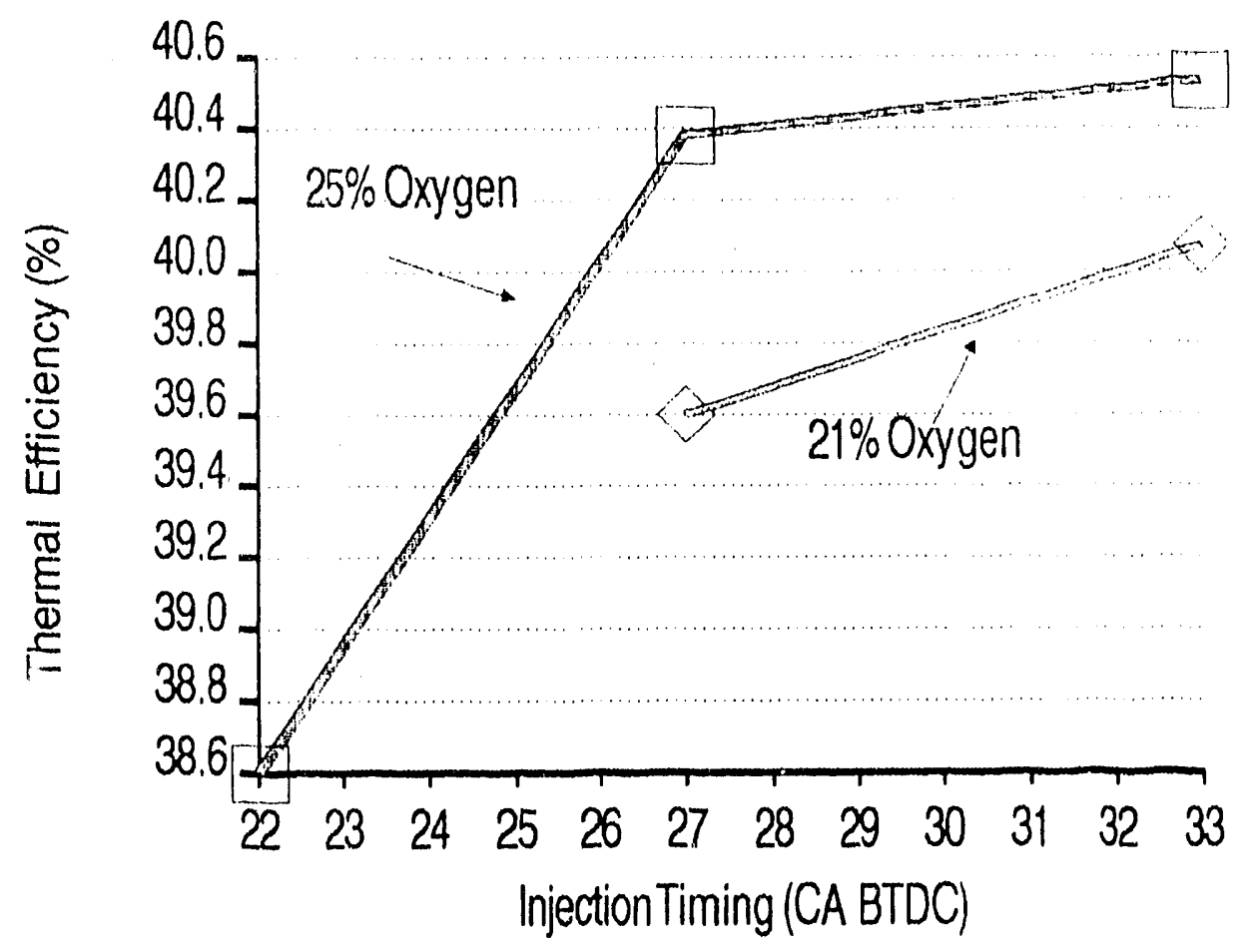

FIGURE B.43 Effect of Injection Timing on Thermal Efficiency, 50 Brake Horsepower, No. 2 Diesel, 5\% Water

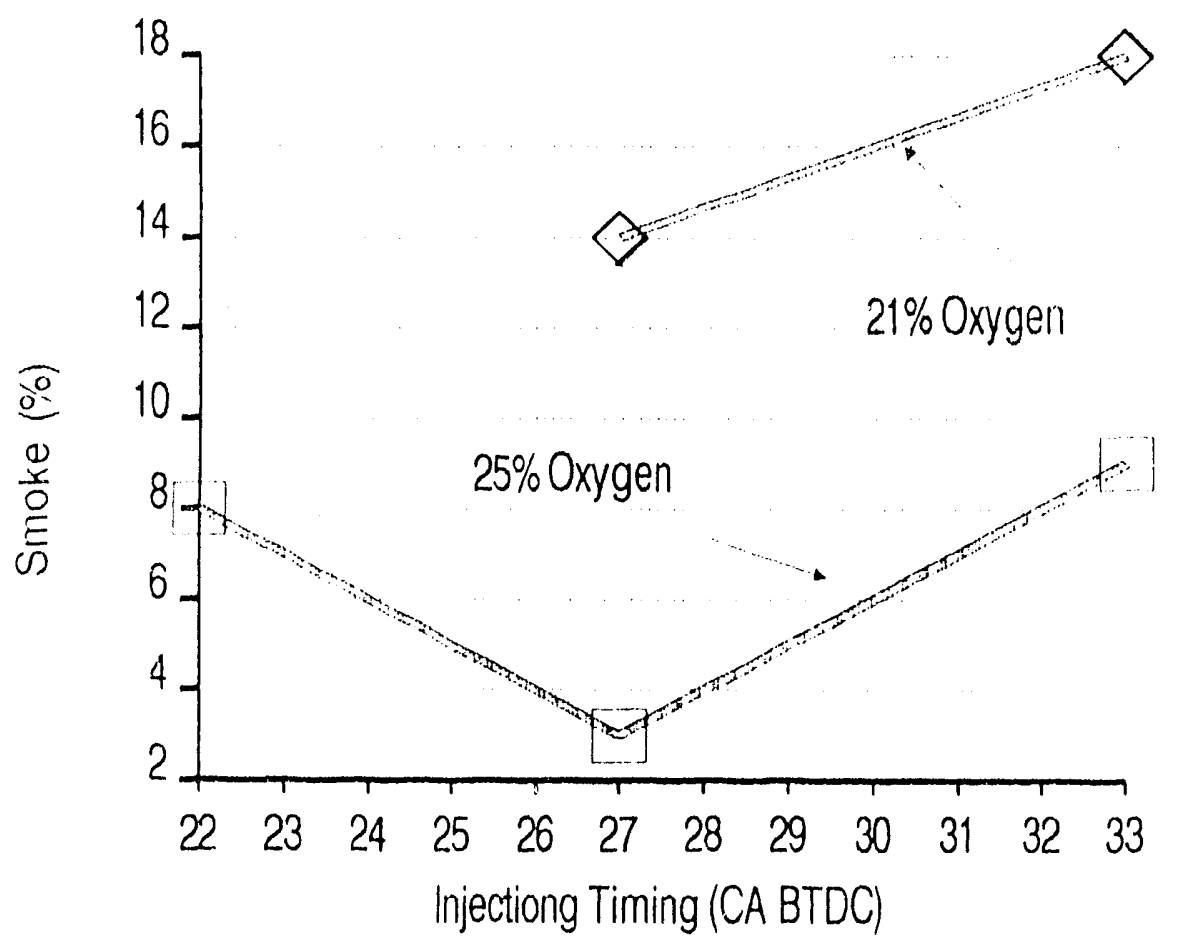

FIGUJRE B.44 Effect of Injection Timing on Smoke, 50 Brake Horsepower, No. 2 Diesel, $5 \%$ Water 


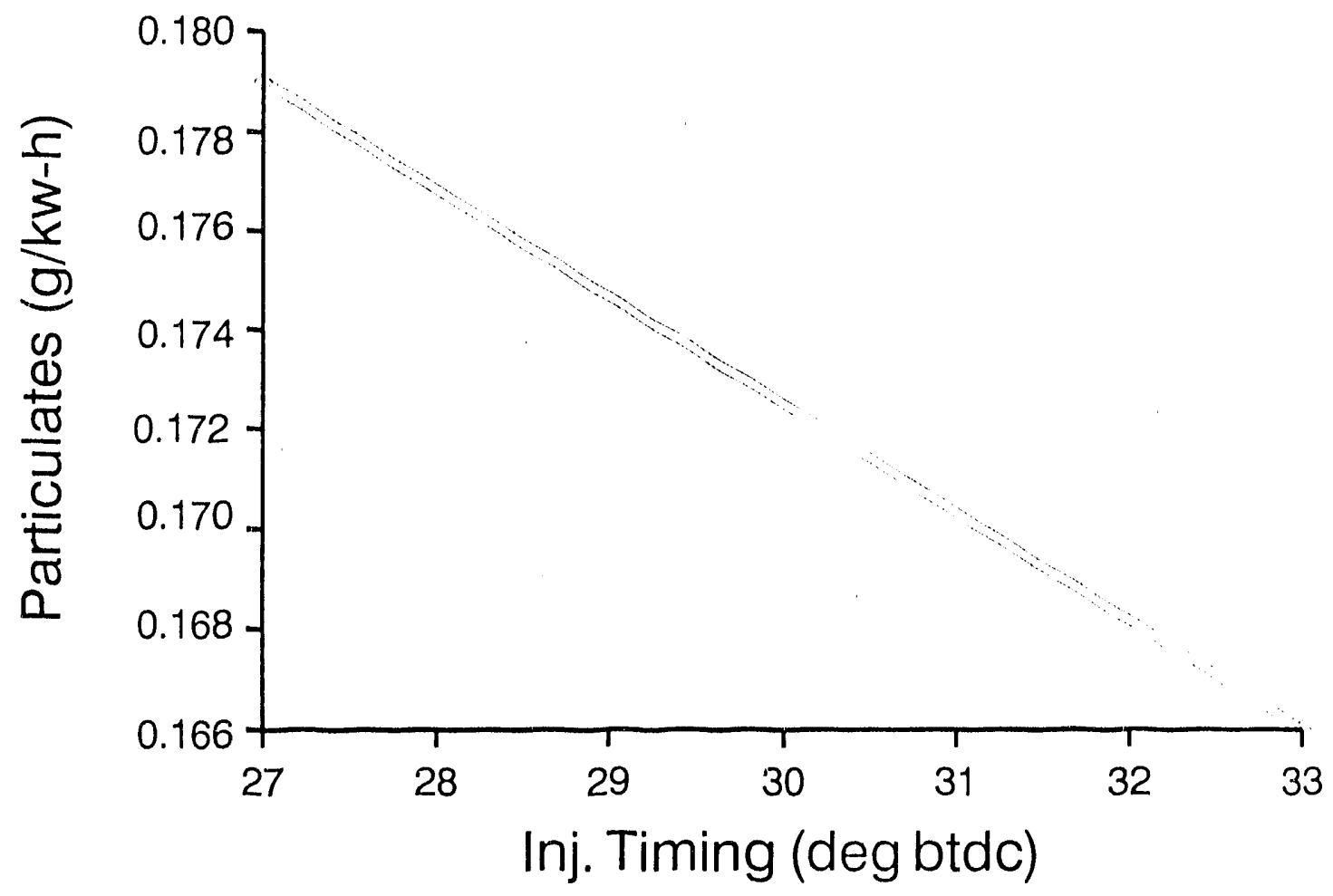

FIGURE B.45 Effect of Injection Timing on Particulate Matter, 50 Brake Horsepower, No. 2 Diesel, $5 \%$ Water, $21 \%$ Oxygen

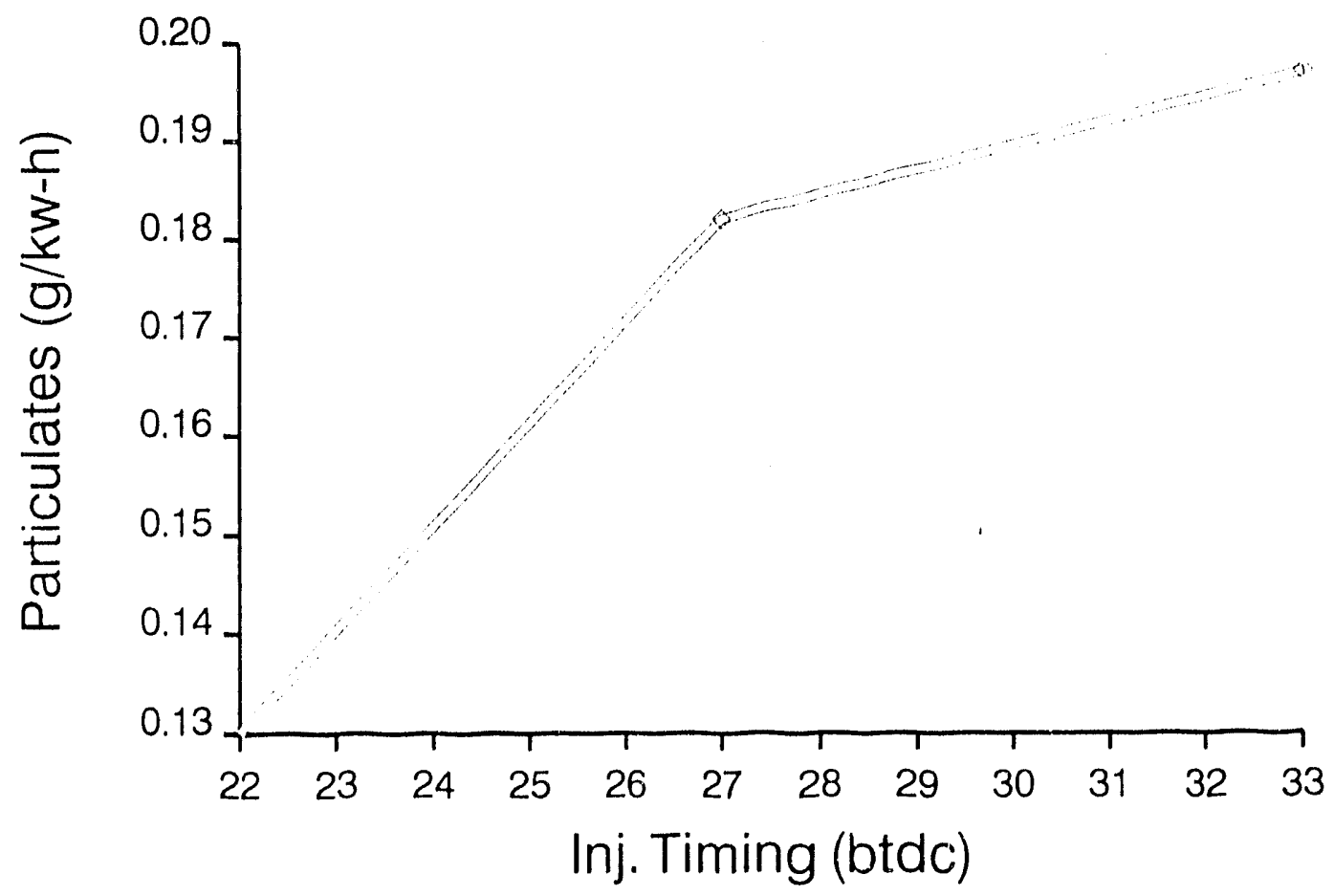

FIGURE B.46 Effect of Injection Timing on Particulate Matter, 50 Brake Horsepower, No. 2 Diesel, $5 \%$ Water, $25 \%$ Oxygen 


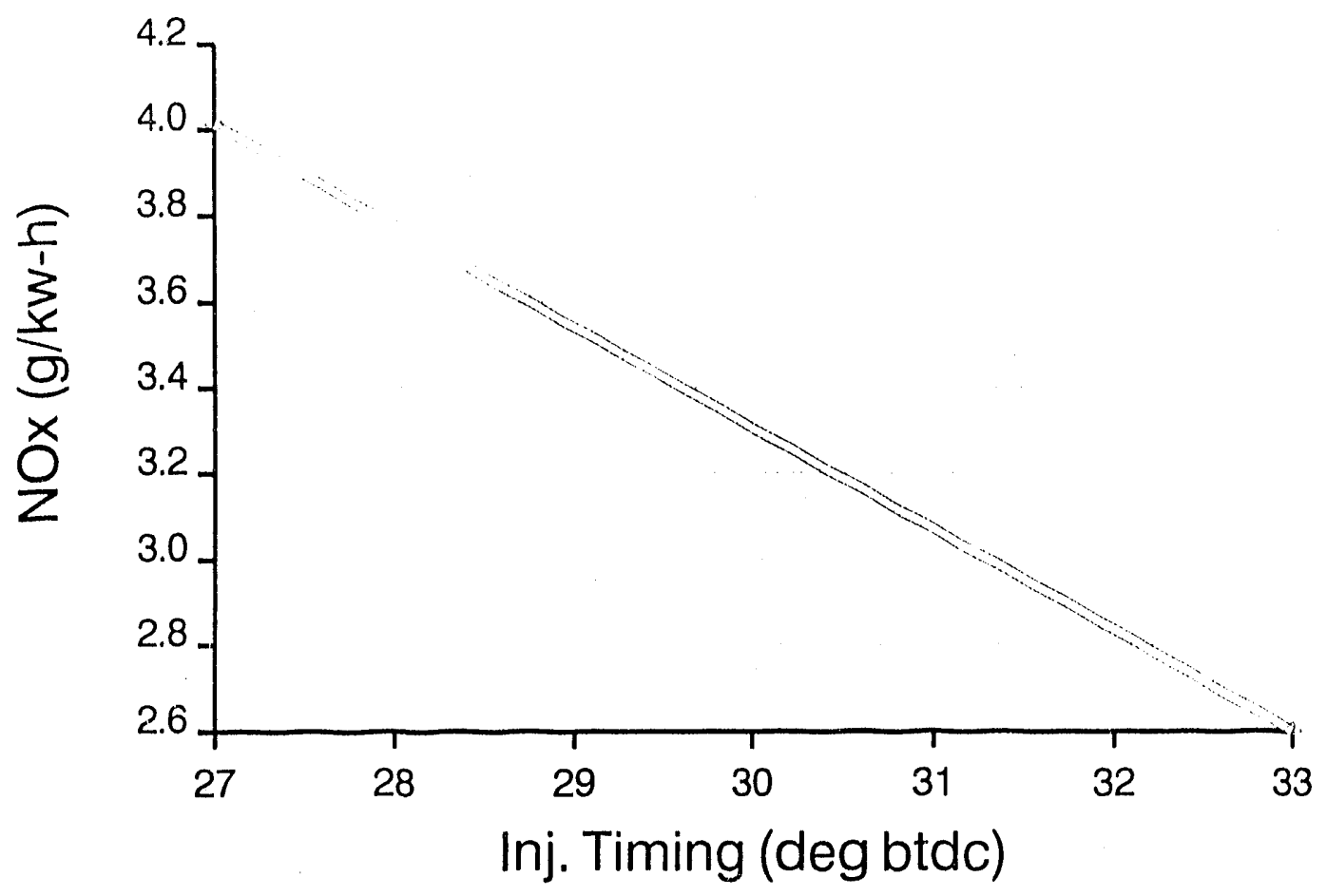

FIGURE B.47 Effect of Injection Timing on $\mathrm{NO}_{x}, 50$ Brake Horsepower, No. 2 Diesel, $5 \%$ Water, $21 \%$ Oxygen

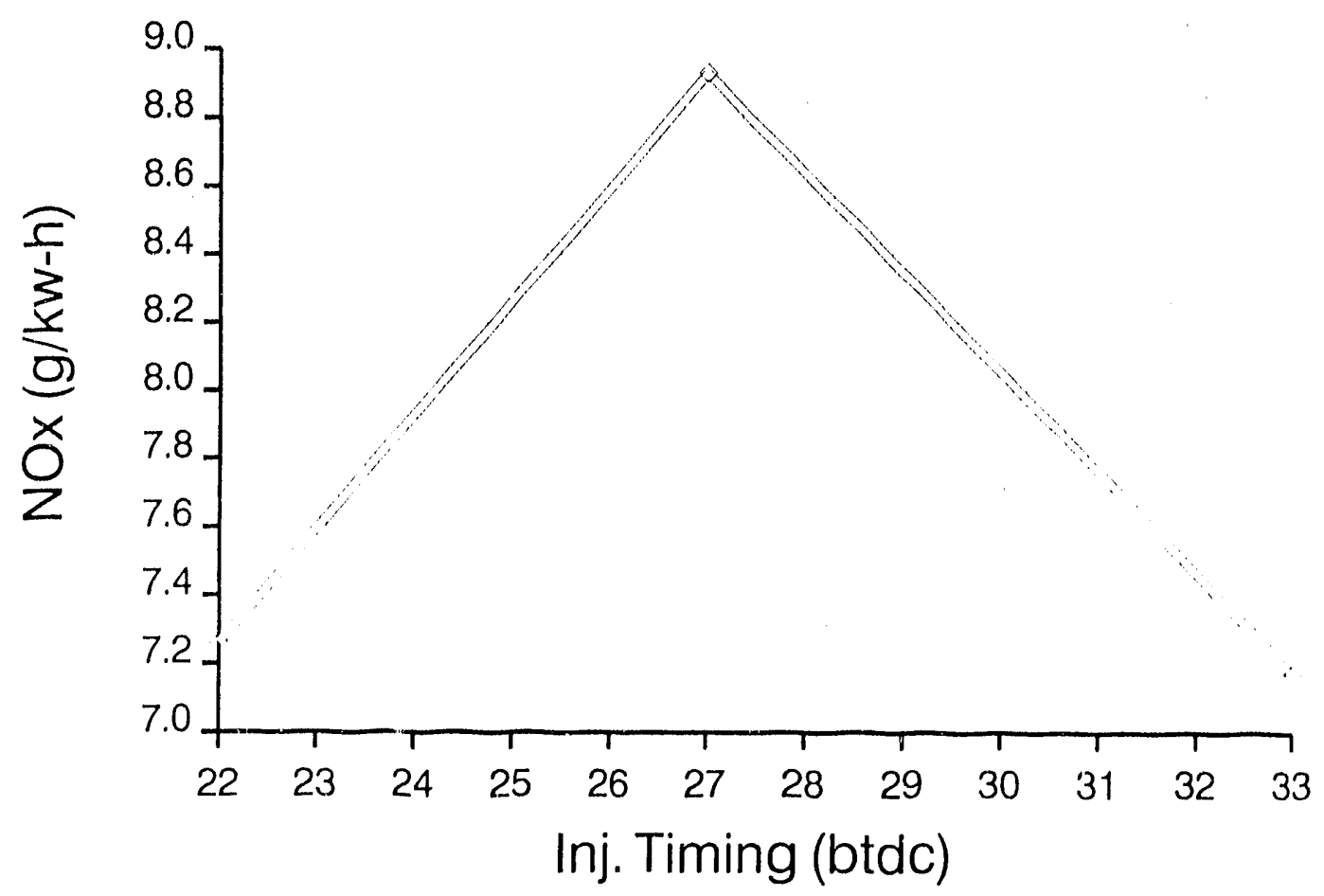

FIGURE B.48 Effect of Injection Timing on $\mathrm{NO}_{x}, 50$ Brake Horsepower, No. 2 Diesel, $5 \%$ Water, $25 \%$ Oxygen 


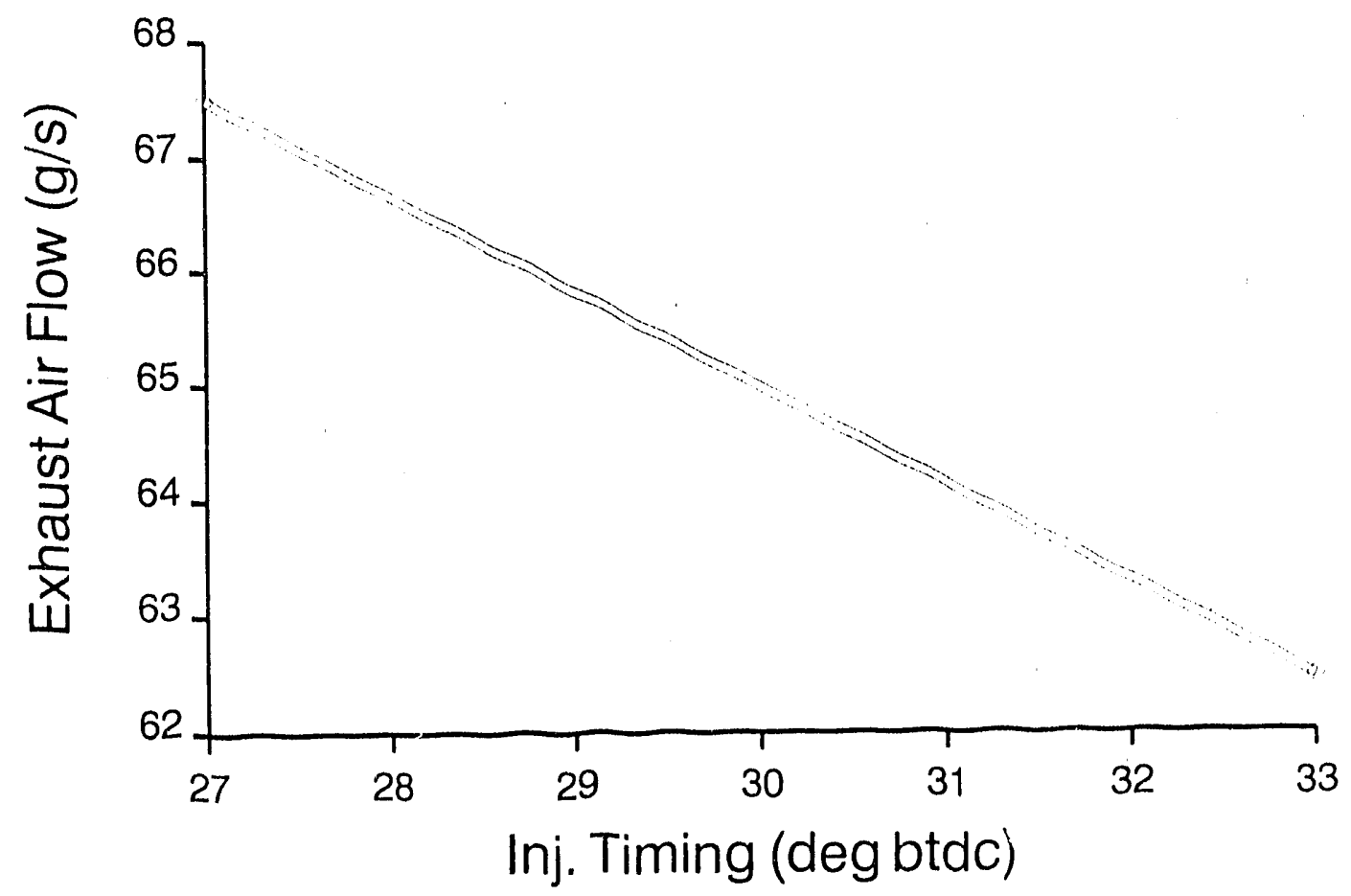

FIGURE B.49 Effect of Injection Timing on Exhaust Air Flow, 50 Brake Horsepower, No. 2 Diesel, $5 \%$ Water, $21 \%$ Oxygen

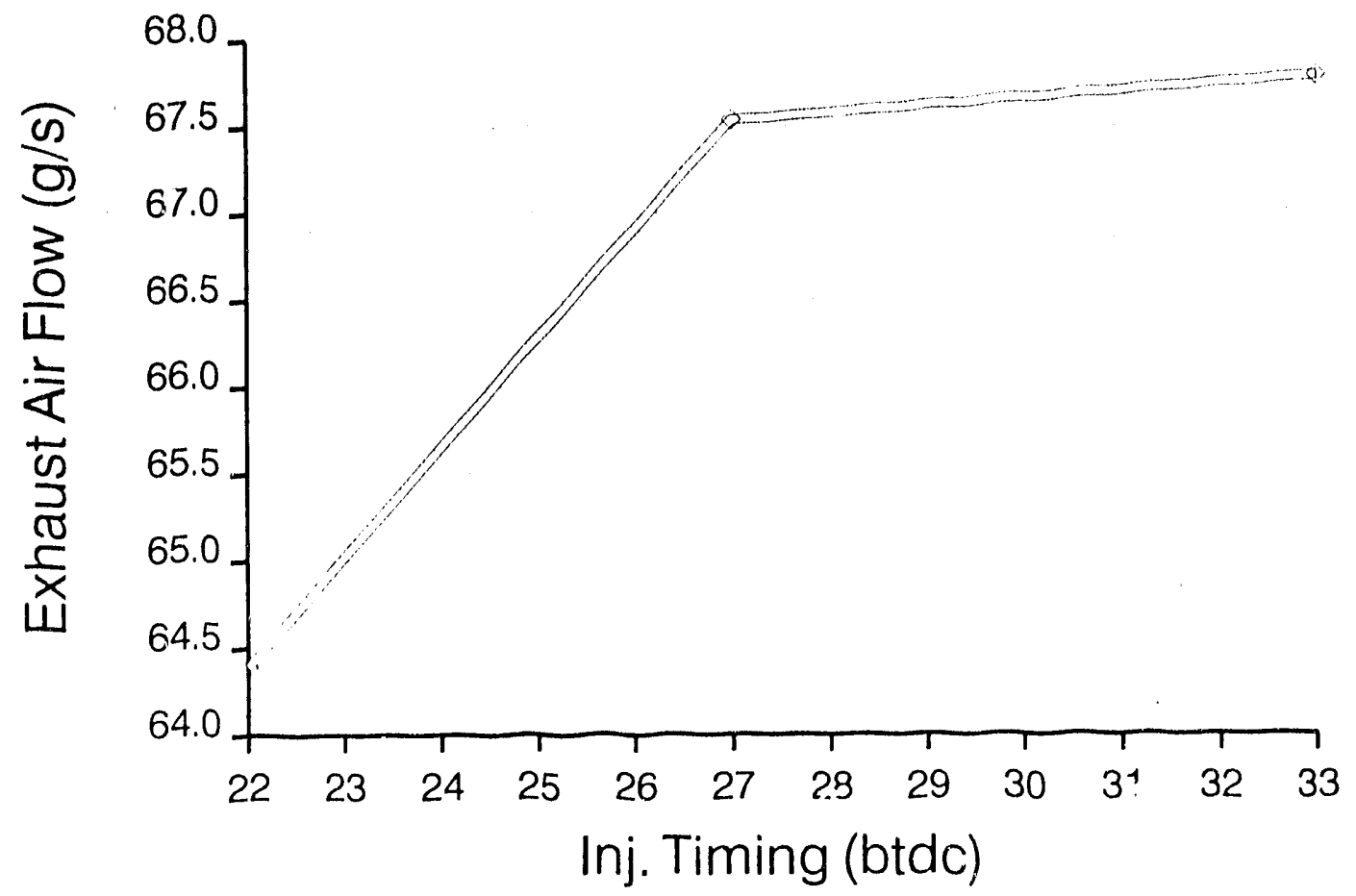

FIGURE B.50 Effect of Injection Timing on Exhaust Air Flow, 50 Brake Horsepower, No. 2 Diesel, $5 \%$ Water, $25 \%$ Oxygen 


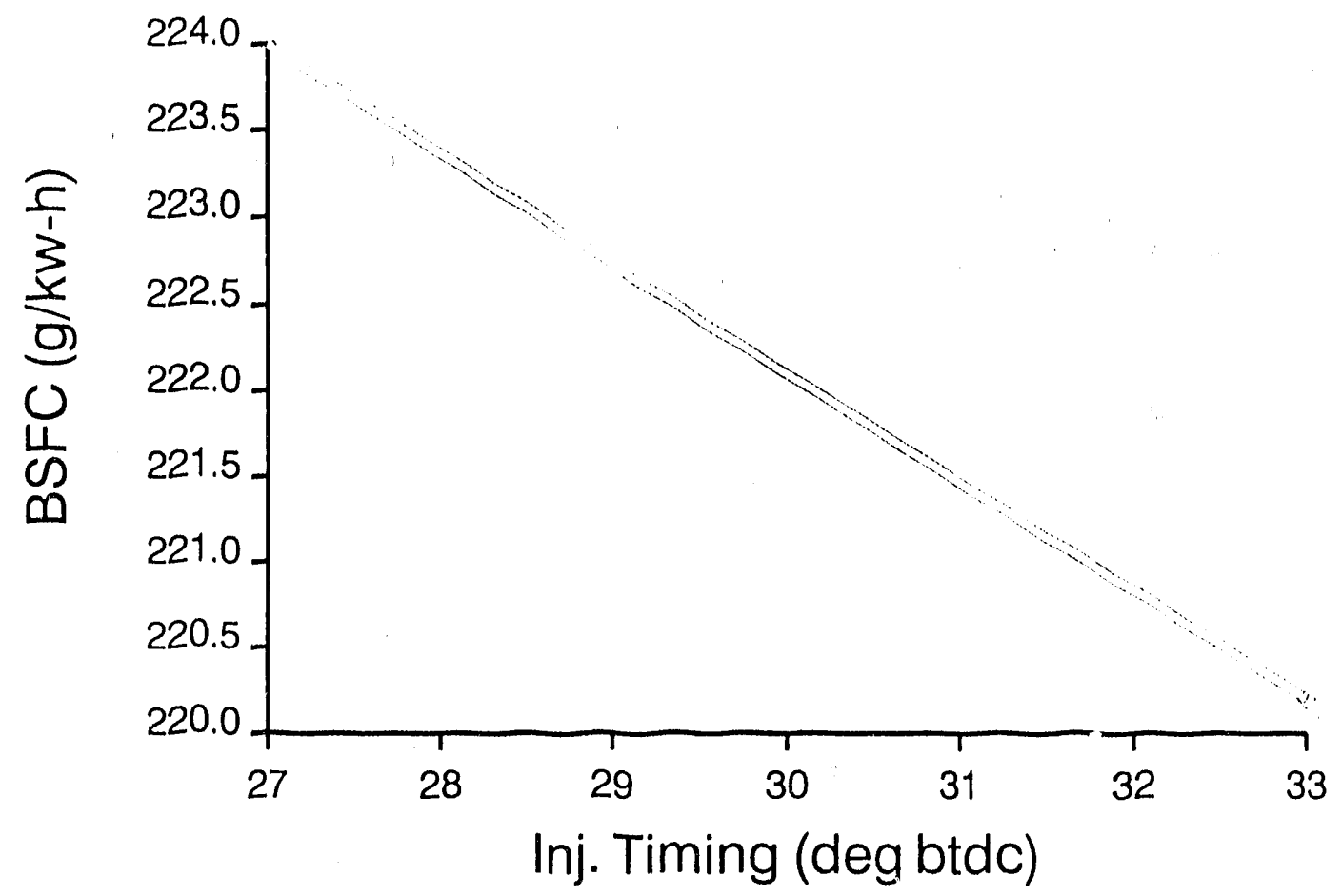

FIGURE B.51 Effect of Injection Timing on Brake-Specific Fuel Consumption, 50 Brake Horsepower, No. 2 Diesel, $5 \%$ Water, $21 \%$ Oxygen

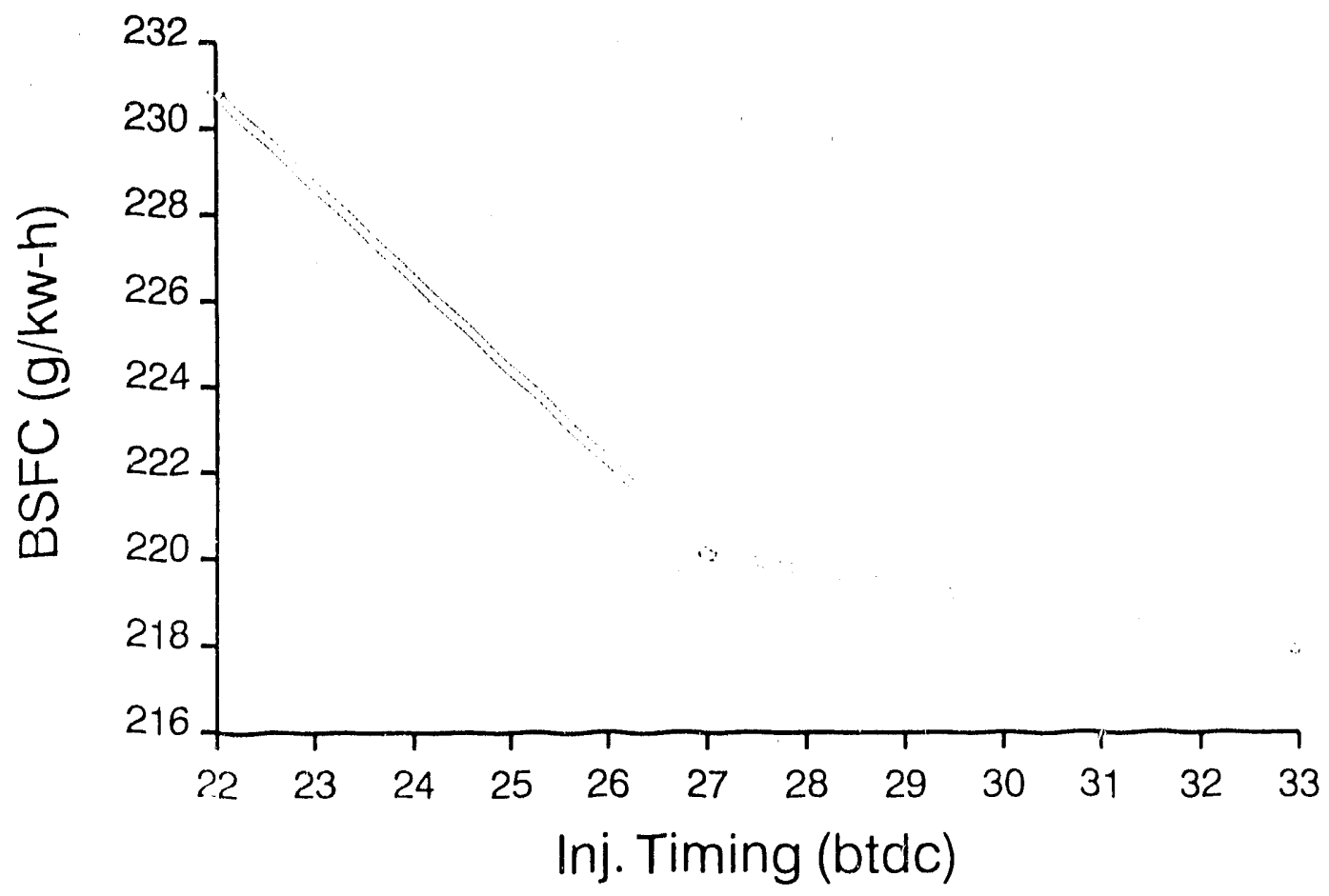

FIGURE B.52 Effect of Injection Timing on Brake-Specific Fuel Consumption, 50 Brake Horsepower, No. 2 Diesel, 5\% Water, 25\% Oxygen 
B.4 Graphic Display of Cylinder Pressure Data and Results of Heat-Release Analysis for No. 2 Fuel 


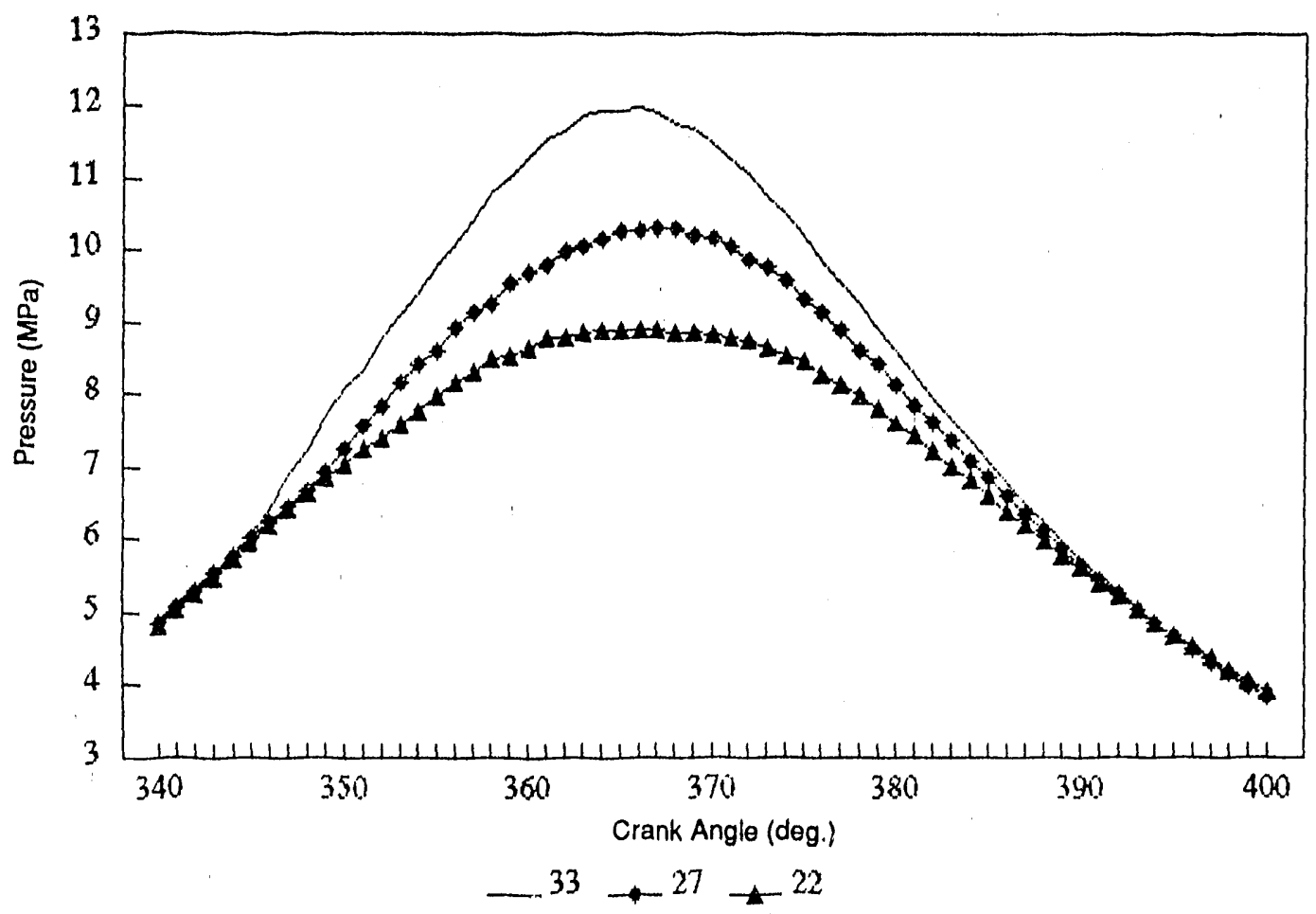

FIGURE B.53 Effect of Fuel Injection Timing on Cylinder Pressure. No. 2 Fuel, Full Load, No Water, $21 \%$ Oxygen

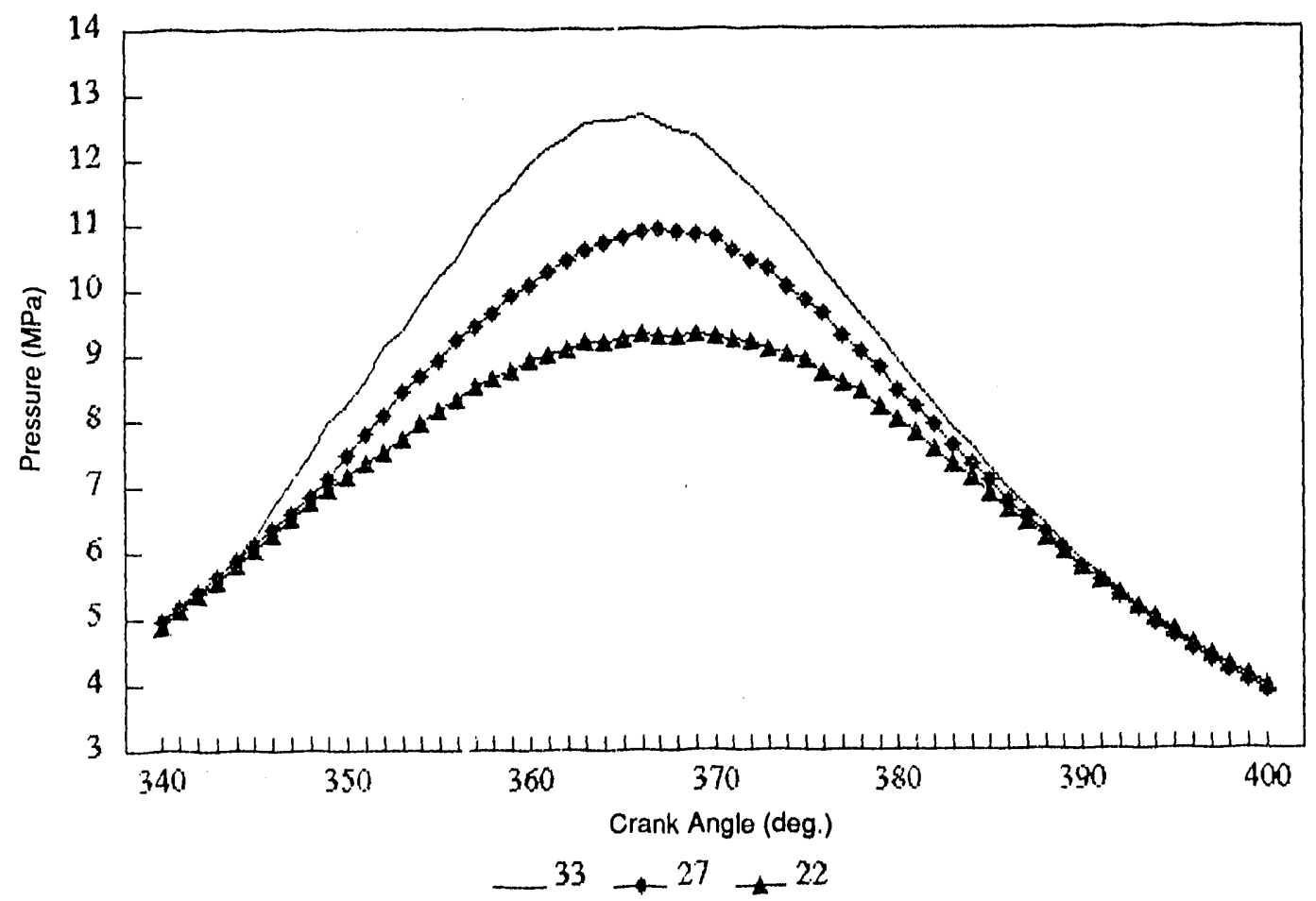

FIGURE B.54 Effect of Fuel Injection Timing on Cylinder Pressure, No. 2 Fuel, Full Load, No Water, $25 \%$ Oxygen 


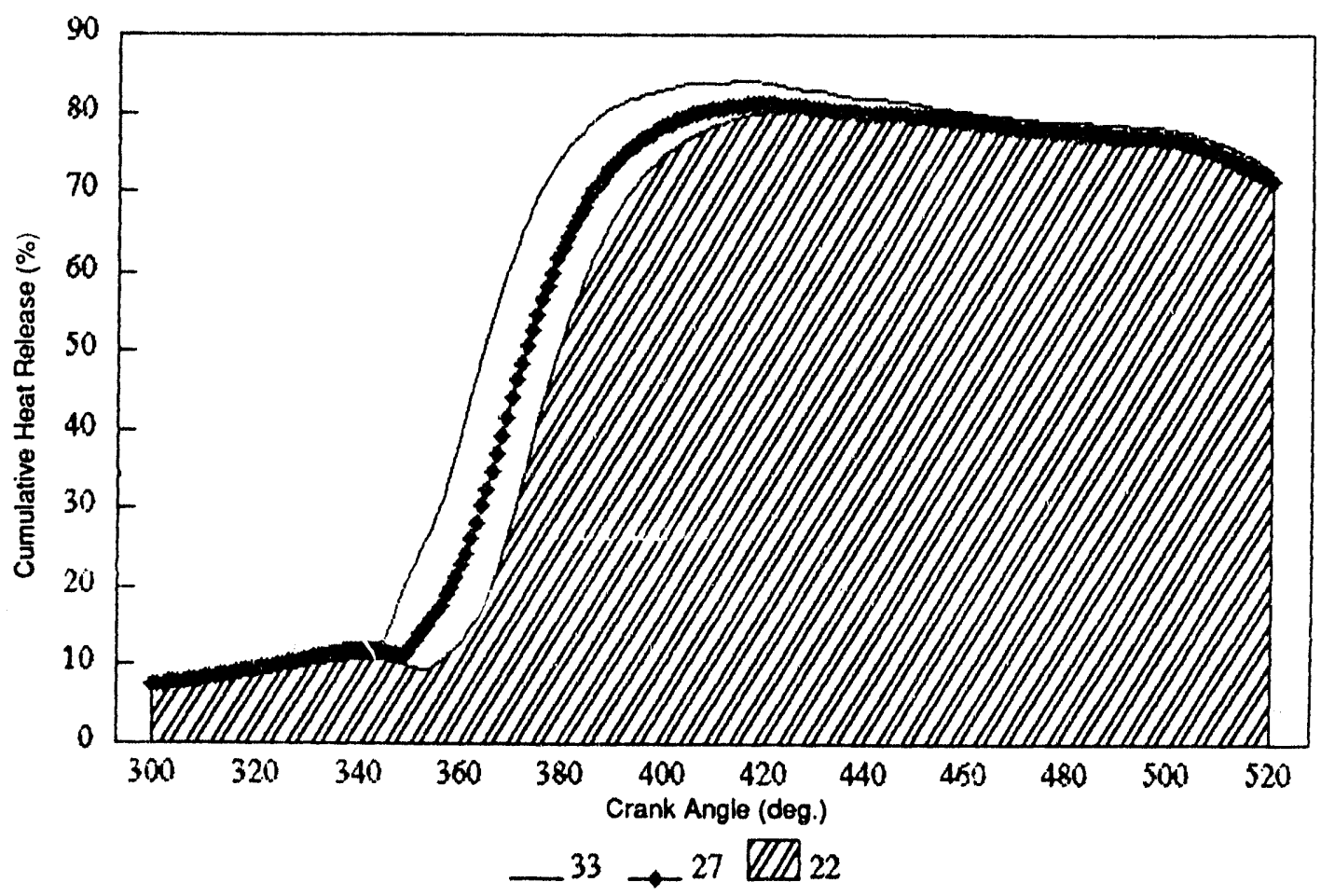

FIGURE B.55 Effect of Fuel Injection Timing on Cumulative Heat Release, No. 2 Fuel, Full Load, No Water, 21\% Oxygen

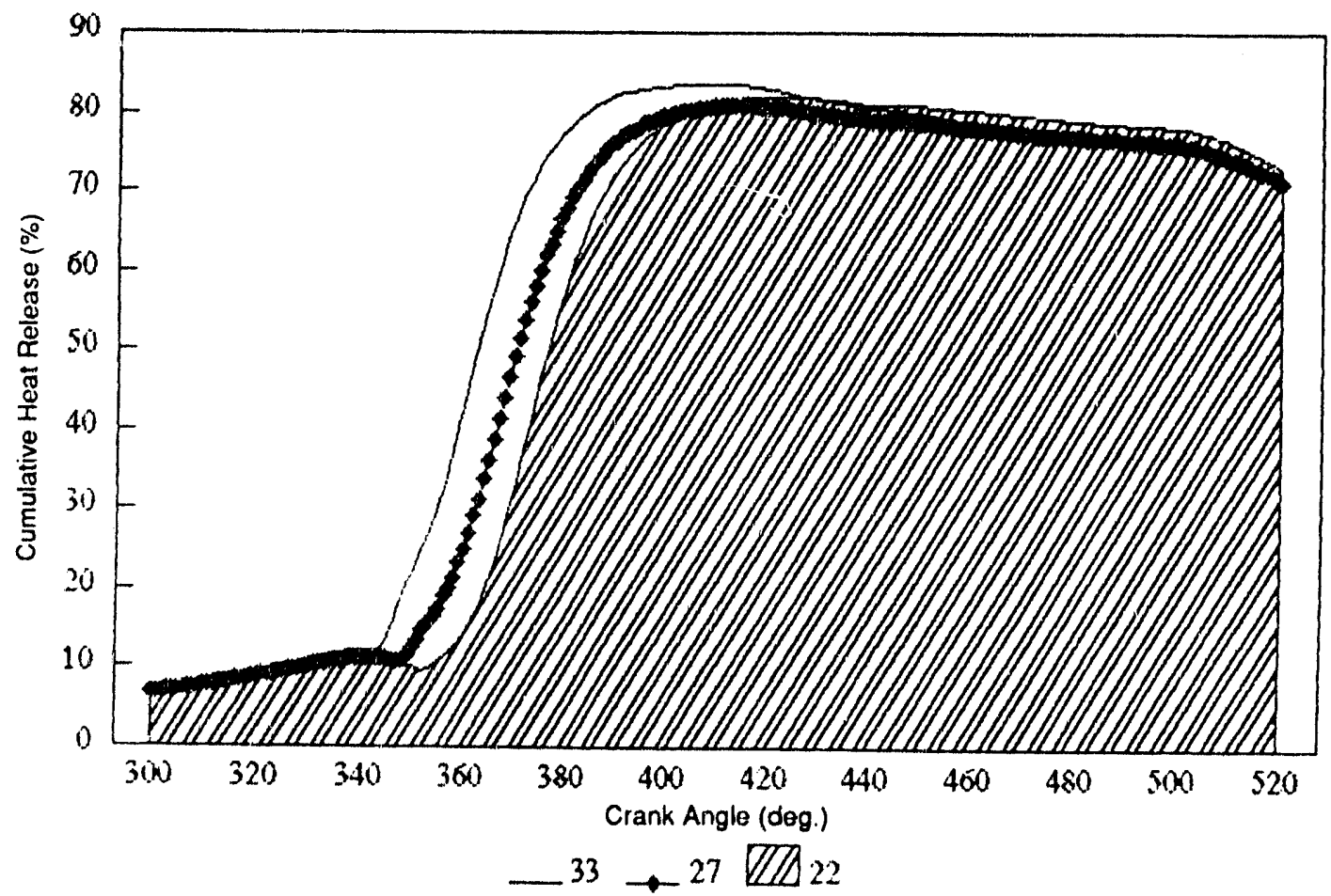

FIGURE B.56 Effect of Fuel Injection Timing on Cumulative Heat Release, No. 2 Fuel, Full Load, No Water, 25\% Oxygen 


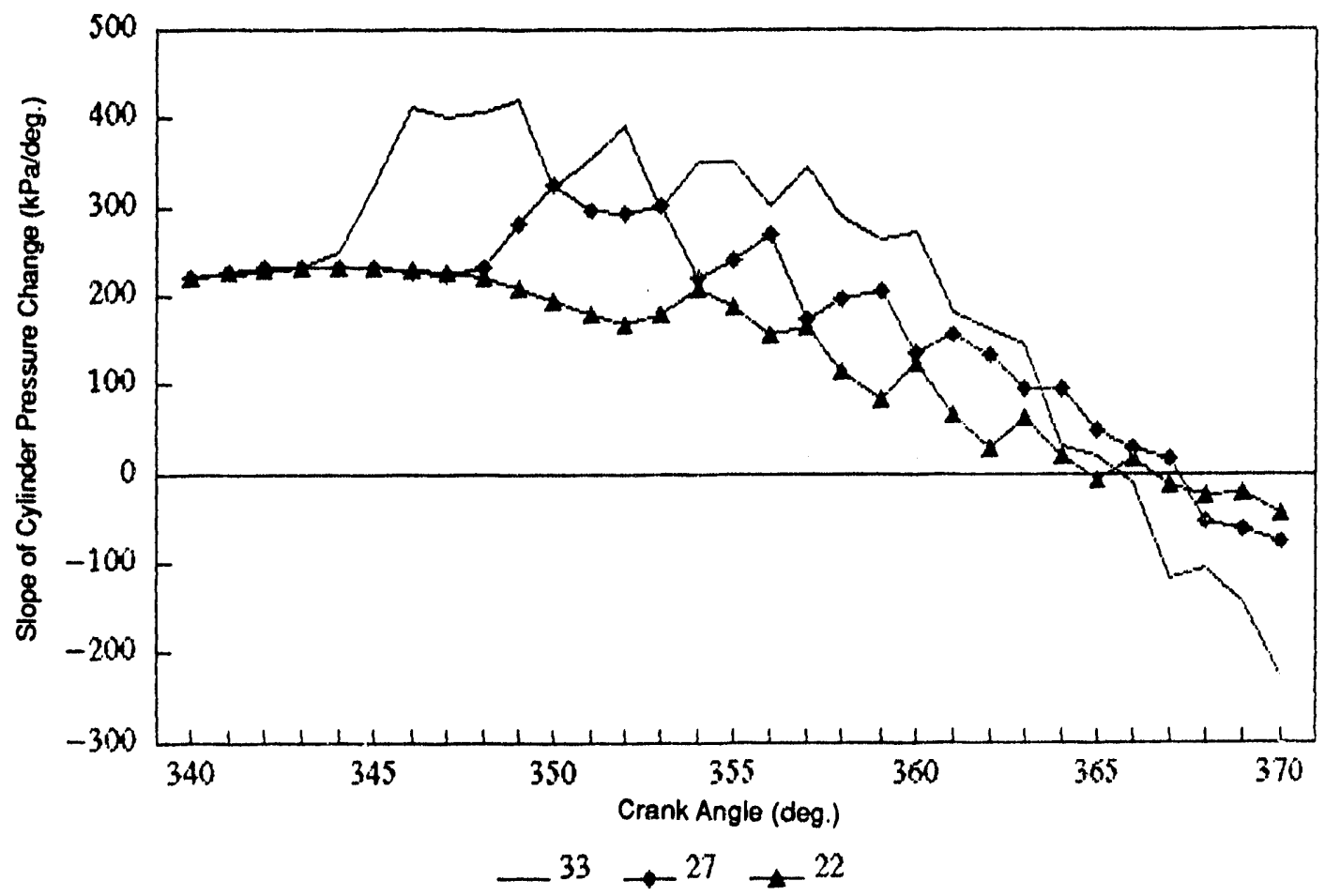

FIGURE B.57 Effect of Fuel Injection Timing on Slope of Cylinder Pressure Change, No. 2 Fuel, Full Load, No Water, 21\% Oxygen

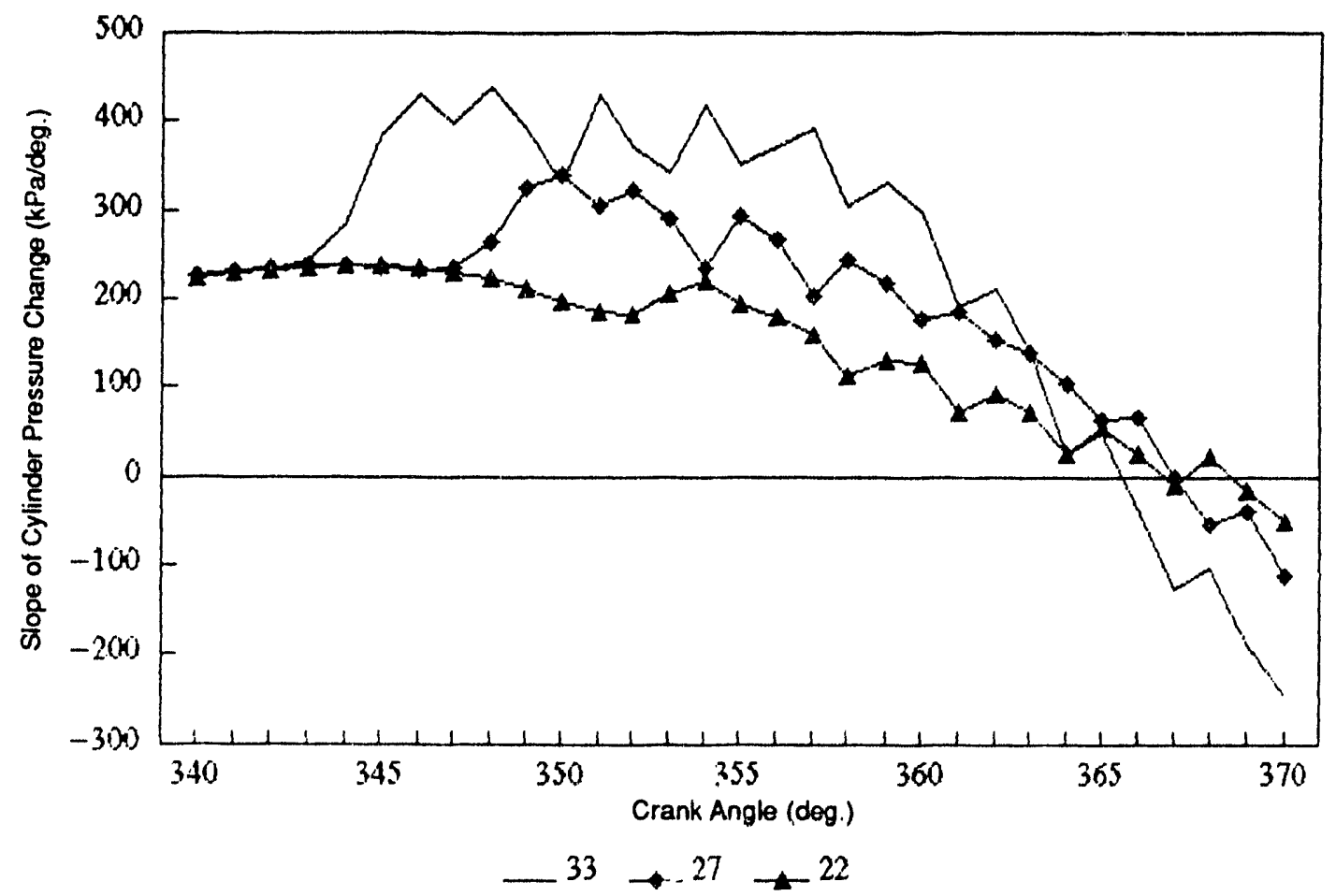

FIGURE B.58 Effect of Fuel Injection Timing on Slope of Cylinder Pressure Change, No. 2 Fuel, Full Load, No Water, $25 \%$ Oxygen 


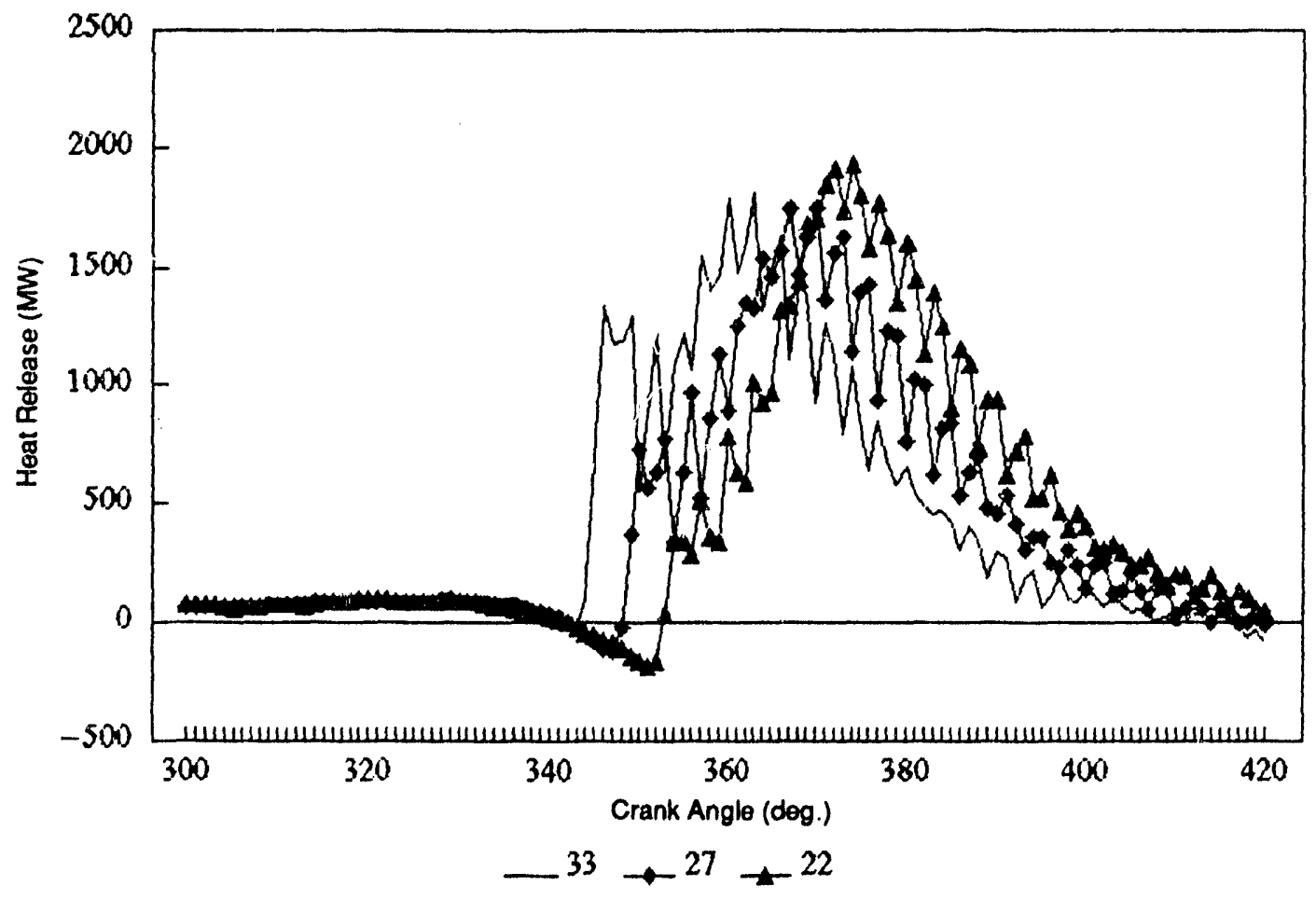

FIGURE B.59 Effect of Fuel Injection Timing on Heat Release, No. 2 Fuel, Full Load, No Water, $21 \%$ Oxygen

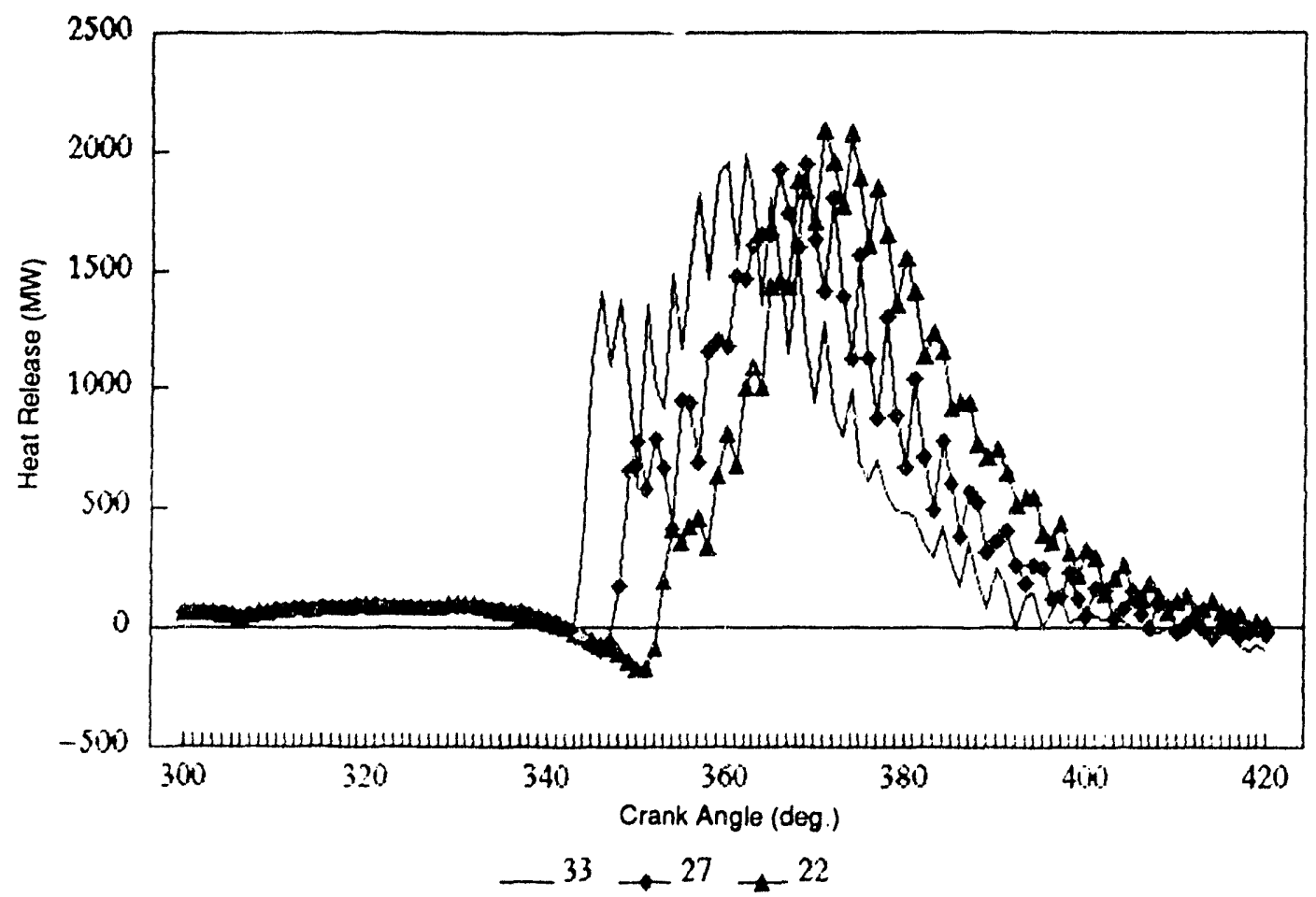

FIGURE B.60 Effect of Fuel Injection Timing on Heat Release, No. 2 Fuel, Full Load, No Water, $25 \%$ Oxygen 


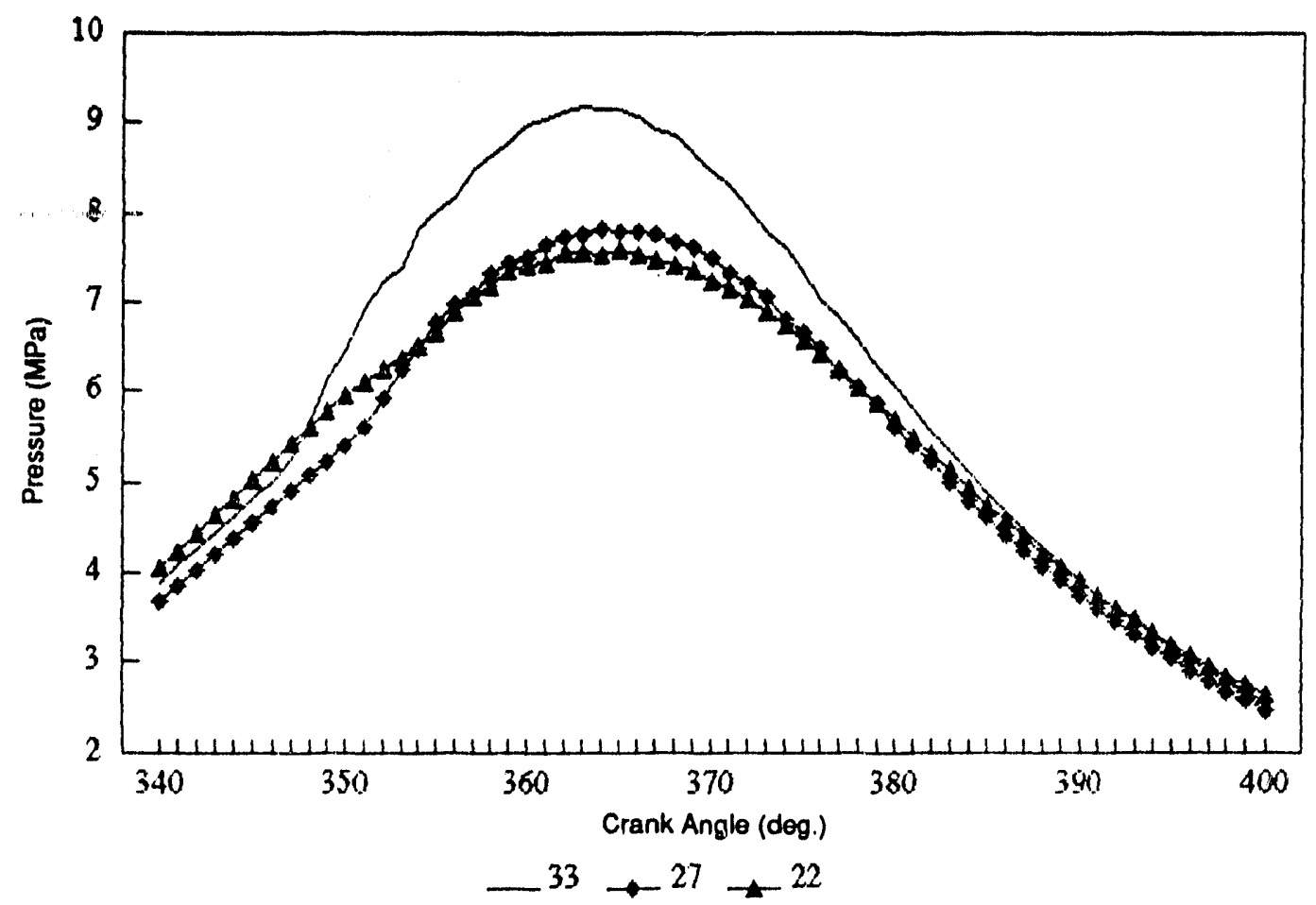

FIGURE B.61 Effect of Fuel Injection Timing on Pressure, No. 2 Fuel, Half Load, No Water, $21 \%$ Oxygen

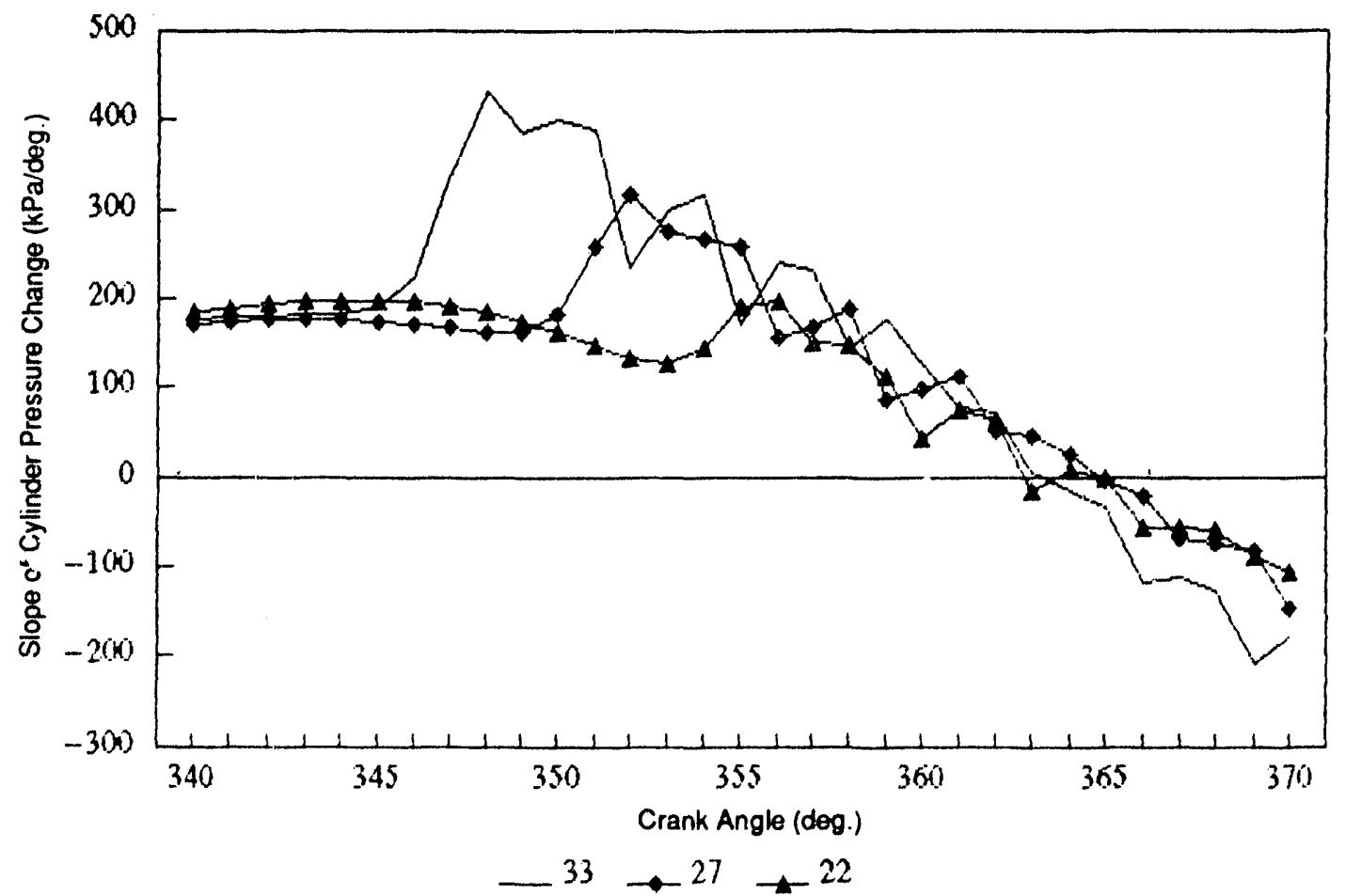

FIGURE B.62 Effect of Fuel Injection Timing on Slope of Cylinder Pressure Change, No. 2 Fusl, Haff Load, No Water, $21 \%$ Oxygen 

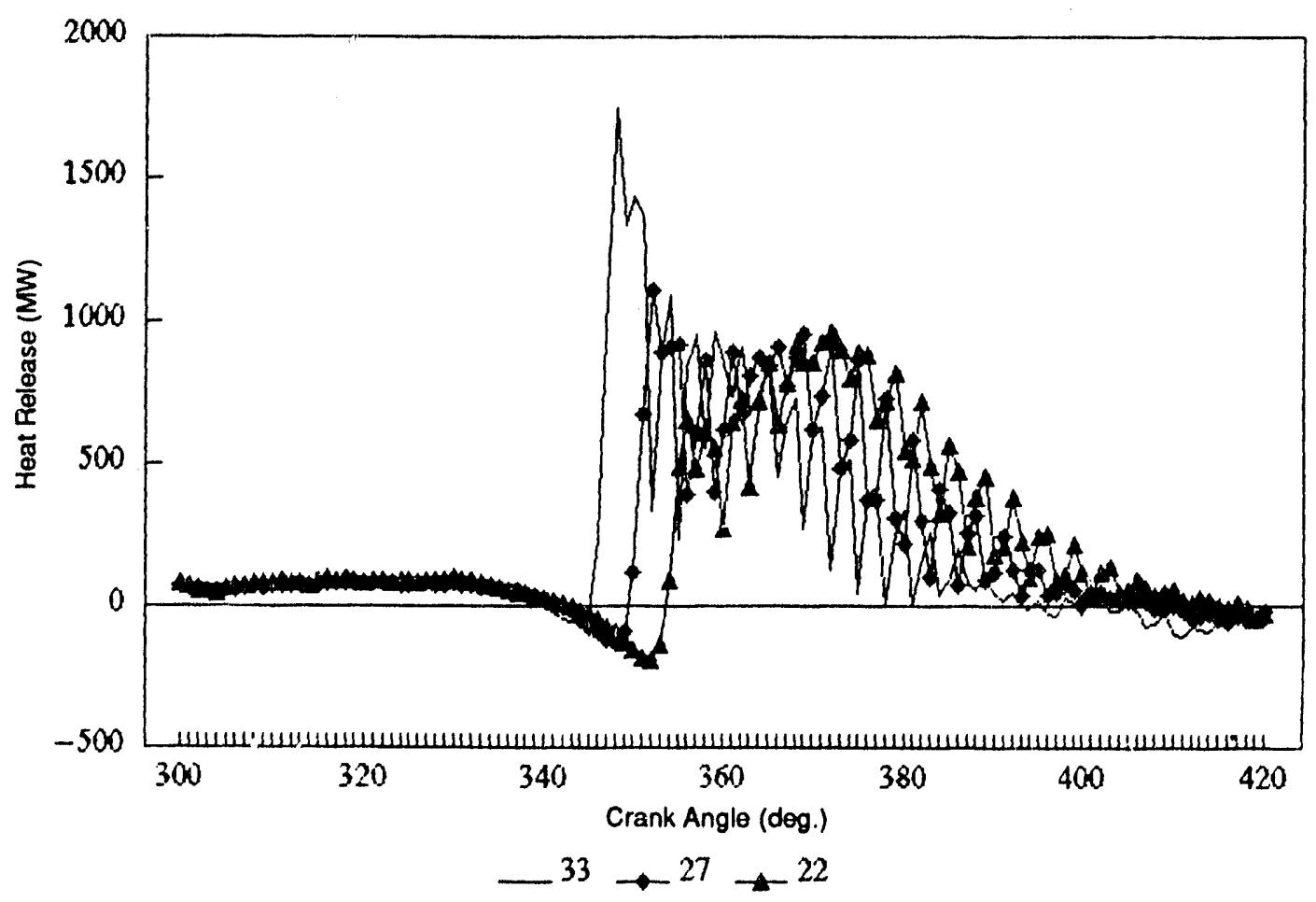

FIGURE B.63 Effect of Fuel Injection Timing on Heat Release, No. 2 Fuel, Half Load, No Water, $21 \%$ Oxygen

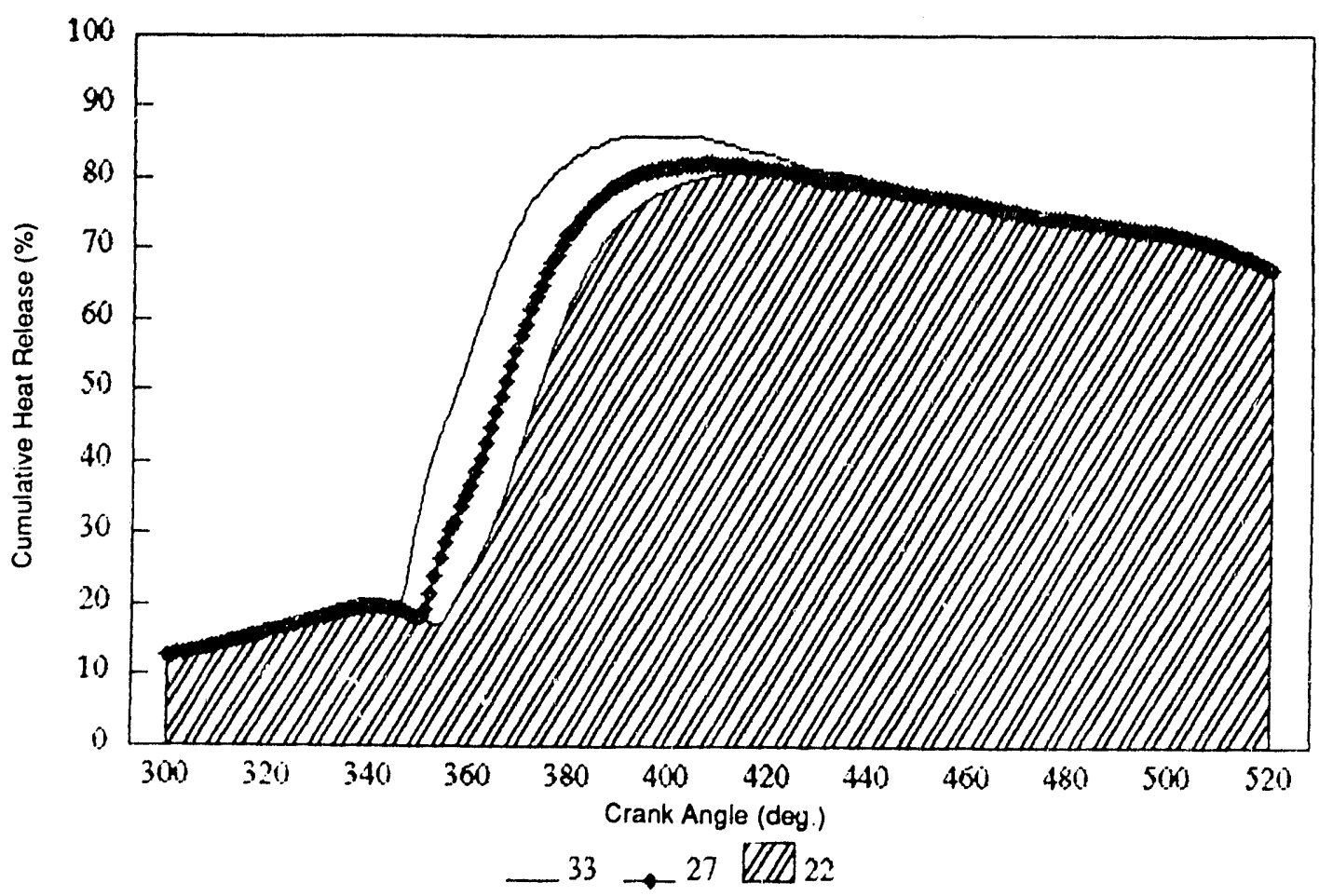

FIGURE B.64 Effect of Fuel Injection Timing on Cumulative Heat Release, No. 2 Fuel, Half Load, No Water, $21 \%$ Oxygen 


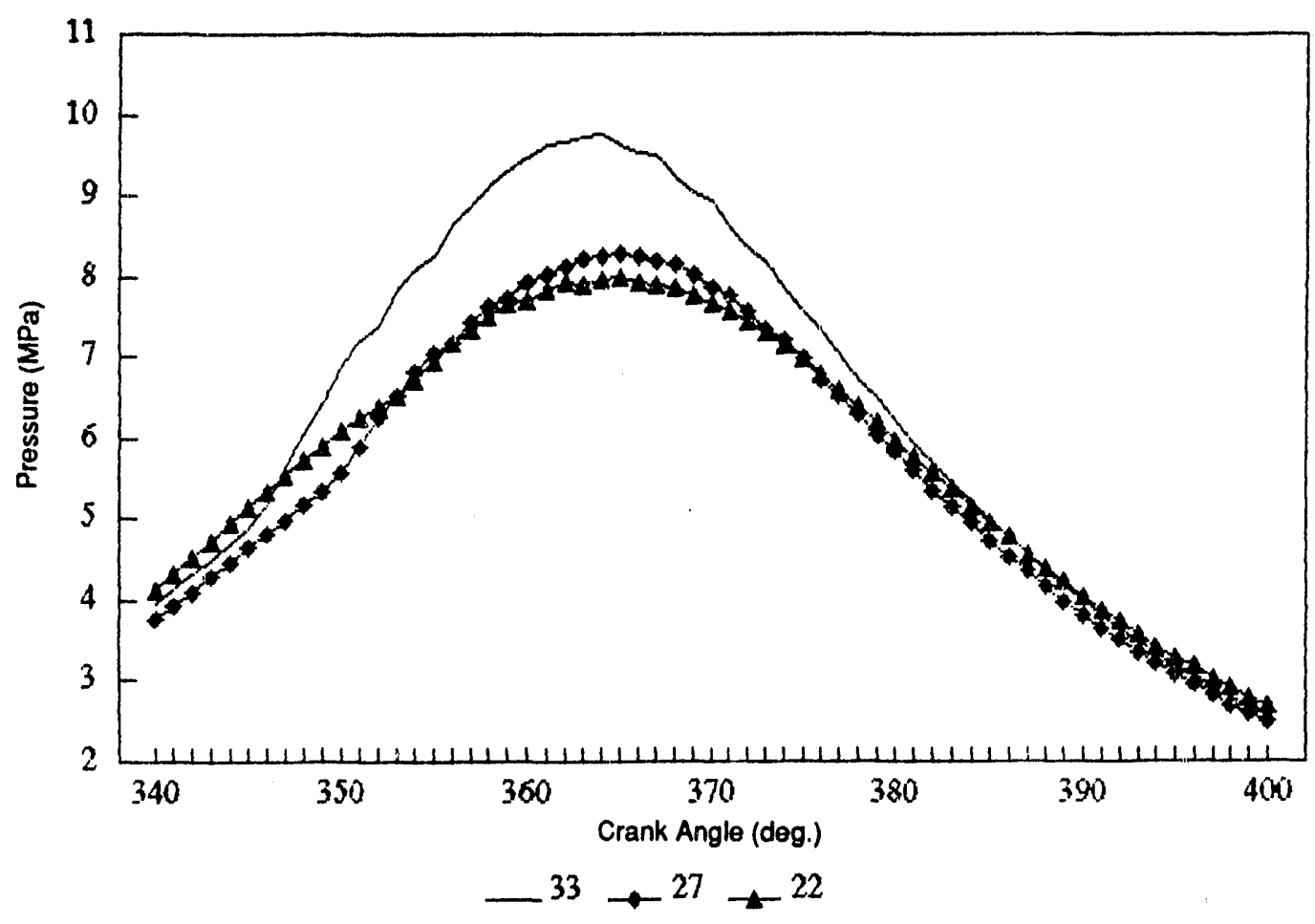

FIGURE B.65 Effect of Fuel Injection Timing on Pressure, No. 2 Fuel, Half Load, No Water, $25 \%$ Oxygen

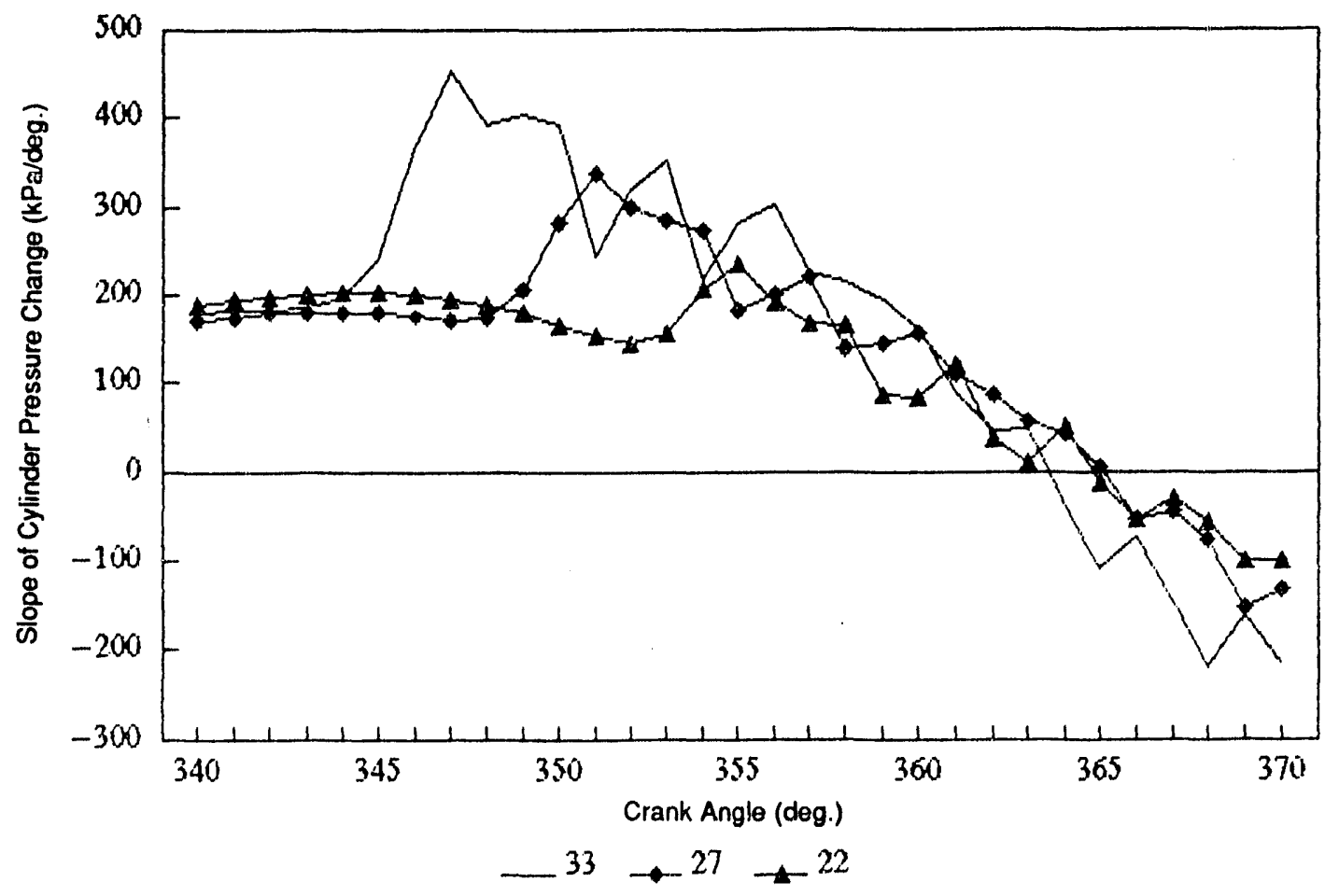

FIGURE B.66 Effect of Fuel Injection Timing on Slope of Cylinder Pressure Change, No. 2 Fuel, Half Load, No Water, $25 \%$ Oxygen 


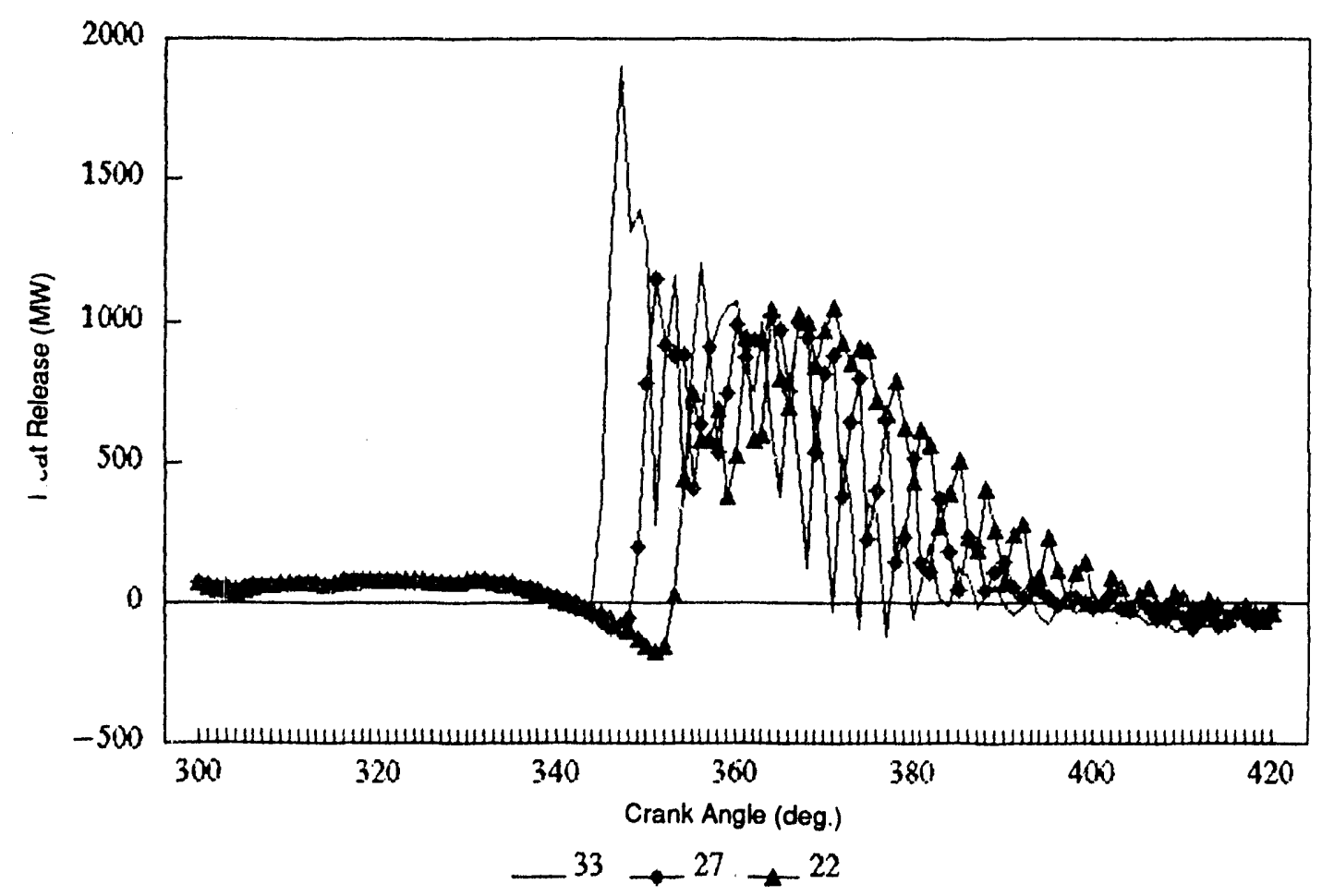

FIGURE B.67 Effect of Fuel Injection Timing on Heat Release, No. 2 Fuel, Hafl Load, No Water, $25 \%$ Oxygen

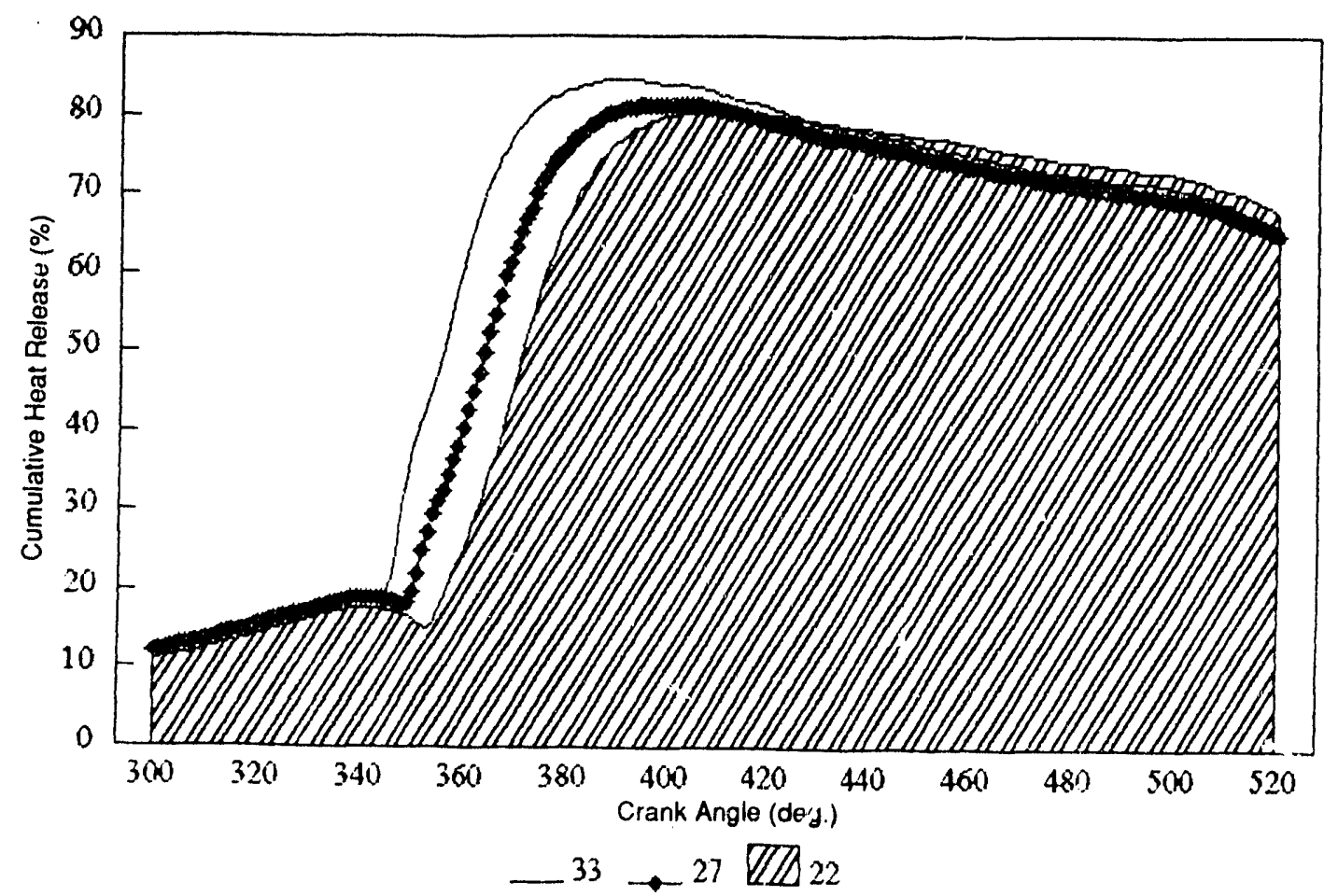

FIGURE B.68 Effect of Fuel Injection Timing on Cumulative Heat Release, No. 2 Fuel, Half Load, No Water, $25 \%$ Oxygen 


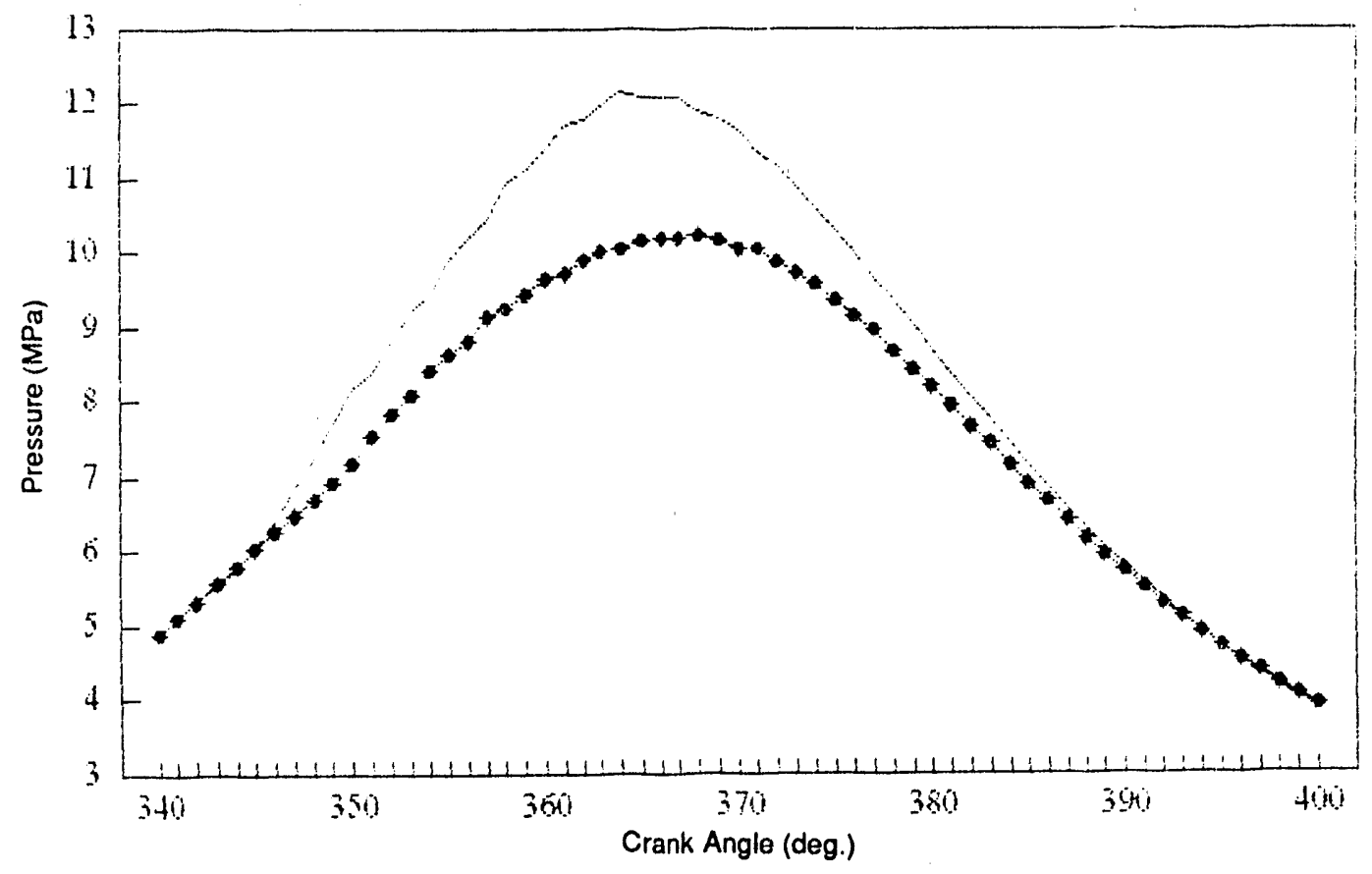

$33 \rightarrow 27$

FIGURE B.69 Effect of Fuel Injection Timing on Pressure, No. 2 Fuel, Full Load, $5 \%$ Water, $21 \%$ Oxygen

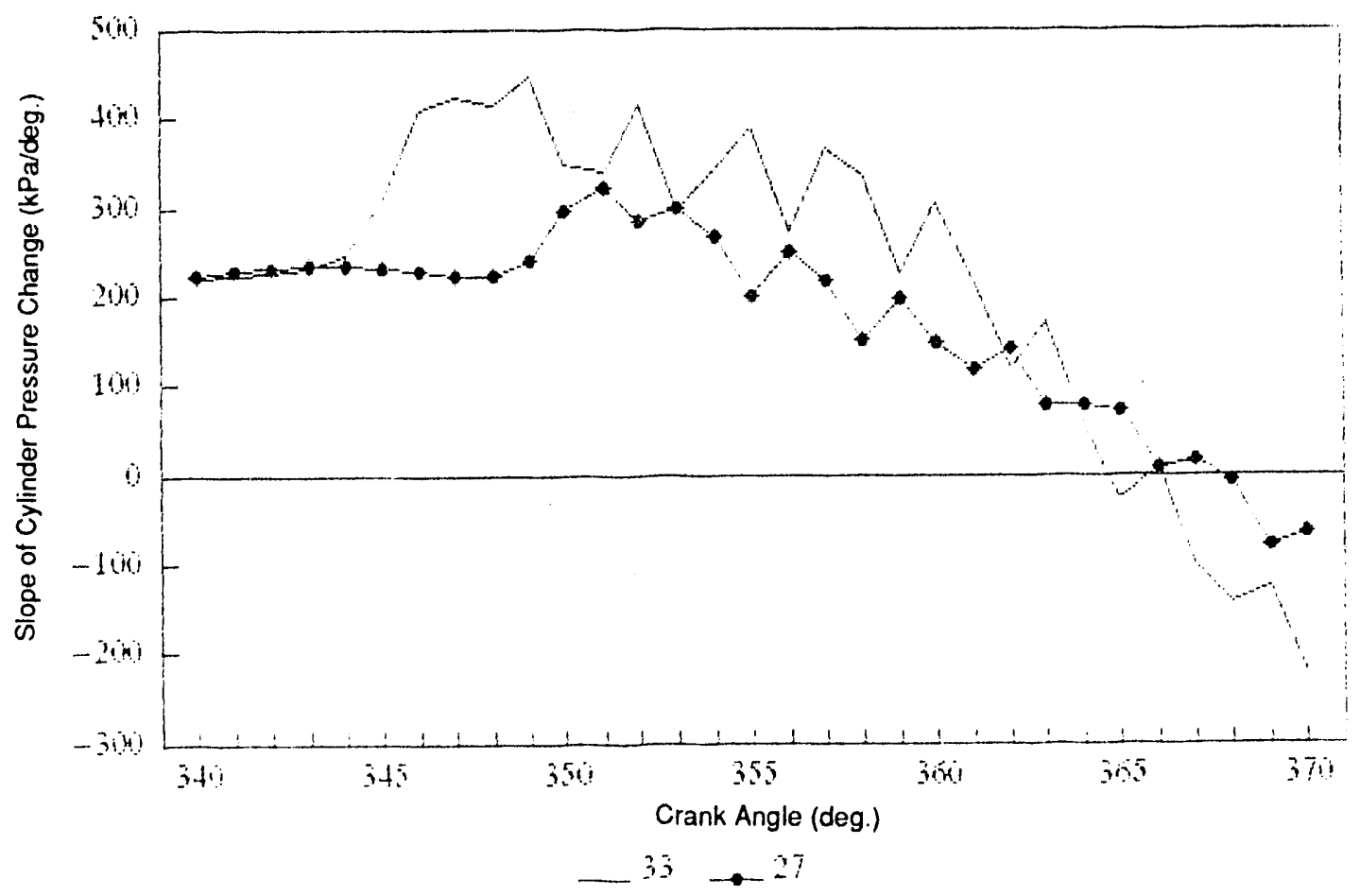

FIGURE B.70 Effect of Fuel Injection Timing on Slope of Cylinder Pressure Change, No. 2 Fuel, Full Load, $5 \%$ Water, 21\% Oxygen 


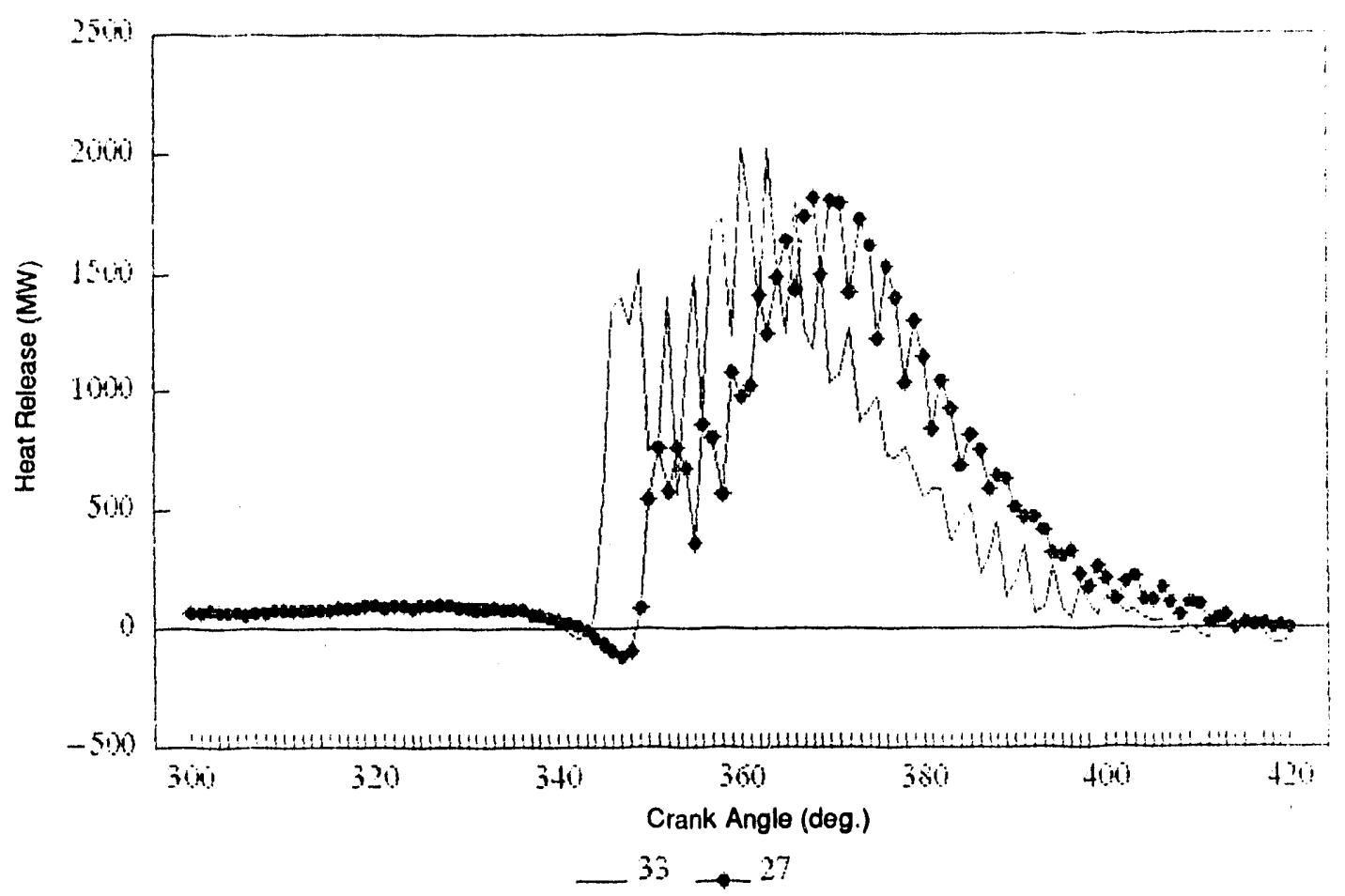

FIGURE B.71 Effect of Fuel Injection Timing on Heat Release, No. 2 Fuel, Full Load, $5 \%$ Water, $21 \%$ Oxygen

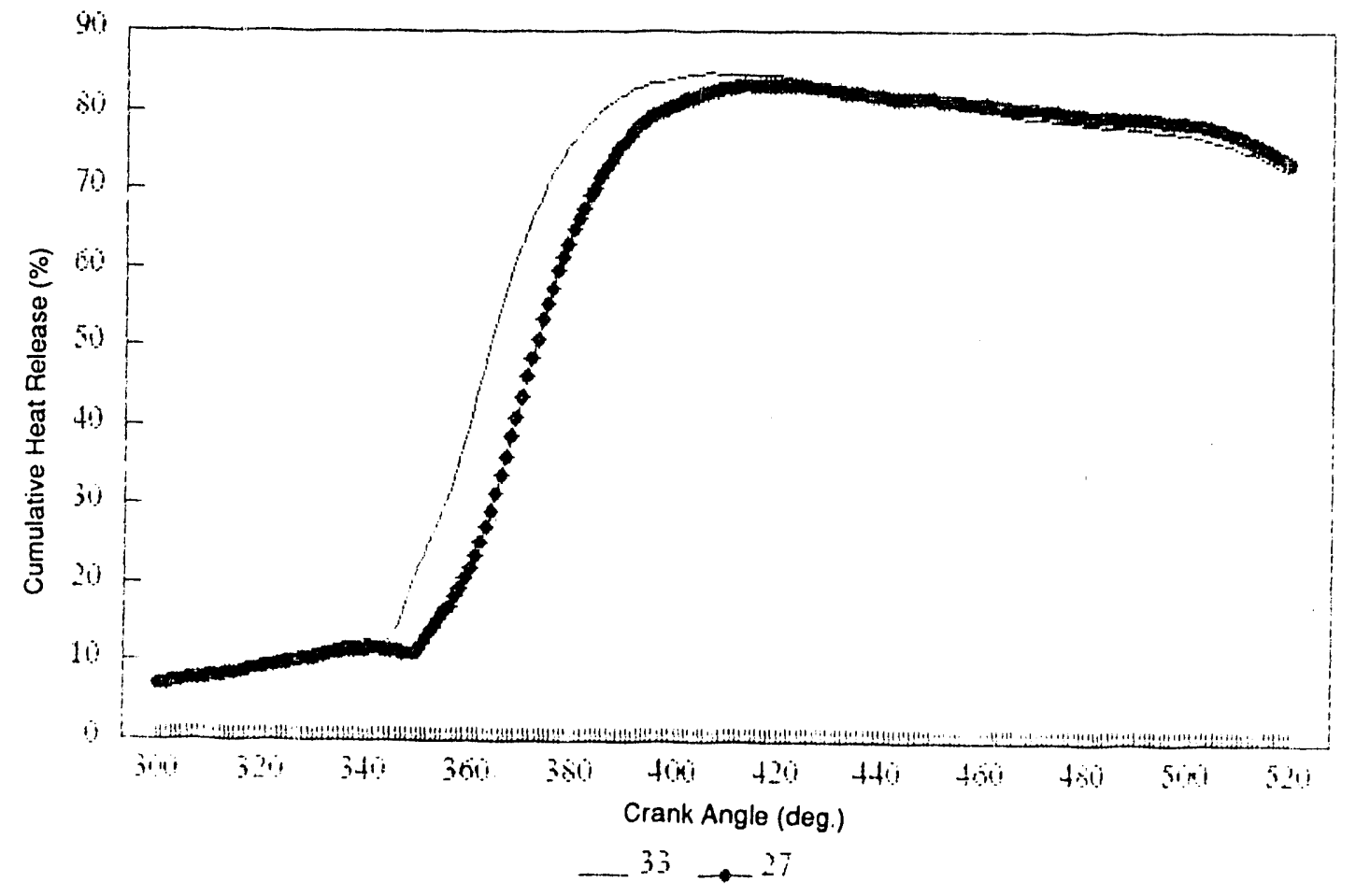

FIGURE B.72 Effect of Fuel Injection Timing on Cumulative Heat Release, No. 2 Fuel, Full Load, $5 \%$ Water, $21 \%$ Oxygen 


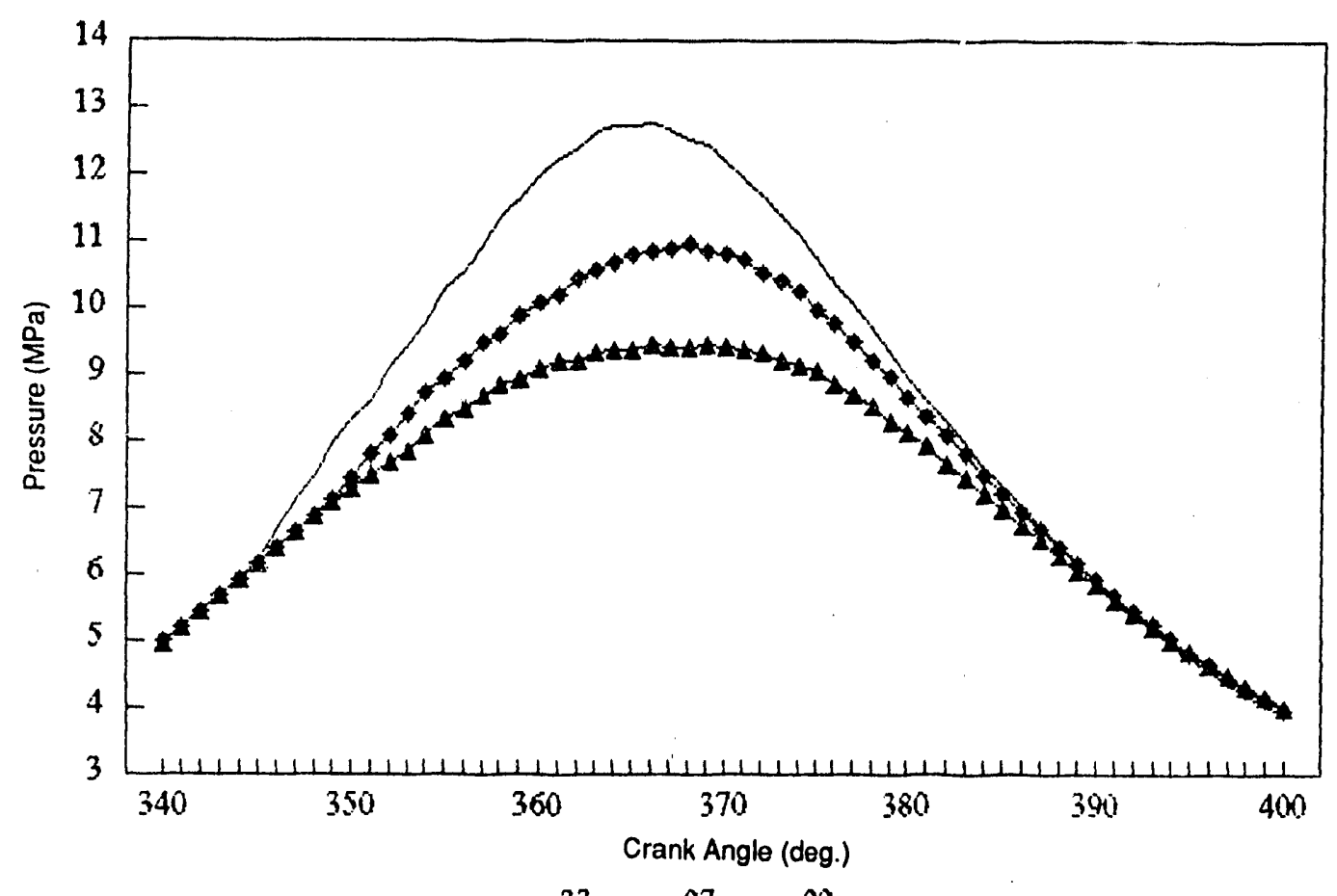

$-33 \multimap 27 \rightarrow 22$

FIGURE B.73 Effect of Fuel Injection Timing on Pressure, No. 2 Fuel, Full Load, $5 \%$ Water, $25 \%$ Oxygen

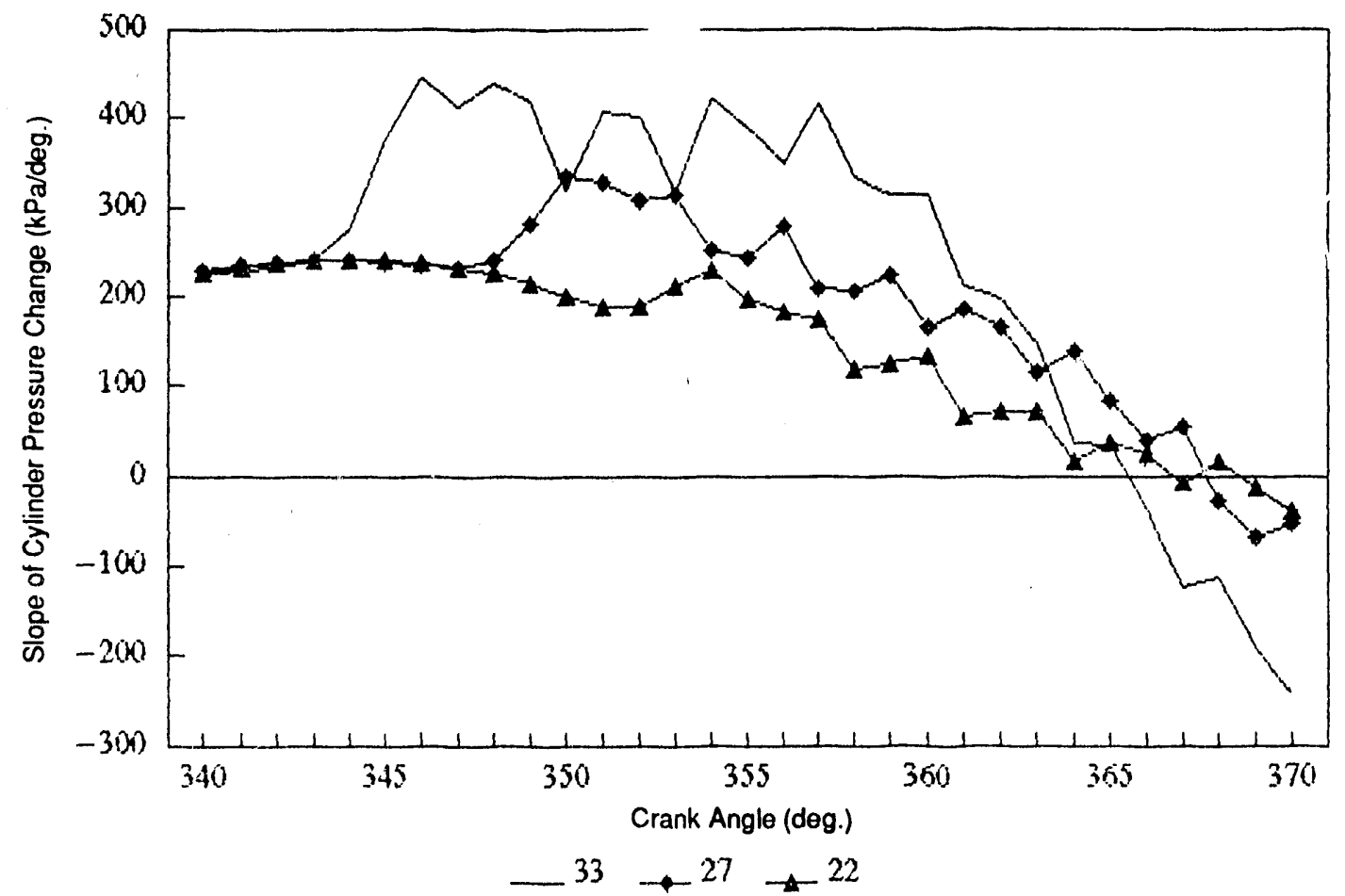

FIGURE B.74 Effect of Fuel Injection Timing on Slope of Cylinder Pressure Change, No. 2 Fuel, Full Load, 5\% Water, 25\% Oxygen 


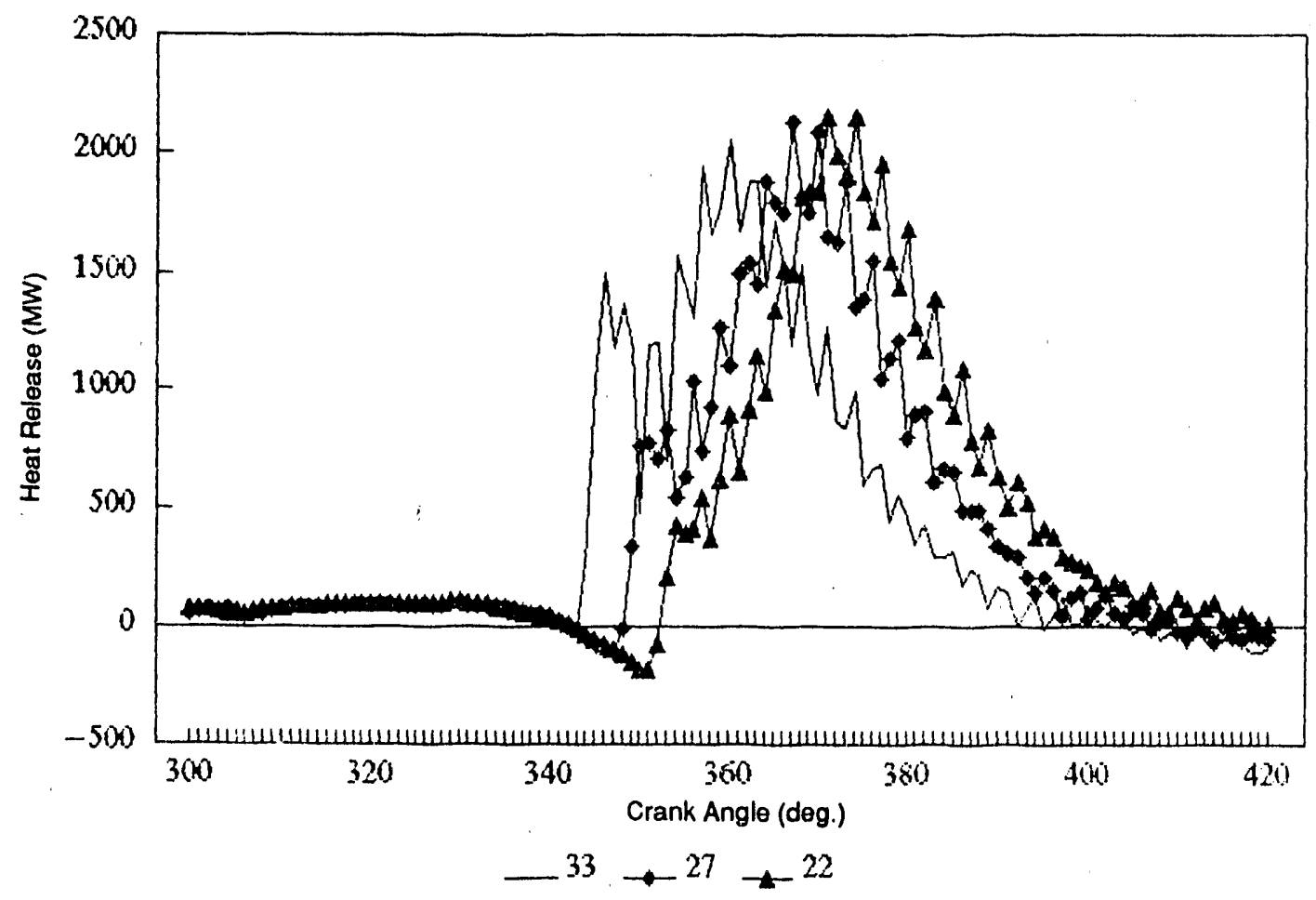

FIGURE B.75 Effect of Fuel Injection Timing on Heat Release, No. 2 Fuel, Full Load, $5 \%$ Water, $25 \%$ Oxygen

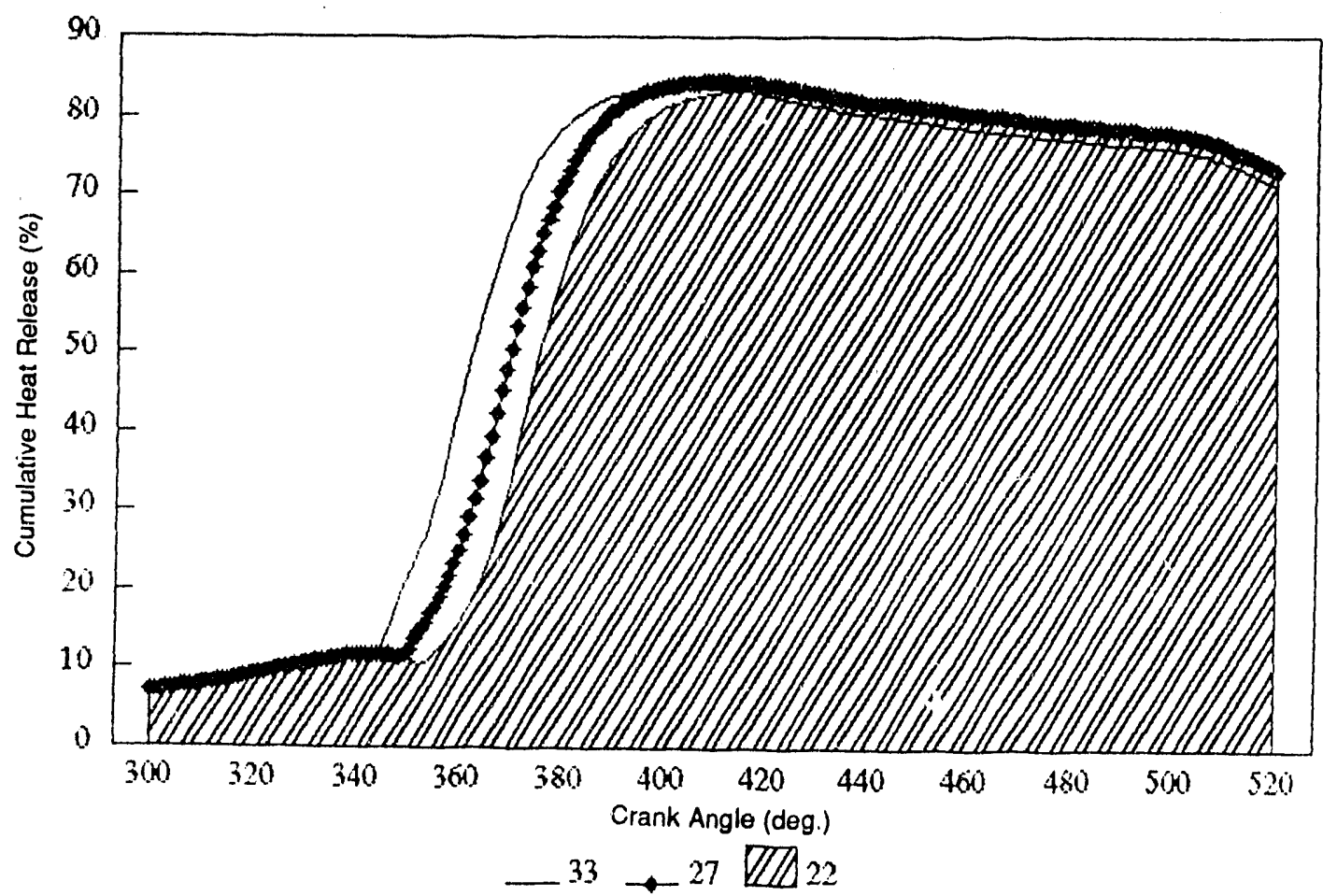

FIGURE B.76 Effect of Fuel Injection Timing on Cumulative Heat Release, No. 2 Fuel, Full Load, $5 \%$ Water, $25 \%$ Oxygen 


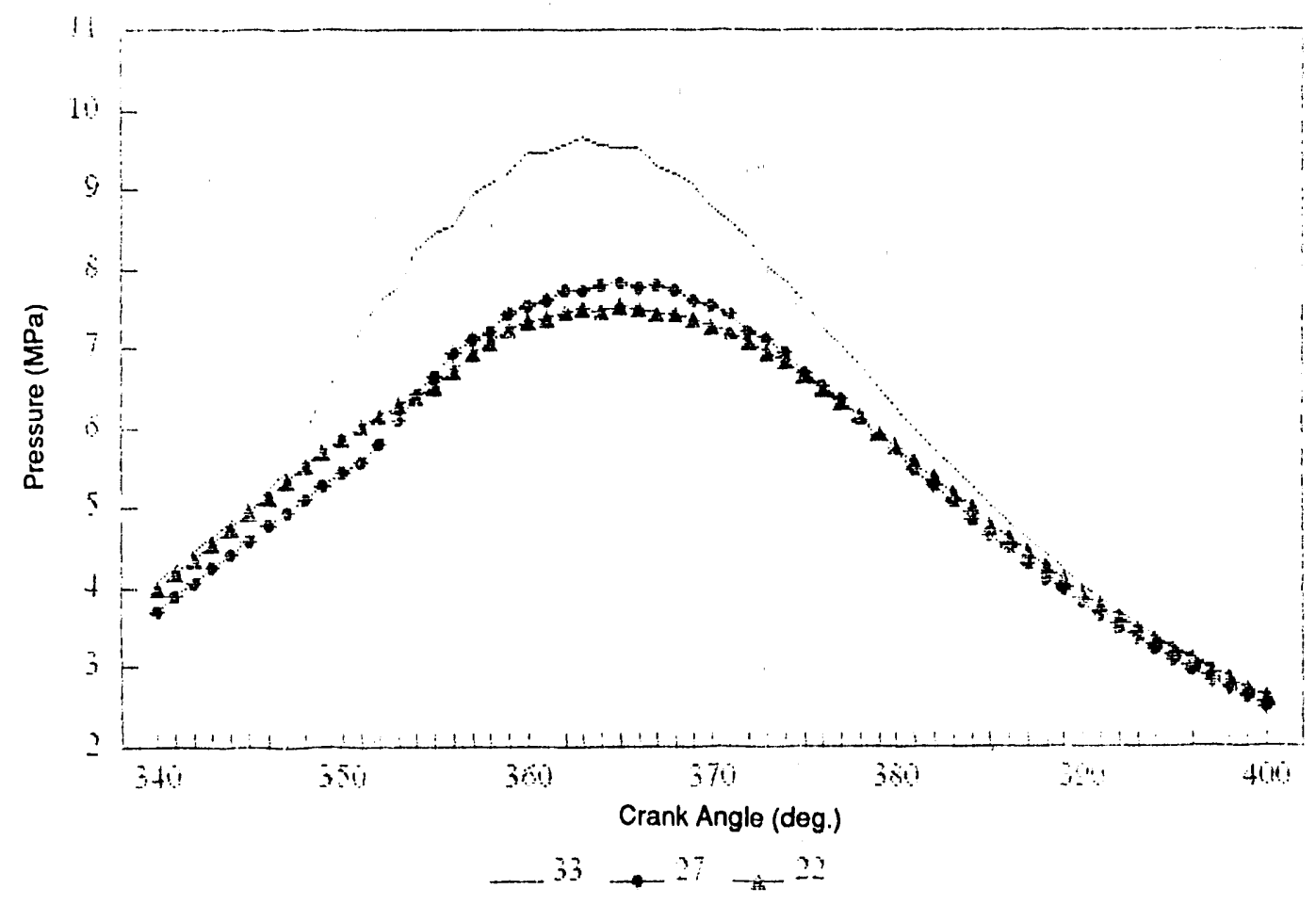

FIGURE B.77 Effect of Fuel Injection Timing on Pressure, No. 2 Fuel, Haff Load, $5 \%$ Water, $21 \%$ Oxygen

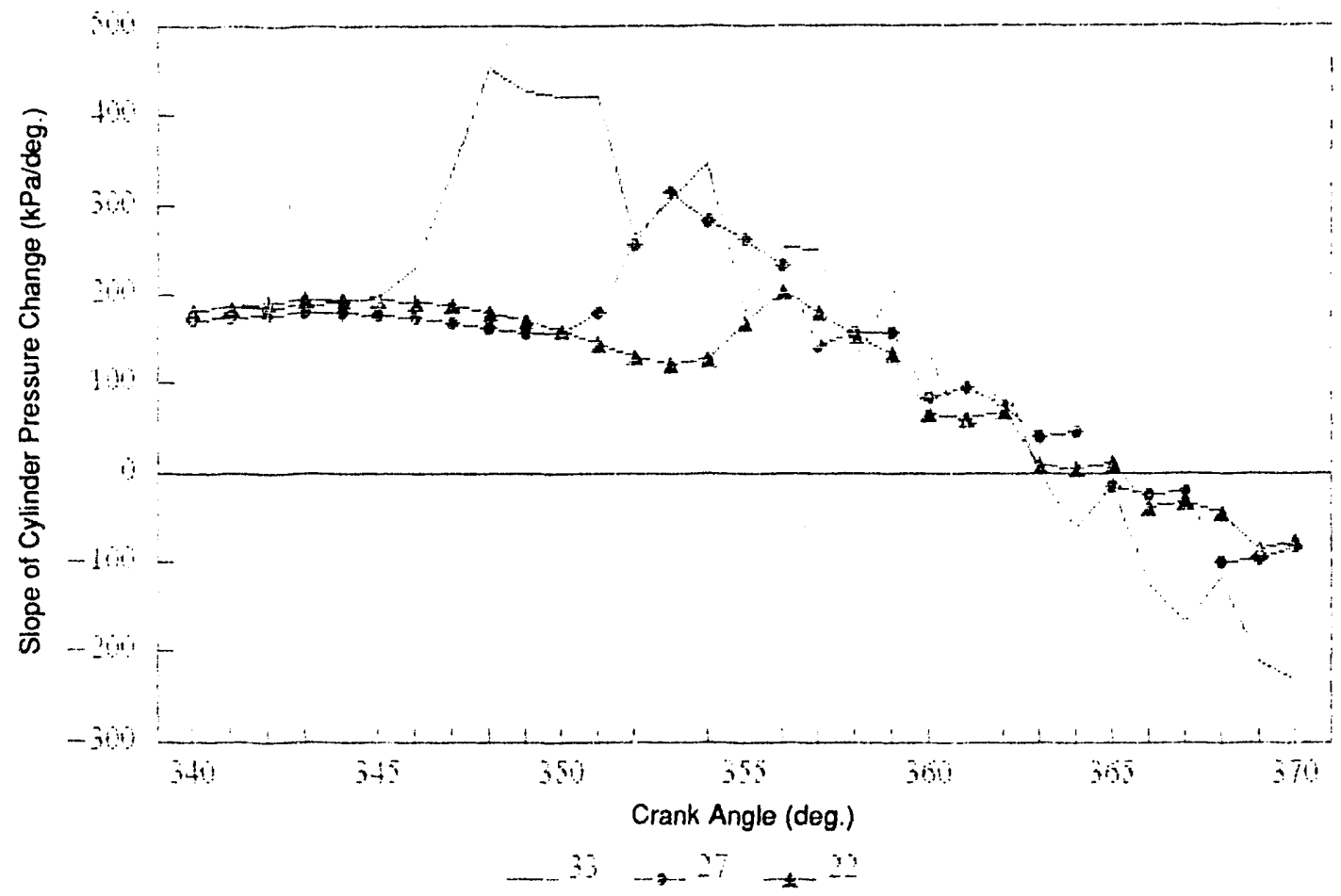

FIGURE B.78 Effect of Fuel Injection Timing on Slope of Cylinder Pressure Change, No. 2 Fuel, Half Load, $5 \%$ Water, $21 \%$ Oxygen 


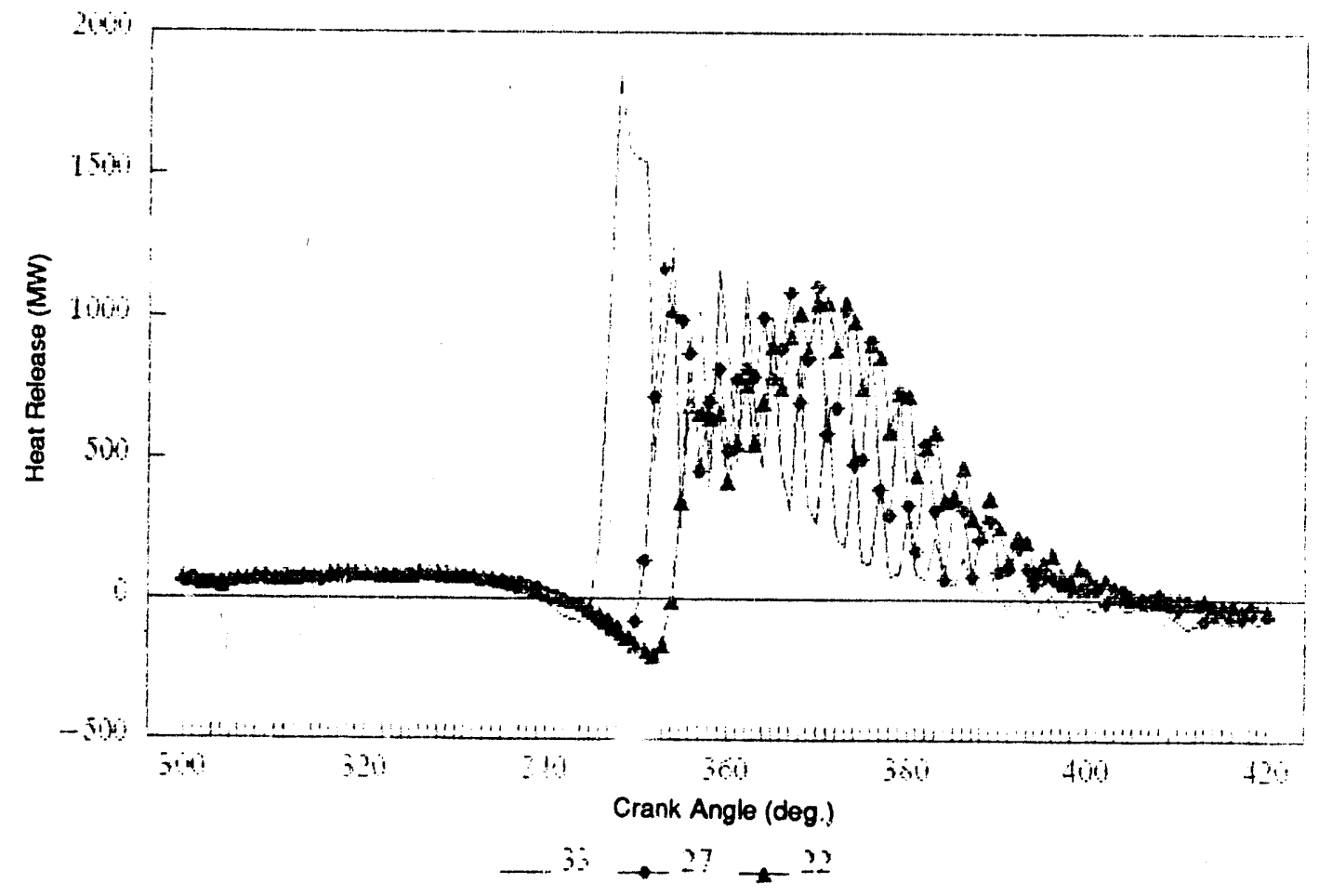

FIGURE B.79 Effect of Fuel Injection Timing on Heat Release, No. 22 Fuel, Half Load, $5 \%$ Water, $21 \%$ Oxygen

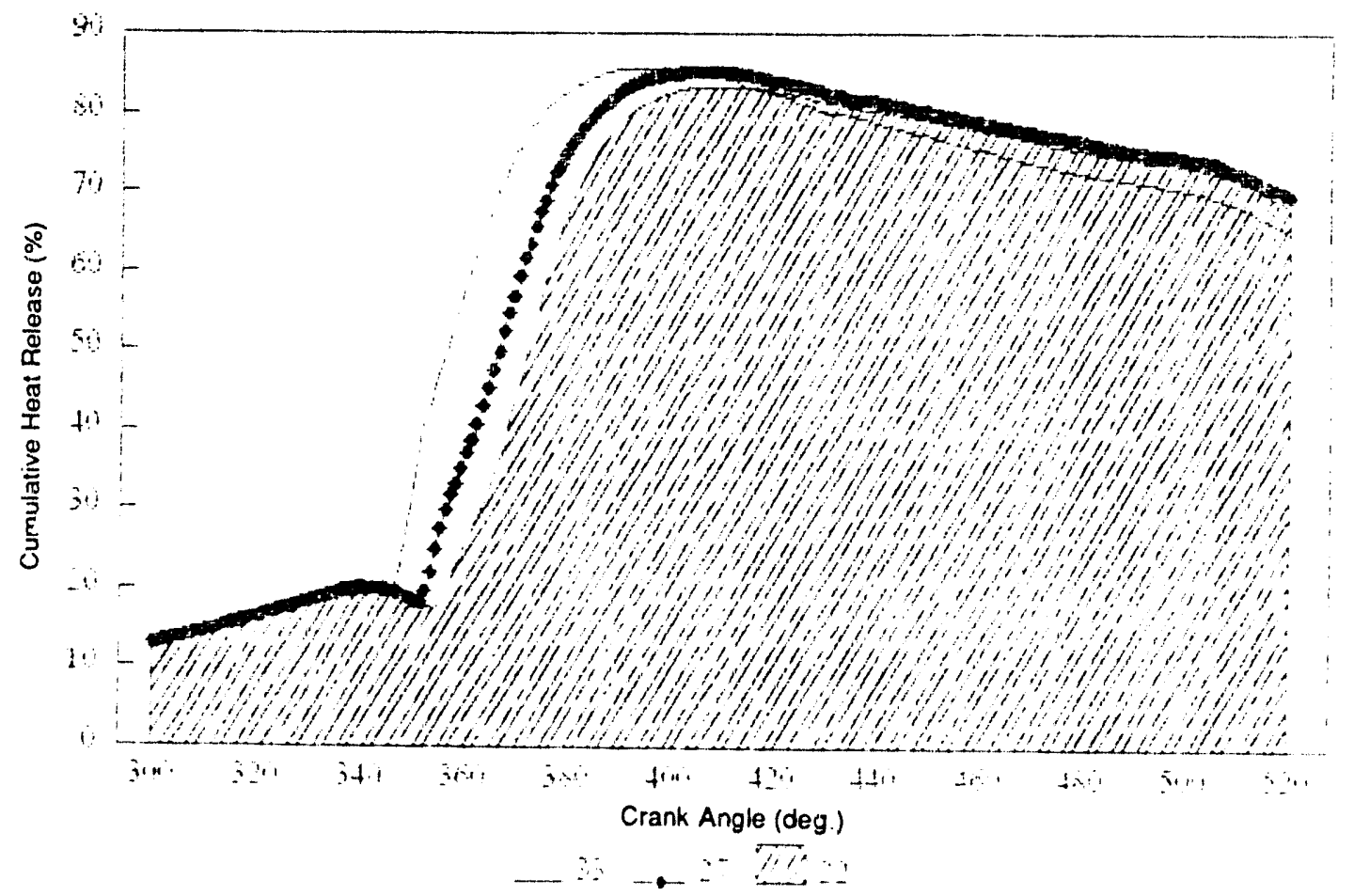

FIGURE B.80 Effect of Fuel Injection Timing on Cumulative Heat Release, No. 2 Fuel, Half Load, $5 \%$ Water, $21 \%$ Oxygen 


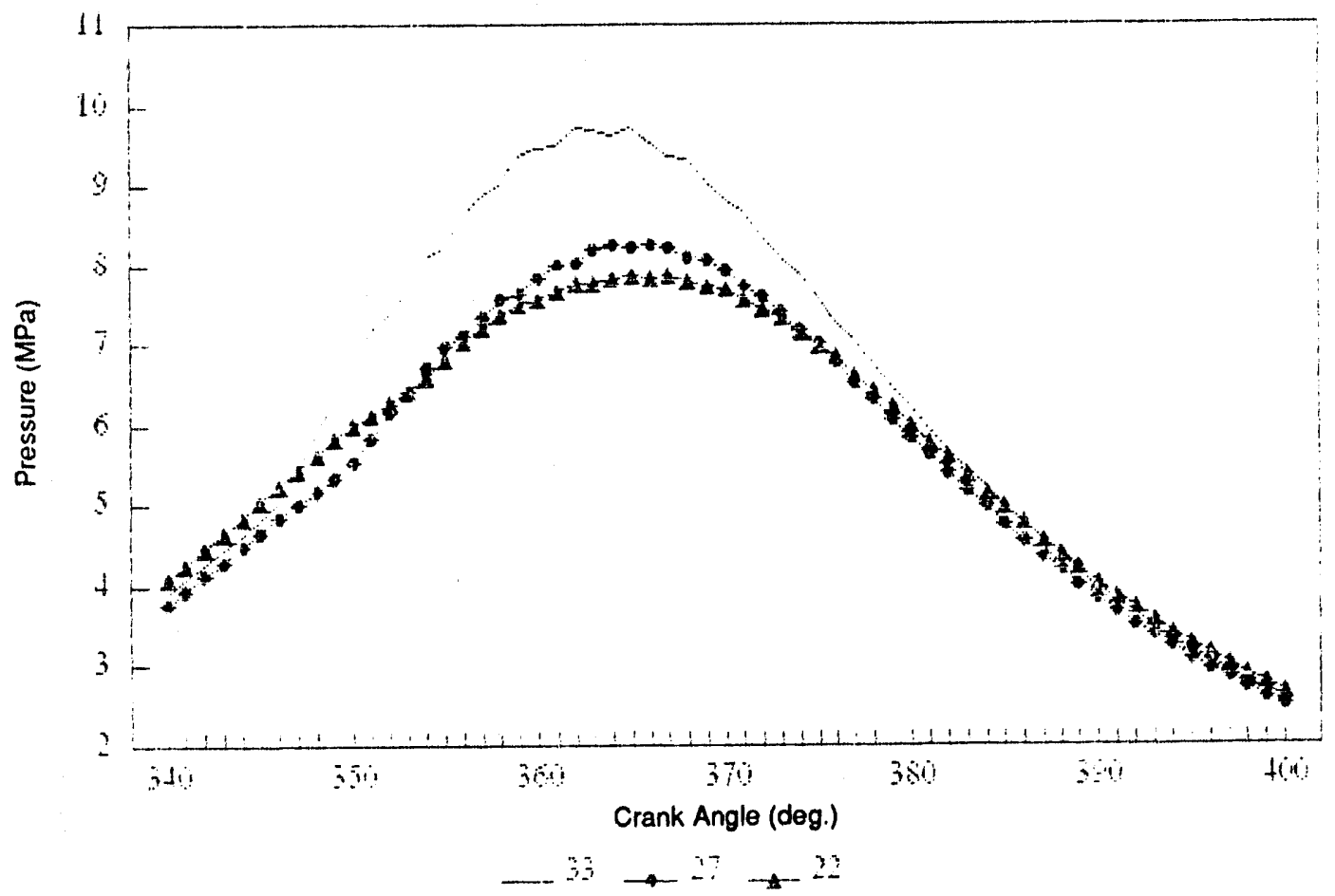

FIGURE B.81 Effect of Fuel Injection Timing on Press' Ire, No. 2 Fuel, Haff Load, $5 \%$ Water, $26 \%$ Oxygen

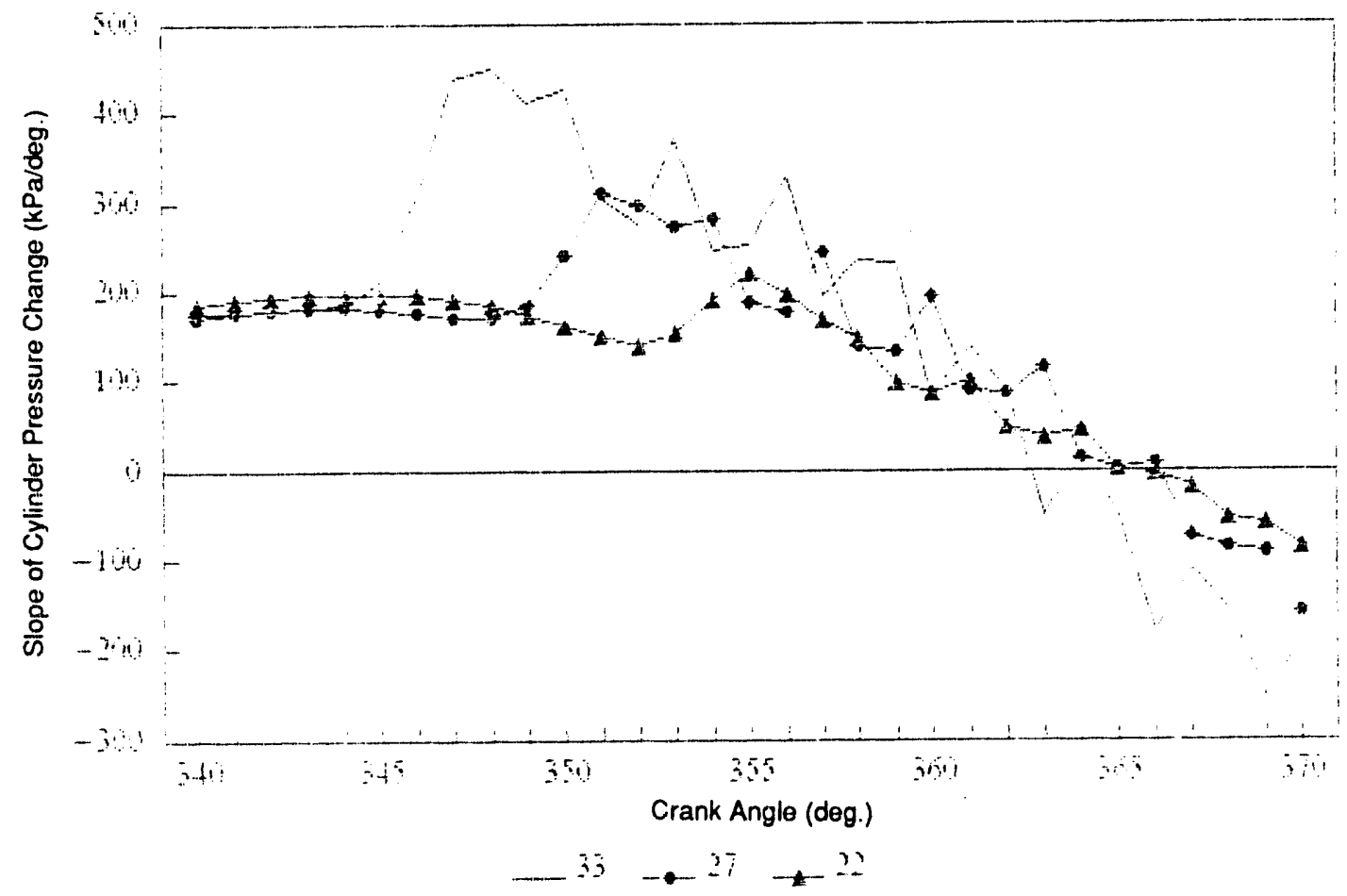

FIGURE B.82 Effect of Fuel Injection Timing on Slope of Cylinder Pressure Change, No. 2 Fuel, Half Load, $5 \%$ Water, 26\% Oxygen 


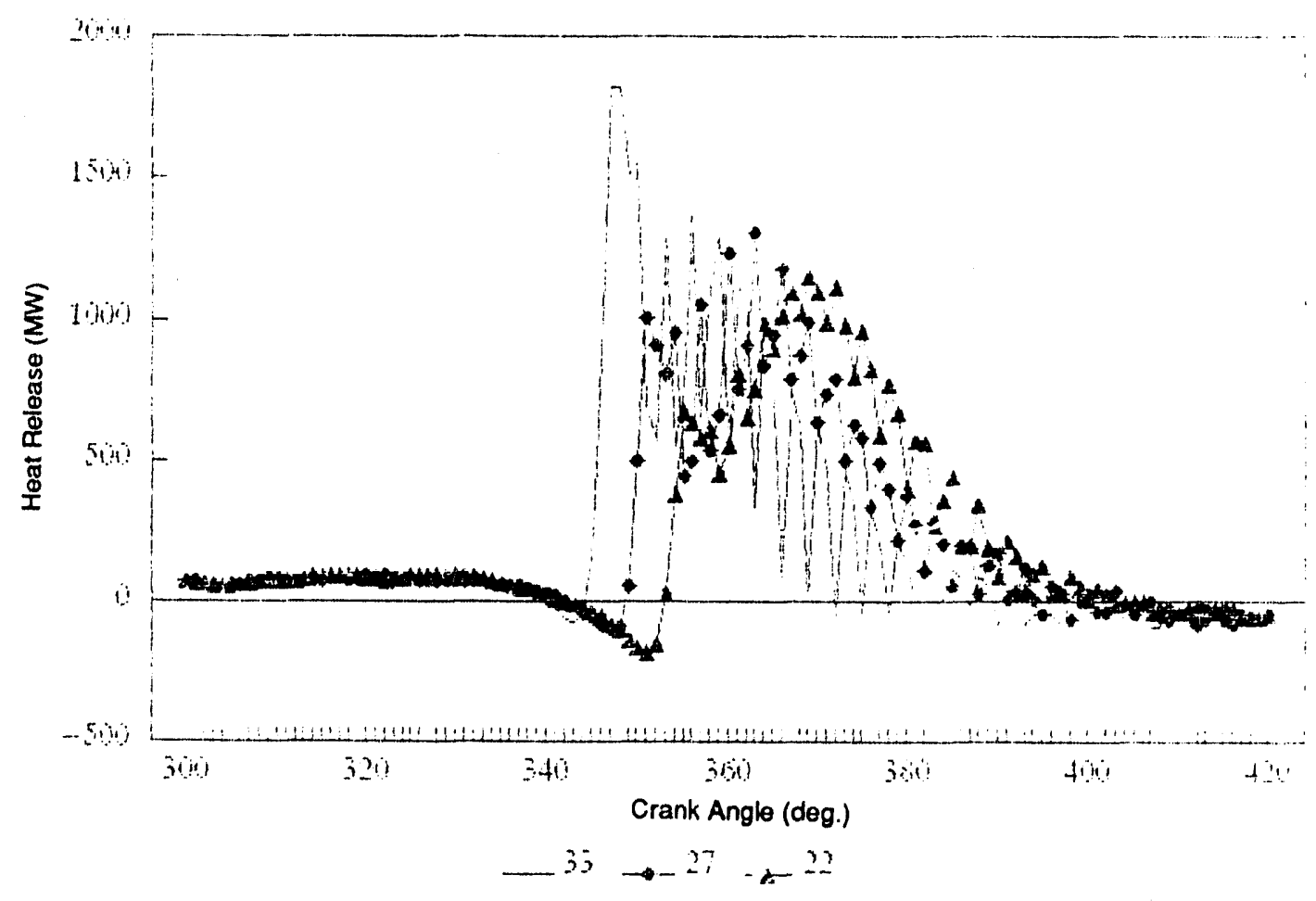

FIGURE B.83 Effect of Fuel Injection Timing on Heat Release, No. 2 Fuel, Half Load, $5 \%$ Water, $26 \%$ Oxygen

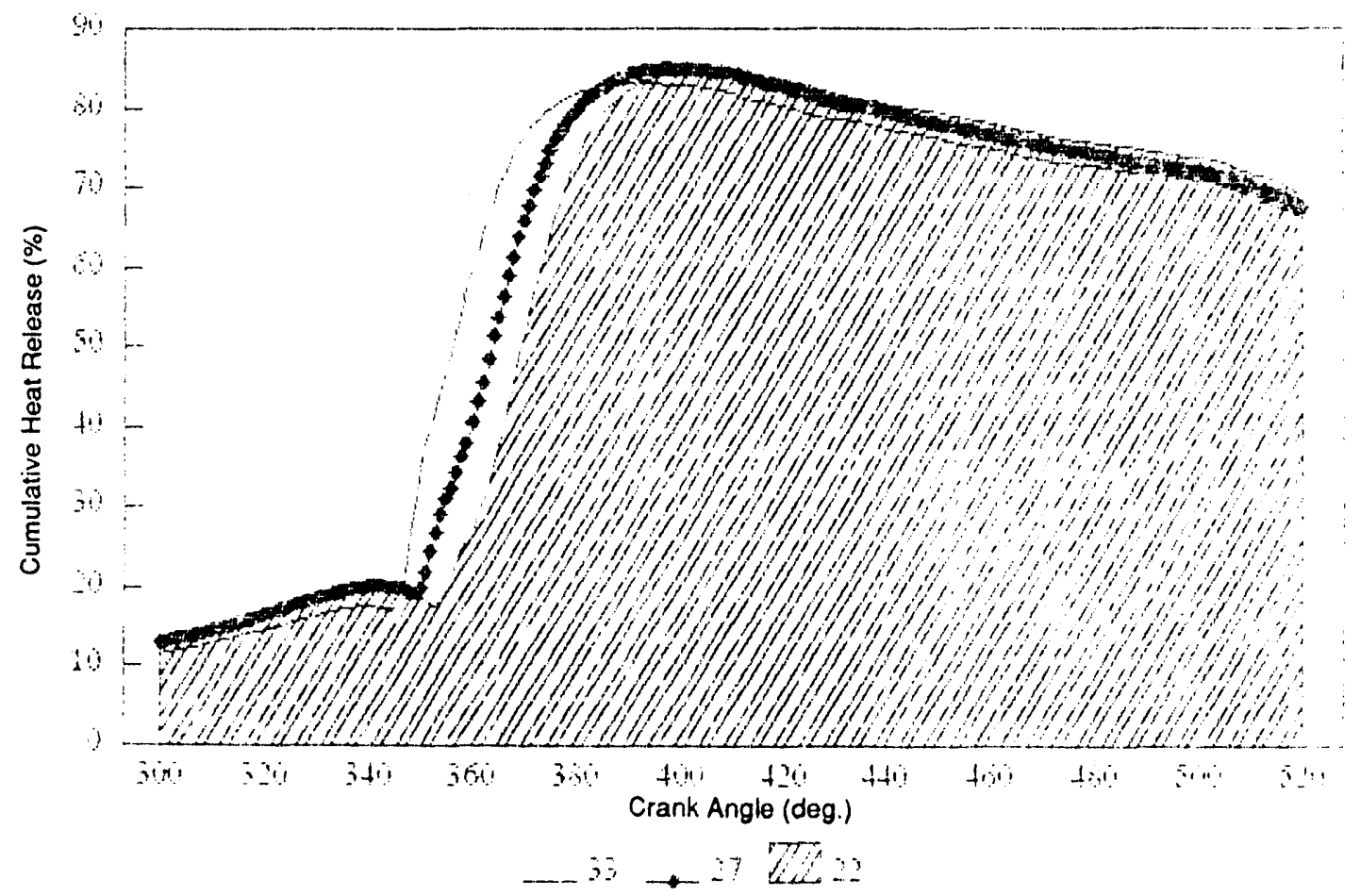

FIGURE B.84 Effect of Fuel Injection Timing on Cumulative Heat Release, No. 2 Fuel, Half Load, 5\% Water, $26 \%$ Oxygen 


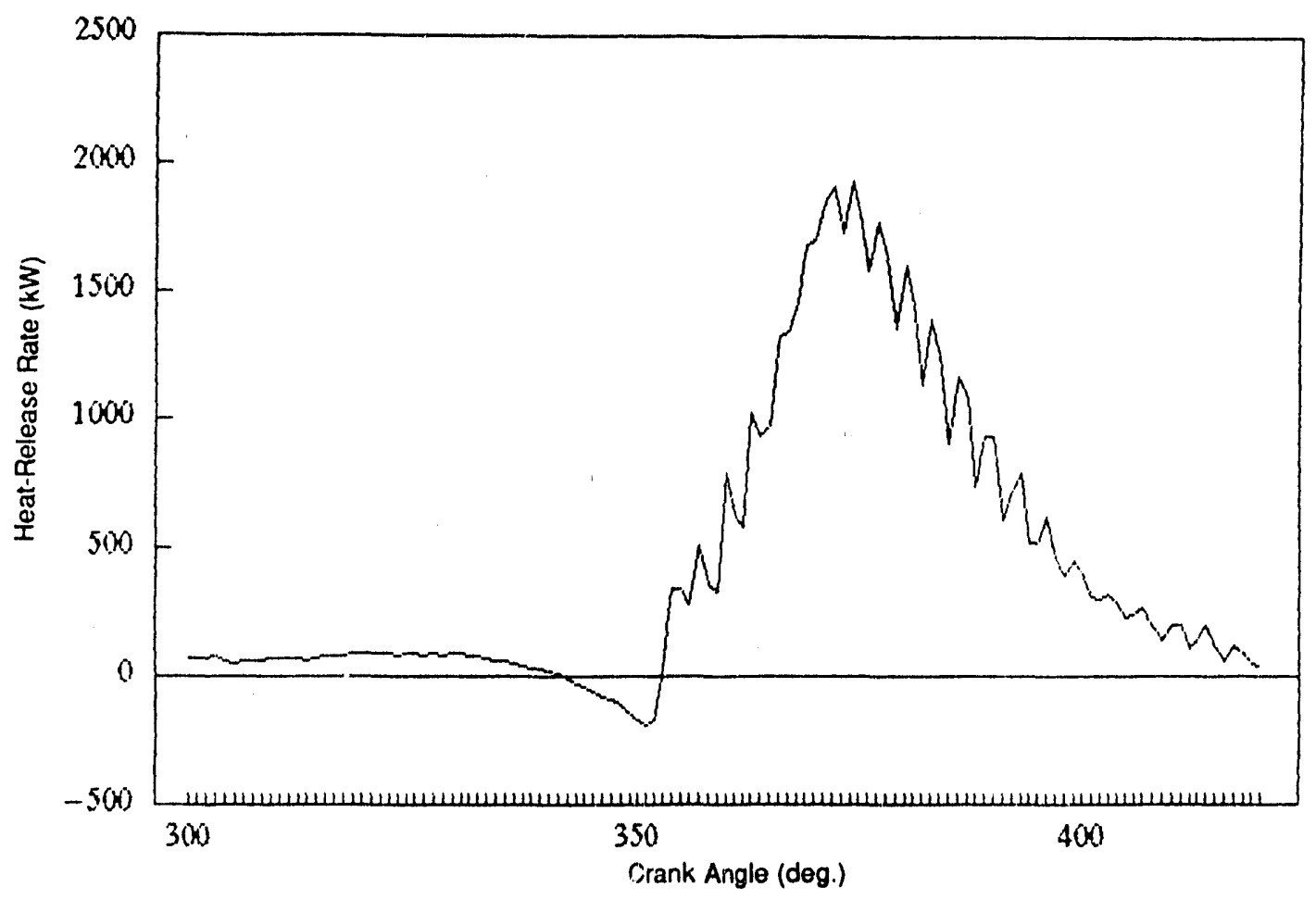

FIGURE B.85 Heat-Release Rate, Run 213

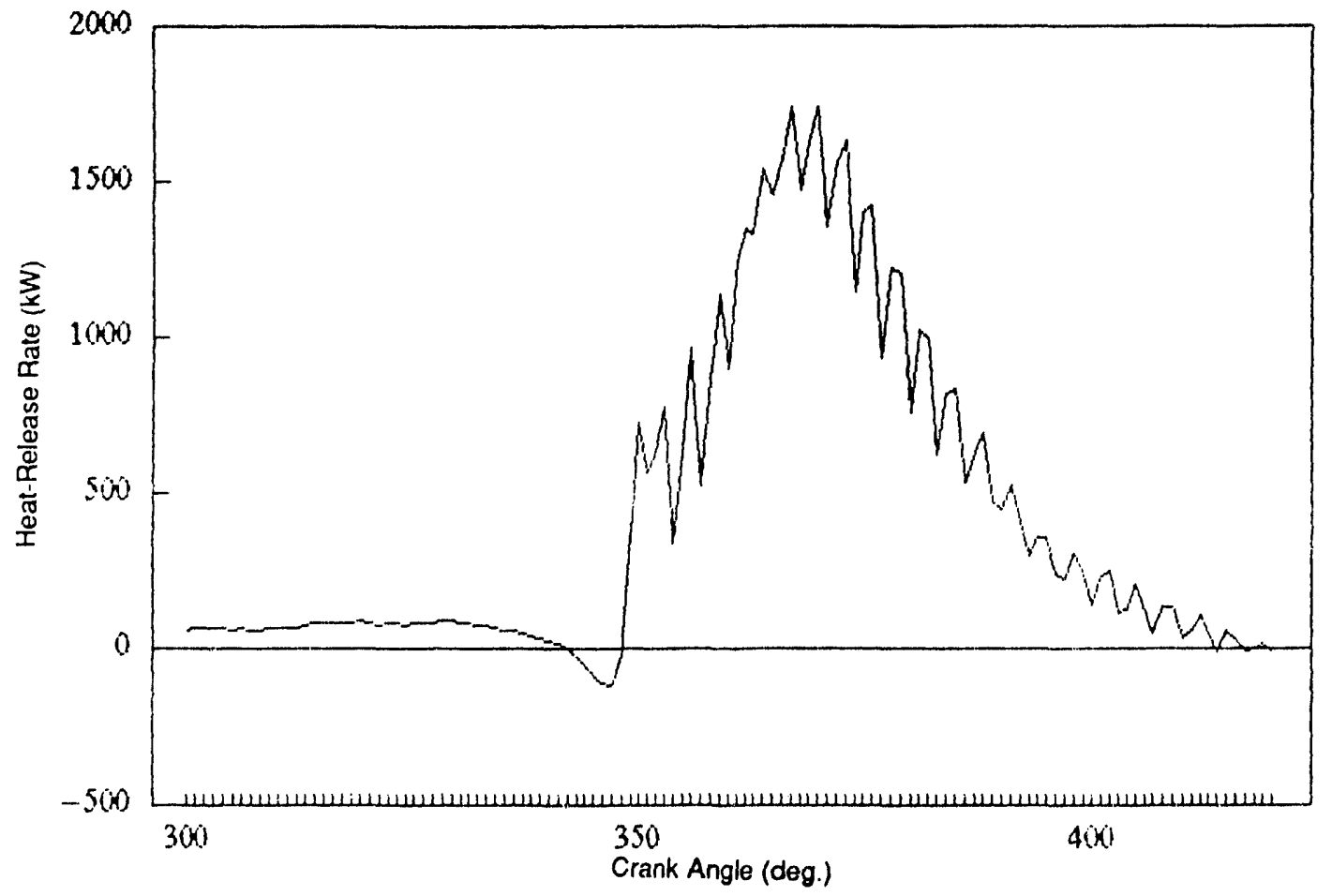

FIGURE B.86 Heat-Release Rate, Run 209 


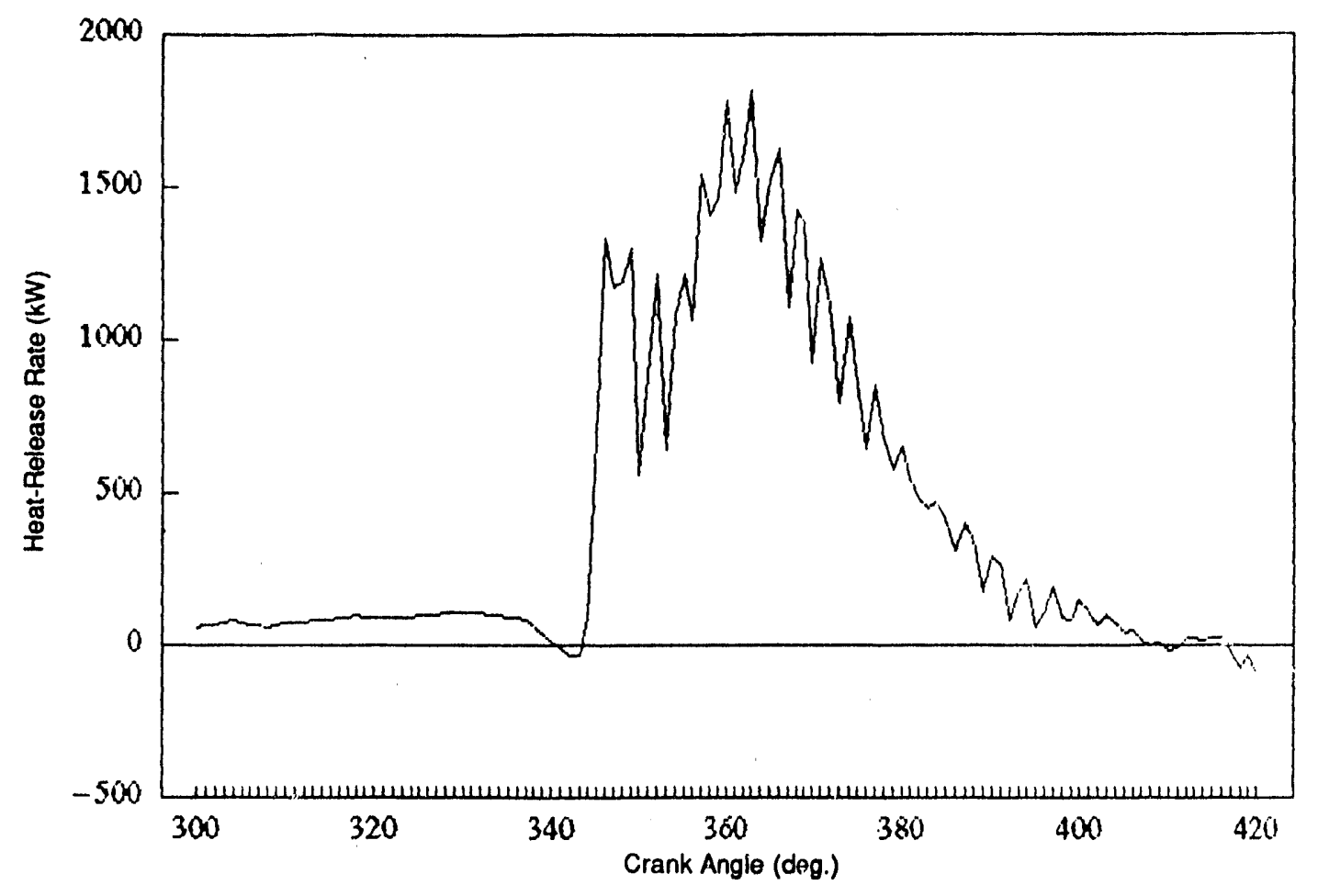

FIGURE B.87 Heat-Release RatA, Run 217

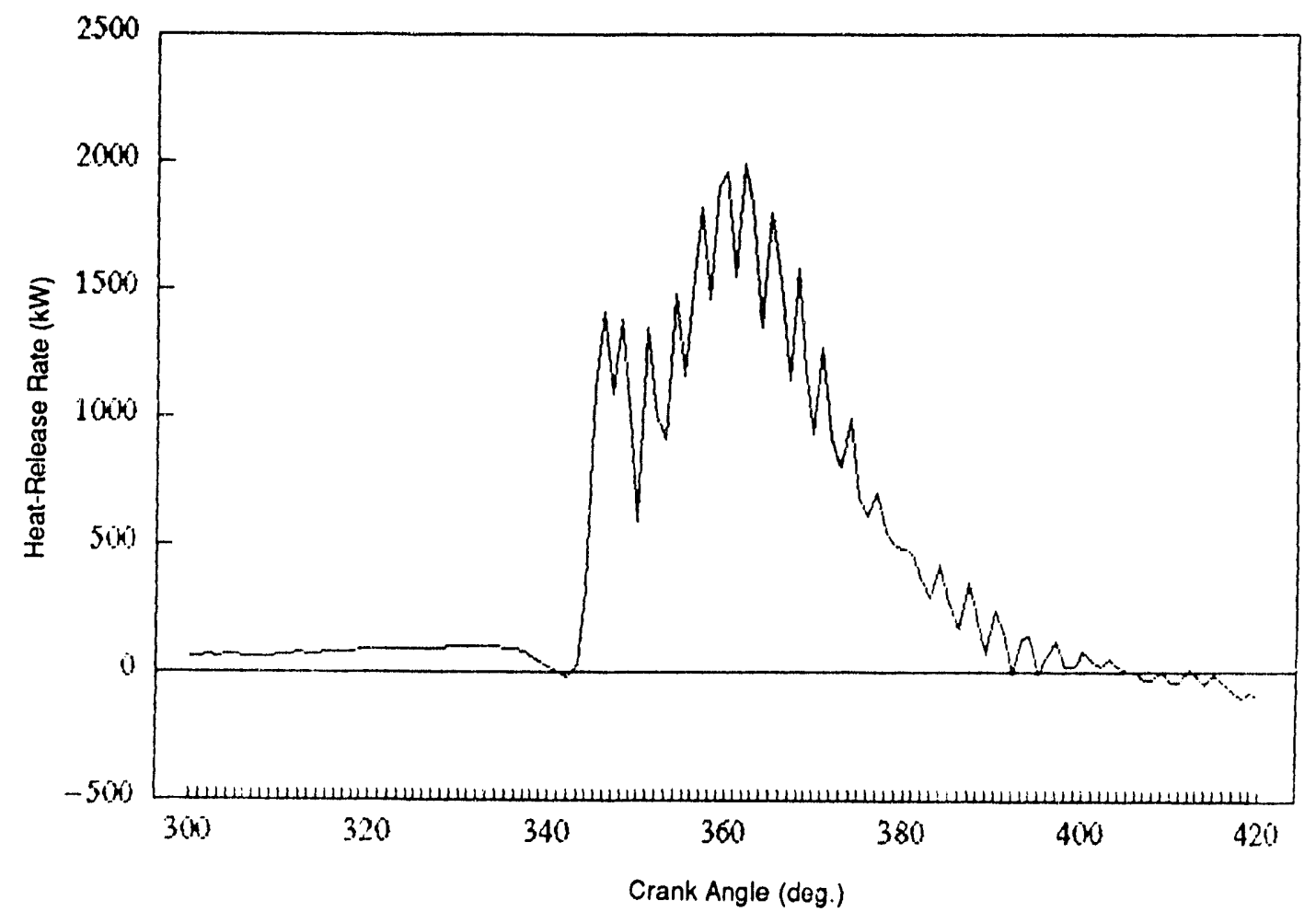

FIGURE B.88 Heat-Release Rate, Run 218 


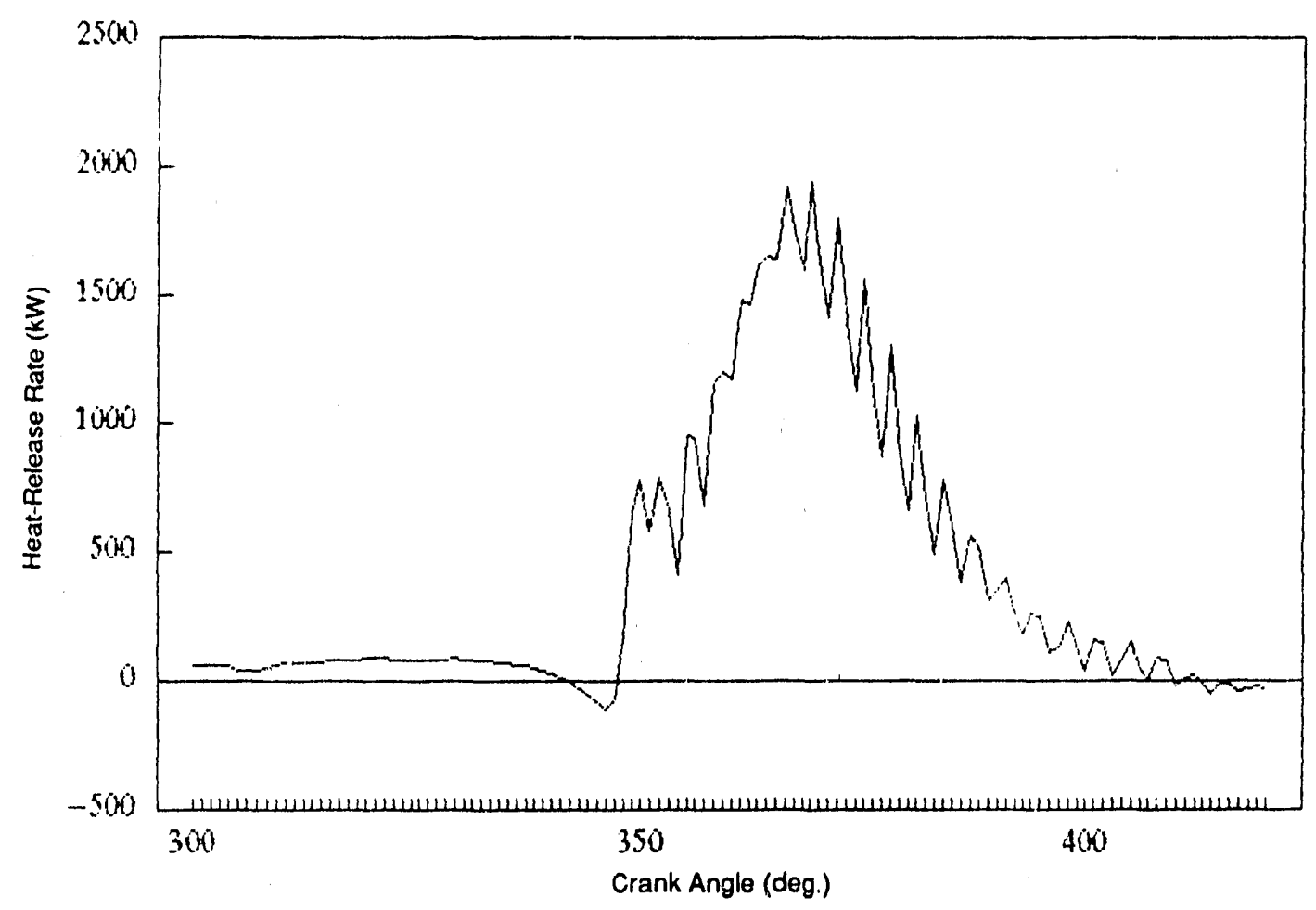

FIGURE B.89 Heat-Release Rate, Run 210

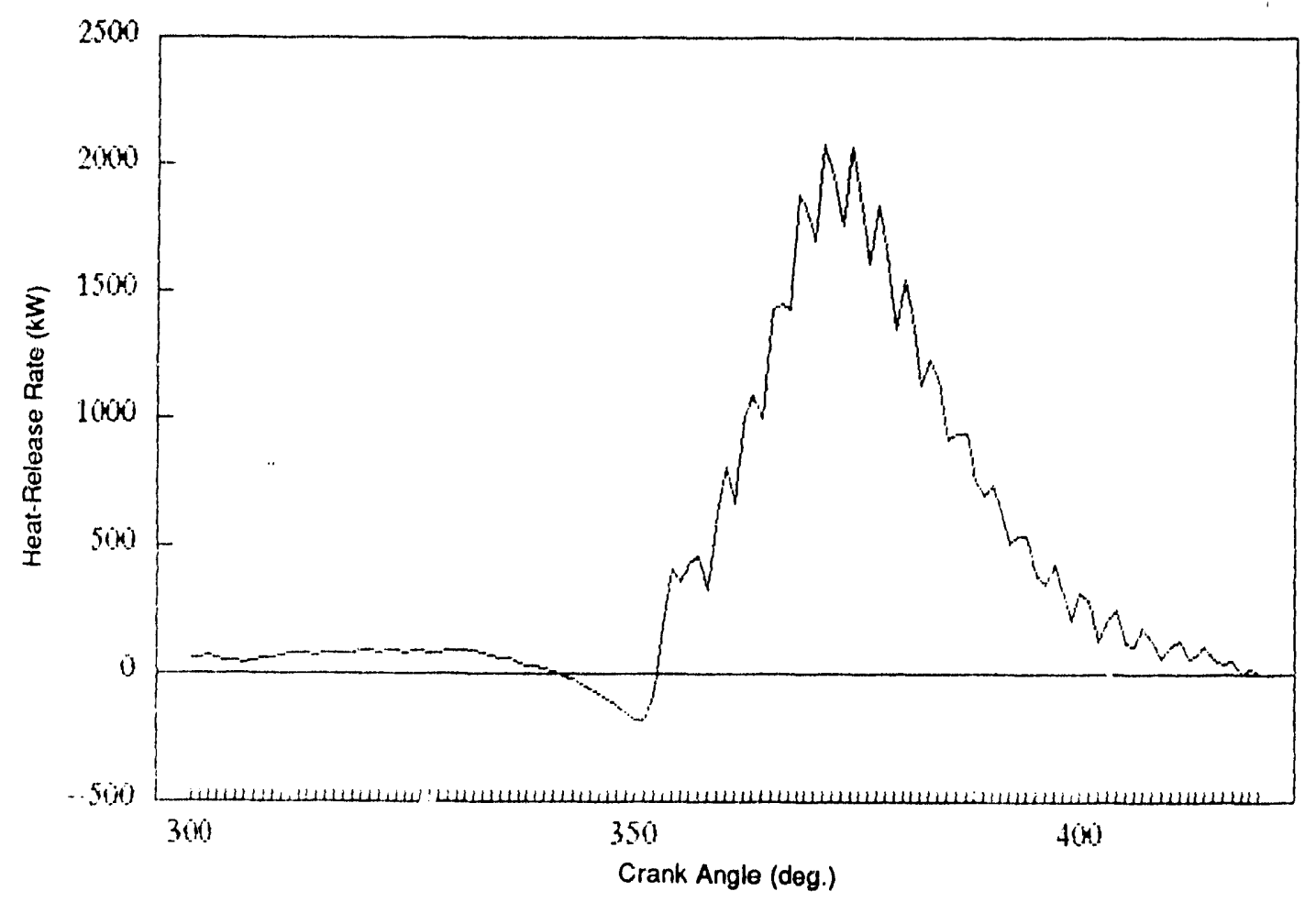

FIGURE B.90 Heat-Release Rate, Run 214 
B.78 
B.5 Graphic Display of Data from Emissions Repeatability Tests 
B-80 


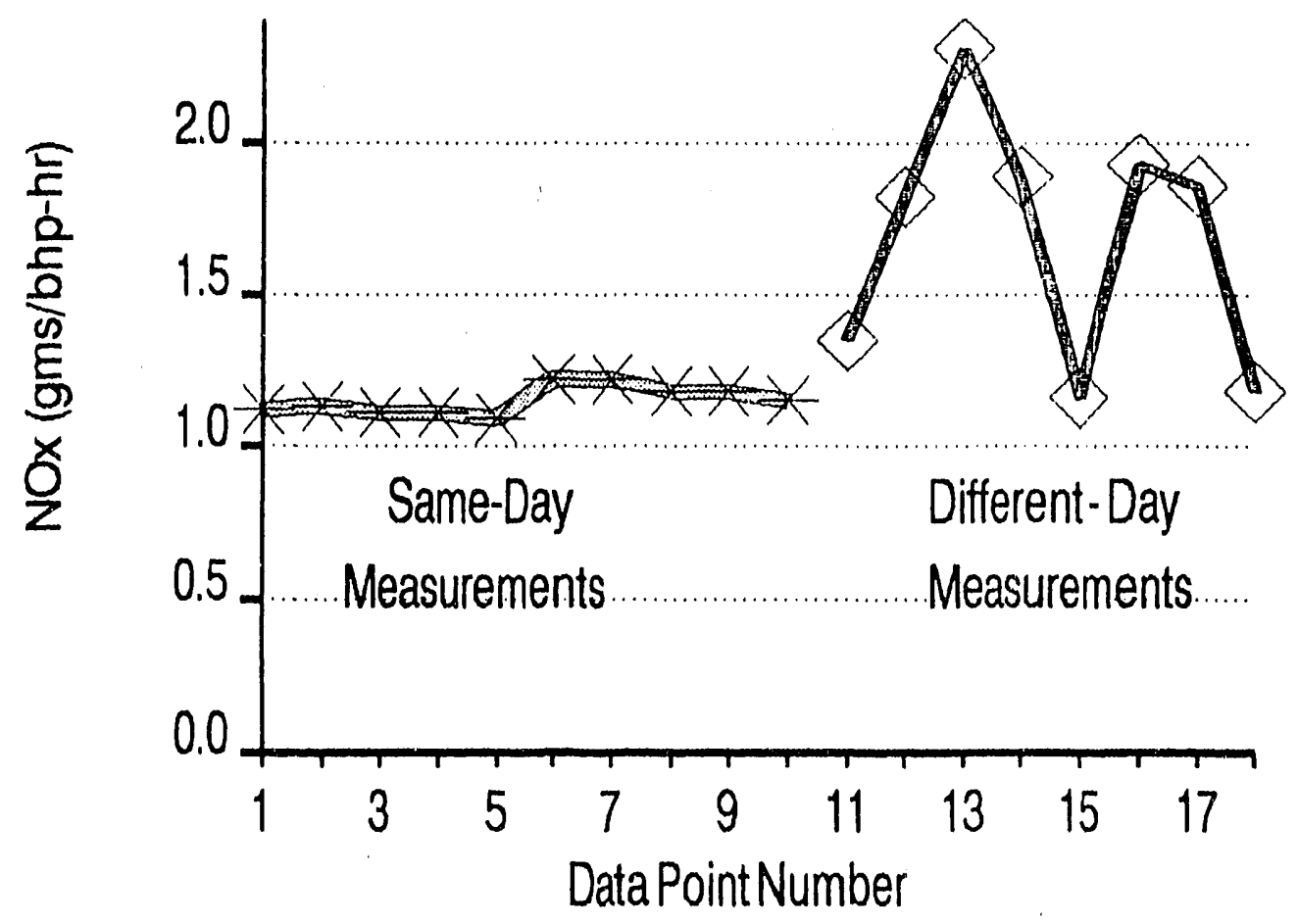

FIGURE B.91 Emission Repeatability Tests, 50 Brake Horsepower, No. 2 Fuel, No Water, $22 \mathrm{deg}$. btdc Injection Timing, NO ${ }_{x}$ Measurements

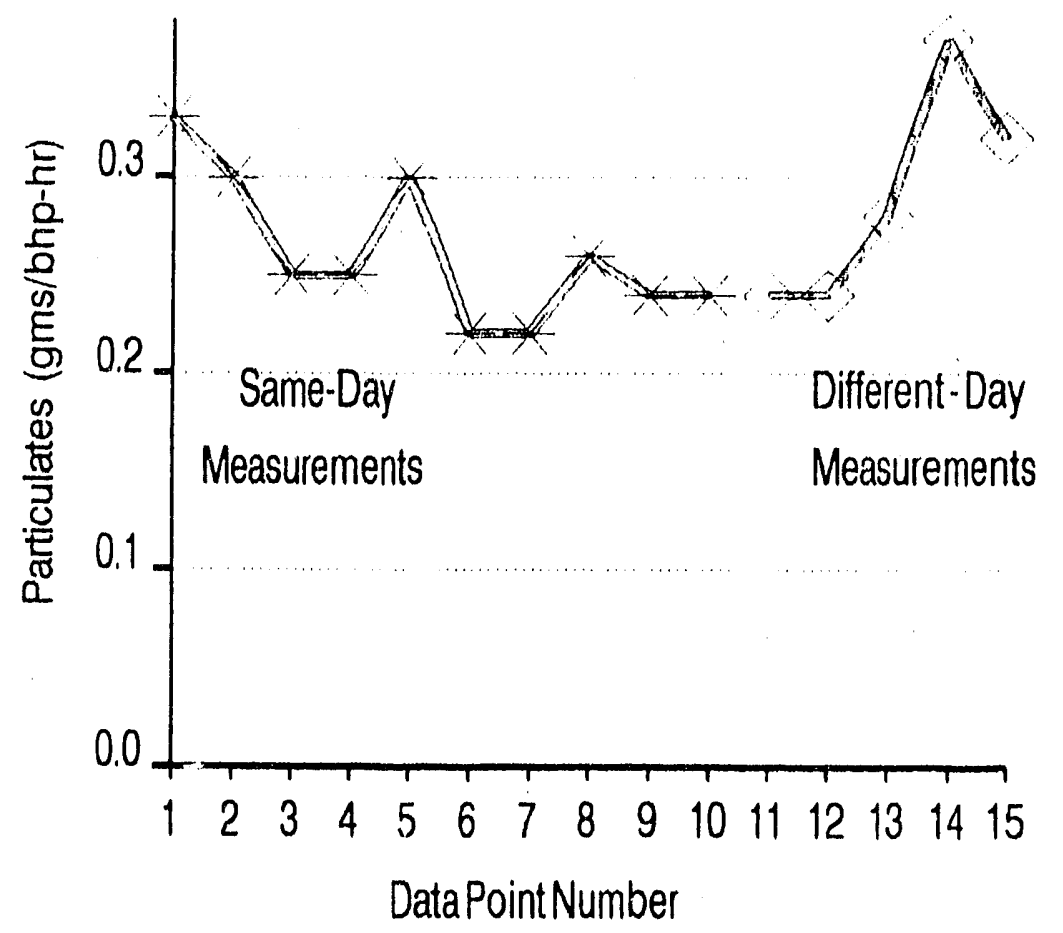

FIGURE B.92 Emission Repeatability Tests, 50 Brake Horsepower, No. 2 Fuel, No Water, 22 deg. btdc Injection Timing, Particulate-Matter Measurements 


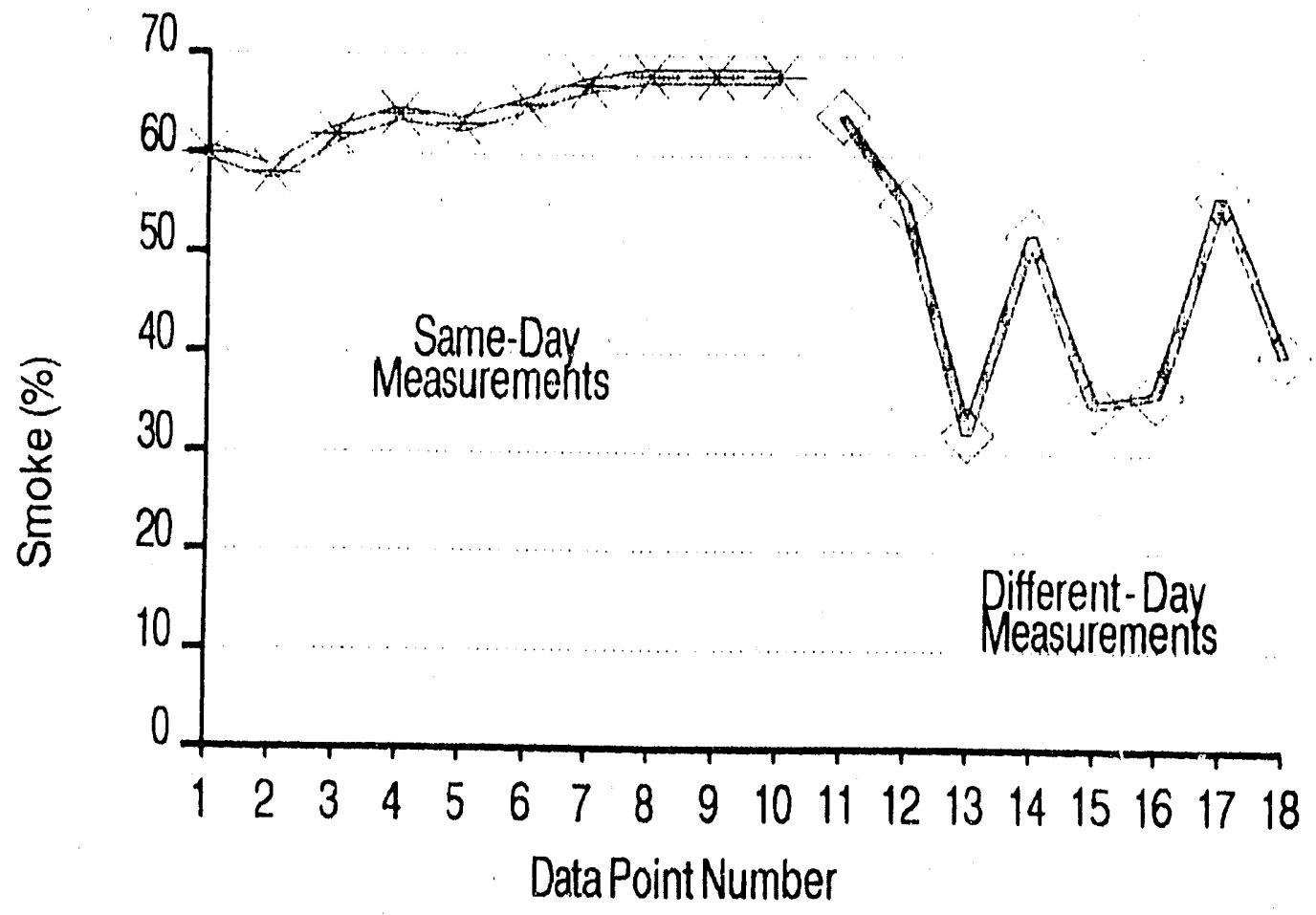

FIGURE B.93 Emission Repeatability Tests, 50 Brake Horsepower, No. 2 Fuel, No Water, 22 deg. btdc Injection Timing, Smoke Measurements

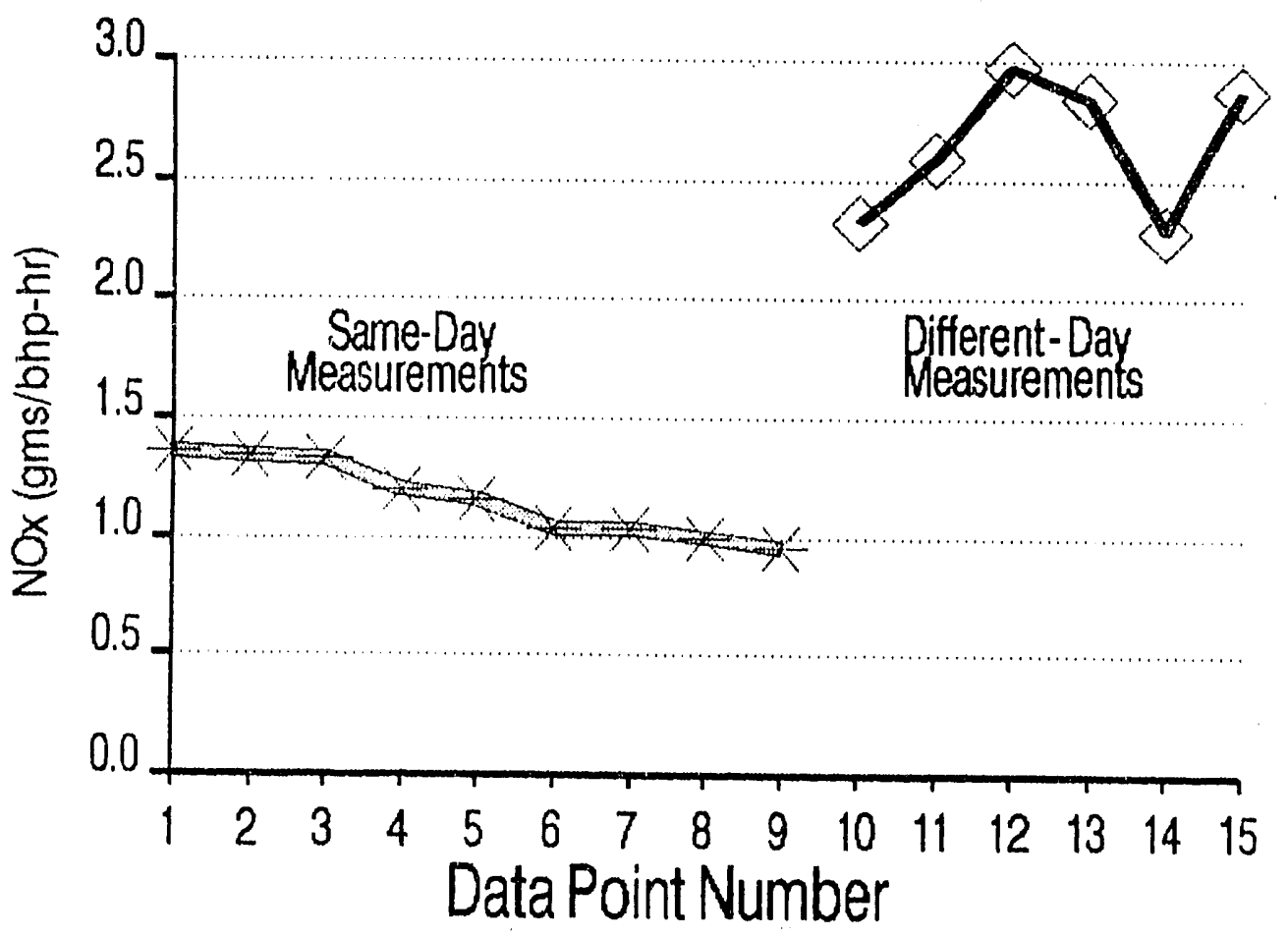

FIGURE B.94 Emission Repeatability Tests, 25 Brake Horsepower, No. 2 Fuel, No Water, 27 deg. btdc Injection Timing, $\mathrm{NO}_{\mathrm{x}}$ Measurements 


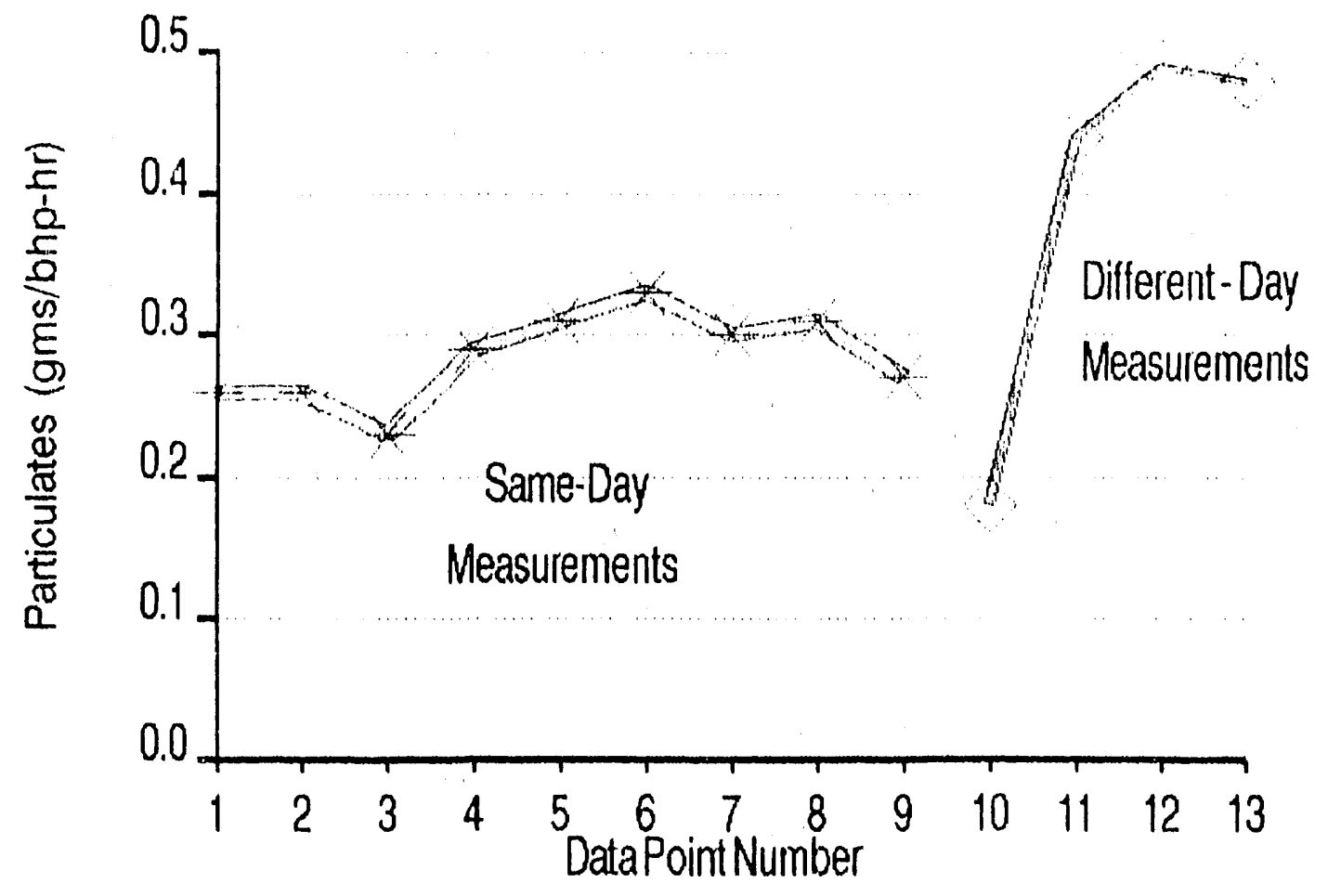

FIGURE B.95 Emission Repeatability Tests, 25 Brake Horsepower, No. 2 Fuel, No Water, $27 \mathrm{deg}$. btdc Injection Timing, Particulate-Matter Measurements

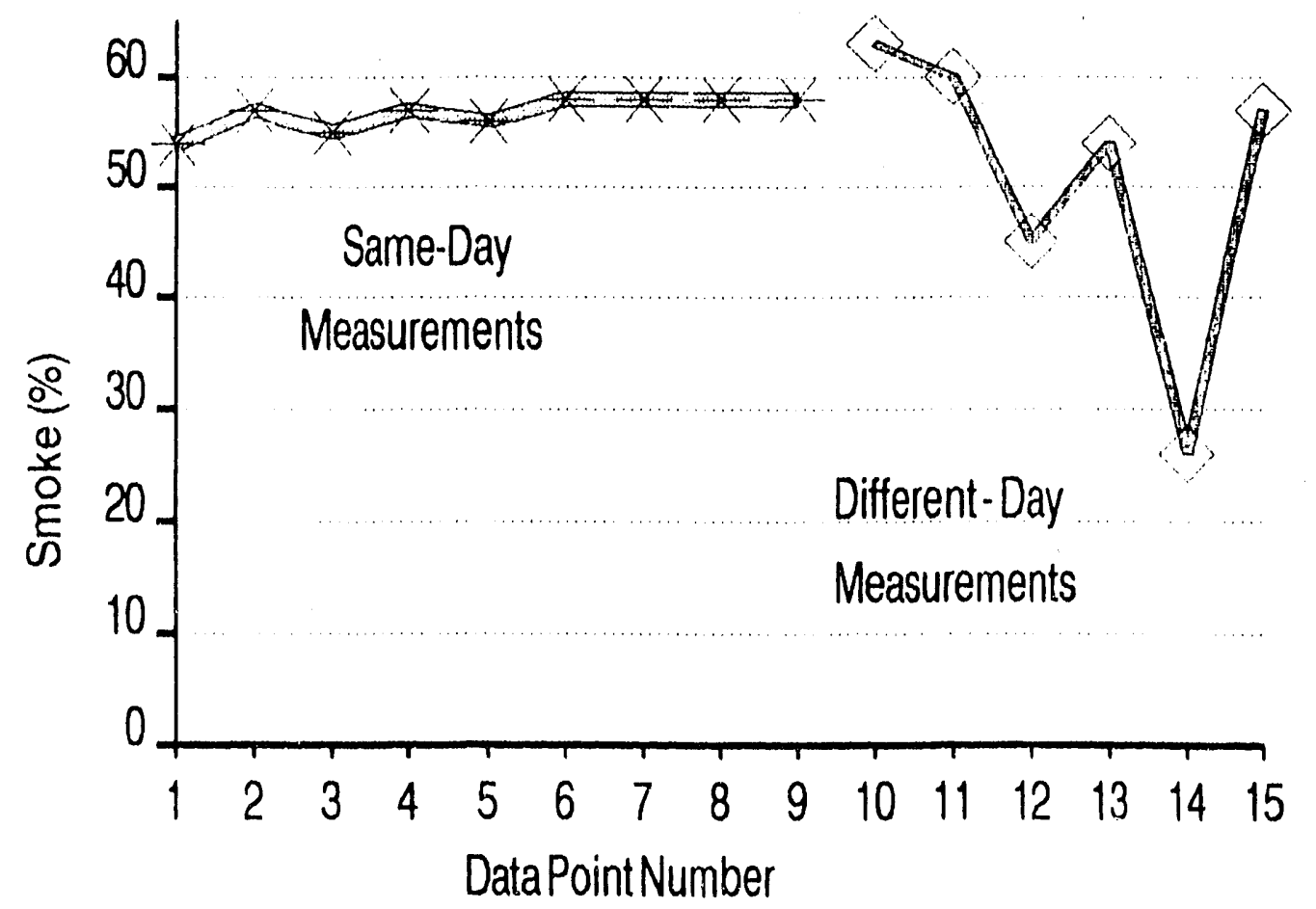

FIGURE B.96 Emission Repeatability Tests, 25 Brake Horsepower, No. 2 Fuel, No Water, $27 \mathrm{deg}$. btod Injection Timing, Smoke Measurements 
B-84 
$B-85$

B.6 Graphic Display of Test Data for No. 4 Fuel 


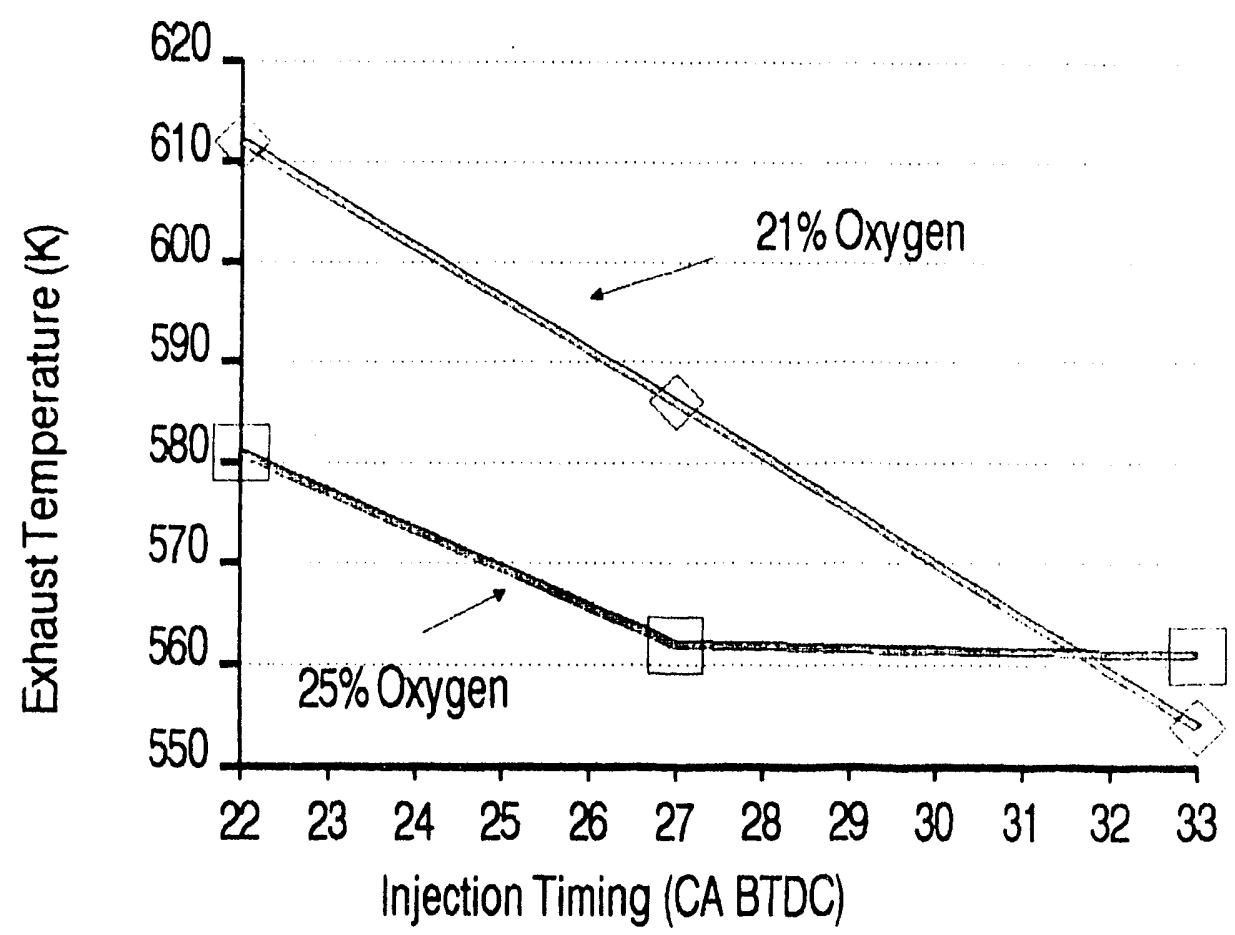

FIGURE B.97 Effect of Injection Timing on Exhaust Temperature, 25 Brake Horsepower, No. 4 Fuel, No Water

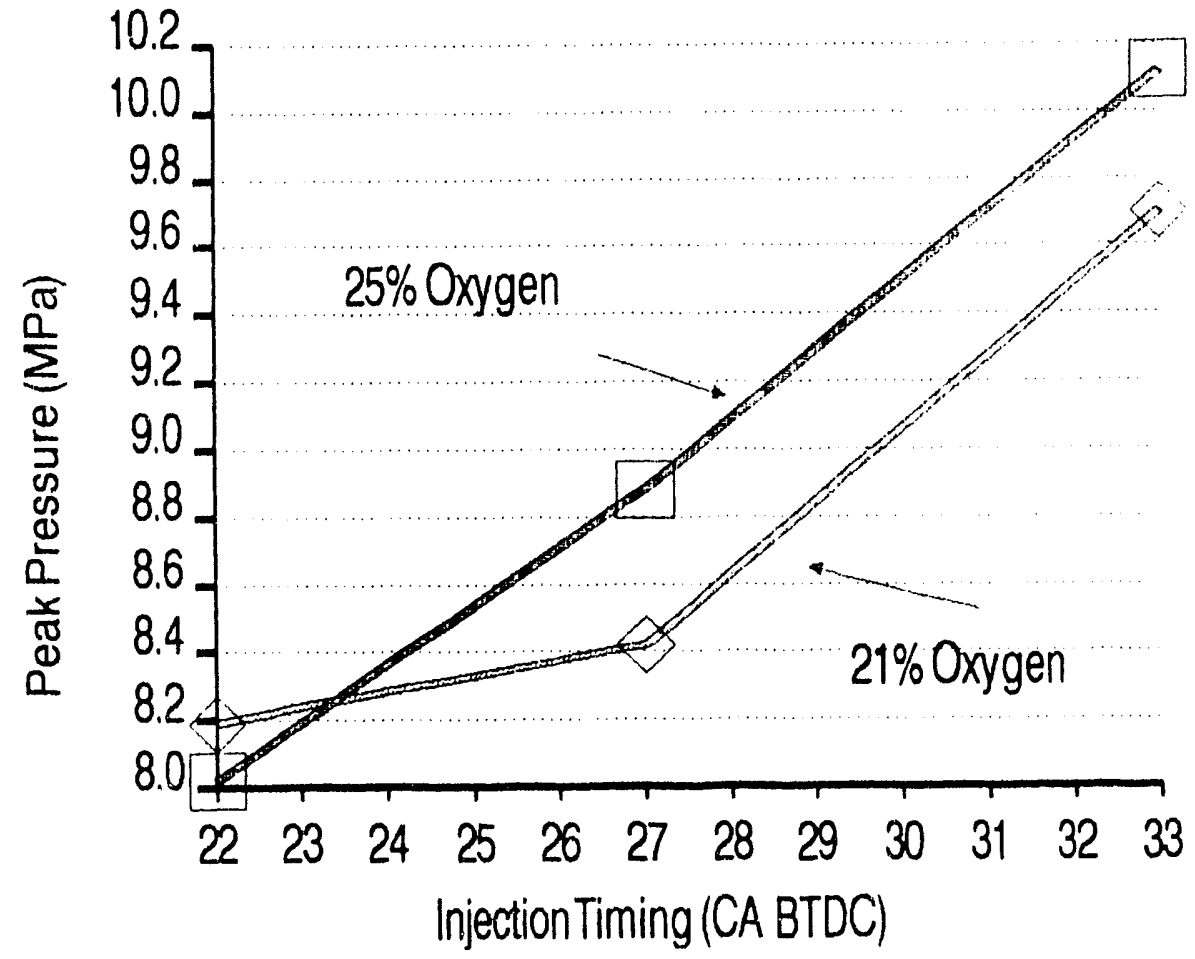

FIGURE B.98 Effect of Injection Timing on Peak Pressure, 25 Brake Horsepower, No. 4 Fuel, No Water 


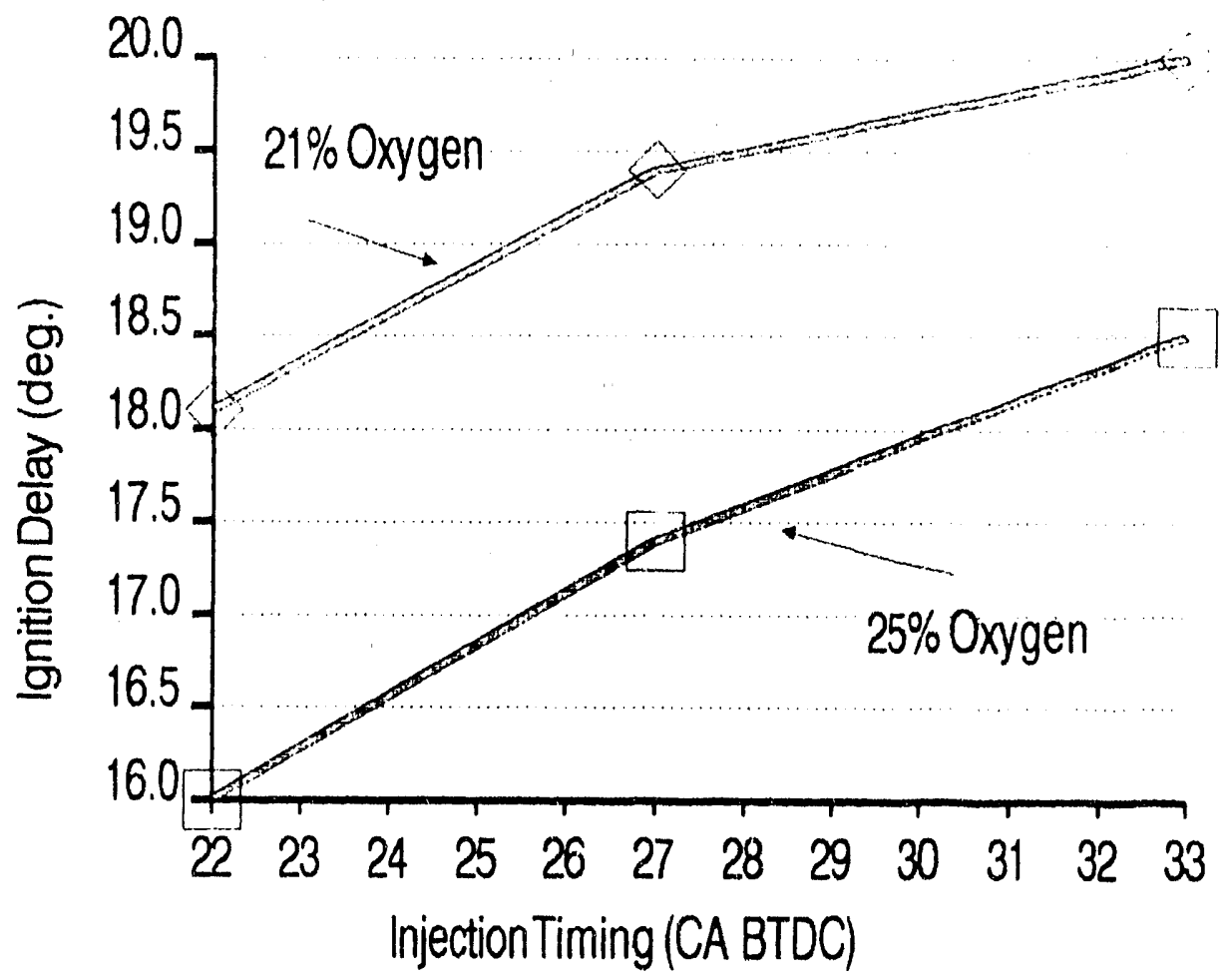

FIGURE B.99 Effect of Injection Timing on Ignition Delay, 25 Brake Horsepower, No. 4 Fuel, No Water

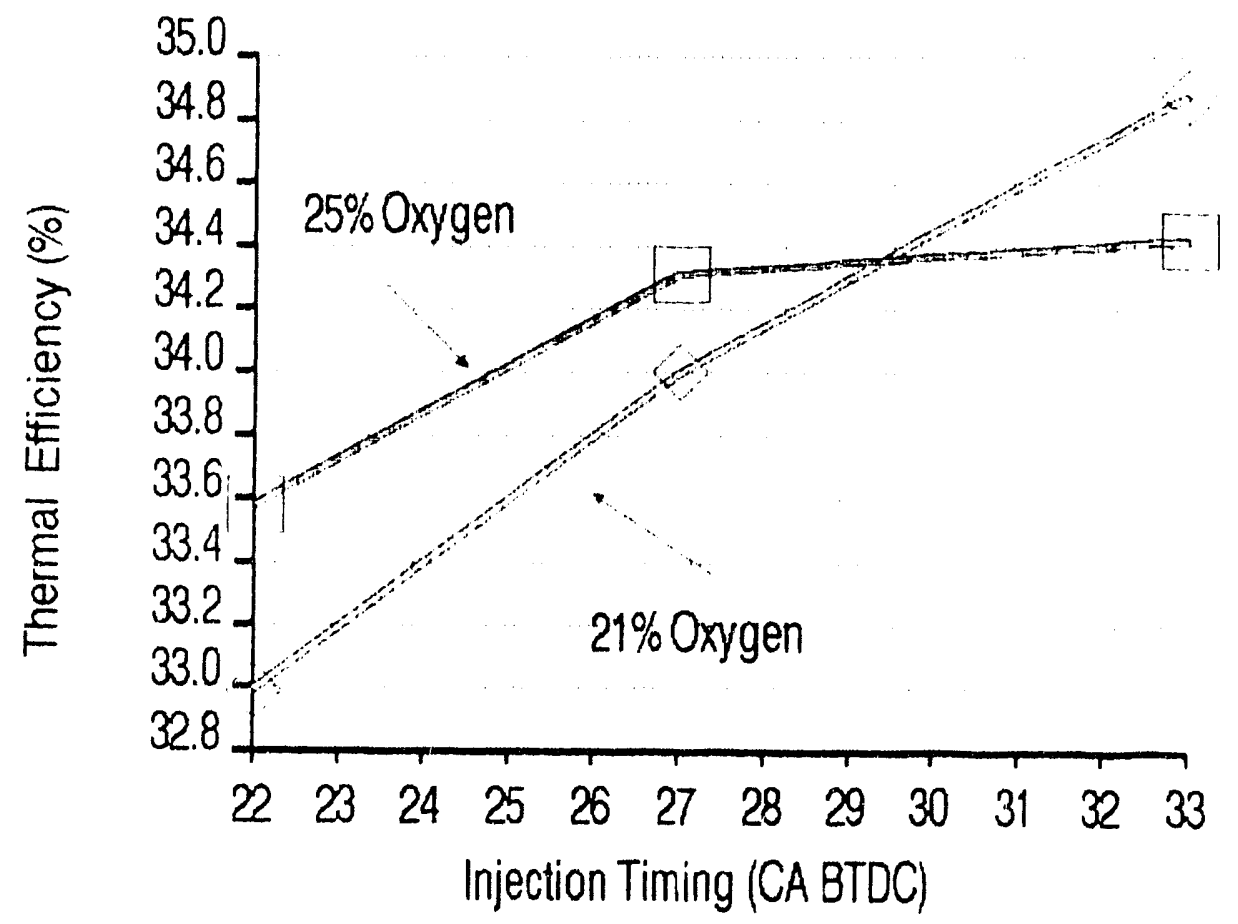

FIGURE B.100 Effect of Injection Timing on Thermal Efficiency, 25 Brake Horsepower, No. 4 Fuel, No Water 


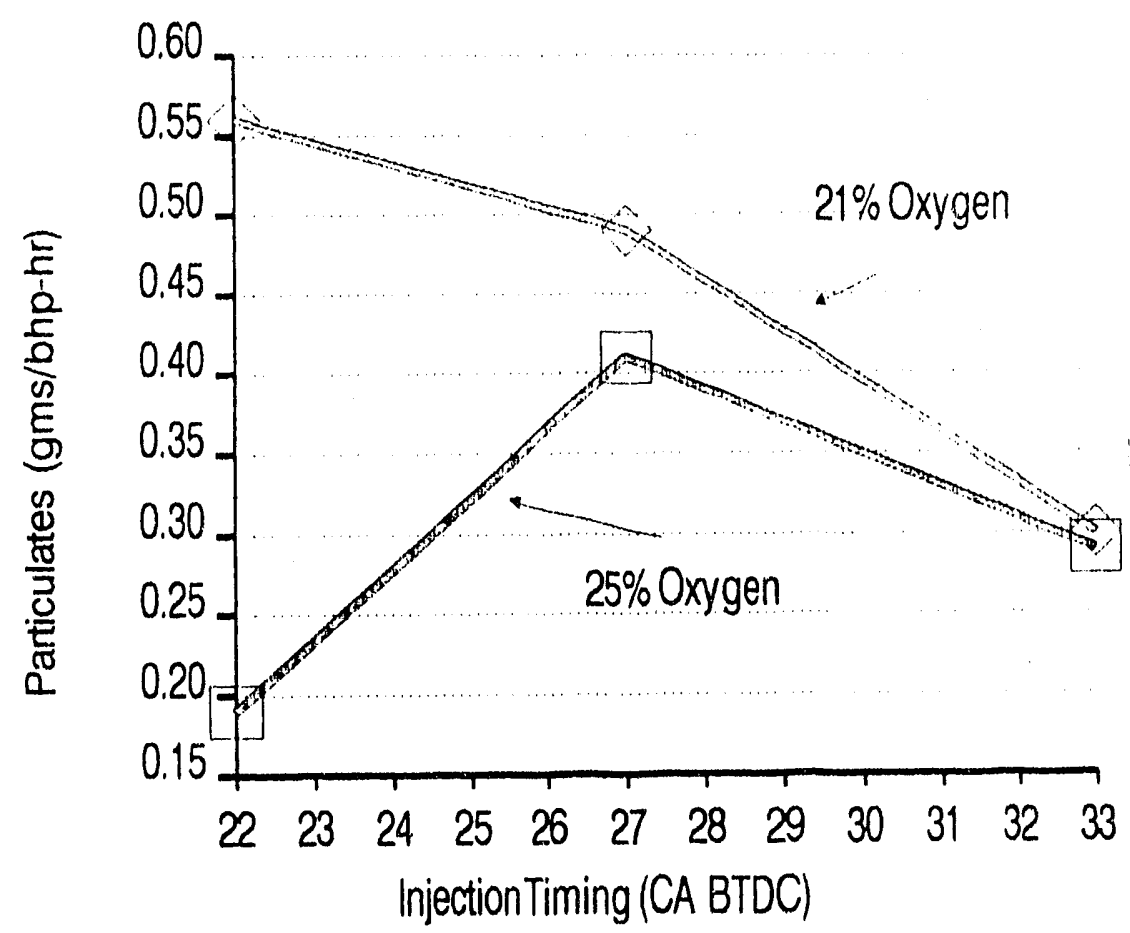

FIGURE B.101 Effect of Injection Timing on Particulate-Matter Emission, 25 Brake Horsepower, No. 4 Fuel, No Water

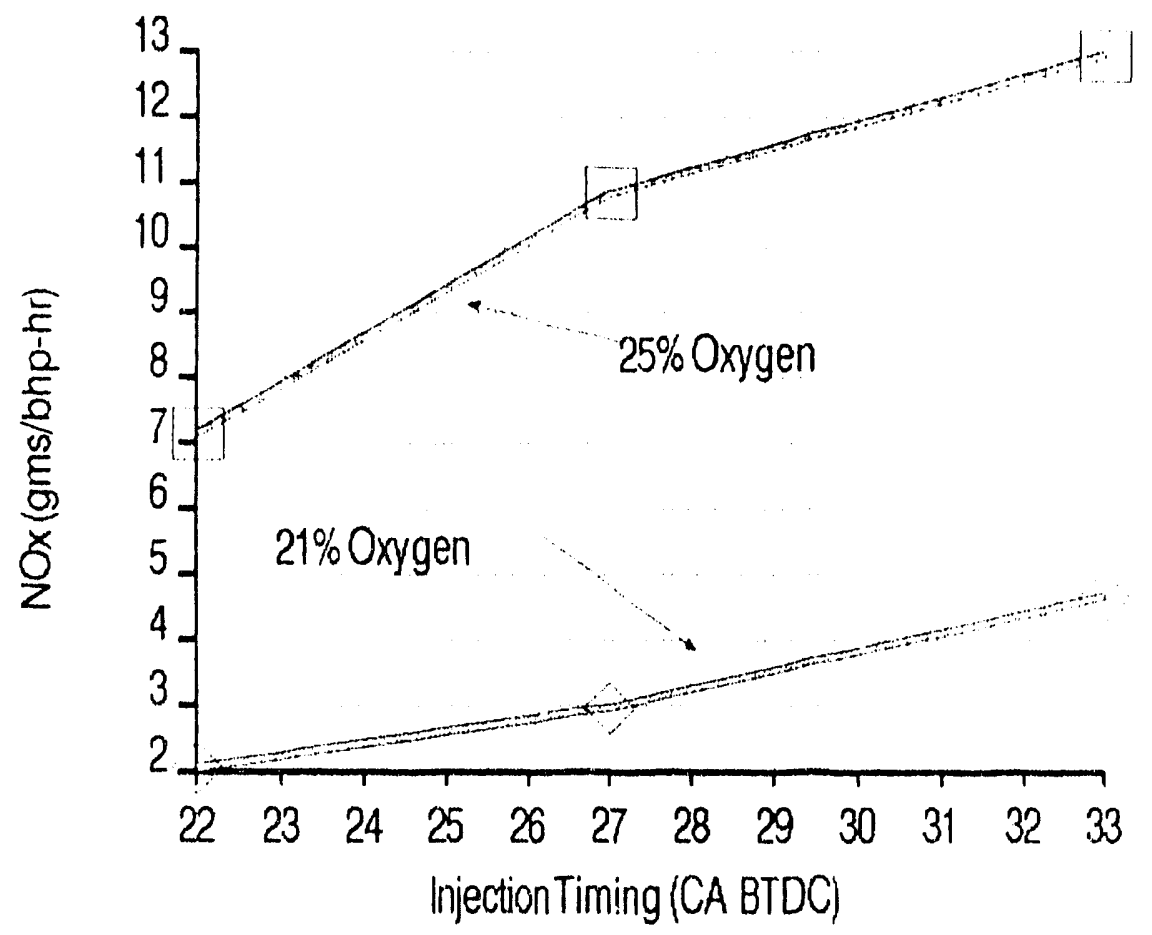

FIGURE B.102 Effect of Injection Timing on $\mathrm{NO}_{x}$ Emission, 25 Brake Horsepower, No. 4 Fuel, No Water 


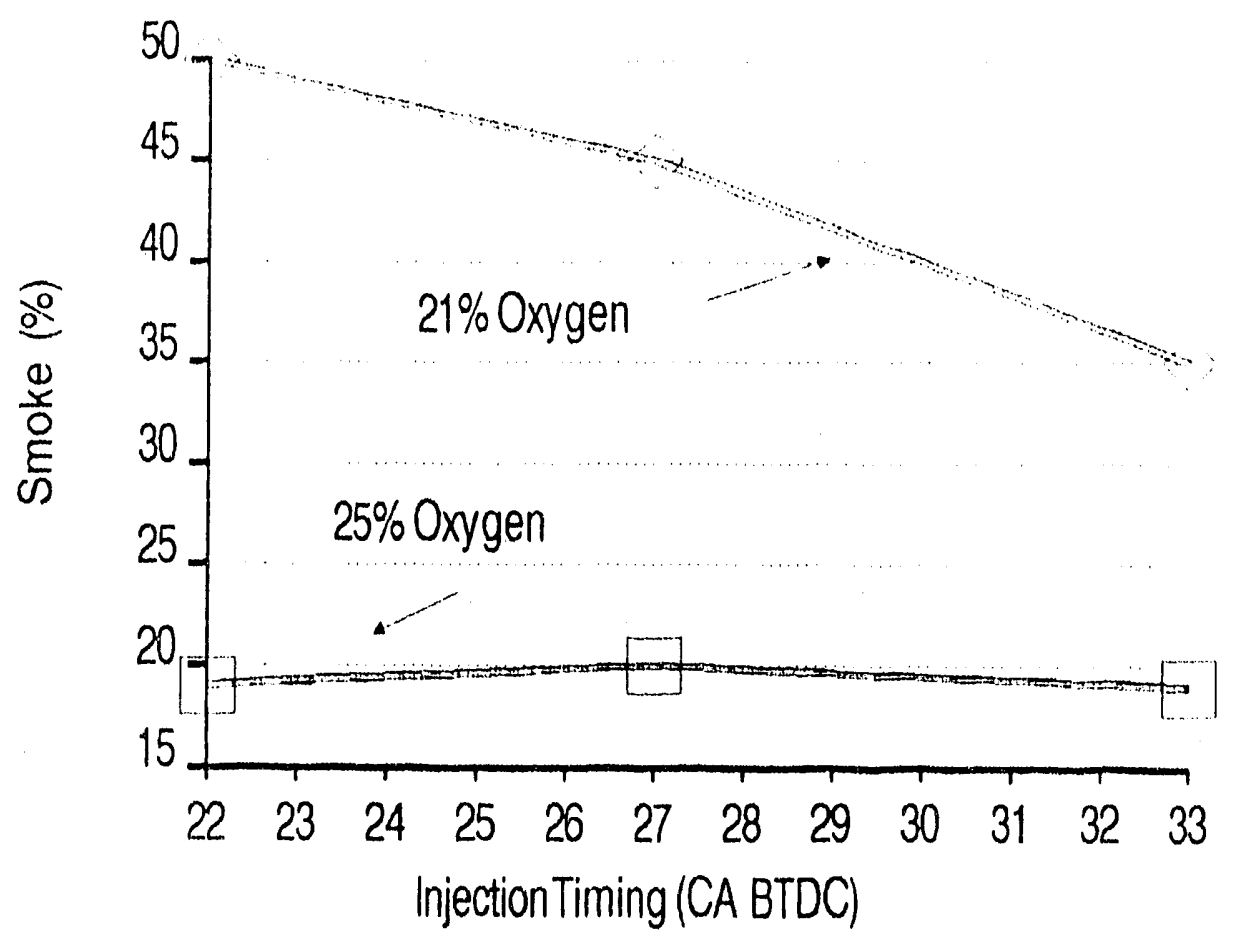

FIGURE B.103 Effect of Injection Timing on Smoke Emission, 25 Brake Horsepower, No. 4 Fuel, No Water

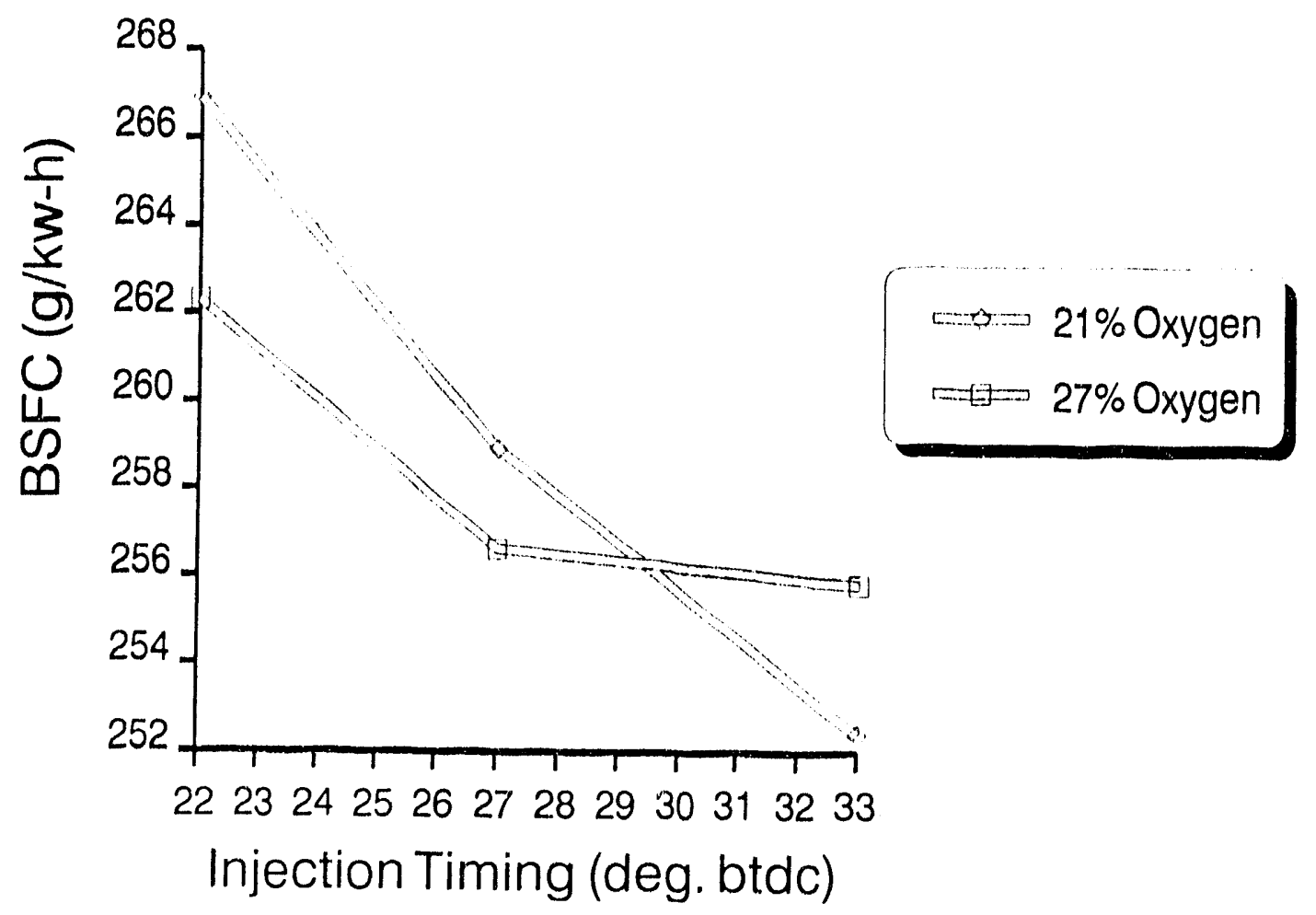

FIGURE B.104 Effects of Injection Timing on Brake-Specific Fuel Consumption, 25 Brake Horsepower, No. 4 Fuel, No Water 


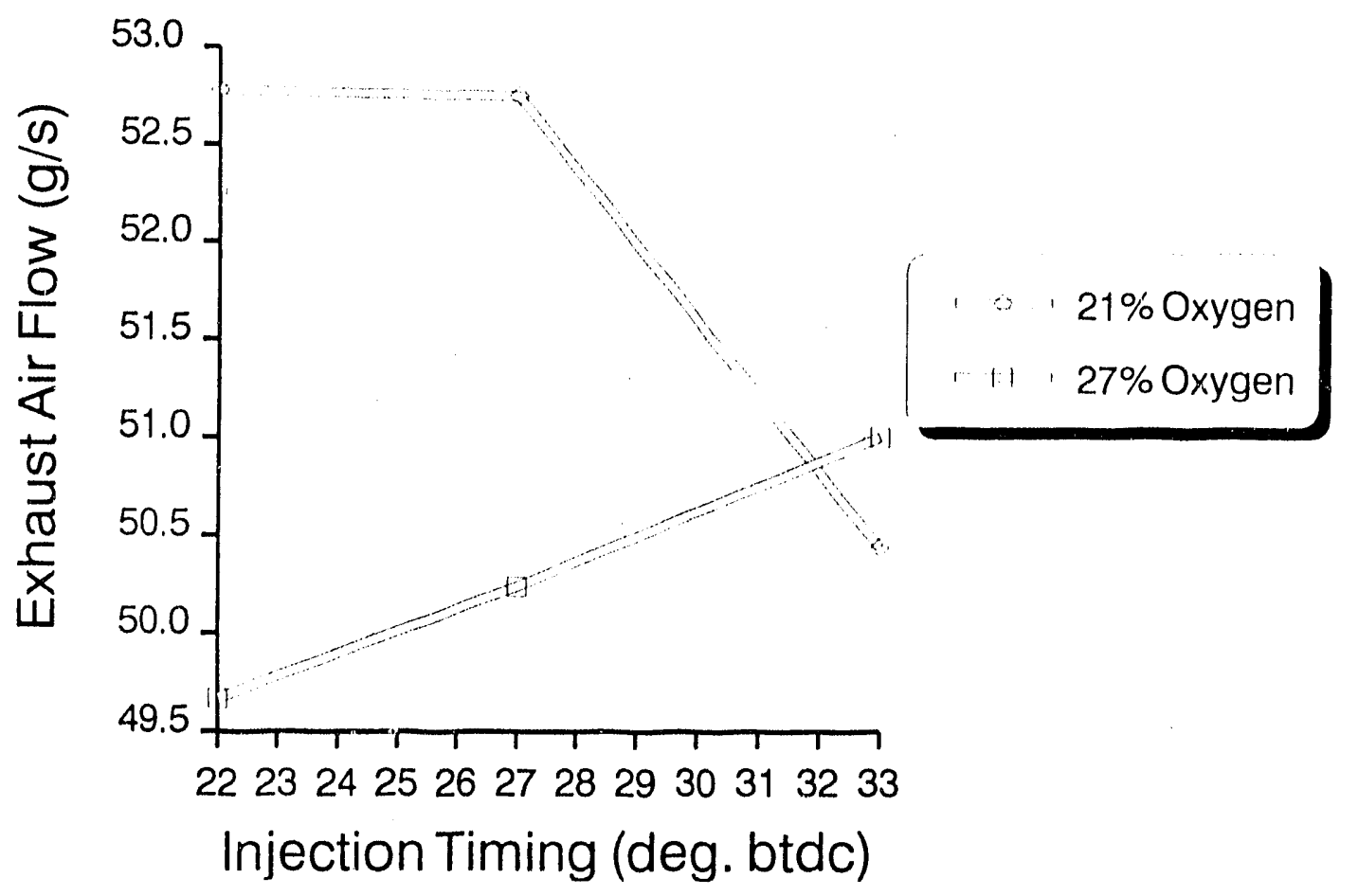

FIGURE B.105 Effects of Injection Timing on Exhaust Air Flow, 25 Brake Horsepower, No. 4 Fuel, No Water

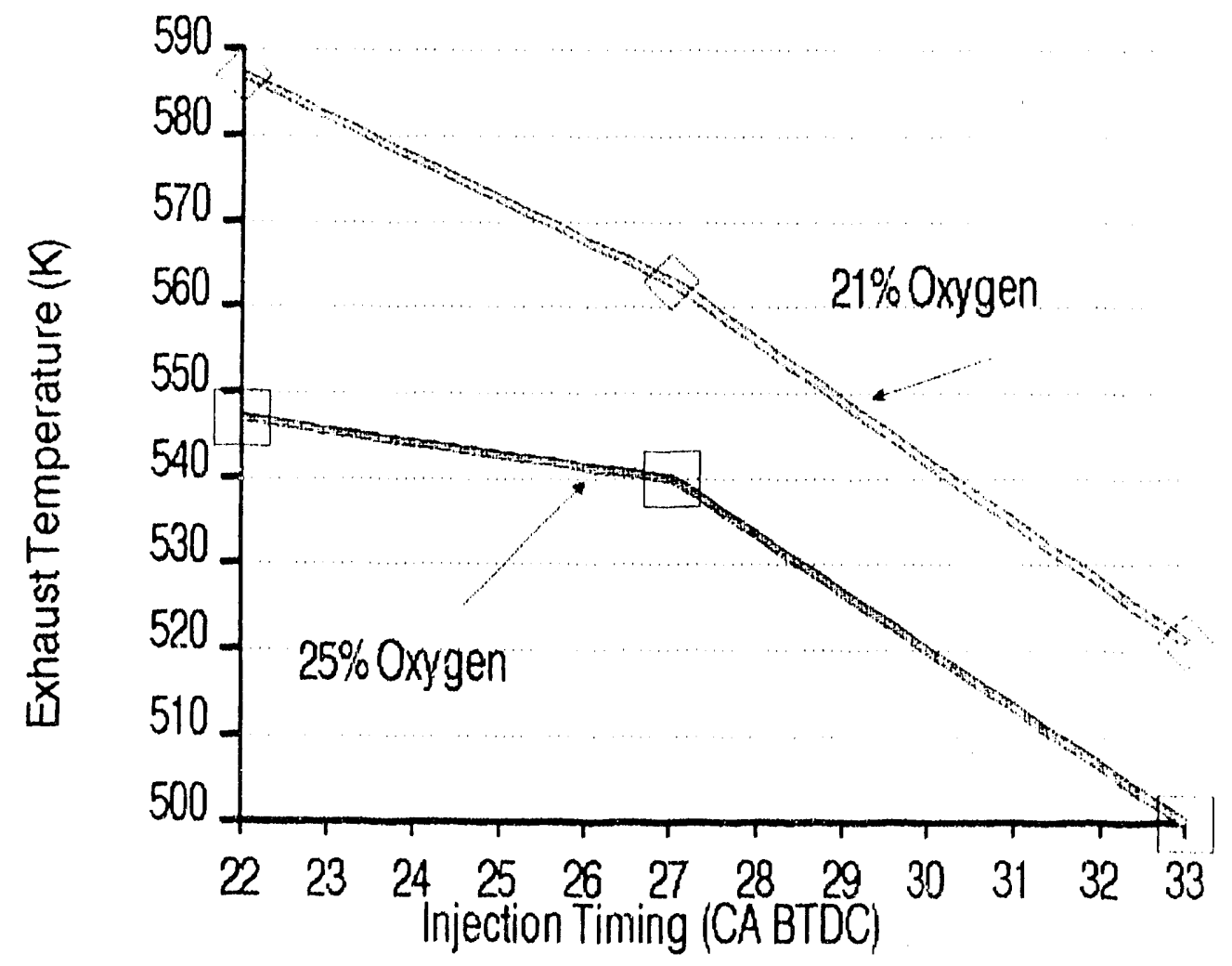

FIGURE B.106 Effect of Injection Timing on Exhaust Temperature, 25 Brake Horsepower, No. 4 Fuel, 5\% Water 


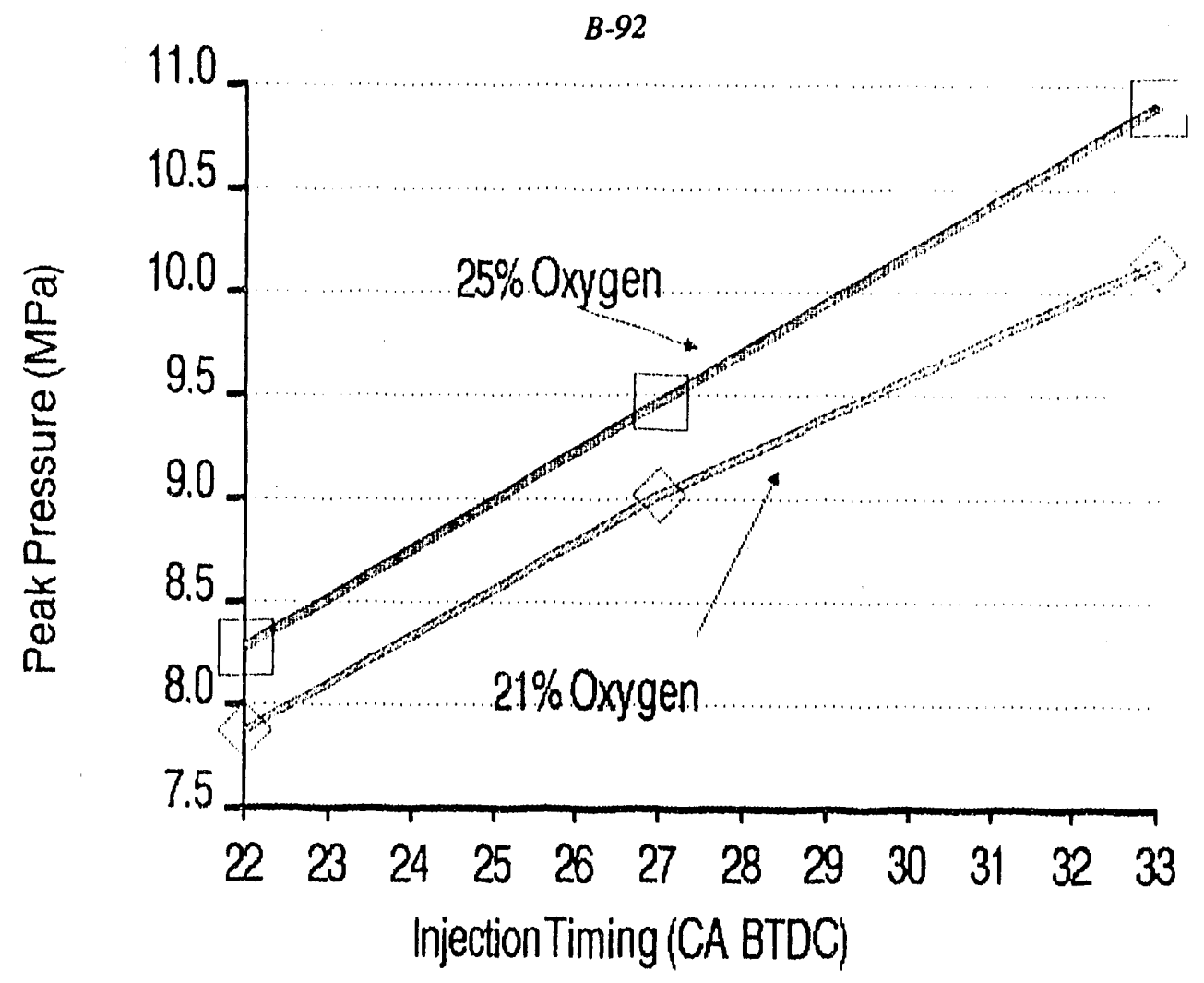

FIGURE B.107 Effect of Injection Timing on Cylinder Pressure, 25 Brake Horsepower, No. 4 Fuel, 5\% Water

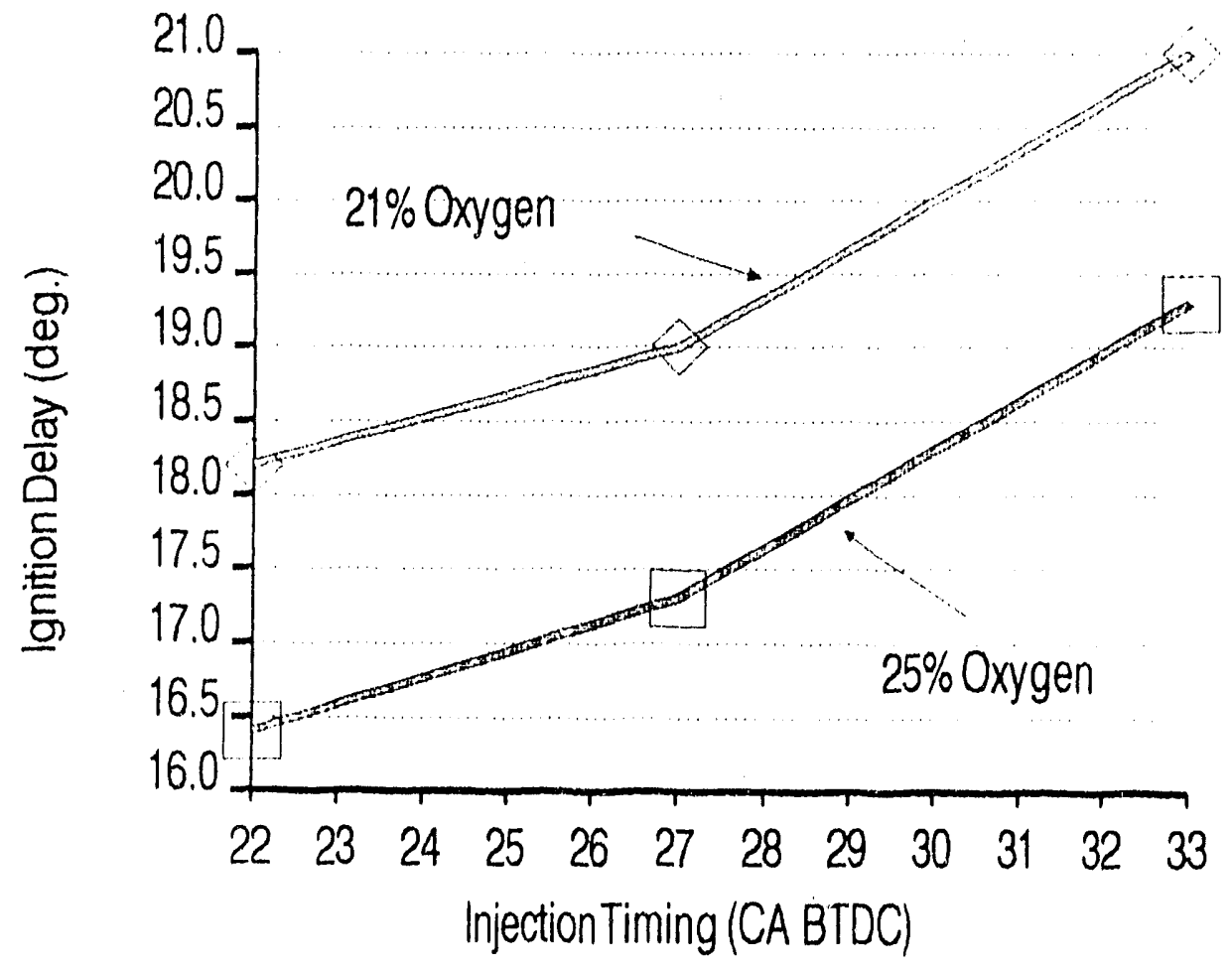

FIGURE B.108 Effect of Injection Timing on Ignition Delay, 25 Brake HorseWusver, No. 4 Fuel, $5 \%$ Water 


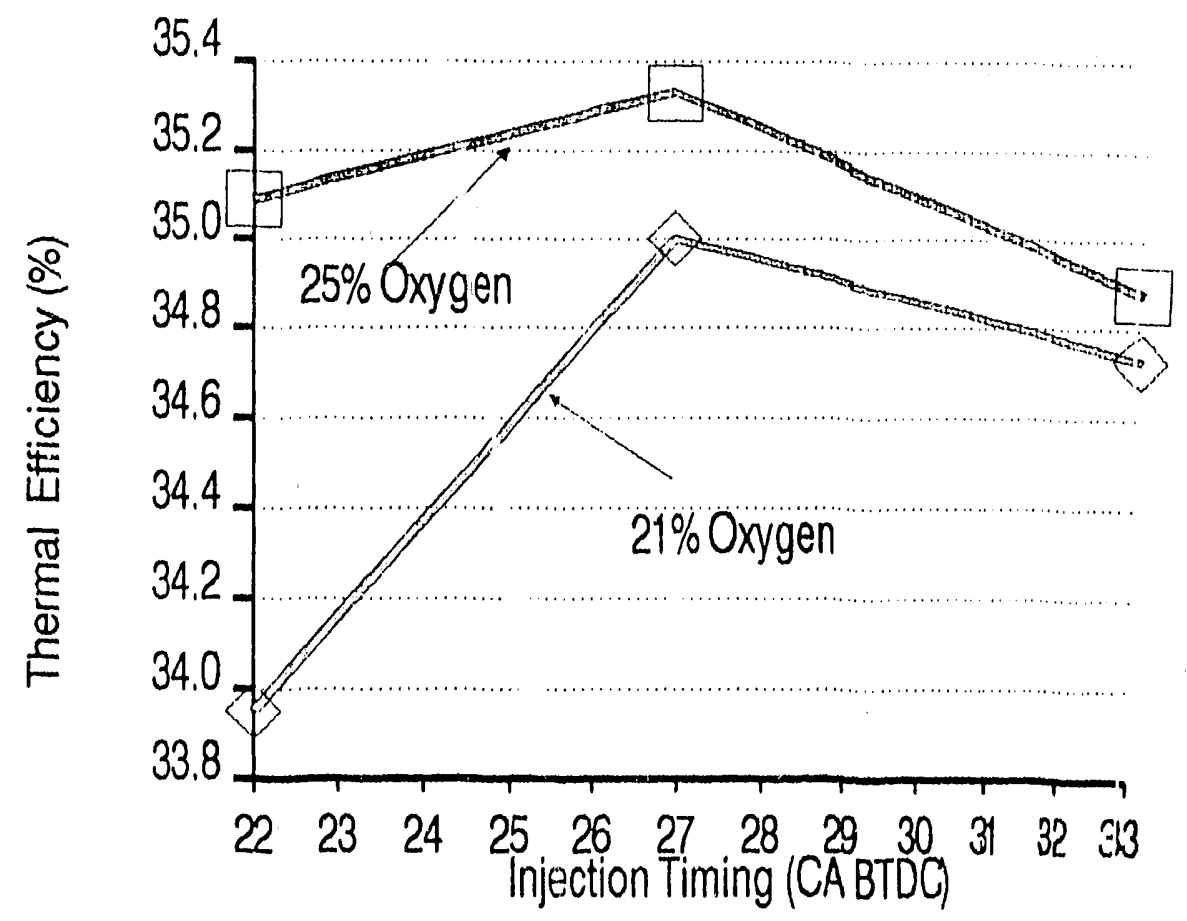

FIGURE B.109 Effect of Injection Timing on Thermal Efficiency, 25 Brake Horsepower, No. 4 Fuel, 5\% Water

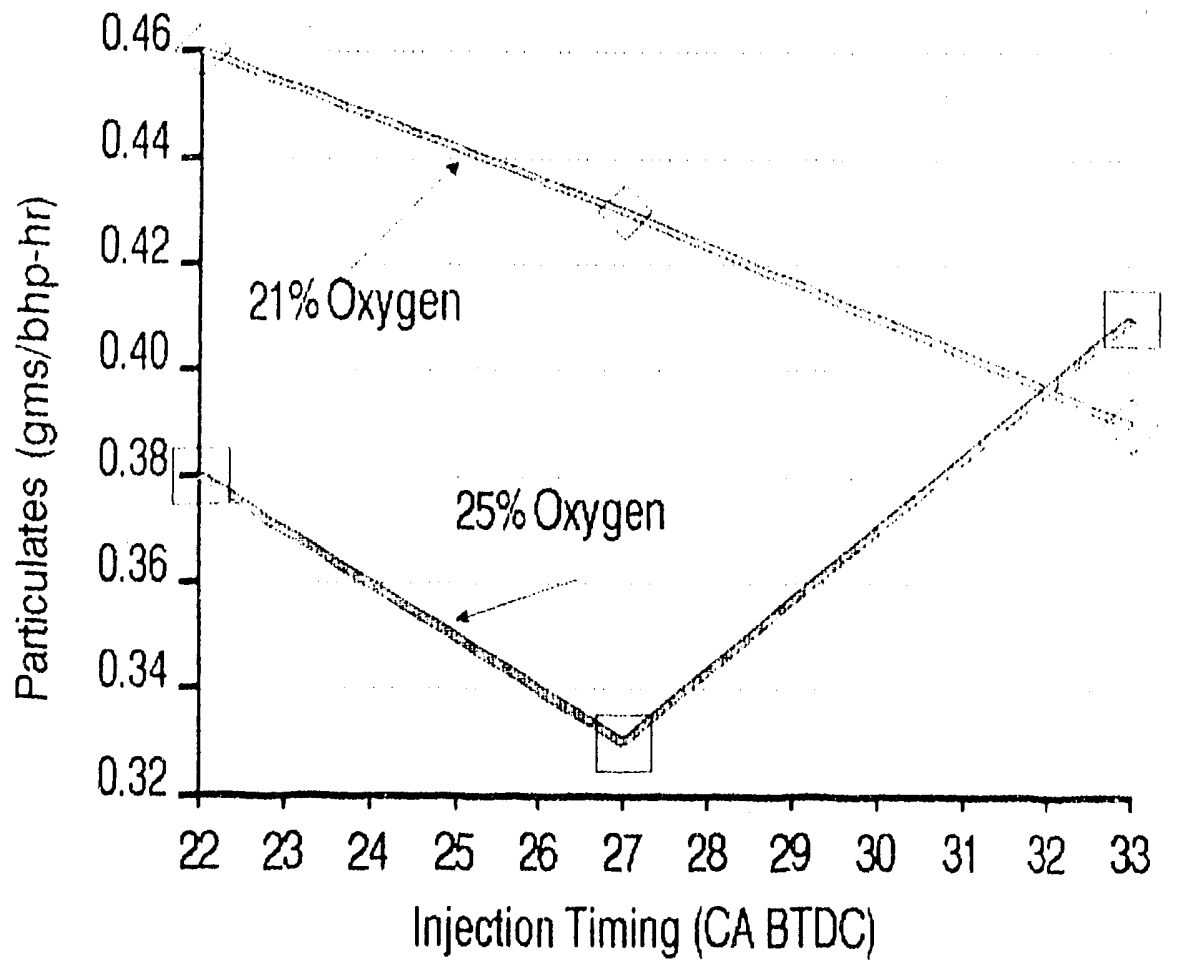

FIGURE B.110 Effect of Injection Timing on Particulate-Matter Emission, 25 Brake Horsepower, No. 4 Fuel, 5\% Water 


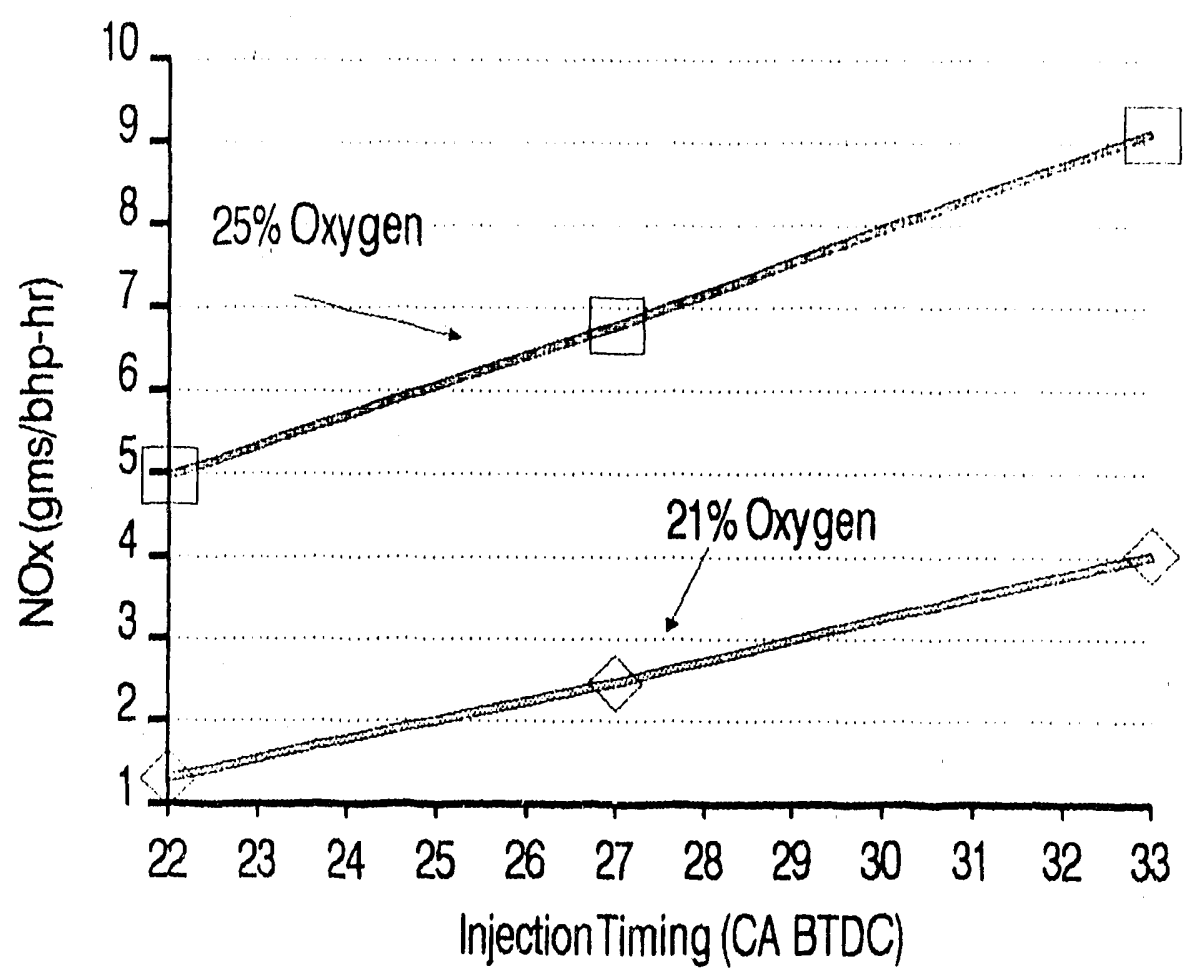

FIGURE B.111 Effect of Injection Timing on $\mathrm{NO}_{x}$ Emission, 25 Brake Horsepower, No. 4 Fuel, 5\% Water

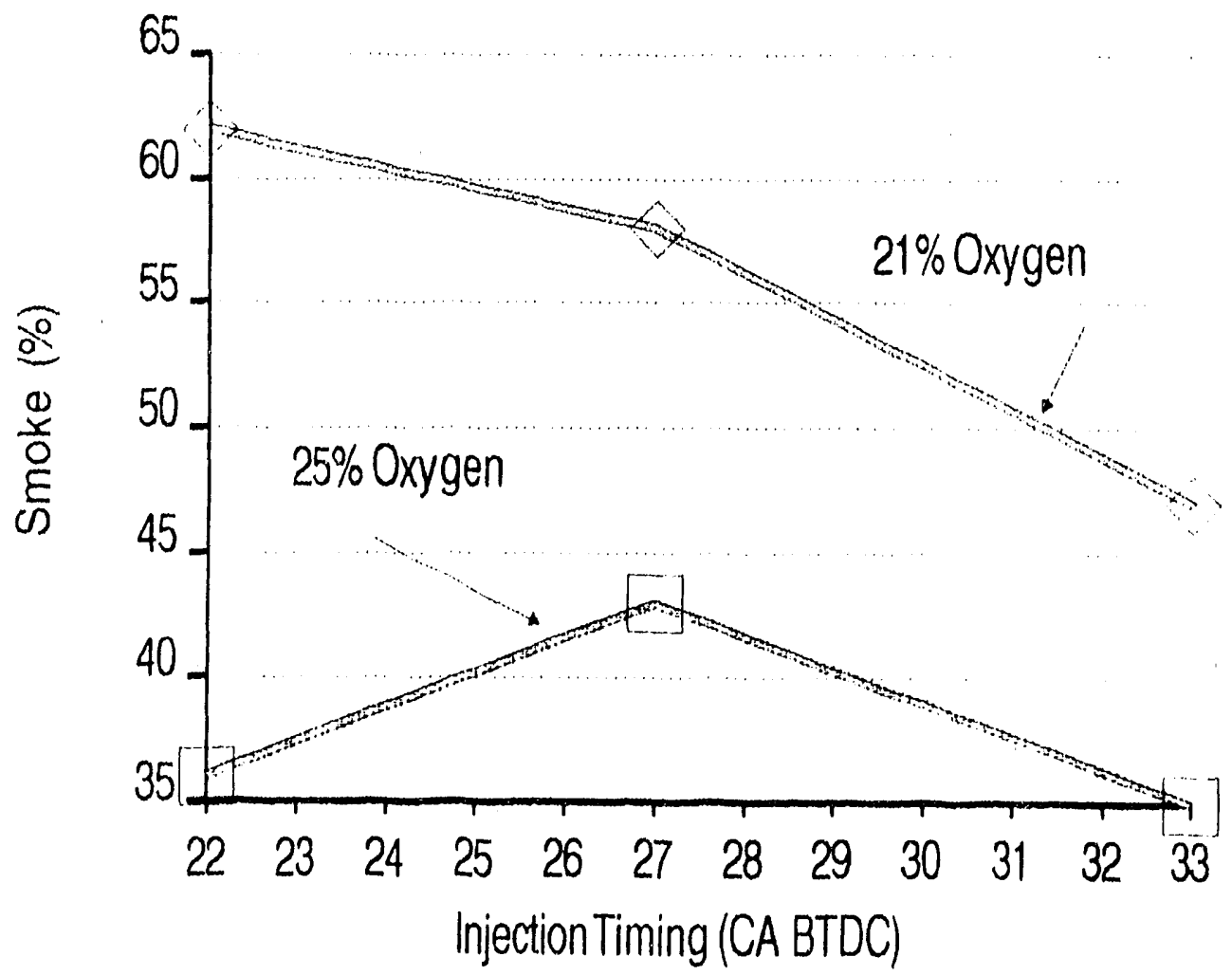

FIGURE B.112 Effect of Injection Timing on Smoke Emission, 25 Brake Horsepower, No. 4 Fuel, 5\% Water 


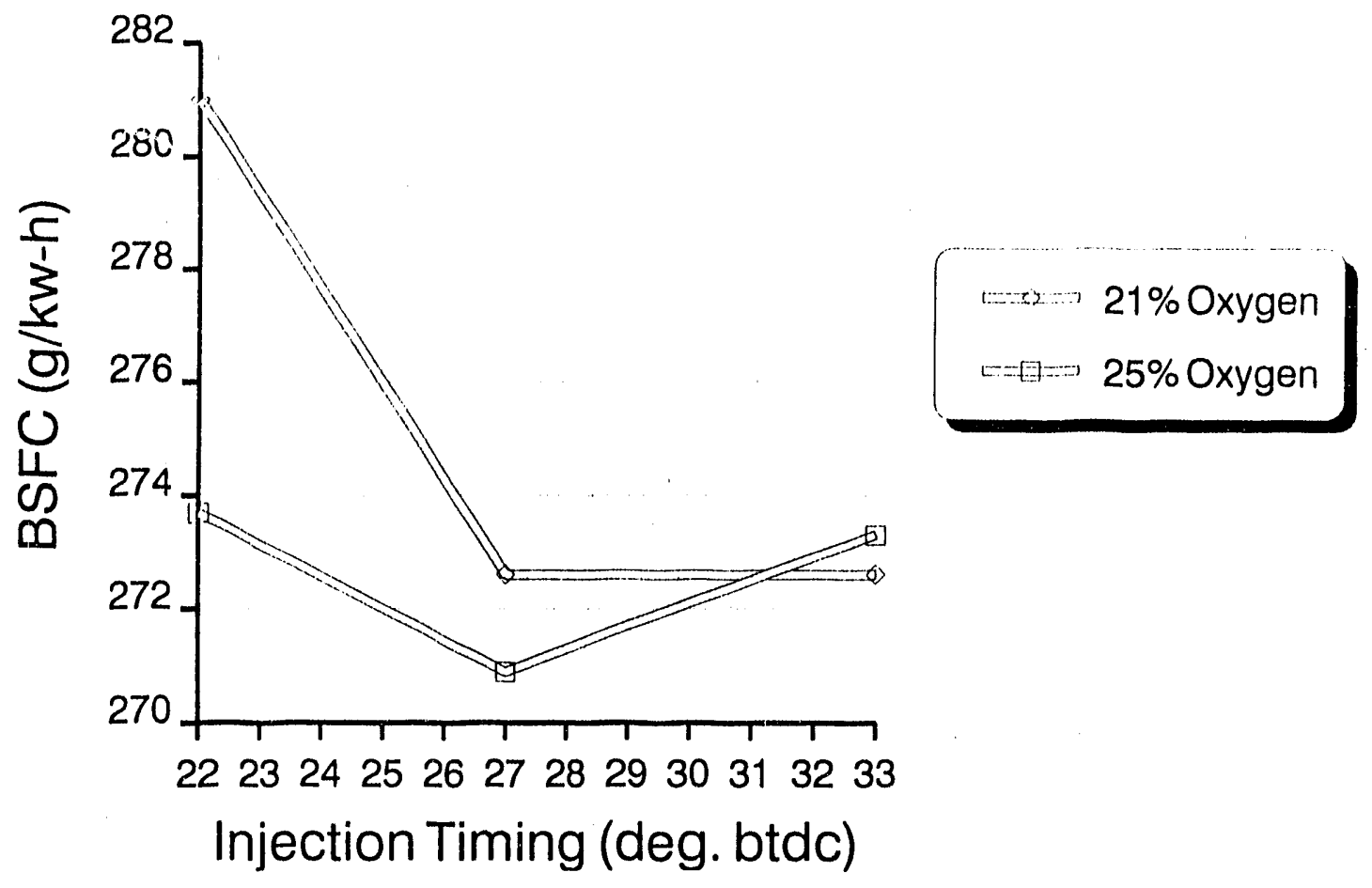

FIGURE B.113 Effect of Injection Timing on Brake-Specific Fuel Cc.Isumption, 25 Brake Horsepower, No. 4 Fuel, 5\% Water

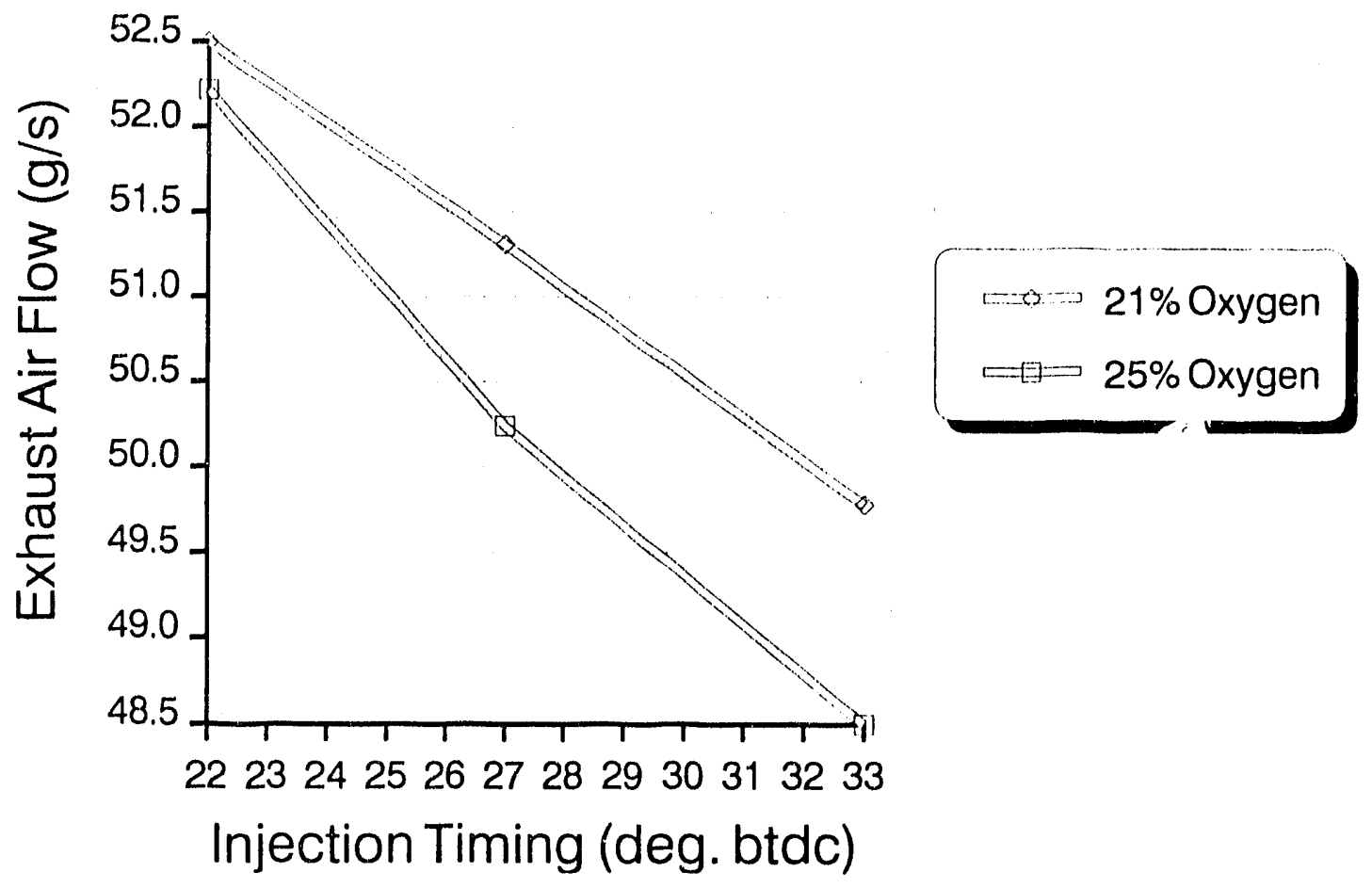

FIGURE B.114 Effect of Injection Timing on Exhaust Air Flow, 25 Brake Horsepower, No. 4 Fuel, $5 \%$ Water 


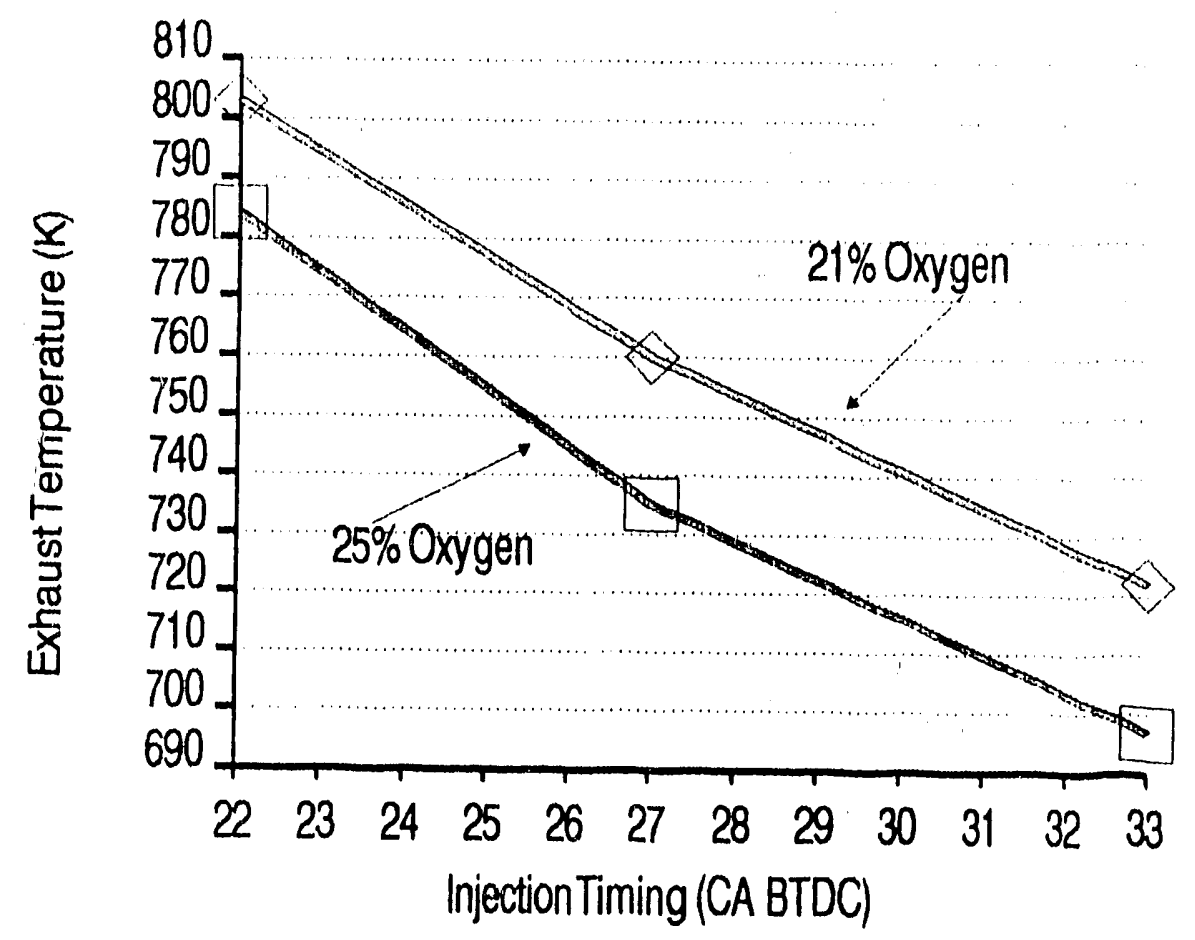

FIGURE B.115 Effect of Injection Timing on Exhaust Temperature, 50 Brake Horsepower, No. 4 Fuel, No Water

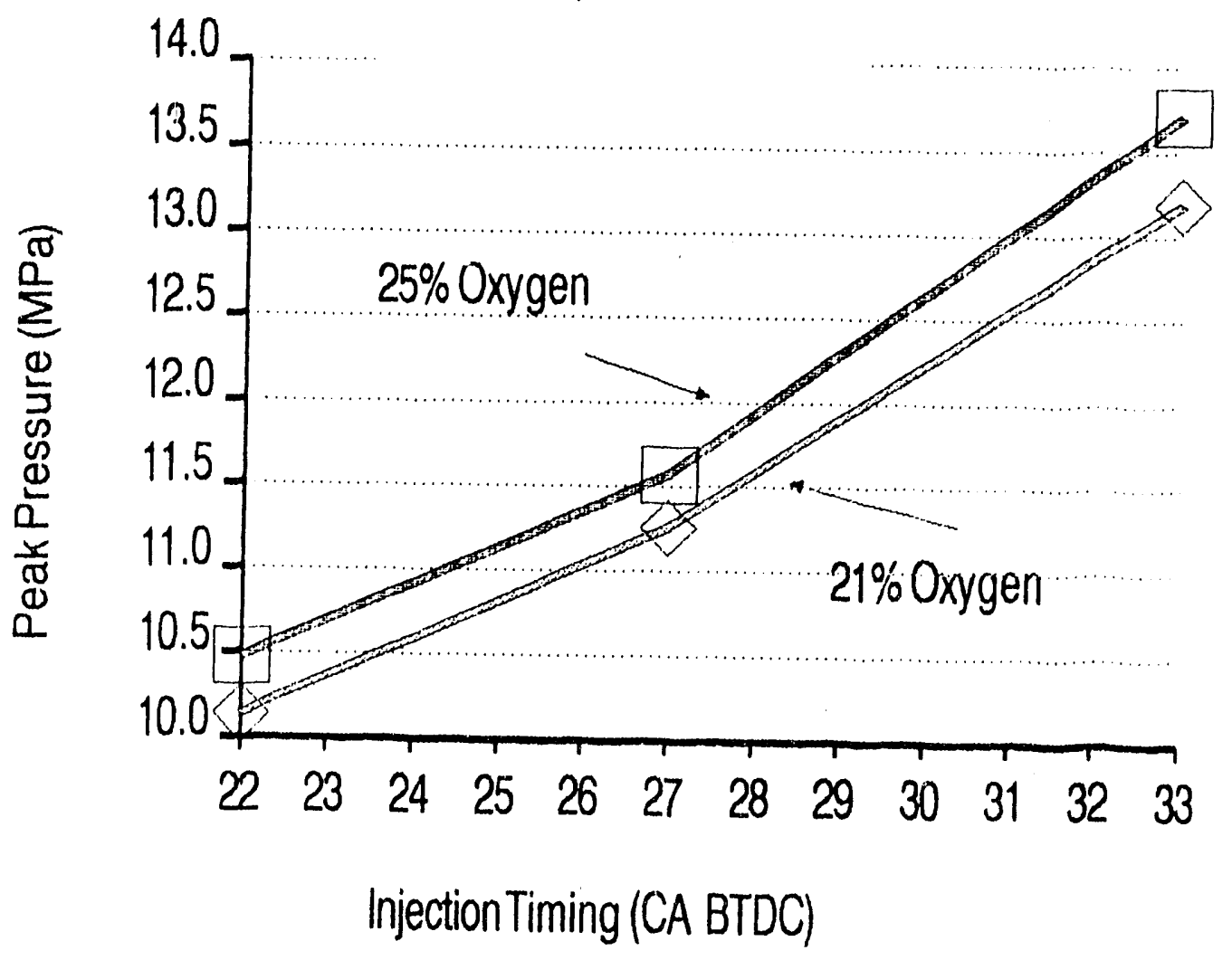
FIGURE B.116 Effect of Injection Timing on Cylinder Pressure, 50 Brake Horsepower,
No. 4 Fuel, No Water 


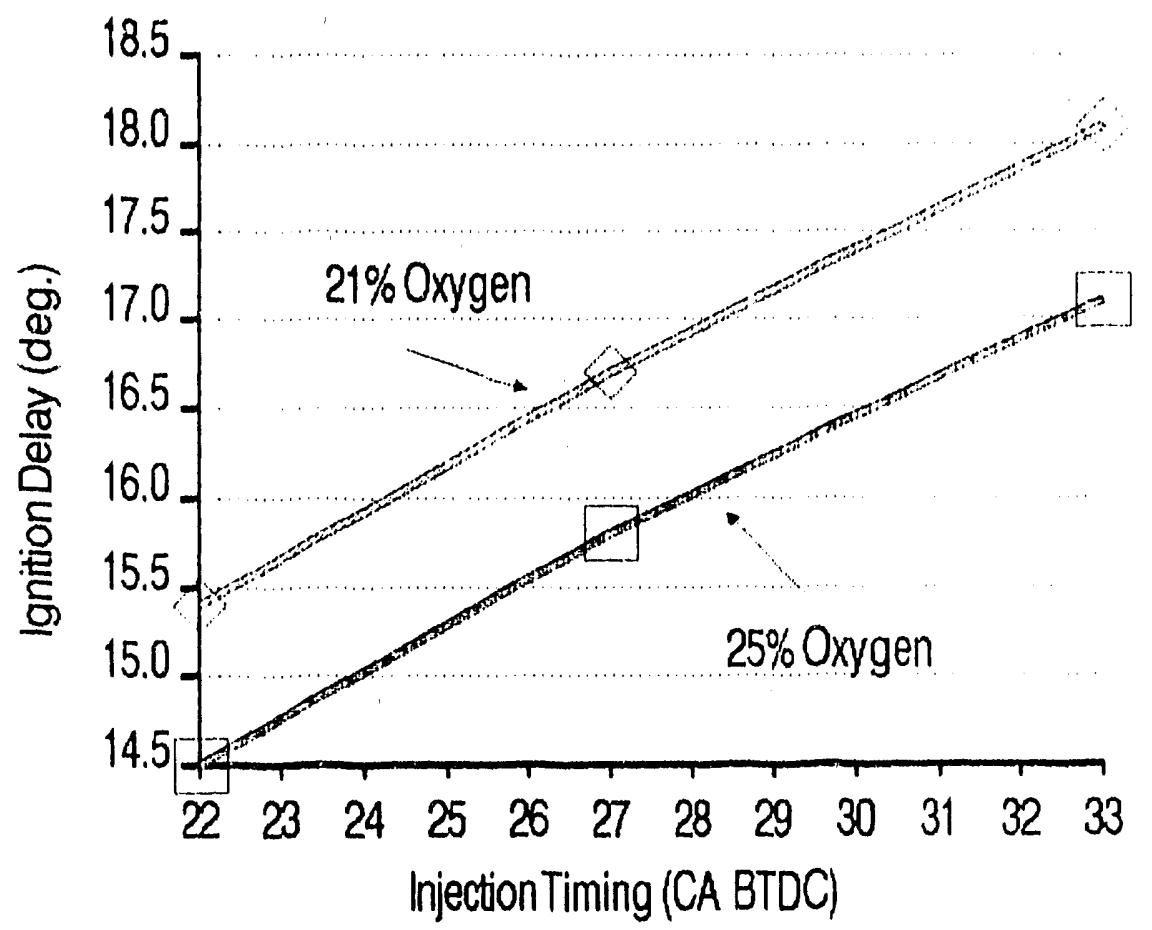

FIGURE B.117 Effect of Injection Timing on Ignition Delay, 50 Brake Horsepower, No. 4 Fuel, No Water

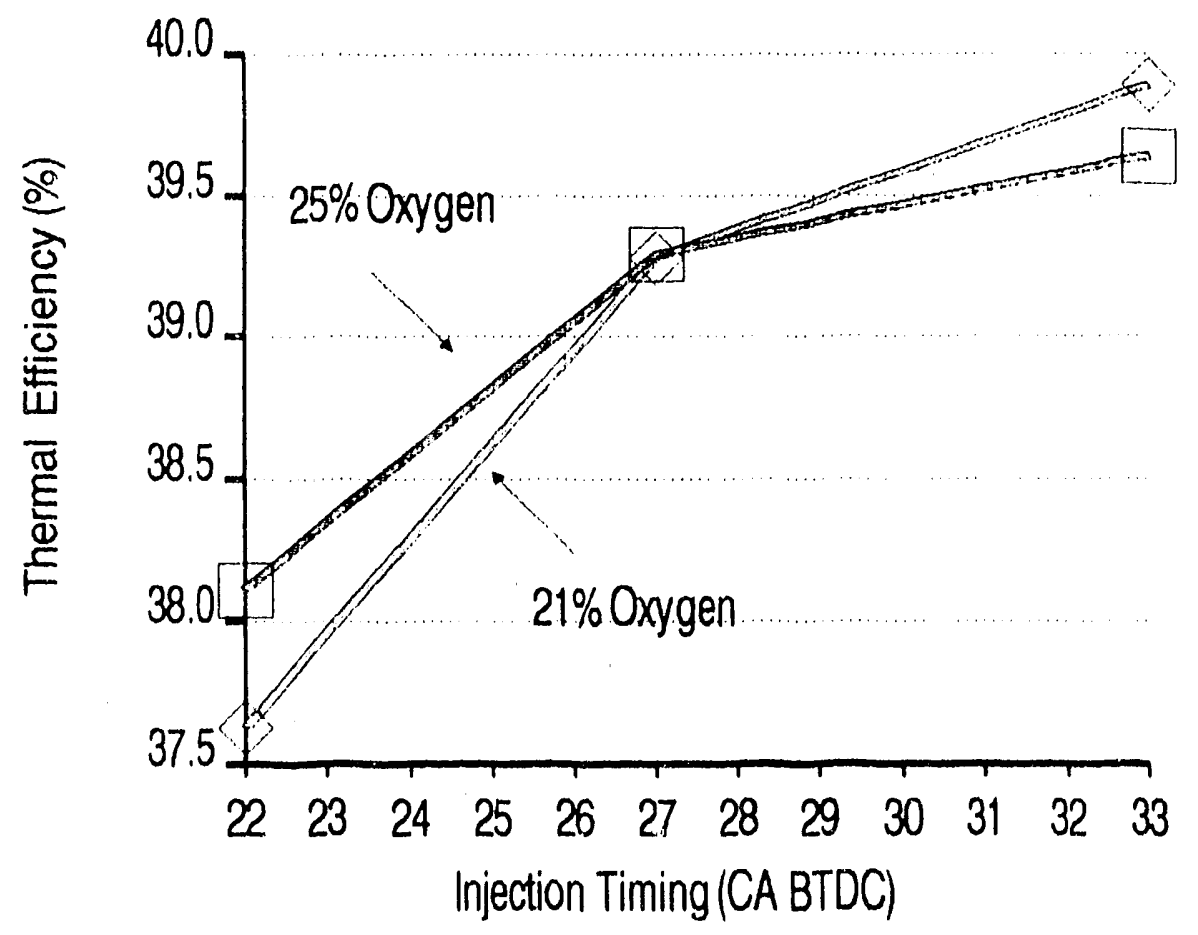

FIGURE B.118 Effect of Injection Timing on Thermal Efficiency, 50 Brake Horsepower, No. 4 Fuel, No Water 


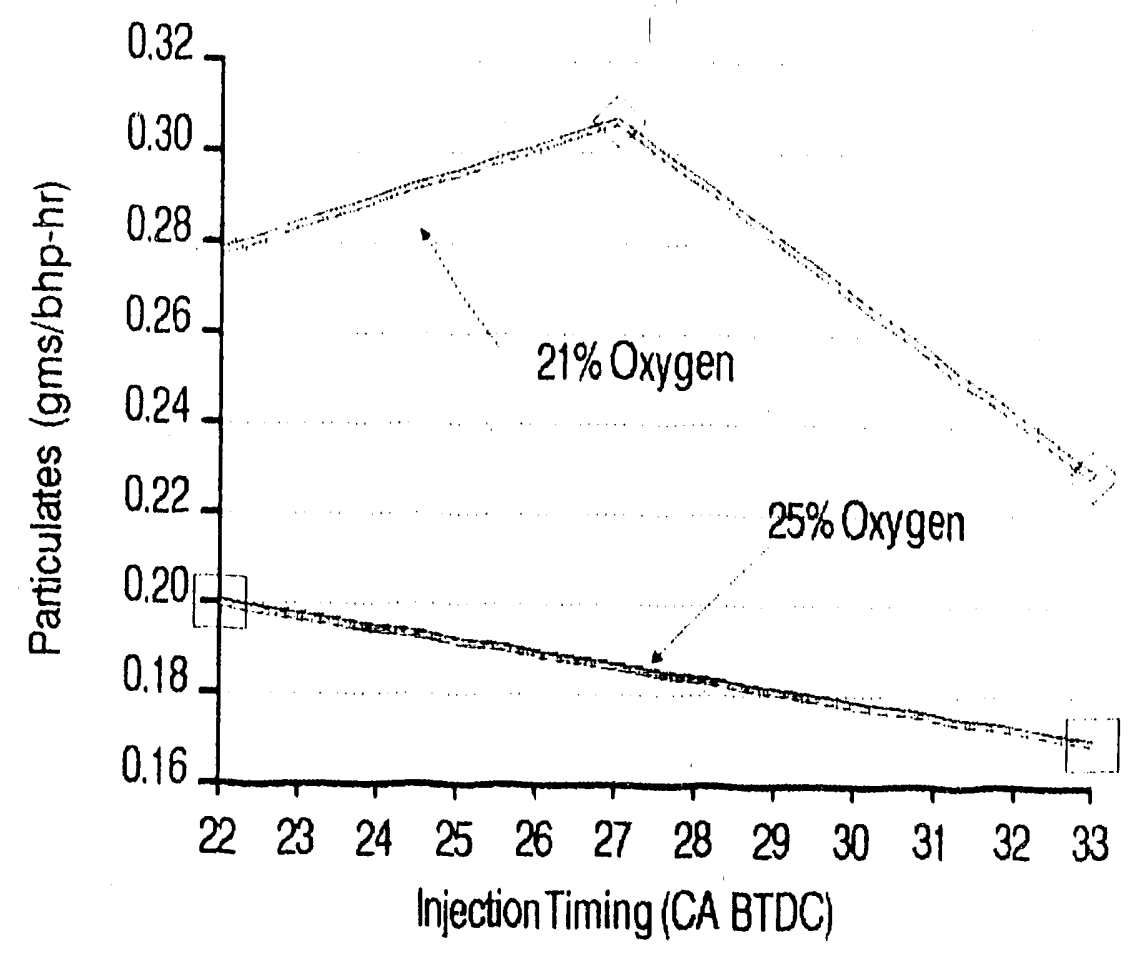

FIGURE B.119 Effect of Injection Timing on Particulate-Matter Emission, 50 Brake Horsepower, No. 4 Fuel, No Water

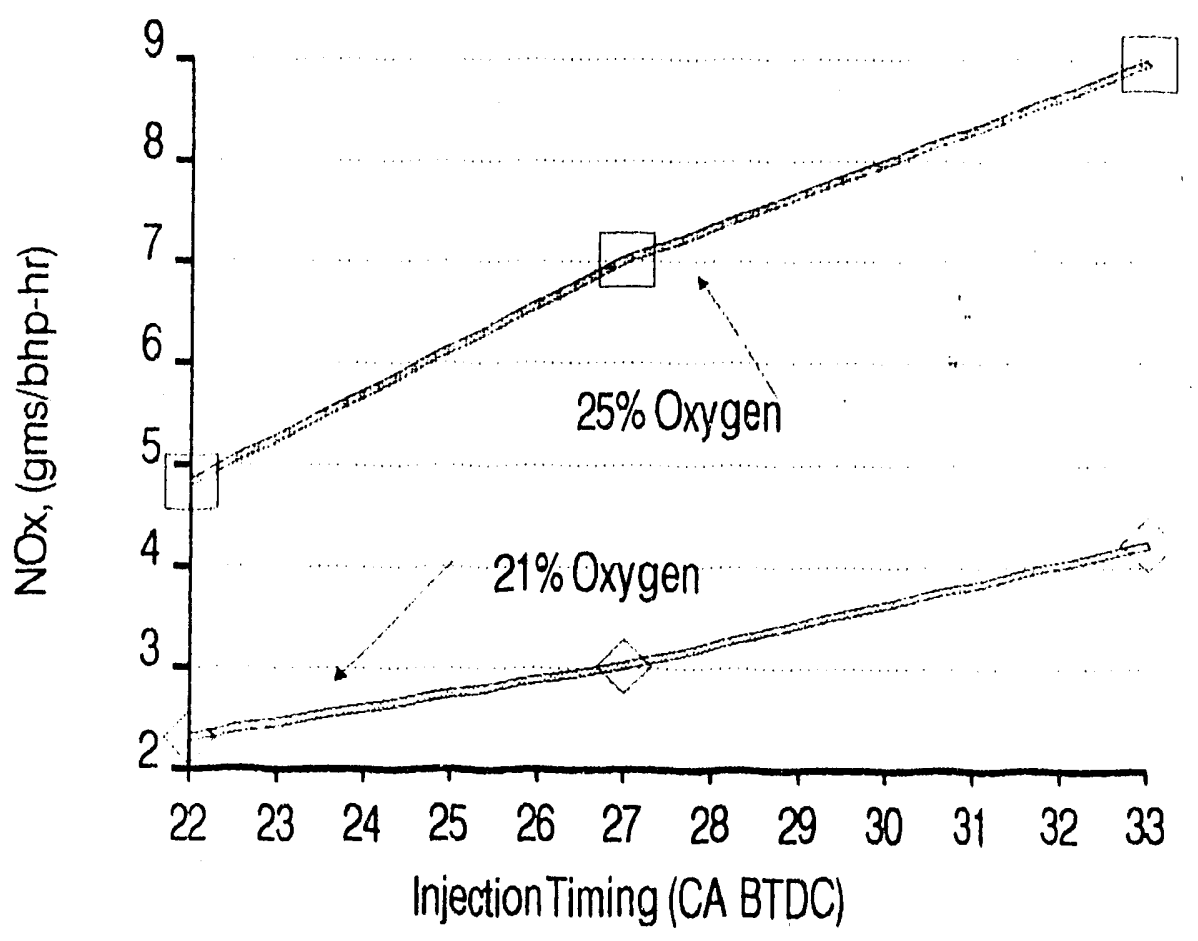

FIGURE B.120 Effect of Injection Timing on $\mathrm{NO}_{x}$ Emission, 50 Brake Horsepower, No. 4 Fuel, No Water 


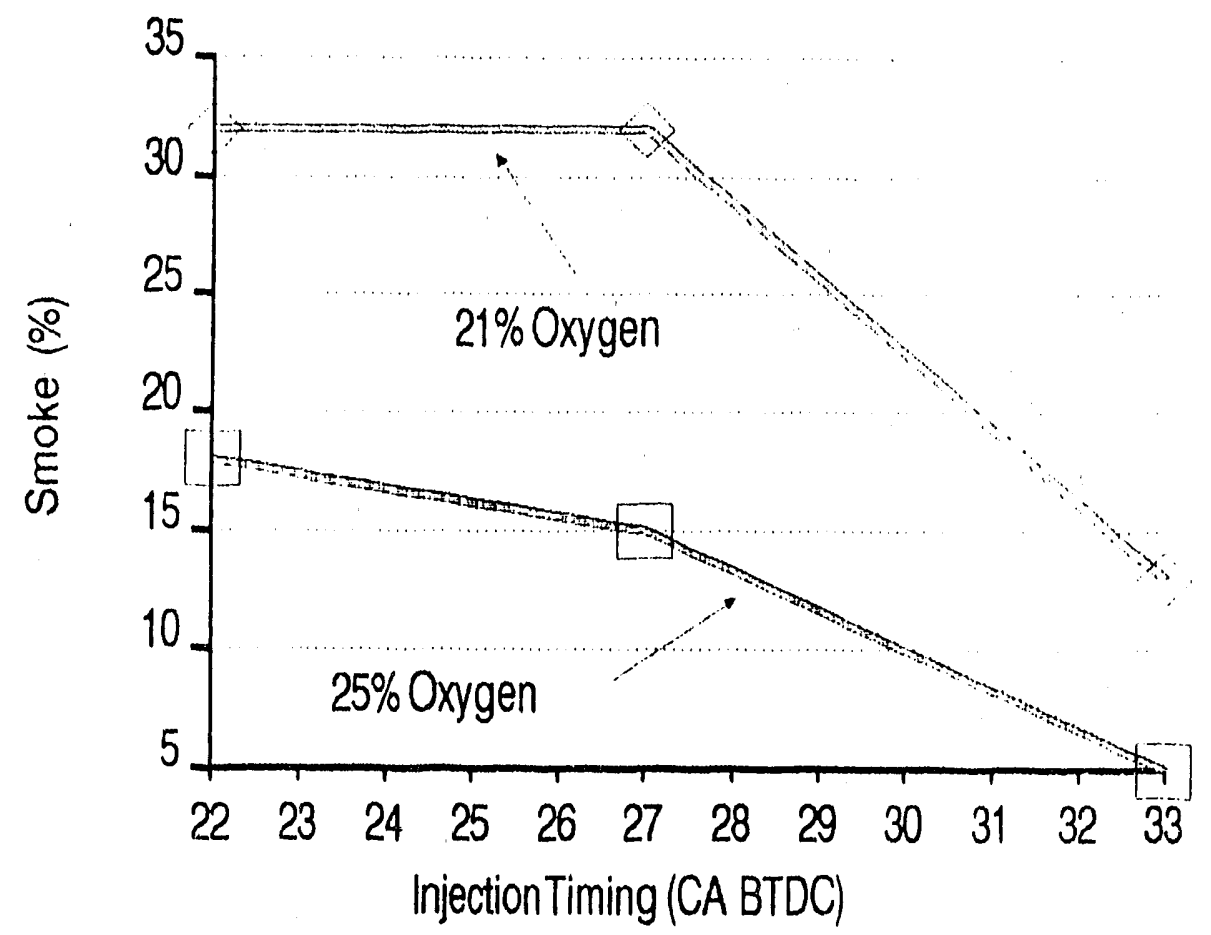

FIGURE B.121 Effect of Injection Timing on Smoke Emission, 50 Brake Horsepower, No. 4 Fuel, No Water

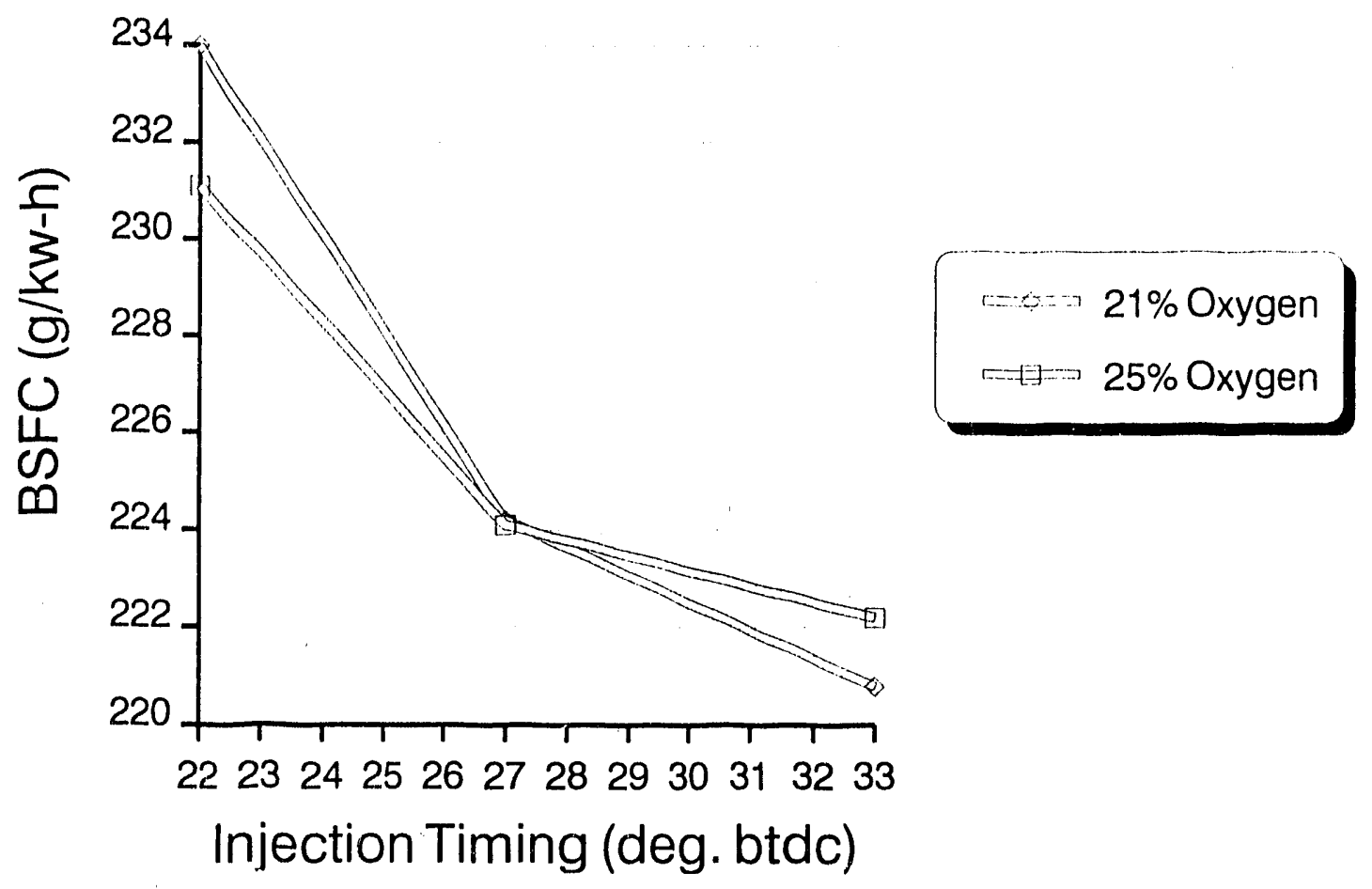

FIGURE B.122 Effect of Injection Timing on Brake-Specific Fuel Consumption, 50 Brake Horsepower, No. 4 Fuel, No Water 


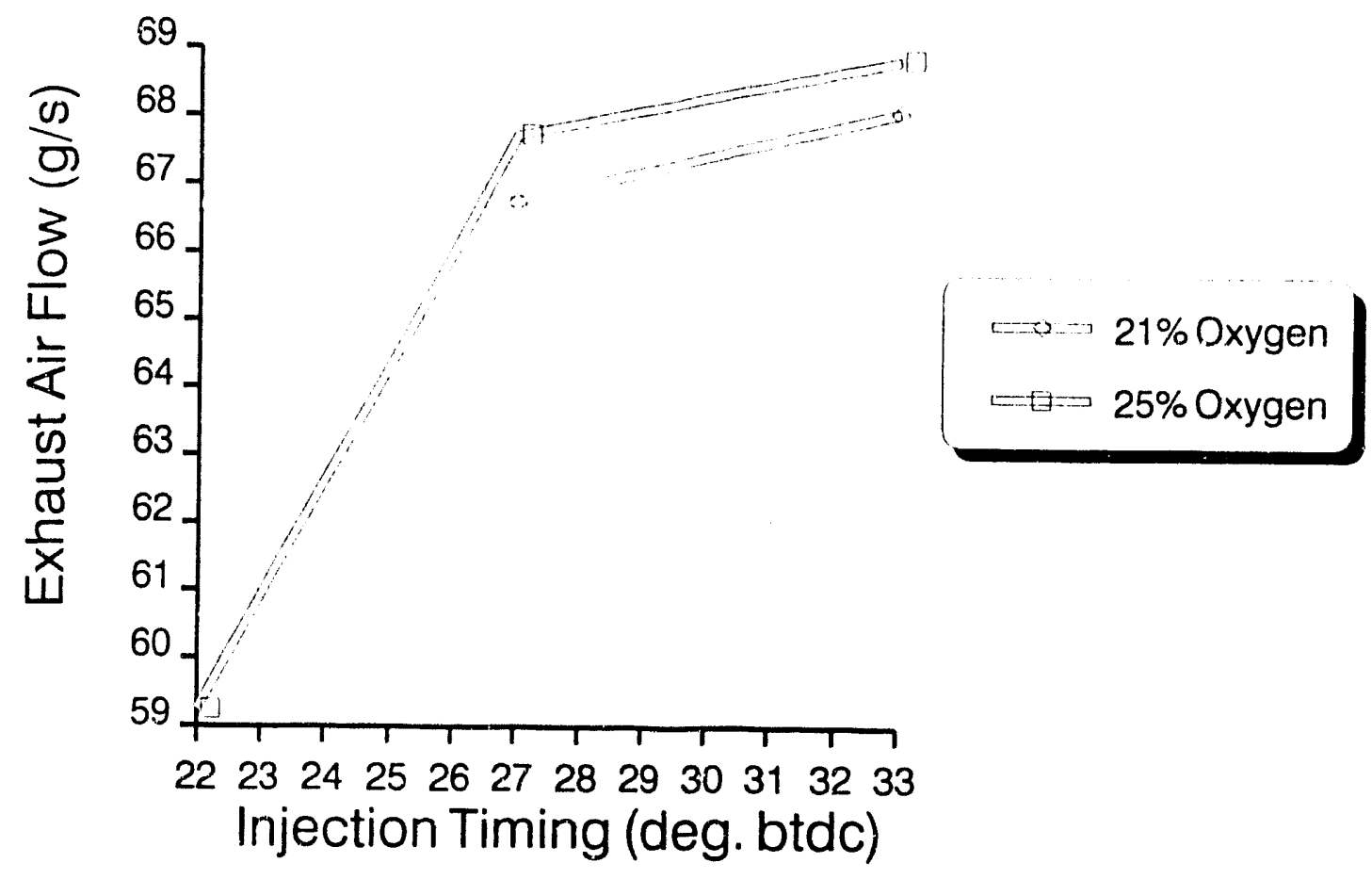

FIGURE B.123 Effect of Injection Timing on Exhaust Air Flow, 50 Brake Horsepower, No. 4 Fuel, No Water

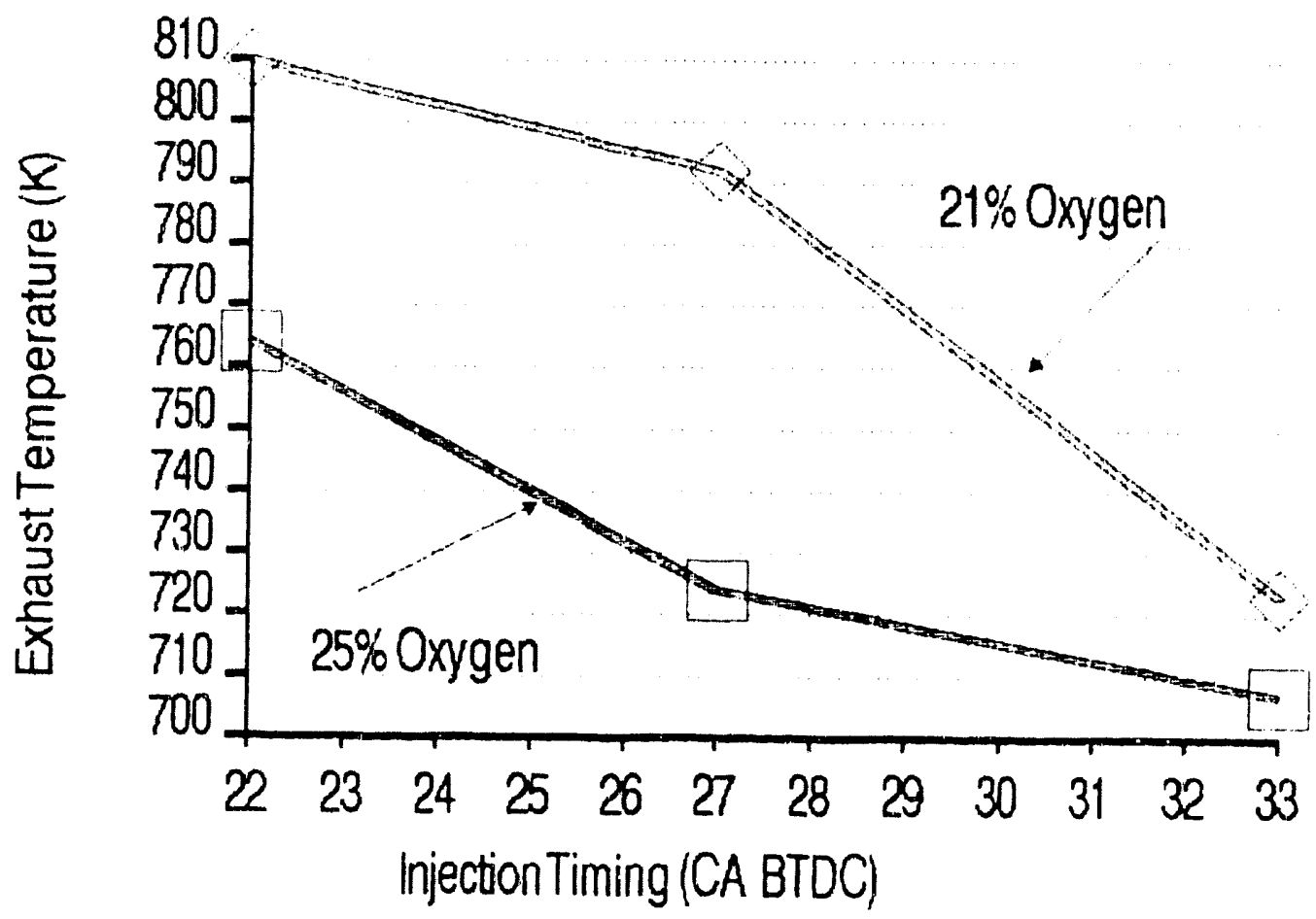

FIGURE B.124 Effect of Injection Timing on Exhaust Temperature, 50 Brake Horsepower, No. 4 Fuel, 5\% Water 


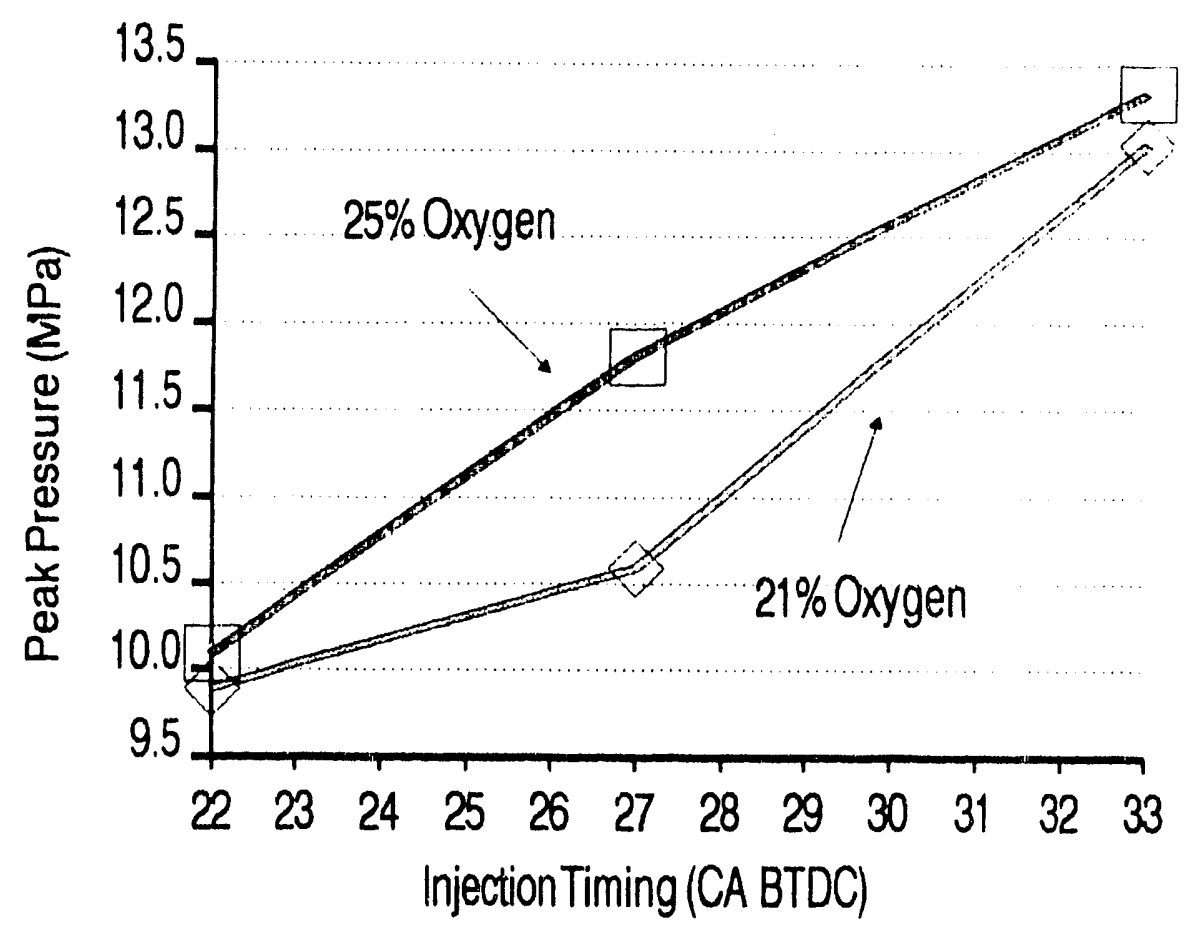

FIGURE B.125 Effect of Injection Timing on Cylinder Pressure, 50 Brake Horsepower, No. 4 Fuel, 5\% Water

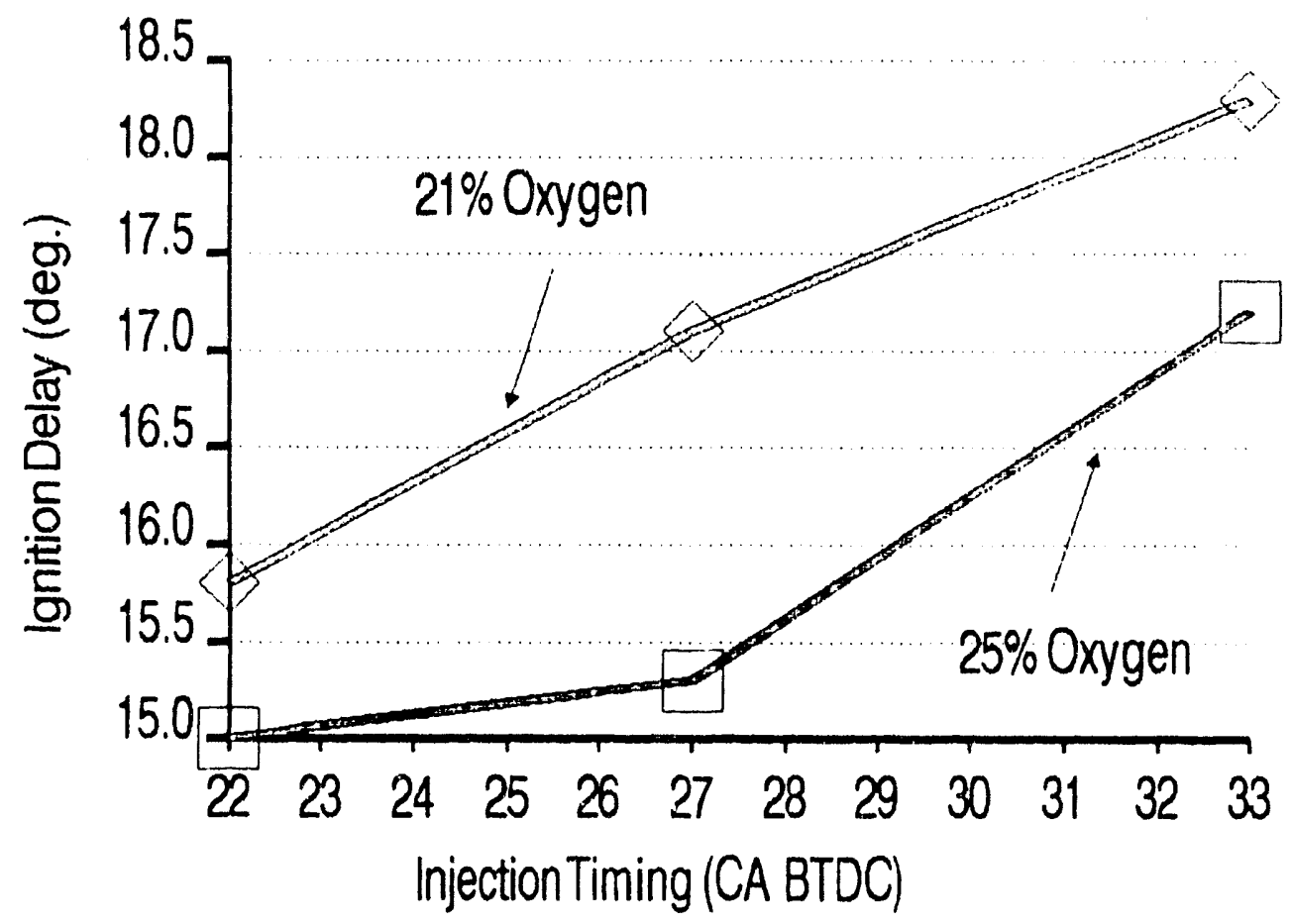

FIGURE B.126 Effect of Injection Timing on Ignition Delay, 50 Brake Horsepower, No. 4 Fuel, $5 \%$ Water 


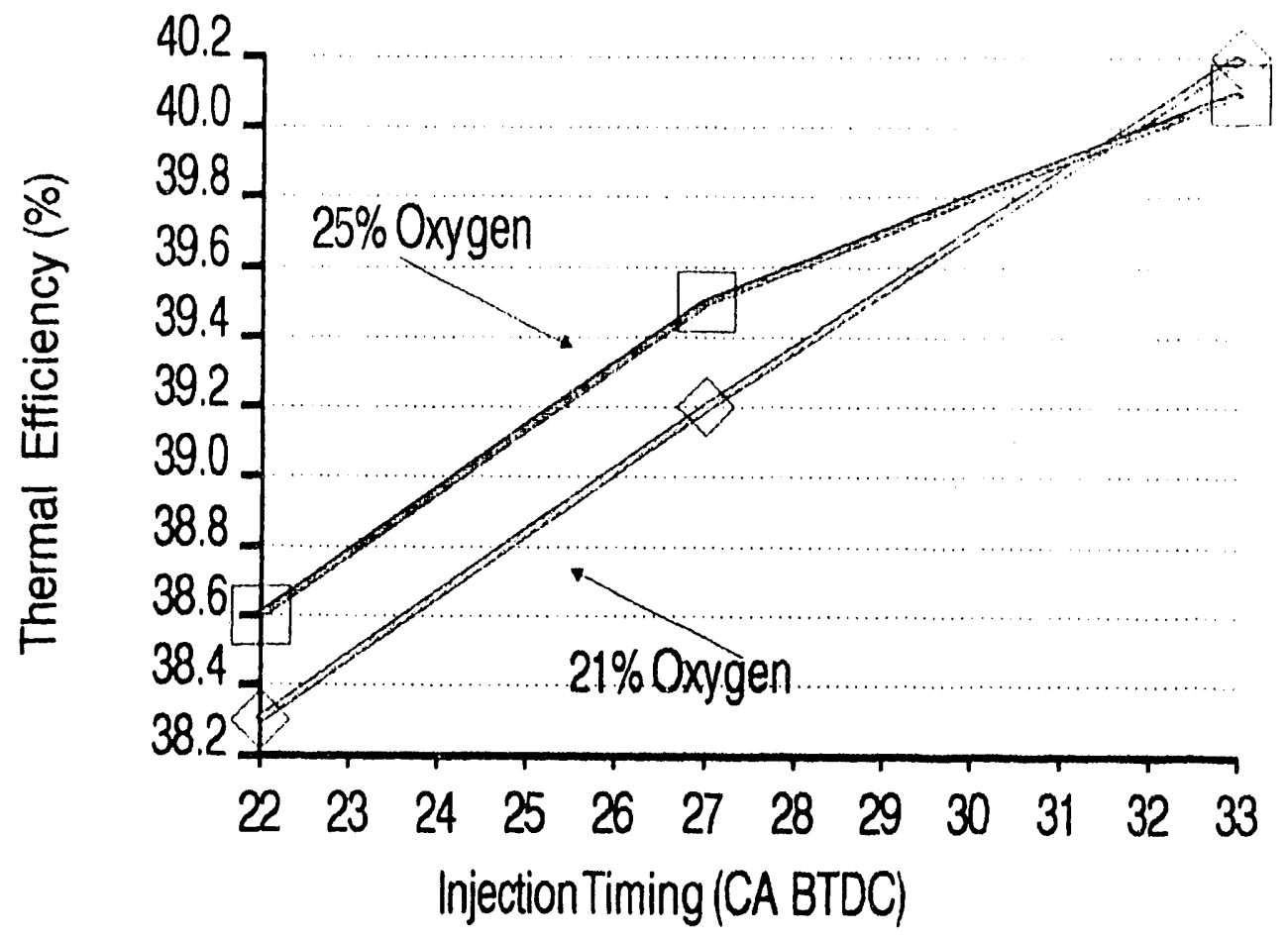

FIGURE B.127 Effect of Injection Timing on Thermal Efficiency, 50 Brake Horsepower, No. 4 Fuel, $5 \%$ Water

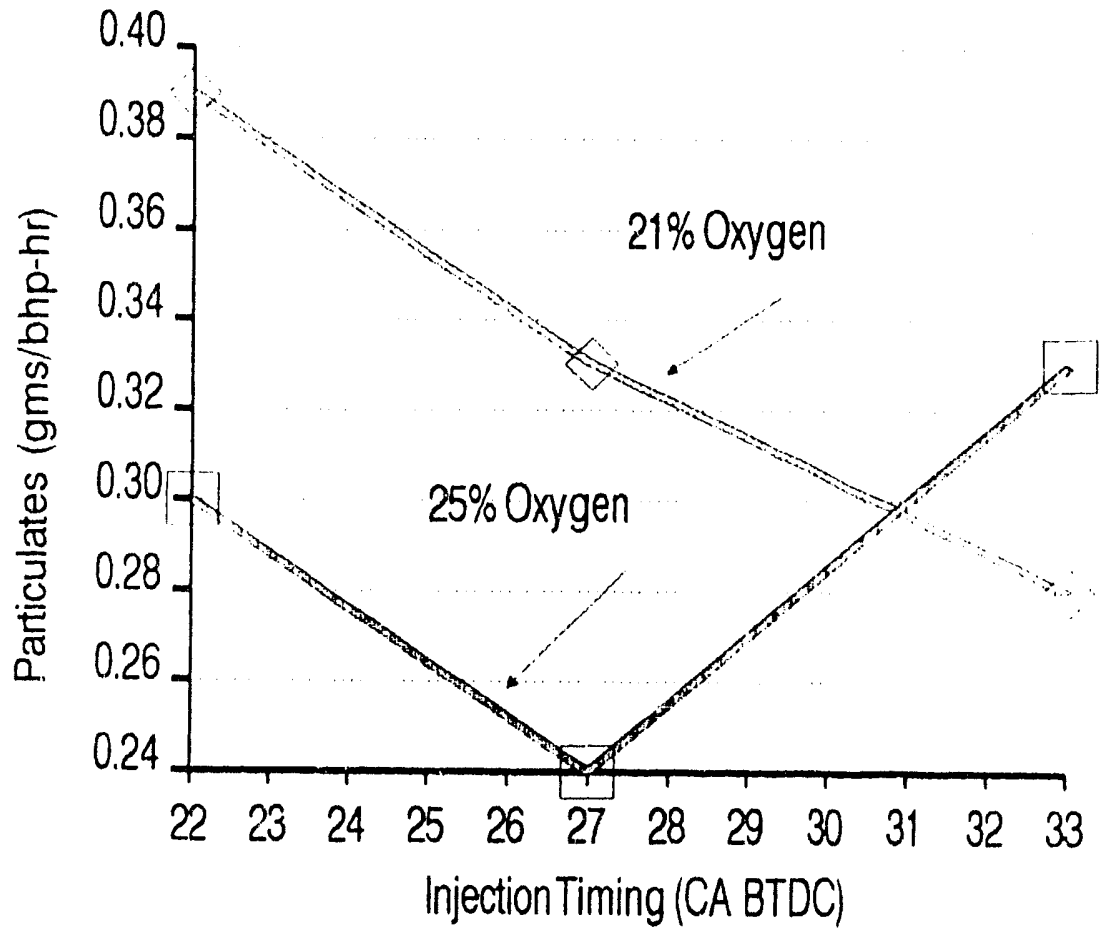

FIGURE B.128 Effect of Injection Timing on Particulate-Matter Emission, 50 Brake Horsepower, No. 4 Fuel, 5\% Water 


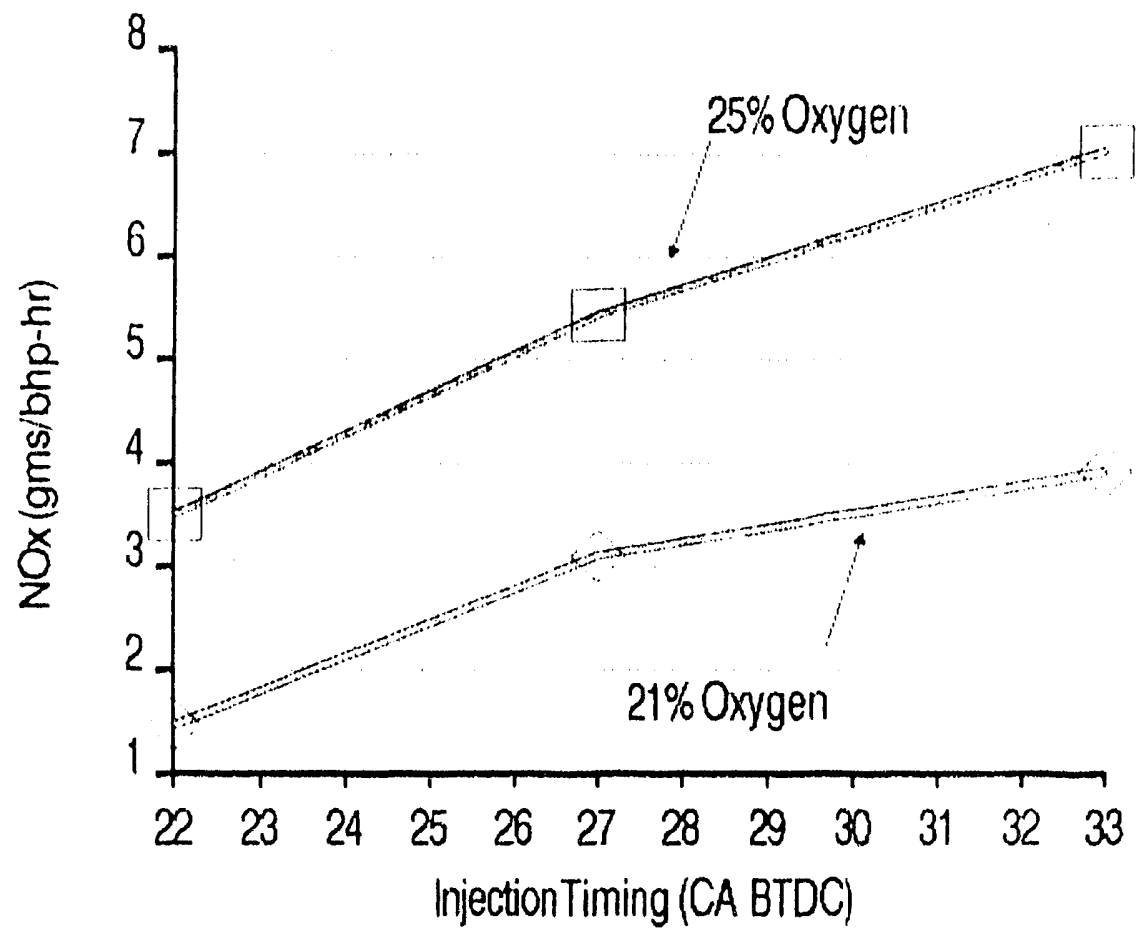

FIGURE B.129 Effect of Injection Timing on $\mathrm{NO}_{x}$ Emission, 50 Brake Horsepower, No. 4 Fuel, 5\% Water

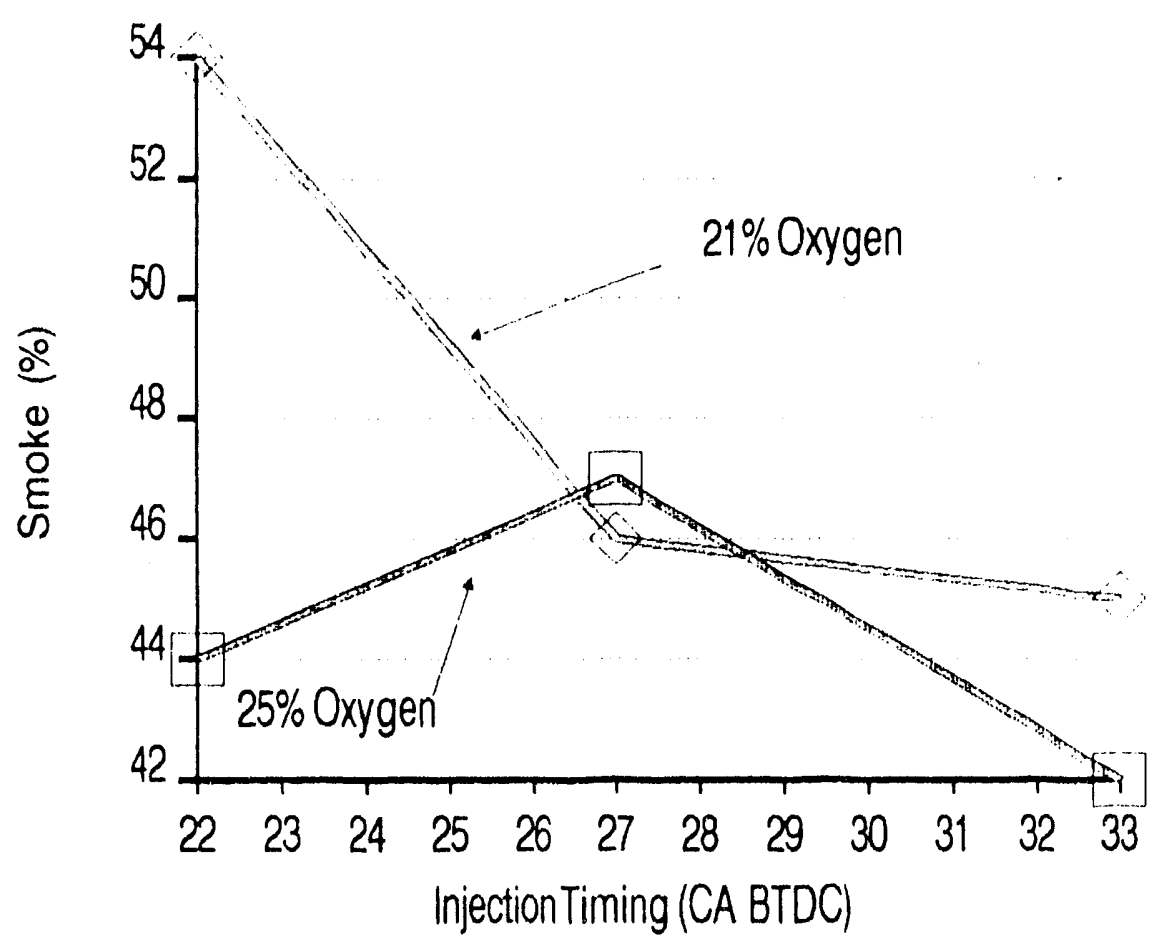

FIGURE B.130 Effect of Injection Timing on Smoke Emission, 50 Brake Horsepower, No. 4 Fuel, $5 \%$ Water 


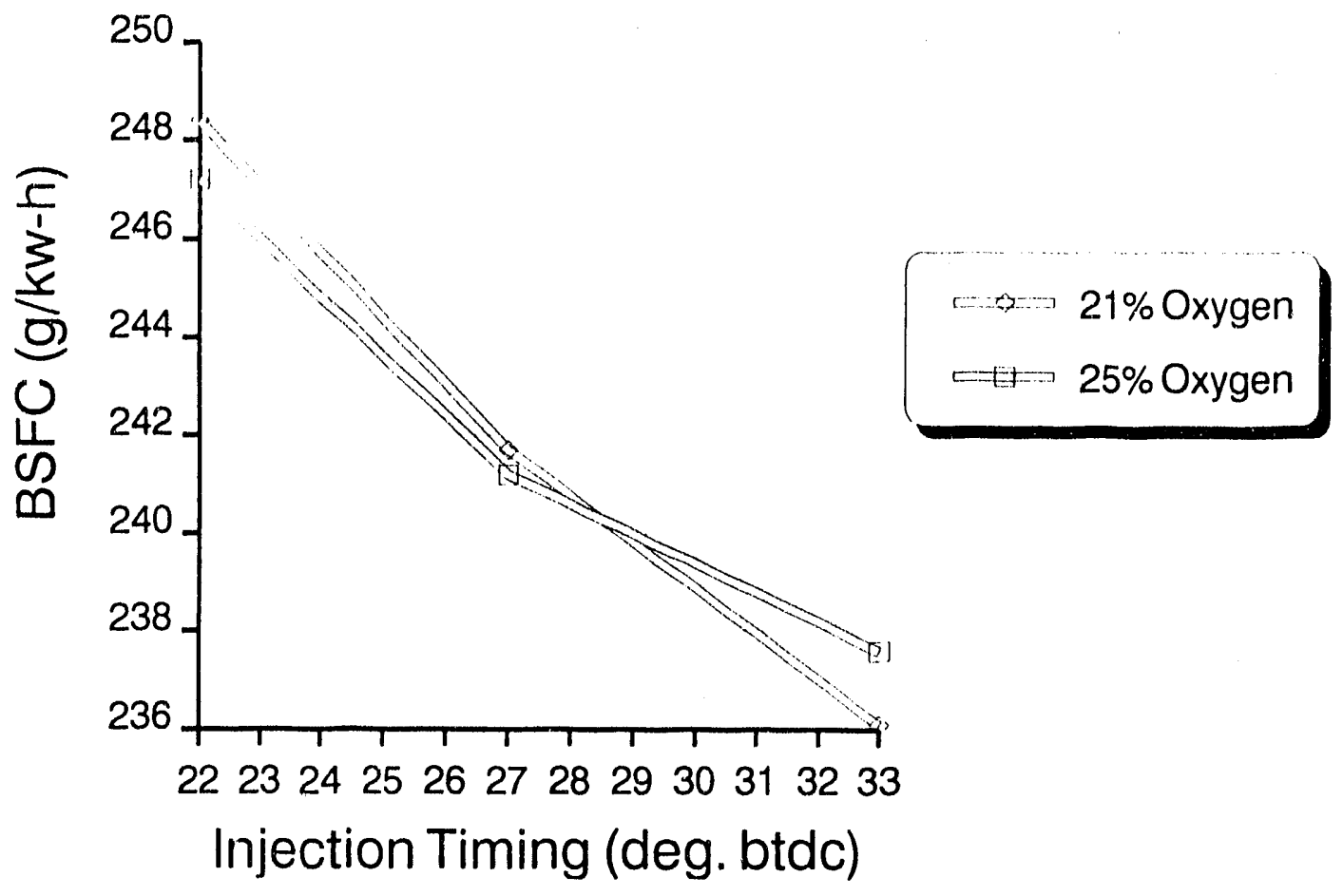

FIGURE B.131 Effect of Injection Timing on Brake-Specific Fuel Consumption, 50 Brake Horsepower, No. 4 Fuel, $5 \%$ Water

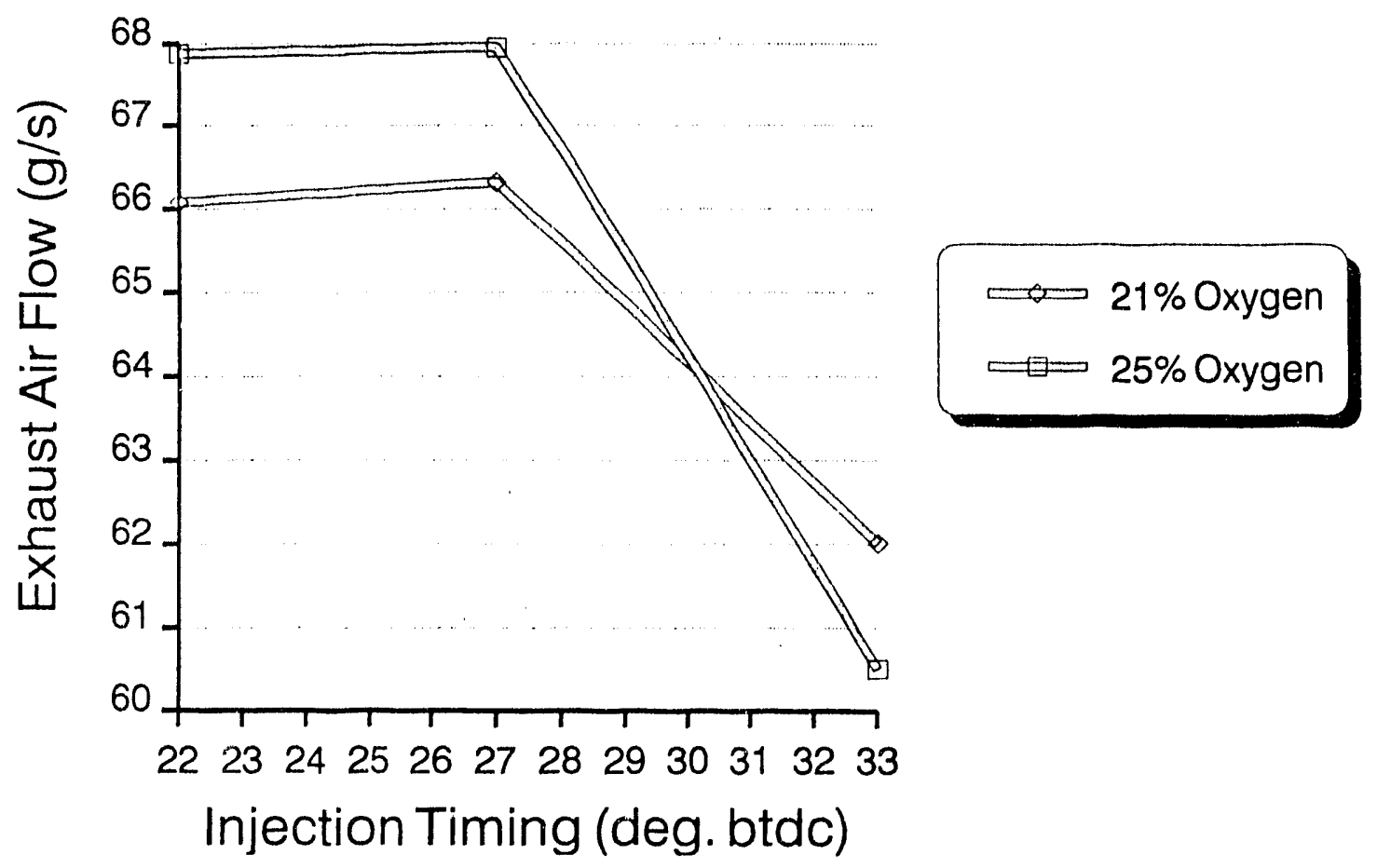

FIGURE B.132 Effect of Injection Timing on Exhaust Air Flow, 50 Brake Horsepower, No. 4 Fuel, $5 \%$ Water 
B.7 Graphic Display of Cylinder Pressure Data and Results of Heat-Release Analysis for No. 4 Fuel 
B. 106 


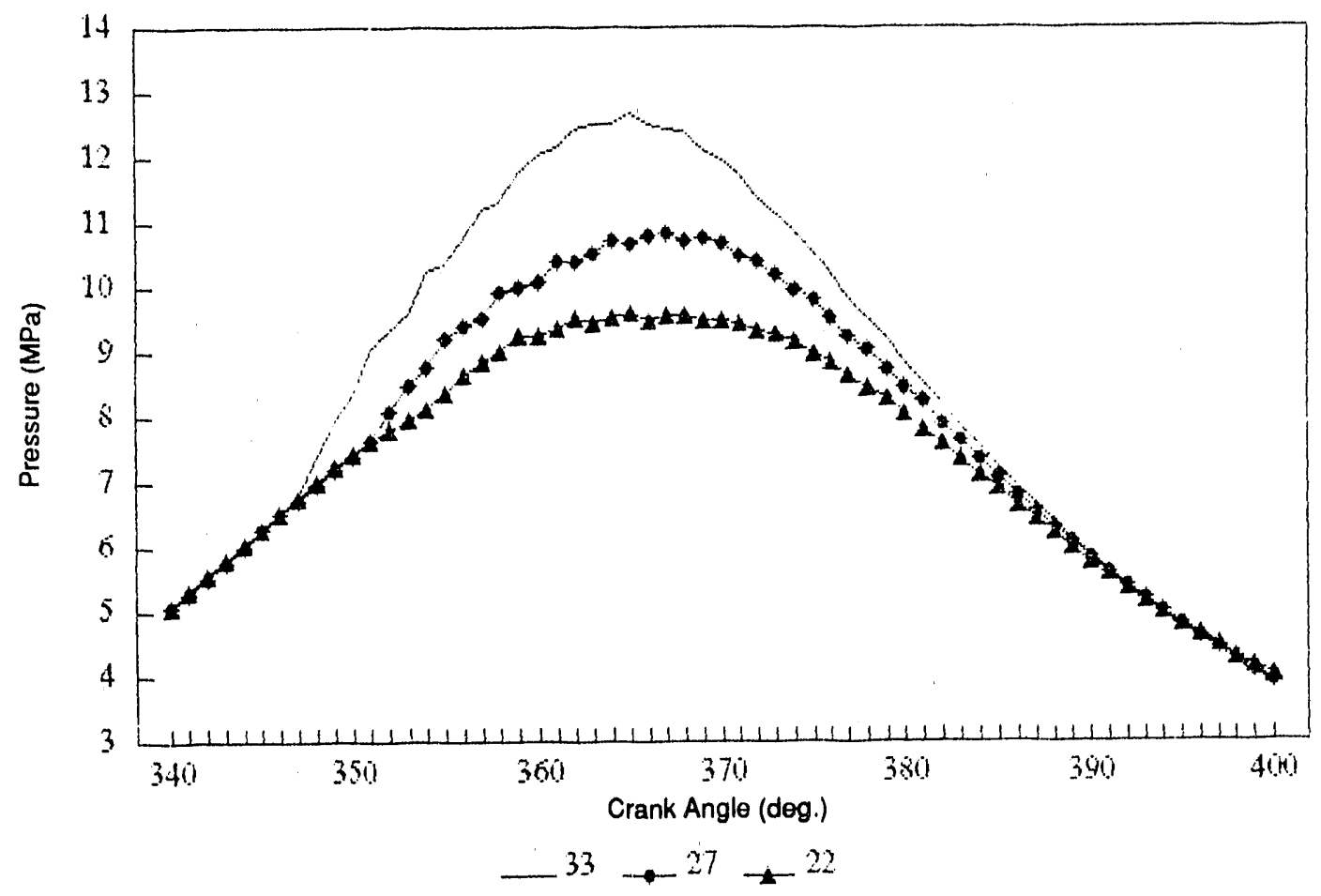

FIGURE B.133 Pressure, No. 4 Fuel, Full Load, No Water, 21\% Oxygen

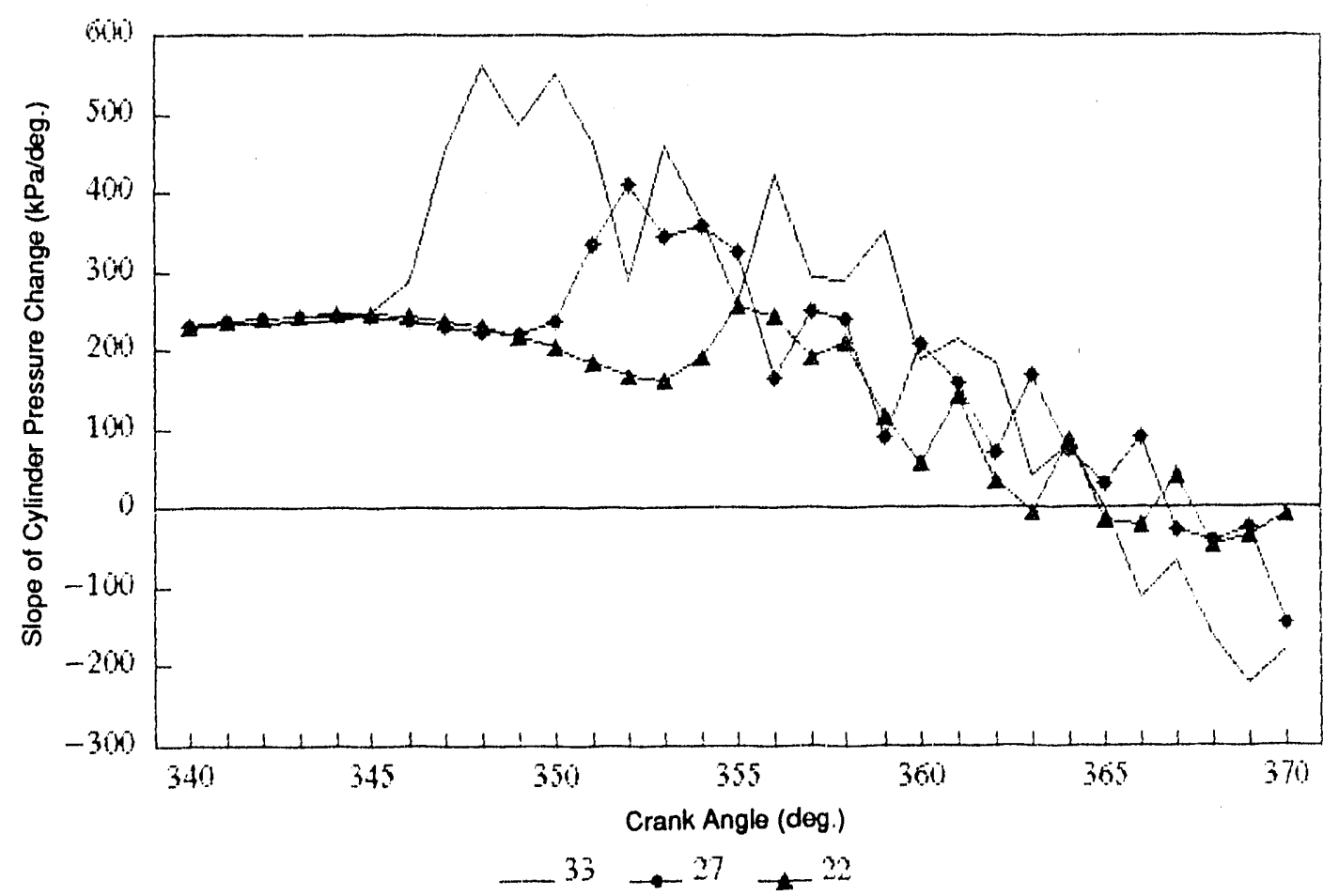

FIGURE B.134 Slope of Cylinder Pressure Change, No. 4 Fuel, Full Load, No Water, $21 \%$ Oxygen 


\section{$B-108$}

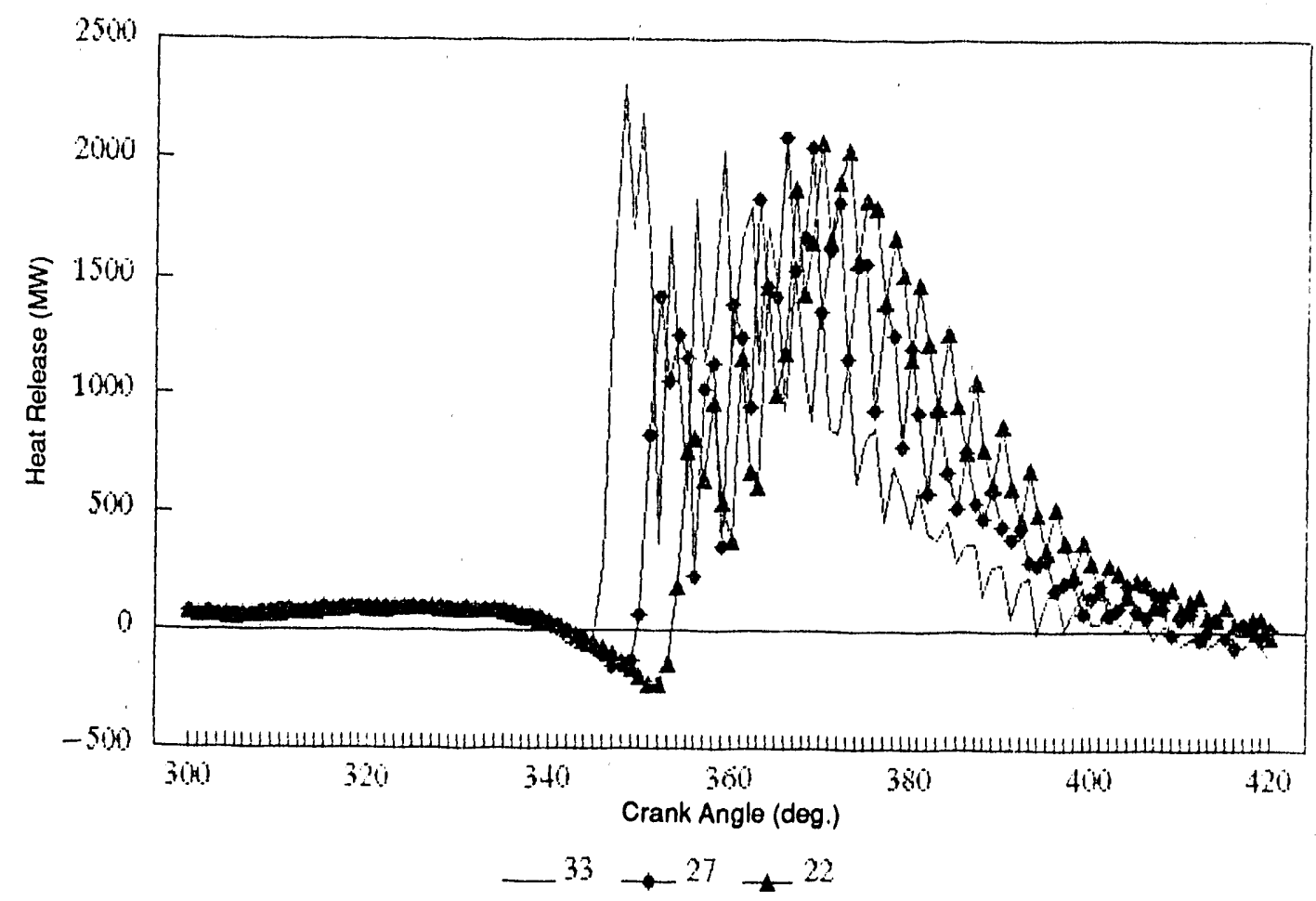

FIGURE B.135 Heat Release, No. 4 Fuel, Full Load, No Water, 21\% Oxygen

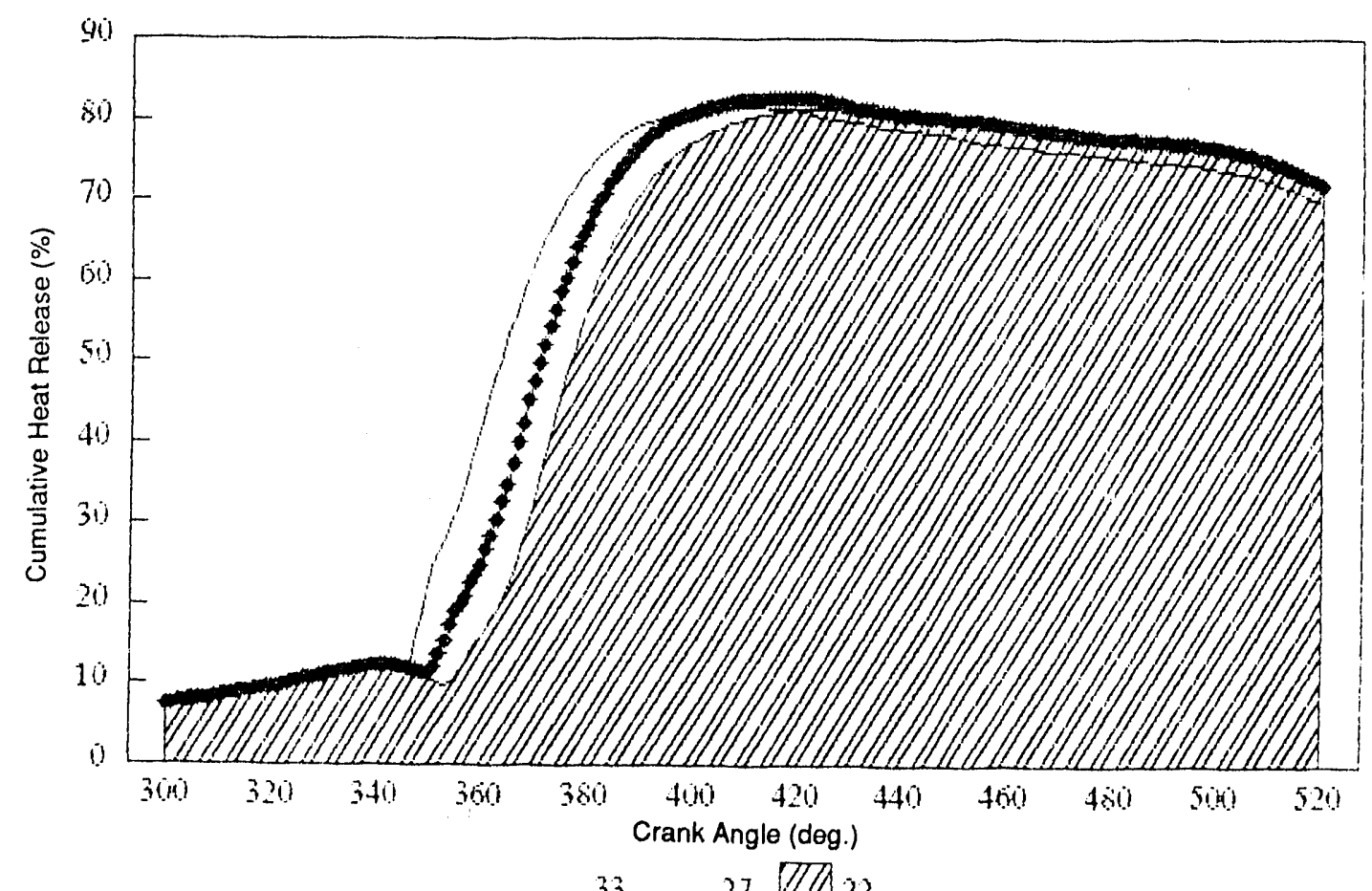

FIGURE B.136 Cumulative Heat Release, No. 4 Fuel, Full Load, No Water, 21\% Oxygen 


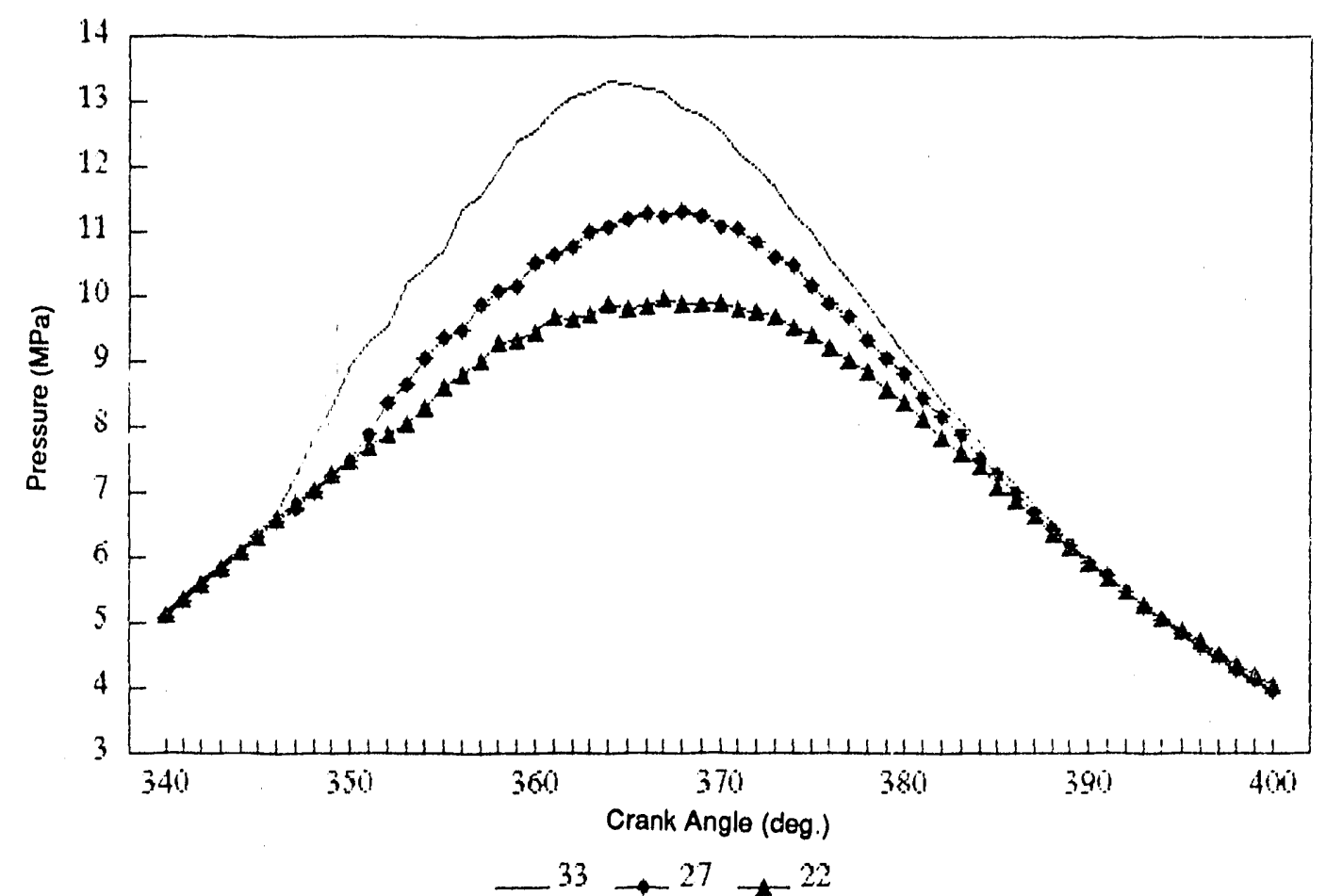

FIGURE B.137 Pressure, No. 4 Fuel, Full Load, No Water, 25\% Oxygen

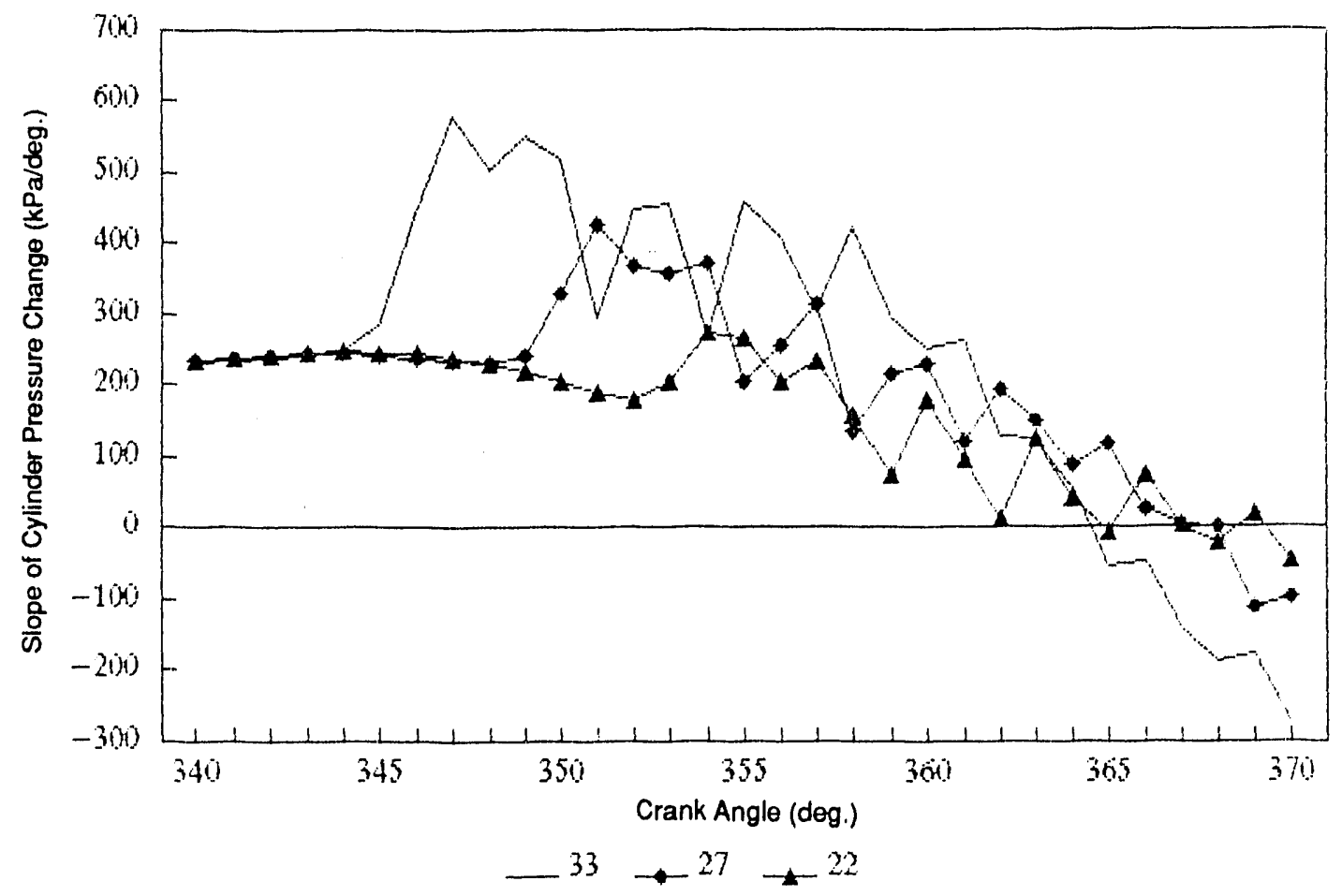

FIGURE B.138 Stope of Cylinder Pressure Change, No. 4 Fuel, No Water, 25\% Oxygen 


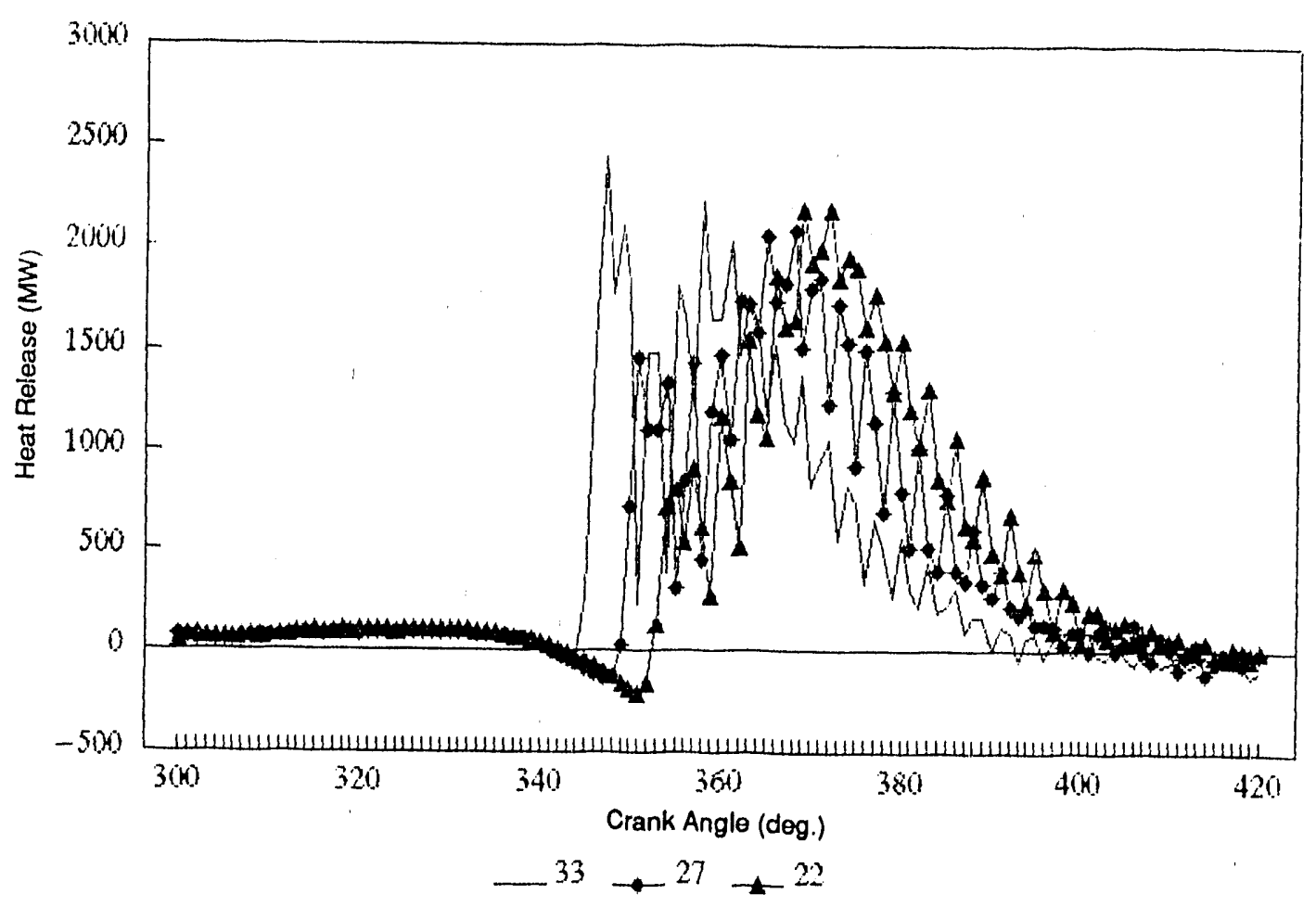

FIGURE B.139 Heat Release, No. 4 Fuel, Full Load, No Water, 25\% Oxygen

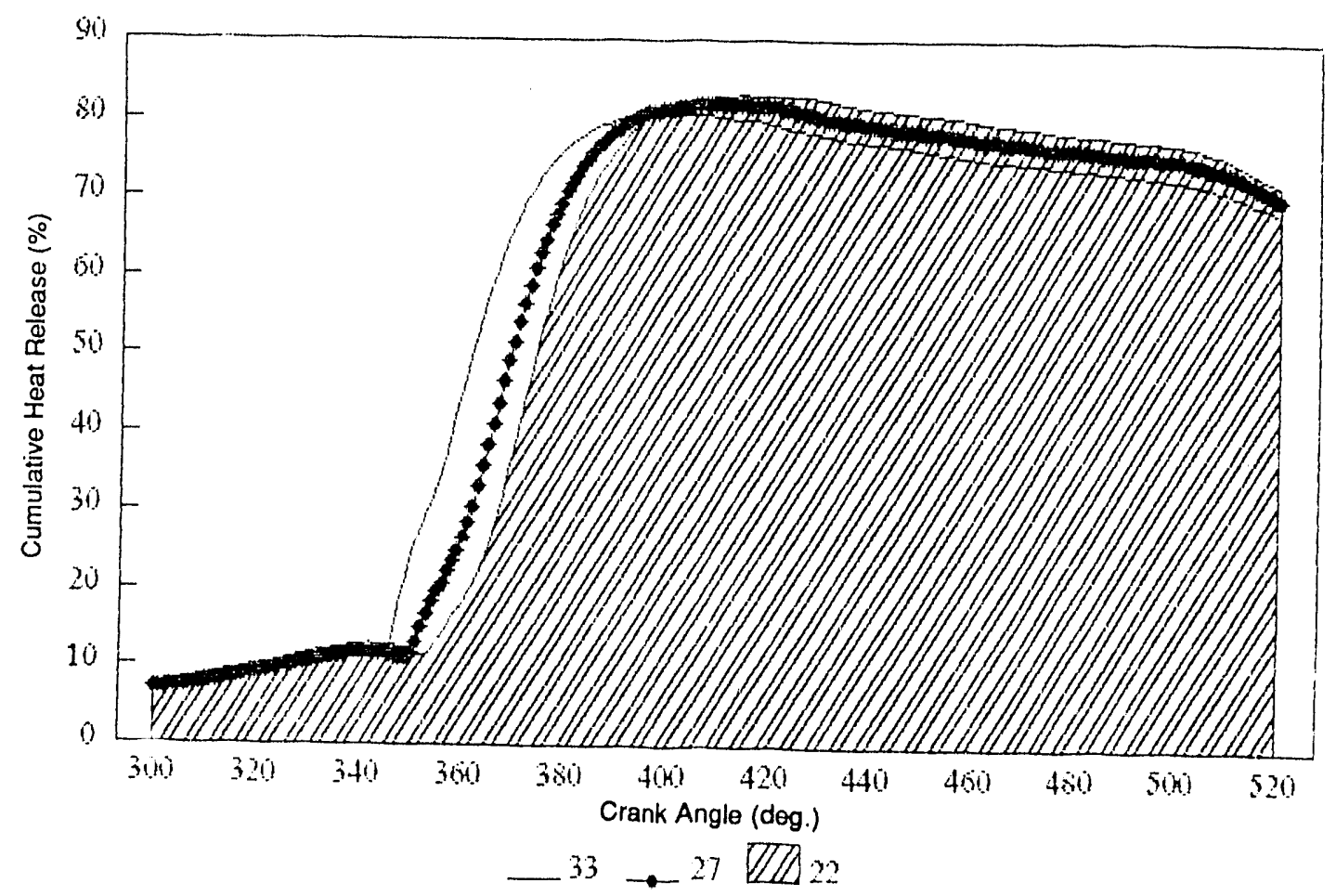

FIGURE B.140 Cumulative Heat Release, No. 4 Fuel, Full Load, No Water, 25\% Oxygen 


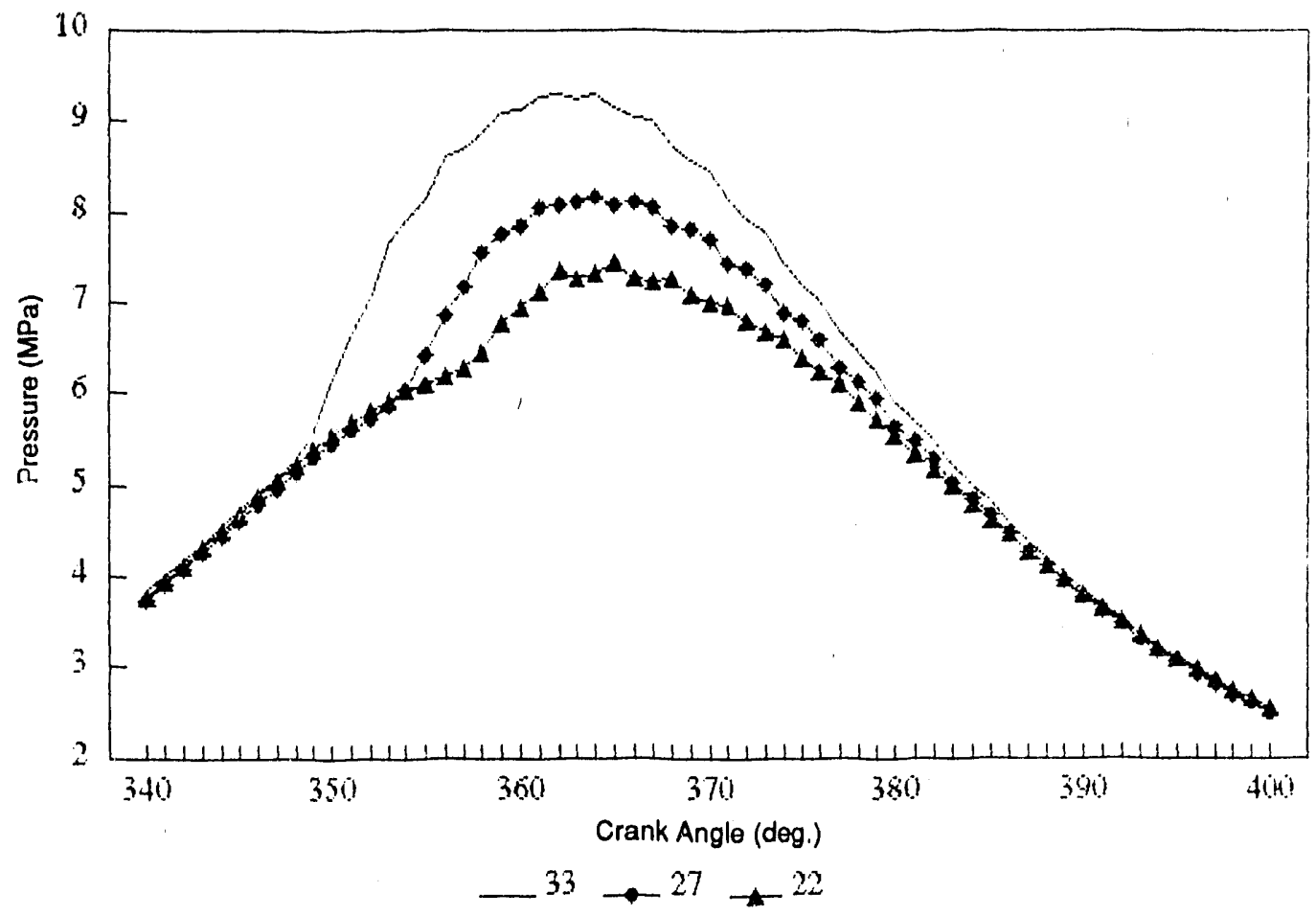

FIGURE B.141 Pressure, No. 4 Fuel, Half Load, No Water, 21\% Oxygen

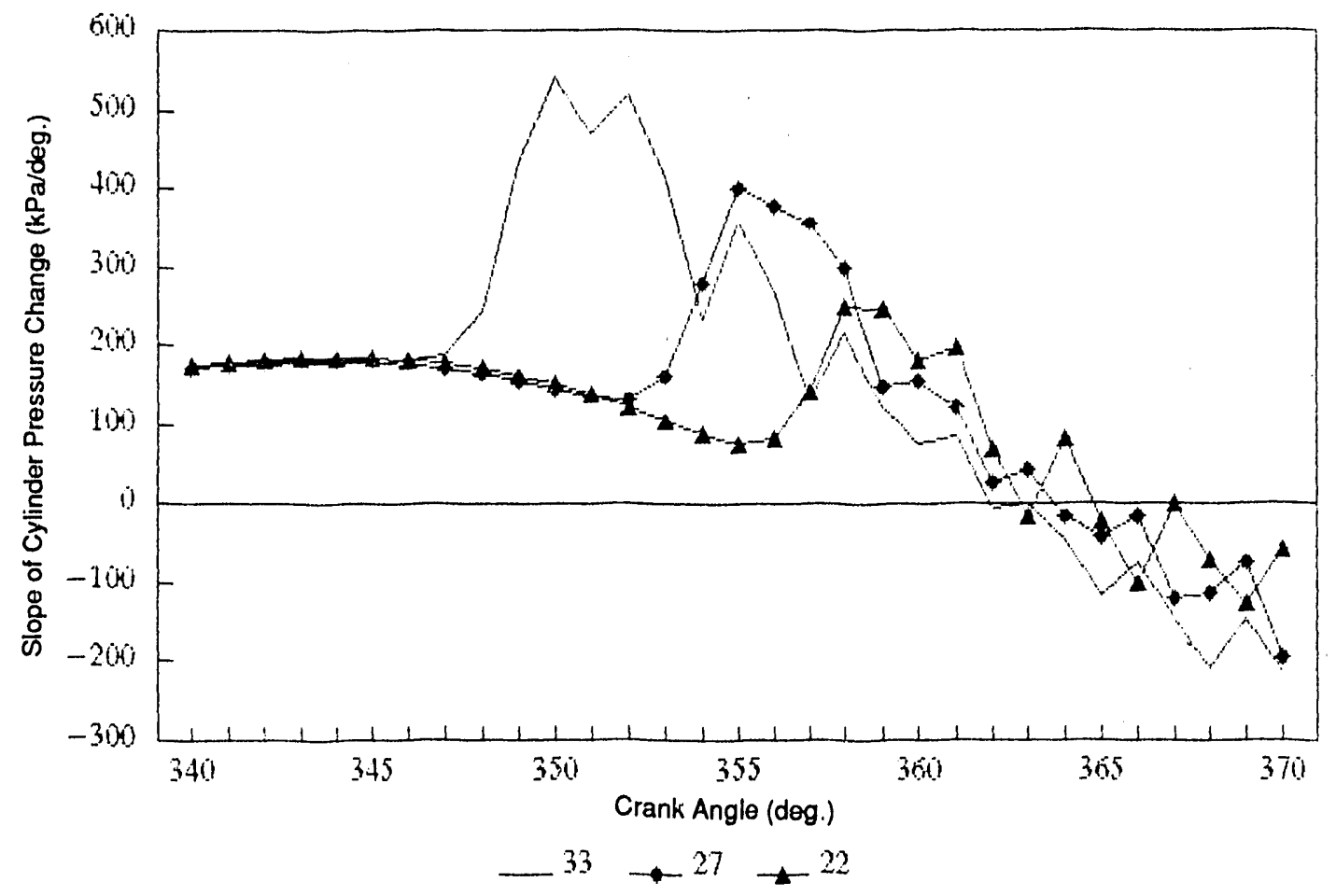

FIGURE B.142 Slope of Cylinder Pressure Change, No. 4 Fuel, Half Load, No Water, $21 \%$ Oxygen 


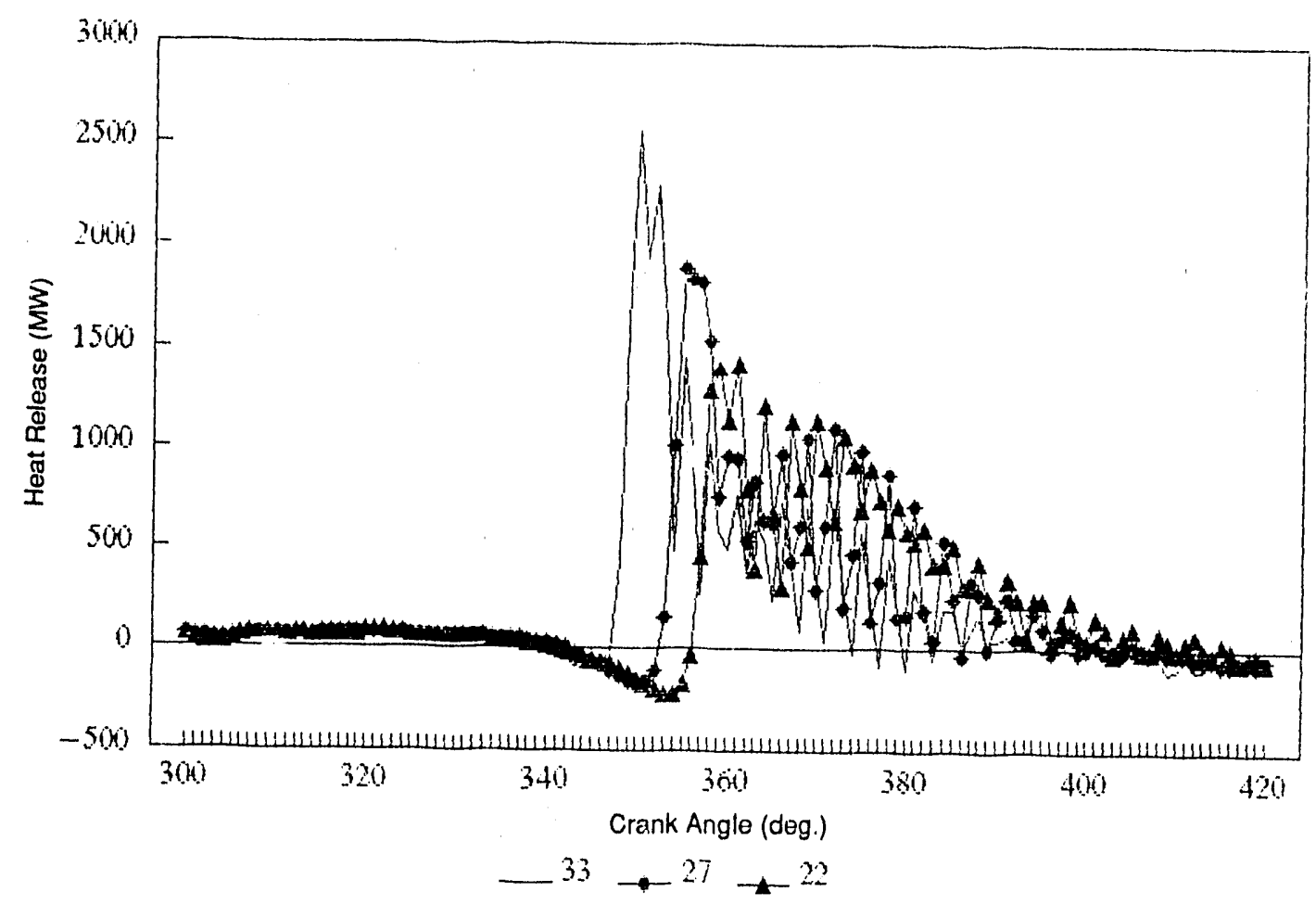

FIGURE B.143 Heat Release, No. 4 Fuel, Half Loar, No Water, 21\% Oxygen

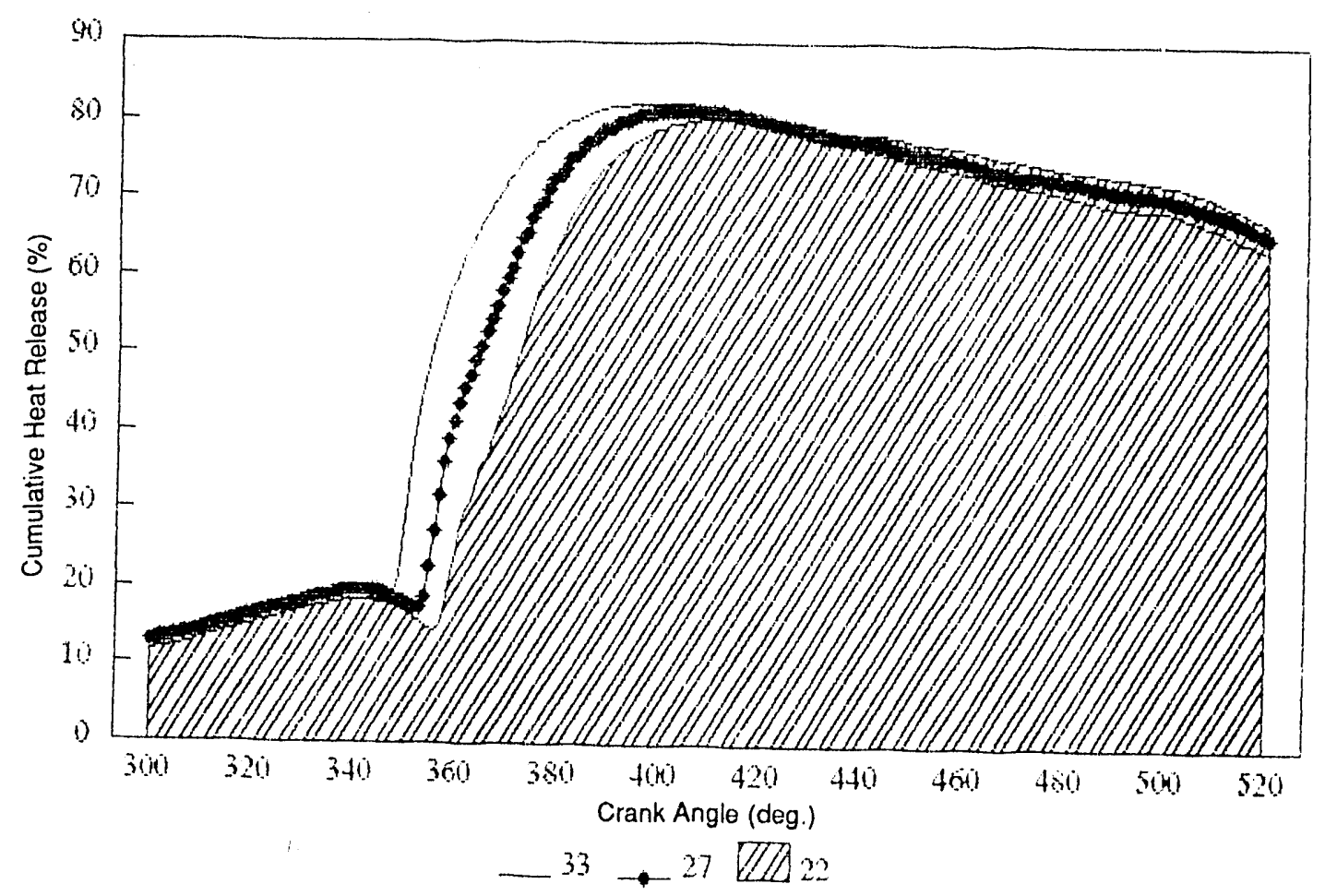

FIGURE B.144 Cumulative Heat Release, No. 4 Fuel, Half Load, No Water, 21\% Oxygen 


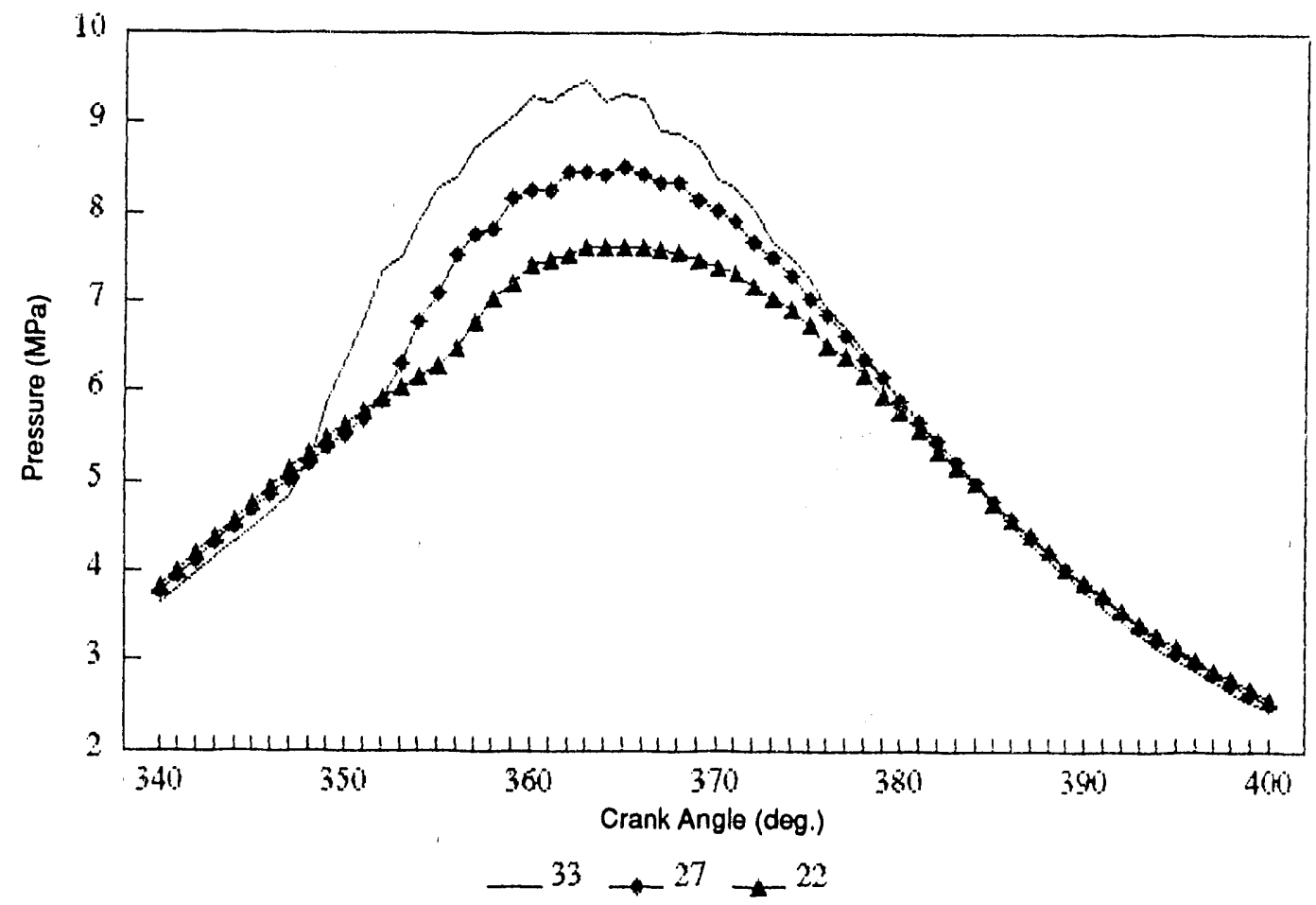

FIGURE B.145 Pressure, No. 4 Fuel, Half Load, No Water, 27\% Oxygen

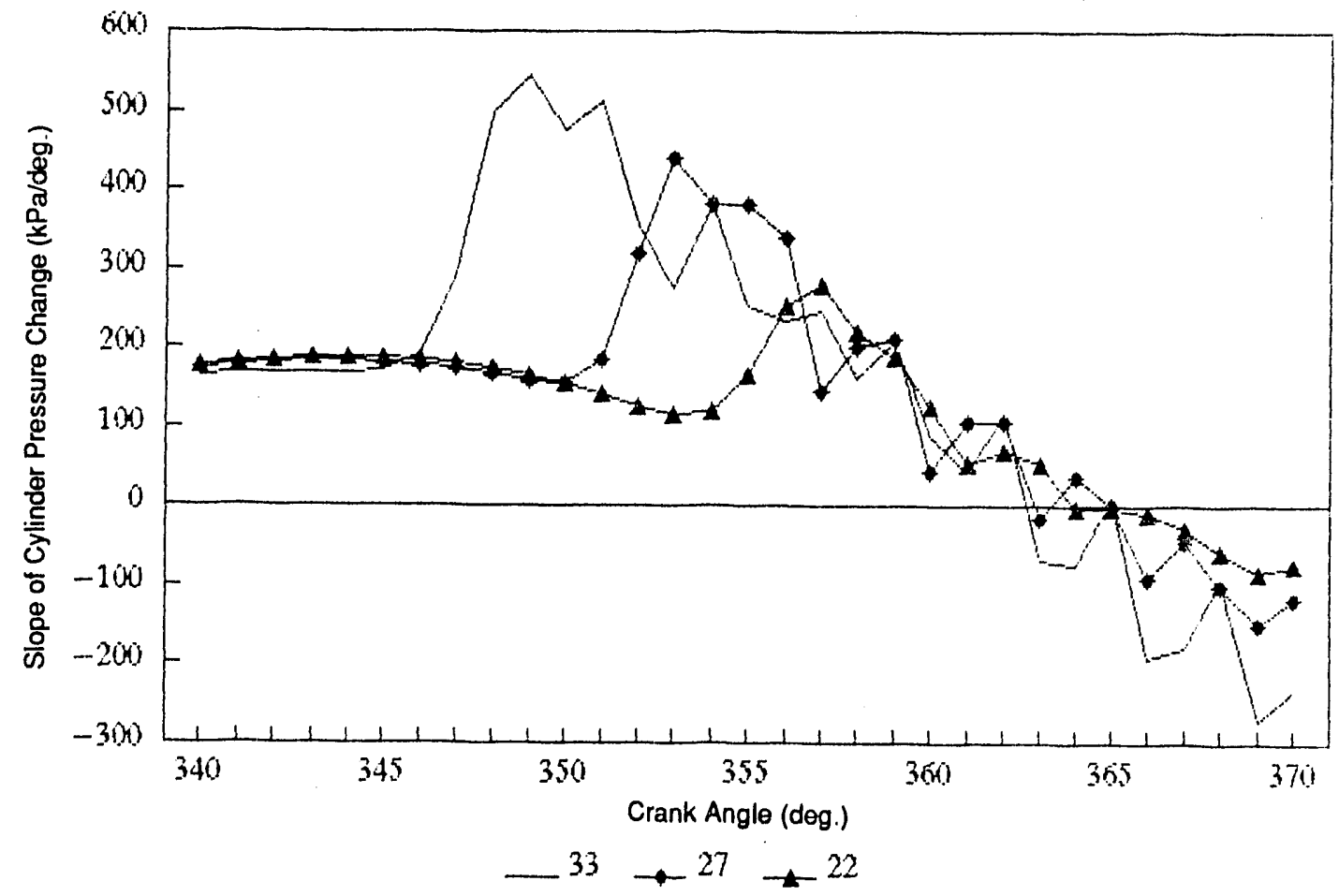

FIGURE B.146 Slope of Cylinder Pressure Change, No. 4 Fuel, Half Load, No Water, $27 \%$ Oxygen 


\section{$B-114$}

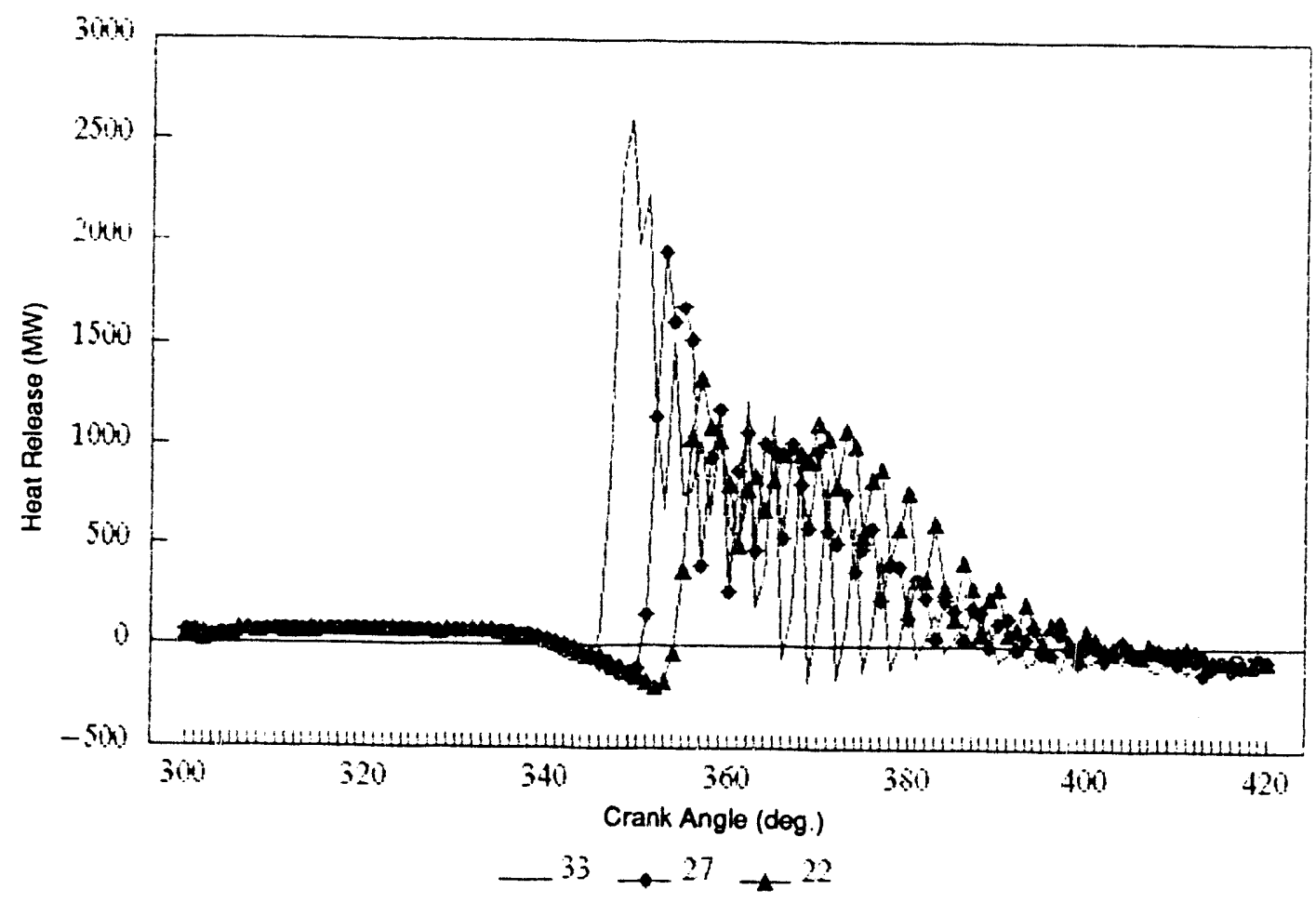

FIGURE B.147 Heat Release, No. 4 Fuel, Half Load, No Wuter, 27\% Oxygen

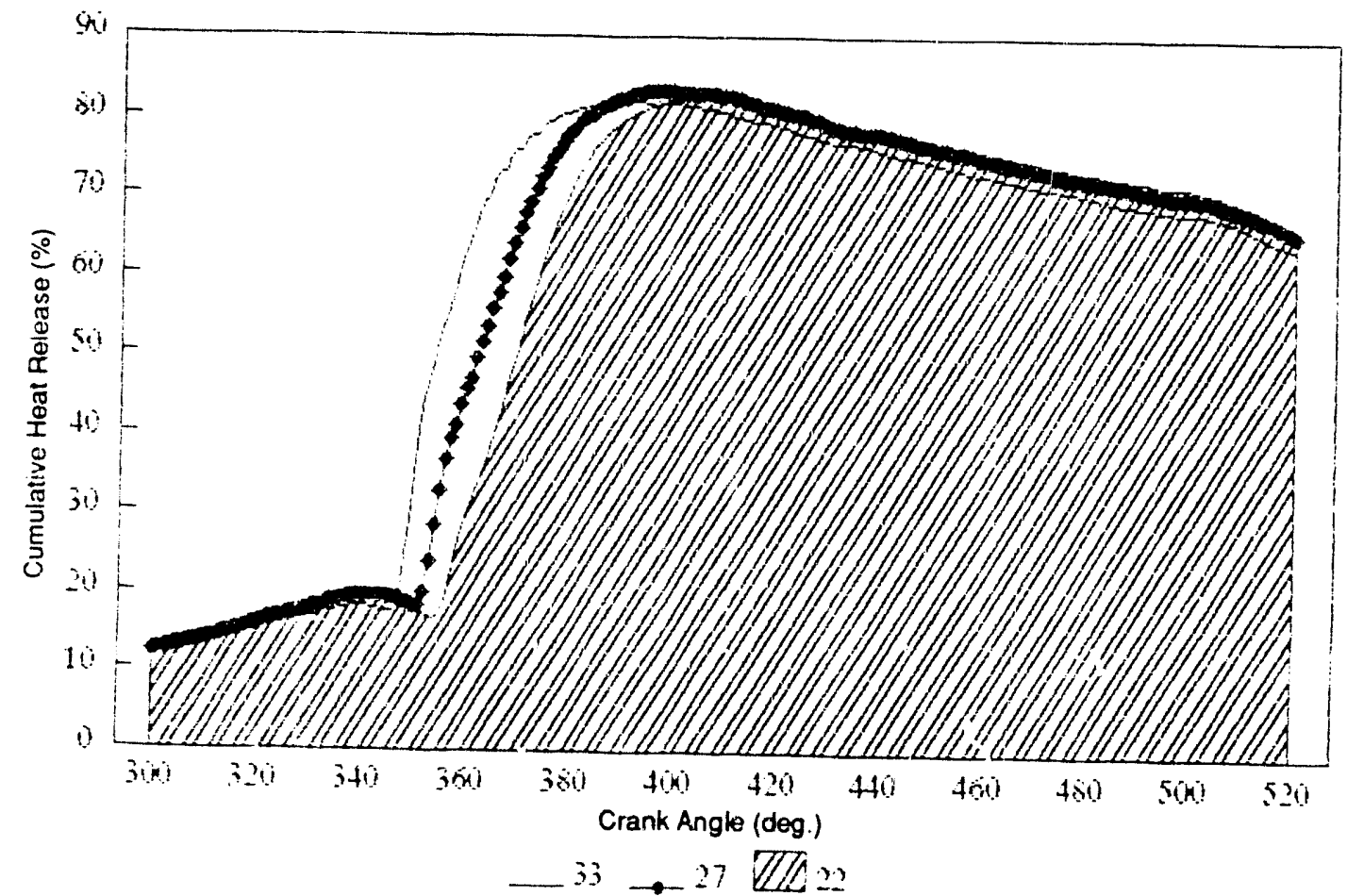

FIGURE B.148 Cumulative Heat Release, No. 4 Fuel, Half Load, No Water, 27\% Oxygen 


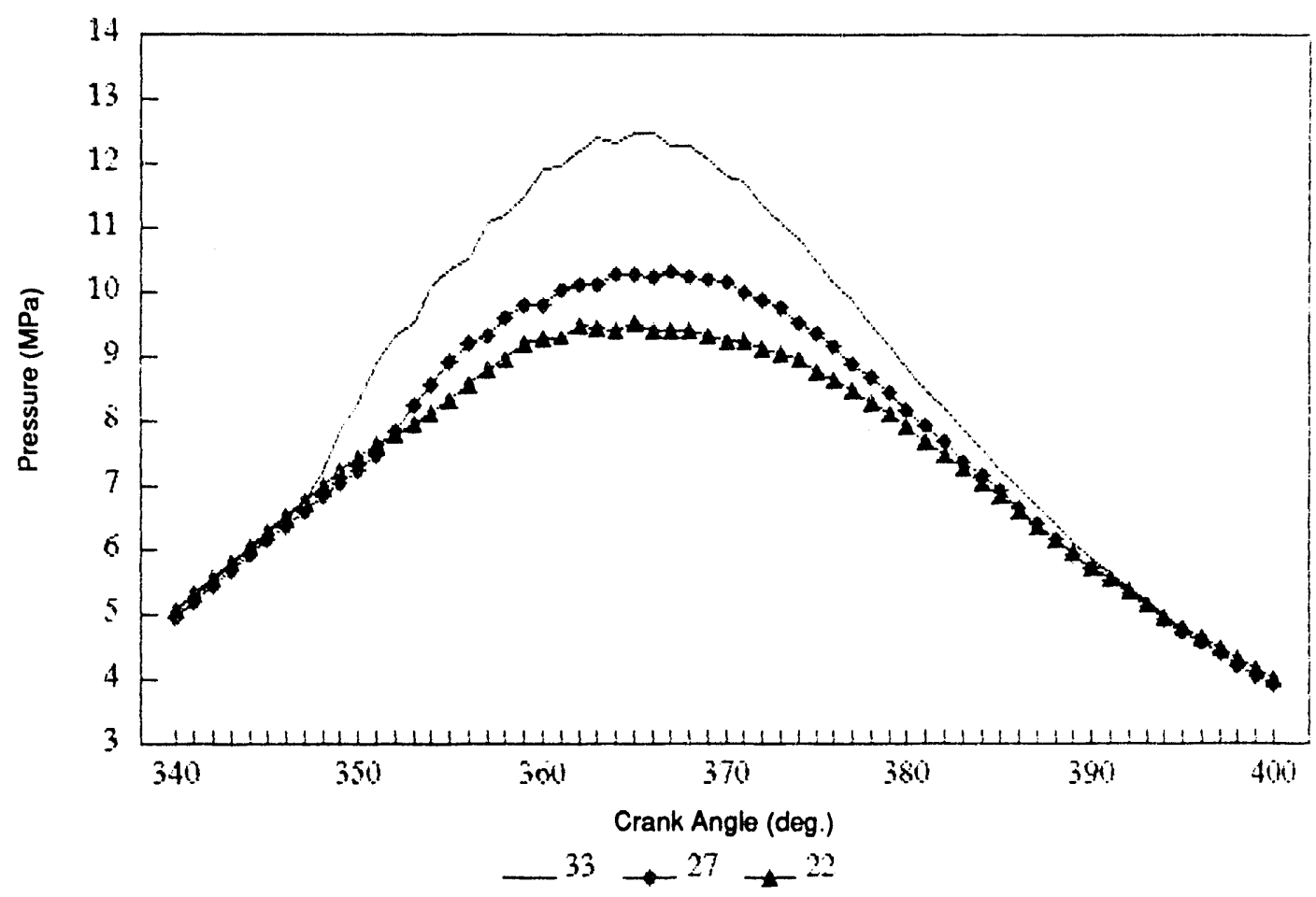

FIGURE B.149 Pressure, No. 4 Fuel, Full Load, 5\% Water, 21\% Oxygen

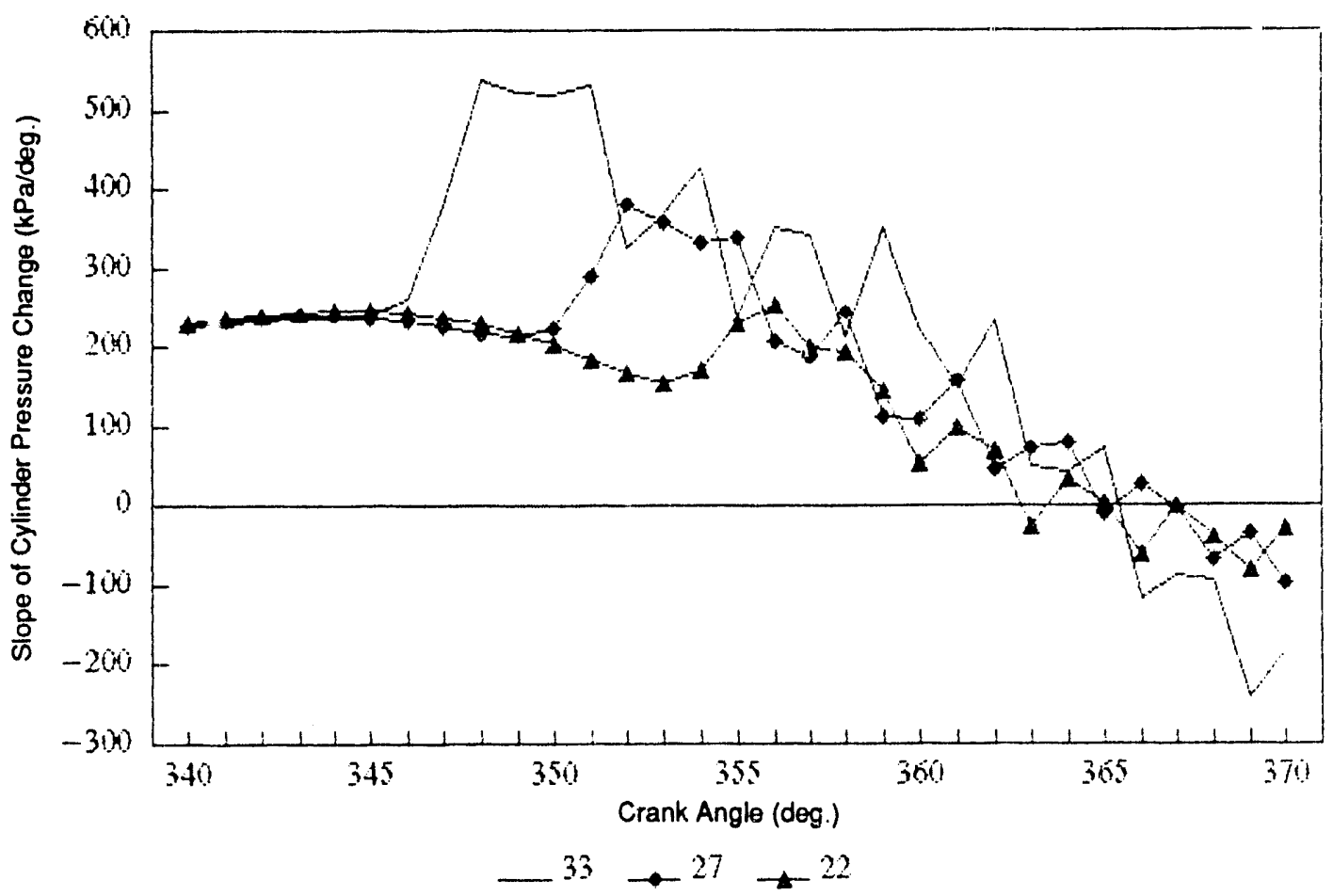

FIGURE B.150 Slope of Cylinder Pressure Change, No. 4 Fuel, Full Load, 5\% Water, $21 \%$ Oxygen 


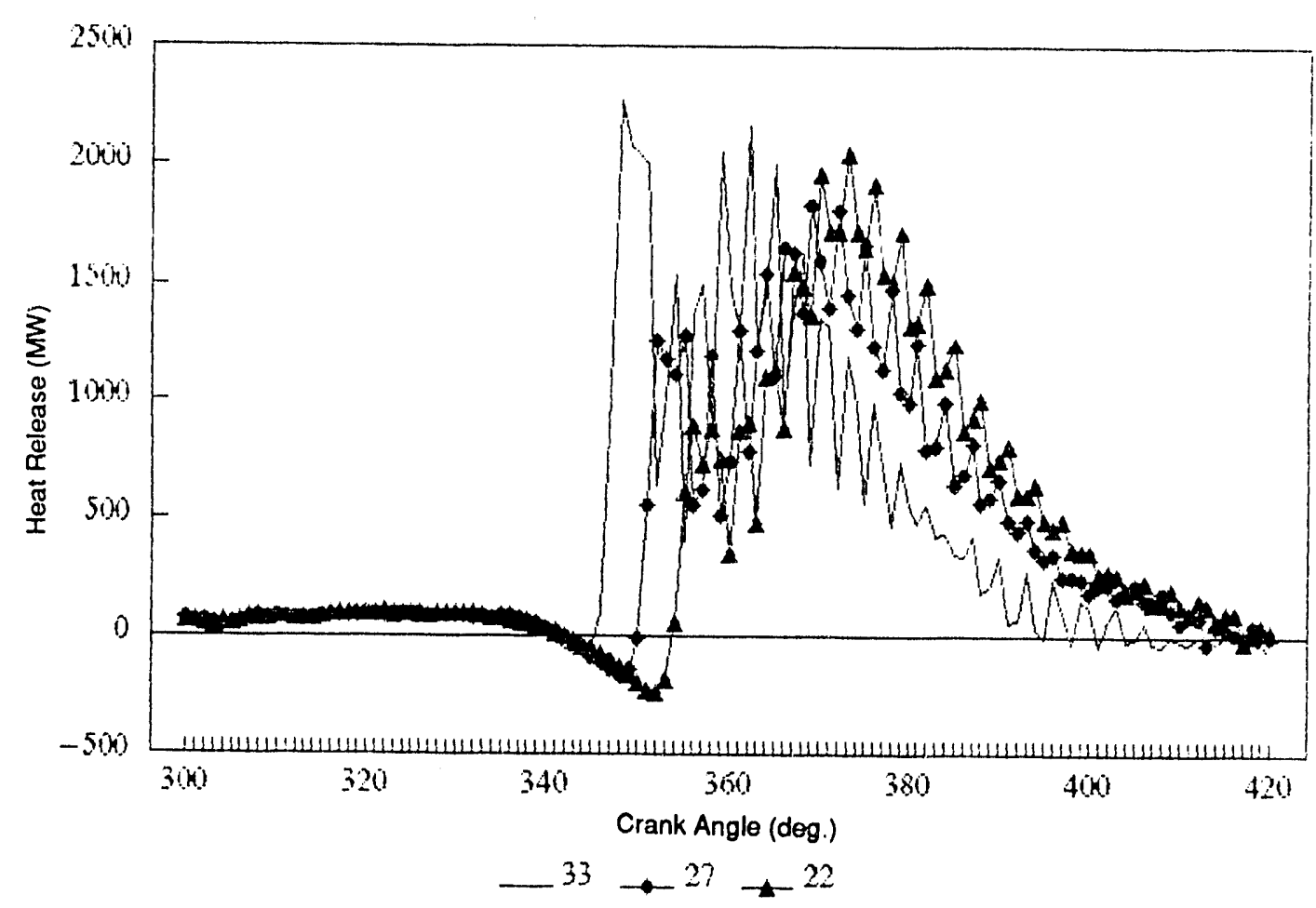

FIGURE B.151 Heat Release, No. 4 Fuel, Full Load, 5\% Water, 21\% Oxygen

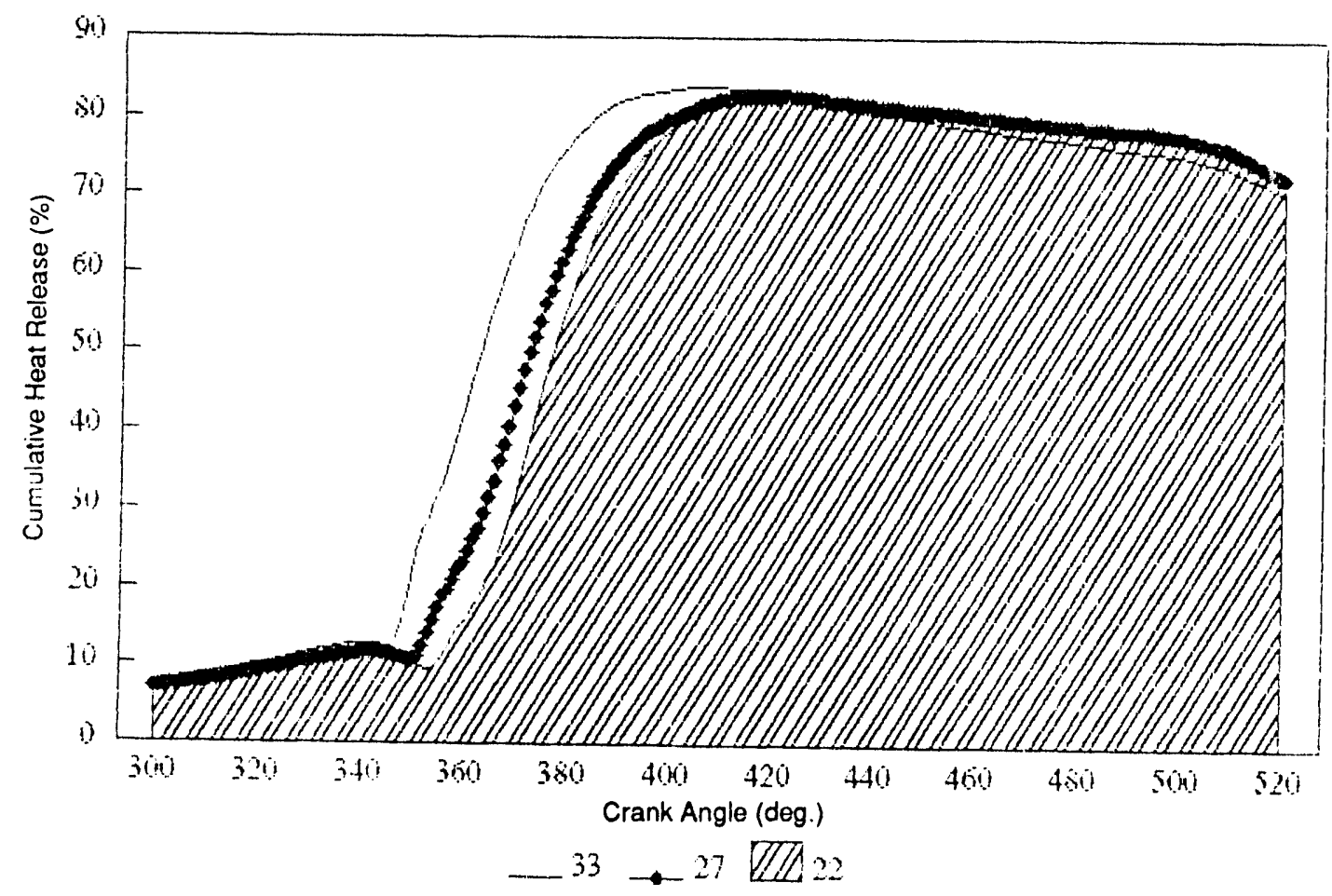

FIGURE B.152 Cumulative Heat Release, No. 4 Fuel, Full Load, 5\% Water, 21\% Oxygen 


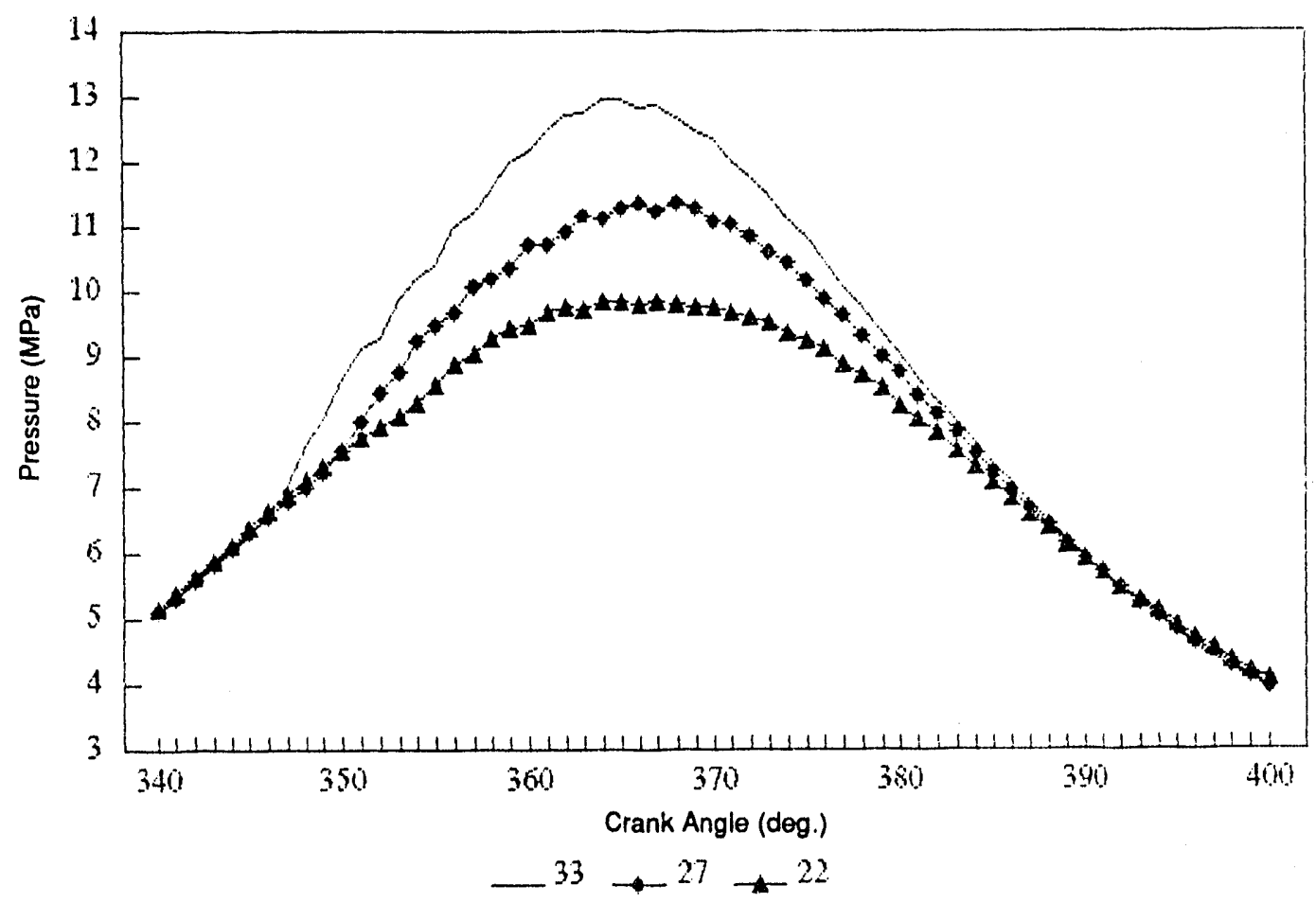

FIGURE B.153 Pressure, No. 4 Fuel, Full Load, 5\% Water, 25\% Oxygen

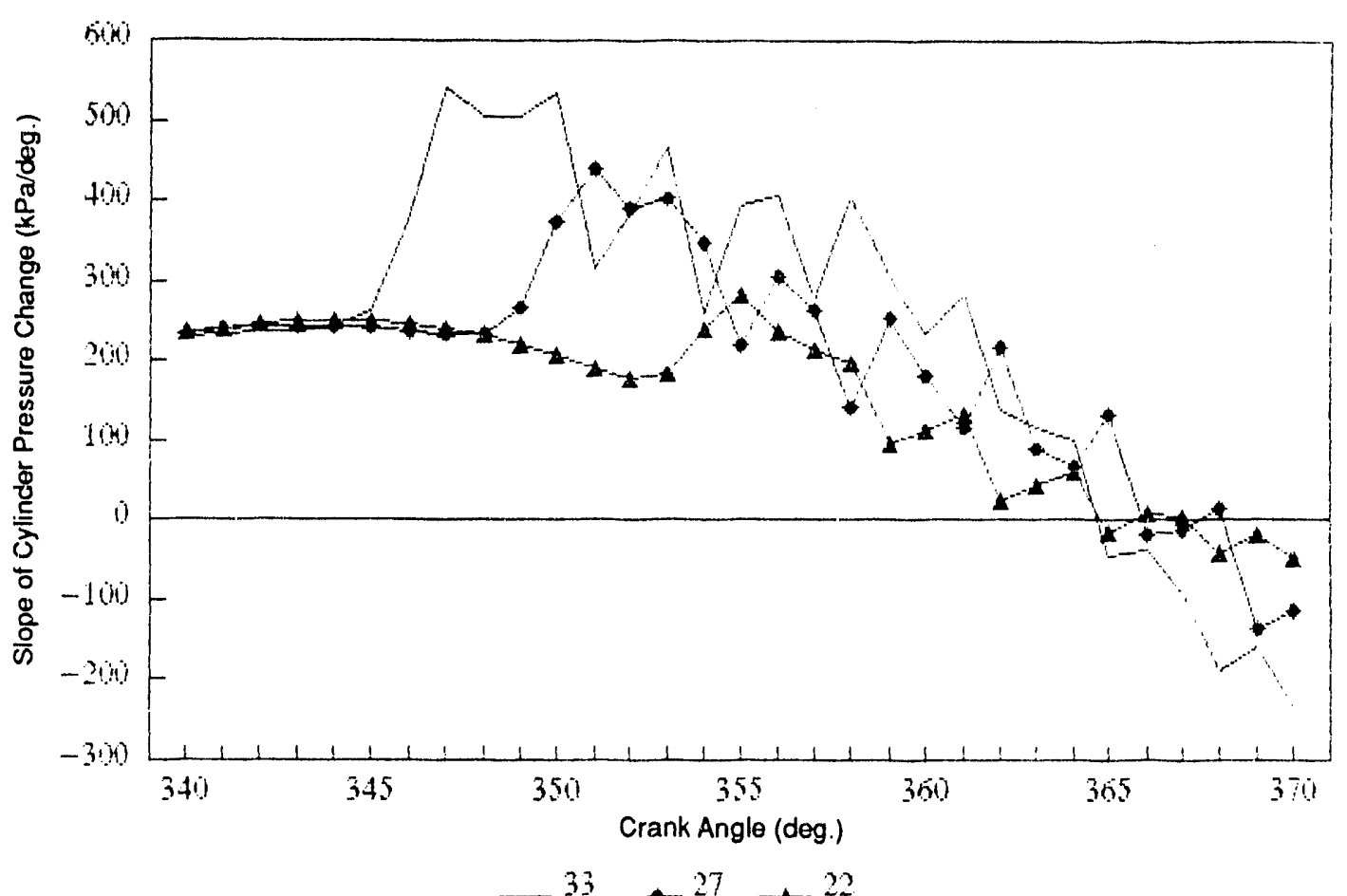

FIGURE B.154 Slope of Cylinder Pressure Change, No. 4 Fuel, Full Load, 5\% Water, $25 \%$ Oxygen 


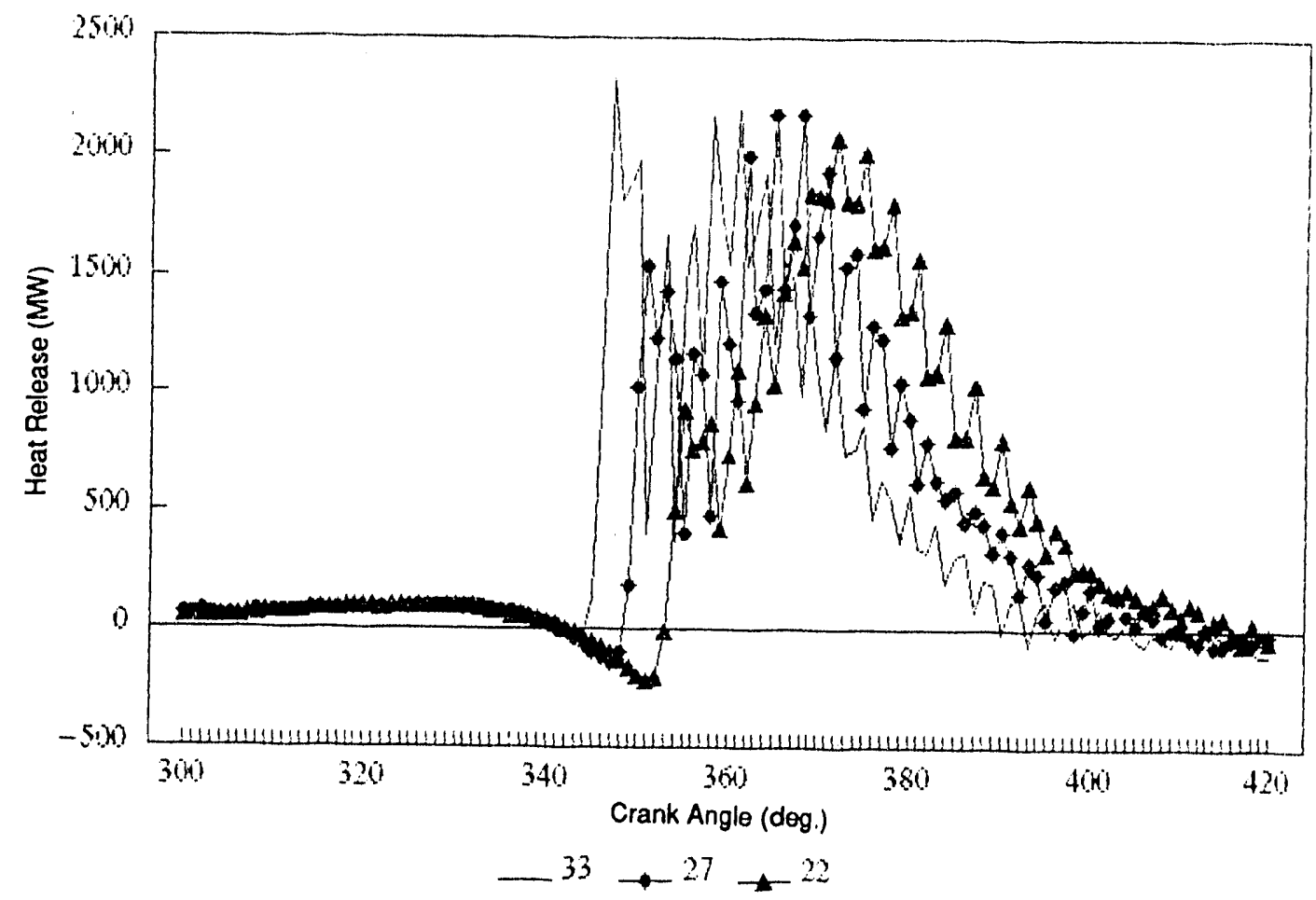

FIGURE B.155 Heat Release, No. 4 Fuel, Full Load, 5\% Water, 25\% Oxygen

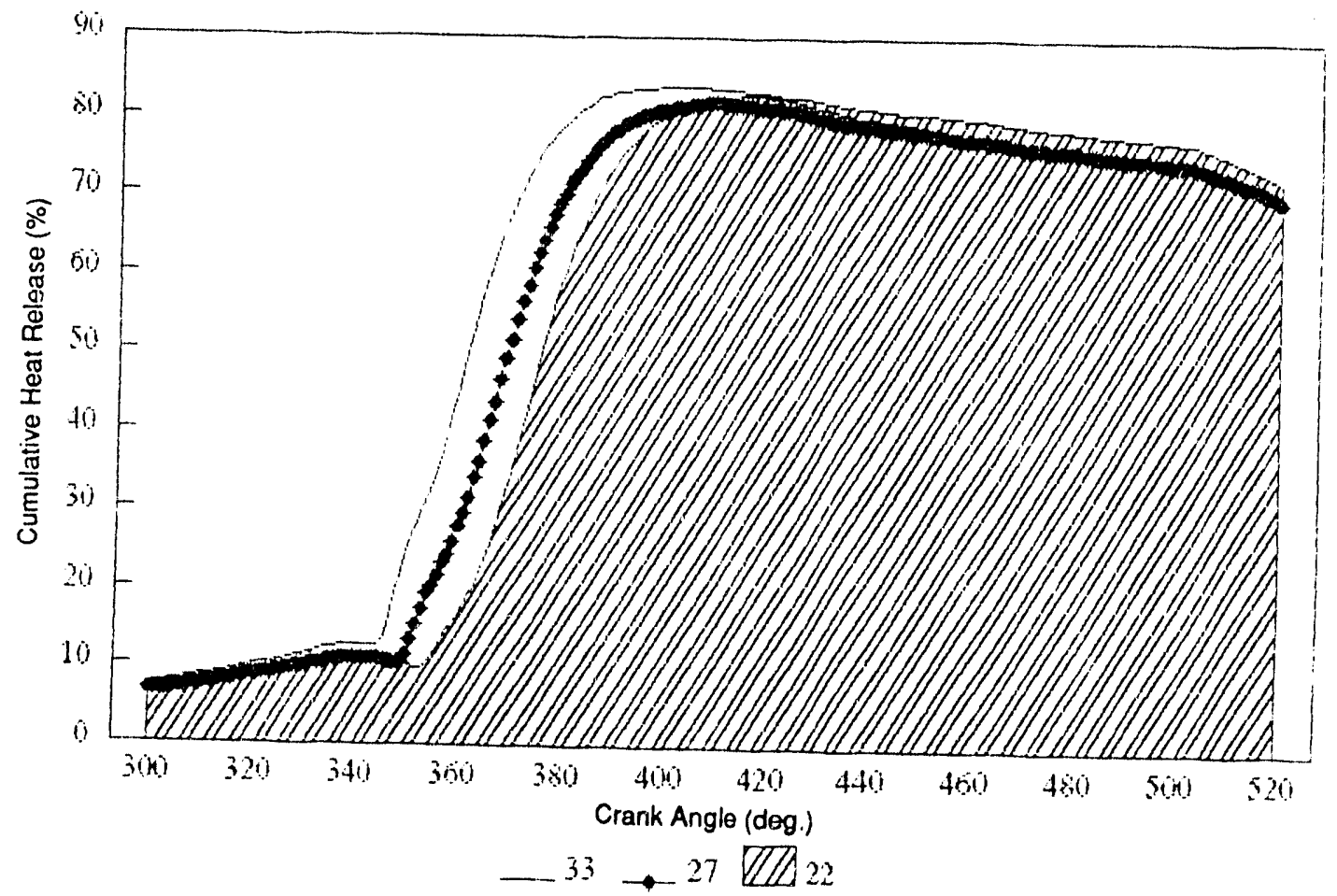

FIGURE B.156 Cumulative Heat Release, No. 4 Fuel, Full Load, 5\% Water, 25\% Oxygen 


\section{$B-119$}

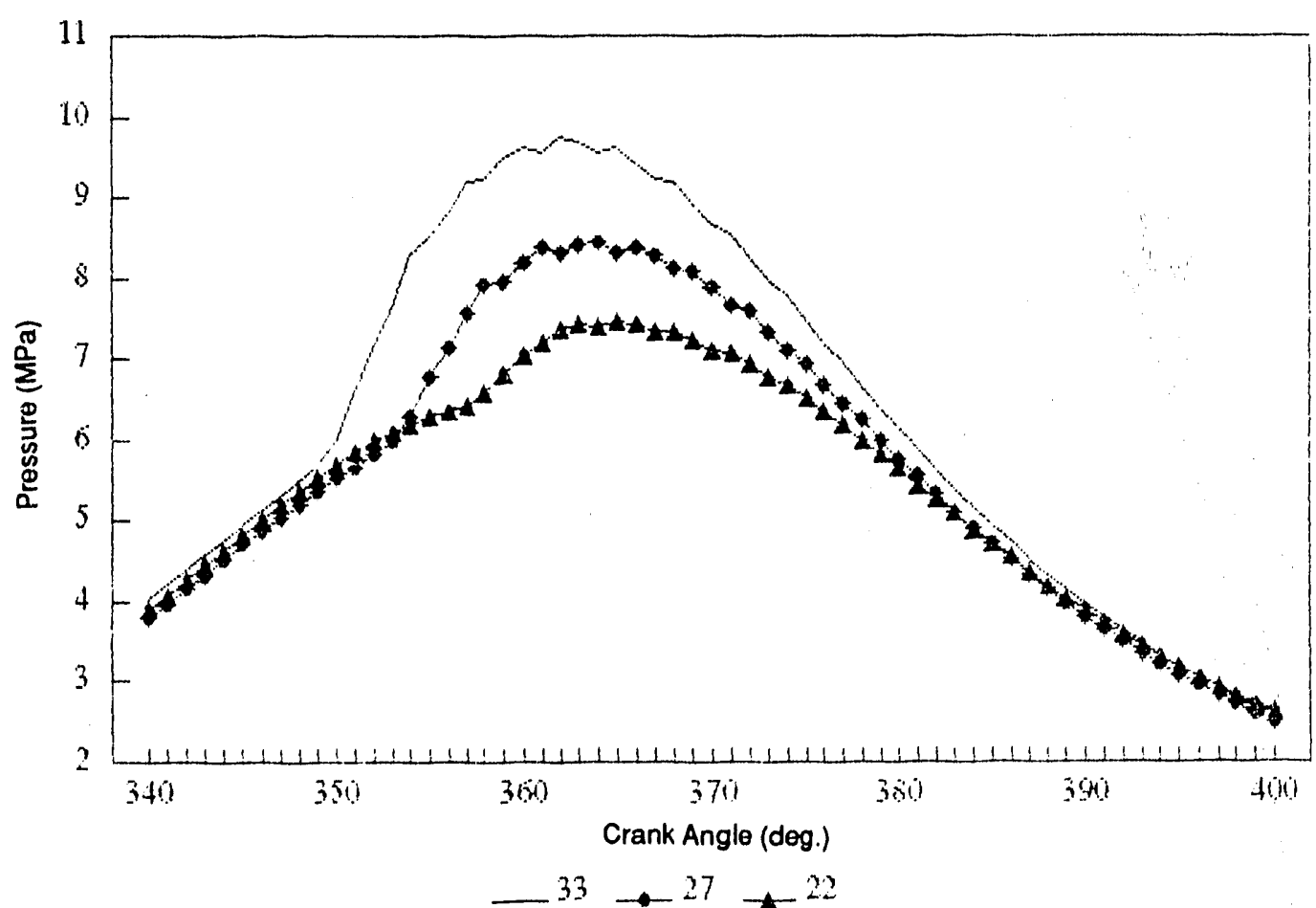

FIGURE B.157 Pressure, No. 4 Fuel, Half Load, 5\% Water, 21\% Oxygen

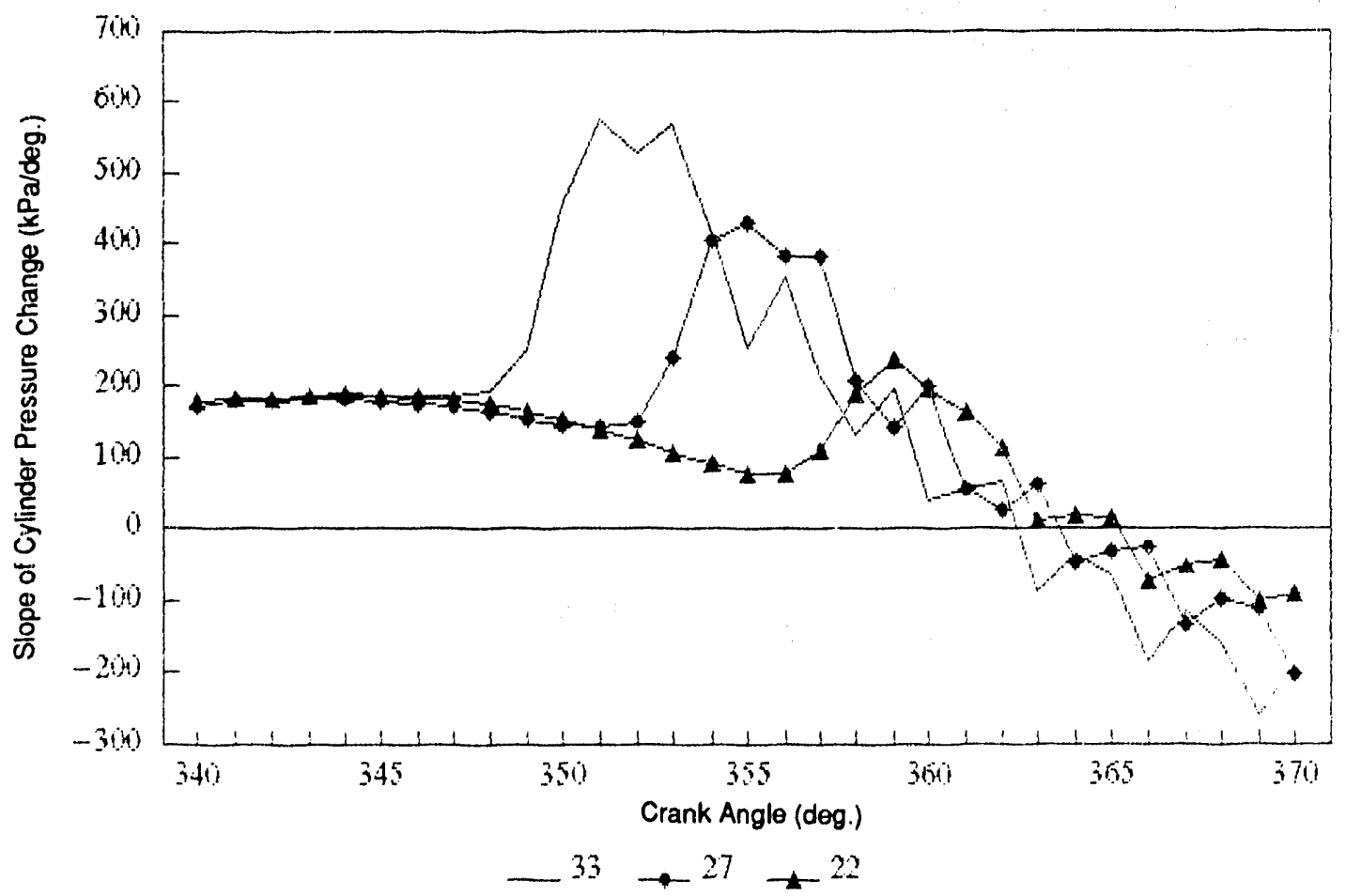

FIGURE B.158 Slope of Cylinder Pressure Change, No. 4 Fuel, Half Load, 5\% Water, $21 \%$ Oxygen 


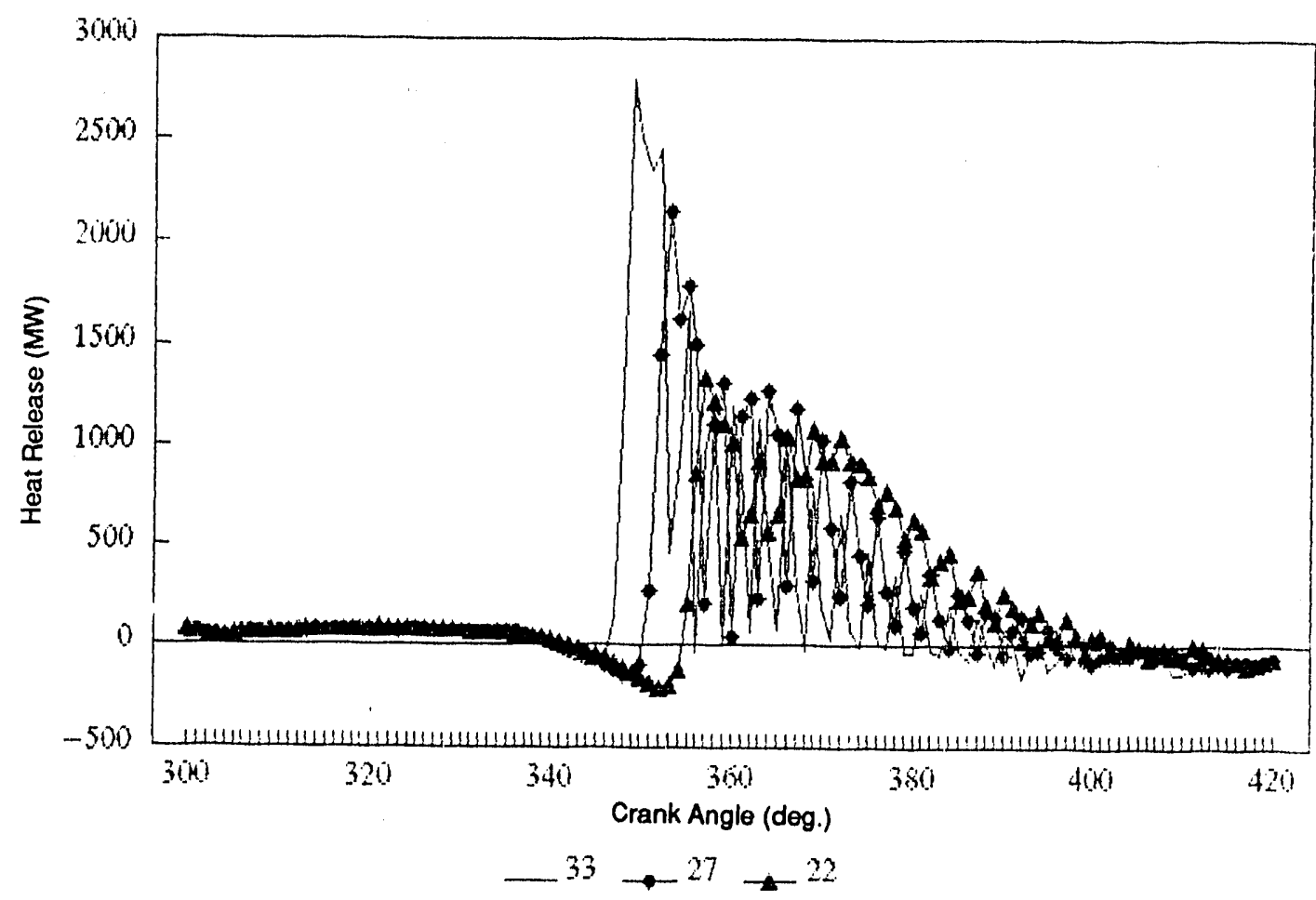

FIGURE B.159 Heat Release, No. 4 Fuel, Half Load, 5\% Water, 25\% Oxygen

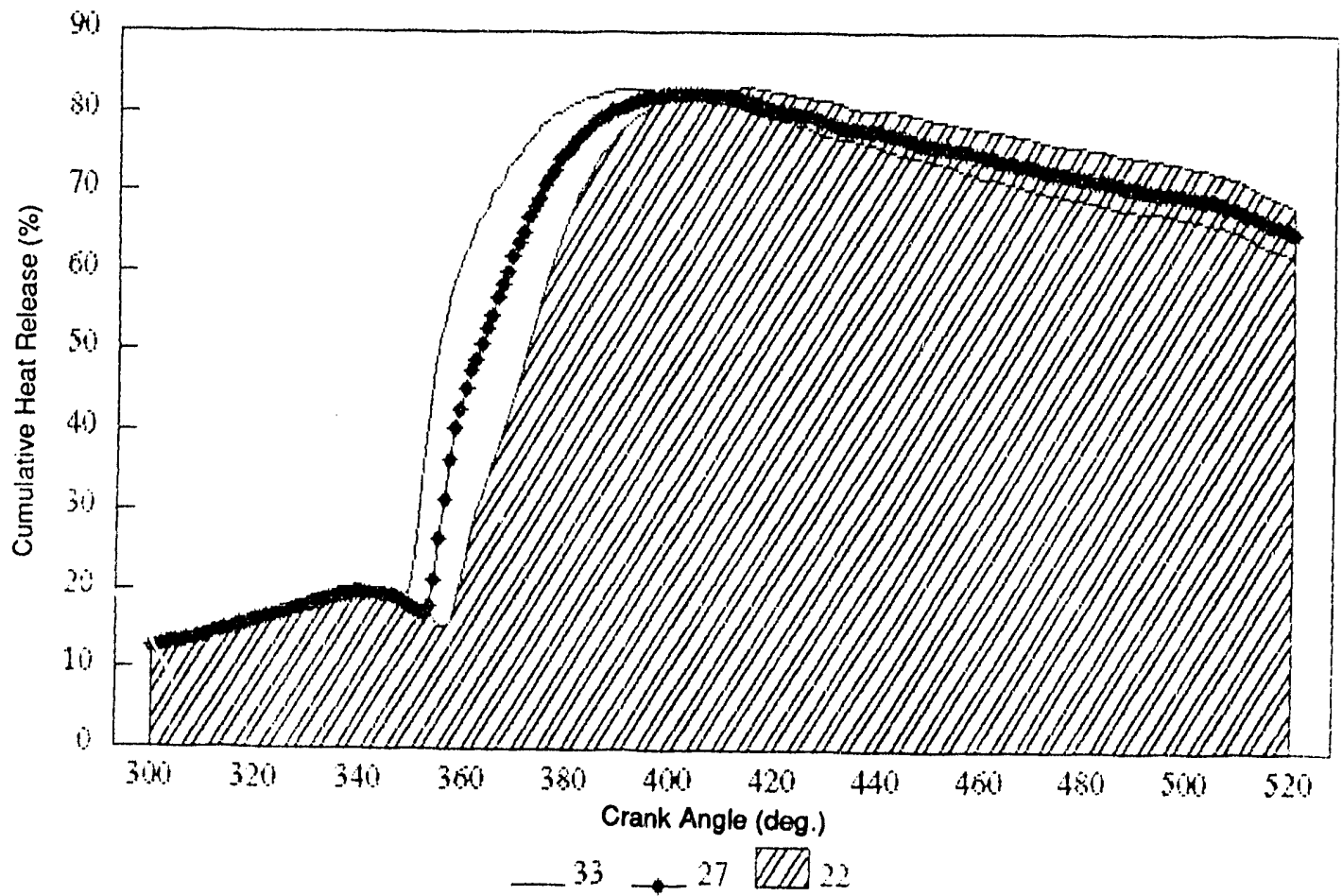

FIGURE B.160 Cumulative Heat Release, No. 4 Fuel, Half Load, 5\% Water, 21\% Oxygen 


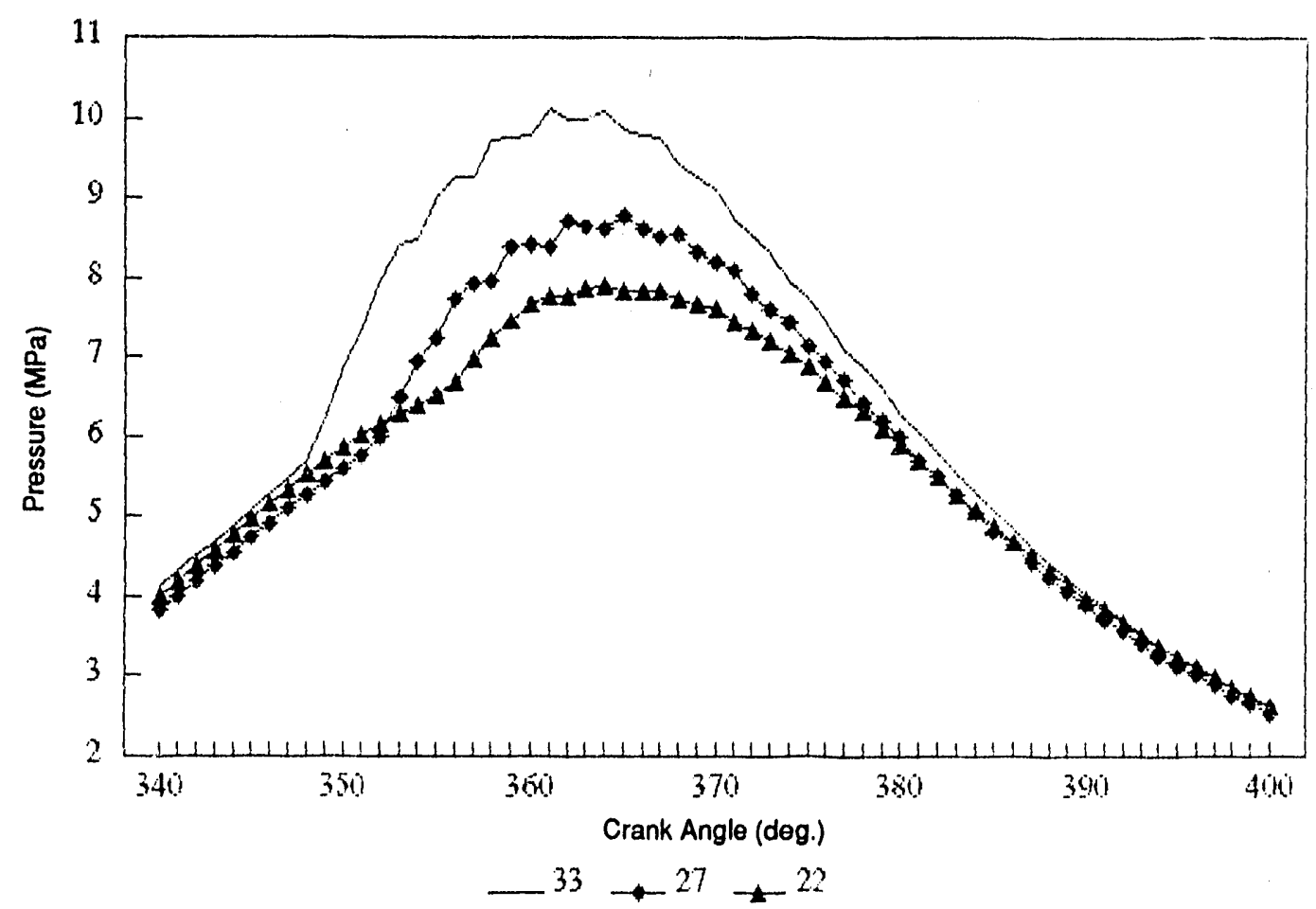

FIGURE B.161 Pressure, No. 4 Fuel, Half Load, 5\% Water, 25\% Oxygen

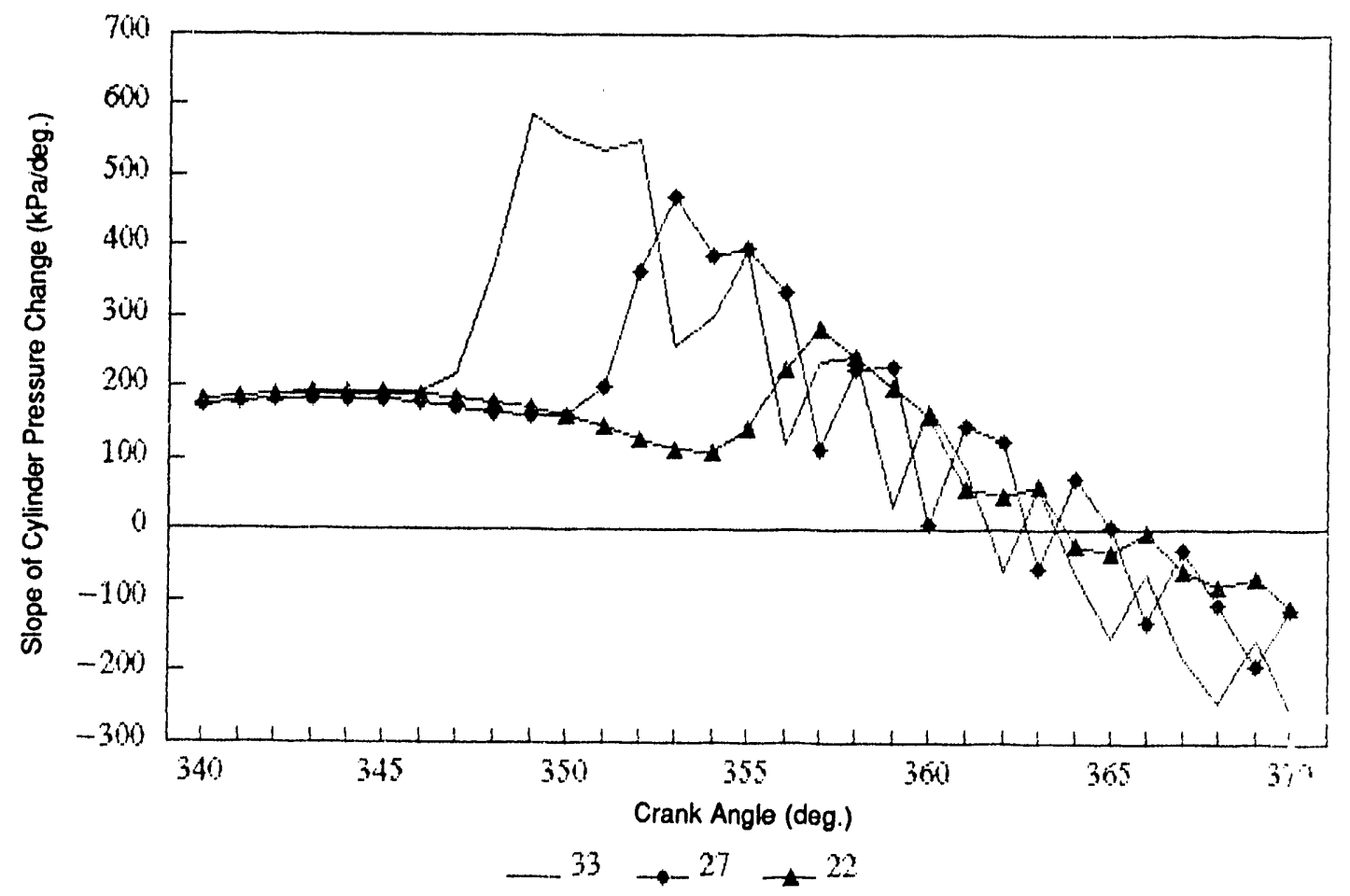

FIGURE B.162 Slope of Cylinder Pressure Change, No. 4 Fuel, Haff Load, 5\% Water, 25\% Oxygen 


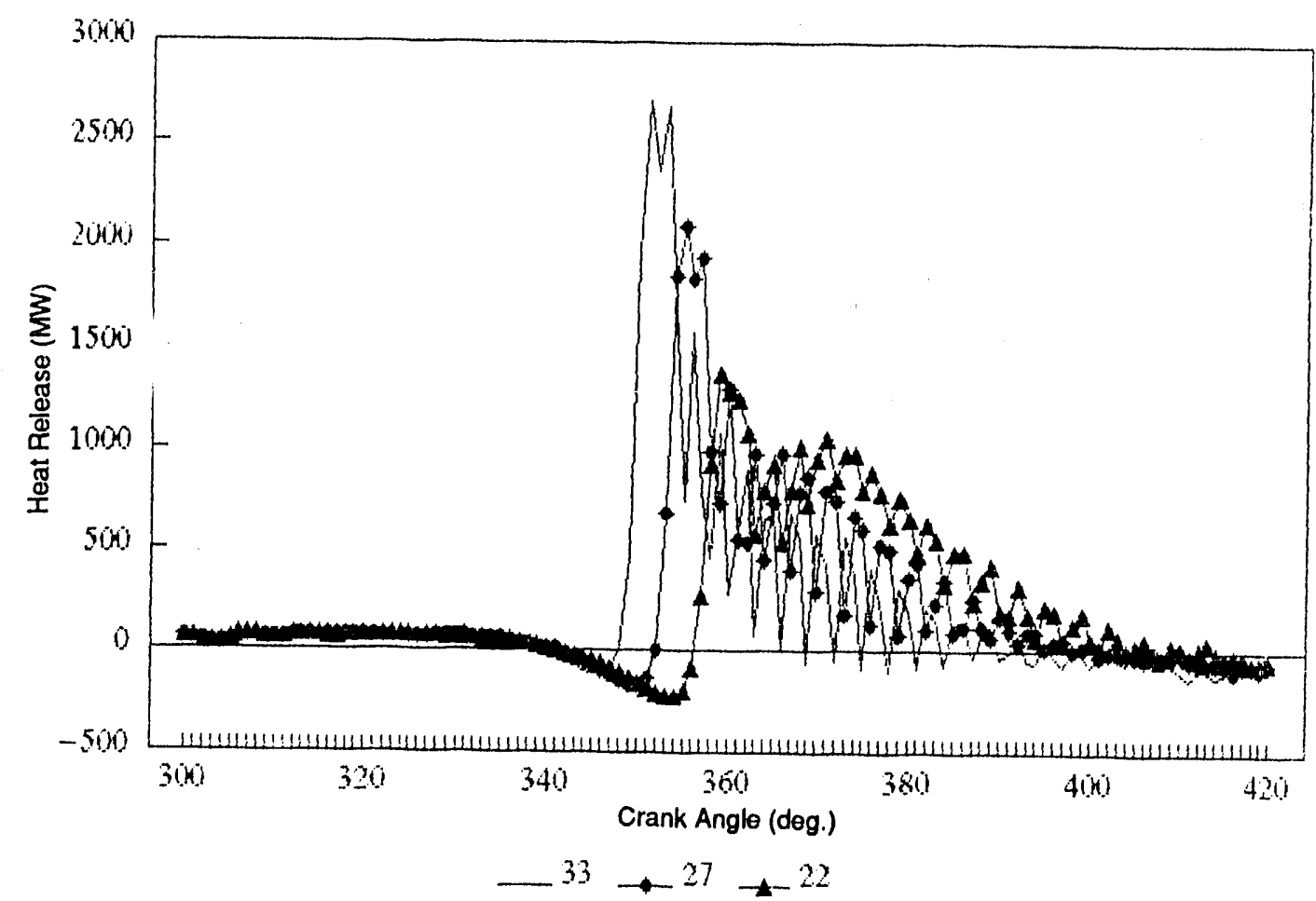

FIGURE B.163 Heat Release, No. 4 Fuel, Half Load, 5\% Water, $21 \%$ Oxygen

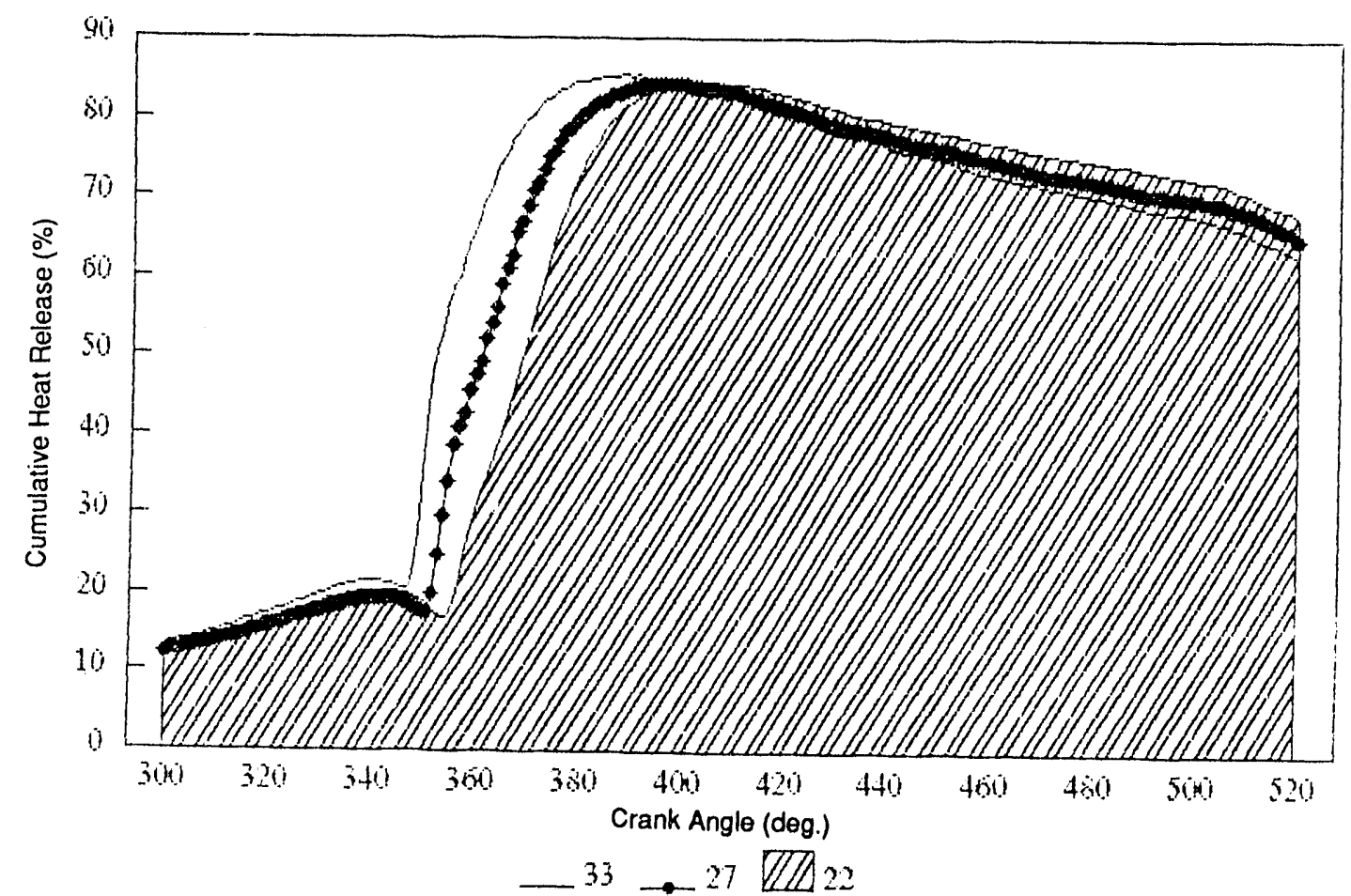

FIGURE B.164 Cumulative Heat Release, No. 4 Fuel, Half Load, 5\% Water, 25\% Oxygen 

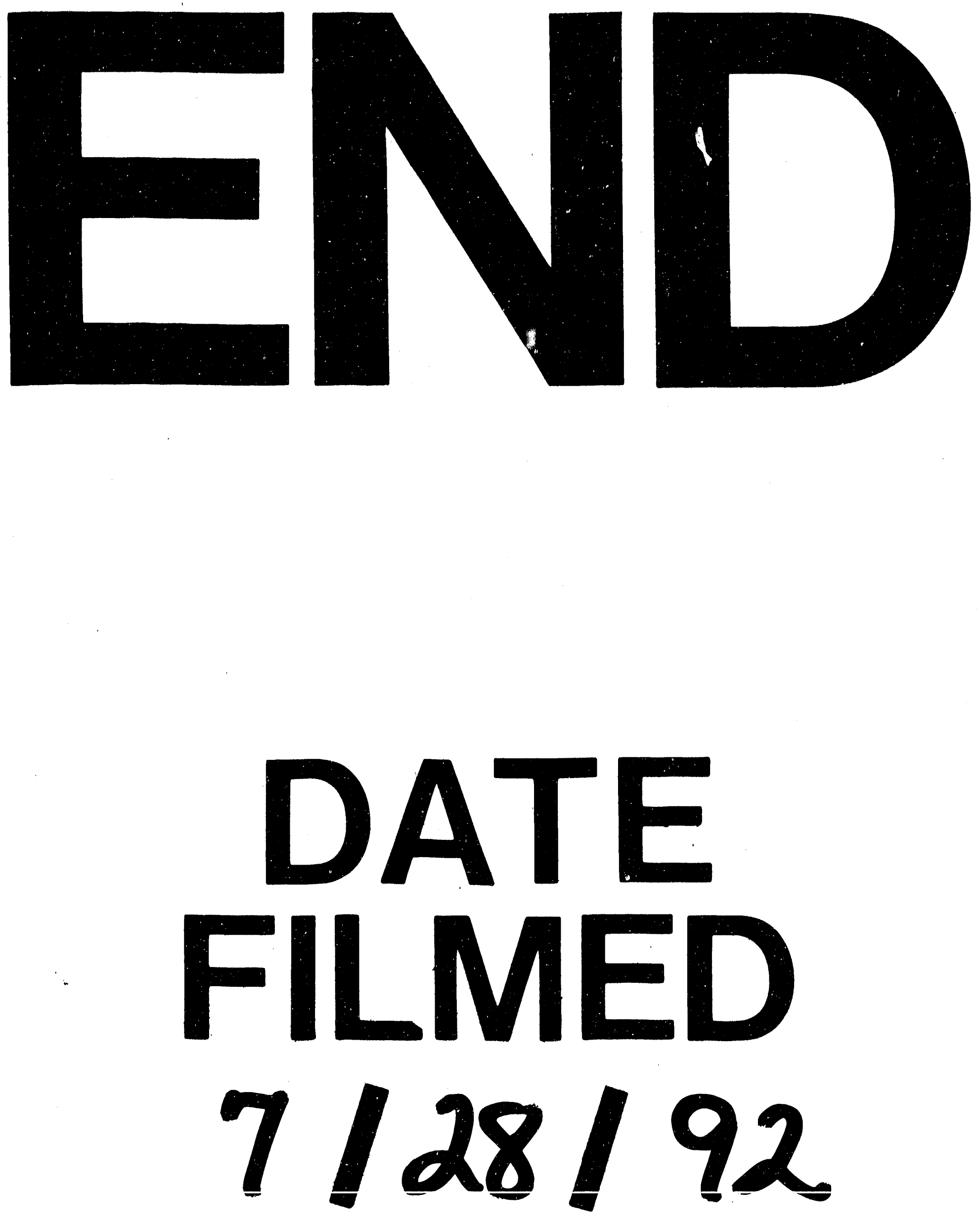
